\title{
When Genetic and Environmental Factors Meet in Modelling Autism
}

\author{
By
}

\section{Michaela Anne Pettie}

\author{
A Thesis \\ Submitted to Victoria University of Wellington \\ In Fulfilment of the Requirements for the Degree of \\ Doctor of Philosophy \\ in Psychology
}

2020 


\begin{abstract}
Autism Spectrum Disorder (ASD) is a neurodevelopmental disorder, characterised by deficits in verbal and non-verbal communication, social interaction and repetitive behaviours (APA, 2013). The aetiology of ASD is mostly unknown, with continued research identifying a multitude of genetic and non-genetic factors. However, it is the interaction between environmental factors and the genetic background of an individual which leads to the development of ASD. There is an urgent need for improved animal models of ASD to further our understanding of the aetiology and particularly its pathophysiology, as this will aid in the development of much needed pharmaceutical treatments to alleviate the impact of adverse symptoms for individuals with ASD. Current animal models of ASD examine the genetic (e.g. serotonin transporter knock out rats) or the environmental (e.g. prenatal exposure to Valproate) contributions to the disorder, and very rarely a combination of the two.

This thesis aimed to improve the Valproate (VPA) induced ASD animal model with a genetic $\times$ environmental interaction approach, as well as optimising chronic administration of the VPA to pregnant rats. To this aim, a non-invasive method of delivering VPA was used, which allowed genetically normal rats to voluntarily consume VPA throughout pregnancy. The prenatal exposure to VPA led to ASD-like behaviours in the offspring (communication delays, increased social behaviour, and social aversion). Next, rats with a genetic deficit in SERT $\left(\mathrm{SERT}^{+/-}\right)$exposed to VPA throughout gestation, with an optimised administration method using gelatine pellets, which allowed for voluntary non-invasive consumption, and a more accurate administration of increased VPA doses. Overall, the chronic prenatal exposure to VPA in $\mathrm{SERT}^{+/-}$rats led to a mild ASD-like phenotype, with rats exhibiting communication delays, abnormal play behaviour, disrupted social preference, and to some extent increased anxiety-like behaviour. The brains of the adult offspring were examined for neuronal changes in the GABA interneurons in brain regions associated with social behaviour (amygdala and hippocampus). However, no significant effects of prenatal VPA exposure, genotype, or sex were found. Thus, the variations GABAergic system is unlikely to underlie the earlier identified behavioural alterations. Ultimately, this thesis has furthered the VPA induced ASD animal model with a genetic $\times$ environmental interaction approach, as well as optimising the chronic administration method for pregnant rats.
\end{abstract}




\section{Acknowledgements}

I would like to express the deepest appreciation to Professor Bart Ellenbroek, for all his guidance, patience, support, and tireless answering of never-ending questions. Thank you for giving me the opportunities and the honour of working in the lab. These thanks are extended to Dr Jiun Youn, for her guidance on all things behavioural and for the alternative perspective. Also, thank you to Dr Joyce Colussi-Mas for all of your assistance and work on the tissue staining and brain extractions (and every other concern or question relating to lab life). I would have been lost without your guidance.

I would like to thank the Behavioural Neurogenetics lab, after being a member for five years, there are so many that have helped my journey. I am indebted to the students who have given their time and energy to me and my project. Thank you for your time, patience, and kind words. Thank you to lab members that have specifically contributed to this work, Meyrick Kidwell, Justine Michelat, Manon Dussol, and Lizzie Summers. Further thanks are extended to Sue Schenk and the Schenk lab (in particular Ross Van de Wetering, Quenten Highgate, \& Alex Mann), who offered their space and support. I would like to thank the animal technicians, Michael Roberts and Aimee Culverhouse, thank you for your unwavering willingness to help and respond to the needs of the animals and students.

Thank you to the postgraduate students that came before me; they forged a path that I was privileged to walk. In particular, thank you to Sophie Müller, she taught me so much relating to behavioural science, lab life, and friendship. Without her help, I would not be the person I am today.

On a personal note, thank you for never-ending support and love from my friends and family, especially to my mother, Karren Kerrisk, for her love, support, and for being there every step of the way. To my family (Treena \& Rob Haylock, Kim \& Glenn Lupo, Lorna \& Waz Hunter, Aaron Waugh), for always being in my corner, and for feeding me when the cupboards were empty. For Grace Walker, Jess Shaw, Emma Ashcroft, Caleb Stone, Jemma Sergent, Aimee (again), and Daniel Chaji, you have been some of my greatest supporters and the loudest voices when it became too difficult; this thesis would not have been possible without you. Finally, I would like to express my sincerest gratitude to my partner, Gareth Minke, for his acceptance and encouragement, the tail end would have been a lot more broken without him. 


\section{Complete Table of Contents}

Abstract

Acknowledgements .......................................................................................................................... V

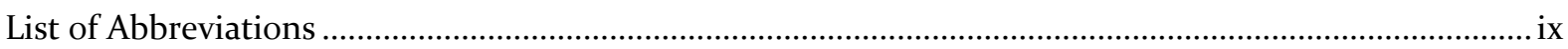

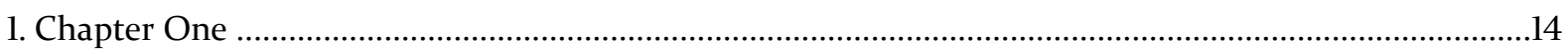

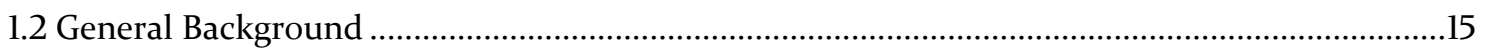

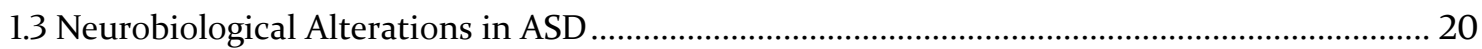

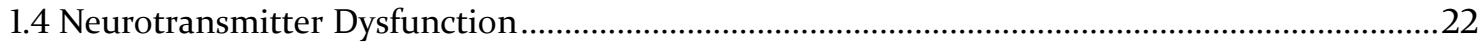

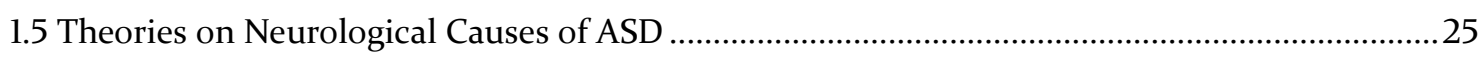

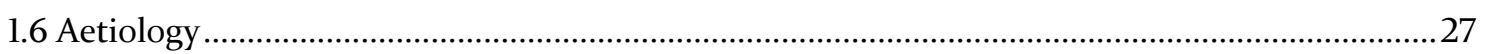

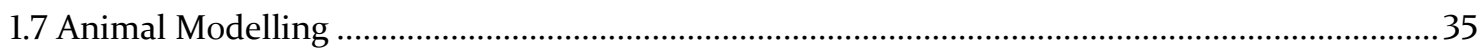

1.8 Environmental approach to Animal Models ...................................................................................

1.9 Chronic VPA Administration in Animals with Reduced SERT as an ASD Animal Model ..........44

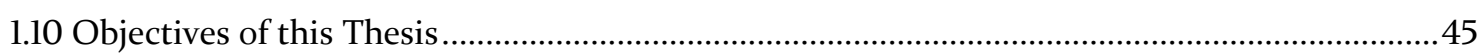

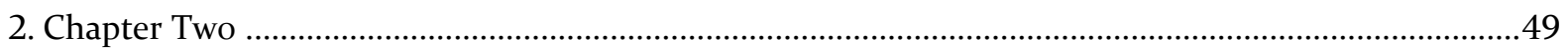

2.1.2 Overall Aims of Chapter Two ………………….....................................................

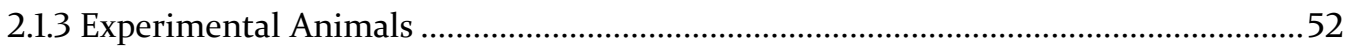

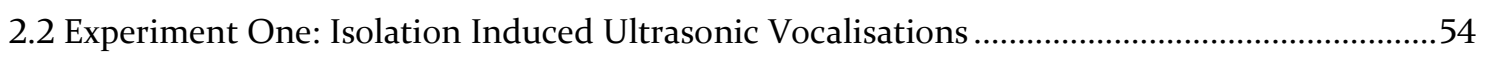

2.2.2 Isolation Induced USV methods .................................................................................56

2.2.3 Isolation Induced USV Results .................................................................................59

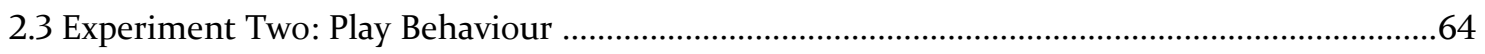

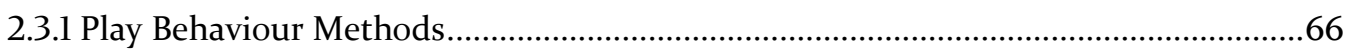

2.3.2 Play Behaviour Results ............................................................................................68

2.4 Experiment Three: Social Approach and Avoidance.................................................................. 73

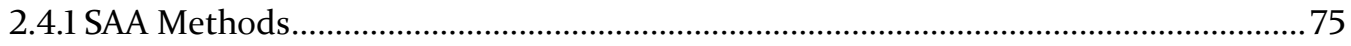

2.4.2 SAA Results for Pre-Pubescent Rats ............................................................................78

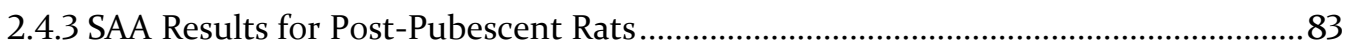

2.4.4 Pre- \& Post-Pubescent Comparison Results ……………………………………............

2.5 Results Summary Table ……………………….................................................................... 90

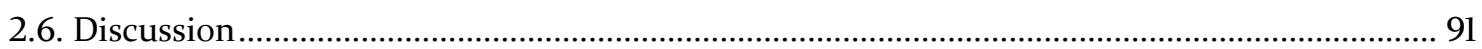

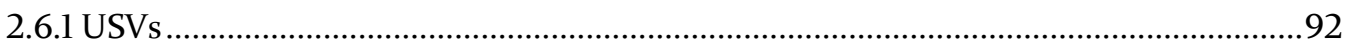

2.6.2 Play Behaviour......................................................................................................... 93

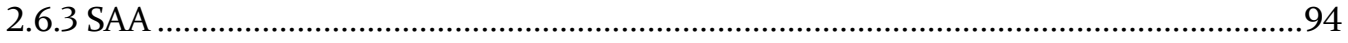

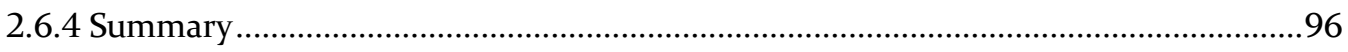

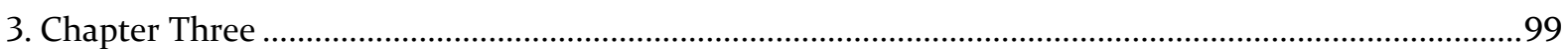

3.1.2 Overall Aims of Chapter three...............................................................................104

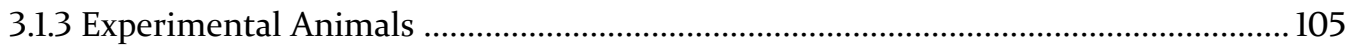

3.2 Experiment One: Isolation Induced Ultrasonic Vocalisations ..................................................... 109 
3.2.1 Isolation Induced USV methods .............................................................................110

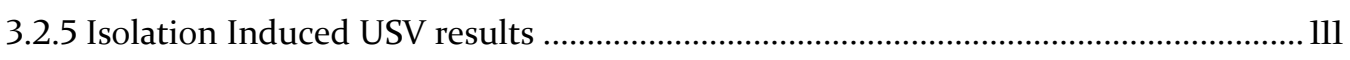

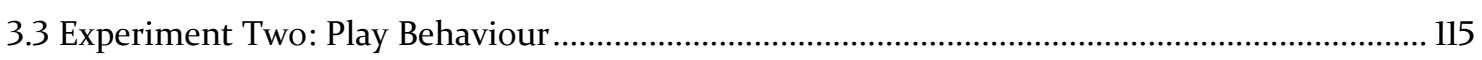

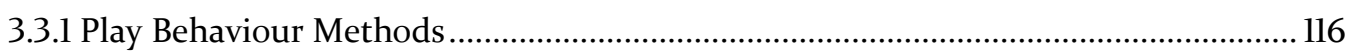

3.3.3 Play Behaviour Results ............................................................................................ 117

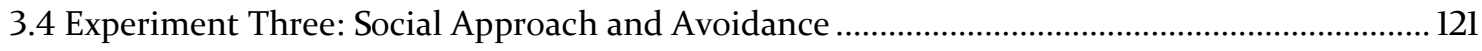

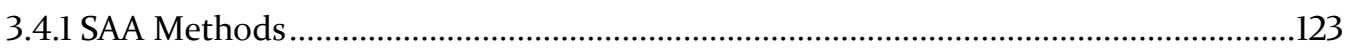

3.4.2 SAA Results for Pre-Pubescent Rats...........................................................................125

3.4.3 SAA Results for Post-Pubescent Rats ..........................................................................134

3.4.4 Pre- \& Post-Pubescent Comparison Results.................................................................143

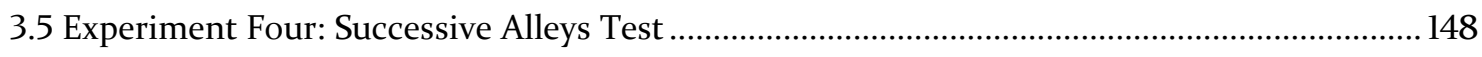

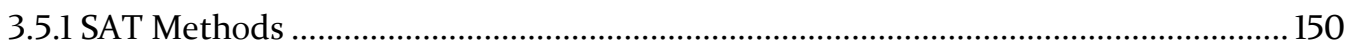

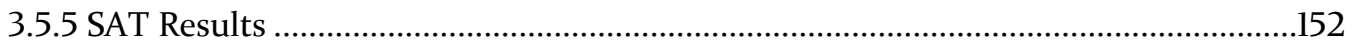

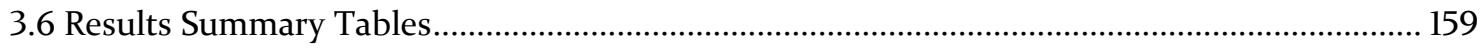

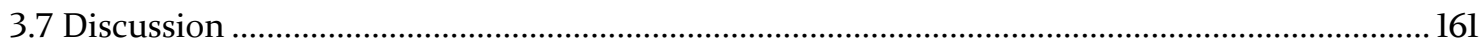

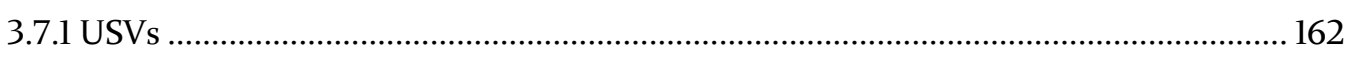

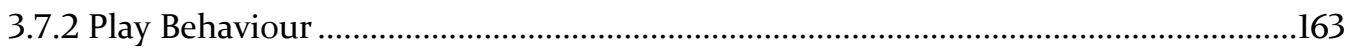

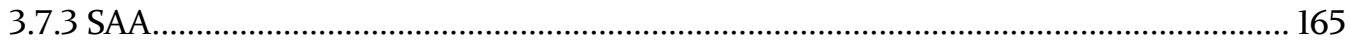

3.7.4 SAT …………………………………………………………………………167

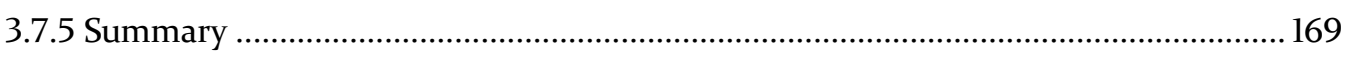

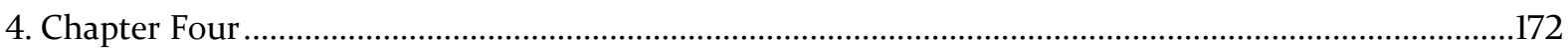

4.2 Parvalbumin Staining Methods ...............................................................................................177

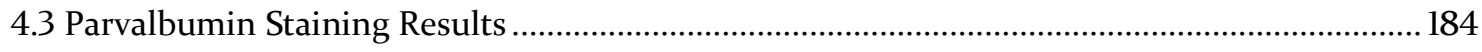

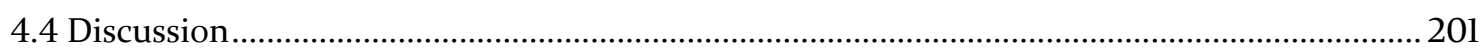

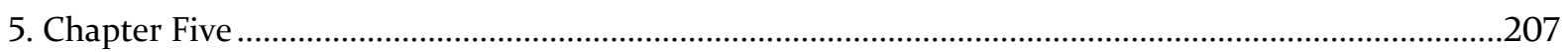

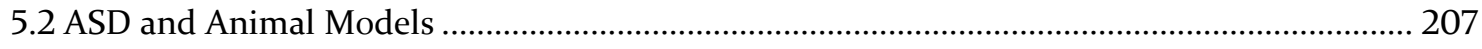

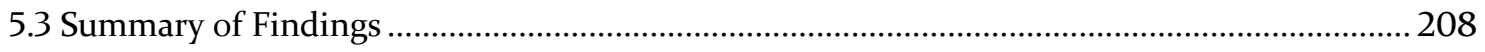

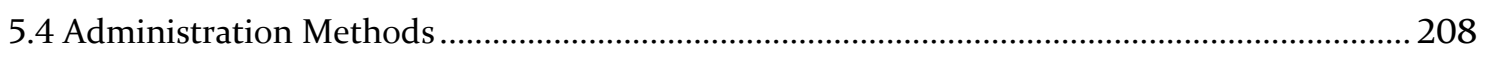

5.5 Gene $\times$ Environment Interaction Approach to the ASD animal model .................................... 210

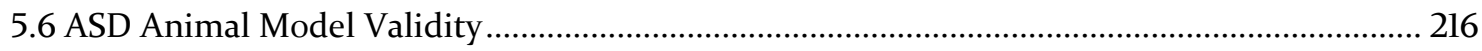

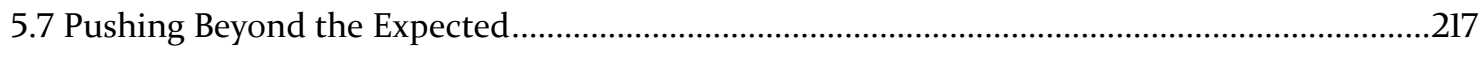

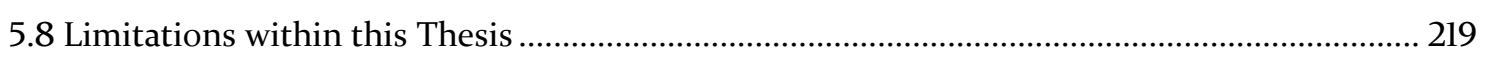

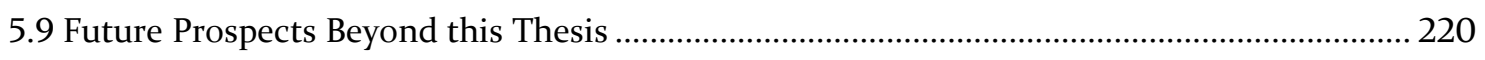

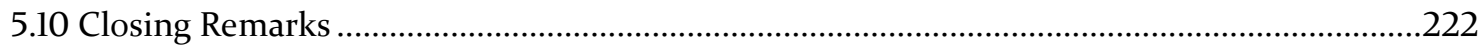

Appendix A - Information Relating to Chapter Three .............................................................................225

Appendix B - Information Relating to Chapter Four …………..........................................................229

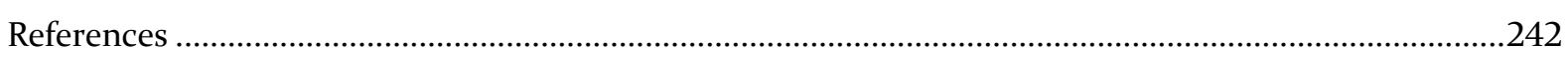




\section{List of Abbreviations}

5-HT 5-hydroxytryptamine, Serotonin

5-HTTLPR Serotonin Transporter Linked Polymorphic region

ADHD Attention Deficit Hyperactivity Disorder

ANOVA Analysis of Variance

ASD Autism Spectrum Disorder (also Autism)

APA American Psychological Association

CDC Centres for Disease Control and Prevention

CNS Central Nervous System

DNA Deoxyribonucleic acid

DSM Diagnostic and Statistical Manual of Mental Disorders

ENU N-ethyl-N-nitrosourea

E:I Excitation : Inhibition

EEG Electroencephalography

fMRI Functional Magnetic Resonance Imaging

GABA gamma-Aminobutyric acid, or $\gamma$-aminobutyric acid

GD Gestational day

GLU Glutamate

HDAC Histone deacetylase

IP Intraperitoneal

IQ Intelligence Quotient

$\mathrm{kHz} \quad$ kilohertz

L-allele 'Long' allele of the 5-HTTLPR

LPS Lipopolysaccharides

MIA Maternal immune activation

MRI Magnetic Resonance Imaging

mRNA Messenger ribonucleic acid

ns Non-significant

PND Postnatal day

PNS Peripheral Nervous System 
Poly I:C Polyinosinic: polycytidylic acid

PV Parvalbumin

PV+ Parvalbumin-positive (parvalbumin-expressing)

S-allele 'Short' allele of the 5-HTTLPR

SAA Social Approach and Avoidance

SAT Successive Alleys Test

SC Subcutaneous Injection

SCM Sweetened Condensed Milk

SERT Serotonin Reuptake Transporter

SERT $^{+/+} \quad$ Wildtype genotype of the SERT Knockout animals

SERT $^{+/-} \quad$ Heterozygous genotype of the SERT Knockout animals

SERT $^{-/-} \quad$ Homozygous genotype of the SERT Knockout animals

SLC6A4 Serotonin transporter gene

SPSS Statistical Package for the Social Sciences

SSRI Selective serotonin reuptake inhibitor

RNA Ribonucleic acid

USV Ultrasonic Vocalisation

VPA Valproic acid, valproate, or 2-propylpentanoic acid 


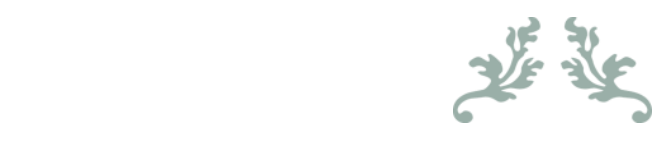

Chapter One

General Introduction

20 


\section{Chapter One Contents}

1.1 Introduction to Chapter One...........................................................................................................

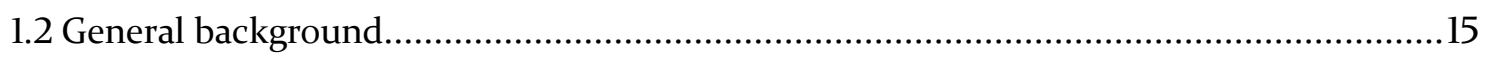

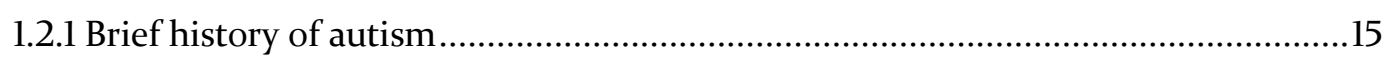

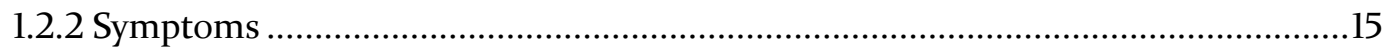

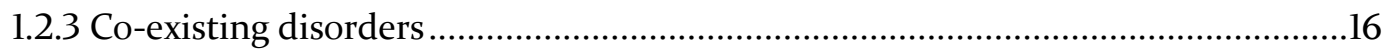

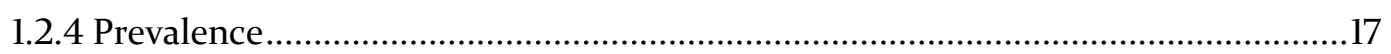

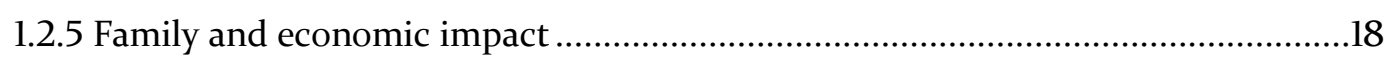

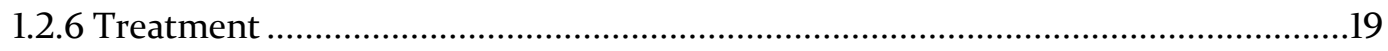

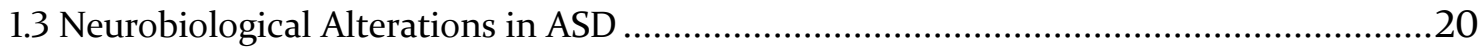

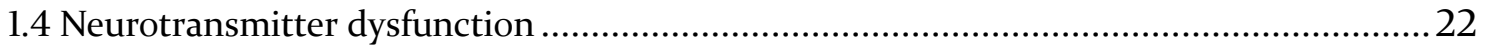

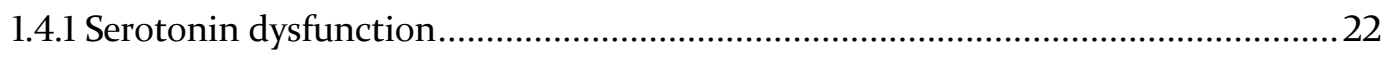

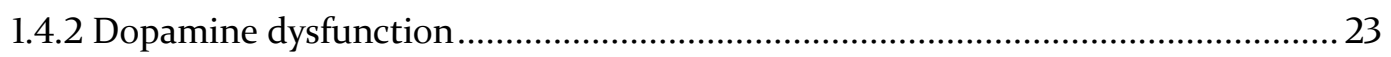

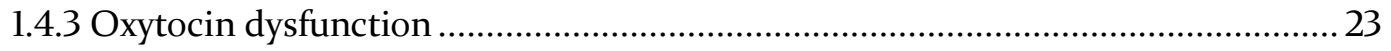

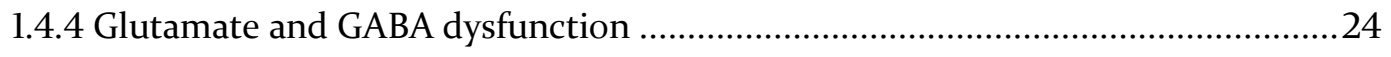

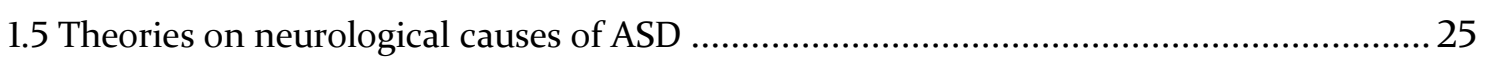

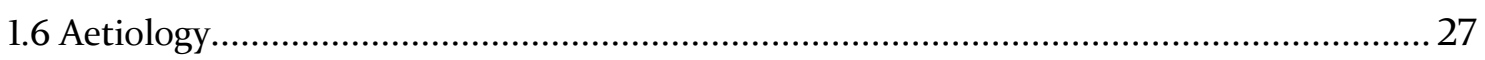

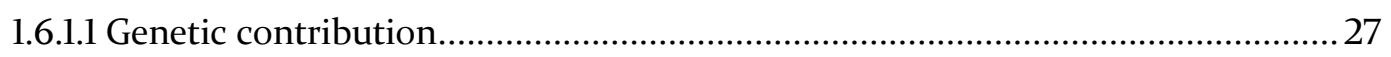

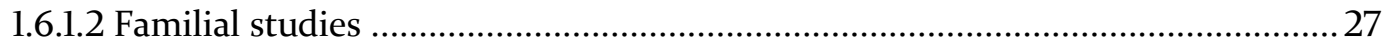

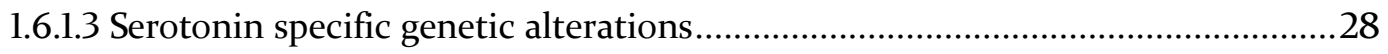

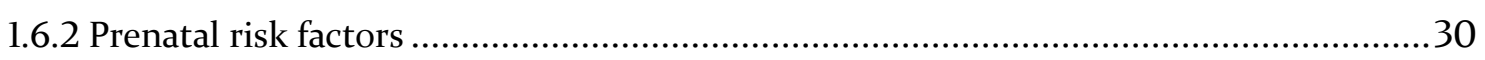

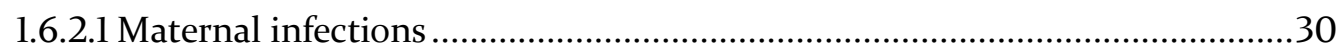

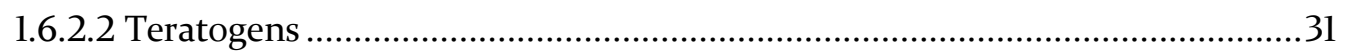

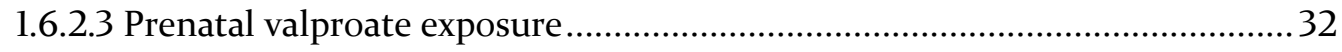

1.6.2.4 Genetic and environmental interactions................................................... 33

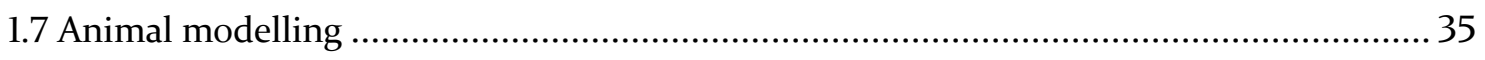

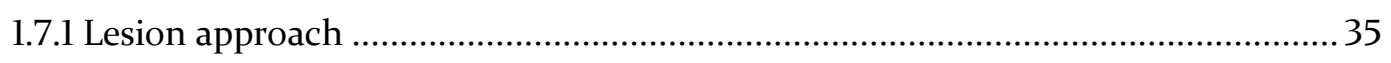

1.7.2 Genetic approach to animal models ………........................................................ 36

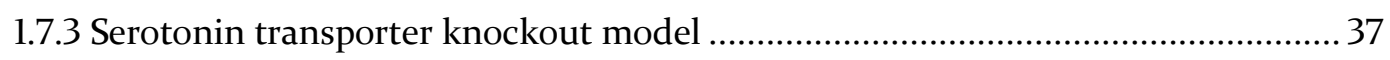

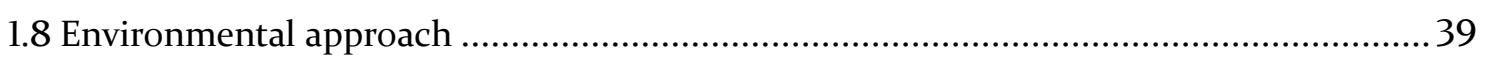

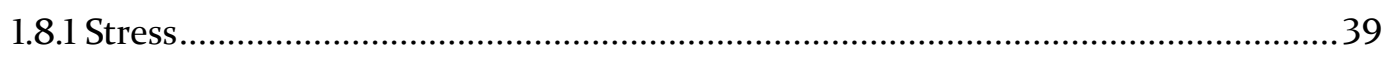

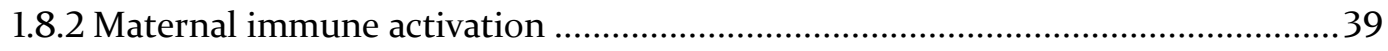

1.8.3 VPA-induced ASD model .................................................................................... 40

1.8.3.1 Effects of single prenatal exposure to VPA .......................................................41

1.8.3.2 Effects of multiple prenatal exposures to VPA................................................42

1.9 Chronic VPA administration in animals with reduced SERT as an ASD model ................44

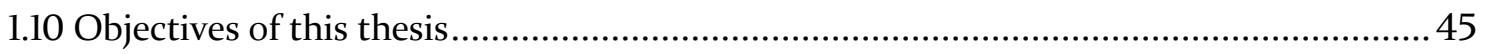




\section{Chapter One}

Autism Spectrum Disorder (ASD) is a complex disorder with multiple behavioural and cognitive abnormalities, which are clinically present from childhood. The Diagnostic and Statistical Manual of Mental Disorders (fifth edition; DSM-5; American Psychological Association [APA], 2013) classifies ASD as a neurodevelopmental disorder. ASD is characterised by pervasive deficits in verbal and non-verbal communication, social interaction, and repetitive and stereotyped behaviours (APA, 2013). One in 59 children had the diagnosis before their eighth birthday (based on 2014 data collection in the United States, Baio et al., 2018). ASD has a substantial lifelong impact, yet the aetiology is mostly unknown. Although the cause of this disorder is not well delineated, adverse environmental influences, genetic factors, and gene $\times$ environment interactions significantly increase an individual's risk of developing ASD. While the symptoms and predisposing factors are becoming more apparent, effective treatment for ASD is yet to be developed (Belzung, Leman, Vourc'h, \& Andres, 2005). It is essential that we further our understanding of the aetiology of ASD, to allow for accurate detection of the disorder, as well as developing targeted pharmacological, and more efficient behavioural treatment options. However, delineating ASD aetiology in humans is difficult due to the heterogeneous nature of the disorder, as well as the complexity of multi-gene and multienvironmental influences. The use of in vivo animal experiments is an important alternative for investigating the genetic and environmental influences that underlie ASD symptoms. However, the progression of an animal model of ASD is hindered due to the limited clinical research which considers gene $\times$ environment interactions along with substantial validity concerns. The work in this thesis intends to advance and explore the validity of the animal model of ASD. 


\subsection{General Background}

\subsubsection{Brief History of Autism}

The pattern of behavioural variation identified as autism has been documented as early as 1938. Kanner (1943) observed the characteristics of eleven children with behavioural abnormalities. The eleven children displayed similar characteristics of repetitive and obsessive behaviours, abnormalities in social interaction, and language development, as well as difficulties with eating, aloofness and sensitivity to loud noises. Kanner (1943) assembled these characteristics into one syndrome, which was further developed by Hans Asperger (1944). Asperger described a 'reduced' form of autism, which characterises the way children had difficulty with social communication and some obsessive or fixated behaviours (Wing, 1981). Asperger (1944) originally described the syndrome as Autistic Psychopathy, but later it became known as Asperger's syndrome. Autism first appeared in the third edition of the DSM (1980) as Infantile Autism, within the category of pervasive developmental disorders (Volkmar \& Reichow, 2013). It was not until 1994 in DSM-4, that it appeared as an individual disorder in the DSM. With the DSM-5 (2013), autism became classified as a spectrum of disorders without division between Autism, Asperger's, and other subtypes. The inclusion of all forms of the disorder into a spectrum was due to studies showing little to no difference between Autism and Asperger's (Macintosh \& Dissanayake, 2004; Sharma, Woolfson, \& Hunter, 2012), along with evidence suggesting these disorders had the same aetiology (Young \& Rodi, 2014). Overall, ASD is a neurodevelopmental disorder and a spectrum of symptoms ranging in severity and impact.

\subsubsection{Symptoms}

ASD is a heterogeneous disorder; the symptoms and impact depend on the individual, developmental stage, age, and the addition of any co-existing disorders. ASD symptoms are typically identified at a young age when children are yet to reach their developmental milestones characteristic for their age (Gabrielsen et al., 2015). These children are often observed as having significant delays in language development, accompanied by a reduced interest in social interaction (APA, 2013; Baird, Douglas, \& Murphy, 2011). In comparison to their same-age peers, children with ASD are likely to have difficulties with initiating or responding to social cues or verbal instructions, repeat words or songs (echolalia; APA, 2013; Baird et al., 201l; Kanner, 1943), and have difficulties with reciprocal conversation (Koegel, Park, \& Koegel, 2014). The reductions in social interest are manifested by difficulty initiating and maintaining eye contact, failure to follow gestures or eye gaze, preference to play alone, and 
physically directing others to objects rather than telling them (e.g. pulling a parents' hand to the object rather than using language to identify the object; Sigman, Mundy, Sherman, \& Ungerer, 1986).

The deficits in communication and social interaction are combined with repetitive behaviours and interests. The behaviours can be grouped into two different categories; the 'lower level' behaviours are simple and typically identified by the repetition of body movements such as body rocking, hand flapping, repetitive object manipulation and self-injurious behaviour (Watt, Wetherby, Barber, \& Morgan, 2008). 'Higher level' behaviours, on the other hand, are typically more complex repetitions which involve insistence on sameness, order, object attachment, and fixation, and also extend to compulsions and ritualistic behaviour (Watt et al., 2008).

Symptoms of ASD change with age, however, the symptoms are still identified by the core characteristics. In adulthood, individuals with ASD are likely to experience deficits related to social-emotional reciprocity, which includes difficulties maintaining back and forth conversation, initiating and joining conversations, responding to gestures, and facial and body language cues (APA, 2013; Baird et al., 2011). An adult with ASD is more likely to have higher level repetitive behaviours and interests (Watt et al., 2008). While this description of symptoms is not exhaustive, it is indicative of the practical impact and appearance of ASD. Ultimately, ASD symptoms across the lifespan have major practical implications, which can significantly impact relationships with others, learning, independent living, employment, and life satisfaction.

\subsubsection{Co-existing Disorders}

ASD is rarely a disorder on its own and is often compounded by other disorders involving cognitive and emotional processing. This makes clear separations in symptoms between ASD and co-existing disorders difficult, which in turn impacts treatment and intervention options (Matson \& Shoemaker, 2009). The most common co-existing disorder with ASD is an intellectual disability, which is characterised by deficits in intellectual functions (such as, reasoning, abstract thought, judgement, and learning) and deficits in adaptive functioning to enable personal independence (such as, communication, social participation, independent living; APA, 2013). Furthermore, the DSM-V recommends that intellectual ability is assessed when assessing an individual for ASD (APA, 2013), as intellectual ability influences performance on psychological assessments. In 2004, the Centers for Disease Control and Prevention (CDC) examined 8-year-old children with ASD across seven areas in the United States (US) and ascertained that $38 \%$ of children with ASD also had an intellectual disability 
(with an Intelligence Quotient [IQ] below 70; Baio et al., 2018). They also found that a further $24 \%$ of the sample had low-to-impairing intellectual ability (IQ between 70 and 85 ). The high co-existence between ASD and intellectual disability has been identified by other researchers as well (La Malfa, Lassi, Bertelli, Salvini, \& Placidi, 2004). The high co-existence of these disorders is indicative of biological underpinnings that give rise to these disorders.

There is also a high rate of comorbidity between ASD and attention-deficit/hyperactivity disorder (ADHD), anxiety, and depression (Simonoff et al., 2008), as well as the co-existence of ASD and epilepsy. Simonoff et al. (2008) examined the co-existence of disorders for 112 children who were diagnosed with ASD (although at the time it was childhood autism and pervasive developmental disorders). The authors showed that $70 \%$ of the children had at least one coexisting disorder, and $41 \%$ had two or more. Furthermore, the co-existence between ASD and epilepsy has been reported in a range from $5 \%$ to $27 \%$, depending on the classifications of ASD and the seizures (Amiet et al., 2008; Jeste \& Tuchman, 2015). Matsuo, Maeda, Sasaki, Ishii, and Hamasaki (2010) examined the cases of 519 patients who had their first seizure before 18 years of age and identified that 79 (15.2\%) also had ASD. It is useful to bear in mind the co-existing disorders, and the potential impact of these disorders on in vivo models, as inducing ASD-like behaviours may induce anxiety- or depression-like behaviours. However, this thesis will be centred on the core behavioural symptoms of ASD (deficits in communication, social interaction, and repetitive behaviours), and efforts will be taken to acknowledge the co-existing disorders.

\subsubsection{Prevalence}

ASD affects about 1 to $2 \%$ of the general population (Baird et al., 2006; Baxter et al., 2015; Christensen et al., 2016). In 2014, the CDC updated the prevalence of ASD in 8-year-old children in the United States (US), showing that prevalence had increased by 15\% from 2012. The authors specify that the overall prevalence of ASD for 8-year-olds in 2014 was one in 59 children (Baio et al., 2018), compared with previous rates of one in 68 in 2010 (Wingate et al., 2014). It is unclear if the increase in ASD prevalence can be attributed to changes in diagnostic criteria, increased awareness of symptoms, changes in methodology, or an actual increase in the frequency of the ASD (APA, 2013). Furthermore, there are distinctive sex differences, as males are 4 to 5 times more likely to be diagnosed with ASD than females (Wingate et al., 2014). The 4 to 1 male to female ratio has remained consistent as of the 2014 prevalence update (Baio et al., 2018). This ratio has been replicated in other populations, including Korean (Kim et al., 2011) and Swedish populations (Gillberg, Cederlund, Lamberg, \& Zeijlon, 2006). Moreover, males and 
females with ASD tend to express ASD symptoms differently (Cridland, Jones, Caputi, \& Magee, 2014). Females tend to express more 'passive-like' symptoms, such as social aloofness, anxiety, and emotion regulation deficits (Mandy et al., 2012; Rynkiewicz \& Łucka, 2015). On the other hand, males tend to express more disruptive behaviours, such as social interaction deficits, repetitive behaviours and hyperactivity (Mandy et al., 2012; Szatmari et al., 2012). Thus, the disruptive nature of symptoms expressed by males likely contributes to the increased male representation in the prevalence of ASD. Furthermore, little attention is directed to the differential presentation of ASD symptoms for males and females. Much more research is needed to explore the difference developmentally for gender-identities other than male, as this would enable treatment advances.

\subsubsection{Family and Economic Impacts}

ASD symptoms are present from an early age and are typically stable throughout the life span. The longevity of the disorder affects the individual's life path (relationships, employment, and life quality), and these individuals require additional assistance and resources throughout their life. Horlin, Falkmer, Parsons, Albrecht, and Falkmer (2014) identified that the cost of ASD to an Australian family is approximately $\$ 34,900$ (AUD, per year). While the cost of ASD was greatly associated with the loss of income, delays in diagnosis also led to significant financial burdens. Individuals diagnosed with ASD often have a lower quality of life (van Heijst \& Geurts, 2015), and are less likely to engage intimately with others (Farley et al., 2018; Humphrey \& Symes, 2010). Using self-reported data, Gotham et al. (2015) showed that adults with ASD are less likely to be employed. The impacts of ASD are also experienced by family members who tend to work less and earn less over their lifespan due to the increased care required for children diagnosed with ASD (Cidav, Marcus, \& Mandell, 2012). Vasilopoulou and Nisbet (2016) showed in a meta-analysis that parents, particularly mothers of children diagnosed with ASD, experienced a lower quality of life. This was also found in a Swedish sample, whereby Järbrink (2007) showed that it takes about 1000 hours per year to care for a child with ASD.

Outside of the individual and their family, the cost of ASD impacts economic, health, and education systems. Buescher, Cidav, Knapp, and Mandell (2014) have identified that the lifetime cost for an individual with ASD was USD 1.4 million in the US, and GBP 0.92 million the United Kingdom (based on 2012 estimates). This cost was considered across multiple life domains; in childhood, the greatest cost was associated with special education and parental income loss, whereas in adulthood the cost was with supported living and the individual's income loss. Based on prevalence rates and associated factors, Leigh and Du (2015) forecasted 
the ASD related costs would rise to USD 450 billion by 2025 due to the increasing number of diagnoses. Furthermore, the global burden of disease for ASD is much greater than other neurodevelopmental disorders, such as ADHD, intellectual disability or foetal alcohol syndrome (Whiteford et al., 2013). Overall, ASD has major economic and societal cost, and the individuals with ASD and their families tend to bear the greatest burden. The broader impacts of ASD emphasise the need for early interventions or treatment options, which would reduce the aforementioned losses.

\subsubsection{Treatment}

Currently, there is no validated or recommended pharmacological treatment for the core symptoms of the disorder (deficits in communication, social interaction and repetitive behaviours). A small percentage of individuals with ASD experience relief from secondgeneration antipsychotics, which help to reduce some of the co-existing symptoms, like irritability, hyperactivity or mood instability (LeClerc \& Easley, 2015). There are two antipsychotics approved by the US Food and Drug Administration (FDA): risperidone and aripiprazole. New Zealand's Ministry of Health permits prescription of risperidone for individuals experiencing irritability, aggression, self-injurious behaviour and severe tantrums (Ministry of Health, 2011). Other options include psychostimulants, like methylphenidate or anti-depressants, such as Selective Serotonin Reuptake Inhibitors (SSRIs; Stepanova, Dowling, Phelps, \& Findling, 2017). While these medications may be given to alleviate symptoms of coexisting disorders, such as ADHD or depression, they do not adequately treat the core symptoms of ASD. Therefore, the current treatment of ASD symptoms rests on behavioural interventions which are effective when implemented at an early age (Stepanova et al., 2017). However, interventions require ongoing enhancements to coincide with an individual's cognitive growth. Ultimately, until new treatment options are developed, individuals with ASD are unlikely to have substantial relief from their symptoms.

While the symptomology and the impacts of ASD have been understood for some time, there are no reliable methods for preventing this disorder. As such, it is important to investigate the contributing factors like genes and the environment and the role they have in ASD, as this will inform methods and avenues for ameliorating ASD symptoms. Furthermore, the aetiology of ASD is not attributable to one source, which makes it likely that it is caused by multiple factors. This chapter will explore the aetiology of ASD and the contributing factors to the disorder; but first, an exploration into the neurobiology of ASD and related theories, as it elucidates the symptoms experienced by individuals. 


\subsection{Neurobiological Alterations in ASD}

ASD is diagnosed solely on behaviours, however, it has been well established that neuronal and biochemical alterations underlie ASD symptoms. Currently, there are no biological or physical markers to diagnose or supplement the diagnosis. However, there are well-replicated abnormalities in ASD populations, such as increased head circumference (Aylward, Minshew, Field, Sparks, \& Singh, 2002), and hyperserotonemia (Cook \& Leventhal, 1996; Hanley, Stahl, \& Freedman, 1977; McBride et al., 1998; Schain \& Freedman, 1961). These characteristic abnormalities are indicative central nervous system (CNS) abnormalities. Particularly with the increased head circumference, which is suggested to be the result of increased myelination or decreased synaptic pruning (Bauman \& Kemper, 2005; Courchesne, Redcay, \& Kennedy, 2004; Hazlett et al., 2005; Herbert, 2005). This suggestion has been supported by Koul (2005) and Deoni et al. (2015). It is proposed that the changes in myelination subsequently alters connectivity within and between brain regions (Kana, Uddin, Kenet, Chugani, \& Müller, 2014; Zikopoulos \& Barbas, 2013). The increased head circumference appears to affect $20 \%$ of ASD children, although the increased head size returns to within the normal range by adulthood (Courchesne et al., 2001).

Additionally, hyperserotonemia, the elevated serotonin (5-hydroxytryptamine; 5-HT) levels in the blood platelets (Cook et al., 1997), affects approximately $30 \%$ of individuals with ASD (Gabriele, Sacco, \& Persico, 2014). Hyperserotonemia is a peripheral nervous system (PNS) measure of 5-HT, and it has been suggested that hyperserotonemia is related to dysfunctional serotonin reuptake transporter (SERT; which also has actions in the CNS), as the SERT is responsible for the 5-HT levels in the blood platelets (Veenstra-VanderWeele et al., 2012). However, increased head circumference and hyperserotonemia only occur in subsets of the ASD population, thus cannot serve as a biomarker for all ASD, but these are indicative of biochemical alterations that are worthy of further exploration.

Individuals with ASD also have neurological abnormalities that distinguish their neural processing from individuals who develop typically (referred to as neurotypicals). The first (known) report of neuronal connectivity dysfunction originated from Rumsey et al. (1985). Using positron emission tomography (PET) to examine cerebral glucose metabolism in 10 adult males with ASD (and 15 adult male controls), Rumsey et al. (1985) demonstrated that men with ASD had widespread elevated brain activity, and some areas were highly active compared to controls. Another early study demonstrated a connectivity deficit between the frontal and parietal lobes of individuals with ASD (Horwitz, Rumsey, Grady, \& Rapoport, 1988). These 
findings spurred further research into the connectivity of ASD brains (meta-analysis on 95 papers reporting fMRI data between 1984 and 2009; Philip et al., 2012). In addition, numerous neuroimaging studies indicated that connectivity between brain regions was dysfunctional for individuals with ASD (Di Martino et al., 2014; Hasson et al., 2009; Kana, Keller, Cherkassky, Minshew, \& Just, 2009). The functional and anatomical abnormalities (as identified earlier in this introduction) reflect alterations in the neural processing, but there are neuropathological changes in the ASD that contribute to the connectivity dysfunction.

Additionally, the structural and functional abnormalities for ASD individuals changes over the lifespan (Ha, Sohn, Kim, Sim, \& Cheon, 2015). The total brain volume for ASD individuals is above average for ASD children (Hazlett et al., 2011), but then rapidly declines in adolescence and adulthood (Ecker et al., 2014; Zielinski et al., 2014). Further to the lifespan changes for ASD individuals, studies have investigated the functional differences for pre- and post-pubescent ASD individuals. Prepubescent individuals with ASD exhibited impaired language development (Wang, Lee, Sigman, \& Dapretto, 2006) and reductions in processing facial and gestures which are critical to social interaction (Kim et al., 2015). Whereas, postpubescent individuals with ASD show deficits in the regions associated with language (Kana, Keller, Cherkassky, Minshew, \& Just, 2006).

Neuronal regions that are associated with emotional and memory processing also show significant alterations. Using a Golgi-Cox stain which visualises 1 to 3\% of neurons and is used to examine the morphology of cells (Zaqout \& Kaindl, 2016); Raymond, Bauman, and Kemper (1995) showed that the hippocampus of two ASD children had fewer dendritic branches when compared with age-matched peers hippocampi. Raymond et al. (1995) suggest that the reduced dendritic branches are indicative of reduced connectivity. Neuronal correlates of emotional or cognitive processing are examined with the use of magnetic resonance imaging (MRI) scans; Sparks et al. (2002) demonstrated that children with ASD have enlarged amygdalae, when compared to neurotypicals (Schumann et al., 2004). Research has shown that the size of the amygdala was correlated with the severity of their social communication impairments (Juranek et al., 2006; Munson et al., 2006).

The differences between ASD and others directs the attention to widespread alterations in the brains, and an excitation and inhibition connectivity imbalance is a theory proposed as the underlying cause of ASD (Markram, Rinaldi, \& Markram, 2007). This has been driven by functional imaging findings which demonstrated that ASD individuals have hypounderconnectivity between brain regions (Di Martino et al., 2014; Hasson et al., 2009), and 
hyper-overconnectivity within regions (Di Martino \& Castellanos, 2003). The connectivity imbalance is further supported at a cellular level, individuals with ASD have a decreased number and size of Purkinje cells, which are the main inhibitory neurons in the cerebellum (Fatemi et al., 2002; Kern, 2003). Therefore, a reduction in the inhibitory neurons leads to 'unchecked' activation of excitation neurons. Additionally, ASD brains show reductions in Parvalbumin (PV), which is a calcium-binding protein that is expressed in GABA interneurons and the PV neurons are the main inhibitory interneurons in the CNS (Gonchar, Wang, \& Burkhalter, 2008). Using post-mortem tissue, Hashemi, Ariza, Rogers, Noctor, and Martínez-Cerdeño (2016) examined prefrontal brain regions of 11 ASD adults and found significant decreases in PV cells when compared to controls. As such, there is mounting evidence to suggest that ASD is characterised by neuronal connectivity deficits, possibly leading to an imbalance between excitation and inhibition. However, in order to develop novel therapeutic targets, it is important to understand how this dysfunctional connectivity is mediated by neurotransmitter changes.

\subsection{Neurotransmitter Dysfunction}

In addition to the neuronal characteristics of ASD, there are substantial alterations to neurotransmitter function. Altered neurotransmitter function changes neuronal activity (such as hyper- or hypo-activity), which is linked to ASD symptoms.

\subsubsection{Serotonin Dysfunction}

Several critical factors underlie the relationship between ASD and the serotonergic (5HTergic) system. Firstly, 5-HT has a critical role in neurodevelopment, particularly regarding neuronal proliferation, neurogenesis, and migration (Alenina \& Klempin, 2015; Banasr, Hery, Printemps, \& Daszuta, 2004). Neurodevelopmental disruption to the serotonergic system may underlie some of the abnormalities found in ASD individuals as well as in animal models of ASD (Aylward et al., 1999; Raymond et al., 1995; Whitaker-Azmitia, 2001). Secondly, 5-HT has a significant role in psychological processes, such as mood, social behaviour and obsessive behaviours (Anderson, 2004; Lesch et al., 1996). Thirdly, pharmacological or dietary alterations suggest a role for 5-HT in ASD symptoms. Thus, a small group of individuals experience relief from ASD symptoms when taking SSRIs (Moore, Eichner, \& Jones, 2004); and dietary restrictions that reduce the 5-HT precursor tryptophan, exacerbate ASD symptoms (McDougle et al., 1996). Finally, as mentioned before, individuals with ASD have hyperserotonemia. The relationship between hyperserotonemia (particularly in the CNS) and ASD is yet to be fully explored (Polleux \& Lauder, 2004). Nonetheless, the accumulated findings point toward 5-HT having a critical role in ASD during the developmental years and across the individual's lifetime. 
Nakamura et al. (2010) found substantial support for the critical role of 5-HT in ASD, with the use of PET scans. Nakamura and colleagues scanned 20 men with ASD and 20 age- and IQmatched controls and identified that ASD individuals had significantly lowered SERT binding throughout the brain and increased dopamine (DA) transporter binding in the orbitofrontal cortex. While 5-HT has a substantial role, other neurotransmitters, like DA, are implicated in ASD.

\subsubsection{Dopamine Dysfunction}

Aside from DA, the other neurotransmitters of interest in relations to ASD, include the neuropeptide Oxytocin (OXY), y-aminobutyric acid (GABA), and Glutamate (GLU; Penn, 2006; Rubenstein \& Merzenich, 2003), and these will be explored in the following sections. While the exact involvement of these neurotransmitters is yet to be understood, there is emerging evidence to suggest that they also have an important role in ASD. Nguyen et al. (2014) showed that the dopaminergic system contributes to the ASD aetiology through interactions of DA genes and the subsequent proteins encoded on the genes. In a review, Staal (2015) found that a single nucleotide polymorphism of the dopamine-3-receptor gene (DRD3) was linked to ASD. Staal (2015) also identified a relationship between high rates of DRD3 expression in the basal ganglia, an area implicated in repetitive and stereotyped behaviour.

\subsubsection{Oxytocin Dysfunction}

Oxytocin is a neuropeptide associated with social behaviours such as attachment to parents, pair bonding, and aggression (Donaldson \& Young, 2008). Oxytocin is synthesised in the hypothalamus and is involved in neurotransmission in brain regions associated with social functioning (such as the amygdala, medial prefrontal cortex, and the insula; Yamasue, 2015). Heinrichs and Domes (2008) and McGraw and Young (2010) suggest that social behaviour is increased by oxytocin because it inhibits the social avoidance and defensive characteristics of humans and rodents (like prairie voles). Children with ASD aged 6-11 years have reduced oxytocin blood levels (Green et al., 2001; Modahl et al., 1998) indicative of low oxytocin levels in the PNS (and possibly the CNS; Green et al., 2001). The authors postulate that the increased unmetabolized peptides are due to genetic deficits in ASD populations. There is emerging evidence demonstrating a strong association between the oxytocin receptor gene and ASD (LoParo \& Waldman, 2015; Yamasue, 2013). Further research has indicated that brain activity in the amygdala (Furman, Chen, \& Gotlib, 2011; Inoue et al., 2010) and insula (Saito et al., 2013) are strongly dependent on oxytocin receptor functioning. Furthermore, there is support from animal studies demonstrating that genetically de-activating the oxytocin receptor gene induces 
social deficits similar to ASD deficits (Winslow \& Insel, 2002; Young, Pitkow, \& Ferguson, 2002). Finally, the intranasal administration of oxytocin was found to alleviate ASD symptoms (Kosaka et al., 2016; Parker et al., 2017; Watanabe et al., 2015). These findings are suggestive of low oxytocin levels for ASD individuals.

\subsubsection{Glutamate and GABA Dysfunction}

As alluded to earlier, there is also evidence to suggest GLU and GABA are affected in ASD populations. These neurotransmitters are responsible for the excitation/inhibition (E:I) balance in the Central Nervous System. Evidence for a dysfunctional E:I balance is supported by high rates of epilepsy, a disorder of electrical brain activity in ASD patients (Amiet et al., 2008). GLU is the major excitatory neurotransmitter, and there is increasing evidence to suggest it has a critical role in ASD symptomology. Hassan et al. (2013) identified that average GLU levels were significantly higher in the anterior cingulate, striatum, cerebellum and frontal lobe in individuals with ASD when compared with controls. Additionally, increased GLU levels have been identified in the blood of adults with ASD (Shinohe et al., 2006). Furthermore, Purcell, Jeon, Zimmerman, Blue, and Pevsner (2001) demonstrated a significant reduction in GLU receptor density in the cerebellum of individuals with ASD.

On the other hand, the primary inhibitory neurotransmitter GABA also shows significant alterations in ASD populations. Neurodevelopmental abnormalities in GABA may underlie some of the developmental changes that result in ASD symptoms. Several studies indicate that GABA receptors are decreased in ASD populations (Blatt et al., 2001; Oblak, Gibbs, \& Blatt, 2010). Fatemi, Reutiman, Folsom, and Thuras (2009), using post-mortem tissue of individuals with and without ASD found that GABA receptors were significantly reduced in the parietal lobe and the cerebellum of ASD individuals, further suggesting GABAergic dysfunction in ASD. Intriguingly, blood tests of ASD and ADHD children, found elevated GABA levels only found in the ASD population (Dhossche et al., 2002). This has been suggested to be indicative of reduced central GABA neurotransmission. The role of GLU and GABA in ASD will be discussed in more detail in chapter four which examines alteration in GABA interneurons in an animal model for ASD. 


\subsection{Theories on Neurological Causes of ASD}

Many theories have been proposed to explain the pathology and origins of ASD symptoms. Most focus on the cognitive aspects, like failure to infer the mental state of others (Baron-Cohen, 2000; Baron-Cohen, Leslie, \& Frith, 1985), and the subsequent theory of weak centralised coherence, which was derived from the Theory of Mind failures (Frith, 2003; Frith \& Happé, 1994), as well as the social motivation theory (Chevallier, Kohls, Troiani, Brodkin, \& Schultz, 2012). Whereas, others look to account for overall dysfunction, such as executive dysfunction/attentional dysfunction (Ozonoff, Pennington, \& Rogers, 1991), and the empathizing-systemizing theory (Baron-Cohen, 2016). However, these theories do not account for the cellular and molecular findings associated with ASD. Two theories that attempt a better account of the cellular aspects of ASD are the connectivity hypothesis and the E:I imbalance; these will be explored in turn.

The connectivity hypothesis postulates that dysfunctional connectivity and synchronisation within and between brain regions leads to ASD symptoms (Belmonte et al., 2004). Belmonte et al. (2004) suggested that ASD brains experience overall under-connectivity, but within regions, there is over-connectivity. Furthermore, ASD symptoms are driven by signal-to-noise abnormalities; where too much connectivity causes noise that cannot be filtered out (Belmonte et al., 2004; Vasa, Mostofsky, \& Ewen, 2016). There is some evidence to support the under-connectivity findings (Di Martino et al., 2014; Just, Cherkassky, Keller, Kana, \& Minshew, 2006; Kana et al., 2009). However, many studies are refuting the stipulation of overall under-connectivity (Mizuno, Villalobos, Davies, Dahl, \& Müller, 2006; Shih et al., 2010). The lack of support for these findings may be attributed to the age of the participants, as children and adolescents exhibit hyperconnectivity within brain regions and hypo-connectivity between regions, but not adults (Farrant \& Uddin, 2016; Nomi \& Uddin, 2015).

Rubenstein and Merzenich (2003) propose a theory of imbalanced E:I in neural systems as the cause of ASD. The authors suggest that imbalance of E:I is driven by genetic factors (genes as well as epigenetic factors), that lead to molecular and neuronal pathway changes which result in ASD abnormalities in language, as well as abnormal social, and affiliative behaviours. Importantly, the E:I imbalance theory rests on dysfunctional GABA and GLU systems. Studies have repeatedly demonstrated the abnormalities associated with GABA signalling in ASD brains (Bailey et al., 1998; Casanova, Buxhoeveden, Switala, \& Roy, 2002; Coghlan et al., 2012; Hussman, 2001; Ritvo et al., 1986). Further to this, Rubenstein and Merzenich (2003) suggest that the changes in the GABAergic system (and others like GLU, 5-HT, and DA) change the 
neuronal activity, leading to overall brain dysfunction. Their suggestion is supported by decreased function in the GABAergic system in ASD brains (Blatt \& Fatemi, 2011; Fatemi et al., 2009). Deficiencies in GABA can increase GLU (via compensatory mechanisms) lead to hyperexcitable and dysfunctional brains (Powell et al., 2003; Shumyatsky et al., 2002; Steele \& Mauk, 1999).

For the moment, both the connectivity and E:I theories further the research into the neuronal underpinnings of ASD, by providing scope for further studies. However, both theories do not adequately describe the situation for ASD individuals. The connectivity hypothesis attempts to encompass a vast range of neurological processes (anatomical, functional connectivity like EEG, neuronal connectivity, computational models of connectivity). Thus, the capacity to accurately describe findings is limited. Whereas, there is emerging evidence demonstrating the E:I imbalance in other psychiatric disorders, such as schizophrenia (Gao \& Penzes, 2015), and ADHD (Selten, van Bokhoven, \& Kasri, 2018). Therefore, E:I imbalance may not be the cause of ASD or other disorders, but rather a functional result of the underlying dysfunction. Ultimately, with the current knowledge and technology, it is possible to examine micro effects (like neuronal changes), and macro effects (like abnormal EEG and fMRIs), however, the capacity to measure the interaction and coordination of the micro and macro effects are limited, therefore, abnormalities are examined in isolation.

The connectivity and E:I theories draw supporting evidence from the GABAergic abnormalities in ASD, by suggesting that connectivity or inhibition is dysfunctional because of GABA changes in ASD brains. The GABAergic abnormalities are consistently found in ASD brains, as well as the ASD animal models, which gives further support to the substantial impact of GABA dysregulation as an underlying mechanism for ASD symptoms. Animal models of ASD will be explored further in section 1.7. 


\subsection{Aetiology}

ASD is a multifactorial disorder, and the genetic and non-genetic contributions are yet to be understood.

\subsubsection{Genetic Contribution}

Twin studies provide insights into the heritability of ASD, as monozygotic (MZ) twins have identical genes and exposure to the same maternal environment; which minimises confounds that are often involved in familial comparisons. Shared genes and environment increase the probability that if one twin develops a disorder, then the other will too, which is known as the concordance rate. The first published twin study for ASD demonstrated MZ (i.e. identical) twins had a concordance rate of $36 \%$. That is, both twins were diagnosed with ASD in a third of the sampled population. However, there was no concordance for dizygotic twins (DZ or fraternal; Folstein \& Rutter, 1977). A high concordance rate was also identified in a twin study from the Nordic countries (Denmark, Finland, Iceland, Norway and Sweden), where in the sample of 21 twin pairs (11 MZ, $10 \mathrm{DZ}$ ), 91\% of MZ were concordant for ASD but not DZ twins (Steffenburg et al., 1989). These findings were supported by Bailey et al. (1995), who found that $69 \%$ of MZ twins were diagnosed with ASD and no concordance for DZ twins. More recent studies show the concordance rates are closer to $88-95 \%$ for MZ twins compared to $31 \%$ in DZ twins (Hallmayer et al., 2011; Rosenberg et al., 2009; Taniai, Nishiyama, Miyachi, Imaeda, \& Sumi, 2008).

\subsubsection{Familial Studies}

Concordance rates in families provide further insights into the genetic contribution for ASD. However, these rates are lower compared to twins, as siblings and family members do not share the pre- and post-natal environments. Examining sibling recurrences, Ritvo, Jorde, Mason-Brothers, Freeman, and Pingree (1989) identified the total recurrence risk for ASD is 8.6\% which suggests that ASD is more likely for siblings when they are born after an autistic child. Familial studies have consistently found that close relatives of individuals with ASD show increased rates of ASD-related symptoms, such as social and communication impairments, and aberrant non-social behaviour (Bolton et al., 1994; Pickles et al., 2000; Piven, Palmer, Jacobi, Childress, \& Arndt, 1997; Tordjman et al., 2001). Furthermore, in a prospective longitudinal study, Ozonoff et al. (2011) showed that sibling recurrence rates were $18.7 \%$, such that, 132 out of 664 infants were diagnosed with ASD in childhood and these infants had at least one older 
sibling with ASD. Ozonoff et al. (2011) found a marked gender difference, which showed that $26.2 \%$ of male infants received an ASD diagnosis compared with $9.1 \%$ of female infants.

Twin and familial studies demonstrate that ASD is highly hereditable. However, the specific genes that increase the risk are not yet known (Neale et al., 2012). To identify potential gene candidates, genome-wide association studies (GWAS) have demonstrated that the risk of ASD increased with increased DNA mutations, like copy number variants (CNV) and sequence variants (Buxbaum et al., 2012; De Rubeis et al., 2014; Glessner et al., 2009). Furthermore, these DNA variants implicate more than 100 genes that were associated with ASD (Buxbaum et al., 2012). GWAS have revealed mutations associated with ASD, that converge on genes that regulate synaptic transmission, chromatin remodelling (Pinto et al., 2014; Yuen et al., 2016), neuronal cell adhesion (Glessner et al., 2009), and excitability transcription (De Rubeis et al., 2014). For example, Sebat et al. (2007) identified that de novo CNVs were significantly associated with ASD and mutations in the germline were a significant risk factor for offspring developing ASD. Further to this, de novo insertions and deletions significantly contributed to increased ASD risk, and the mutations were strongly associated with the paternal chromosomes (Dong et al., 2014). This association may be related to epigenetic changes, such as changes to DNA methylation found in the sperm of fathers that already had a child diagnosed with ASD (Feinberg et al., 2015). The combination of concordance and familial research confirms the presence of a strong genetic component that underlies the aetiology of ASD.

\subsubsection{Serotonin Specific Genetic Alterations}

Of the alleles mentioned above, the SLC6A4 allele, which codes for the SERT and psychological disorders has been repeatedly examined in relation to ASD (Taylor et al., 2006; Way \& Taylor, 2010). The relationship between serotonin and ASD has been investigated because of the serotoninergic alterations in ASD populations and is the focus of this thesis (refer to introduction sections 1.7.6, 1.8, and chapter three). The SERT protein modulates 5-HT transmission by terminating and recycling 5-HT back into the pre-synaptic neuron (Lesch et al., 1994). This SLC6A4 gene is located in chromosome region 17ql1.2 (Gelernter, Pakstis, \& Kidd, 1995), and has a polymorphism in the promoter region (5-HT transporter gene-linked polymorphic region; 5-HTTLPR). This polymorphism is related to 20-23 DNA base-pairs, which is repeated 16 times ('long' variant) or 14 times ('short' variant; Taylor et al., 2006). The short variant leads to decreased function of the SERT (Coutinho et al., 2004; Heils et al., 1996), which has implications for the availability of 5-HT in the synapse as well as the binding sites for medications such as SSRIs. Studies have reported a link between this variant and depression 
(Taylor et al., 2006), anxiety (Lesch et al., 1996), and ASD (Cook et al., 1997; Devlin et al., 2005; Yirmiya et al., 2001). In this respect, it is interesting that depression and anxiety are frequently comorbid with ASD (see section 1.2.3).

However, studies examining the link between 5-HTTLPR and ASD have not been consistent (Coutinho et al., 2004; Ramoz et al., 2006). Devlin et al. (2005), Conroy et al. (2004) and Cook et al. (1997) have found that the short variant is a predictor for ASD behaviours, whereas Kluck, Poustka, Benner, Lesch, and Poustka (1997) and Tordjman et al. (2001), found that the long variant was more common among ASD populations. Others have found no association or link between either variant and ASD (Betancur et al., 2002; Coutinho et al., 2004; Maestrini et al., 1999; Persico et al., 2000; Ramoz et al., 2006).

Additionally, there is another polymorphism in the second intron of the SLC6A4 gene which is associated with ASD. The variant consists of a variable number of tandem repeats (STin2 VNTR) and has three common alleles, STin2.9, STin2.10, STin2.12 (9, 10, and 12 denote the number of repeated units in the gene; Lesch et al., 1994). Studies have reported the STin2.12 is more common in ASD populations (Huang \& Santangelo, 2008; Kluck et al., 1997) and may contribute to the increased risk for hyperserotonemia (Coutinho et al., 2004; Coutinho et al., 2007). However, an association between the Stin 2.12 and ASD is, again, not always consistent (Brune et al., 2006), and contribution of the Stin2.12 to hyperserotonemia has also not been replicated (Betancur et al., 2002).

Thus, the research examining the SLC6A4 gene and ASD have not revealed consistent results (which in itself is not unique and generally holds true for every gene associated with ASD). This may in part be due to small sample sizes and measurement error (Border et al., 2019). Border et al. (2019) and Risch, Herrell, Lehner, Liang, and Merikangas (2009) suggest that psychiatric disorders (such as depression and ASD) are polygenic and therefore, isolating a single gene is by definition erroneous. Additionally, it is clear that psychiatric disorders are the result of multifactorial interactions, with contribution from both genes and the environment. In this respect, Nijmeijer et al. (2010) and Hecht et al. (2016), found that while the 5-HTTLPR alone did not significantly increase the risk for ASD, in interaction with prenatal stress or maternal smoking, a significant association was found. 


\subsubsection{Prenatal Risk Factors}

In addition to genetic factors, environmental factors have a critical impact on neurodevelopment and the risk for ASD. In particular, prenatal risk factors like exposure to teratogens or maternal infections are known to increase the risk of ASD for the offspring. The prenatal period is critical for optimal neuronal development, and adverse effects have profound impacts during this time with long-term consequences. The research on prenatal factors has centred on maternal stress, exposure to teratogens, and infections during gestation. Kinney, Miller, Crowley, Huang, and Gerber (2008) found an increased risk of ASD in the children of pregnant mothers exposed to a storm (hurricane or cyclone) during the middle or end of the gestation period. This parallels the findings of Laplante, Brunet, Schmitz, Ciampi, and King (2008) who identified a negative association between prenatal maternal stress and lower cognitive and language abilities in 5-and-half-year-old children whose mothers had been exposed to the 1998 Quebec ice storm. In a review, Kinney, Munir, Crowley, and Miller (2008) suggested that stress during gestation and just after birth is a significant risk factor for ASD. Furthermore, in a review of multiple prenatal stress studies in laboratory animals, Kinney et al. (2008) summarised that prenatal stress does not need to be chronic or severe to result in significant postnatal developmental changes.

\subsubsection{Maternal Infections}

Maternal infections can have a substantial impact on the development of the offspring. Libbey, Sweeten, McMahon, and Fujinami (2005) postulated that maternal infection could interact with genetic susceptibility leading to the symptomology of ASD. This interaction aligns with the view that aetiology of ASD is multi-factorial and lacks a singular cause. Atladottir et al. (2010) found that early gestation infection, particularly with the influenza virus, increases the risk of ASD. This was based on a review of Denmark Medical Birth Register, including births from 1980 to 2005 with an examination of the hospital admissions and the diagnosis of the offspring. Another virus that was associated with ASD development in children was the in utero exposure to rubella (Chess, 1971). Maternal antibodies created in response to viruses could be crossing the placental barrier, interacting with the foetus, and thereby enhancing ASD risk.

Animal studies have furthered our knowledge of maternal infections and the increased risk for ASD. Polyriboinosinic - polyribocytidilic acid (PolyI:C) and lipopolysaccharide (LPS) are two commonly used compounds that simulate viral or bacterial infections in laboratory animals (Patterson, 2011). Non-human primate studies also demonstrate the impact of maternal immune activation (MIA). Bauman et al. (2013) administered an adapted version of Poly I:C to 
pregnant macaques (non-human primates) and found that the offspring exhibited decreased social interaction, communication and repetitive behaviours. Similar results have been found when Rhesus monkeys were prenatally exposed to Poly I:C (Machado, Whitaker, Smith, Patterson, \& Bauman, 2015). Corresponding effects of MIA have also been found in rats and mice. That is, rodents that were prenatally exposed to Poly I:C exhibit behavioural deficits which are similar to ASD symptoms, such as reduced communication and deficits in social behaviour (Malkova, Collin, Hsiao, Moore, \& Patterson, 2012; Schwartzer et al., 2013; Shi, Fatemi, Sidwell, \& Patterson, 2003). The ASD-like effects of prenatal exposure to Poly I:C also extend to the neuronal abnormalities, particularly in the hippocampus (Ohkawara, Katsuyama, Ida-Eto, Narita, \& Narita, 2015) and the amygdala (Li et al., 2018). Similar ASD-like behaviours are found in offspring prenatally exposed to LPS (Kirsten, Queiroz-Hazarbassanov, Bernardi, \& Felicio, 2015). Importantly, Smith, Li, Garbett, Mirnics, and Patterson (2007) and Ashdown et al. (2006) have demonstrated that ASD-like behaviour of rodents prenatally exposed to Poly I:C or LPS is likely to the be the result of maternal immune response rather than the active infection crossing the placental barrier. Overall, the behavioural variations in the prenatally exposed offspring align with diagnostic criteria for ASD.

\subsubsection{Teratogen Exposure}

Teratogens are usually defined as compounds that cause developmental disturbances and often malformation of the foetus (Ranger \& Ellenbroek, 2015). Medications such as thalidomide and Valproic acid have stood out in the adverse effects on foetus development because of their pharmacological insult during sensitive neurodevelopmental periods. Thalidomide was a medication used in the 1950s-60s to treat anxiety and morning sickness. However, the ingestion of this medication while pregnant resulted in physical and neurological deformities for the children. When ingested around gestational day (GD) 20-24, the children were more likely to experience neurodevelopmental deficits (Miller, Strömland, Gillberg, Johansson, \& Nilsson, 1998). GD 20-24 is a sensitive period, as it is associated with neural tube closure in the developing foetus, and exposure during this time critically impairs the developing tissue (Detrait et al., 2005). Accumulated evidence demonstrated that women taking this medication while pregnant were much more likely to have a child with ASD when compared to the population averages (Stromland, Nordin, Miller, Akerstorm, \& Gillberg, 1994). This has been attributed to thalidomide crossing the placenta barrier and altering the development of neurons (Rodier, Ingram, Tisdale, \& Croog, 1997). The association of thalidomide and ASD has been supported by animal studies, where prenatal exposure to thalidomide leads to changes in the serotonergic system, similar to the elevated 5-HT levels in ASD populations (Miyazaki, Narita, 
\& Narita, 2005; Narita et al., 2002). However, prenatal exposure to thalidomide in rats has not conclusively resulted in ASD-like behaviours. Narita et al. (2010) showed that thalidomide exposed rats had no significant social interaction deficits but exhibited some learning deficit in a memory task. Thus, it is likely that thalidomide-induced ASD model is unsuitable for examining social alterations in rats.

\subsubsection{Prenatal Valproate Exposure}

More importantly, for the current thesis, another medication that has teratogenic effects relevant to ASD is Valproic acid (valproate, VPA, 2-Propylpentanoic acid). This medication is primarily prescribed to treat epilepsy but is also effective in treating bipolar disorder, migraines, and mood disturbances (Bollino, Balan, \& Aurelian, 2015; Lloyd, 2013). There is considerable evidence to demonstrate that the prenatal exposure to VPA significantly increases the risk of the offspring developing ASD (Adab et al., 2004; Christensen et al., 2013; Christianson, Chester, \& Kromberg, 1994; Dean et al., 2002; Moore et al., 2000; Smith \& Brown, 2014; Viinikainen et al., 2006; Williams \& Hersh, 1997). Prenatal exposure to VPA is linked to birth abnormalities, changes in cognitive function and developmental deficits (Lloyd, 2013; Roullet, Lai, \& Foster, 2013). Further to this, VPA was found to increase the risk for ASD by $4.42 \%$ when women were taking it while pregnant (Christensen et al., 2013). Rasalam et al. (2005) identified that children who had been exposed to VPA in utero were significantly more likely to be diagnosed with ASD compared with children who had been exposed to other anticonvulsants. The authors went on to specify that children exposed to VPA in utero demonstrated a distinctive pattern of cognitive delays, especially in language and motor milestones, which aligns with a diagnosis of ASD. Additionally, when VPA is the only medication taken during pregnancy, the risk of ASD-like symptoms in the children is seven times higher (Bromley et al., 2008). It is from this association between in utero exposure to VPA and ASD in humans that an in vivo animal model of ASD has been generated to test the behavioural and neurological features of ASD (this model is detailed in section 1.8.3).

Pharmacodynamically, VPA increases GABA in the brain (Löscher, 2002), and regulates neurotransmission by blocking voltage-dependent sodium channels, which prevent cells from completing action potentials (Bollino et al., 2015). VPA crosses the placental barrier and can have teratogenic effects on developing foetus (Dickinson, Harland, Lynn, Smith, \& Gerber, 1979; Nau, Koch, Häuser, \& Helge, 1981). The teratogenic mechanism of VPA is attributed to histone deacetylase (HDAC) inhibition, which leads to epigenetic alterations in the developing foetus (Gurvich et al., 2005; Ranger \& Ellenbroek, 2015). Additionally, VPA increases reactive oxygen 
species (ROS), endogenous molecules that can cause damage to DNA, proteins, and cellular communication (Defoort, Kim, \& Winn, 2006; Na, Wartenberg, Nau, Hescheler, \& Sauer, 2003; Tung \& Winn, 2011). In foetuses, the antioxidant systems that normally detoxify ROS are immature, thus making the foetus more susceptible, particularly during organogenesis (Tung \& Winn, 2011). VPA has complex mechanisms of action which may lead to an interactive effect, whereby HDAC inhibition leads to increase ROS levels, ultimately resulting in increased cell damage (Carew, Giles, \& Nawrocki, 2008).

\subsubsection{Genetic and Environmental Interactions}

This chapter has examined studies that demonstrate 5-HT contributes to ASD symptoms (see section 1.3.1.4), and the significant impact of prenatal VPA leading to an increased risk of ASD (see section 1.6.2.3), Furthermore, studies have shown that prenatal VPA exposure alters 5-HTergic development and functioning (Miyazaki et al., 2005, Tsujino et al., 2007, Oyabu et al., 2013). Thus, it is important to examine whether the 5-HTergic system and the prenatal exposure to VPA interact with each other.

Despite the evidence that suggests that ASD results from gene-environment interactions (Kim \& Leventhal, 2015), the current state of research on in vivo models of ASD relies on either purely genetic mutations (Banerjee-Basu \& Packer, 2010; Moy et al., 2008; Moy et al., 2007), or singular environmental challenges (Fatemi et al., 2008; Schneider \& Przewlocki, 2005; Takahashi, Haglin, \& Kalin, 1992). In rare cases, studies have examined a genetic factor in interaction with prenatal exposure to VPA. Kim et al. (2019) examined mice with contactinassociated-protein-like 2 knock-outs (Cntnap2 KO) that were prenatally exposed to 100 or $300 \mathrm{mg} / \mathrm{kg}$ of VPA on GD 10. The Cntnap2 is a member of the neurexin gene family which is involved in axonal differentiation and guidance, and there is some evidence to suggest it is disrupted in ASD populations(Anney, 2013). Cntnap2 KO mice show disruptions to neurodevelopment and ASD-like behaviours(Hali et al., 2020). Kim et al., found that Cntnap2 KO mice prenatally exposed to VPA had improved social deficits but heighten seizure susceptibility. The authors examined amplitudes of excitatory postsynaptic currents in the pyramidal neurons of the prefrontal cortex and found that Cntnap2 KO mice prenatally exposed to VPA equivalent to controls, but the Cntnap2 KO mice and the VPA-exposed mice had abnormal neural transmission. Thus, Kim et al., found that the interaction of a genetic factor and an environmental factor lead to the recovery of the ASD-like phenotypes of the singlefactors. 
Ellenbroek, August, and Youn (2016) examined ASD-like behaviours following the prenatal exposure of heterozygous serotonin transporter knockout (SERT KO) rats to prenatally to $400 \mathrm{mg} / \mathrm{kg}$ VPA on GD 12.5. They were unable to find significant gene-environment interaction but did find that VPA-exposed offspring had increased anxiety and reductions in cognition. The authors suggested that the lack of interactions may be attributed to the timing of the environmental challenge, and that perhaps offspring with genetically compromised SERT function are more sensitive earlier or later in gestation.

Ultimately, this thesis will advance the work of Ellenbroek et al. (2016) and will examine the relationship between VPA and 5-HT with the use of SERT KO animals. This thesis will assess ASD-like behaviours following chronic prenatal exposure to VPA as an environmental risk factor when combined with the genetic vulnerability contributed by the reduction in the SERT activity in the SERT KO strain. However, before examining the research on the animal model of ASD by prenatal exposure to VPA (section 1.8.3), but it is first relevant to examine animal modelling more broadly. 


\subsection{Animal Modelling}

Animal models of human conditions should fulfil meaningful validity criteria, of which there are three relevant items (Belzung \& Lemoine, 2011; van der Staay, 2006; van der Staay, Arndt, \& Nordquist, 2009). First, construct validity refers to how the disorder and its aetiology is modelled in the animal (Hulbert \& Jiang, 2016; Willner, 1984). To fulfil the construct validity criterion, an ASD animal model ideally has similar genetic and/or environmental factors that are known to contribute to ASD. The second validity criterion is face validity, this refers to whether the animal model accurately represents the anatomical, behavioural, and biochemical characteristics of the disorder (Nestler \& Hyman, 2010). The phenotype or the observable characteristics of an ASD animal model should include altered communication and social interaction. The final validity criterion is predictive validity, and this requires the model to respond to pharmacological treatments as a human would respond (Nestler \& Hyman, 2010). Unfortunately, for ASD, given the very limited effectiveness of pharmacological therapy, this criterion is very difficult to assess.

This thesis aims to examine and further advance the animal modelling of ASD. Before examining the current literature on VPA animal model of ASD, I will review other animal models for ASD. Animal models of ASD have been constructed in three major ways; a lesion approach, genetic basis (e.g. selective breeding for phenotypes or mutations), and the use of environmental manipulations (e.g. prenatal challenges).

\subsubsection{Lesion Approach to Animal Modelling of ASD}

The first method for modelling ASD behaviours in animals is through lesion studies, in which experimenters chemically or physically remove or lesion particular brain regions and examine the behavioural consequences of this lesion. Individuals with ASD do not have one specific brain region that is said to underlie the disorder; therefore, it is challenging to model ASD using lesion based techniques, and the construct validity of these models can be questioned. However, these models have contributed to our understanding of how alterations in specific brain regions lead to ASD-like behaviours in animals.

From non-human primate studies, the removal of the medial temporal lobe in young macaque monkeys resulted in ASD-like deviations in sociability, particularly decreased social initiation, and increases stereotyped behaviours (Bachevalier, 1996). In rats, Wolterink et al. (2001) showed that lesions to the amygdala or hippocampus during the early postnatal period (postnatal day, PND 7) or later on (PND 21), resulted in decreases of pinning behaviours, social 
exploration, and approach behaviours in the play behaviour assay. More specifically, social behaviour was decreased when lesions were applied to the amygdala, rather than the ventral hippocampus (Daenen, Wolterink, Gerrits, \& Van Ree, 2002). Furthermore, Schneider and Koch (2005) demonstrated that lesions in the medial prefrontal cortex led to a reduction in play behaviour and social exploration. Taken together, these studies show that midbrain and cortical structures are necessary for sociability and that dysfunction in these regions result in sociobehavioural alterations that are consistent with social dysfunction symptoms in ASD (Amaral, Schumann, \& Nordahl, 2008; Barnea-Goraly et al., 2014; Carper \& Courchesne, 2005).

\subsubsection{Genetic Approach to Animal Models of ASD}

In contrast to lesion models, altering the genetic makeup in animals has resulted in a large number of in vivo animal models for ASD. The genetic approach to animal models includes the use of inbred strains of animals (forward genetics), which have been specifically bred due to their expression of behavioural traits, such as the BTBR $\mathrm{T}+\mathrm{tf} / \mathrm{J}$ (BTBR) mice that were bred for their reduced sociability (McFarlane et al., 2008). Whereas, animal models are also based on targeted mutations in specific genes (reverse genetics), such as the Shank mutated mice or mice and rats with mutations in the Slc6a4 gene (Blakely \& Veenstra-VanderWeele, 2011).

The BTBR mice are used as an animal model for ASD because of their reduced sociability and aberrant vocalisations (Defensor et al., 2011; McFarlane et al., 2008; Moy et al., 2007). These behaviours are attributed to neuronal reductions in the hippocampus and an almost complete removal of the corpus callosum (Wahlsten, Metten, \& Crabbe, 2003). BTBR are an inbred strain and the specific genetic locus (or more likely loci) that underlies the behavioural expression has yet to be determined. BTBR mice have transcript alterations in the hippocampus and amygdala (Nadler et al., 2006), polymorphisms associated with serotonin regulation (McFarlane et al., 2008), mutation in the protein encoded by Disrupted in schizophrenia gene (Discl; Meyza et al., 2013), and further mutations in the exostosin 1 (Extl) gene which is required for the molecules vital to forming the corpus callosum (Meyza et al., 2013). Furthermore, administering fluoxetine (an SSRI) to BTBR mice increases their sociability to match that of controls (Chadman, 2011; Gould et al., 2011). This model exhibits good face validity, as the behavioural expressions align with ASD symptoms; and there is emerging evidence to suggest the neurobiological factors (such as a lack of the corpus callosum, and reduced white matter) are aligned with findings from ASD populations, which supports the construct validity of this model (Chadman \& Guariglia, 2012). 
An example of reverse genetics is the SHANK animal model, which has been derived from evidence showing that the Shank family genes are altered in ASD populations. There are three Shank genes (Shankl-3) which encode proteins involved in synaptic scaffolding and dysfunction of these genes leads to reduced neuronal connectivity, and as such Shank genes have been attributed a critical role in ASD (Gauthier et al., 2009; Peça et al., 2011; Yoo, Bakes, Bradley, Collingridge, \& Kaang, 2014). Mice with Shankl mutations display deficits in communication and exhibit anxious-like behaviours. However, Shankl mutants do not exhibit the ASD symptom triad, as social interaction and repetitive behaviours are no different when compared to mice with functional Shankl gene (Silverman et al., 201l; Wöhr, Roullet, Hung, Sheng, \& Crawley, 2011). In contrast, mice with Shank2 (Schmeisser et al., 2012; Won et al., 2012) and Shank3 (Peça et al., 2011; Wang et al., 2011) mutations demonstrate reduced communication, social interaction, and increased repetitive behaviours. The disassociation between Shankl and Shank2-3 indicate that these genes individually contribute to ASD-like behaviours.

In addition to the BTBR and Shank mice models, many other animal models of ASD have been developed. In vivo models with ASD-like behaviours (i.e. reductions in communication, social interaction and repetitive behaviours) includes animals with mutations in neuroligins (Nlgn 1 - 4; Blundell et al., 2010; Radyushkin et al., 2009; Tabuchi et al., 2007), and the Fmrl gene (Bernardet \& Crusio, 2006; Mineur, Huynh, \& Crusio, 2006; Pietropaolo, Guilleminot, Martin, D'Amato, \& Crusio, 2011). Further, heterozygous Mecp-2 null mice models Rett syndrome, which is a disorder that is closely associated with ASD. These mice exhibit reductions in social behaviour and significant repetitive behaviours (Gemelli et al., 2006; Moretti, Bouwknecht, Teague, Paylor, \& Zoghbi, 2004).

\subsubsection{Serotonin Transporter Knockout Model}

In addition to the models mentioned above and with particular relevance to this thesis, a SERT KO model has been developed, allowing for the investigation of the role of the SERT in behavioural and physiological processes. The SERT is particularly relevant to the examination of ASD aetiology and the exhibited behaviours, as the 5-HT system has a critical role in ASD, and by examining the SERT KO animals in laboratory context we can discern the SERT contribution to behaviours in experimental settings. The original SERT KO model was generated in mice, where animals were mutated with homologous recombination that resulted in mice with a non-functional SERT (Lira et al., 2003). The mice with non-functional SERT genes $\left(\right.$ SERT $\left.^{-/}\right)$exhibit increased anxiety and depressive-like behaviours which have been 
replicated within and across laboratories (Bregman et al., 2018; Carroll et al., 2007; Holmes, Murphy, \& Crawley, 2003; Lira et al., 2003).

A SERT KO has also been generated in Wistar rats. This model was generated using $\mathrm{N}$ ethyl- $N$-nitrosourea (ENU); a compound with high mutagenicity (Smits et al., 2006). The process of development of the SERT KO strain required Wistar males to be treated with ENU for three weeks, and this significantly mutated the spermatogonial stem cells (pre-sperm cells). These treated males were subsequently mated with genetically-unaltered females, and the resulting offspring were genotyped to identify mutated genes (Smits et al., 2006). One of the genetic mutations identified was a premature stop-codon in the Slc6a4 gene. The offspring with this mutation was backcrossed with wildtype $\left(\mathrm{SERT}^{+/+}\right)$animals over multiple generations, thus cleaning up the genetic background while maintaining the mutation in the gene of interest (Smits et al., 2006). The SERT mutation results in a gene dependent deficit in the SERT function, where homozygous (SERT ${ }^{-/}$) have a $100 \%$ reduction, while heterozygous $\left(\mathrm{SERT}^{+/-}\right.$) rats have a $40-60 \%$ reduction compared with $\mathrm{SERT}^{+/+}$rats that do not express the mutation (Homberg et al., 2007). The authors also demonstrated that there were no compensatory changes in tryptophan hydroxylase (which is a 5-HT synthesising enzyme) or monoamine oxidase (which is involved in metabolising 5-HT) activity. Furthermore, there were no alterations in dopamine and noradrenaline reuptake mechanisms.

The genetic mutation in the SERT protein leads to social behaviour changes. Homberg, Schiepers, Schoffelmeer, Cuppen, and Vanderschuren (2007) showed that juvenile SERT ${ }^{-/-}$rats had significant reductions in play behaviour and exhibited aberrant play behaviours patterns when compared with their wild type controls. The reduced social behaviours exhibited by SERT ${ }^{-}$ I- rats was replicated by Muller, Olivier, and Homberg (2010). The findings in rats are similar to the reductions in social initiation behaviours that were identified in SERT knockout mice (Kalueff, Fox, Gallagher, \& Murphy, 2007). Together, these findings suggest that the SERT has a significant role in social behaviour and more importantly, the SERT KO rat model is suitable for examining ASD-like behaviours (as shown in chapter three).

In addition to the social behaviour alterations in SERT KO animals, other studies have examined the impact of the 5-HT mutations on the animal's behaviour. SERT ${ }^{-1-}$ rats exhibit increased anxiety in a range of behavioural tests (Homberg et al., 2007), which we have also found in our own lab (Kidwell, 2018). Holmes, Li, Murphy, Gold, and Crawley (2003) using SERT $^{-/-}$mice, also found increased anxiety-like behaviour, in line with others (Adamec, Burton, Blundell, Murphy, \& Holmes, 2006; Moy et al., 2009). 


\subsection{Environmental approach to Animal Models}

In humans, there is an established association between prenatal exposure to environmental challenges and the development of ASD. Therefore, ASD-like behaviours in animals have been investigated in response to such environmental challenges. Common prenatal environmental challenges used in laboratory settings are stress, maternal immune activation, and teratogens; these will be explored in the following section.

\subsubsection{Prenatal Exposure to Stress}

Stress during prenatal development in rats significantly affects behaviour in later life. Takahashi et al. (1992) administered a tail shock to pregnant females every second day of gestation from gestational day two. This environmental stress resulted in offspring exhibiting significant delays in initiating social interaction and showed increased anxious-like behaviour (increased freezing after a foot shock) when compared with offspring unexposed to prenatal stress. In a spatial memory task, Lemaire, Koehl, Le Moal, and Abrous (2000) demonstrated that prenatally stressed offspring had learning delays. Similar effects were found in prenatallystressed adult mice (Son et al., 2006). In a gene $\times$ environment interaction study, Jones et al. (2010) examined the reaction of the offspring from pregnant mice with reduced SERT function $\left(\mathrm{SERT}^{+/}\right)$that were exposed to chronic prenatal stress. The authors suggest that their results demonstrate that the prenatally stressed offspring of SERT ${ }^{+-}$had significant deviations in communication and social interaction. However, close inspection of their data actually shows that the offspring of SERT ${ }^{+/+}$mothers were more sensitive to prenatal stress. Nonetheless, the study supports the evidence suggesting that prenatal stress increases the risk for ASD.

\subsubsection{Maternal Immune Activation}

As mentioned in the earlier section on maternal infections as a prenatal risk factor (1.6.2.1), another way to induce ASD-like behaviours in animals is with MIA, using the influenza virus, Poly I:C, or LPS. Shi et al. (2003) infected pregnant mice at GD 9 with the influenza virus and showed that the influenza-exposed offspring exhibited significant deficits in social interaction as well as reductions Purkinje cells. Fatemi et al. (2008) administered the influenza virus on GD 18, which resulted in a significant reduction in 5-HT levels. As identified earlier, maternal antibodies also significantly alter the brain and behaviour of prenatally-exposed offspring (Bauman et al., 2013). Both Poly I:C and LPS have been used to model ASD-like behaviours in rodents, with success. A prenatal administration of Poly I:C leads to deficits in behaviours relevant ASD, such as communication, social interaction and repetitive behaviours 
(Bauman et al., 2014; Malkova et al., 2012; Patterson, 2011). These behaviours have also been observed in offspring that were prenatally exposed to LPS (Kirsten, Taricano, Maiorka, PalermoNeto, \& Bernardi, 2010; Ohkawara et al., 2015; Shi et al., 2009).

However, the MIA model is not specific to ASD and is often associated with schizophrenia-like behaviours (McAlonan, Li, \& Cheung, 2010). There is mounting evidence to suggest that infection mid-to-late pregnancy contributes to the risk of developing schizophrenia (Cooper, 1992; Karlsson \& Dalman, 2019; Mednick, Huttunen, \& Machón, 1994). While there is an indication that MIA increases the risk of ASD, the association between MIA and schizophrenia is much stronger (McAlonan et al., 2010). Therefore, this thesis will focus on the influence of VPA as a teratogen which imparts the environmental factor contributing to ASD.

\subsubsection{Valproate-induced ASD Model}

The VPA-induced ASD model was based on clinical studies that prenatal exposure to VPA significantly increases the risk for children to develop ASD (Christensen et al., 2013), as discussed in prenatal risk factors section (1.6.2.3). This link led to the VPA induced animal model for ASD (Rodier et al., 1997; Rodier, Ingram, Tisdale, Nelson, \& Romano, 1996).

In the 1980s, VPA was chronically administered to pregnant rats to examine the teratogenicity. This research has established appropriate doses for rats and options for chronic administration of VPA. Nau, Zierer, Spielmann, Neubert, and Gansau (1981) used doses of 0, $200,400,800 \mathrm{mg} / \mathrm{kg}$ of VPA from GD 7 to 15 via subcutaneous injection in mice. The authors found that high doses of VPA $(400-800 \mathrm{mg} / \mathrm{kg}$ ) resulted in significantly reduced foetal weight, increased incidence of malformation, and increased resorptions or deaths at birth (Nau et al., 1981; Nau et al., 1981). These findings were supported by the work of Vorhees (1987), who used an oral gavage to administer doses of VPA $(0,150,200,300,400$, or $600 \mathrm{mg} / \mathrm{kg})$ to pregnant Sprague-Dawley (SD) rats between GD 7 to 18 . The $600 \mathrm{mg} / \mathrm{kg}$ repeated dose was lethal for two out of four dams and was lethal to all offspring. The $400 \mathrm{mg} / \mathrm{kg}$ repeated dose led to $53 \%$ of the offspring being resorbed or dead. In contrast, doses of 150,200 , and $300 \mathrm{mg} / \mathrm{kg}$ resulted in better survival rates but also increased birth abnormalities, such as limb, cardiovascular, and skeletal defects. In another study, Vorhees (1987) used oral gavage to administer 0, 150, and $200 \mathrm{mg} / \mathrm{kg}$ of VPA to pregnant SD rats on GD 7-18, and found that offspring exposed to VPA had decreased locomotor activity, increased working memory errors in a water maze and reduced startle responses. Furthermore, these deficits were worse for the $200 \mathrm{mg} / \mathrm{kg}$ than the $150 \mathrm{mg} / \mathrm{kg}$ condition. From the teratogenicity studies, it is clear that high doses of VPA have an 
embryo-toxicity that is lethal to dams and pups. However, lower doses do not have the same effect, and can be tolerated by the dam.

The prenatal exposure to VPA has become a common method for inducing ASD, although the time point of gestational exposure is a critical variable to consider when developing a VPA model for ASD. Clinical findings have suggested that children from women taking VPA during the first trimester of pregnancy had a significantly increased risk of ASD (Christensen et al., 2016; Moore et al., 2000). The first trimester is a critical time for organogenesis and neural tube development and closure. This critical period roughly translates to gestational days 8-15 in rats and mice (Ergaz, Weinstein-Fudim, \& Ornoy, 2016). As such, studies have commonly used GD 12 as the day of administration (Bringas, Carvajal-Flores, López-Ramírez, Atzori, \& Flores, 2013; Kim et al., 2011; Loohuis et al., 2017; Schneider \& Przewlocki, 2005; Stanton, Peloso, Brown, \& Rodier, 2007). Other days for administration have been used with similar effects, such as GD 9 (Dufour-Rainfray et al., 2010; Tsujino et al., 2007) or GD 13 (Kataoka et al., 2013; Wagner, Reuhl, Cheh, McRae, \& Halladay, 2006). Using different time points during gestation, Kim et al. (2011) showed that the most effective time point was GD 12 for mice and rats. However, the fact that in rats a single dose is typically used, while in humans VPA is taken throughout pregnancy reduces the construct validity of these animal models.

\subsubsection{Effects of Single Prenatal Exposure to VPA}

VPA-exposed animals have reductions in social interactions. For example, Schneider and Przewlocki (2005) showed that adolescent rats prenatally exposed to $600 \mathrm{mg} / \mathrm{kg}$ of VPA on GD 12 showed a marked decrease in the frequency of pinning. During adulthood, VPA-exposed rats were slower to socially interact, also had decreases in interaction events. These results were supported by Olde Loohuis et al. (2015). Additionally, social interaction deficits in the VPA animal model have been identified through the use of the social approach-avoidance assay. Dufour-Rainfray et al. (2010) exposed rats to VPA on GD 9. They showed that juvenile offspring rats exhibited reduced social initiation (decreased visits to social stimulus) when compared with control offspring. VPA exposure at GD 12 showed a similar effect (Bambini-Junior et al., 2011; Kim et al., 2011; Markram, Rinaldi, La Mendola, Sandi, \& Markram, 2008).

However, the ASD-like behaviours in animals prenatally exposed to VPA are not always consistent. Studies using behavioural assays to measure social behaviour of prenatally exposed VPA and control animals have shown increases (Cohen, Varlinskaya, Wilson, Glatt, \& Mooney, 2013; Stefanik, Olexova, \& Krskova, 2015) or no significant difference (Narita et al., 2010). These 
differences may be attributed to the day of administration or the dose. Narita et al. (2010) used an oral gavage to administer $800 \mathrm{mg} / \mathrm{kg}$ of VPA at GD 9, and the adult offspring showed no significant difference in the social interaction measures of sniffing, pinning, chasing and avoidance. The lack of difference for these animals may be attributed to the oral gavage (which can induce stress) combined with a high VPA dose. However, Cohen et al. (2013) used an Intraperitoneal (IP) injection of $350 \mathrm{mg} / \mathrm{kg}$ on GD 13 and found increased social investigation and play fighting. Similar increases in social behaviour was found by Stefanik et al. (2015) who used a single IP injection of $600 \mathrm{mg} / \mathrm{kg}$ of VPA on GD 12. Both Cohen et al.'s and Stefanik et al.'s GD, administration, and dose were similar to methods of Schneider and Przewlocki (2005), thus the conflicting findings are not attributed to the administration factors. Further research is required to delineate ASD-like behaviours in animals prenatally exposed to VPA, which is included in the aims of this thesis, and will be explored in chapter two and three.

This brings to light the significant validity concerns relating to the VPA administration method, as well as the lack of consideration of the genetic contribution to this animal model. An essential element of this model is the administration of VPA. As identified earlier, the validity of an animal model is based on the construct, face, and predictive validity. Currently, prenatal exposure to VPA results in ASD-like behaviours, which supports the face validity of this model. However, the construct validity is weaker, because women typically ingest VPA daily throughout pregnancy, while the current animal model typically uses a single dose. Therefore, to enhance the construct validity of the VPA-induced ASD animal model, improvements must be made to the administration methods as well as the consideration for the genetic component. Both of these factors will be targeted in this thesis.

\subsubsection{Effects of Multiple Prenatal Exposures to VPA}

Some researchers have attempted to enhance the construct validity of the VPA-ASD model by delivering multiple VPA doses during gestation. Chapman and Cutler (1984) administered $160-180 \mathrm{mg} / \mathrm{kg} /$ day of VPA in drinking water to mice throughout gestation and lactation periods. Intriguingly, the offspring exhibited no behavioural changes compared with controls until adulthood, when the offspring showed increased social investigation. This finding is the opposite of the majority of the behavioural results from single VPA exposure offspring, although there some evidence to suggest that a single VPA administration can increase social behaviour (Cohen et al., 2013; Stefanik et al., 2015). It is, therefore, important to more systematically investigate the long-term effect of repeated VPA exposure during pregnancy. Interestingly, the repeated administration of VPA in the drinking water also resulted in 
epigenetic changes and dysregulation of neuronal function (Fujimura, Mitsuhashi, Shibata, Shimozato, \& Takahashi, 2016; Semmler et al., 2017), which aligns with findings from ASD populations.

Administering multiple doses of VPA has also been accomplished using other ingestion methods. During gestation, Main and Kulesza (2017) delivered and VPA (800mg/kg) mixed with peanut butter to pregnant Sprague-Dawley rats on GD 10 and GD 12 and found that VPAexposed rats weighed less, with smaller brains, and exhibited significant deficits in motor coordination tasks (such as rotorod, beam walking, and gait patterns). The authors suggest that disrupted cerebellar development underlies these deficits in coordination. Upon examining the cerebellum, they found that VPA exposed animals had significantly smaller Purkinje cells which are the cells responsible for motor coordination in the cerebellum. Further, mice exposed to multiple oral of VPA doses on GD 12 to 14, this resulted in neurogenesis deficits and abnormal hippocampal morphology but the anxiety and cognitive measures showed little difference between VPA exposed and controls (Juliandi et al., 2015). However, these authors did not study social communication or social interaction.

Intraperitoneal injections are an alternative method to chronically administered VPA. Sabers, Bertelsen, Scheel-Kruger, Nyengaard, and Moller (2014) used IP administration of $20 \mathrm{mg} / \mathrm{kg} /$ day or $100 \mathrm{mg} / \mathrm{kg} /$ day of VPA to pregnant Wistar rats from GD 12.5 to GD 21. Treatment also continued for the mothers during the lactation period until PND 23. Sabers et al. (2014) found that offspring prenatally and postnatally exposed to VPA had increased neocortical cells and increased cortical thickness, which suggests that VPA exposure significantly disrupts cortical growth and organisation, alongside disruptions in the cerebellum. More recently, Bertelsen et al. (2017) administered 20 or $100 \mathrm{mg} / \mathrm{kg} /$ day of VPA via IP injections to Wistar rats from GD 12 to birth (and did not continue treatment), and found that prepubescent male rats exposed to $20 \mathrm{mg} / \mathrm{kg} /$ day engaged in play behaviour significantly less than controls, however, there were no differences between controls and $100 \mathrm{mg} / \mathrm{kg} / \mathrm{day}$. This finding is at odds with the findings of a single administration of VPA during gestation. The authors suggest that $100 \mathrm{mg} / \mathrm{kg} /$ day from GD 12 to birth, activates biochemical changes (histone deacetylation, DNA alterations, and increased GABA activity) that increase (or normalise) abnormal behaviour. Further, another multiple dose study administered VPA via IP injection between GD 11 to 13 resulted in no sociability changes in rats (Dendrinos, Hemelt, \& Keller, 2011). Overall, although far less studied, multiple VPA exposures (via drinking water, oral/digestion, and IPs) seems to lead to different (and even opposite) effects compared to a single injection (see chapter two, table 2.1). 
Given that women ingest VPA throughout pregnancy, and their children have a significantly increased risk of developing ASD, chronically exposed to VPA throughout gestation in rats would represent a much higher construct validity than a single administration. Therefore, in this thesis, a detailed investigation of the continuous prenatal exposure in rodents was undertaken.

\subsection{Chronic VPA Administration in Animals with Reduced SERT as an ASD Animal Model}

The VPA-ASD animal model has limited construct validity when examining ASD symptomology in animals, as there are significant factors that separate the animal model from the clinical situation in humans. While researchers who have administered multiple VPA doses have enhanced the construct validity of the model, the results in terms of face validity have been disappointing so far. Furthermore, the VPA model of ASD does not consider the influence of the genetic factor in the aetiology of ASD.

Therefore, I intend to advance the animal model of ASD using a gene $\times$ environment interaction method. With the accumulated evidence that implicates serotonin in ASD (as discussed in sections 1.4.1, 1.6.1.3, and 1.7.3), I will make use of the SERT knock-out rat model, in particular, the wild-type and heterozygous $\left(\mathrm{SERT}^{+/}\right.$) genotype animals. SERT ${ }^{+/-}$have a $40-$ $60 \%$ reduction in the SERT function, similar to the levels seen in humans with the short allele of the 5-HTTLPR, which has been associated with ASD (as reviewed in section 1.6.1.4; Lesch \& Gutknecht, 2005). Furthermore, the SERT genetic background will be combined with chronic administration of VPA throughout gestation (see section 1.8.3).

More specifically, pregnant SERT ${ }^{+/+}$rats that were previously mated with SERT ${ }^{+/}$, will receive oral VPA doses for the 21 days of gestation. It is hypothesised that the genetic susceptibility (SERT ${ }^{+-}$donated by the father) will interact with the prenatal exposure to VPA. This interaction will result in an in vivo model that more closely aligns with the clinical picture of the disorder identified in humans. 


\subsection{Objectives of this Thesis}

This thesis investigates the hypothesis that offspring with genetically compromised SERT and exposed to VPA throughout gestation will exhibit significant behavioural and neuronal alterations akin to ASD in humans.

This hypothesis will be examined, firstly, by investigating the behavioural impacts of chronic prenatal VPA exposure in genetically normal rats (chapter two). Chapter two examines the efficacy of continuous administration of VPA throughout gestation, by investigating the communication and social behaviour over the lifespan of the male and female offspring. It is hypothesised that prenatal exposure to the high dose of VPA will result in reduced communication and social behaviour, particularly for the males. Such that, the males exposed to $300 \mathrm{mg} / \mathrm{kg} /$ day will exhibit reductions in communication and social behaviour, beyond that of male exposed to the control dose.

Secondly, the results of chapter two will be expanded to examine the behavioural impact of the gene $\times$ environment interaction with the use of SERT ${ }^{+-}$with prenatal chronic VPA exposure (chapter three). Chapter three examines the communication and social behaviour of male and female $\mathrm{SERT}^{+/+}$and SERT ${ }^{+/}$offspring that were exposed to VPA. It is hypothesised that the SERT reduction will interact with the prenatal VPA exposure, particularly for the males. Such that, the male SERT ${ }^{+-}$offspring exposed to a high dose will exhibit reductions in communication and social behaviour, beyond that of male SERT ${ }^{+/+}$exposed to VPA or male SERT $^{+/-}$exposed to the control dose.

Brain tissue from the offspring in the second study will then be used to examine inhibitory GABA interneurons, in the context of the gene $\times$ environment interaction $\left(\mathrm{SERT}^{+/-}\right.$by prenatal chronic VPA exposure; chapter four). Chapter four examines the GABA interneurons in the hippocampus and the amygdala of male and female SERT ${ }^{+/}$and SERT ${ }^{+/-}$offspring that were exposed to VPA. It is hypothesised that the SERT reduction will interact with the prenatal VPA exposure, particularly for the males. Such that, the male SERT ${ }^{+-}$offspring exposed to a high dose will exhibit reductions in the GABA interneurons in the hippocampus and the amygdala, beyond that of male SERT ${ }^{+/+}$exposed to VPA or male SERT ${ }^{+/-}$exposed to the control dose. 


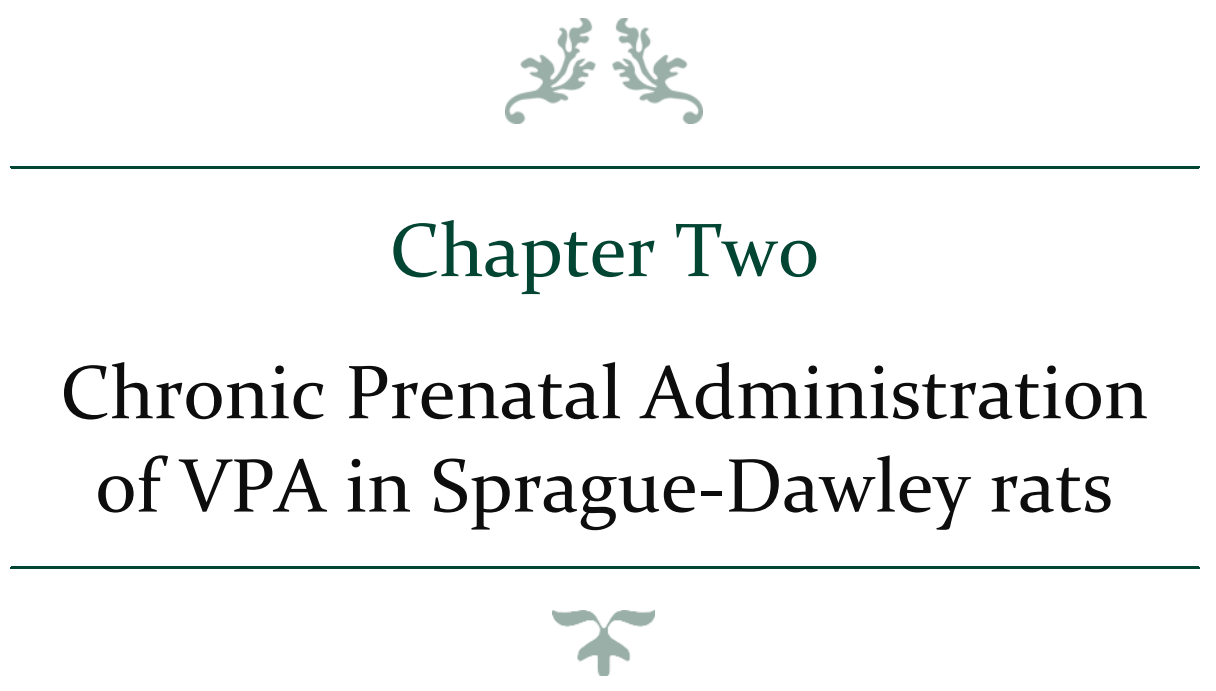




\section{Chapter Two Contents}

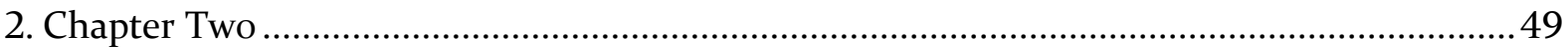

2.1.2 Overall aims of Chapter Two ....................................................................... 51

2.1.3 Experimental animals ................................................................................. 52

2.2 Experiment One: Isolation induced Ultrasonic Vocalisations .................................. 54

2.2.2 Isolation induced USV methods .................................................................... 56

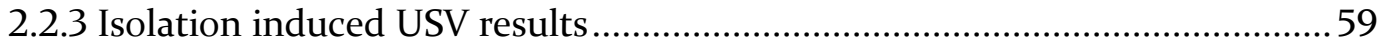

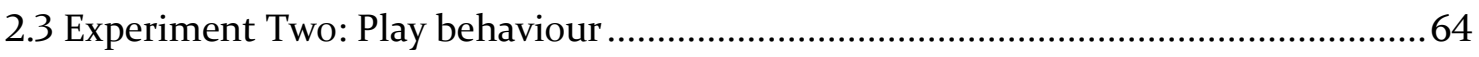

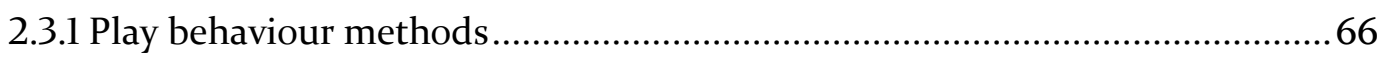

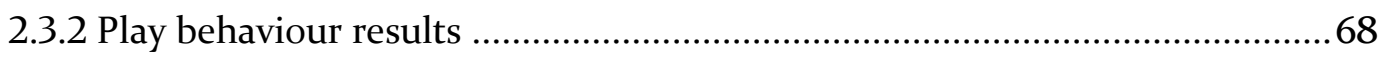

2.4 Experiment Three: Social Approach and Avoidance .................................................. 73

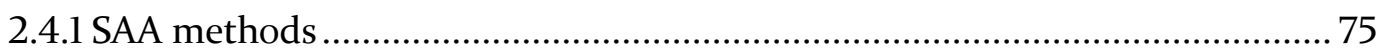

2.4.2 SAA results for pre-puberty rats ................................................................. 78

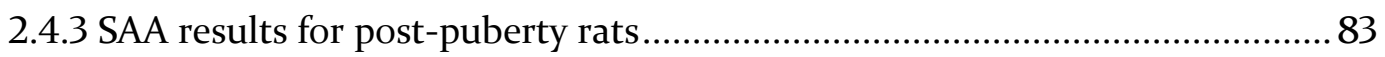

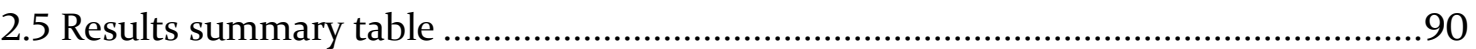

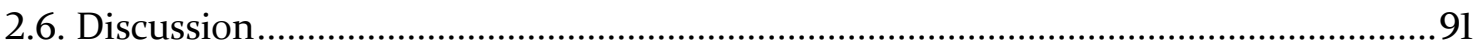

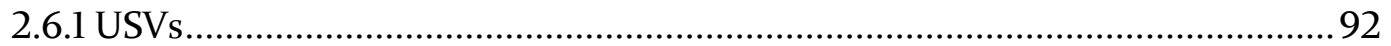

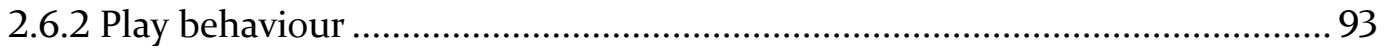

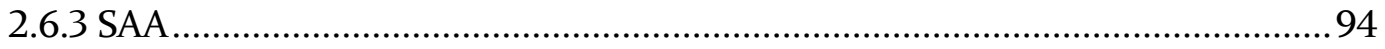

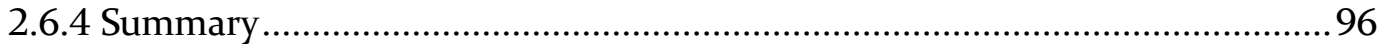




\section{Chapter Two}

The relationship between in utero exposure to VPA and ASD in humans led to the development of an in vivo animal model of ASD (Rodier et al., 1997). The typical method for generating this model is to inject VPA into pregnant rats, halfway through gestation (as assessed by Kim et al., 2011). Using this method Schneider and Przewlocki (2005) showed that the offspring exposed to VPA on GD 12.5 demonstrated deficits in social behaviours, repetitive movements and hyperactivity with reduced exploration when compared with control animals. Similar to the findings of Schneider and Przewlocki (2005), investigations into the VPA-induced animal model of ASD in our lab have demonstrated significant reductions in the social investigation of novel social stimuli and increased anxiety. In particular, VPA exposed rats exhibit decreased social behaviour (Ranger, 2016), as well as increased anxiety-like behaviour including an increased time in the closed arms of the elevated plus maze and increased latency to consume novel food in the novelty suppressed feeding test (Ellenbroek et al., 2016). Together, the data suggest that the VPA model has high face validity as an effective model for ASD.

As explored in chapter one (section 1.8.3), the construct validity of VPA-induced animal model for ASD is weakened by the disparity in administration methods of VPA. That is, a woman would ingest VPA throughout her pregnancy, which increases the risk of her child developing ASD (Christensen et al., 2013). Yet, the prenatal exposure to VPA for animals is commonly a single dose, and often through IP injection. Thus, the actions that lead to ASD in humans following prenatal exposure to VPA and the methods used in animal models do not align. As such, this chapter will explore the efficacy of continuous VPA exposure throughout gestation for rats.

Previous research has attempted to reduce this disparity between human and animals by repeatedly exposing pregnant females to VPA (see table 2.1), with a variety of administration methods, days of exposure, as well as the overall findings. As explored in chapter one (section 1.8.3.2), the behavioural findings from multiple prenatal exposures to VPA are inconsistent. For examples, Chapman and Cutler (1984) and Wellmann, Varlinskaya, and Mooney (2014) found increased social behaviour, Bertelsen et al. (2017) found decreased social behaviour, whereas Dendrinos et al. (2011) found no change. On the other hand, single exposure to VPA has more consistently lead to decreased social behaviour (Dufour-Rainfray et al., 2010; Gandal et al., 2010; Markram et al., 2008; Olde Loohuis et al., 2015; Schneider \& Przewlocki, 2005; Schneider et al., 2008). Together, these data suggest that repeated or continuous VPA may lead to different (if 
not opposite) effects when compared to a single injection. Given that clinically, mothers ingest VPA throughout pregnancy, a more thorough investigation of the continuous prenatal exposure in rodents is warranted.

Table 2.1. The findings of studies which used multiple prenatal VPA exposure in rodents, including VPA doses and administration routes.

\begin{tabular}{|c|c|c|c|c|c|}
\hline Reference & Species & $\begin{array}{l}\text { VPA dose } \\
\mathrm{mg} / \mathrm{kg} / \text { day }\end{array}$ & $\begin{array}{l}\text { Admin } \\
\text { Route }\end{array}$ & $\begin{array}{c}\text { GD } \\
\text { exposure }\end{array}$ & Findings \\
\hline $\begin{array}{l}\text { Chapman } \\
\text { \& Culter } \\
(1984)\end{array}$ & Mice & $160-180$ & $\begin{array}{l}\text { Drinking } \\
\text { water }\end{array}$ & $\begin{array}{l}\text { Conception } \\
\text { to birth (\& } \\
\text { lactation to } \\
\text { weaning) }\end{array}$ & $\begin{array}{l}\uparrow \text { Social investigation in } \\
\text { adulthood }\end{array}$ \\
\hline $\begin{array}{l}\text { Frisch et } \\
\text { al. (2009) }\end{array}$ & Rats & 825 & $\begin{array}{l}\text { Drinking } \\
\text { water }\end{array}$ & $\begin{array}{l}\text { Conception } \\
\text { to birth }\end{array}$ & $\begin{array}{l}\downarrow \text { Water maze spatial memory } \\
\downarrow \text { Rotorod motor } \\
\text { coordination }\end{array}$ \\
\hline $\begin{array}{l}\text { Dendrinos } \\
\text { et al. } \\
\text { (2011) }\end{array}$ & Rats & 200 & $\begin{array}{c}\text { IP } \\
\text { injections }\end{array}$ & 11 to 13 & No social behaviour changes \\
\hline $\begin{array}{l}\text { Sabers et } \\
\text { al. }(2014)\end{array}$ & Rats & 20 or 100 & $\begin{array}{c}\text { IP } \\
\text { injections }\end{array}$ & $\begin{array}{l}12 \text { to } 21(\& \\
\text { lactation to } \\
\text { weaning) }\end{array}$ & $\begin{array}{l}\uparrow \text { Neocortical cells } \\
\uparrow \text { Cortical thickness }\end{array}$ \\
\hline $\begin{array}{l}\text { Wellmann } \\
\text { et al. } \\
\text { (2014) }\end{array}$ & Rats & 200 & $\begin{array}{c}\mathrm{IP} \\
\text { injections }\end{array}$ & 12 to 13 * & $\begin{array}{l}\downarrow \text { Number of USVs } \\
\uparrow \text { Locomotor activity } \\
\uparrow \text { Play behaviour }\end{array}$ \\
\hline $\begin{array}{l}\text { Juliandi et } \\
\text { al. (2015) }\end{array}$ & Mice & 300 & Oral $^{* *}$ & 12 to 14 & $\begin{array}{l}\downarrow \text { Learning and memory } \\
\downarrow \text { Contextual and fear } \\
\text { associations } \\
\uparrow \text { HDAC inhibition }\end{array}$ \\
\hline $\begin{array}{l}\text { Fujimura } \\
\text { et al. } \\
(2016)\end{array}$ & Mice & $\begin{array}{l}\text { Not } \\
\text { specified } \\
* * *\end{array}$ & $\begin{array}{l}\text { Drinking } \\
\text { water }\end{array}$ & $\begin{array}{l}\text { Conception } \\
\text { to birth }\end{array}$ & $\begin{array}{l}\uparrow \text { Epigenetic changes } \\
\text { Altered neuronal } \\
\text { proliferation }\end{array}$ \\
\hline $\begin{array}{l}\text { Bertelsen } \\
\text { et al. } \\
(2017)\end{array}$ & Rats & 20 or 100 & $\begin{array}{c}\text { IP } \\
\text { injections }\end{array}$ & 12 to 21 & $\begin{array}{l}\downarrow \text { Play behaviour for pre- } \\
\text { pubescent male exposed to } \\
20 \mathrm{mg} / \mathrm{kg} / \text { day }\end{array}$ \\
\hline $\begin{array}{l}\text { Semmler } \\
\text { et al. } \\
(2017)\end{array}$ & Rats & $500 \& 825$ & $\begin{array}{l}\text { Drinking } \\
\text { water }\end{array}$ & $\begin{array}{l}\text { Conception } \\
\text { to birth }\end{array}$ & $\begin{array}{l}\uparrow \text { Hippocampal neurons } \\
\downarrow \text { Astrocytes } \\
\text { No epigenetic changes }\end{array}$ \\
\hline $\begin{array}{l}\text { Kinjo et } \\
\text { al. (2019) }\end{array}$ & Rats & 100 or 200 & $\begin{array}{c}\text { IP } \\
\text { injections }\end{array}$ & 12 to birth & $\begin{array}{l}\uparrow \text { Locomotor activity } \\
\uparrow \text { Time in open arms of EPM } \\
\uparrow \text { Hippocampal proliferation }\end{array}$ \\
\hline
\end{tabular}

* Wellmann et al. (2014) administered an additional dose on GD 12 (pm). ** Juliandi et al. (2015) did not specify exact oral administration methods. ${ }^{* * *}$ Fujimura et al. (2016) administered VPA at a concentration of $0.4 \%$ in the drinking water, achieving VPA plasma levels of 10 to $60 \mu \mathrm{g} / \mathrm{ml}$ in the dams. ' $\uparrow$ ' denotes increase, and ' $\downarrow$ ' decrease from controls. 


\subsubsection{Overall Aims of Chapter Two}

This chapter investigates the efficacy of administering VPA continuously throughout gestation and examines the behavioural phenotypes of the offspring. The VPA doses of 0,100 , $200,300 \mathrm{mg} / \mathrm{kg} /$ day were selected, as previous research had shown this would avoid potentially fatal effects for the dam and the offspring, which occurs at $400-800 \mathrm{mg} / \mathrm{kg}$ (Vorhees, 1987). The selected doses permit an investigation of dose-response alterations in the behaviour of the offspring. Furthermore, sweetened condensed milk (SCM) was selected as the vehicle of administration for the VPA doses. SCM provides a method for delivering VPA to dams every day of gestation while minimising stress and using a method that aligns with human ingestion of oral doses. In a pilot study, female rats were more likely to consume diluted (50\% water) SCM in a ceramic dish in the home cage, over SCM delivered in a syringe or peanut butter delivered in a ramekin or syringe (data not shown).

The overall aim of this series of experiments is to assess the communication and social behaviours of rats prenatally exposed to VPA throughout gestation. Further to this, the experiments are intended to examine a dose-response relationship in the behavioural deficits. Thus, it is predicted that:

i. Males and females prenatally exposed to VPA will exhibit decreased communication and sociability, compared with males and females prenatally exposed to the control dose,

ii. The decreased behaviours will differ as a function of prenatal VPA dose, such that the greatest deficits will be exhibited by males and females exposed to $300 \mathrm{mg} / \mathrm{kg} /$ day compared with those exposed to 100 , or $200 \mathrm{mg} / \mathrm{kg} /$ day,

iii. Finally, the decreased behaviour will be persistent over the lifespan of the offspring. 


\subsubsection{Experimental Animals}

Nine adult males and 21 adult nulliparous female Sprague-Dawley rats were randomly selected from stock at VUW vivarium. Five days prior to mating, all females were handled by the experimenter for 15 minutes and exposed to Sweetened Condensed Milk (SCM) in a ramekin for 30 minutes. On the sixth day, one female and one male were paired in polycarbonate cages which included a metal grid lining the bottom of the cage. The grid enabled the detection of a vaginal mucus plug. Plugs were checked within 24 hours after pairing, and each day following for a further 10 days. If no mucus plug was identified, the female was returned to the animal stock. When a mucus plug had been detected, the male and female were separated. The male was then placed in group housing for later pairing or euthanised; the female was randomly allocated into a dose condition of $0,100,200$, or $300 \mathrm{mg} / \mathrm{kg} /$ daily of VPA, weighed then housed individually to raise their own litters until postnatal day (PND) 21. Day one of gestation was the day the mucus plug was detected, and also the first delivery of SCM containing a dose of VPA (see section 2.1.5 VPA administration for further details).

The pregnant females were weighed twice a week to adapt the VPA dosage for weight increases due to foetal growth. Additional weights were not taken to prevent inducing unnecessary stress or handling during gestation. Females were checked for delivery every day. The day of birth was termed PND 1. At PND 21, the dam was removed from the cage and euthanised. The offspring were separated in sex- and litter-specific groups until they weighed approximately $150-200 \mathrm{~g}$, at which point they were housed in groups of 2 or 3 in each cage.

\subsubsection{Housing Conditions for All Animals}

The housing environment was maintained at a temperature of $21^{\circ} \mathrm{C}( \pm 2)$ and a humidity level of 55-60\%, with reversed night/day cycle (lights on 1900-0700 hours). Animals were housed in conventional wire top cages $(44 \mathrm{~cm} \times 28 \mathrm{~cm} \times 21 \mathrm{~cm})$ which were lined with pine or paper bedding (with the exception of metal grids lining the tray bottom for breeding pairs). Breeding pairs and lactating dams had ad libitum access to water and speciality laboratory chow (Speciality Feeds Rat and Mouse Cubes, Glen Forest, Aus). The stock and offspring animals had ad libitum access to water and laboratory chow (Diet 86, Sharpes Stock Feed). All animals were bred and housed at the VUW vivarium. All procedures followed the VUW Animal Ethics guidelines (Animal Ethics Committee number 000022477). 


\subsubsection{VPA Administration}

The regularly updated females' weights were used to tailor the VPA dose. Each day of gestation, the experimenter combined a diluted mixture of SCM $(1.5 \mathrm{ml})$ and water $(1.5 \mathrm{ml})$ with the individually tailored dose of VPA in a ramekin. The ramekin was placed in the female's home cage for 30 minutes, after which time the ramekins were collected and cleaned to be prepared for the next dose.

\subsubsection{Experimental Animal Breeding Outcomes}

Twenty-one females were bred in this study, 19 of them producing a litter. There were no fatalities of the dams, and there were no significant differences in the number or gender of pups born (data not shown). A total of 205 offspring were born. However, one dam who had received $100 \mathrm{mg} / \mathrm{kg} /$ day produced three blind pups. Furthermore, two pups from a $100 \mathrm{mg} / \mathrm{kg} /$ day litter, and one pup from a $200 \mathrm{mg} / \mathrm{kg} /$ day litter, were still-born. For the surviving offspring, there were no identifiable physical abnormalities, such as tail kinks. Such abnormalities were expected following previous reports (Edalatmanesh, Nikfarjam, Vafaee, \& Moghadas, 2013; Vorhees, 1987) 


\subsection{Experiment One: Isolation Induced Ultrasonic Vocalisations}

Ultrasonic vocalisations (USVs) are an optimal way to measure communication in rats, as it is non-invasive and minimises the stress on the animal. Vocalisations made by rats are broadly classified into three classes of vocalisations, which are defined by the frequencies of 22 kilohertz (kHz), 50kHz and 40kHz (Brudzynski, 2005). $22 \mathrm{kHz}$ vocalisations commonly occur in the range of $18-32 \mathrm{kHz}$ (Portfors, 2007). While the $50 \mathrm{kHz}$ vocalisations occur between 35 and $70 \mathrm{kHz}$ (Knutson, Burgdorf, \& Panksepp, 2002). Both 22kHz and 50kHz calls are emitted by adult rats and mice (Portfors, 2007). The $22 \mathrm{kHz}$ calls are identified as relating to distress or negative states because these vocalisations are commonly emitted following foot stock, drug withdrawal, and restraint. In comparison, $50 \mathrm{kHz}$ vocalisations are associated with positive states as these calls are emitted in response to, or anticipation of a reward; such as being tickled, and anticipation of a food reward. Vocalisations are affected by an animal's age, environment, and affective state (Portfors, 2007). The 40kHz (roughly between 40 and $65 \mathrm{kHz}$ ) vocalisations are commonly emitted by pre-weaning pups and are identified as distress calls (Brudzynski, Kehoe, \& Callahan, 1999). The distress calls are a response to separation from the dam as well as loss in body temperature (Hahn \& Lavooy, 2005), and these $40 \mathrm{kHz}$ calls will be examined in this experiment.

Pre-weaning rodents will produce the largest number of USVs in the first 21 days of life, with the highest number of vocalisations produced around the seventh day (Branchi, Santucci, \& Alleva, 2001). Socially isolating offspring is known to induce vocalisations, typically eliciting a behavioural response from the dam (e.g. pup retrieval or returning the pup to the nest). Wöhr and Schwarting (2008) used a playback experiment, where exposing a dam to $40 \mathrm{kHz}$ vocalisations led to the dam demonstrating searching behaviours which was not seen when a $40 \mathrm{kHz}$ tone or white noise was played. This supports the notion that $40 \mathrm{kHz}$ calls are a signal to the dam from pre-weaning pups that are isolated or in distress.

As of 2019, research on the pre-weaning vocalisations of offspring exposed to VPA is very limited; however, it has been shown to decrease USVs. Gandal et al. (2010) administered $600 \mathrm{mg} / \mathrm{kg}$ of VPA on GD 13 to pregnant mice and found their offspring had a reduction in the number of USVs. Additionally, Servadio et al. (2018) found that rats exposed to 400 and $500 \mathrm{mg} / \mathrm{kg}$ on GD 12, made fewer vocalisation on PND 9, but showed no difference at PND 5. As identified in these studies, the isolation-induced USVs provide an early measure of developmental changes. This forms the first experiment with two measures of USVs taken at PND 7 and 14 for the offspring continuously exposed to VPA throughout gestation. 
Continuing with the overall aims of this chapter (section 2.1.2), this experiment examines the quantity and quality of the USV communication in pre-weaning pups that were prenatally exposed to VPA throughout gestation. With the pre-weaning pups, the detection of sexes was not clear, and for the first experiment, there is no sex separation in the analyses. Nevertheless, it was predicted that;

i. The pre-weaning pups prenatally exposed to VPA will exhibit decreased communication (number of calls, length, and other measures), compared with pups prenatally exposed to the control dose,

ii. The decreased communication will differ as a function of prenatal VPA dose, such that the greatest deficits will be exhibited by those exposed to $300 \mathrm{mg} / \mathrm{kg} /$ day compared with those exposed to 100 , or $200 \mathrm{mg} / \mathrm{kg} /$ day,

iii. Finally, the decreased communication exhibited by pups prenatally exposed to VPA will be consistent from PND 7 to PND 14. 


\subsubsection{Isolation Induced USV methods}

\subsubsection{USV Apparatus}

An UltraMic (250K 16 bi; Java Sound) microphone was used to record the vocalisations of the offspring. This microphone was suspended above an isolation container lined with clean bedding. A Dell processor laptop was used to operate the Raven Pro software (Cornell University, Ithaca, NY) which detected and recorded the vocalisations for 300 seconds.

\subsubsection{USV Procedure}

On PND 7 and 14, pups were randomly selected from the litter and transported into the testing room. During the testing phase, the dam remained with the litter. Transport was in a cylindrical container lined with clean bedding. When the pup and container were in place under the microphone, the 300-second recording of the vocalisations was initiated. Afterwards, the pup was taken out of the cylinder, weighed, and a general health observation was made (eyeopening, ear unfolding and digit separation, data not shown). The pup was then marked with an indelible marker to prevent selection of the same pup when it was returned to the litter. The pup was placed back in the cylinder and transported to the housing room and returned to the litter and dam. The next pup was then selected, and the process was repeated until 6 to 8 pups had been recorded. At the end of the recording experiment, all remaining pups were weighed, checked for health and marked with an indelible marker.

\subsubsection{USV Data Analysis}

In the analysis of the USVs, the Raven Software was manually adjusted for clear detection of vocalisations. As analysis required the spectrogram file, the window size of 840 points was selected because it achieves the balance between computer processing and visibility of the vocalisations. A randomised selection of recordings was taken from the overall sample to keep consistency across all doses. Data was collected through manual inspection of the recorded USVs. Observation and selection were completed with the use of the spectrogram file, which enabled the selection of individual vocalisations (see figure 2.1). 


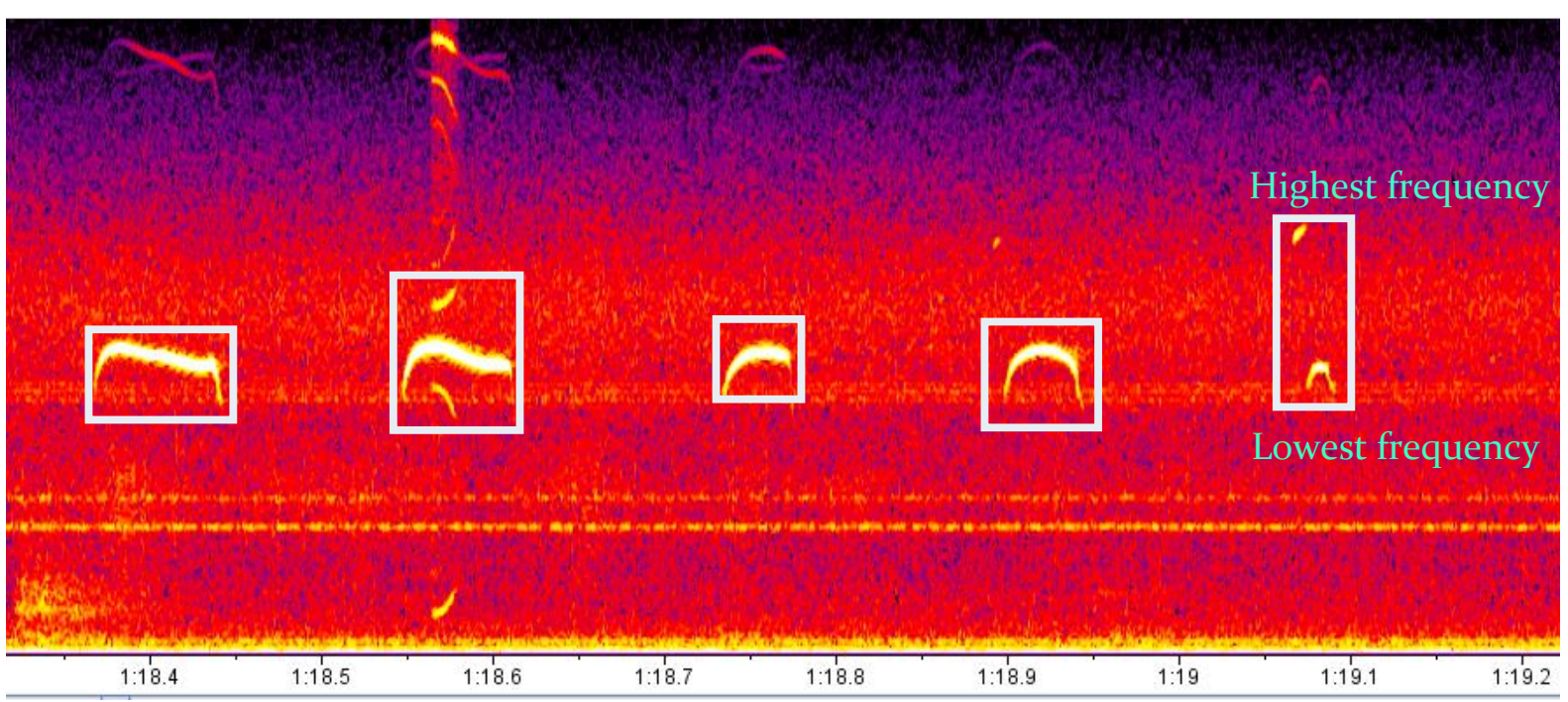

Figure 2.1. Representative spectrograph displaying the sonographic features of five vocalisations emitted by a pup exposed to $100 \mathrm{mg} / \mathrm{kg} /$ day (litter ID 3.C). The last call shows the limits of the highest and lowest frequency of that call.

Once all visible and identifiable vocalisations had been made (such as those presented in figure 2.1), USVs were measured using several parameters. For the analysis of vocalisations the number of vocalisations (count), duration (seconds), low and high frequency (Hz), and overall frequency range of vocalisations $(\mathrm{Hz}$; a measure of the change between low and high in frequency within a single call) was used. It is useful to note that frequency is the pitch of the sound.

The USV parameters were examined with a 4 (Prenatal dose: $0 \mathrm{mg} / \mathrm{kg} / \mathrm{day}$, $100 \mathrm{mg} / \mathrm{kg} /$ day, $200 \mathrm{mg} / \mathrm{kg} /$ day, 300mg/kg/day) as the between-subject factor; by 2 (PND: 7, 14) as the within-subjects factor, repeated measures ANOVA. A Greenhouse-Geisser correction was used on all measures due to violations of sphericity. The repeated measures ANOVAs were used to analyse the number, duration, low and high frequencies as well as the range in frequencies of vocalisations over time.

All data were analysed for statistical significance with SPSS (version 22-25). Partial eta squared $\left(\eta_{p}{ }^{2}\right)$ was used for the effect size. All significant analyses were followed up with Tukey HSD post hoc tests, and/or planned comparisons were used to follow up potential effect comparing specific prenatal dose or genotype conditions with others using Student's t-test. The alpha level for statistical significance was set at $p<.05$.

\subsubsection{Sample Selection for Comparative Analysis}

All USV recordings had unique identifiers which detailed the recording order (from 1 to 8) and litter, which allowed the recordings to PND 7 to be matched with PND 14. The data was 
selected from 18 offspring of each prenatal dose condition, resulting in a total of 72 recordings which had corresponding analysis. The total number of recordings were reduced due to statistical outliers, data corruption, and to ensure the PND 7 and PND 14 corresponding data points.

More specifically for the control dose litters, there were 17 recordings from five litters and one recording was removed due to data corruption. There were 16 recordings from five litters exposed to $100 \mathrm{mg} / \mathrm{kg} /$ day, two recordings were removed due to outliers. For the $200 \mathrm{mg} / \mathrm{kg} /$ day, there were 13 recordings from three litters, no calls were presented in four recordings and another recording was an outlier. Finally, 13 recordings from three litters exposed to $300 \mathrm{mg} / \mathrm{kg} /$ day, three recordings were removed due to discrepancies in pup identifiers and two recordings were significant outliers.

Please note, a recording was considered to be an outlier when the average value/measurement was three times the mean of the condition. For example, of the $100 \mathrm{mg} / \mathrm{kg} /$ day litters, two recordings were removed as their values/averages were three times higher or lower than the average for the remaining recordings of the $100 \mathrm{mg} / \mathrm{kg} /$ day litters. As such, outliers were determined based on the mean value of the variables being three times the mean of the prenatal dose condition. 


\subsubsection{Isolation Induced USV Results}

\section{Number of vocalisations emitted on PND7 compared with PND 14}

The number of vocalisations decreased from PND 7 to $14\left(F(1,55)=22.09, p=.001, \eta_{\mathrm{p}}{ }^{2}\right.$ $=.29$, see figure 2.2$)$, but there was no interaction with prenatal dose exposure $(P N D \times$ prenatal dose: $F(3,55)=.42, p=.741, \eta_{\mathrm{p}}{ }^{2}=.02$, ns; prenatal dose main effect: $F(3,55)=.18, p=.911, \mathrm{\eta}_{\mathrm{p}}{ }^{2}=$ $.01, n s)$.

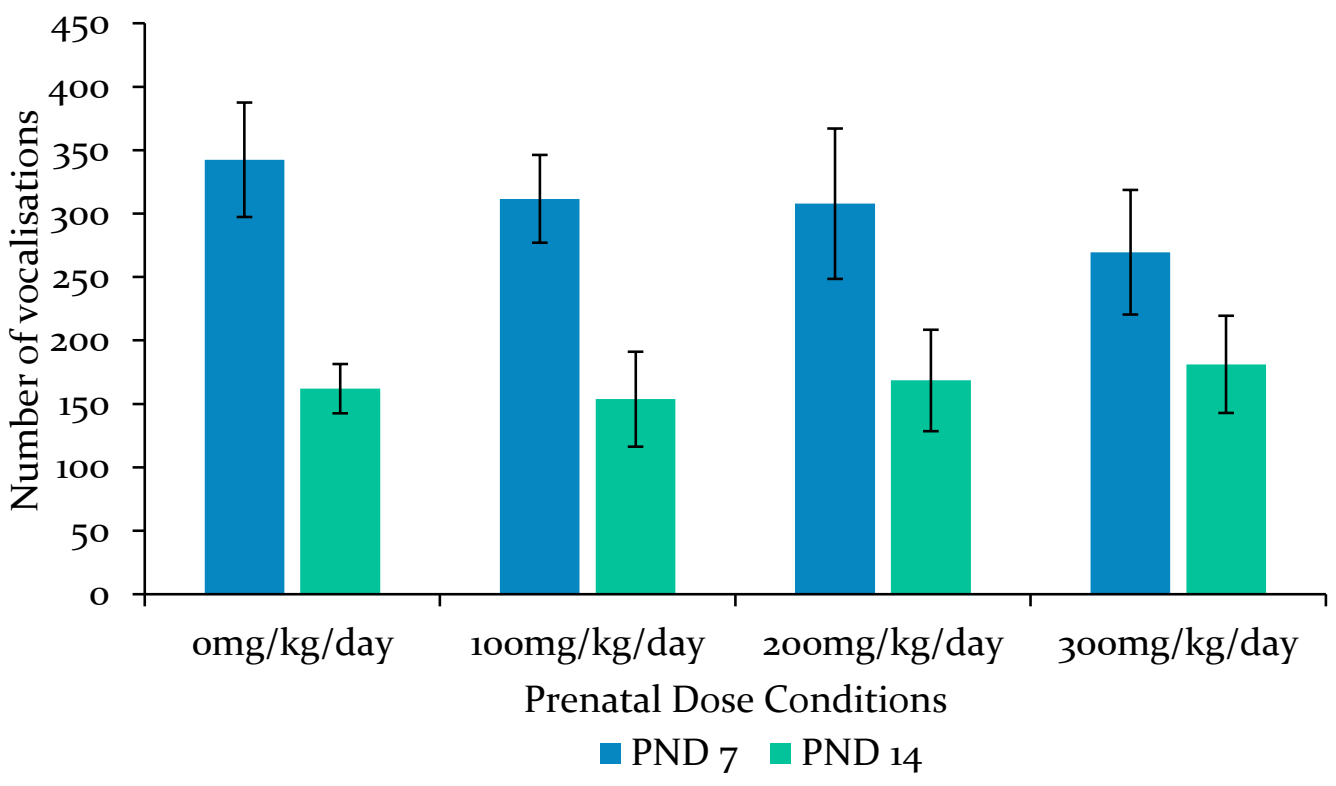

Figure 2.2. The average number of vocalisations emitted on PND 7 and 14 by pups prenatally exposed to $0 \mathrm{mg} / \mathrm{kg} /$ day, $100 \mathrm{mg} / \mathrm{kg} /$ day, $200 \mathrm{mg} / \mathrm{kg} /$ day, $300 \mathrm{mg} / \mathrm{kg} /$ day of VPA. Error bars represent \pm SEM. 


\section{Duration of vocalisations emitted on PND 7 compared with PND 14}

The length of the vocalisations increased from PND 7 to $14\left(F(1,55)=7.17, p=.010, \mathrm{\eta}_{\mathrm{p}}{ }^{2}\right.$ $=.12$, see figure 2.3$)$, but there was no interaction with prenatal dose exposure $(P N D \times$ prenatal dose: $\left.F(3,55)=1.39, p=.257, \eta_{\mathrm{p}}^{2}=.07\right)$. There was a non-significant trend toward an effect for the prenatal doses (prenatal dose main effect: $F(3,55)=2.37, p=.081, \eta_{\mathrm{p}}{ }^{2}=.11, n s$ ). The follow up analysis showed that the vocalisations of pups exposed to the $300 \mathrm{mg} / \mathrm{kg} /$ day dose emitted vocalisations that were significantly shorter at PND 7 than PND 14 ( $p=.008$, see figure 2.3$)$, and this was the only prenatal dose condition to show a significant effect.

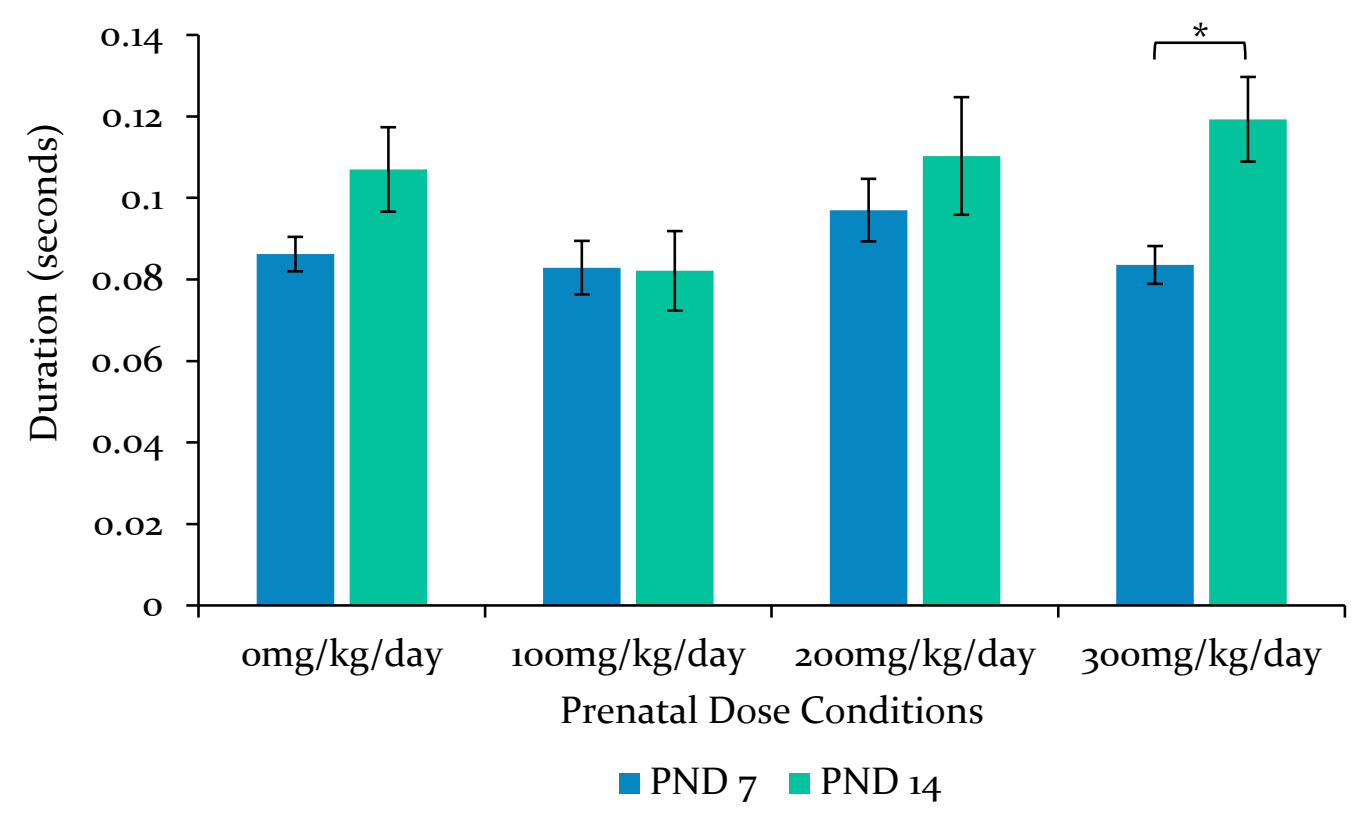

Figure 2.3. The average duration of vocalisation emitted on PND 7 and 14 by pups prenatally exposed to $0 \mathrm{mg} / \mathrm{kg} /$ day, $100 \mathrm{mg} / \mathrm{kg} /$ day, $200 \mathrm{mg} / \mathrm{kg} /$ day, $300 \mathrm{mg} / \mathrm{kg} /$ day of VPA. Pups exposed to the $300 \mathrm{mg} / \mathrm{kg} /$ day doses emitted significantly longer vocalisations on PND 14 compared to PND 7. * indicates significance to $p<.05$. Error bars represent \pm SEM. 


\section{Frequency of vocalisations emitted on PND 7 compared with PND 14}

There was a significant increase in the average lowest frequency from PND 7 to 14 ( $F(1$, $55)=33.96, p=.001, \mathrm{\eta}_{\mathrm{p}}{ }^{2}=.38$, see figure 2.4 ), and there was a significant interaction between the exposure to the prenatal doses the PND on lowest frequency $(P N D \times$ prenatal doses: $F(3,55)$ $\left.=4.61, p=.006, \eta_{\mathrm{p}}^{2}=.20\right)$, but no main effect of the prenatal doses $\left(F(3,55)=1.08, p=.367, \eta_{\mathrm{p}}^{2}\right.$ $=.06, n s)$.

The follow up analysis for the lowest frequency showed that pups exposed to $0 \mathrm{mg} / \mathrm{kg} /$ day and $100 \mathrm{mg} / \mathrm{kg} /$ day exhibited significant increases in the lowest frequency of their vocalisations from PND 7 to 14 ( $p=.004$, and $p=.001$, respectively), whereas, the pups exposed to $200 \mathrm{mg} / \mathrm{kg} /$ day and $300 \mathrm{mg} / \mathrm{kg} /$ day did not exhibit this increase $(p=.107$, and $p=.353$, ns, respectively).

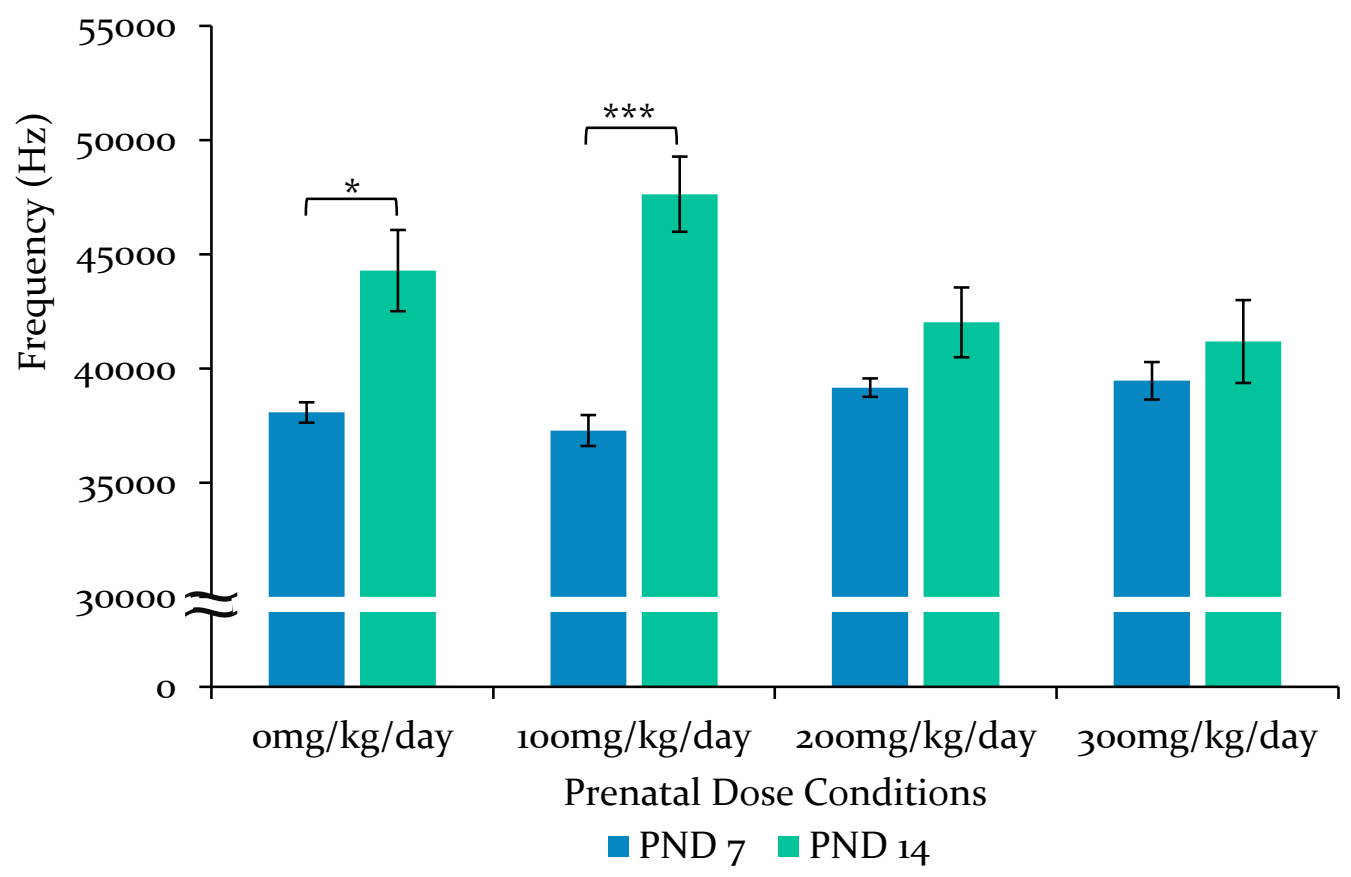

Figure 2.4. The average lowest frequencies of vocalisations emitted on PND 7 and 14 by pups prenatally exposed to $0 \mathrm{mg} / \mathrm{kg} /$ day, $100 \mathrm{mg} / \mathrm{kg} /$ day, $200 \mathrm{mg} / \mathrm{kg} /$ day, $300 \mathrm{mg} / \mathrm{kg} /$ day of VPA. The shortened $y$-axis shows the small difference in the low frequency of the $200 \mathrm{mg} / \mathrm{kg} / \mathrm{day}$ and $300 \mathrm{mg} / \mathrm{kg} /$ day from PND 7 to PND 14 . * indicates significance to $p<.05$, *** indicates significance to $p=.001$. Error bars represent \pm SEM. 
There was a significant increase in the average highest frequency from PND 7 to 14 ( $F(1$, $55)=52.98, p=.001, \eta_{\mathrm{p}}{ }^{2}=.49$, see figure 2.5$)$, and there was a significant interaction between the exposure to the prenatal doses the PND on highest frequency $(P N D \times$ prenatal doses: $F(3$, $\left.55)=4.54, p=.007, \eta_{\mathrm{p}}^{2}=.20\right)$, but no main effect of the prenatal doses $(F(3,55)=.82, p=.490$, $\left.\eta_{\mathrm{p}}^{2}=.04, n s\right)$.

The follow up analysis for the average highest frequency showed that pups exposed to $0 \mathrm{mg} / \mathrm{kg} /$ day, $100 \mathrm{mg} / \mathrm{kg} /$ day and $200 \mathrm{mg} / \mathrm{kg} /$ day exhibited significant increases in the highest frequency of their vocalisations from PND 7 to 14 ( $p=.001, p=.001$, and $p=.029$, respectively), whereas, the pups exposed to $300 \mathrm{mg} / \mathrm{kg} /$ day did not exhibit this increase $(p=.115, n s)$.

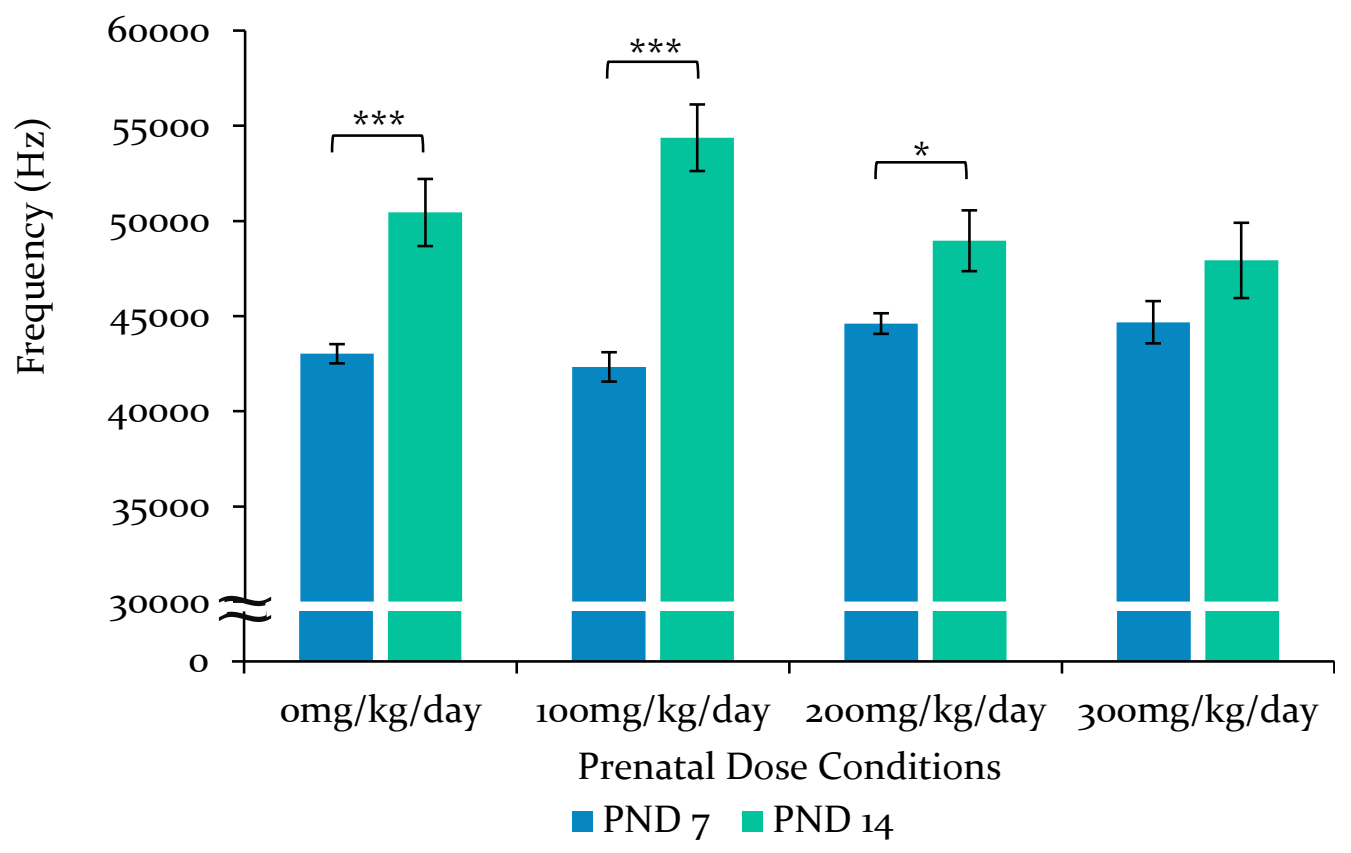

Figure 2.5. The average highest frequencies of vocalisations emitted on PND 7 and 14 by pups prenatally exposed to $0 \mathrm{mg} / \mathrm{kg} /$ day, $100 \mathrm{mg} / \mathrm{kg} /$ day, $200 \mathrm{mg} / \mathrm{kg} /$ day, $300 \mathrm{mg} / \mathrm{kg} /$ day of VPA. The shortened y-axis shows the small difference in the high frequency of the $300 \mathrm{mg} / \mathrm{kg} / \mathrm{day}$ from PND 7 to PND 14. * indicates significance to $p<.05$, ${ }^{* * *}$ indicates significance to $p=.001$. Error bars represent \pm SEM. 
The final measure of frequency is the overall frequency range of vocalisations. There was a significant increase in the range of frequency from PND 7 to $14\left(F(1,55)=50.66, p=.001, \eta_{\mathrm{p}}^{2}\right.$ $=.48$, see figure 2.6 ), but there was no significant interaction between the exposure to the prenatal doses the PND $\left(P N D \times\right.$ prenatal doses: $\left.F(3,55)=.26, p=.851, \mathrm{\eta}_{\mathrm{p}}{ }^{2}=.01, n s\right)$, and no main effect of the prenatal doses $\left(F(3,55)=1.33, p=.274, \mathrm{n}_{\mathrm{p}}^{2}=.07, n s\right)$.

Overall, the frequency measures extend the common count and duration measures, and demonstration that offspring prenatally exposed to VPA exhibit communication changes.

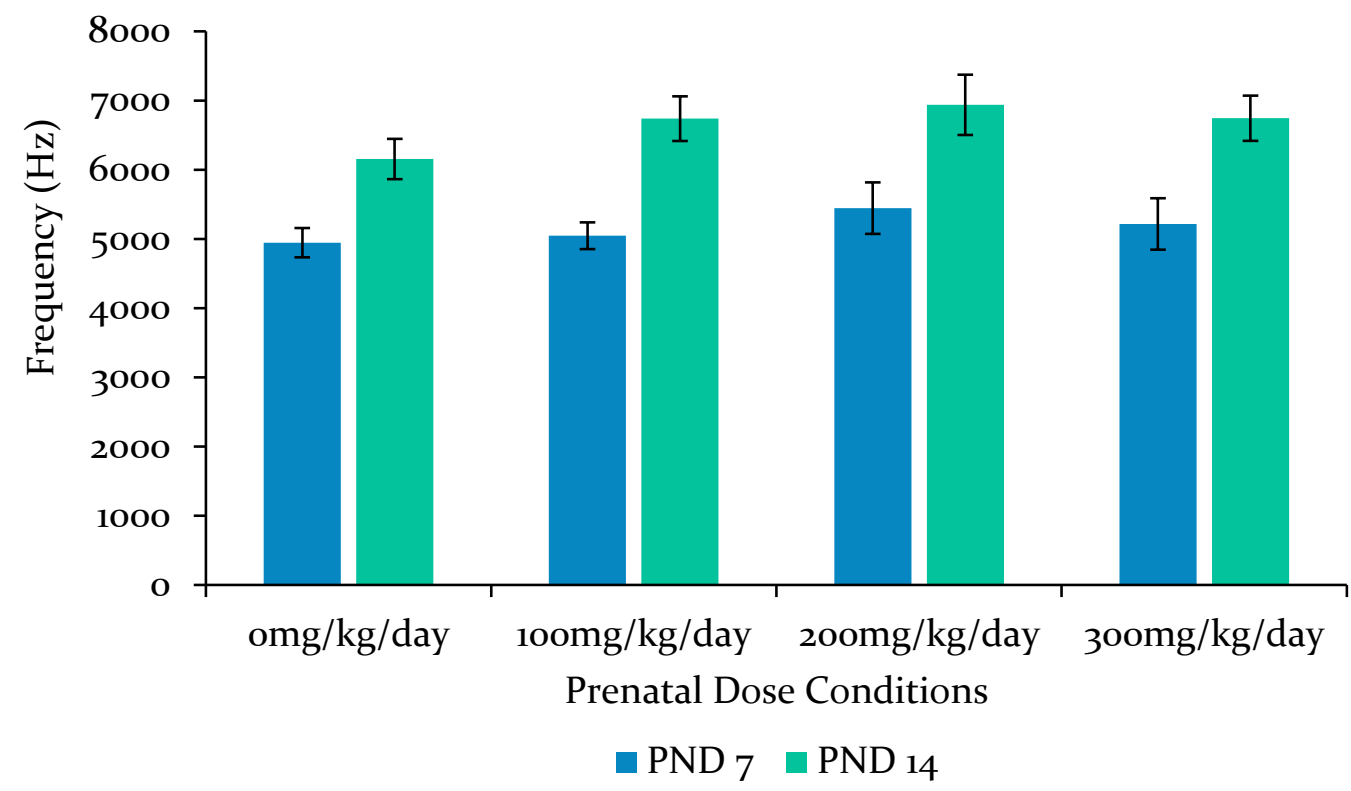

Figure 2.6. The average range of frequency for vocalisations emitted on PND 7 and 14 by pups prenatally exposed to $0 \mathrm{mg} / \mathrm{kg} /$ day, $100 \mathrm{mg} / \mathrm{kg} /$ day, $200 \mathrm{mg} / \mathrm{kg} /$ day, $300 \mathrm{mg} / \mathrm{kg} /$ day of VPA. All offspring emitted vocalisations with a larger ranger frequency at PND 14. Error bars represent \pm SEM. 


\subsection{Experiment Two: Play Behaviour}

The purpose of this experiment was to investigate ASD-like deficits in social interaction with the measure of play behaviour of juvenile offspring that were prenatally exposed to VPA.

Social play behaviour deficits exhibited by rats are often in response to pharmacological or environmental manipulations (e.g., O'Shea, McGregor, \& Mallet, 2006; Sams-Dodd, 1995). Rats are highly social species, and social play is vital to cognitive and social development (Meng, Li, Han, Shao, \& Wang, 2010; Van den Berg et al., 1999). Thus, deficits in social play behaviour are indicative of changes to cognitive and social development.

ASD-like behaviour is exhibited by rats prenatally exposed to VPA. Wang et al. (2013) prenatally exposed rats to a single dose of $500 \mathrm{mg} / \mathrm{kg}$ of VPA on GD 12.5 and these animals engaged in social behaviour for a shorter period with fewer interactions, when compared with controls. Similarly, the deficits in social play behaviour have been replicated in other studies, which have used the same dose and PND as above (Markram et al., 2008; Olde Loohuis et al., 2015), as well as an increased dose $600 \mathrm{mg} / \mathrm{kg}$ on GD 12 (Sandhya, Sowjanya, \& Veeresh, 2012). However, other studies have shown increases in sociability (Cohen et al., 2013), or no difference between prenatally VPA exposed and control offspring (Narita et al., 2010). Therefore, the impact of prenatal exposure on play behaviour in adolescence has revealed inconsistent results.

The studies above have examined social play behaviour deficits by placing two conspecific animals together in an arena for a set time (5 to 15 minutes), and experimenters specify the onset and offset (code) the rats' specific behaviours. Social play behaviour was operationalised by Bolles and Woods (1964), which was further expanded on by Meaney and Stewart (1979) and continues to be developed. A commonly examined behaviour is pinning, (Panksepp \& Beatty, 1980; Thor \& Holloway, 1984; Trezza, Baarendse, \& Vanderschuren, 2010) which is where one animal is 'standing over' its play mate who has rotated to its dorsal surface. Another behaviour is pouncing, which is used to measure play initiation, where the initiating rat attempts to make contact with the neck of the play partner, typically approaching from behind (Vanderschuren, Niesink, \& Van Pee, 1997). Further to this, a commonly observed behaviour is chasing, where one animal follows another to initiate or evade a play sequence (Grant, 1963; Trezza et al., 2010). Pinning, pouncing, and chasing behaviours have clear onset and ending, and provide objective measures of social play behaviour (Panksepp \& Beatty, 1980). Other behaviours such as boxing or wrestling, as well as less active behaviours like social grooming and sniffing, have also been used to examine social investigation behaviour (Vanderschuren et al., 1997) 
Overall, measuring animals' social behaviour is, technically, a relatively simple and effective measure for examining behavioural changes. Also, deficits in social interaction and communication are integral to the diagnosis of ASD. Thus it is crucial to examine the social interaction in the animal model for ASD, and previous studies have found reduced social play behaviour (Markram et al., 2008; Olde Loohuis et al., 2015; Sandhya et al., 2012; Wang et al., 2013).

Continuing with the overall aims of this chapter (section 2.1.2), this experiment examines the social play behaviour in juvenile offspring that were prenatally exposed to VPA throughout gestation. It was predicted that;

i. The juvenile male and female offspring prenatally exposed to VPA would exhibit decreased play behaviours (duration and frequency of pinning, chasing, wrestling and passive social behaviours), compared with pups prenatally exposed to the control dose, and

ii. The decreased play behaviour will differ as a function of prenatal VPA dose, such that the greatest reductions will be exhibited by those exposed to $300 \mathrm{mg} / \mathrm{kg} / \mathrm{day}$ compared with those exposed to 100 , or $200 \mathrm{mg} / \mathrm{kg} /$ day. 


\subsubsection{Play Behaviour Methods}

\subsubsection{Play Behaviour Apparatus}

This experiment took place in a testing arena $(40 \times 25 \times 30 \mathrm{~cm})$ made from black polycarbonate plastic. The arena was cleaned with $70 \%$ ethanol and paper towels, and then a thin layer of clean bedding was spread evenly over the bottom of the arena. Habituation and experimental testing were conducted under red light; the source was from a red-light bulb suspended 60 to $80 \mathrm{~cm}$ above the arena. All experimental testing was recorded with EthoVision $\left(\mathrm{XT}^{\mathrm{TM}}\right)$ software (Noldus, Netherlands). EthoVision allows the experimenter to score the animals' behaviour manually, then to determine the duration (time in seconds) and frequency (number of events) of those manually scored behaviours.

\subsubsection{Play Behaviour Procedure}

The following method was derived from Vanderschuren et al. (2008). After weaning on PND 21, same-sex littermates (within prenatal dose condition) were paired (denoted by corresponding tail marks with indelible marker). In their pairs, animals were habituated to the testing room for 10 minutes and then immediately habituated to the testing arena for 10 minutes. Finally, the animals were placed back in their home cage in the housing room. This process was repeated on PND 22 and 24.

On testing days (PND 23 and 25), in pairs animals were habituated to the room for 10 minutes, then the arena for 10 minutes, and then each animal was placed in a clean polycarbonate cage by themselves for 3.5 hours. The social isolation period increases the likelihood and motivation to engage in social play (Niesink \& Van Ree, 1989; Van Hasselt et al., 2012). The cages were kept in their housing room for this duration, and this served as a social deprivation period. Three hours and 20 minutes after the animals had finished their arena habituation, the isolated animals were placed in the testing room for 10 minutes. Then animals were paired and placed in the testing arena for 10 minutes to examine their behaviour. Following the test period, animals were weighed and checked for general health development, then returned their home cage in the housing room.

During the testing phase, the behaviours were manually scored by one experimenter, who observed and scored the onset and offset of pinning, chasing, wrestling, and passive social behaviours. A pinning behaviour was defined as one animal of the pair 'standing over' the other animal which had its dorsal side against the floor. Chasing was defined as following one rat following the other to initiate play. Wrestling (also incorporates boxing) was defined as both 
animals standing upright on the hind legs pawing at each other (boxing), as well as rolling or moving over each another (wrestling; Meaney \& Stewart, 1979). Finally, passive social behaviour was defined as the animals being in close contact without active social investigation (such as, sniffing or grooming themselves or the other; File \& Hyde, 1978).

\subsubsection{Play Behaviour Data Analysis}

The variables of interest were the duration and frequency of pinning, chasing, wrestling and passive social behaviour during the 10-minute test. The frequency measure is a count of every instance of the behaviour (such as pinning, or chasing) and the duration measure is the total length of time the behaviour, (e.g. the length of time that a pair were engaged in pinning).

Once all recordings were coded and extracted from EthoVision, SPSS (version 22-25) was used for statistical analysis. One-way 4(Prenatal dose; 0mg/kg, 100mg/kg, 200mg/kg, $300 \mathrm{mg} / \mathrm{kg}$ ) ANOVAs were run on the duration and frequency of pinning, chasing, wrestling, and passive social behaviours. These ANOVAs were run on the behavioural measures of the males separate from females.

All data were analysed for statistical significance with SPSS (version 22-25). Partial eta squared $\left(\mathrm{n}_{\mathrm{p}}^{2}\right)$ was used for the effect size. All significant analyses were followed up with Tukey HSD post hoc tests, and/or planned comparisons were used to follow up potential effect comparing specific prenatal dose or genotype conditions with others using Student's t-test. The alpha level for statistical significance was set at $p<.05$. 


\subsubsection{Play Behaviour Results}

With no difference occurring between PND 23 and 25, the analysis was pooled together testing days, then averaged over. Of the 142 trials, there were 42 trials from the $0 \mathrm{mg} / \mathrm{kg} / \mathrm{day}, 42$ from the $100 \mathrm{mg} / \mathrm{kg} /$ day offspring, 28 from the $200 \mathrm{mg} / \mathrm{kg} /$ day, and 30 from the $300 \mathrm{mg} / \mathrm{kg} /$ day. There were no eliminated trials.

Table 2.2. The number of play behaviour pairs recorded based on prenatal dose and sex, and separated by the postnatal day of recording. Grey cells refer to the sample sizes used in the following analysis.

\begin{tabular}{c|c|c|c|c|c|c}
\hline \hline & \multicolumn{2}{c|}{ PND 23 } & \multicolumn{2}{c|}{ PND 25 } & \multicolumn{2}{c}{ Total } \\
\cline { 2 - 7 } & Males & Females & Males & Females & Males & Females \\
\hline 0mg/kg/day & 11 & 10 & 11 & 10 & 22 & 20 \\
\hline $100 \mathrm{mg} / \mathrm{kg} /$ day & 11 & 11 & 10 & 10 & 21 & 21 \\
\hline $200 \mathrm{mg} / \mathrm{kg} /$ day & 8 & 6 & 8 & 6 & 16 & 12 \\
\hline $300 \mathrm{mg} / \mathrm{kg} /$ day & 8 & 7 & 8 & 7 & 16 & 14 \\
\hline \hline
\end{tabular}

\subsubsection{Pinning}

\section{Males pinning behaviour}

The prenatal doses almost had a significant effect on the duration of pinning for males $\left(F(3,71)=2.60, p=.059, \eta_{\mathrm{p}}^{2}=.10, n s\right)$. A planned comparisons analysis showed that males exposed to $200 \mathrm{mg} / \mathrm{kg} /$ day spent significantly more time engaged in pinning behaviours, compared with the control dose males ( $p=.048$, see figure 2.7 ). Interestingly, males exposed to $300 \mathrm{mg} / \mathrm{kg} /$ day showed increased pinning compared with the control dose males, although it missed significance $(p=.062, n s)$.

There was also a trend toward significance on the frequency of pinning behaviour for males prenatally exposed to VPA $\left(F(3,71)=2.54, p=.063, \mathrm{\eta}_{\mathrm{p}}{ }^{2}=.10, n s\right)$, data displayed in figure 2.7 (right panel). To follow up the non-significant trend, the analysis showed that the males exposed to $200 \mathrm{mg} / \mathrm{kg} /$ day had significantly more pinning events compared with the control dose males $(p=.022)$. 


\section{Females pinning behaviour}

There was a significant effect on the duration of pinning for females prenatally exposed to VPA $\left(F(3,63)=3.86, p=.013, \eta_{\mathrm{p}}^{2}=.16\right)$. The follow up analysis showed that the females exposed to $200 \mathrm{mg} / \mathrm{kg} /$ day and $300 \mathrm{mg} / \mathrm{kg} /$ day spent significantly more time engaged in pinning compared with $100 \mathrm{mg} / \mathrm{kg} /$ day ( $p=.049, p=.042$, respectively), however, there was no difference between VPA exposure and controls (see left graph in figure 2.7).

Furthermore, there was a significant effect on the number of pinning events that females engaged in $\left(F(3,63)=2.93, p=.040, \mathrm{n}_{\mathrm{p}}^{2}=.12\right.$, right panel in figure 2.7$)$. The post hoc analysis revealed non-significant differences, where the number of pinning events for females exposed to $300 \mathrm{mg} / \mathrm{kg} /$ day was increased (albiet not significantly) over the control dose females ( $p=$ $.084, n s)$ and the females exposed to $100 \mathrm{mg} / \mathrm{kg} /$ day $(p=.083)$.
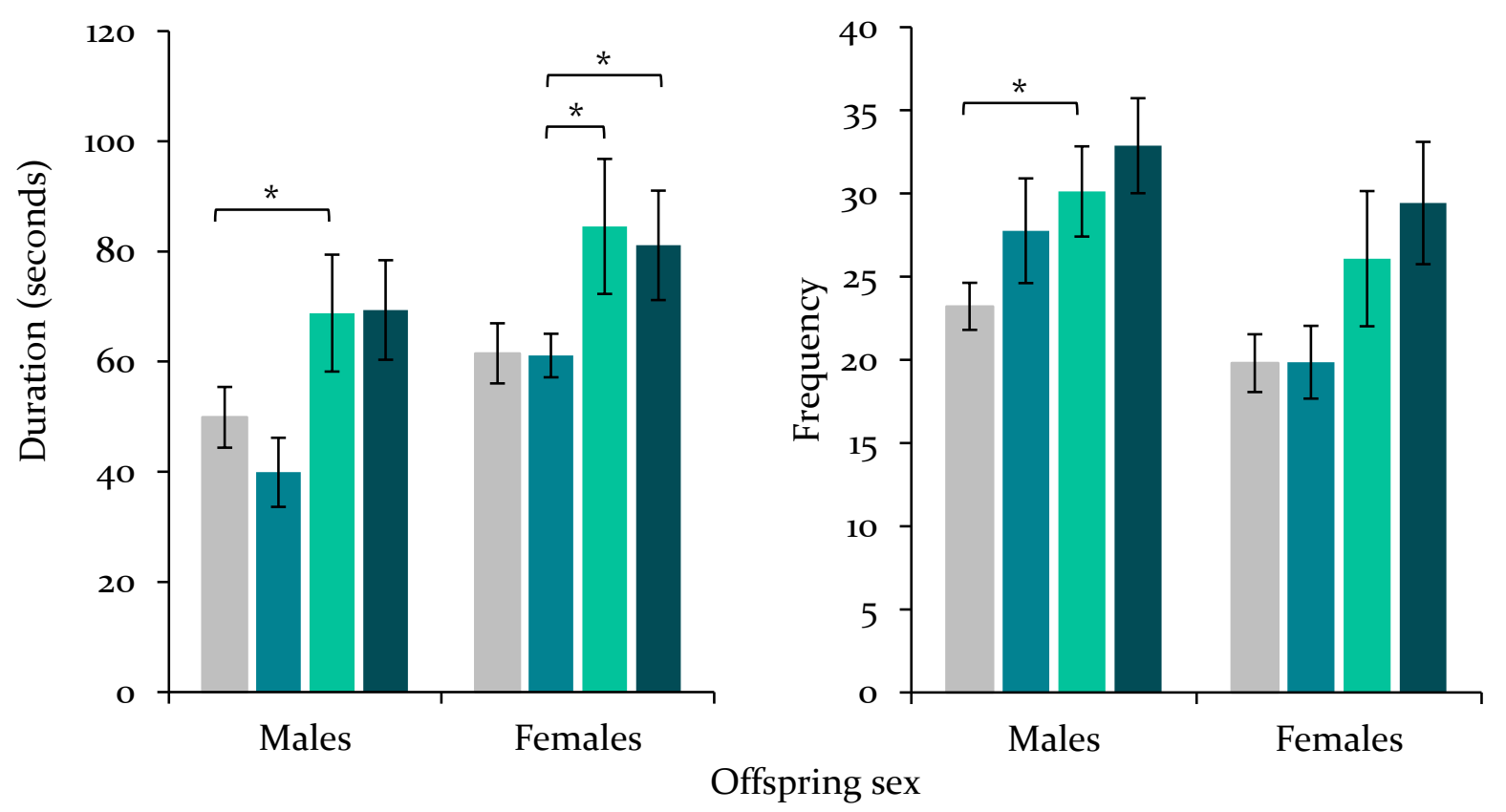

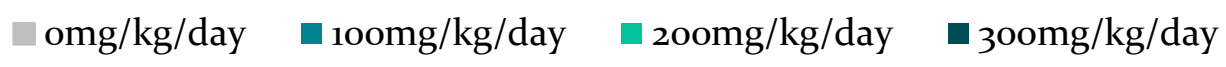

Figure 2.7. The average duration (left) and frequency (right) of pinning behaviours for the male and female offspring prenatally exposed to $0 \mathrm{mg} / \mathrm{kg} /$ day, $100 \mathrm{mg} / \mathrm{kg} /$ day, $200 \mathrm{mg} / \mathrm{kg} / \mathrm{day}$, $300 \mathrm{mg} / \mathrm{kg} /$ day of VPA. Displaying that juvenile males exposed to $200 \mathrm{mg} / \mathrm{kg} /$ day of VPA exhibit increased duration and frequency of pinning behaviours. ${ }^{*}$ indicates significance to $p<.05$. Error bars represent \pm SEM. 


\subsubsection{Chasing}

\section{Males duration and frequency of chasing}

The prenatal exposure to VPA had no overall effect on the duration of chasing behaviours for males $\left(F(3,71)=1.60, p=.196, \eta_{\mathrm{p}}^{2}=.06, n s\right)$. The same was also true for the number of chasing events for males $\left(F(3,71)=1.63, p=.190, \eta_{\mathrm{p}}^{2}=.06, n s\right.$, see figure 2.8$)$.

\section{Females duration and frequency of chasing}

The prenatal doses had no significant effect on the duration of chasing for females $(F(3$, $\left.63)=2.24, p=.09, \eta_{\mathrm{p}}^{2}=.10, n s\right)$, and there were no trends or effects revealed in the post hoc analysis. Although, there was a close to significant effect on the number of chasing events that females engaged in $\left(F(3,63)=2.53, p=.065, \mathrm{n}_{\mathrm{p}}^{2}=.11, n s\right.$, see figure 2.8$)$. The post hoc analysis showed that the close to significant effect on the frequency of chasing was due to a nonsignificant increase in females exposed to $300 \mathrm{mg} / \mathrm{kg} /$ day compared to the control dose $(p=$ $.094, n s)$.
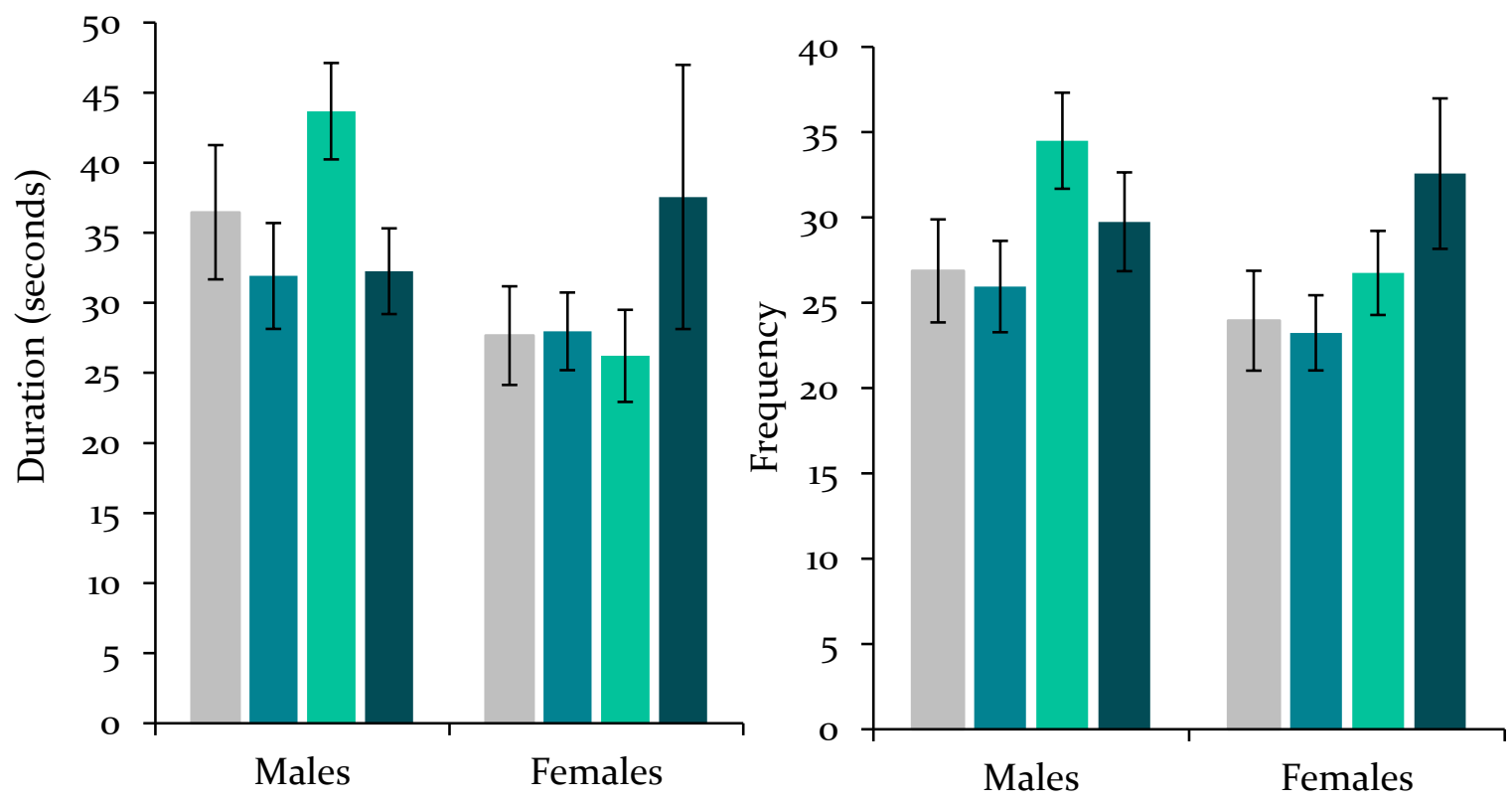

Offspring sex

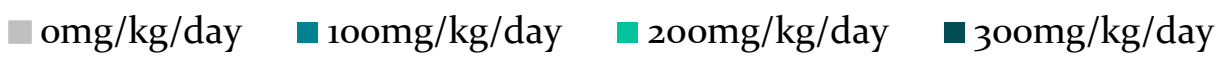

Figure 2.8. The average duration (left) and frequency (right) of chasing behaviours for the male and female offspring prenatally exposed to $0 \mathrm{mg} / \mathrm{kg} /$ day, $100 \mathrm{mg} / \mathrm{kg} / \mathrm{day}, 200 \mathrm{mg} / \mathrm{kg} / \mathrm{day}$, $300 \mathrm{mg} / \mathrm{kg} /$ day of VPA. Error bars represent \pm SEM. 


\subsubsection{Wrestling}

\section{Males duration and frequency of wrestling}

The prenatal exposure to VPA had no significant effect on the duration of wrestling behaviours for males $\left(F(3,71)=1.67, p=.181, \mathrm{\eta}_{\mathrm{p}}^{2}=.07, n s\right)$.

There was a significant effect of the prenatal doses on the frequency of chasing in males $\left(F(3,71)=3.46, p=.021, \eta_{\mathrm{p}}^{2}=.13\right)$. The post hoc analysis showed that males exposed to $300 \mathrm{mg} / \mathrm{kg} /$ day engaged in significantly more wrestling events than those in the control condition $(p=.019$, see figure 2.9$)$.

\section{Females duration and frequency of wrestling}

The prenatal exposure to VPA had no significant effect on the duration of wrestling behaviours for females $\left(F(3,63)=1.81, p=.154, \mathrm{\eta}_{\mathrm{p}}{ }^{2}=.08, n s\right)$, nor on the number of wrestling events for females $\left(F(3,63)=1.46, p=.235, \mathrm{n}_{\mathrm{p}}{ }^{2}=.07, n s\right.$, see figure 2.9$)$.

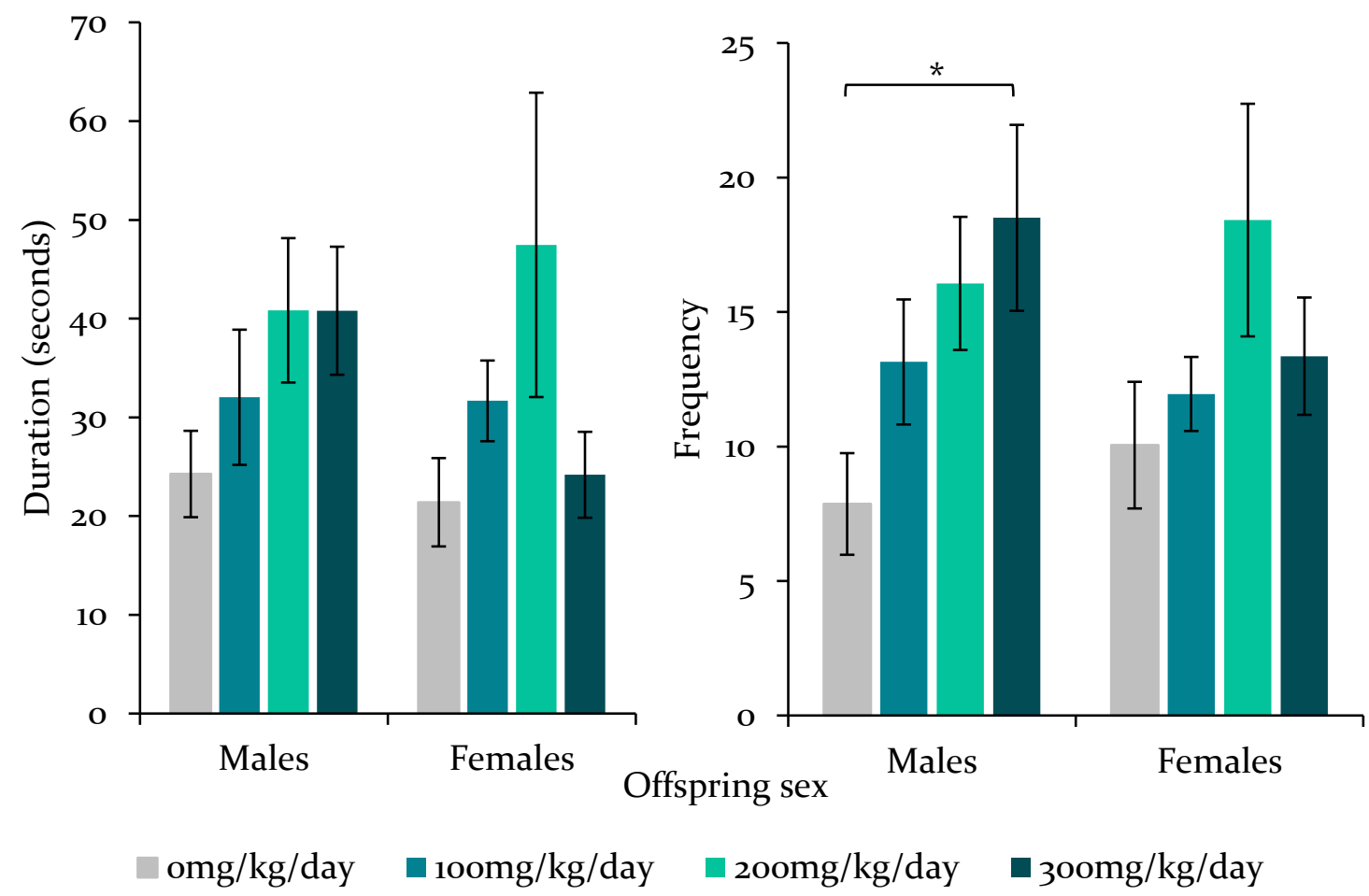

Figure 2.9. The average duration (left) and frequency (right) of wrestling behaviours for the male and female offspring prenatally exposed to $0 \mathrm{mg} / \mathrm{kg} /$ day, $100 \mathrm{mg} / \mathrm{kg} / \mathrm{day}, 200 \mathrm{mg} / \mathrm{kg} / \mathrm{day}$, $300 \mathrm{mg} / \mathrm{kg} /$ day of VPA, displaying the increased frequency of wrestling behaviour for males exposed to $300 \mathrm{mg} / \mathrm{kg} /$ day of VPA. ${ }^{*}$ indicates significance to $p<.05$. Error bars represent \pm SEM. 


\subsubsection{Passive Social Behaviour}

\section{Males duration and frequency of passive social behaviour}

The prenatal exposure to VPA had no significant effect on the duration of passive social behaviours for males $\left(F(3,71)=1.72, p=.171, \mathrm{n}_{\mathrm{p}}{ }^{2}=.07, n s\right)$, nor was there an effect of the prenatal doses on the number of passive social behaviours for males $\left(F(3,71)=2.11, p=.107, \mathrm{\eta}_{\mathrm{p}}{ }^{2}=.08, n s\right.$, see figure 2.10).

\section{Females duration and frequency of passive social behaviour}

The prenatal doses had a near to significant effect on the duration of passive social interaction for females $\left(F(3,63)=2.48, p=.069, \mathrm{n}_{\mathrm{p}}{ }^{2}=.11, n s\right)$. This non-significant effect is likely due to the decreased passive duration exhibited by the females exposed to $200 \mathrm{mg} / \mathrm{kg} / \mathrm{day}$, compared with the control dose females $(p=.102, n s)$. Additionally, there was no effect on the number of passive social interaction events that females engaged in $\left(F(3,63)=2.11, p=.107, \mathrm{\eta}_{\mathrm{p}}^{2}\right.$ $=.08, n s$, see figure $2 \cdot 10)$.
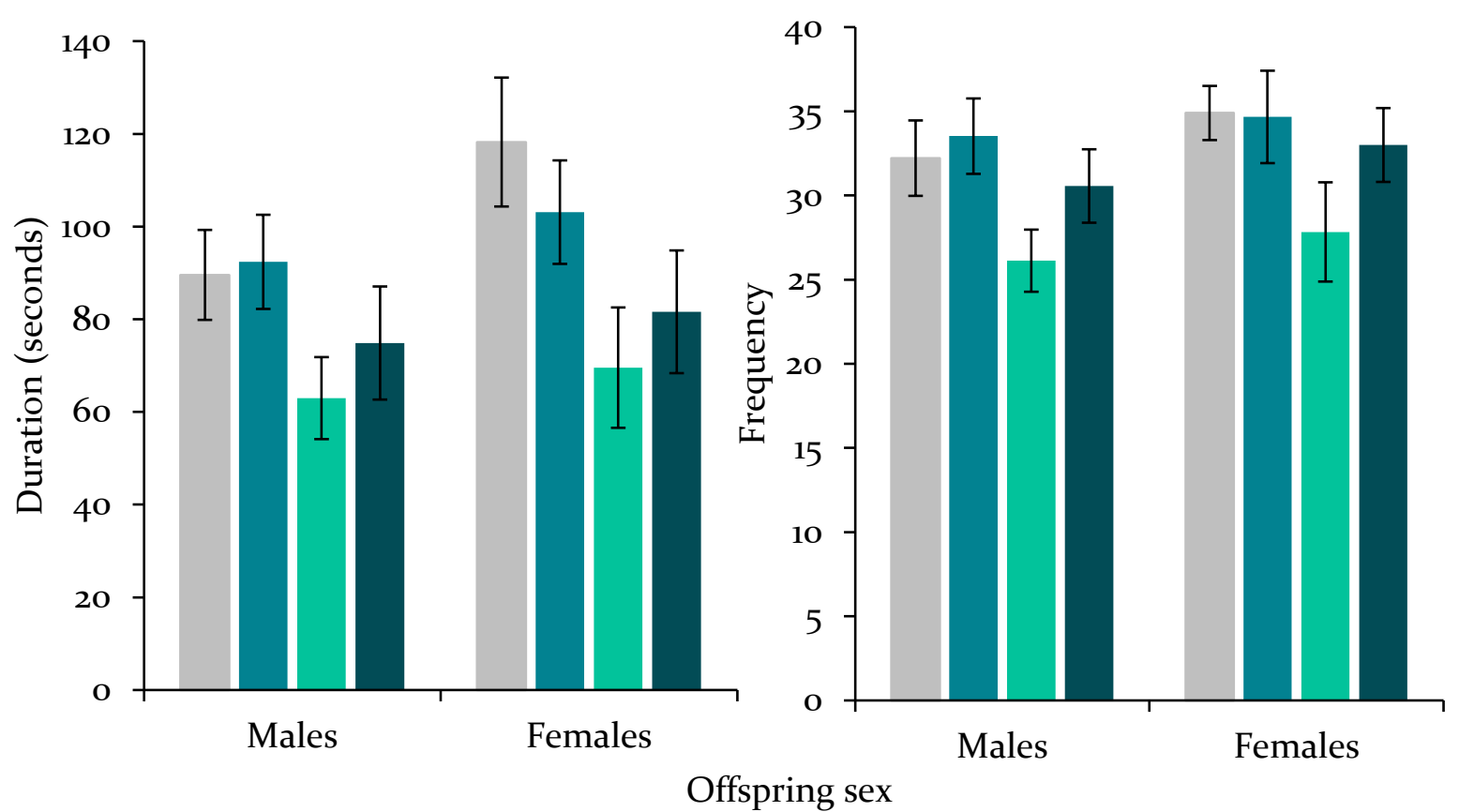

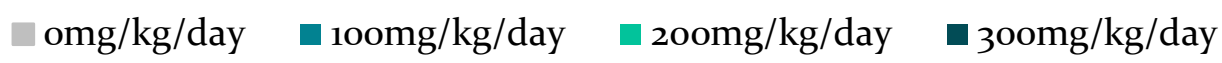

Figure 2.10. The average duration (left) and frequency (right) of passive social behaviours for the male and female offspring prenatally exposed to $0 \mathrm{mg} / \mathrm{kg} / \mathrm{day}, 100 \mathrm{mg} / \mathrm{kg} / \mathrm{day}$, $200 \mathrm{mg} / \mathrm{kg} /$ day, $300 \mathrm{mg} / \mathrm{kg} /$ day of VPA, no statistical effects detected. Error bars represent \pm SEM. 


\subsection{Experiment Three: Social Approach and Avoidance}

Rodents with social behaviour deficits are likely to avoid or delay interaction with novel partners. These behaviours can be examined through the social approach and avoidance paradigm, also known as the three-chamber test, which was originally established by Nadler et al. (2004). This paradigm allows for the investigation of an animal's social behaviour without direct influence from the conspecific's behaviour and more accurate detection of low levels of sociability (Crawley, 2004), making this a valid paradigm for the measurement of ASD-like behaviours in rodents.

Social Approach and Avoidance (SAA) measures the proximity of a freely moving test animal to restricted conspecifics, which act as social stimuli. The test is divided into different phases. Phase one measures the tendency of the test animal to approach and interact with a social stimulus. This phase allows the test animal to develop social recognition and familiarity with the social stimulus (Kogan, Frankland, \& Silva, 2000). Due to the highly social nature of rodents, rats typically spend more time interacting with the social than the non-social stimulus. Phase two measures social novelty. Here, the time the test animal spends with the social stimulus from the familiar phase is compared to the time spent with a newly introduced social stimulus. The animal has the freedom to interact with either familiar and novel stimulus or can spend time alone in the central zone. Because of the social familiarity established in the first phase, the preference for social novelty over familiarity can be assessed. Phase two animals prefer novel stimuli, and most control animals will spend more time with the novel social stimulus than the familiar one.

The face validity of this test has led to its extensive use when examining the behavioural alterations in rodents prenatally exposed to VPA. A single injection of VPA during mid-gestation leads to animals spending less time with the novel stimulus and increased time in the non-social areas or with the familiar stimulus (Bambini-Junior et al., 2011; Kerr, Downey, Conboy, Finn, \& Roche, 2013; Kim et al., 2014; Kim et al., 2011). However, these results were not uniformly replicated. Stefanik et al. (2015) exposed rats to $600 \mathrm{mg} / \mathrm{kg}$ of VPA on GD 12.5 and measured SAA at PND 25, 46, and 76. They found that VPA exposed rats spent more time in proximity to the stranger rat and were quicker to interact. It is of note that Stefanik et al.'s SAA test differed from the methods established by Nadler et al. (2004) and Moy et al. (2008). The social stimulus used by Stefanik et al. was an adult male from the parents' generation, and a second phase was not implemented for comparison of social novelty. An age disparity between the test animal and the social stimulus may have impacted the approach behaviours of the test animal, and thus, 
the findings must be interpreted with caution. While Stefanik et al.'s methodology was different from the procedure used in the following experiment, it demonstrates that prenatal exposure to VPA impacts the offsprings' social interaction behaviour.

In addition to the Stefanik et al. (2015) findings, Dufour-Rainfray et al. (2010) also found that offspring of rats exposed to $600 \mathrm{mg} / \mathrm{kg}$ of VPA on GD 9 spend significantly more time with the novel social stimulus in adulthood. Dufour-Rainfray et al. (2010) methodology also differed from the typical protocol, as they did not divide the test into two phases. Instead, in a single trial, the authors used littermates as familiar social stimulus and animals from different litters as unfamiliar stimuli. Thus, differences in the protocol may have affected the outcome of the social approach-avoidance tests.

As ASD is a neurodevelopmental disorder with symptoms present from a young age and persisting into adulthood, we would expect to see ASD-like behaviours in animals persisting throughout life. However, this trajectory is rarely investigated. Stefanik et al. (2015) investigated age effects of VPA exposure by measure SAA at weaning, puberty and adulthood. They found a significant effect of age, such that weaning aged offspring were quicker to interact with the social stimulus than pubescent and adult controls. The lifespan trajectory will be examined in this experiment, by comparing the rats' sociability scores from prepuberty to postpuberty, which will allow the investigation of persistence of abnormal behaviours.

Continuing with the overall aims of this chapter (section 2.1.2), this experiment examines the social behaviour in the SAA assay of pre- and post-pubescent offspring that were prenatally exposed to VPA throughout gestation. To examine if the ASD-like behaviours are consistent pre- and post-puberty, the SAA experiment was run twice. The pre-puberty measure was taken on PND 30, and the post-puberty measure was taken on PND 60. It was predicted that:

i. The male and female offspring prenatally exposed to VPA will exhibit decreased social novelty compared with offspring prenatally exposed to the control dose,

ii. The decreased social novelty will differ as a function of prenatal VPA dose, such that the greatest reductions will be exhibited by those exposed to $300 \mathrm{mg} / \mathrm{kg} / \mathrm{day}$ compared with those exposed to 100 , or $200 \mathrm{mg} / \mathrm{kg} / \mathrm{day}$, and

iii. The decreased social novelty will be consistent pre- and post-puberty. 


\subsubsection{SAA Methods}

\subsubsection{SAA Apparatus}

Testing occurred in a T-maze $(116 \mathrm{~cm} \times 90 \mathrm{~cm} \times 40 \mathrm{~cm})$ made from black polycarbonate plastic. Before the onset of each individual test, the arena was cleaned with $70 \%$ ethanol and paper towels, which was allowed to dry before the next trial. All experimental testing was recorded with EthoVision $\left(\mathrm{XT}^{\mathrm{TM}}\right)$ software, which was used to manually score the animal's behaviour and track each animal's nose, body and tail during the session.

\subsubsection{SAA Procedure}

On PND 30, a test animal was selected from its home cage, transferred into a temporary cage and placed in the experimental room for 10 minutes to habituate to the environment. Animals serving as conspecifics (experimentally naive Sprague-Dawley juveniles aged between PND 20-25 selected from the breeding colony) were also moved into the experimental room before the trials to allow for habituation to the environment.

The test animal was placed in the T-maze for 10 minutes with an empty wire mesh cylinder in each arm. After 10 minutes, the test animal was removed from the apparatus and placed in a temporary cage, while a conspecific juvenile was placed under a cylinder. The placement of the first juvenile alternated for each test animal. The test animal was returned to the arena for 10 minutes to interact with the juvenile enclosed in the cylinder (phase one, see figure 2.11). The test animal was removed again from the arena, and another conspecific juvenile was enclosed under the opposite cylinder. With the addition of the second conspecific juvenile, the first conspecific juvenile becomes known as the 'familiar' social stimulus, and the newly added conspecific becomes the 'novel' social stimulus, indicating that the test animal has had time to familiarise itself to the first conspecific but not the second.

Finally, the test animal was returned the T-maze for a 10-minute test session (phase two, see figure 2.11). During this phase, the animal was tracked for its location in the arena. Upon completion of this session, test animals were weighed and returned to their home cage. The conspecifics were returned to a temporary cage if more sessions were to occur in the day or they were returned to their home cage. The arena was cleaned with ethanol, and the next test animal was placed in the experimental room for habituation. Phase two is the critical phase for the assessment of familiarity and novelty, and due to an experimental error, phase one was not recorded this experiment. Therefore, data extraction and analysis were exclusively performed from phase two. 

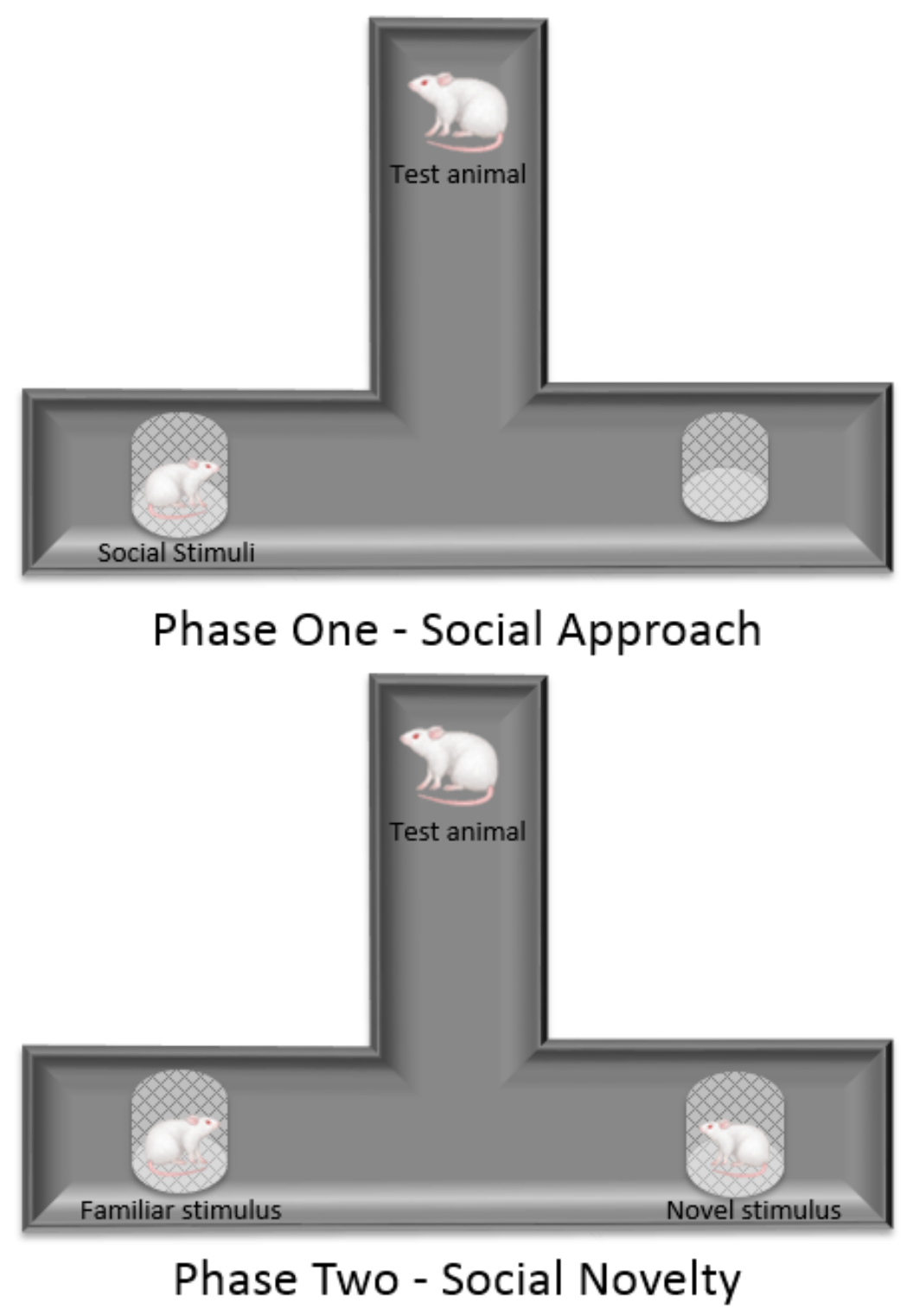

Figure 2.11. T-maze set up for the SAA paradigm. Depicting phase one and two with the placement of familiar and novel social stimuli. 


\subsubsection{SAA data analysis}

Once all recordings were coded and extracted from EthoVision $\left(\mathrm{XT}^{\mathrm{TM}}\right)$, SPSS (version 2225) was used for statistical analysis. The trials were then inspected for error or deviations, the elimination of trials is detailed in the next section (2.4.2).

When analysing the SAA data, an ANOVA on time spent in proximity to the familiar and novel stimulus is typically used. However, these measures are not independent as proximity to one stimulus requires the absence of proximity to the other, thus violating the assumptions of the ANOVA. Therefore we first used the sociability score based on work by Brodkin, Hagemann, Nemetski, and Silver (2004). For every second the rat spends with the novel social stimulus the sociability score is increased by 1 , while for every second it spends with the familiar social stimulus the sociability score is decreased by 1 . Thus, a positive sociability score indicates the rat spent more time with the novel stimulus, while a negative sociability score indicates the rat spent more time with the familiar stimulus. The sociability score was analysed with ANOVAs.

Additionally, to replicate the standard SAA analysis procedure, we used a repeated measures ANOVA to analyse the duration spent in proximity to the social and non-social stimuli (as per Crawley, 2004; Moy et al., 2008; Moy et al., 2007).

To examine the changes from pre- to post-puberty, additional repeated measures ANOVAs were used to analyse the sociability score between PND 30 and PND 60. Tukey posthoc analysis was used to examine significant prenatal dose effects, along with planned comparisons.

The ANOVAs in this experiment were separated by sex leading to four between-subjects levels (prenatal dose; 0mg/kg/day, 100mg/kg/day, 200mg/kg/day, 300mg/kg/day). When significant main effects were found, follow up analyses used Tukey HSD post hoc test. Partial eta squared $\left(\mathrm{\eta}_{\mathrm{p}}{ }^{2}\right)$ was used for the effect size. Also, when required, planned comparisons were used to follow up potential effects by comparing specific prenatal dose conditions with others using independent t-tests. The alpha level for statistical significance was set at $p<.05$. 


\subsubsection{SAA Results for Pre-Pubescent Rats}

A total of 90 trials were recorded for SAA when the rats reached PND 30. After reviewing the trials, the data for seven animals were removed. The reasons for exclusion were: the data from two animals were excluded due to tracking errors, three were excluded as the animals did not leave the start zone and a further two animals' data was ruled out because animals climbed on top of the apparatus rather than interacting with the conspecifics. Table 2.3 details the total number of animals used in this analysis.

Table 2.3. The number of animals used in SAA at PND 30 separated by prenatal dose and sex.

\begin{tabular}{c|c|c}
\hline & & PND 30 \\
\cline { 2 - 3 } & Males & Females \\
\hline $0 \mathrm{mg} / \mathrm{kg} /$ day & 9 & 9 \\
\hline $100 \mathrm{mg} / \mathrm{kg} /$ day & 11 & 11 \\
\hline $200 \mathrm{mg} / \mathrm{kg} /$ day & 12 & 12 \\
\hline $300 \mathrm{mg} / \mathrm{kg} /$ day & 11 & 8 \\
\hline \hline
\end{tabular}




\section{Sociability score of PND 30 males and females}

This score measures the preference for social novelty (positive) or familiarity (negative) toward the social stimulus. The prenatal exposure to VPA had no significant effect on the overall sociability score for males $\left(F(3,38)=.17, p=.919, \mathrm{\eta}_{\mathrm{p}}^{2}=.02\right.$, $n s$, see figure 2.12$)$.

For the females, the prenatal exposure to VPA also had no significant effect on the overall sociability score $\left(F(3,37)=1.10, p=.363, \mathrm{n}_{\mathrm{p}}{ }^{2}=.08, n s\right.$, see figure 2.12$)$.

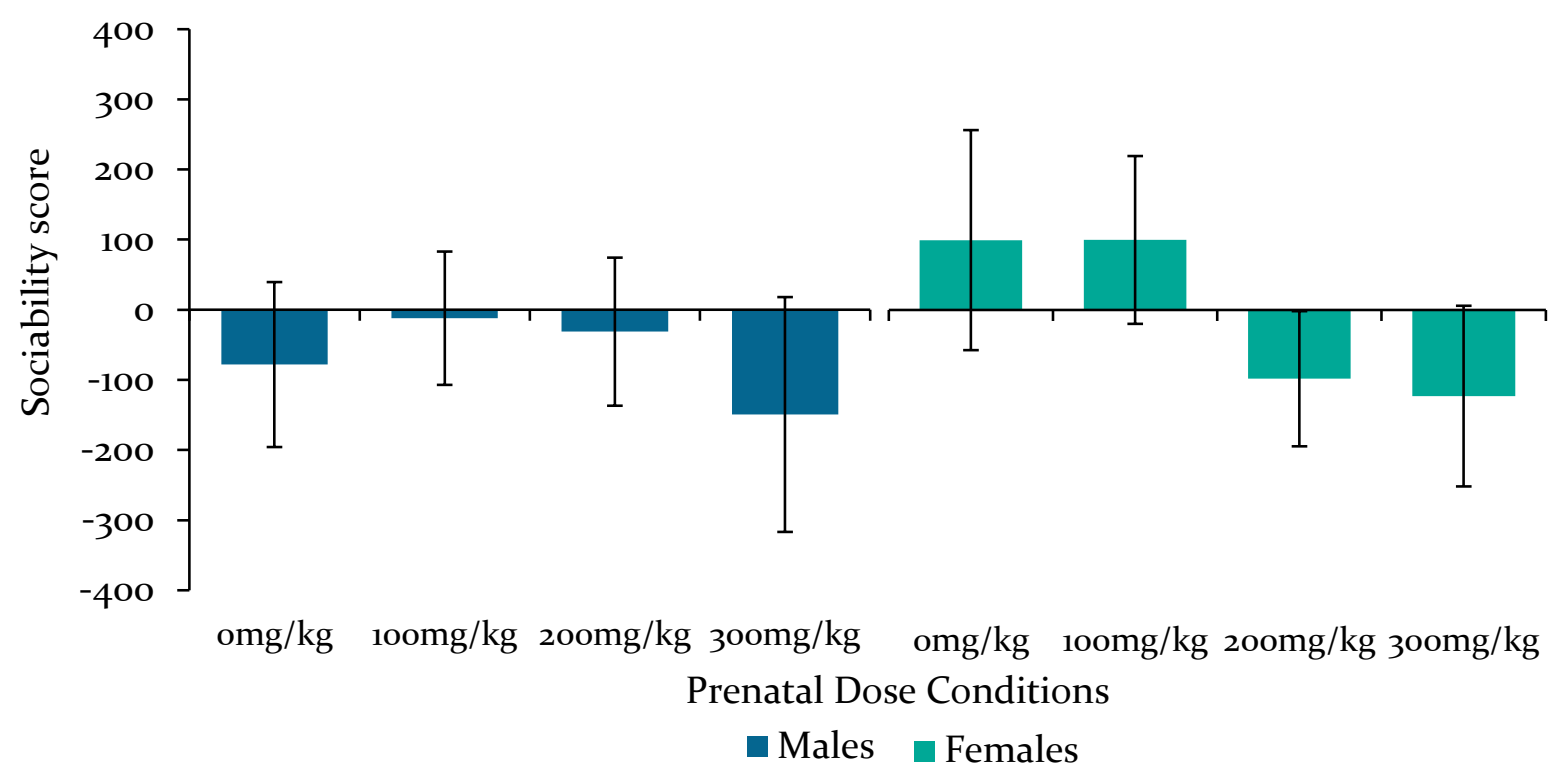

Figure 2.12. The average sociability score of males (left) and females (right) prenatally exposed to VPA (doses are measured in $\mathrm{mg} / \mathrm{kg} /$ day). Positive values indicate a preference for social novelty. No statistical differences found. Error bars represent \pm SEM. 


\section{Males duration of time spent in proximity to familiar or novel stimuli}

Investigation of the time males spent in proximity to the familiar or novel stimuli revealed no significant results. This analysis showed no significant preference for the familiar or novel areas $\left(F(1,38)=.93, p=.342, \eta_{\mathrm{p}}{ }^{2}=.02, n s\right)$, the prenatal dose conditions did not show differential preferences $\left(F(3,38)=.17, p=.919, \mathrm{\eta}_{\mathrm{p}}{ }^{2}=.02, n s\right)$, and there was no overall effects of the prenatal dose $\left(F(3,38)=.51, p=.680, \mathrm{n}_{\mathrm{p}}{ }^{2}=.04, n s\right.$; see figure 2.13$)$.

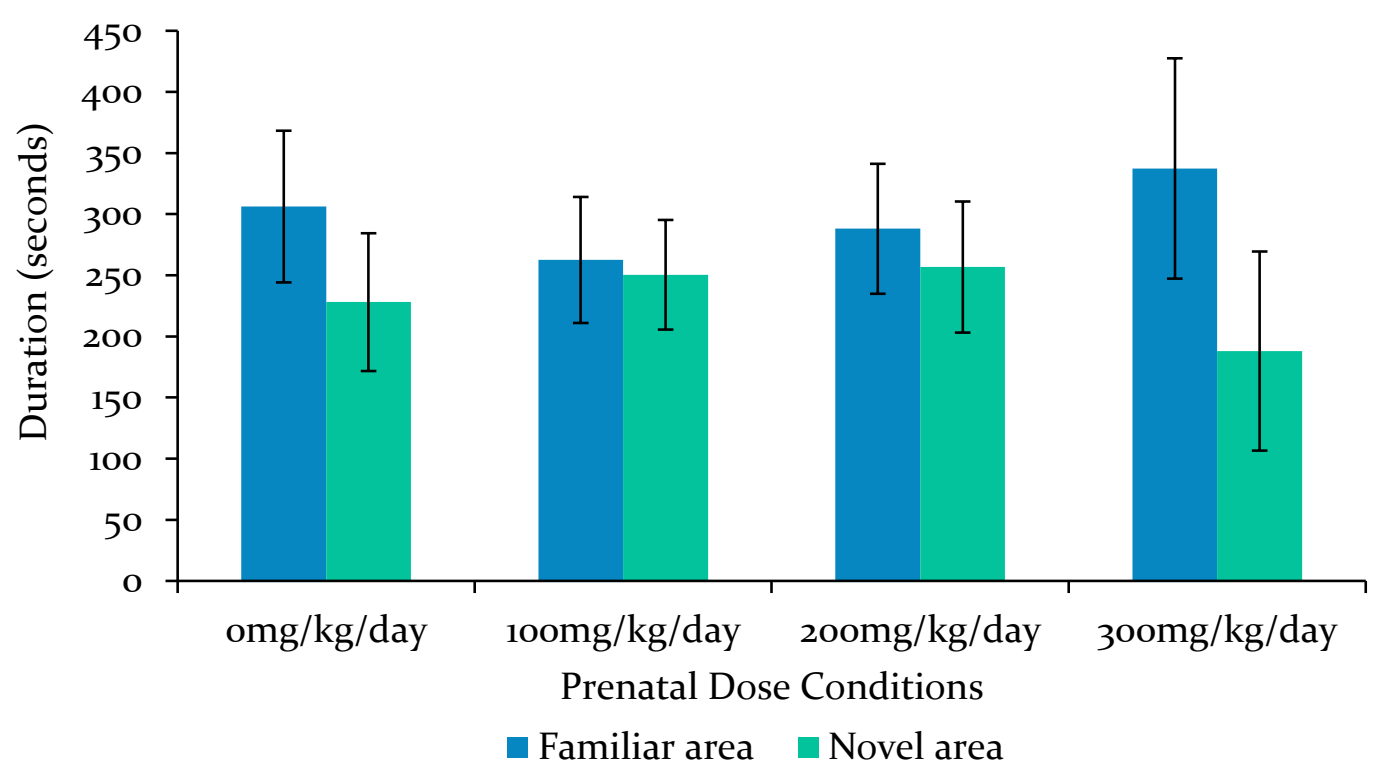

Figure 2.13. The average duration spent in proximity to the familiar or novel stimulus for males prenatally exposed to $0 \mathrm{mg} / \mathrm{kg} /$ day, $100 \mathrm{mg} / \mathrm{kg} /$ day, $200 \mathrm{mg} / \mathrm{kg} /$ day, $300 \mathrm{mg} / \mathrm{kg} /$ day of VPA, no statistical effects detected. Error bars represent \pm SEM. 


\section{Females duration of time spent in proximity to familiar or novel stimuli}

This analysis of time females spent in proximity to familiar or novel stimuli showed no significant preference for the familiar or novel areas $\left(F(1,37)=.00, p=.999, \eta_{\mathrm{p}}^{2}=.00, n s\right)$, the prenatal dose conditions did not interact with the area preferences $\left(F(3,37)=1.10, p=.363, \eta_{\mathrm{p}}^{2}\right.$ $=.08, n s)$, and there was no overall effects of the prenatal dose $\left(F(3,37)=1.49, p=.233, \eta_{\mathrm{p}}{ }^{2}=.11\right.$, ns; see figure 2.14).

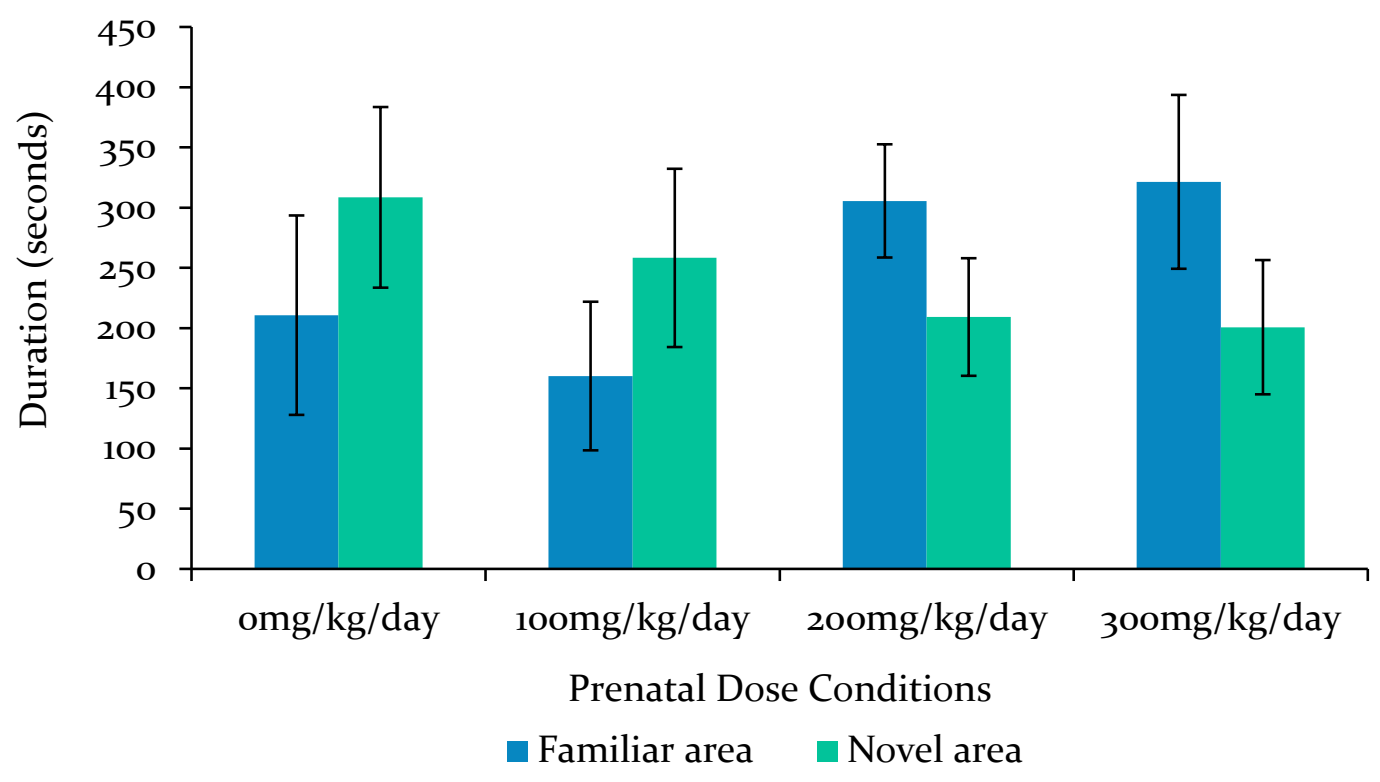

Figure 2.14. The average duration spent in proximity to familiar or novel stimulus for females prenatally exposed to $0 \mathrm{mg} / \mathrm{kg} /$ day, $100 \mathrm{mg} / \mathrm{kg} /$ day, $200 \mathrm{mg} / \mathrm{kg} /$ day, $300 \mathrm{mg} / \mathrm{kg} /$ day of VPA, no statistical effects detected. Error bars represent \pm SEM. 


\section{Distance travelled by males}

The prenatal doses did not have a significant impact on the total distance travelled on PND 30 for the male offspring $\left(F(3,38)=.30, p=.825, \eta_{\mathrm{p}}{ }^{2}=.02\right.$, $n s$, see figure 2.15$)$.

\section{Distance travelled by females}

The prenatal doses did not have a significant impact on the total distance travelled on PND 30 for the female offspring $\left(F(3,37)=1.04, p=.385, \eta_{\mathrm{p}}{ }^{2}=.08, n s\right.$, see figure 2.15$)$.

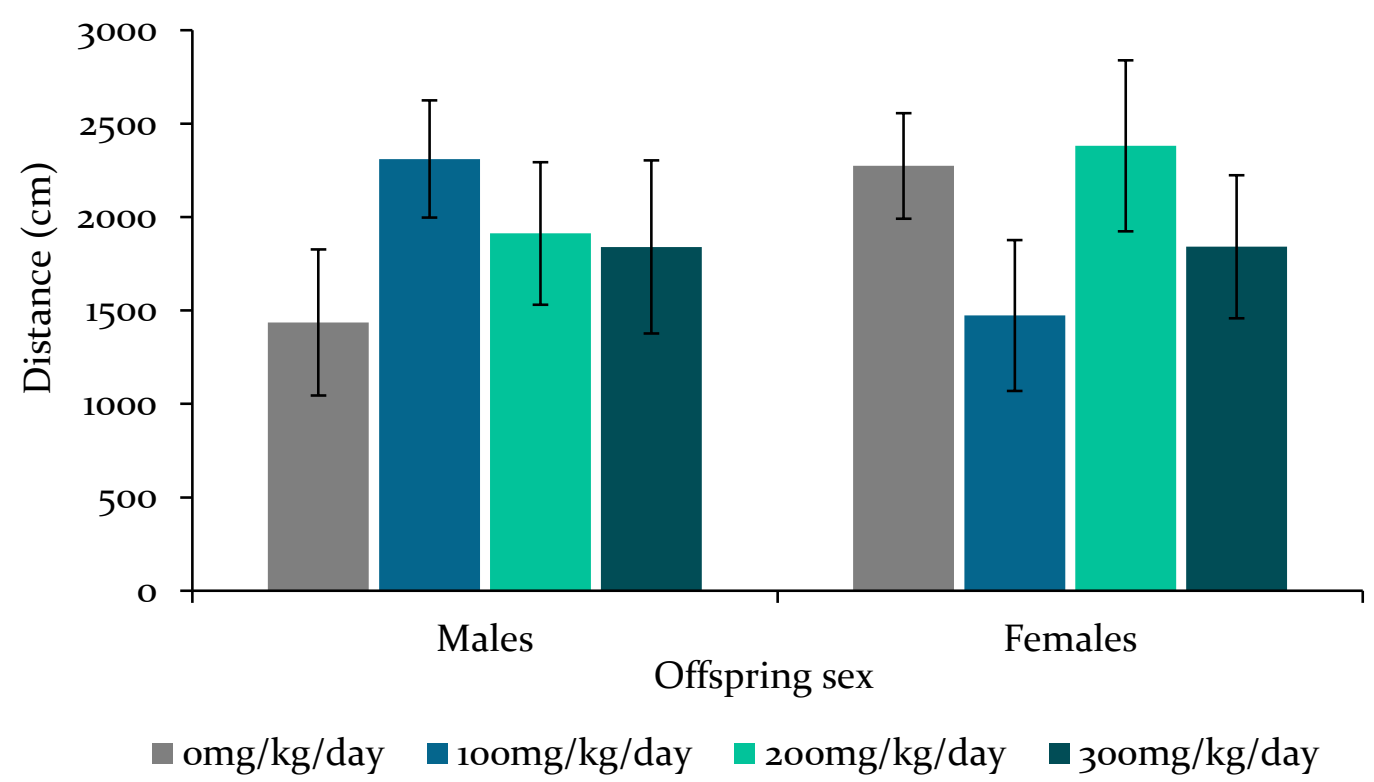

Figure 2.15. The average distance travelled by males (left) and females (right) prenatally exposed to $0 \mathrm{mg} / \mathrm{kg} /$ day, $100 \mathrm{mg} / \mathrm{kg} /$ day, $200 \mathrm{mg} / \mathrm{kg} /$ day, $300 \mathrm{mg} / \mathrm{kg} /$ day of VPA, no statistical effects detected. Error bars represent \pm SEM. 


\subsubsection{SAA Results for Post-Pubescent Rats}

A total of 89 trials were recorded for SAA on PND 60. After reviewing the trials, the data for four animals were excluded due to the following reasons: the data from one animal exposed to $200 \mathrm{mg} / \mathrm{kg} /$ day was removed due tracking errors, two animals did not leave the starting zone (one was exposed to $100 \mathrm{mg} / \mathrm{kg} /$ day and the other was exposed to $200 \mathrm{mg} / \mathrm{kg} /$ day), and the data from a $100 \mathrm{mg} / \mathrm{kg} /$ day exposed rat was excluded because the animal climbed on top of the apparatus. Table 2.4 below details the total number of animals used in this analysis.

Table 2.4. The number of test animals used in SAA at PND 60 divided by prenatal dose conditions and sex.

\begin{tabular}{c|c|c|}
\hline \multirow{2}{*}{} & \multicolumn{2}{c}{ PND 60 } \\
\cline { 2 - 3 } & Males & Females \\
\hline Omg/kg/day & 9 & 10 \\
$100 \mathrm{mg} / \mathrm{kg} /$ day & 8 & 12 \\
$200 \mathrm{mg} / \mathrm{kg} /$ day & 11 & 11 \\
$300 \mathrm{mg} / \mathrm{kg} /$ day & 12 & 12 \\
\hline \hline
\end{tabular}




\section{Sociability score of males}

There was a trend toward significance for the prenatal exposure to VPA on the overall sociability score for males $\left(F(3,36)=2.28, p=.096, \mathrm{\eta}_{\mathrm{p}}{ }^{2}=.16, n s\right)$. The approach to significant effect was likely due to a (non-significant) increase sociability score of the males exposed to $300 \mathrm{mg} / \mathrm{kg} /$ day compared with the males exposed to $200 \mathrm{mg} / \mathrm{kg} /$ day $(p=.081$, $n s$; see figure 2.16). Furthermore, the planned comparisons also showed that the sociability score of $100 \mathrm{mg} / \mathrm{kg} /$ day was increased compared with $200 \mathrm{mg} / \mathrm{kg} /$ day, albeit not significantly $(p=.074$, $n s)$. However, none of the VPA doses differed significantly from the control condition.

\section{Sociability score of females}

The females prenatally exposed to VPA exhibited novelty preference but there were no significant differences in the sociability score between the prenatal dose conditions $(F(3,40)=$ $.27, p=.844, \eta_{\mathrm{p}}^{2}=.02, n s$, see figure 2.16 ).

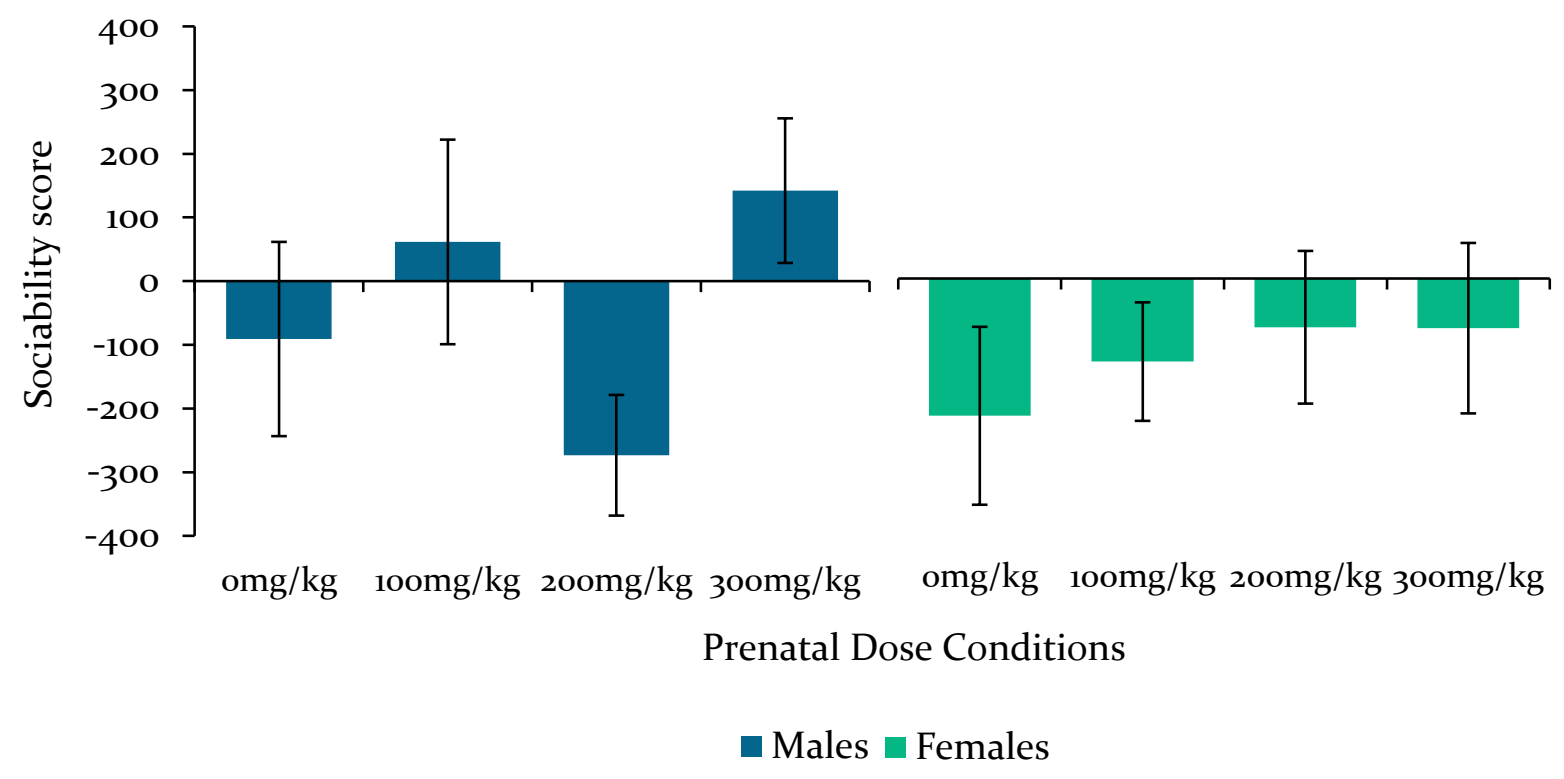

Figure 2.16. The average sociability score at PND 60 of males (left) and females (right) prenatal exposed to VPA (doses are measured in $\mathrm{mg} / \mathrm{kg} /$ day). The positive values reflect social novelty preference. Error bars represent \pm SEM. 


\section{Males duration of time in proximity to familiar or novel stimuli}

In the analysis of time males spent in proximity to familiar or novel stimuli, there were no overall preferences for the stimuli $\left(F(1,36)=.39, p=.536, \mathrm{\eta}_{\mathrm{p}}{ }^{2}=.01, n s\right)$, nor was there an overall effect of the prenatal dose $\left(F(3,36)=1.05, p=.383, \mathrm{n}_{\mathrm{p}}^{2}=.08, n s\right)$.

However, there was a trend towards a significant interaction between the prenatal dose condition and the stimuli area $\left(F(3,36)=2.28, p=.096, \mathrm{\eta}_{\mathrm{p}}^{2}=.16, n s\right)$. To investigate this trend further, the side preferences was examined within each of the prenatal dose conditions, this revealed a significant effect for males exposed to $200 \mathrm{mg} / \mathrm{kg} /$ day alone $(F(1,10)=8.33, p=.016$, $\eta_{\mathrm{p}}{ }^{2}=.45$, see figure 2.17$)$.

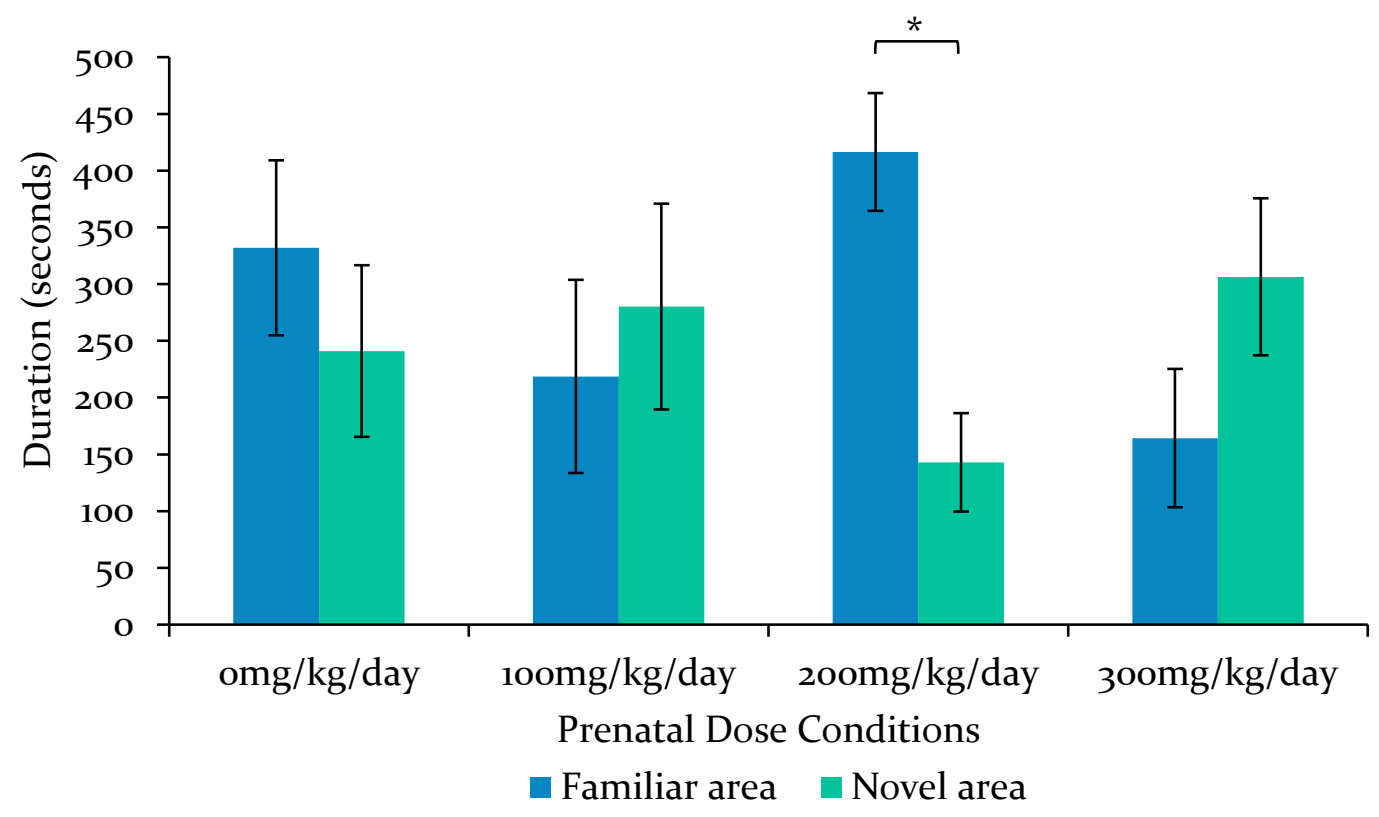

Figure 2.17. The average duration spent in proximity to the familiar or novel stimulus at PND 60 of males prenatally exposed to $0 \mathrm{mg} / \mathrm{kg} /$ day, $100 \mathrm{mg} / \mathrm{kg} /$ day, $200 \mathrm{mg} / \mathrm{kg} /$ day, $300 \mathrm{mg} / \mathrm{kg} / \mathrm{day}$ of VPA. With the $200 \mathrm{mg} / \mathrm{kg} /$ day males exhibiting a significant familiar area preference. * indicates significance to $p<.05$. Error bars represent \pm SEM. 


\section{Females duration of time in proximity to familiar or novel stimuli}

On the other hand, females showed no overall prenatal dose effect $(F(3,40)=.53, p=$ $\left..663, \mathrm{\eta}_{\mathrm{p}}{ }^{2}=.04, n s\right)$, and there was no interaction between prenatal dose conditions and area preference $\left(F(3,40)=.27, p=.844, \eta_{\mathrm{p}}^{2}=.02, n s\right)$, but there was a significant area preference $\left(F(1,40)=4.26, p=.046, \eta_{\mathrm{p}}^{2}=.10, n s ;\right.$ see figure 2.18$)$.

The follow up analysis on the area preference showed that the females exposed to the control and $100 \mathrm{mg} / \mathrm{kg} /$ day doses exhibited a close to significant preference for the familiar area ( $p=.079, p=.094, n s$, respectively). Whereas, the females exposed to VPA did not exhibit a preference for either area $(p=.269 ; p=.287, n s$, respectively). Thus, prenatal exposure to VPA is disrupting the preference formation for adult females.

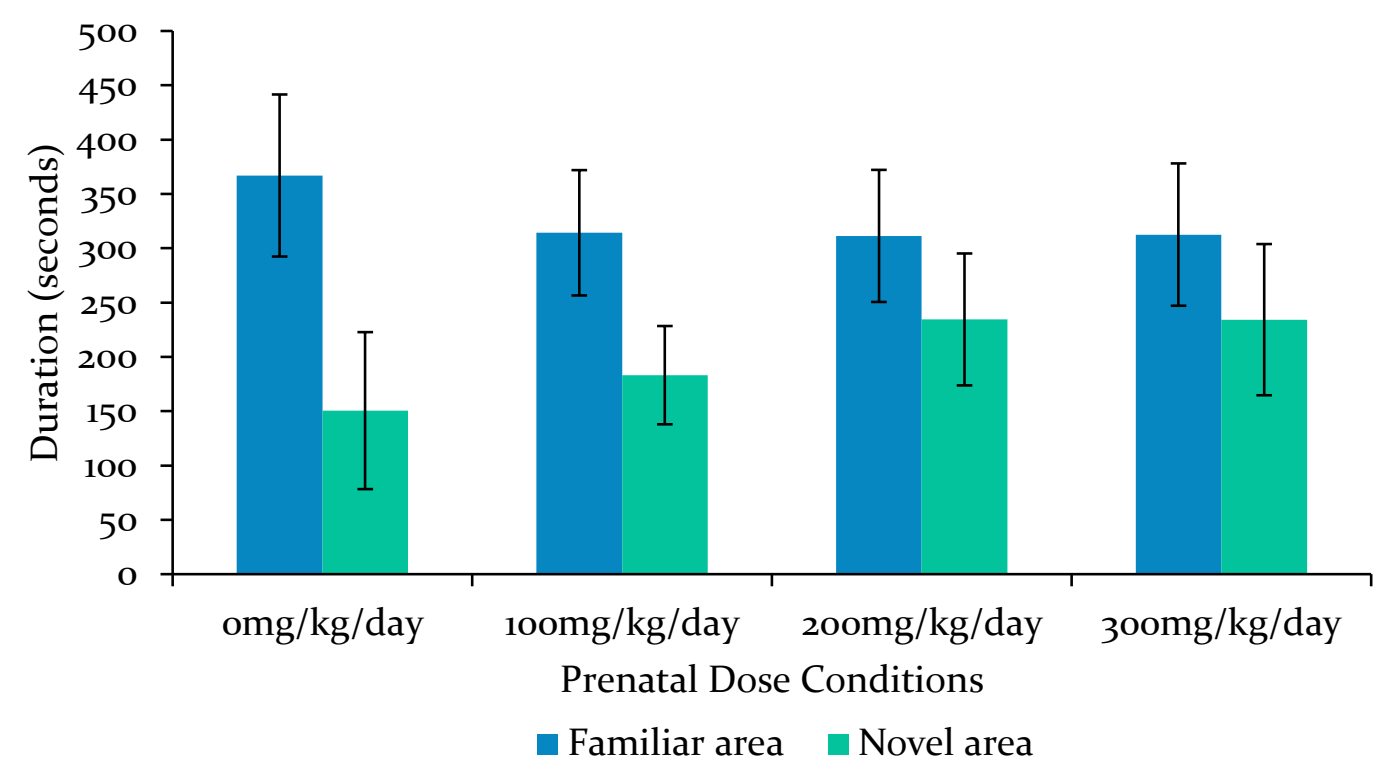

Figure 2.18. The average duration spent in proximity to the familiar or novel stimulus at PND 60 of females prenatally exposed to $0 \mathrm{mg} / \mathrm{kg} / \mathrm{day}, 100 \mathrm{mg} / \mathrm{kg} /$ day, $200 \mathrm{mg} / \mathrm{kg} / \mathrm{day}$, $300 \mathrm{mg} / \mathrm{kg} /$ day of VPA. The females exposed to higher doses of VPA exhibit no preference. Error bars represent \pm SEM. 


\section{Distance travelled by males}

In addition to the sociability measures, general locomotor activity was recorded in SAA. The prenatal doses did not have a significant impact on the total distance travelled on PND 60 for the male offspring $\left(F(3,36)=1.09, p=.366, \eta_{\mathrm{p}}{ }^{2}=.08\right.$, $n s$, see figure 2.19).

\section{Distance travelled by females}

The prenatal doses did not have a significant impact on the total distance travelled on PND 60 for the female offspring $\left(F(3,40)=1.47, p=.236, \eta_{\mathrm{p}}{ }^{2}=.10\right.$, $n s$, see figure 2.19$)$.

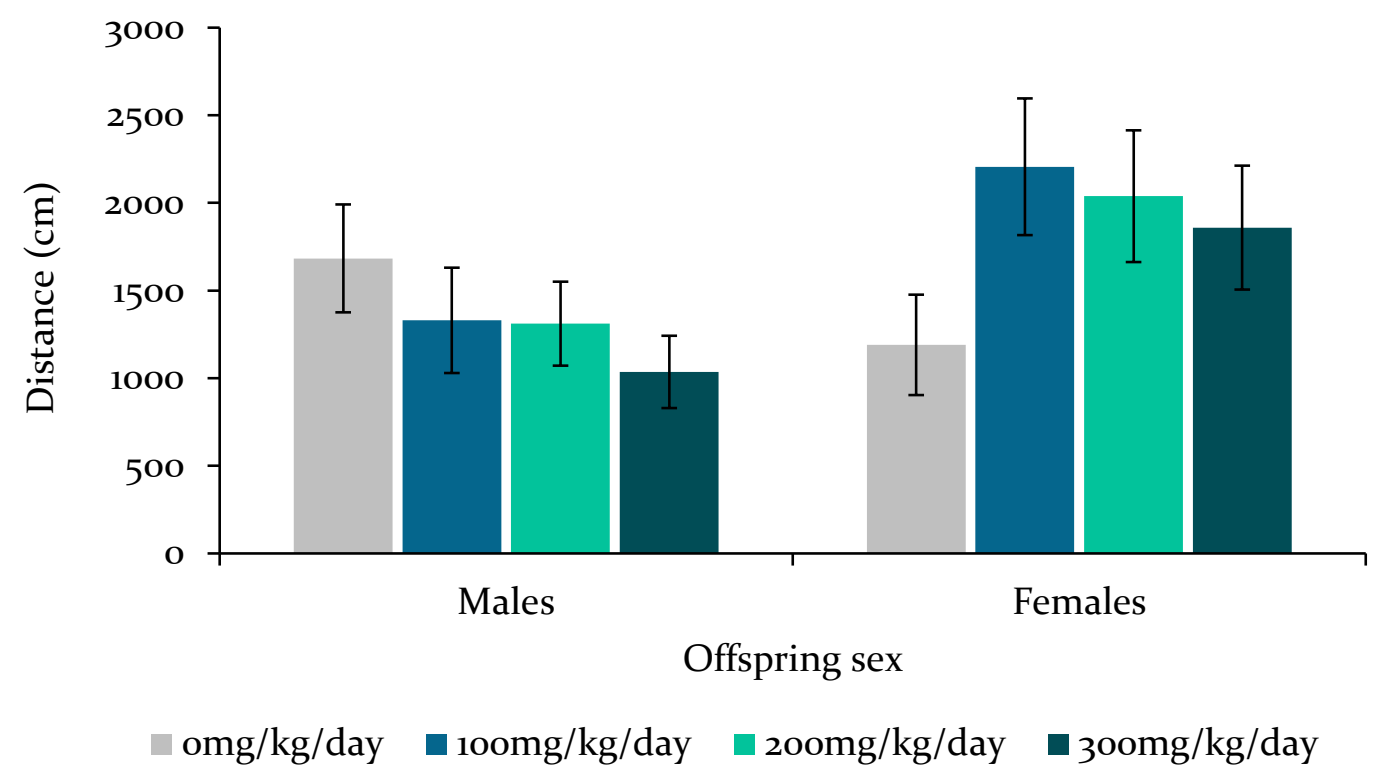

Figure 2.19. The distance travelled by males and females prenatally exposed to $0 \mathrm{mg} / \mathrm{kg} / \mathrm{day}$, $100 \mathrm{mg} / \mathrm{kg} /$ day, $200 \mathrm{mg} / \mathrm{kg} /$ day, $300 \mathrm{mg} / \mathrm{kg} /$ day of VPA. No detectable differences found. Error bars represent \pm SEM. 


\subsubsection{Pre- \& Post-Pubescent Comparison Results}

The social behaviour of males and females prenatally exposed to VPA examined prepuberty (PND 30) and post-puberty (PND 60). A 2(PND: 30, 60) by 4(Dose: 0mg/kg/day, $100 \mathrm{mg} / \mathrm{kg} /$ day, $200 \mathrm{mg} / \mathrm{kg} /$ day, $300 \mathrm{mg} / \mathrm{kg} /$ day) repeated measures ANOVA was run on the sociability score.

\section{Sociability score for males from PND 30 to PND 60}

The sociability score for males did not significantly change over time (see figure 2.20). There was no significant effect of time on the sociability score $\left(F(1,32)=.05, p=.835, \eta_{\mathrm{p}}{ }^{2}=.00\right.$, $n s)$, no significant effect of the prenatal dose conditions $\left(F(3,32)=.24, p=.869, \eta_{\mathrm{p}}{ }^{2}=.02, n s\right)$, and the prenatal doses did not have an overall effect $\left(F(3,32)=.62, p=.605, \eta_{\mathrm{p}}{ }^{2}=.06, n s\right)$.

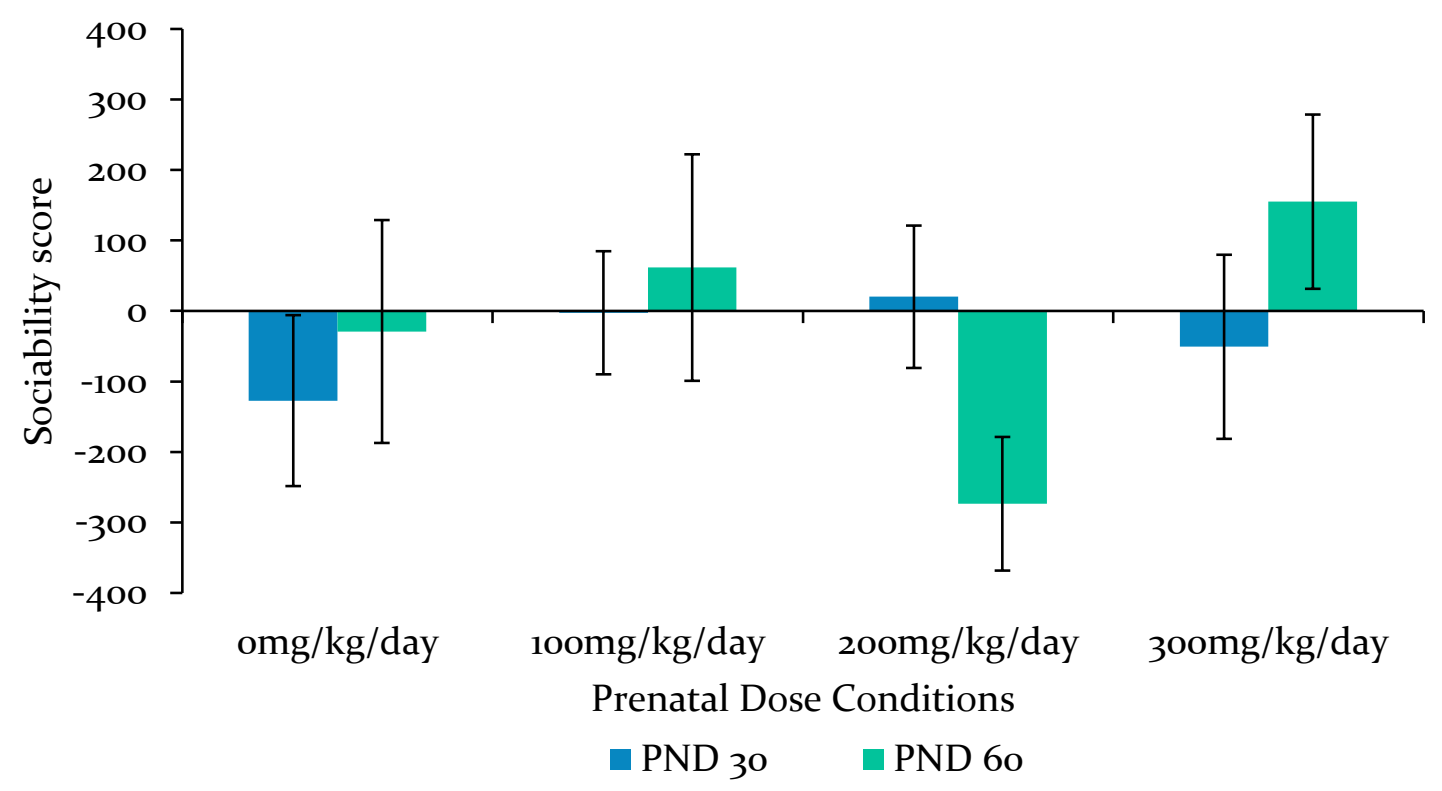

Figure 2.20. The average sociability score at PND 30 and PND 60 of males prenatally exposed to $0 \mathrm{mg} / \mathrm{kg} /$ day, $100 \mathrm{mg} / \mathrm{kg} /$ day, $200 \mathrm{mg} / \mathrm{kg} /$ day, $300 \mathrm{mg} / \mathrm{kg} /$ day of VPA. The positive values reflect social novelty preference. No significant changes over time. Error bars represent \pm SEM. 


\section{Sociability score for females from PND 30 to PND 60}

The sociability score for females did not significantly change over time (see figure 2.21). There was no significant effect of time on the sociability score $\left(F(1,32)=2.81, p=.104, \eta_{\mathrm{p}}{ }^{2}=.08\right.$, $n s)$, there was no differential effect of the prenatal dose conditions $\left(F(3,32)=.36, p=.780, \eta_{\mathrm{p}}^{2}\right.$ $=.03, n s)$, and the prenatal doses did not have an overall effect $\left(F(3,32)=.24, p=.869, \eta_{\mathrm{p}}{ }^{2}=\right.$ $.02, n s)$.

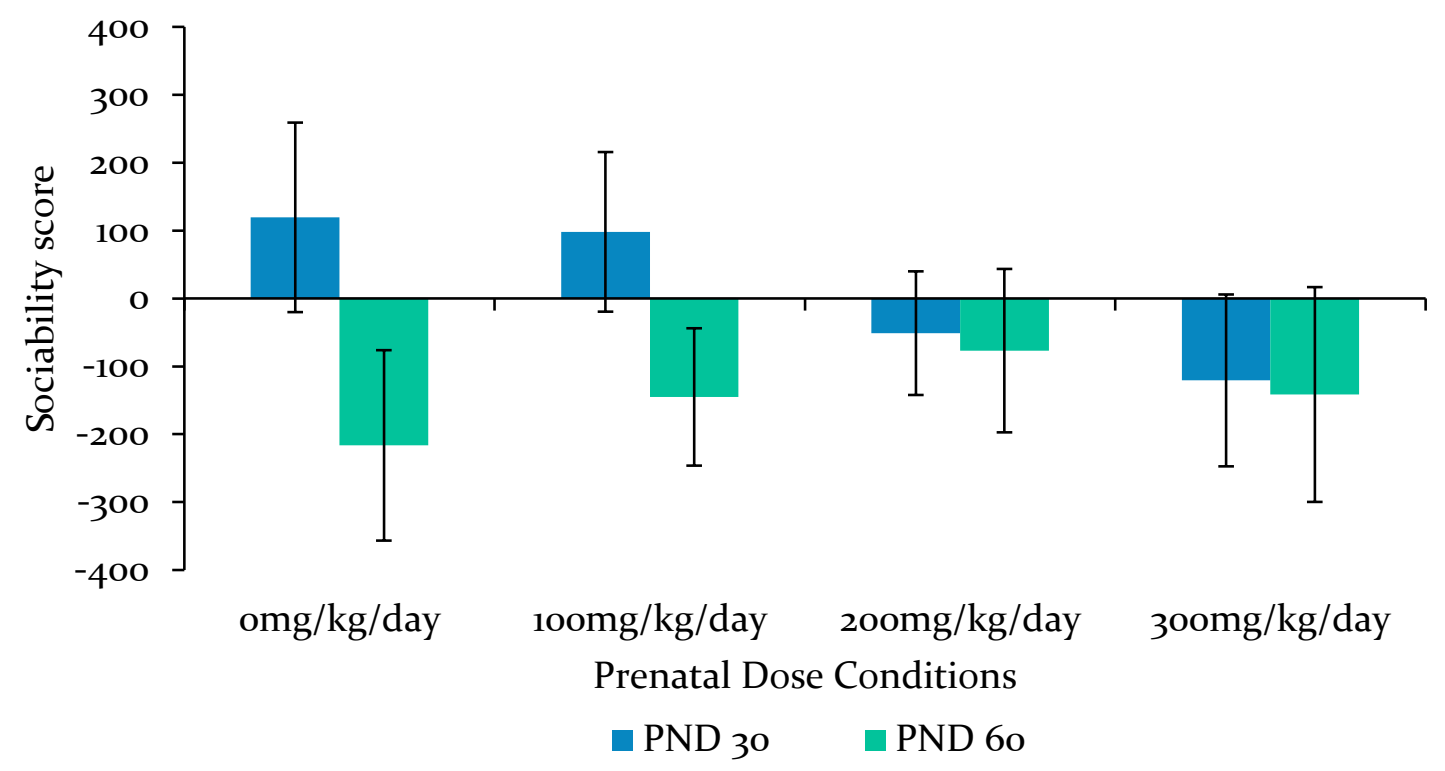

Figure 2.21. The average sociability score at PND 30 and PND 60 of females prenatally exposed to $0 \mathrm{mg} / \mathrm{kg} /$ day, $100 \mathrm{mg} / \mathrm{kg} /$ day, $200 \mathrm{mg} / \mathrm{kg} /$ day, $300 \mathrm{mg} / \mathrm{kg} /$ day of VPA. The positive values reflect social novelty preference. No change in sociability from PND 30 to PND 60 for females exposed to $200 \mathrm{mg} / \mathrm{kg} /$ day and $300 \mathrm{mg} / \mathrm{kg} /$ day. Error bars represent \pm SEM. 


\subsection{Results Summary Table}

Table 2.5. Complete summary of significant differences in the experiments of chapter two for the offspring prenatally treated with VPA. The table includes directional dose/group comparisons.

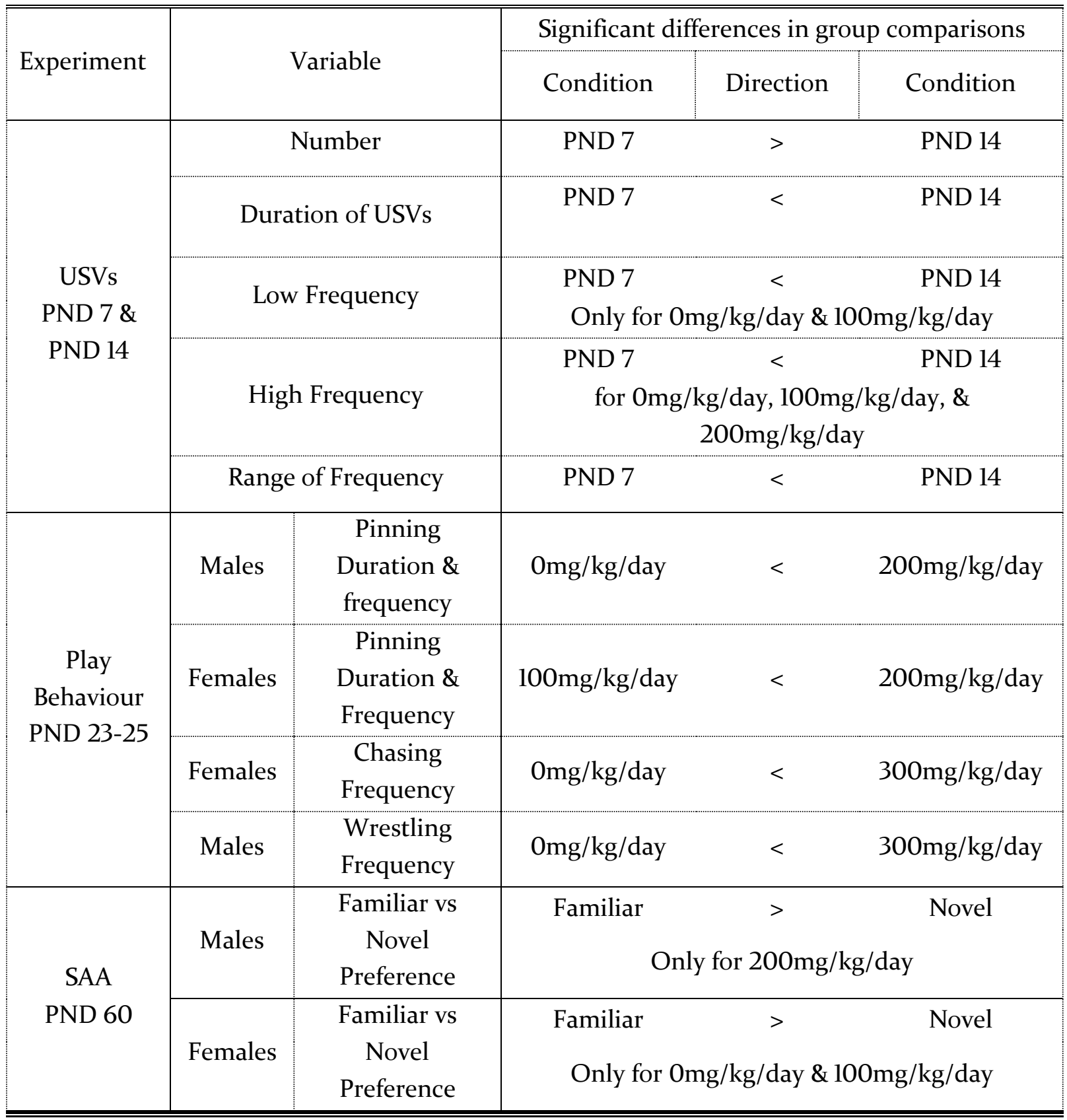

Note: '>' refers to less than, e.g. there were fewer vocalisations at PND 7 than PND 14. 


\subsection{Discussion}

The aim of study one was to investigate the efficacy of administering VPA continuously throughout gestation and to test the hypothesis that continuous gestational exposure would result in dose-dependent alterations in communication and social interaction. Three experiments were run pre-puberty, and one was run post-puberty, all intended to capture ASDlike deficits. Overall, this study found several behavioural alterations following the continuous prenatal exposure to VPA, although the dose effects do not appear to be consistent, both in terms of dose-response and direction of the socio-behavioural alterations.

Nineteen litters were born with no fatalities to the dams and no substantial physical impairment to the offspring. The lack of fatalities and impairments indicate that the administration of VPA daily with SCM at doses of 0, 100, 200, and 300mg/kg were below the level of toxicity for the dams and the offspring. This complements the findings in Vorhees (1987), who showed that oral gavage doses of 400-800 mg/kg were toxic to dams and offspring. While the doses in this study were below toxic levels, they were expected to result in significant and consistent behavioural deficits, based on previous literature discussed in the introduction.

It was hypothesised that there would be dose-dependent alterations in communication and social behaviour following daily administration of $100,200,300 \mathrm{mg} / \mathrm{kg} /$ day in comparison with controls $(0 \mathrm{mg} / \mathrm{kg} /$ day). While indeed some effects were observed, the results do not overwhelmingly support the hypothesis. The offspring exposed to 200 and 300mg/kg/day showed altered behaviours across the four experiments. The offspring exposed to the highest dose of VPA (300mg/kg/day) had increased vocalisation length (PND 7 and PND 14), increased active behaviours (pinning and wrestling, PND 25), and a trend toward of social novelty aversion (PND 30, 60). Some differences between males and females were also found. Thus, especially the females exposed to $200 \mathrm{mg} / \mathrm{kg} /$ day exhibited an increase in active play behaviour (pinning and wrestling, PND 23, 25), as well as a pattern of social novelty aversion (PND 30, 60).

Overall, the social aversion patterns align with previous studies that have examined the social behaviour of offspring prenatally exposed to VPA. The work of Bambini-Junior et al. (2011), along with Kim et al. (2011), Kerr et al. (2013), and Kim et al. (2014), who have all shown that VPA-exposed animals spend less time with the novel stimulus and increased time with familiar stimulus. This pattern has been identified in this study at both time points, pre- and post-puberty. However, USV and play behaviour effects are less clear in comparison to current literature. The following is a more specific discussion and review of the results found in the above experiments. 


\subsubsection{USVs}

The first experiment examined the USVs of the pre-weaning offspring. To date, the vast majority of published studies examine the total number of vocalisations, and at times, the duration of USVs (Bronzuoli et al., 2018; Melancia et al., 2018; Servadio et al., 2018). Based on previous literature, prenatal exposure to VPA was expected to reduce the number of vocalisations. However, there were no significant differences between the prenatal doses conditions, although, in line with previous research, we did find a significant decrease from PND 7 to 14 (Branchi et al., 2001; Elsner, Suter, \& Alder, 1990). Close inspection of figure 2.2 indicates that the reduction was not as clear for the $300 \mathrm{mg} / \mathrm{kg} /$ day (although this failed to reached significance).

While the number of vocalisations decreases with age, the duration of those vocalisations increases, that is, the pre-weaning pups will emit vocalisations that increase in duration as they age (Elsner et al., 1990). This increase duration with age pattern was only found in the pups exposed to a high dose of VPA (figure 2.3). Interestingly, the $100 \mathrm{mg} / \mathrm{kg} / \mathrm{day}$ show no change between PND 7 and PND 14. The driving effect of this is unclear, as other measures for these offspring indicate similar trends as the control offspring, with the exception of the duration of USVs.

In addition to the analysis of the number and duration of USVs, several frequency measures were also examined. The frequency measures the pitch of a call, more specifically the highest and lowest pitch as well as the range was analysed, to get a measure of the quality of the sounds made by the pups. The frequency measures provide further insight into the USVs, as rodent vocalisations are distinguished on frequency range as well as the sonographic features, or the shape of the calls (Brudzynski, 2015). Overall, USVs can be broadly categorised into short or long flat calls (typically seen at $22 \mathrm{kHz}$ ), $50 \mathrm{kHz}$ flat, $50 \mathrm{kHz}$ step up or step-downs, and trills (Brudzynski, 2015; Portfors, 2007; Wright, Gourdon, \& Clarke, 2010). Furthermore, figure 2.1 displays typical sonographic features of pre-weaning pups in this study. The frequency measures in this study show that the pups exposed to higher doses of VPA $(200 \mathrm{mg} / \mathrm{kg} / \mathrm{day}$ and $300 \mathrm{mg} / \mathrm{kg} /$ day) are exhibiting a delayed maturation at PND 14. The pups exposed to $200 \mathrm{mg} / \mathrm{kg} /$ day and $300 \mathrm{mg} / \mathrm{kg} /$ day did not exhibit an increase in the low and high frequencies (figures 2.4 and 2.5), and therefore not achieving similar USV frequency as the controls. This is further compounded by the lack of clear reduction in the total number of vocalisations emitted by the pups exposed to the higher VPA doses. 
The results of the first experiment demonstrated that daily administration of VPA throughout gestation leads to a qualitative change in the vocalisations emitted by the offspring. The qualitative changes in USVs provide an early indicator of neurobehavioural alterations (Lester, 1987; Servadio, Vanderschuren, \& Trezza, 2015). To date, the vast majority of the literature has focused on the number and duration of the ultrasonic vocalisations, while paying little attention to more qualitative changes. The current study suggests that this quantitative focus may lead to a significant underestimation of the effects of prenatal exposure of VPA on the subsequent nature of the USVs (Brudzynski et al., 1999). With more recent software developments have led to automated USV classification with the use of software like MUPET (Van Segbroeck, Knoll, Levitt, \& Narayanan, 2017) and DeepSqueak (Coffey, Marx, \& Neumaier, 2019) which would allow for a more detailed examination of the qualitative changes.

\subsubsection{Play Behaviour}

To examine the social interaction of the offspring chronically exposed to VPA during gestation, two experimental sessions of play behaviour were taken at PND 23 and 25. Increases in the active behaviours were identified, and there were significant effects for males and females, although not consistent across all measures. In particular, for the active behaviours (chasing, pinning, and wrestling), the offspring prenatally exposed to $200 \mathrm{mg} / \mathrm{kg} /$ day and $300 \mathrm{mg} / \mathrm{kg} /$ day of VPA showed an increase in play behaviour (pinning: figure 2.7; and wrestling: figure 2.9), although there was some inconsistency as the effects were sometimes observed in frequency and sometimes in duration of the behaviour.

The increase in social play behaviour exhibited by the offspring prenatally exposed to VPA is surprising as it does not follow the expected outcomes for this animal model; at least based on the previous research that had used a single VPA exposure during gestation. Previous studies showed that rats prenatally exposed to VPA exhibit decreased social interaction (Markram et al., 2008; Olde Loohuis et al., 2015; Sandhya et al., 2012; Wang et al., 2013). The results of the present study show that, if anything, chronic prenatal exposure to VPA leads to the opposite. This clearly warrants further investigation, as it raises significant concerns with respect to the validity of the rat VPA model for ASD. It would be important to further investigate the nature of the social interaction both prepubertal as well as postpubertal. One important aspect that could be investigated is the nature of the "partner" in the play behaviour. It is well known from clinical studies that children with ASD respond differently to familiar or unfamiliar people. Thus, it would be interesting to see whether VPA treated rats play differently with littermates compared with unknown conspecifics. Likewise, and similar to the USV analysis, it 
would be important to look at the quality of the play behaviour. Play behaviour is highly ritualised (Pellis \& Pellis, 2007) and even in the absence of significant differences in the individual items that compose play behaviour, the sequence may be disrupted.

\subsubsection{SAA}

SAA allows for the investigation of social behaviour of VPA exposed offspring in a context where the conspecifics cannot directly affect the offspring's behaviour. There were trends towards social novelty aversion in the $200 \mathrm{mg} / \mathrm{kg} /$ day and $300 \mathrm{mg} / \mathrm{kg} /$ day (see sociability score, figure 2.12 for PND 30 and figure 2.16 for PND 60). However, the offspring in the control condition did not exhibit any preference for the novel stimulus, which a complication for the analysis and interpretation. Previous studies have shown that offspring that were prenatally exposed to a saline injection exhibit a preference for the novel stimulus (when compared with the familiar stimulus; Favre et al., 2015). The control offspring in this chapter did not exhibit a clear social preference, which may be in part be due to the apparatus used or the lack of social isolation prior to the experiment. SAA was originally designed using a three-chamber apparatus, whereas, this experiment used a T-maze. Compared to the three-chamber approach the choice between the two arms in the T-maze may be less deliberate (as the animals do not have to cross the "neutral" zone), though, at the same time, the distance between the two stimuli is larger in the T-maze. As discussed in the introduction, methodological parameters can have a large impact on the outcome in the SAA, and perhaps it would, therefore, be useful to repeat these experiments using a different set-up to ensure that the control animals show a clear preference for the novel stimulus. Perhaps the social housing had carry-over effects for these rats. Overall, it is possible that lack of preference exhibited by the control rats may have masked VPA effects, as controls should serve as the baseline from which the behaviour of the VPA offspring should deviate from.

In SAA, social preference for familiarity or novelty is generally assessed by the duration in proximity to the familiar or novel social stimulus (Crawley, 2004). The female offspring exposed to the control dose and $100 \mathrm{mg} / \mathrm{kg} /$ day showed increased time spent with the familiar stimulus (although, the dose effects did not reach significance, figure 2.14). More importantly, there were dose-related decreases in the time spent with the novel stimulus, such that the offspring exposed to $300 \mathrm{mg} / \mathrm{kg} /$ day spent even less time with the novel stimulus than the familiar, and also show the greatest decrease in social preference. The increased duration with the familiar stimulus (see figure 2.14) was exhibited by the females exposed to $300 \mathrm{mg} / \mathrm{kg} / \mathrm{day}$ compared with the females exposed to the control dose. These trends align with the findings of 
Kim et al. (2011). Their experiment demonstrated that 400mg/kg VPA at GD 12 led to the offspring spending the least amount of time with the novel stimulus and significantly more time with the familiar stimulus compared to controls.

The rats prenatally exposed to VPA exhibited very few statistically different behaviours in the post-puberty (PND 60) measure of SAA. Although a significant familiarity preference was found in the males exposed to $200 \mathrm{mg} / \mathrm{kg} /$ day (figure 2.17). Furthermore, the sociability of these rats was examined pre- and post-puberty (figures 2.20 and 2.21), and there were divergent profiles for the males and females. The males exhibited preference at PND 60 that they had not shown at PND 30, however, this was in opposing direction for the $200 \mathrm{mg} / \mathrm{kg} / \mathrm{day}$ and $300 \mathrm{mg} / \mathrm{kg} /$ day (figure 2.20). Whereas, the females showed perseverance of behaviour (or no change from PND 30 to PND 60), which aligns with the hypothesis and provides further support for the impact of the chronic prenatal VPA exposure in rats. 


\subsubsection{Summary}

Overall, this study has confirmed that chronic prenatal exposure to VPA impacts social behaviour. While some of the findings aligned with the literature, such as the VPA offspring exhibiting reductions in social novelty in SAA, there were also inconsistencies. Notably with VPA offspring in this study showing increased social behaviour in the play behaviour paradigm, whereas based on previous research, decreases in play behaviour have been reported after VPA treatment. Ultimately, the chronic prenatal treatment with VPA led to a relatively mild phenotype. This was surprising given that previous studies (Schneider \& Przewlocki, 2005; Vorhees, 1987) found substantial effects on behaviour following an oral gavage administration with slightly higher VPA doses. As such, the mild phenotype in this study may be a result of low VPA doses combined with an administration method that would likely lead to slower increases in VPA blood levels than seen after an IP injection or oral gavage. Furthermore, inconsistencies between the behaviours identified in this study and the literature may be attributable to strain differences. For example, Vorhees (1987) used Sprague-Dawley rats, whereas Schneider and Przewlocki (2005) used Wistar rats. While the current study also used Sprague Dawley rats, this is an outbred strain and as such genetically different from other Sprague Dawley strains. In this respect, it is important to remember, as discussed in the introduction that ASD has a strong genetic component and is likely the result of gene $\times$ environment interactions. Therefore, the genetic contribution needs to be considered. In the next study, we examined this further with a different strain, increased dose, and a more controlled and measurable method of delivering VPA to rats with genetically induced serotonin deficits. 


\section{Chapter Three}

Chronic Prenatal Exposure to VPA in SERT Deficient Rats 


\section{Chapter Three Contents}

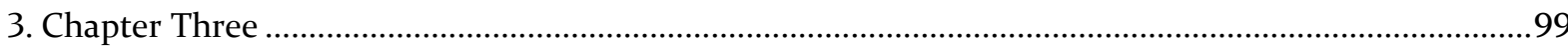

3.1.2 Overall aims of Chapter three ........................................................................ 104

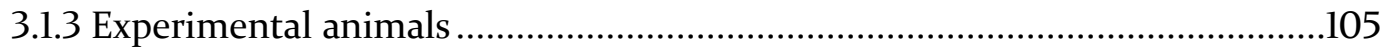

3.2 Experiment One: Isolation induced Ultrasonic Vocalisations .................................. 109

3.2.1 Isolation induced USV methods .................................................................. 110

3.2.5 Isolation induced USV results .................................................................... 111

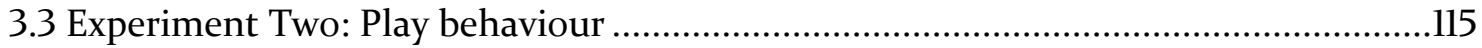

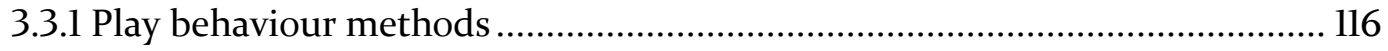

3.3.3 Play behaviour results .................................................................................117

3.4 Experiment Three: Social Approach and Avoidance .................................................121

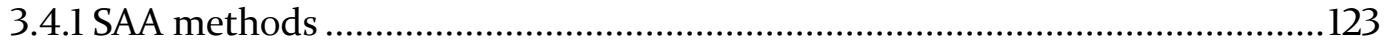

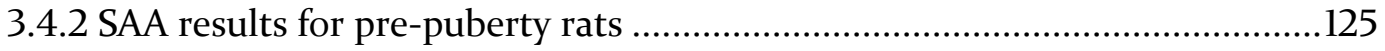

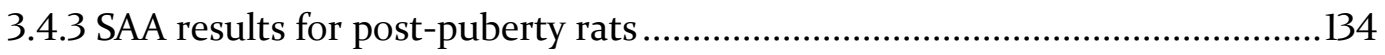

3.4.4 Pre- and post-puberty comparison results .................................................... 143

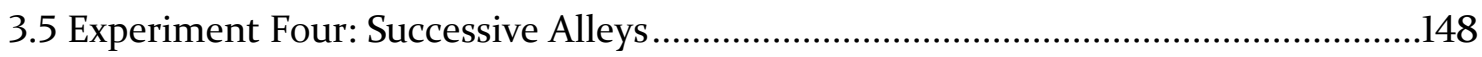

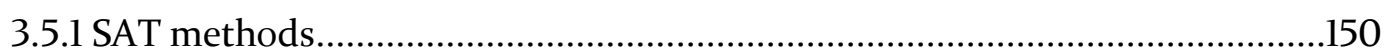

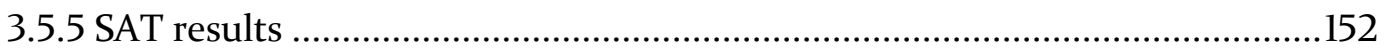

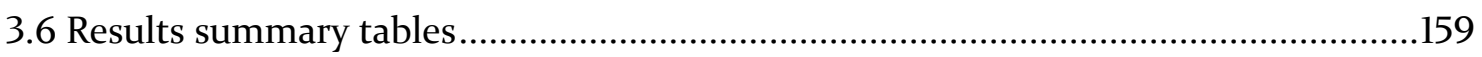

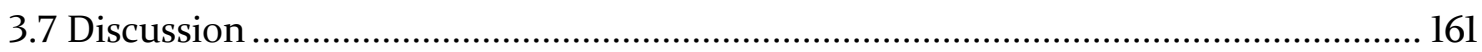

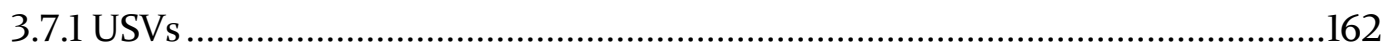

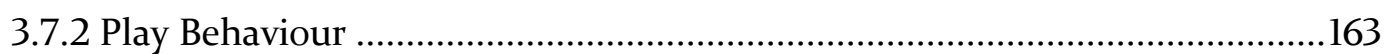

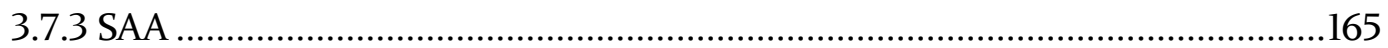

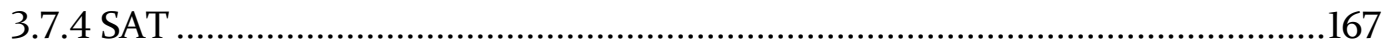

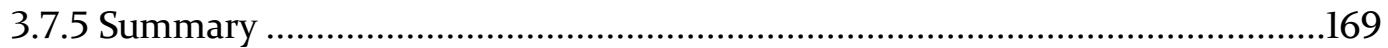




\section{Chapter Three}

The following study adopts a gene $\times$ environment interaction method to continue the advancement of the VPA-induced animal model of ASD. The following introduction highlights previous research relevant to the methods used in this study, then the methods and findings will be detailed in sections $2-4$, followed by a discussion of the results in this chapter.

As established in chapter one, the serotonergic system is markedly altered in ASD populations. There is a subset of ASD individuals who have hyperserotonemia, which is the elevated levels of 5-HT in blood platelets (Cook \& Leventhal, 1996; Gabriele et al., 2014; Janušonis, 2008; McBride et al., 1998). Platelets are incapable of synthesising 5-HT; instead, 5HT accumulates in platelets via SERT (Veenstra-VanderWeele et al., 2012). Thus, the subset of ASD individuals with hyperserotonemia, they are likely to have a hyperactive SERT in the PNS, resulting in the increased 5-HT levels in the blood platelets. However, a dysfunctional SERT is yet to be confirmed as the cause of hyperserotonemia. Other researchers have suggested that hyperserotonemia is the result of reduced 5-HT clearance from the platelets (Cook \& Leventhal, 1996), or it is the increased synthesis in the intestine, where $95 \%$ of the 5 -HT is synthesised (Croonenberghs, Verkerk, Scharpe, Deboutte, \& Maes, 2005).

While hyperserotonemia provides indications of biological factors in the PNS, it does not necessarily imply that similar 5-HT changes occur in the central nervous system (CNS). It is theorised that individuals with ASD have low intracellular serotonin levels in the CNS (Harrington, Lee, Crum, Zimmerman, \& Hertz-Picciotto, 2013). A deficit in the tryptophanto-5-HT metabolism has been found in a subset of individuals with ASD (Adams et al., 2011; Boccuto et al., 2013; Naushad, Jain, Prasad, Naik, \& Akella, 2013). The relationship between low 5-HT and ASD symptoms was further highlighted when adults with ASD were given a low tryptophan diet (which decreases 5-HT precursor, and subsequently 5-HT levels) for 24 hours, and this resulted in a significant worsening of ASD symptoms (McDougle et al., 1996). Furthermore, the relationship between low 5-HT levels and ASD symptoms is supported by a subset of ASD individuals who experience symptom relief following treatment with SSRIs, which increases the extracellular availability of 5-HT and therefore the 5-HT levels in the CNS (Hollander et al., 2012; McDougle et al., 1996; Moore et al., 2004). Altogether, these findings are indicative of significant serotonergic alterations underlying and contributing to ASD symptoms. 
Given the influence of the serotonergic system on ASD symptomology, studies have examined 5-HT aberrations in the VPA induced ASD animal model. Dufour-Rainfray et al. (2010) examined behaviour and serotonergic alterations, in rats that were prenatally exposed to $600 \mathrm{mg} / \mathrm{kg}$ (via IP injection) of VPA on GD 9. Gestational day nine was selected because the 5HTergic cells are present at this point but are not yet fully formed (Zhou \& Iacovitti, 2000). Dufour-Rainfray et al. (2010) found that adult rats prenatally exposed to VPA had a 46\% decrease in serotonin levels in the hippocampus but no difference between VPA exposed and controls was observed in the cortex or cerebellum. In comparison, Narita et al. (2010) examined the 5 -HT levels in adult offspring that were prenatally exposed to $800 \mathrm{mg} / \mathrm{kg}$ of VPA on GD 9 (via oral gavage). They found increased 5-HT levels in the hippocampus, cerebellum and plasma but, again, no differences in the cortex. On the other hand, Tsujino et al. (2007) found higher levels of 5-HT in the frontal cortex of offspring that were prenatally exposed to $800 \mathrm{mg} / \mathrm{kg}$ administered orally on GD 9. Both Tsujino et al. (2007) and Miyazaki et al. (2005) showed offspring prenatally exposed to VPA have a significant shift in the location of 5-HT cells in the dorsal raphe nucleus, with the cell bodies being more caudally situated after VPA. The shift in location suggests that prenatal exposure to VPA changes neuronal migration. This has been supported by Kuwagata, Ogawa, Shioda, and Nagata (2009), who found that the oral administration of $800 \mathrm{mg} / \mathrm{kg}$ on GD 11 resulted in GD 16 foetuses exhibiting abnormal 5-HT migration. Overall, these studies demonstrate the prenatal exposure to VPA has a profound impact on 5-HT neurotransmission in the CNS.

The 5-HT system also plays an essential role in social behaviour. Following the release of 5-HT, the principal metabolite 5-hydroxyindoleacetic acid (5-HIAA) is produced, which can be detected in cerebrospinal fluid and can be used as an in vivo measure of CNS 5-HT levels (Semerdjian-Rouquier, Bossi, \& Scatton, 1981). In particular, a negative relationship between levels of 5-HIAA and aggressive behaviours has been found in humans, primates, and rodents (Fairbanks, Melega, Jorgensen, Kaplan, \& McGuire, 2001; Higley \& Linnoila, 1997; Maestripieri, McCormack, Lindell, Higley, \& Sanchez, 2006). This relationship also extends to non-aggressive social behaviour, where Tõnissaar, Philips, Eller, and Harro (2004) found a similar negative relationship between time spent in social interaction and the 5-HIAA levels in the frontal cortex of Wistar rats. Thus, emphasizing the role of 5-HT in social behaviour.

In addition, there is genetic evidence to further support a role for 5-HT in social behaviour. Watson, Ghodasra, and Platt (2009) examined non-human primates (Rhesus macaques) for the 5-HTTLPR polymorphism and the relationship with social responses. They found that macaques, heterozygous for the s-allele (which have reduced SERT function) 
exhibited more anxiety-like behaviour than macaques homozygous for the L-allele when viewing images of high-status males and conspecifics. This research shows that the variants of 5-HTTLPR differentially contribute to anxiety behaviours.

Moreover, social deficits have been described in animals with genetic deletion or reduction of the SERT. Kalueff et al. (2007) found that in dyadic interactions, SERT ${ }^{-/}$pairs showed reduced social sniffing and following compared to SERT ${ }^{+++}$mice pairs. Further to this, Moy et al. (2009) showed that SERT ${ }^{-/}$mice have decreased social preference, as these mice spent significantly less time with a social stimulus in phase one compared the SERT ${ }^{+/+}$and $\mathrm{SERT}^{+/-}$males; there were no effects for females, or in phase two. However, social behaviour in mice should be interpreted with caution, as social play and related behaviours is much less developed in mice when compared to rats (Pellis \& Pasztor, 1999; Poole \& Fish, 1975); as such, rats provide a better model for social behaviour in humans (Wöhr \& Scattoni, 2013).

Similar to social deficits found in SERT ${ }^{-/-}$mice, rats with genetic deletion leading to nonfunctional SERT also exhibit social deficits. Using adolescent rats (PND 28-35), Homberg et al. (2007) found that $\mathrm{SERT}^{-/-}$rats exhibit decreases in active play behaviours (defined as pinning, pouncing, and boxing), but did not exhibit differences in exploring their playmate. Also, these SERT $^{-/-}$rats exhibited interest in the play partner (defined as sniffing or licking/grooming play partner) but little play initiation (defined by the active play behaviours; Homberg et al., 2007). However, Olivier et al. (2008) showed that SERT ${ }^{-/-}$rats had increased anxiety levels in an open field test, elevated plus maze, and in a novelty suppressed feeding task. Therefore, it cannot be excluded that the reduction in play behaviour of the $\mathrm{SERT}^{-/-}$rats was secondary to increased anxiety levels. These studies indicate that animals with a genetic deletion of the SERT show reduced social behaviour reminiscent of ASD. However, such complete genetic deletions (SERT ${ }^{-}$ I-) have not been observed in humans, as the reduced 'short' (s) form of the 5-HTTLPR leads to reduction in SERT expression and function(Lesch et al., 1996). This is similar to the reduction observed in SERT ${ }^{+/-}$rats and mice, and therefore, this chapter examines the effects of gestational VPA exposure on SERT ${ }^{+/+}$and $\mathrm{SERT}^{+/-}$rats.

The combination of genetic alterations in the SERT and environmental challenges has, so far, rarely been explored. Schipper, Nonkes, Karel, Kiliaan, and Homberg (2011) examined the effects of early-life exposure to construction stress (infrequent and unpredictable noise and vibrations) in SERT ${ }^{+/+}$and SERT ${ }^{-/-}$rats. The SERT ${ }^{-/-}$rats exposed to construction stress showed increased anxiety-like behaviours in the elevated plus maze EPM, which was expected based on unstressed SERT ${ }^{--}$and previous research (Olivier et al., 2008). The genotype $\times$environment 
interaction effects were found in a reversal-learning task, where the $\mathrm{SERT}^{-/-}$exposed to construction stress exhibited increased cognitive flexibility, and the stressed SERT ${ }^{+/+}$exhibited decreased flexibility. The authors suggest the environmental effects interacting with the genetic background can result in positive outcomes (e.g. increased cognitive flexibility) as well as negative outcomes (e.g. increased anxiety behaviours). Also, exposure to stress early in life, decreased the stress response (or hypothalamic-pituitary-adrenal axis activity) in SERT ${ }^{-/-}$rats (Van der Doelen et al., 2014). With particular relevance to this chapter, Ellenbroek et al. (2016) examined ASD related behaviours following the interaction of SERT ${ }^{+/-}$and the exposure to $400 \mathrm{mg} / \mathrm{kg}$ of VPA on GD 12. The authors were unable to find a significant gene-environment interaction but found that VPA-exposed offspring had increased anxiety and reductions in cognitive processing. They suggest the lack of interaction may be attributed to the timing of the environmental challenge, whereby offspring with genetically compromised SERT function are more sensitive earlier or later in gestation. Thus, a continuous administration of VPA as the environmental challenge would capture multiple sensitive periods.

Further work has been undertaken in our laboratory to examine the SERT KO with the prenatal VPA exposure. Ranger (2016) paired SERT ${ }^{+/+}$females with either SERT ${ }^{+/+}$or SERT SE- $^{-/-}$ males, to produced litters with SERT ${ }^{+/+}$and SERT ${ }^{+/}$offspring. The mothers were administered $400 \mathrm{mg} / \mathrm{kg}$ of VPA or saline on GD 12. The offspring were examined for changes in communication, social interaction, and repetitive behaviours. The study showed no changes in communication for pre-weaning offspring, but there were SERT and VPA interactions in social and repetitive behaviours of the adult offspring. The VPA exposure for $\mathrm{SERT}^{+/-}$rats resulted in increased active social behaviours, whereas the $\mathrm{SERT}^{+/+}$exposed to VPA exhibited significant decreases. This pattern was also found in the measure of repetitive behaviours, where the $\mathrm{SERT}^{+/-}$exposed to VPA made significantly fewer entries into previously explored areas, compared with the SERT ${ }^{+/+}$exposed to VPA which made significantly more entries. Thus, the SERT $^{+/}$exposed to VPA, did not show the expected decreases in these measures and the author suggests that SERT ${ }^{+/-}$were, in fact, more resilient to prenatal VPA exposure. Ultimately, Ranger (2016) showed that compromised SERT function in utero is perhaps protective against environmental challenges, like prenatal VPA exposure.

Overall, research in our laboratory, as well as the inconsistencies in the literature, highlights the difficulty of investigating the consequences of a single prenatal VPA administration, particularly in combination with a genetic factor such as the reduction in SERT activity. The experiments in this chapter assess ASD-like behaviours following chronic prenatal exposure to VPA as an environmental risk factor combined with the genetic vulnerability of 
reduced SERT activity. It is relevant to point out that studies examining the social behaviour of SERT animals have typically been done in SERT ${ }^{-/}$rats and mice, not in SERT ${ }^{+/}$animals. As such, the extent of the social deficits in SERT ${ }^{+/}$animals is unclear. The experiments in this chapter follow the work of Ellenbroek et al. (2016) and Ranger (2016). The following experiments replicate behavioural assays and methods established in chapter two, with the inclusion of an anxiety measure (successive alleys test, section 3.5). Furthermore, the VPA administration method was amended and optimised for this chapter (section 3.1.5). Additionally, the VPA doses in this chapter were increased to $300 \mathrm{mg} / \mathrm{kg} /$ day and $600 \mathrm{mg} / \mathrm{kg} /$ day. The VPA doses were increased as the behavioural phenotype identified in chapter two was relatively mild and pregnant rats were able to tolerate the increased dose without dam fatality (based on a pilot study, data not shown).

With the continued aim of chronically administering VPA in a non-stressful manner, and to overcome the limitations of the previous administration method, a novel voluntary administration method was implemented for this chapter, with the use of gelatine pellets. Gelatine pellet delivery is considered a superior option over syringe feeding (Atcha et al., 2010), peanut butter (Main \& Kulesza, 2017), and SCM methods (which was explored in chapter two). Gelatine pellet delivery is a superior option as it is more similar to the oral intake of medication for people (especially chronic exposure), as well as the pellets provide a more precise and less invasive method for administering a dose to rodents (Zhang, 2011). Gelatine pellets were developed based on the work of Zhang et al. (2010), who used a gelatine pellet delivery method for three weeks to administer rimonabant (an inverse agonist of cannabinoid CB1 receptor, antiobesity drug) to neuropeptide Y (NPY) knockout mice, and successfully showed that blocking CBl and NPY lead to reductions in body weight not bone mass. Subsequently, Zhang (2011) detailed the protocol for gelatine pellets as a method for oral administration of drugs in mice. The protocol was successfully implemented by Cheng, Spiro, Jenner, Garner, and Karl (2014) who showed that mice tolerated long term (8 months) administration of phytocannabinoid cannabidiol (CBD), which led to the successful prevention of memory deficits in Alzheimer's disease transgenic mice. We, therefore, used this gelatine pellet oral delivery method in this chapter (see 3.1.5 VPA administration for further details). 


\subsubsection{Overall Aims of Chapter three}

The experiments in this chapter will examine the behaviours of rats with a genetic reduction in SERT that were chronically exposed to VPA throughout gestation. This will advance the VPA-induced ASD animal model, by combining gene and environmental factors to examine the impact on ASD-like behaviours, as well as advancing the chronic administration method for delivering VPA throughout gestation.

The overall aim of this series of experiments is to assess the communication, social interaction and anxiety-like behaviours of rats with genetic deficits in the SERT following chronic prenatal exposure to VPA. Based on previous literature, it is typically the SERT ${ }^{-/}$ genotype that exhibits phenotypic differences in comparison to $\mathrm{SERT}^{+/+}$rats. As such, it is uncertain if the SERT ${ }^{+/}$genotype will exhibit deficits in communication, social interaction, and anxiety-like behaviours. Thus, it is hypothesised that the interaction of the SERT deficits with chronic VPA prenatal exposure will result in a more pronounced ASD-like phenotype. Therefore, it is predicted that:

i. Males and females prenatally exposed to VPA will exhibit decreased communication and sociability with increased anxiety-like behaviours, compared to rats prenatally exposed to the vehicle,

ii. There will be an interaction between SERT reduction and chronic exposure to VPA. As such, SERT $^{+/}$prenatally exposed to VPA will exhibit a stronger decrease in sociability and increased anxiety-like behaviours compared to $\mathrm{SERT}^{+/+}$exposed to VPA,

iii. Finally, the decreases exhibited by male and female $\mathrm{SERT}^{+/+}$and $\mathrm{SERT}^{+/-}$ prenatally exposed to VPA will persistent over their lifespan. 


\subsubsection{Experimental Animals}

Seven adult heterozygous (SERT ${ }^{+/}$) males and 19 adult nulliparous wildtype (SERT ${ }^{+/+}$) female Wistar rats were randomly selected from breeding stock at VUW vivarium. All females were handled by the experimenter for five minutes per day for three days before mating, to mitigate the handling stress during gestation. During the handling phase, the females were given gelatine pellets with a range of flavours to overcome neophobia, which could interfere with VPA ingestion during gestation.

Four days after the first handling and gelatine exposure session, each female was paired with one male, in polycarbonate cages which included a metal grid lining the tray bottom. The grid enabled the detection of vaginal mucus plugs. Plugs were checked once 24 hours after pairing, and each day following for a further seven days. If no mucus plug was discovered, the female was returned to the animal stock. When a mucus plug was detected, the male and female were separated. The male was then placed in group housing for later pairing or euthanising and the female was randomly allocated into a dose condition of 0,300 , or $600 \mathrm{mg} / \mathrm{kg} /$ daily of VPA weighed then housed individually. On the day the mucus plug was detected (GD 1), the dams were given their first gelatine pellet containing a dose of VPA (or control dose; see section 3.1.5 VPA administration for further detail).

Females were checked daily for delivery of the litter. The day of birth was considered PND 1. Mostly, the dam and pups were left undisturbed until weaning on PND 21, with the exceptions of USV recordings on PND 7 and 14 (see section 3.2) and weekly cleaning of the cage. On PND 21 the dam was removed from the offspring and euthanised. The offspring had an RFID tag subcutaneously implanted, and a small ear punch ( $2 \mathrm{~mm}$ diameter) was taken for genotyping (TransnetYX, Cordova, TN). The offspring were separated in sex-specific cages where they were housed in litter specific groups until they weighed approximately $150-200 \mathrm{~g}$, at which point they were housed in groups of two or three in each cage.

\subsubsection{Housing Conditions for All Animals}

The housing environment was maintained at a temperature of $21^{\circ} \mathrm{C}( \pm 2)$ and a humidity level of 55-60\%, with reversed night/day cycle (lights on 1900-0700 hours). Animals were housed in conventional wire top cages were lined with pine or paper bedding (with the exception of metal grids lining the tray bottom for breeding pairs). All animals, including breeding pairs and offspring, had ad libitum access to water and laboratory chow (Speciality Feeds Rat and Mouse Cubes, Glen Forest, Aus). All animals were bred and housed at the VUW 
vivarium. All procedures followed the VUW Animal Ethics guidelines (AEC number 000022477).

\subsubsection{VPA Administration}

The female's weight was recorded on GD 1 and every four days following, to adapt the VPA doses to the specific weight of each female. Batches of gelatine pellets were made for each female so that the VPA dose was tailored to her weight, and dose condition. Each day of gestation in the morning (0700-0900 hours) and evening (1700-1900 hours) the experimenter weighed a VPA (or control) gelatine pellet and placed it in the cage for the pregnant females to voluntarily consume. The remains of the gelatine pellet were collected and weighed before the next dose was placed in the cage. This allowed for a calculation of the actual dose consumed for each individual female (see table 3.1 for average consumption).

Morning and evening doses were intended to maintain VPA blood levels by reducing the time between doses. Furthermore, it reduced the concentration of VPA in each pellet, and the resulting doses were more palatable for the females, as VPA has an unpleasant taste (Cloyd et al., 1992). Potentially because of the unpleasant taste, the females did not reliably consume the entire pellet. To increase consumption of VPA and to avoid pellets from drying out, new batches were created every four days with a rotation of different flavours (orange, strawberry, banana, caramel, and blueberry; GoBake 'Favacol'). Flavours were changed in each batch to avoid the adverse association of VPA taste with pellet. The average weight of the pellets was 4.2 grams. Figure 3.1 displays the typical size and shape of the VPA gelatine pellets.

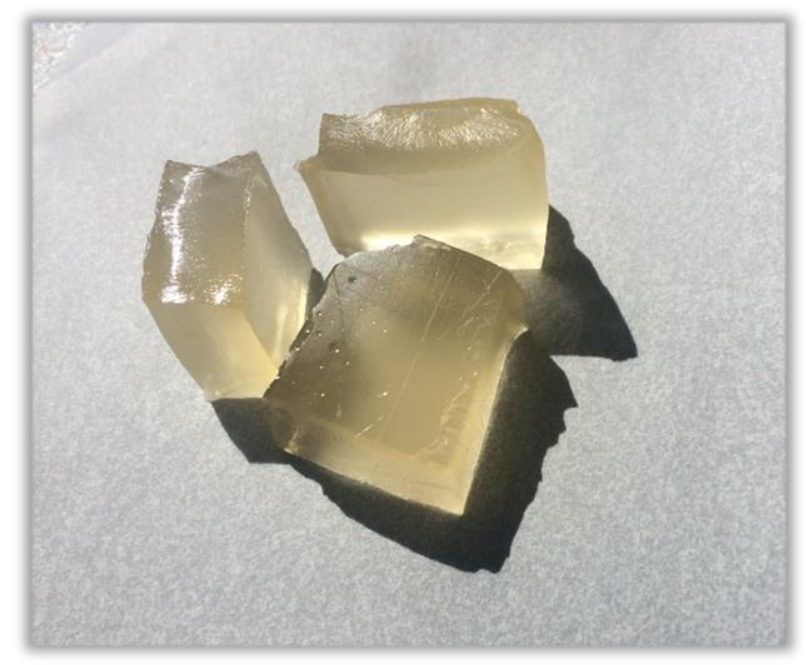

Figure 3.1. Banana flavoured VPA gelatine pellets, representative of typical doses given morning and evening to pregnant females. 


\subsubsection{Experimental Animal Breeding Outcomes}

Nineteen litters were bred in this study, which produced a total of 198 offspring. Two females that were given $600 \mathrm{mg} / \mathrm{kg} /$ day birthed stillborn pups (litter identifier, ID 004 and 010, see table 3.1). Also, one female from the $600 \mathrm{mg} / \mathrm{kg} /$ day group produced a litter of 15 pups (ID 008) and all except one died in the two days following birth. The remaining pup was euthanised due to the abnormalities of the litter and associated (potential) differences in maternal care. A female in the $300 \mathrm{mg} / \mathrm{kg} /$ day produced only a single pup (ID 001), the singular pup was euthanised with the dam at weaning. Additionally, litter ID $002(300 \mathrm{mg} / \mathrm{kg} / \mathrm{day})$ and 003 $(600 \mathrm{mg} / \mathrm{kg} /$ day) were removed from the analysis due to inconsistent consumption of the gelatine pellets. Overall, across all litters, there were no other dose-related physical abnormalities, such as tail kinks or webbed toes. Such abnormalities were expected following reports in previous studies (Edalatmanesh et al., 2013; Vorhees, 1987).

VPA consumption was calculated from the total VPA doses given, minus the amount left in the remains of the gelatine pellets. The calculations separated the litters based on a range of consumed doses, subsequently divided into low and high prenatal dose conditions. From these calculations, one female (ID 019) from the $600 \mathrm{mg} / \mathrm{kg} /$ day group consumed considerably less VPA and was therefore placed in the low dose condition. Similarly, one female (ID 017) consumed a considerably lower dose than the remainder of the females in the $300 \mathrm{mg} / \mathrm{kg} /$ day group, and the litter was therefore removed from the analysis. As a result, the low dose condition included five litters, with an average intake of 218 to $299 \mathrm{mg} / \mathrm{kg} /$ day. The high dose condition consists of three litters, with an average intake between 351 to $394 \mathrm{mg} / \mathrm{kg} /$ day (see table 3.1 for details on consumption and resulting offspring). These prenatal dose conditions are compared with the control offspring from four litters that received the gelatine pellets without VPA. 
Table 3.1. Litter information displaying intended and consumed doses with resulting genotype and litter size. The shaded rows refer to included litters (control dose - light green, low dose green, and high dose - purple), ' ' refers to approximation, and '-' refers to unavailable information.

\begin{tabular}{|c|c|c|c|c|c|c|c|c|}
\hline \multirow{3}{*}{$\begin{array}{l}\text { Litter } \\
\text { ID }\end{array}$} & \multirow{3}{*}{$\begin{array}{l}\text { Given dose } \\
(\mathrm{mg} / \mathrm{kg} / \text { day })\end{array}$} & \multirow{3}{*}{$\begin{array}{c}\text { Average } \\
\text { consumed } \\
\text { dose } \\
(\mathrm{mg} / \mathrm{kg} / \text { day })\end{array}$} & \multirow{3}{*}{$\begin{array}{c}\text { Allocated } \\
\text { Dose } \\
\text { Group }\end{array}$} & \multicolumn{5}{|c|}{ Offspring } \\
\hline & & & & \multicolumn{2}{|c|}{ Males } & \multicolumn{2}{|c|}{ Females } & \multirow{2}{*}{ Total } \\
\hline & & & & SERT $^{+/+}$ & SERT $^{+/-}$ & SERT $^{+/+}$ & SERT $^{+/-}$ & \\
\hline 005 & 0 & 0 & \multirow{4}{*}{ Control } & 3 & 5 & 2 & 1 & 11 \\
\hline 009 & 0 & 0 & & 4 & 2 & 1 & 3 & 10 \\
\hline 015 & 0 & 0 & & 1 & 6 & 5 & 2 & 13 \\
\hline 016 & 0 & 0 & & 2 & 10 & 0 & 1 & 13 \\
\hline 001 & 300 & -183 & & - & - & - & - & 1 \\
\hline 002 & 300 & -186 & & 1 & 1 & 3 & 4 & 9 \\
\hline 006 & 300 & 218 & \multirow{6}{*}{ Low } & 5 & 2 & 1 & 6 & 14 \\
\hline 007 & 300 & 264 & & 6 & 2 & 1 & 6 & 15 \\
\hline 012 & 300 & 259 & & 5 & 8 & 2 & 1 & 16 \\
\hline 013 & 300 & 299 & & 1 & 1 & 5 & 3 & 10 \\
\hline 017 & 300 & 184 & & $z$ & 3 & 4 & $z$ & $H$ \\
\hline 019 & 600 & 277 & & 4 & 2 & 4 & 2 & 13 \\
\hline 003 & 600 & -267 & & $\theta$ & 1 & 6 & 1 & 8 \\
\hline 004 & 600 & $\sim 410$ & & $=$ & $=$ & $=$ & $=$ & $\begin{array}{c}\text { Deceased at } \\
\text { birth }\end{array}$ \\
\hline 008 & 600 & 394 & & - & $=$ & - & - & $\begin{array}{c}15- \\
\text { Deceased }\end{array}$ \\
\hline 010 & 600 & 355 & & $=$ & $=$ & $=$ & $=$ & Deceased \\
\hline 011 & 600 & 394 & \multirow{3}{*}{ High } & 3 & 4 & 6 & 3 & 16 \\
\hline 014 & 600 & 351 & & 4 & 3 & 3 & 1 & 11 \\
\hline 018 & 600 & 376 & & 5 & 6 & 1 & 1 & 13 \\
\hline
\end{tabular}




\subsection{Experiment One: Isolation Induced Ultrasonic Vocalisations}

As discussed in chapter two (section 2.2), isolation-induced ultrasonic vocalisations (USVs) are an effective method of measuring communication between rats. Given that communication deficits are a core symptom of ASD, this assay allows for examination of early developmental alterations in animals.

Previous research has shown that rats exposed to doses of 400-500mg/kg of VPA at GD 12 show significant reductions in the total number of USVs when recorded at PND 9 (Servadio et al., 2018), similar has also been found in mice (Gandal et al., 2010). Chapter two showed that chronic prenatal exposure of VPA in Sprague Dawley rats impacted the quality rather than the quantity of vocalisations emitted by 7 - and 14-day-old pups. With specific relevance to Wistars and the SERT animals, previous research in our laboratory has recorded and examined the USVs of pre-weaning SERT rats. Ranger (2016) prenatally exposed SERTs to VPA and found no interaction, treatment, or genotype effect. Overall, there are divergent findings relating to the pre-weaning USVs emitted by pups prenatally exposed to VPA. This may be related to a lack of investigation into the quality of USVs, as most researchers only examine the number and length of USVs (see chapter two, section 2.2 for further details).

Continuing with the overall aims of this chapter (section 3.1.2), this experiment aims to examine the communication of pre-weaning pups that were prenatally exposed to VPA throughout gestation. As pups were genotyped at weaning, the following experiment does not account for the genetic reduction of the SERT ${ }^{+/-}$pups. Therefore, it is hypothesised that;

i. Rats prenatally exposed to VPA will exhibit decreased communication (number and duration of calls, and decreased pitch) compared with rats prenatally exposed to the control dose,

ii. The decreased communication exhibited by pups prenatally exposed to VPA will be consistent from PND 7 to PND 14. 


\subsubsection{Isolation Induced USV methods}

USV methods are identical to those described in study one, section 2.1.

\subsubsection{USV data analysis}

As genotyping was not completed until PND 21, the genotype of the offspring was not considered in the USV analysis. The USV parameters were examined with a 3 (Prenatal dose: Control, Low, High) as the between-subject factor; by 2 (PND: 7, 14) as the within-subjects factor, repeated measures ANOVA. A Greenhouse-Geisser correction was used on all measures due to violations of sphericity. The repeated measures ANOVAs were used to analyse the number, duration, low and high frequencies as well as the range in frequencies of vocalisations over time. Partial eta squared $\left(\eta_{\mathrm{p}}{ }^{2}\right)$ was used for the effect size. All significant analyses were followed up with Tukey HSD post hoc tests. The alpha level for statistical significance was set at $p<.05$.

\subsubsection{Sample Selection for Comparative Analysis}

Seventy-two pups were isolated and recorded for this experiment, 66 recordings were subsequently analysed. In total, 24 offspring were selected from the four control litters. Two recordings from a control litter (ID 009) were excluded from analysis due to a lack of vocalisations, thus reducing the number of analysed recordings to 22 . There were 30 offspring recorded from the low dose condition (five litters). However, a recording from a litter (ID 019) and a recording from 007 was excluded due to a lack of vocalisations, two recordings from a litter (ID 006) were not available due to data corruption; thus 26 recordings were analysed. Additionally, there were 18 recordings from the high dose condition (three litters), with one recording excluded (from litter 018) due to a lack of vocalisations, leading to 17 recordings analysed from the high dose condition. 


\subsubsection{Isolation Induced USV results}

\section{Number of vocalisations emitted on PND 7 compared with PND 14}

The number of vocalisations increased from PND 7 to $14\left(F(1,62)=12.14, p=.001, \eta_{\mathrm{p}}{ }^{2}=\right.$ .16), but there was no interaction with prenatal dose exposure (PND $\times$ prenatal dose: $F(1,62)=$ $1.18, p=.314, \mathrm{\eta}_{\mathrm{p}}{ }^{2}=.04$; prenatal dose main effect: $\left.F(1,62)=1.53, p=.224, \mathrm{\eta}_{\mathrm{p}}{ }^{2}=.05\right)$. The follow up analysis showed that the PND effect was due to the low dose offspring emitting significantly more vocalisations on PND 14 compared to PND 7 ( $p=.001$, see figure 3.2). Neither the controls nor the high dose exhibited statistically significant increases from PND 7 to 14 .

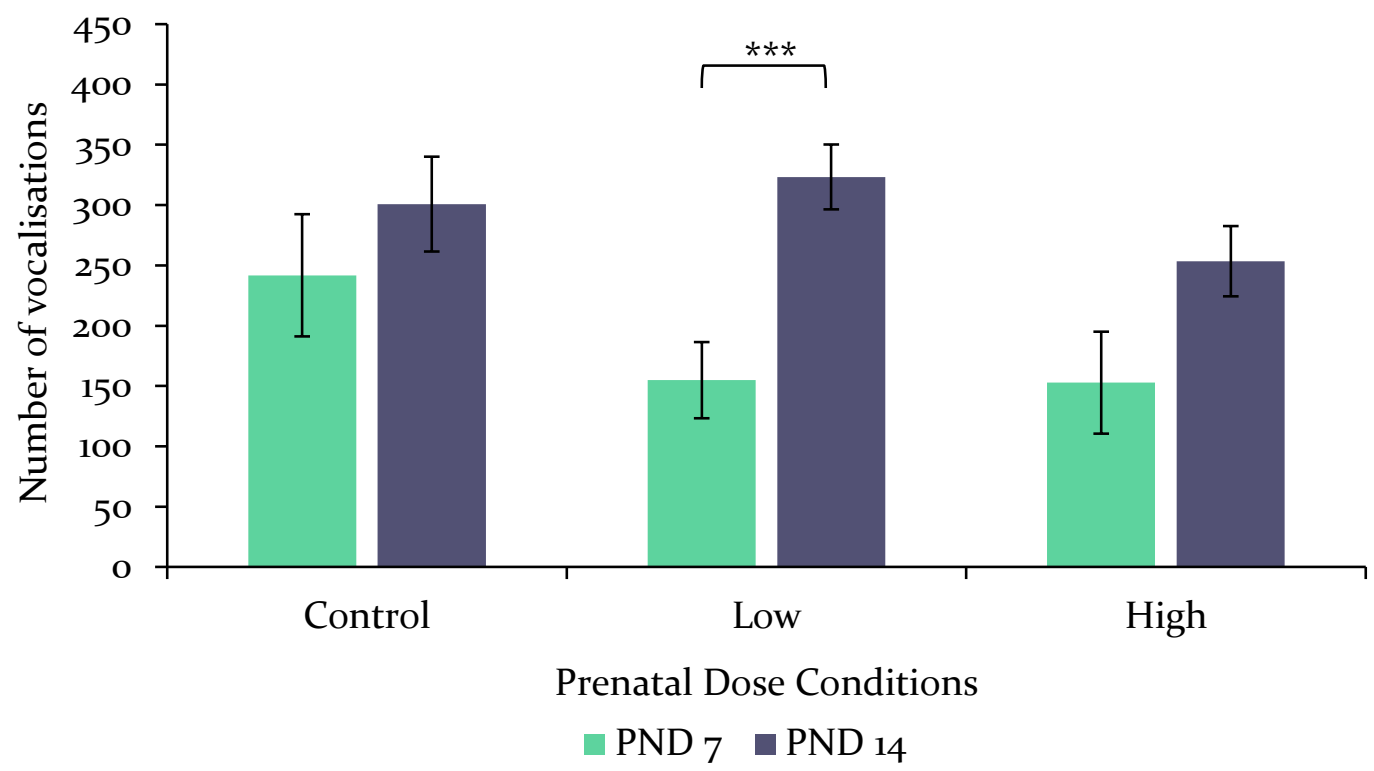

Figure 3.2. The average number of vocalisations emitted on PND 7 and 14 by pups prenatally exposed to control, low, or high doses of VPA. Pups exposed to the low doses emitted significantly more vocalisations on PND 14 compared to PND 7. ${ }^{* * *}$ indicates significance to $p$ $<$.001. Error bars represent \pm SEM. 


\section{Duration of vocalisations emitted on PND 7 compared with PND 14}

The length of the vocalisations increased from PND 7 to $14(F(1,62)=48.58, p=.001$, $\left.\eta_{\mathrm{p}}{ }^{2}=.44\right)$, there was a close to significant interaction with the prenatal doses $(F(2,62)=2.89, p$ $\left.=.063, \mathrm{\eta}_{\mathrm{p}}^{2}=.09, n s\right)$, and there was an overall main effect of the prenatal doses $(F(2,62)=3.55$, $\left.p=.035, \eta_{\mathrm{p}}^{2}=.10\right)$. The follow up analysis showed that the vocalisations of pups exposed to the high dose were significantly longer than the vocalisation of low dose pups ( $p=.032$ ).

To follow up the close to significant interaction effect between PND and prenatal doses, the length of vocalisations was examined on PND 7 and 14 separately. The prenatal dose conditions did not significantly affect the length of vocalisation on PND $7(F(1,62)=.79, p=$ $\left..46, \eta_{\mathrm{p}}{ }^{2}=.02, n s\right)$, but it did on PND $14\left(F(1,62)=4.11, p=.021, \eta_{\mathrm{p}}{ }^{2}=.12\right)$. The post hoc analysis confirmed the overall findings, where the vocalisations of pups exposed to the high dose were significantly longer compared with the pups exposed to the low dose $(p=.016$, see figure 3.3$)$.

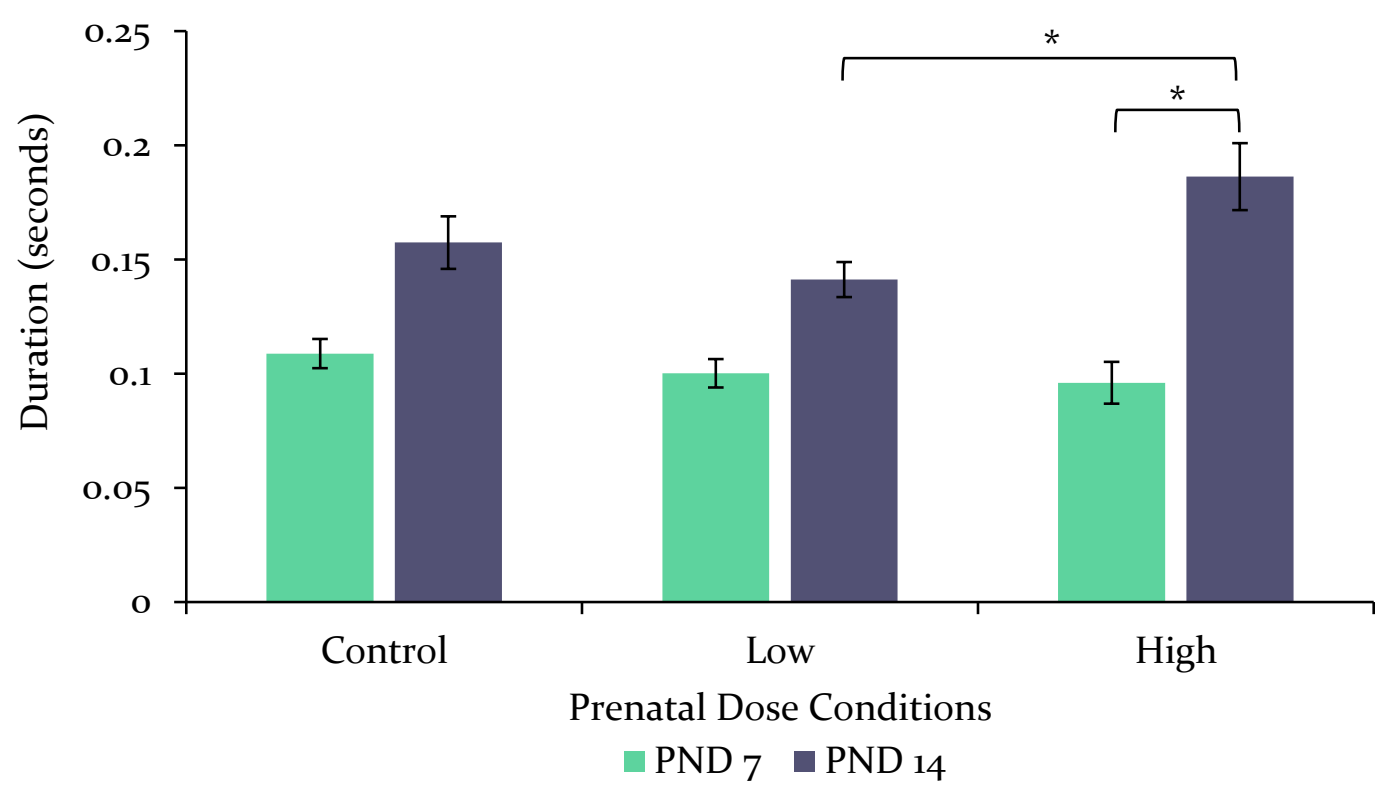

Figure 3.3. The average duration (or length) of vocalisations emitted on PND 7 and 14 by pups prenatally exposed to control, low, or high doses of VPA. The vocalisations were longer on PND 14 , and the high dose emitted longer vocalisations than low dose pups. * indicates significance to $p<.05$. Error bars represent \pm SEM. 


\section{Frequency of vocalisations emitted on PND 7 compared with PND 14}

In the analysis of the lowest frequency of the vocalisations, there was no change from PND 7 to $14\left(F(1,62)=2.46, p=.122, \eta_{\mathrm{p}}^{2}=.04, n s\right)$, the exposure to the prenatal doses did not affect the lowest frequency $\left(F(2,62)=1.20, p=.307, \mathrm{n}_{\mathrm{p}}{ }^{2}=.04, n s\right)$, and there was no interaction between PND and prenatal doses $\left(F(2,62)=1.35, p=.268, \eta_{\mathrm{p}}^{2}=.04, n s\right.$, data not shown).

In comparison, analysis of the highest frequency showed a significant effect of PND ( $F(1$, $\left.62)=23.34, p=.001, \eta_{\mathrm{p}}{ }^{2}=.27\right)$, and of prenatal doses $\left(F(1,62)=3.19, p=.048, \eta_{\mathrm{p}}{ }^{2}=.09\right)$, as well as an interaction between PND and prenatal doses $\left(F(2,62)=4.31, p=.018, \eta_{\mathrm{p}}{ }^{2}=.12\right)$. The post hoc analysis showed that vocalisations emitted by the high dose pups had a significantly lower high frequency than those of low dose pups $(p=.047)$, but there was no difference between VPA exposure and the control dose.

To further explore the interaction of the prenatal doses and PND, the highest frequency was examined within each of the prenatal dose conditions, separately. There was no significant change in the high frequency for the pups prenatally exposed to a high dose $(F(1,16)=1.38, p=$ $\left..258, \mathrm{\eta}_{\mathrm{p}}{ }^{2}=.08, n s\right)$. However, the pups exposed to the control $\left(F(1,21)=4.83, p=.039, \mathrm{\eta}_{\mathrm{p}}{ }^{2}=.19\right)$ and low $\left(F(1,25)=28.58, p=.001, \eta_{\mathrm{p}}^{2}=.53\right)$ doses exhibited a significant increase in the high frequency from PND 7 to 14 (see figure 3.4).

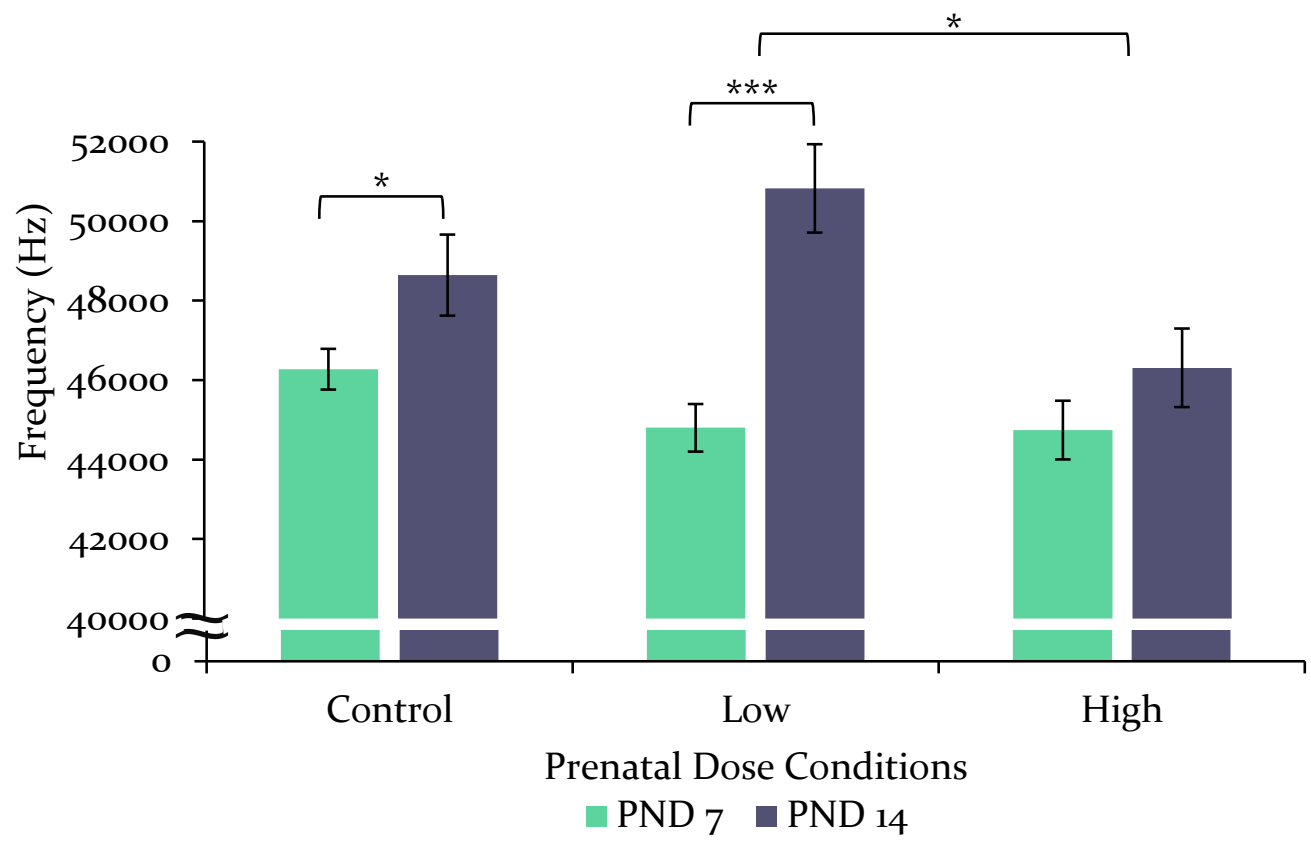

Figure 3.4. The average highest frequencies of vocalisations emitted on PND 7 and 14 by pups prenatally exposed to control, low, or high doses of VPA. The shortened y-axis shows the small difference in the high frequency of the high dose group across PND. * indicates significance to $p<.05$, and ${ }^{* * *}$ indicates significance to $p<.001$. Error bars represent \pm SEM. 
Finally, the overall frequency range of vocalisations (the total frequency difference between the low and high frequency within a single call) showed a significant main effect of $\operatorname{PND}\left(F(1,62)=80.24, p=.001, \eta_{\mathrm{p}}^{2}=.56\right)$, and prenatal doses $\left(F(1,62)=6.97, p=.002, \mathrm{\eta}_{\mathrm{p}}^{2}=\right.$ $.18)$, as well as an interaction between PND and prenatal dose conditions $(F(2,62)=6.97, p=$ $\left..002, \mathrm{\eta}_{\mathrm{P}}{ }^{2}=.18\right)$. The frequency range increased from PND 7 to PND 14 ( $p=.001$, see figure 3.5), and the post hoc analysis showed that the frequency range of the high dose pups was significantly smaller than that of low dose pups $(p=.011)$.

To explore the interaction further, the frequency range was examined within each of prenatal dose conditions, separately. All prenatal dose conditions exhibited a significant increase in the frequency range from PND 7 to 14 (control: $F(1,21)=23.21, p=.001, \eta_{\mathrm{p}}{ }^{2}=.53$; low: $F(1,25)=60.40, p=.001, \eta_{\mathrm{p}}^{2}=.71$; high: $F(1,16)=11.37, p=.004, \eta_{\mathrm{p}}{ }^{2}=.42$; see figure 3.5$)$.

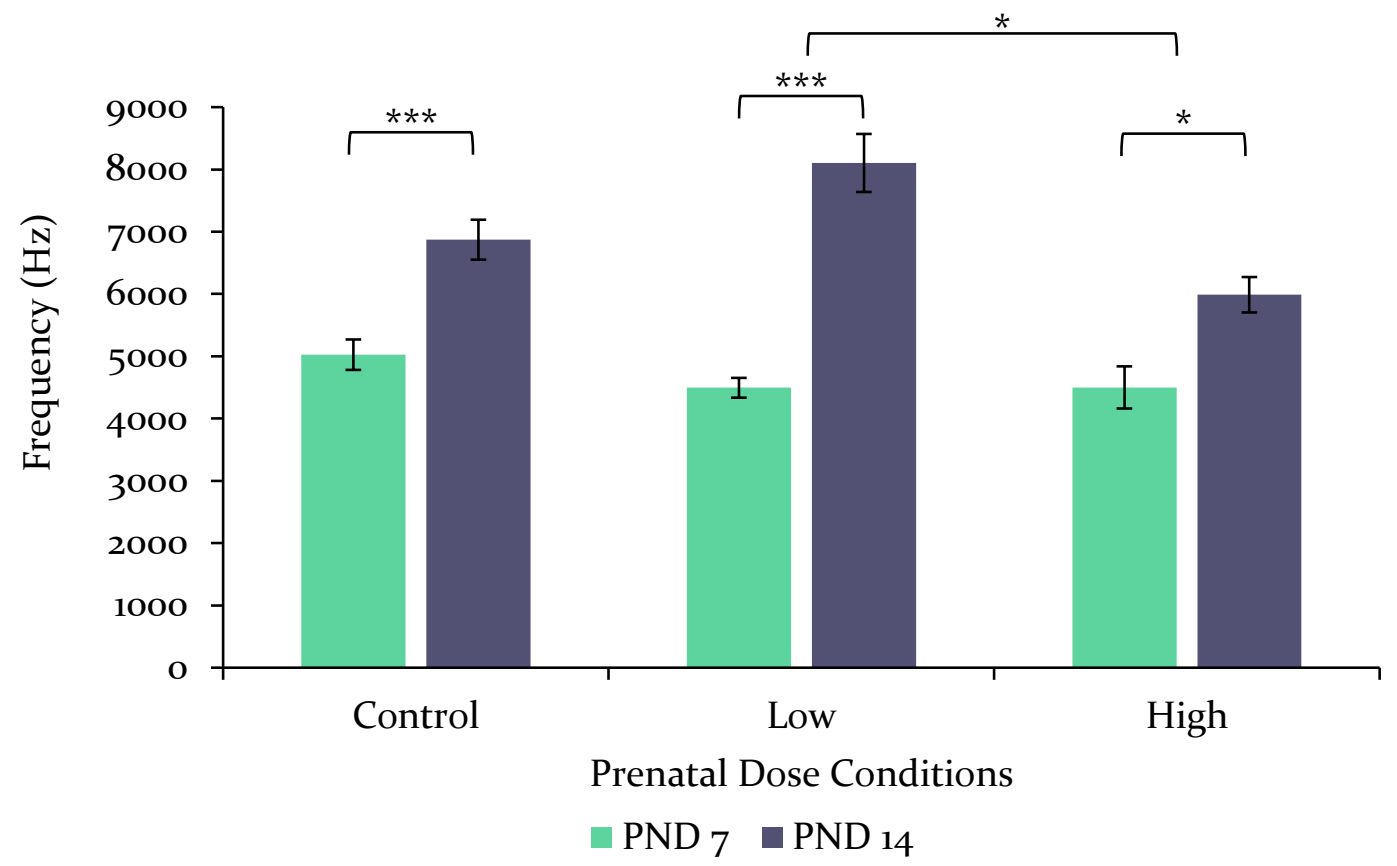

Figure 3.5. The average range of frequency for vocalisations emitted on PND 7 and 14 by pups prenatally exposed to control, low, or high doses of VPA. Pups exposed to high dose emitted calls that were of a narrower range than the pups exposed to the low dose. * indicates significance to $p<.05$, and ${ }^{* *}$ indicates significance to $p<.001$. Error bars represent \pm SEM. 


\subsection{Experiment Two: Play Behaviour}

The purpose of this experiment was to investigate the duration and frequency of social interactions between cage mates of SERT ${ }^{+/+}$and $\mathrm{SERT}^{+/-}$juvenile rats that were chronically exposed to VPA during gestation. The literature and research supporting the investigation of play behaviour in rats, specifically those prenatally exposed to VPA is outlined in chapter two (section 2.3), and the play behaviour of SERT KO animals has been outlined in chapter one (section 1.7.3).

This experiment aimed to examine the play behaviour of juvenile SERT ${ }^{+-}$and SERT ${ }^{++}$ males and females prenatally exposed to VPA throughout gestation. However, there was a confound with genotype pairing for this experiment. As the sample tissue for the genotyping analysis was taken on PND 21, the results were not available at the time of pairing the animals for the experiment, thus pairs were randomly assigned without consideration for their genotype (see table 3.3 for a full break down of the number of pairs). Therefore, the genotypes of the offspring could not be considered for this experiment. Given this situation and the overall aim of this chapter, it is hypothesised that:

i. Males as well as females prenatally exposed to VPA will exhibit decreased active play behaviours (chasing and pinning) compared with rats prenatally exposed to the control dose

ii. The decrease in behaviour will differ as a function of the prenatal dose, where the rats prenatally exposed to the high dose will exhibit a greater decrease in behaviour compared to the rats prenatally exposed to the low dose. 


\subsubsection{Play Behaviour Methods}

Play behaviour methods were closely matched with methods described in chapter two, section 2.3.1. Briefly, after weaning on PND 21, animals were habituated to the testing room for 10 minutes, then same-sex and within litter pairs were habituated to the testing arena for 10 minutes. Then returned to their home cage in the housing room. This habituation process was repeated on PND 22.

Unlike the methods detailed in chapter two, there was a single testing day between PND 22-25 for this experiment, as the double recording in chapter two did not substantially enhance the analysis. On the testing day, animals were habituated to the room (10 minutes) and the arena (10 minutes). Subsequently, the animals were placed in a clean cage by themselves for three and a half hours. After three hours and 20 minutes, the isolated animals were then returned to the room for 10 minutes and placed in the arena for an additional 10 minutes to record their behaviour in the test situation. During the testing phase, the behaviours (pinning, chasing, and passive social behaviour) were manually scored by one experimenter.

\subsubsection{Play behaviour data analysis}

The data analysis methods follow those described in chapter two, section 2.3.1. For this experiment, the variables of interest were the duration and frequency of pinning, chasing, and passive social behaviour. Data were analysed for statistical significance with SPSS (version 2225).

As the experiment examined the effect of prenatal exposure to VPA in males and females separately, two 3 (Dose; control, low, high) by 3 (Genotype of pairs; $\mathrm{SERT}^{+/+} \mathrm{x} \mathrm{SERT}^{+/+}$, $\mathrm{SERT}^{+/+}$ $\mathrm{x} \mathrm{SERT}^{+-}, \mathrm{SERT}^{+/-} \mathrm{x} \mathrm{SERT}^{+-}$) ANOVAs was run for each dependent variable; one for males, and one for females. However, due to the limitations of this experiment the genotype was excluded, and the focus on the analysis was on a 3 (Dose; control, low, high) ANOVAs, separated for males and females. Partial eta squared $\left(\mathrm{n}_{\mathrm{p}}^{2}\right)$ was used for the effect size. All significant analyses were followed up with Tukey HSD post hoc tests. The alpha level for statistical significance was set at $p<.05$. 


\subsubsection{Play Behaviour Results}

As mentioned above, the genotype pairs were randomly assigned without consideration for their genotype. This led to three genotype pair groups of SERT ${ }^{+/+}$with SERT ${ }^{+/+}$, $\mathrm{SERT}^{+/+}$with SERT $^{+/-}$, or SERT ${ }^{+/-}$with SERT ${ }^{+/-}$. With the random allocation and the exclusion of litters, some conditions had few samples, and the analyses were run on the males and females exposed to control, low, and high doses, regardless of the genotype (see figure 3.3 for a full break down of the number of pairs). Additionally, figures appendix A section A, illustrate the average duration of chasing, pinning, and passive play behaviour with genotype information included.

Table 3.3. The number of play behaviours pairs recorded based on prenatal dose, sex, and the genotype pairing, with the subsequently combined totals.

\begin{tabular}{c|c|c|c|c|c|}
\hline \hline \multicolumn{1}{c|}{} & $\begin{array}{c}\text { SERT }^{+/+} \mathrm{x} \\
\text { SERT }^{+/+}\end{array}$ & $\begin{array}{c}\text { SERT }^{+/+} \mathrm{x} \\
\text { SERT }^{+/-}\end{array}$ & $\begin{array}{c}\text { SERT }^{+/-} \mathrm{x} \\
\text { SERT }^{+/-}\end{array}$ & Combined \\
\hline \multirow{4}{*}{ Control } & Males & 1 & 7 & 7 & 15 \\
\cline { 2 - 7 } & Females & 2 & 3 & 1 & 6 \\
\cline { 2 - 7 } & Combined & 3 & 10 & 8 & 21 \\
\hline \multirow{4}{*}{ Low } & Males & 4 & 5 & 3 & 12 \\
\cline { 2 - 7 } & Females & 1 & 7 & 4 & 12 \\
\cline { 2 - 7 } & Combined & 5 & 12 & 7 & 21 \\
\hline \multirow{3}{*}{ High } & Males & 3 & 4 & 3 & 10 \\
\cline { 2 - 7 } & Females & 2 & 3 & 1 & 6 \\
\cline { 2 - 7 } & Combined & 5 & 7 & 4 & 16 \\
\hline \hline
\end{tabular}




\section{Male chasing behaviour}

There was a significant main effect of prenatal dose on the duration of chasing behaviours $\left(F(2,34)=3.99, p=.028, \eta_{\mathrm{p}}^{2}=.19\right)$. The follow up analyses showed that the offspring prenatally exposed to the high dose spent more time chasing than the offspring exposed to the control dose $(p=.030)$. The prenatal exposure to VPA also increased the frequency of chasing events $\left(F(2,34)=3.83, p=.032, \mathrm{\eta}_{\mathrm{p}}^{2}=.18\right)$, with the follow up analysis also showing higher frequencies in the offspring exposed to the high dose compared to the control dose offspring ( $p$ $=.037$, see figure 3.6 ).

\section{Female chasing behaviour}

The prenatal exposure to VPA significantly increased the time spent engaged in chasing behaviours $\left(F(2,34)=3.99, p=.028, \mathrm{n}_{\mathrm{p}}^{2}=.19\right)$. The frequency of chasing events did not reach significance $\left(F(2,24)=1.89, p=.175, \eta_{\mathrm{p}}{ }^{2}=.15, n s\right)$, though figure 3.7 shows a similar doseresponse curve. The prenatal dose effect on duration was due to females prenatally exposed to the low dose, which had a close to significant increase, compared with offspring exposed to the control dose ( $p=.054, n s$; see figure 3.6).
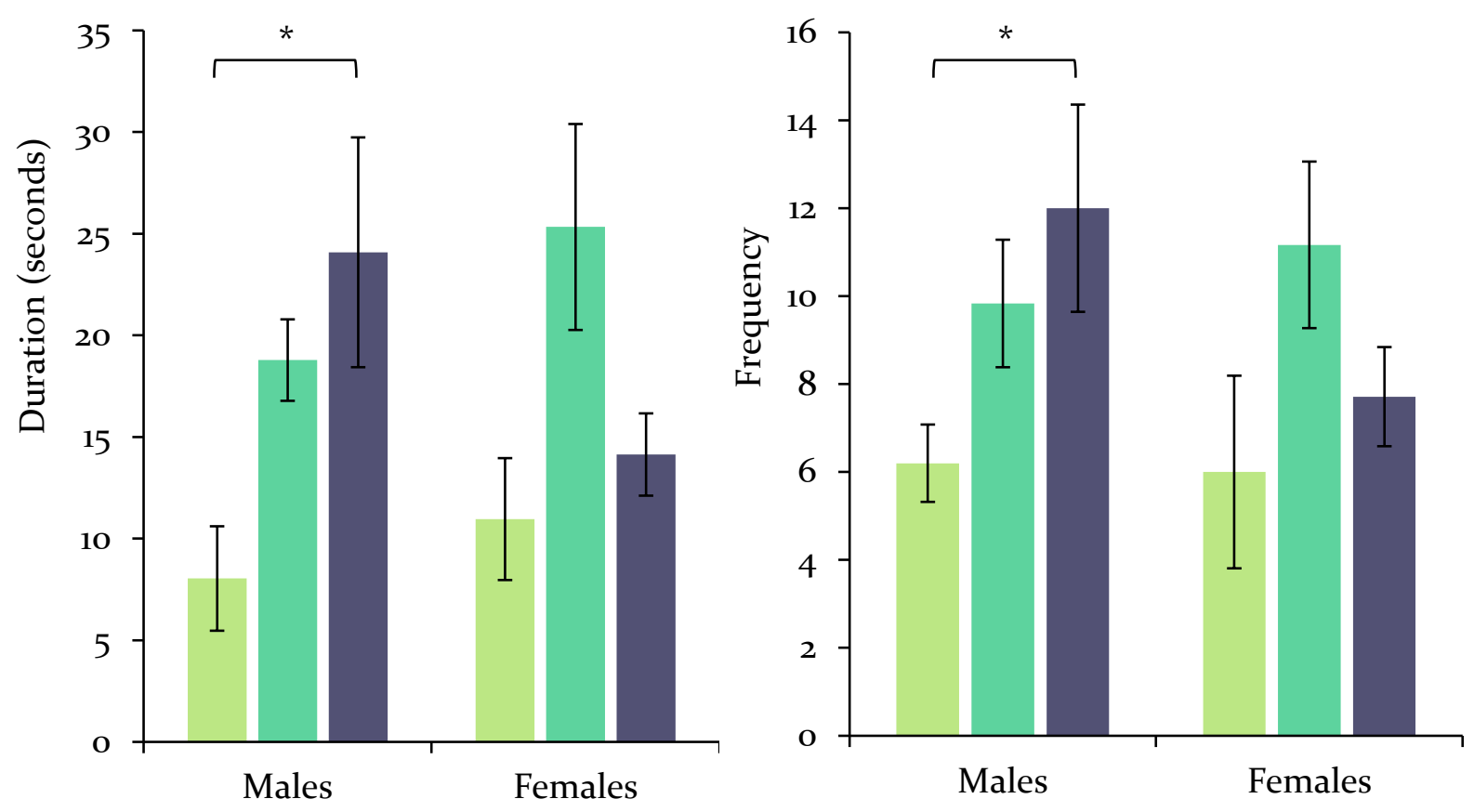

Offspring sex
Control $\square$ Low $\quad$ High

Figure 3.6. The duration (left) and frequency (right) of chasing behaviours for the offspring exposed to control, low, or high doses of VPA. Significant increases for high dose males and low dose females. * indicates significance to $p<.05$. Error bars represent \pm SEM. 


\section{Male pinning behaviour}

The prenatal exposure to VPA did not significantly affect the duration $(F(2,34)=.24, p$ $\left.=.791, \mathrm{\eta}_{\mathrm{p}}{ }^{2}=.01, n s\right)$ or frequency $\left(F(2,34)=.13, p=.878, \mathrm{\eta}_{\mathrm{p}}{ }^{2}=.0, n s\right)$ of pinning behaviour in males (displayed in figure 3.7).

\section{Female pinning behaviour}

The prenatal exposure to VPA did not significantly affect the duration $(F(2,24)=2.22$, $\left.p=.134, \mathrm{\eta}_{\mathrm{p}}{ }^{2}=.17, n s\right)$ or frequency $\left(F(2,24)=2.10, p=.147, \mathrm{\eta}_{\mathrm{p}}{ }^{2}=.17, n s\right)$ of pinning behaviours in females (see figure 3.7).

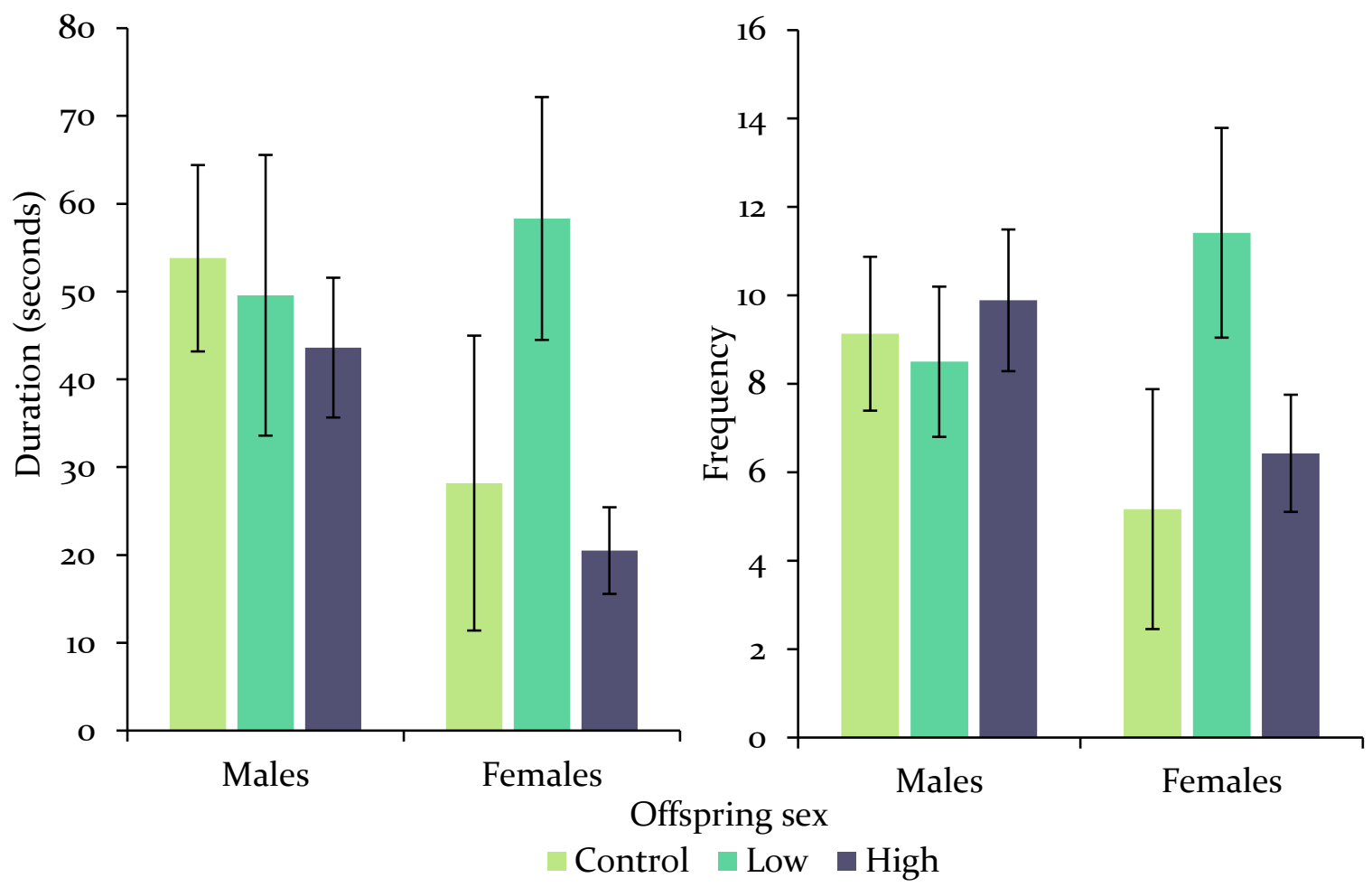

Figure 3.7. Duration (left) and frequency (right) of pinning behaviours for the offspring exposed to control, low, or high doses of VPA. No statistical effects for males or females prenatally exposed to VPA. Error bars represent \pm SEM. 


\section{Male passive social behaviour}

The prenatal exposure to VPA did not affect the duration $\left(F(2,34)=2.51, p=.096, \mathrm{n}_{\mathrm{p}}{ }^{2}\right.$ $=.13, n s)$; nor the frequency $\left(F(2,34)=.85, p=.438, \mathrm{n}_{\mathrm{p}}{ }^{2}=.05, n s\right)$ of passive social behaviour in males (see figure 3.8).

\section{Female passive social behaviour}

The prenatal exposure to VPA did not affect the duration $\left(F(2,24)=2.28, p=.128, \eta_{\mathrm{p}}^{2}\right.$ $=.18, n s)$; nor the frequency $\left(F(2,24)=1.15, p=.337, \eta_{\mathrm{p}}{ }^{2}=.10, n s\right)$ of passive social behaviour in females (see figure 3.8).

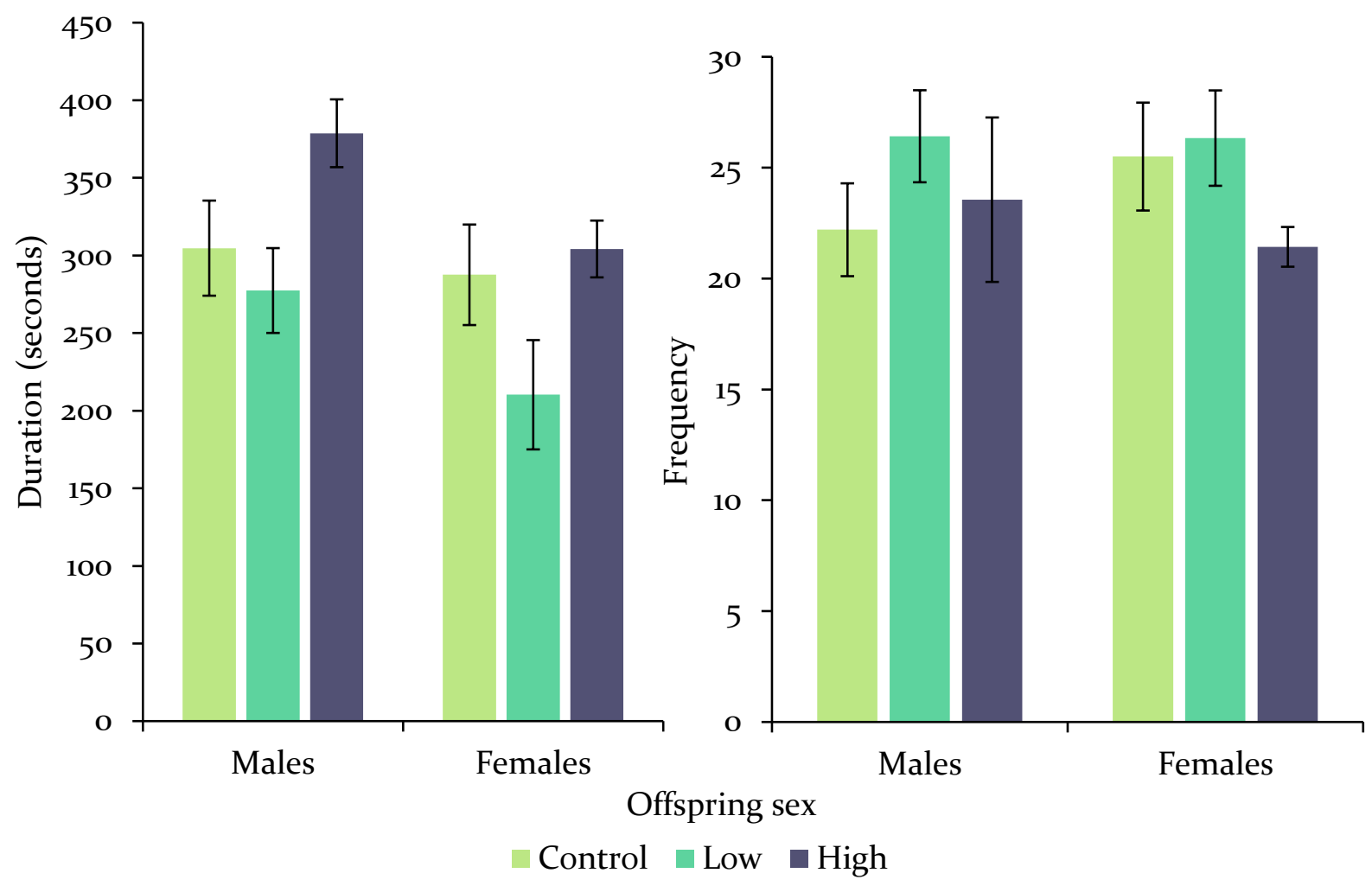

Figure 3.8. Duration (left) and frequency (right) of passive interaction for the offspring exposed to control, low, or high doses of VPA. No statistical effects for males or females prenatally exposed to VPA. Error bars represent \pm SEM. 


\subsection{Experiment Three: Social Approach and Avoidance}

The purpose of this experiment was to investigate the social preference of pre-pubescent and post-pubescent SERT ${ }^{+/+}$and SERT ${ }^{+/}$rats that were chronically exposed to control, low, or high doses of VPA during gestation. This experiment uses the methods established in chapter two (section 2.4).

SAA examines a rodents preference or avoidance of social stimuli, and as such, it has been extensively used to examine ASD-like behaviours of animals; especially as deficits in social behaviour is a core symptom of ASD. The social approach and avoidance paradigm allows the experimenter to examine the social behaviour of a singular test animal, unlike play behaviour, which involves two animals actively interacting. Furthermore, it allows for the comparison of preference and approach to social stimuli (Crawley, 2004; Moy et al., 2008). These factors make this paradigm invaluable for assessing social deficits in rodents, particularly in rats.

As discussed in chapter two (section 2.4), this paradigm has been used to show that rats exposed to VPA during gestation exhibit decreased time with a novel stimulus and increased time with a familiar stimulus or alone (Bambini-Junior et al., 2011; Bronzuoli et al., 2018; Kerr et al., 2013; Kim et al., 2014; Kim et al., 2011). However, this finding has not been consistent across the literature, with some researchers finding increased time with the social stimulus or the novel social stimulus (Dufour-Rainfray et al., 2010; Stefanik et al., 2015). Reductions in social approach or time spent with the novel social stimulus would be expected for animals that were exposed to VPA during gestation. The addition of the genotype is expected to potentiate the effects of prenatal VPA exposure, such that the SERT ${ }^{+/-}$exposed to the high dose are likely to show deficits beyond that seen in the high dose SERT ${ }^{+/+}$.

Previous research in our laboratory was the first to our knowledge to examine the social behaviour of SERT rats that were prenatally exposed to VPA. Ranger (2016) examined the social behaviours of adolescent (PND 35-60) male SERT ${ }^{+/+}$and SERT ${ }^{+/-}$that were prenatally exposed to $400 \mathrm{mg} / \mathrm{kg}$ on GD 12, and these rats did not show differences in social exploration or social novelty from controls. While VPA did reduce exploration of the novel stimulus, this effect was seen both in SERT ${ }^{+/}$and SERT ${ }^{+/+}$animals., and thus no genotype interactions were found. In the present study, in addition to using chronic VPA treatment, females were also included to assess sex-related changes. 
Therefore, this experiment aims to examine the social behaviour in the SAA assay of preand post-pubescent $\mathrm{SERT}^{+/-}$and $\mathrm{SERT}^{+/+}$males and females prenatally exposed to VPA throughout gestation. It is hypothesised that:

i. Males and females that were prenatally exposed to VPA will exhibit decreased sociability (as seen in phase one) and a decreased preference for social novelty (as seen in phase two), compared with rats prenatally exposed to the control dose,

ii. There will be an interaction between SERT reduction and chronic exposure to VPA. As such, SERT ${ }^{+/-}$prenatally exposed to VPA will exhibit decreased sociability and social novelty preference compared with SERT ${ }^{+/+}$exposed to the control dose,

iii. Finally, the decreases exhibited by SERT ${ }^{+/-}$and SERT ${ }^{+/+}$prenatally exposed to VPA will be seen both pre- and post-puberty 


\subsubsection{SAA Methods}

\subsubsection{SAA Apparatus}

The apparatus and experiment set up replicates the protocol in chapter two, section 2.4.1.

\subsubsection{SAA Procedure}

SAA methods were closely matched with methods described in chapter two, section 2.4.1. Briefly, a test animal was selected from its home cage and transferred into the experimental room for a 10-minute habituation period. Conspecific (but not of the same genotype) animals serving as the social stimuli were also transferred into the experimental room for habituation. The test animal was then placed in the T-maze for 10 minutes with empty wire mesh cylinders in each arm.

After the habituation to the room and T-maze, the test animal was placed in a temporary cage while a conspecific juvenile was enclosed in one of the cylinders. The test animal was returned to the T-maze for 10 minutes to interact with the juvenile (phase one). Once again, the test animal was placed in a temporary cage, and a second juvenile was enclosed in the opposite cylinder, and the test animal was returned to the T-maze for a final 10 minutes (phase two). Including the second conspecific in the cylinder, allows the first conspecific juvenile to become known as the 'familiar' and the newly added conspecific becomes the 'novel' stimulus, indicating that the test animal has had time to familiarise itself to the first conspecific but not the second.

At the end of phase two, the test animal was weighed and returned to its home cage. The social stimuli were returned to their temporary cage, and the arena was cleaned with $70 \%$ ethanol and paper towels, and the next test animal was placed in the experimental room for habituation.

Based on data collected in the first study, the following experiment will include the data from phase one, allowing for the examination of social approach (phase one) and social preference (phase two).

\subsubsection{Data Analysis for SAA}

To analyse the effect of prenatal exposure to VPA in $\mathrm{SERT}^{+/+}$and SERT ${ }^{+/-}$, factorial ANOVAs 3 (Prenatal dose; control, low, high) by 2 (Genotype; SERT ${ }^{+/+}, \mathrm{SERT}^{+/}$) was run separately for male and female offspring. These factorial ANOVAs were run on the dependent variables of the sociability score, and distance travelled. 
As described in section 2.4.1.3, the preference of familiarity was coded into a sociability score to analyse the preference without violating the assumptions of ANOVAs. For this experiment, phase one examined sociability, with positive values $(+1)$ assigned to the time spent with the social stimulus, and negative values (-1) assigned to the time in the empty space. Whereas in phase two, positive values were assigned to time spent with the novel stimulus, and negative values to time with the familiar stimulus. In either phase, the assigned values were added together, resulting in a sociability score.

Furthermore, a repeated measures ANOVA with areas (phase one: social, empty; phase two: familiar, novel) as the repeated factor was used, as it is a typical method for analysing data from SAA.

Finally, to examine the overall change in the sociability over time, a repeated measures 3(Dose: control, low, high), 2(Genotype: SERT ${ }^{+/+}$and SERT ${ }^{+/}$), 2(PND: 30, 60) ANOVA, with PND as the repeated factor. This analysis was run on the sociability score in phase one, and the sociability score in phase two.

All data were analysed for statistical significance with SPSS (version 22-25). Partial eta squared $\left(\eta_{\mathrm{p}}{ }^{2}\right)$ was used for the effect size. All significant analyses were followed up with Tukey HSD post hoc tests, and/or planned comparisons were used to follow up potential effect comparing specific prenatal dose or genotype conditions with others using Student's t-test. The alpha level for statistical significance was set at $p<.05$. 


\subsubsection{SAA Results for Pre-Pubescent Rats}

A total of 104 rats were tested in the SAA paradigm, the test session occurring between PND 30 and 35. One SERT ${ }^{+/-}$female exposed to the high dose was removed from the analysis due to lack of movement or exploration. Table 3.4 provides the number of samples for each condition, sex, genotype.

Table 3.4. The number of test animals used in SAA PND 30-35 divided by prenatal dose, sex and genotype.

\begin{tabular}{c|c|c|c|c|}
\hline \hline \multirow{3}{*}{ Control } & & SERT $^{+/+}$ & SERT $^{+/-}$ & Combined \\
\cline { 2 - 5 } & Males & 9 & 11 & 20 \\
\cline { 2 - 5 } & Females & 8 & 7 & 15 \\
\hline \multirow{3}{*}{ Low } & Combined & 17 & 18 & 35 \\
\cline { 2 - 5 } & Males & 10 & 13 & 23 \\
\cline { 2 - 5 } & Females & 7 & 9 & 16 \\
\cline { 2 - 5 } High & Combined & 17 & 21 & 38 \\
& Males & 9 & 12 & 8 \\
\hline \hline & Females & 5 & 3 & 29 \\
\cline { 2 - 5 } & Combined & 14 & 15 & \\
\hline
\end{tabular}

\subsubsection{Phase One Analysis}

Firstly, a sociability score was calculated to analyse the social preference of the SERT ${ }^{+/+}$ or SERT ${ }^{+/-}$males and females prenatally exposed to VPA. This score measures the preference (positive) or avoidance (negative) of the social stimulus in the first phase. 


\section{Male sociability score}

The prenatal exposure to VPA did not significantly affect the sociability score $(F(2,58)$ $\left.=.21, p=.808, \mathrm{\eta}_{\mathrm{p}}{ }^{2}=.00, n s\right)$. Furthermore, there was no effect of genotype $(F(1,58)=.04, p=$ $\left..837, \mathrm{\eta}_{\mathrm{p}}{ }^{2}=.00, n s\right)$ and no interaction $\left(F(2,58)=2.02, p=.141, \mathrm{\eta}_{\mathrm{p}}{ }^{2}=.065\right)$. Figure 3.9 displays the pattern of sociability score for males.

\section{Female sociability score}

The females prenatally exposed to VPA exhibited a significant decrease in the sociability score $\left(F(2,33)=3.79, p=.033, \mathrm{\eta}_{\mathrm{p}}^{2}=.19\right)$. The post-hoc analysis demonstrated that there was a trend for a decreased sociability score in high dose females compared with the control dose ( $p$ $=.075, n s)$. Furthermore, there was a trend toward significance in the sociability score of the genotypes $\left(F(1,33)=3.90, p=.057, \mathrm{\eta}_{\mathrm{p}}^{2}=.11, n s\right)$. However, there was no interaction between prenatal VPA exposure and the genotypes on the sociability score $\left(F(2,33)=2.21, p=.125, \mathrm{\eta}_{\mathrm{p}}{ }^{2}=\right.$ .12 , ns; see figure 3.9).

As there was a significant VPA effect with an almost significant effect of genotype, the sociability score of the prenatal dose conditions was examined in the $\mathrm{SERT}^{+/+}$and $\mathrm{SERT}^{+/-}$ females separately. There was no effect of prenatal VPA exposure for the $\operatorname{SERT}^{+/+}(F(2,17)=0.38$, $\left.p=.692, \mathrm{\eta}_{\mathrm{p}}^{2}=.04, n s\right)$, but there was a significant effect for the $\operatorname{SERT}^{+/-}$females $(F(2,16)=6.75$, $\left.p=.008, \mathrm{\eta}_{\mathrm{p}}^{2}=.46\right)$. Particularly, the $\mathrm{SERT}^{+/-}$females exposed to the high dose exhibited significantly decreased sociability scores compared to the control SERT ${ }^{+-}(p=.006)$ and the low dose SERT $^{+-}(p=.048)$ females.
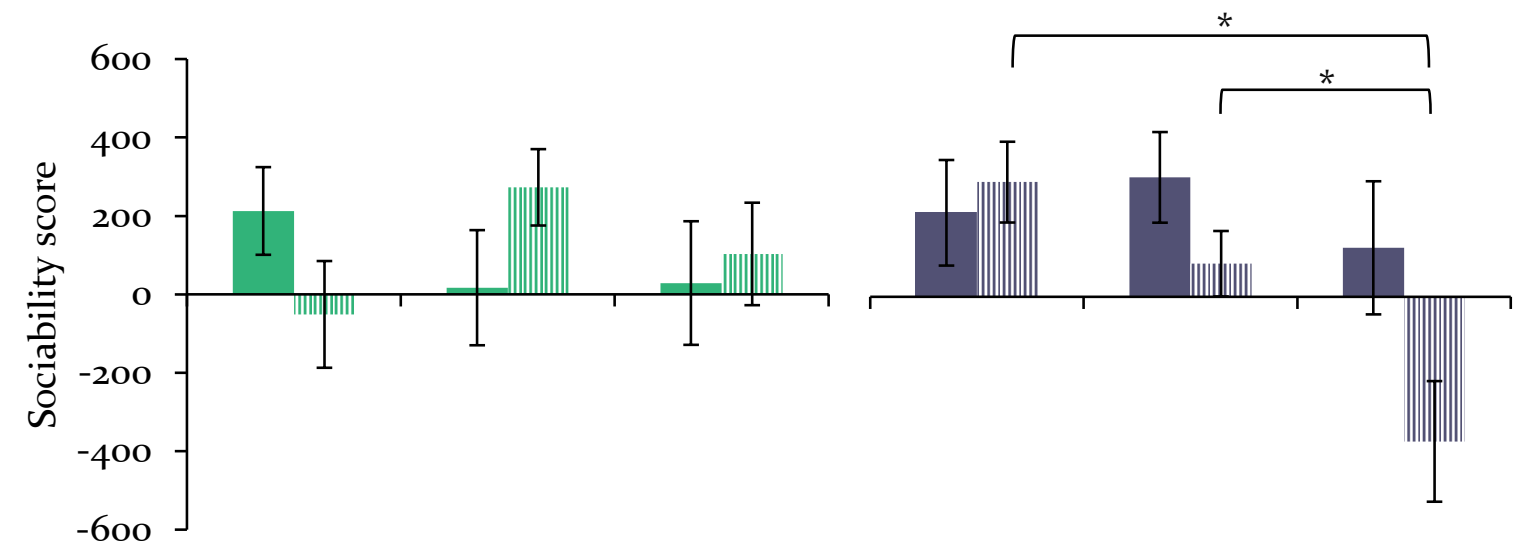

$$
\begin{aligned}
& \text { Control Low High Control Low High }
\end{aligned}
$$

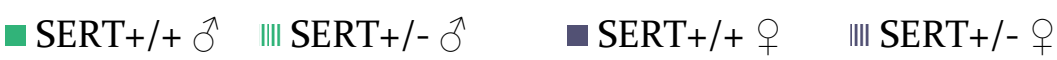

Figure 3.9. The average sociability scores for males (left) and females (right) prenatally exposed to control, low, or high doses of VPA, separated by genotype. Positive values indicate time spent with social stimulus. High dose females show significantly decreased sociability score. * indicates significance to $p<.05$. Error bars represent \pm SEM. 


\section{Male time spent in social and non-social areas}

The male SERT ${ }^{+/+}$and SERT ${ }^{+/}$offspring showed a preference for the social area, although this was not significant (area; $F(1,58)=3.31, p=.074, \eta_{\mathrm{p}}{ }^{2}=.05$, $n s$; see figure 3.10 for average duration of males). The area preference showed no interaction with prenatal dose (area $\times$ prenatal dose: $\left.F(2,58)=.214, p=.808, \eta_{\mathrm{p}}^{2}=.00, n s\right)$, genotype (area $\times$ genotype: $F(1,58)=.043$, $p=.837, \eta_{\mathrm{p}}^{2}=.00, n s$ ), or the interaction between prenatal dose and genotype (area $\times$ prenatal dose $\times$ genotype: $\left.F(2,58)=2.02, p=.141, \eta_{\mathrm{p}}^{2}=.06, n s\right)$. Furthermore, there were no significant main effects (prenatal dose: $F(2,58)=.91, p=.410, \eta_{\mathrm{p}}^{2}=.03$, ns; genotype: $(1,58)=.001, p=.969$, $\left.\eta_{\mathrm{p}}{ }^{2}=.00, n s\right)$, nor was there an interaction of prenatal dose and genotype $(F(2,58)=.607, p=$ $\left..549, \mathrm{\eta}_{\mathrm{p}}^{2}=.020, n s\right)$.

Overall, the control SERT ${ }^{+/+}$males showed a preference for the social area (albeit not significantly, $p=.09, n s)$, while the control SERT ${ }^{+-}$did not $(p=.357, n s)$. Exposure to VPA eliminated this preference in the SERT ${ }^{+/+}$males. Thus, prenatal exposure to VPA tended to inhibit social preference in the $\mathrm{SERT}^{+/+}$males.

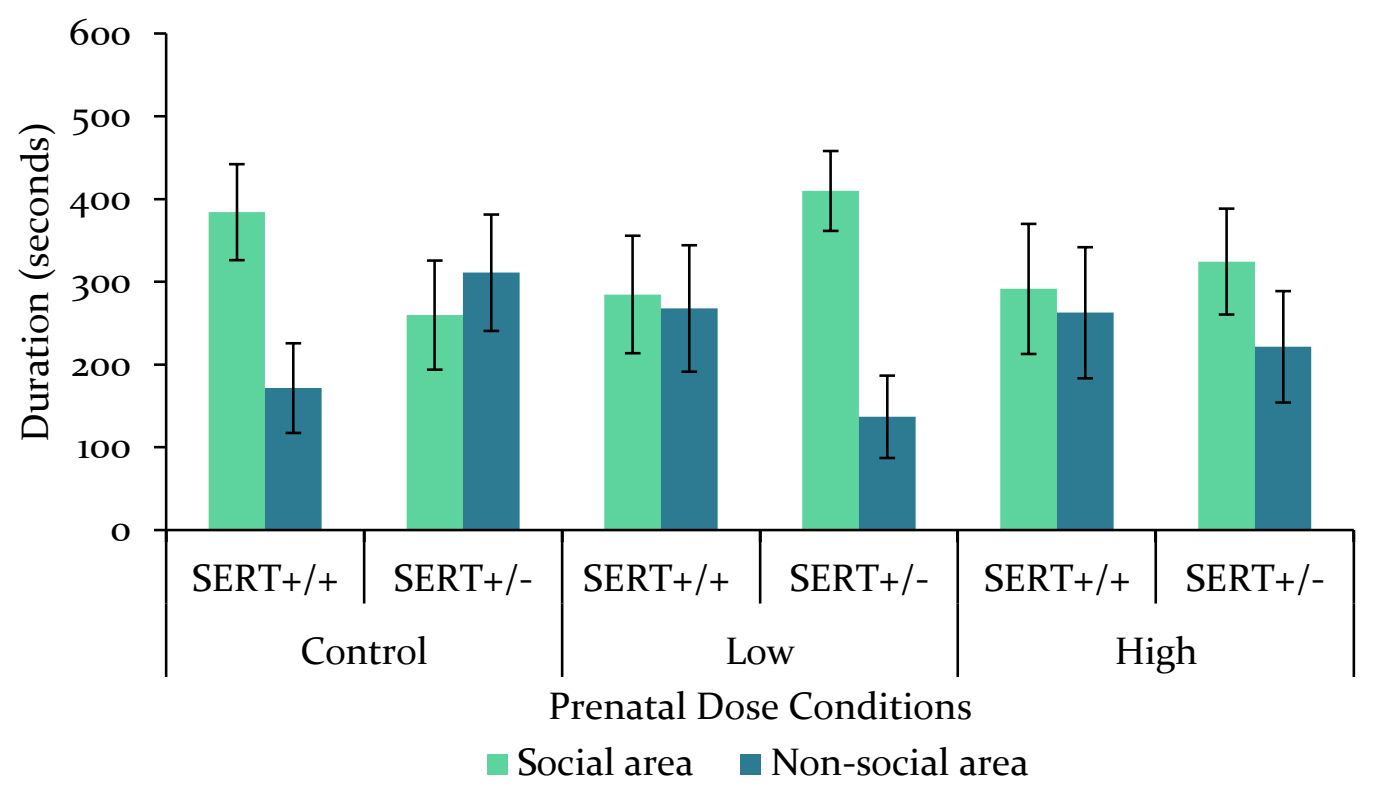

Figure 3.10. The average duration of time spent in the social and non-social area for SERT ${ }^{+/+}$and $\mathrm{SERT}^{+/-}$males prenatally exposed to control, low, or high VPA doses. Overall trends for social area preference, prenatal VPA exposure eliminated area preference for $\mathrm{SERT}^{+/+}$. Error bars represent \pm SEM. 


\section{Female duration of time spent in social and non-social areas}

The female SERT ${ }^{+/+}$and SERT ${ }^{+/-}$offspring showed a significant preference for the social area (area: $\left.F(1,33)=4.10, p=.05, \eta_{\mathrm{p}}^{2}=.11\right)$. Furthermore, there was significant interaction between the prenatal dose condition and the area preference (area $\times$ prenatal dose: $F(2,33)=$ $\left.3.79, p=.033, \mathrm{\eta}_{\mathrm{p}}{ }^{2}=.19\right)$, and there was a close to significant interaction of prenatal doses and genotypes for the females (prenatal dose $\times$ genotype: $F(1,33)=3.16, p=.056, \eta_{\mathrm{p}}^{2}=.16, n s$ ).

The interaction of prenatal dose on area preference was examined in the $\mathrm{SERT}^{+/+}$and $\mathrm{SERT}^{+/-}$females separately. This analysis showed that the SERT ${ }^{+/+}$females exposed to the control dose exhibited a trend toward a preference for the social area $(p=.077, n s)$, and there was a significant social area preference for the low dose SERT ${ }^{+/+}$females $(p=.019)$, however the high dose SERT ${ }^{+/+}$showed no preference $(p=.251, n s)$. Similar to SERT ${ }^{+/+}$, the control SERT ${ }^{+/}$had a significant preference for the social area $(p=.015)$, and opposing the SERT ${ }^{+/+}$, the low dose SERT $^{+/-}$exhibited no preference $(p=.170, n s)$, and the high dose SERT $^{+/-}$exhibited a close to significant preference for the non-social area $(p=.063, n s$, see figure 3.11$)$.

Overall, the prenatal exposure to VPA in the $\mathrm{SERT}^{+/+}$females impacts the social preference for the high dose $\mathrm{SERT}^{+/+}$but not for the controls or low dose $\mathrm{SERT}^{+/+}$, whereas the prenatal exposure to VPA in the SERT ${ }^{+/}$females more clearly disrupted the social preference formation and approach to social stimuli.

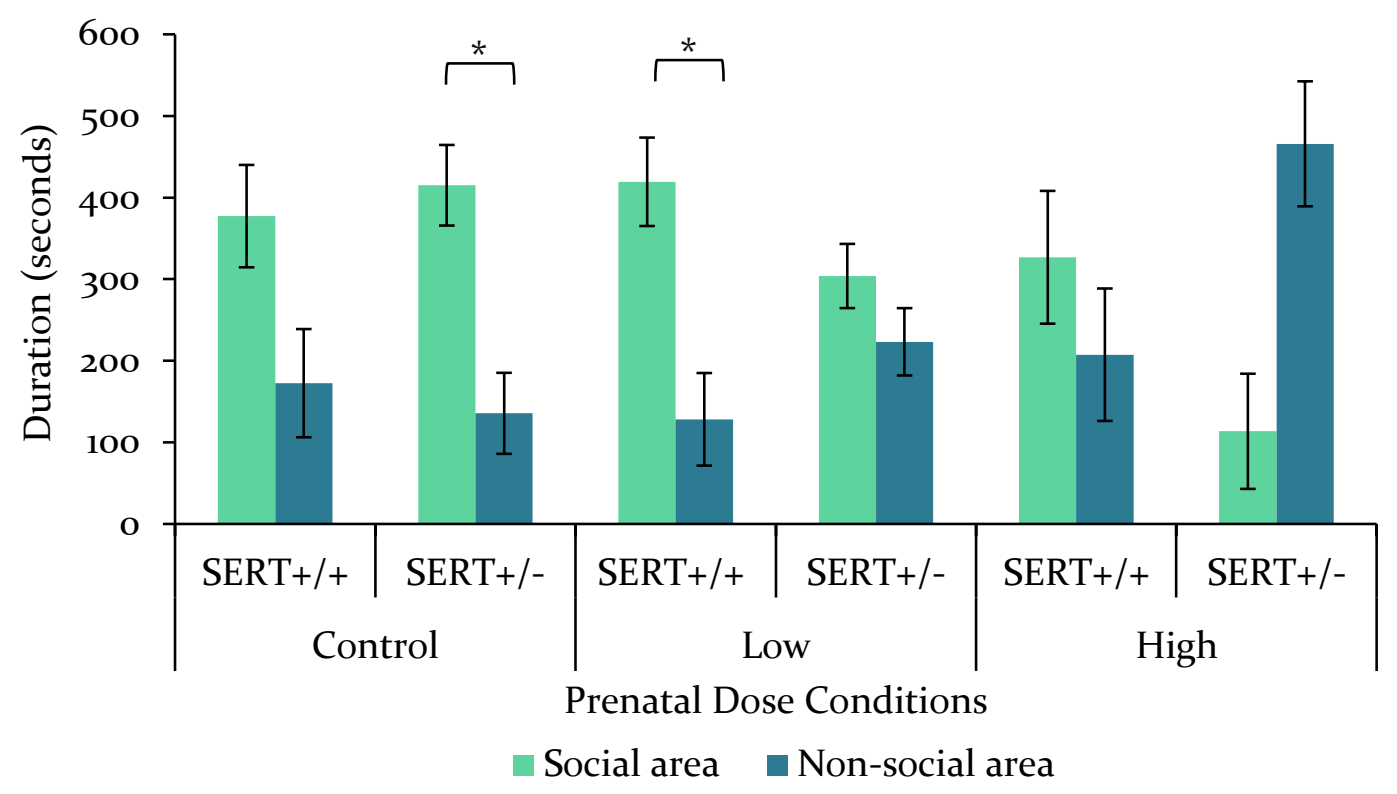

Figure 3.11. The average duration of time spent in the social and non-social area for SERT ${ }^{+/+}$ and SERT $^{+/}$females that were prenatally exposed to control, low, or high VPA doses. Prenatal VPA exposure for SERT ${ }^{+/-}$females disrupts preference. * indicates significance to $p<.05$. Error bars represent \pm SEM. 


\subsubsection{Phase Two Analysis}

Phase two of SAA introduces a new social stimulus, allowing the test animal to interact with either the familiar stimulus (the stimulus introduced in phase one) or a new novel social stimulus. To analyse the preference for social novelty in the SERT ${ }^{+/+}$or SERT ${ }^{+/-}$prenatally exposed to VPA, the sociability score was calculated, with positive values assigned to time spent with the novel social stimulus and negative values assigned to time with the familiar.

\section{Males sociability score}

The male SERT ${ }^{+/+}$and SERT ${ }^{+/-}$prenatally exposed to VPA exhibited no differences in their sociability scores in phase two at PND 30 (prenatal dose: $F(2,58)=1.83, p=.169, \mathrm{\eta}_{\mathrm{p}}{ }^{2}=.05$, ns; genotype: $F(1,58)=.11, p=.738, \eta_{\mathrm{p}}^{2}=.00, n s$; prenatal dose $\times$ genotype: $F(2,58)=2.07, p=$ $\left..135, \mathrm{\eta}_{\mathrm{p}}{ }^{2}=.067, n s\right)$, see figure 3.12 .

\section{Females sociability score}

For the females, there was no effect of genotype $\left(F(1,33)=1.26, p=.270, \mathrm{n}_{\mathrm{p}}{ }^{2}=.04, n s\right)$ nor an interaction (prenatal dose $\times$ genotype: $\left.F(2,33)=.19, p=.829, \eta_{\mathrm{p}}{ }^{2}=.01, n s\right)$. However, the females had a close to significant effect of the prenatal doses on the sociability score $(F(2,33)=$ 2.91, $\left.p=.06, \mathrm{\eta}_{\mathrm{p}}^{2}=.14, n s\right)$. The follow up analysis showed that females prenatally exposed to the low dose exhibited increased sociability compared with the control dose females $(p=.054, n s)$, but this did not reach significance, see figure 3.12.

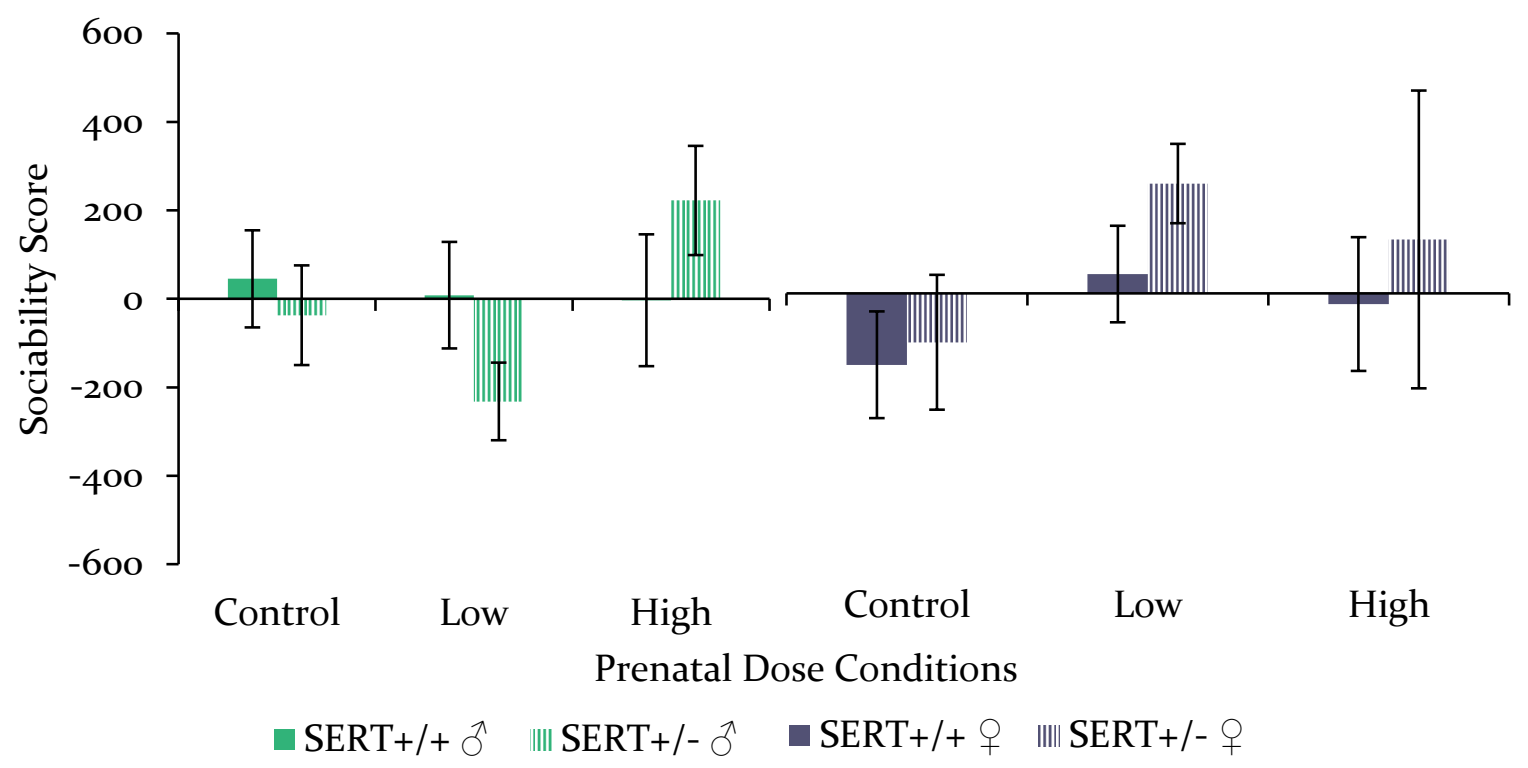

Figure 3.12. The average sociability scores of males (left) and females (right) SERT' ${ }^{+/+}$and $\mathrm{SERT}^{+/-}$ prenatally exposed to control, low, and high doses of VPA. Negative values indicate a preference for familiarity. No effect for males but females exposed to the low dose of VPA exhibited increased sociability. Error bars represent \pm SEM. 


\section{Males time spent in familiar and novel social area}

The male SERT ${ }^{+/+}$and SERT ${ }^{+/-}$offspring showed no significant preference for the familiar or novel stimuli, see figure 3.13. There was no preference for side $\left(F(2,58)=.00, p=.99, \eta_{\mathrm{p}}^{2}=\right.$ $.00, n s)$, there was no interaction between stimuli preference and prenatal doses $(F(2,58)=1.83$, $\left.p=.169, \mathrm{\eta}_{\mathrm{p}}^{2}=.06, n s\right)$, nor did the genotypes $\left(F(1,58)=.11, p=.738, \mathrm{\eta}_{\mathrm{p}}^{2}=.00, n s\right)$ and there was no interaction between the prenatal dose, genotypes and time spent with the stimuli $(F(2,58)$ $\left.=2.07, p=.135, \mathrm{\eta}_{\mathrm{p}}^{2}=.06, n s\right)$. There were no overall effects (prenatal dose: $F(2,58)=1.46, p=$ $.241 \mathrm{\eta}_{\mathrm{p}}^{2}=.53$, ns; genotype: $F(2,58)=.03, p=.862, \mathrm{\eta}_{\mathrm{p}}^{2}=.00, n s$, and prenatal dose $\times$ genotype: $\left.F(2,58)=.21, p=.814, \mathrm{\eta}_{\mathrm{p}}^{2}=.00, n s\right)$.

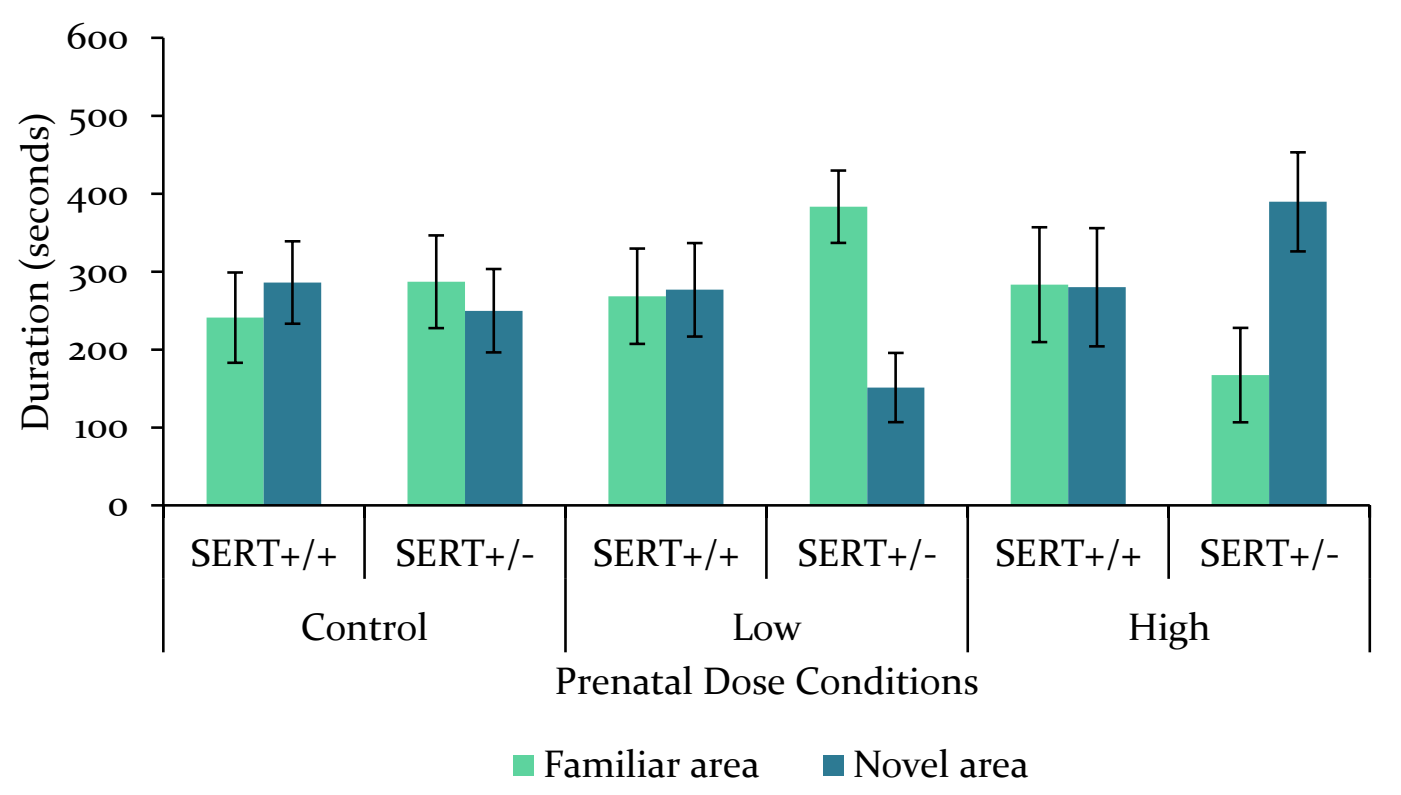

Figure 3.13. The average duration of time spent in the familiar or novel area for $\mathrm{SERT}^{+/+}$and $\mathrm{SERT}^{+/-}$males that were prenatally exposed to control, low, or high VPA doses. No statistically significant effects were found. Error bars represent \pm SEM. 


\section{Females time spent in familiar and novel social area}

The prenatal VPA exposure did not have a significant effect (prenatal dose: $F(2,33)=$ $\left..334, p=.719, \mathrm{n}_{\mathrm{P}}^{2}=.02, n s\right)$, but there was a trend toward an interaction between prenatal doses and preference (prenatal dose $\times$ stimuli: $F(2,33)=2.55, p=.093, \eta_{\mathrm{p}}^{2}=.13, n s$ ). The nonsignificant interaction effect was followed up by examining the stimuli preference in each of the prenatal dose conditions, revealing a close to significant novel preference for the low dose females $\left(F(1,14)=4.36, p=.056, \eta_{\mathrm{p}}^{2}=.24, n s\right.$, see figure 3.14$)$.

Furthermore, there was a significant difference between female SERT ${ }^{+/+}$and SERT ${ }^{+/-}$ (genotype: $F(1,33)=10.41, p=.003, \mathrm{n}_{\mathrm{p}}{ }^{2}=.24$, see figure 3.14). The $\mathrm{SERT}^{+/+}$females exhibited no clear preference for the familiar or novel stimuli, across all prenatal dose conditions $(F(1,17)=$ $\left..41, p=.530, \mathrm{n}_{\mathrm{p}}{ }^{2}=.02, n s\right)$. However, $\mathrm{SERT}^{+/-}$females revealed a different pattern. The control dose SERT ${ }^{+/-}$showed no preference $(p=.248, n s)$, whereas the low dose SERT ${ }^{+/-}$had a significant preference for the novel stimulus $(p=.010)$. While a similar effect was seen at the high dose (no stimuli preference, $p=.376$ ), this failed to reach significance, presumably due to the sample size, coupled to a large variability (as evidenced in figure 3.14).

The contrast between the control and low dose SERT ${ }^{+/-}$females is indicative of earlier findings, suggesting that the prenatal exposure to VPA disrupted the social preference in $\mathrm{SERT}^{+/}$ females.

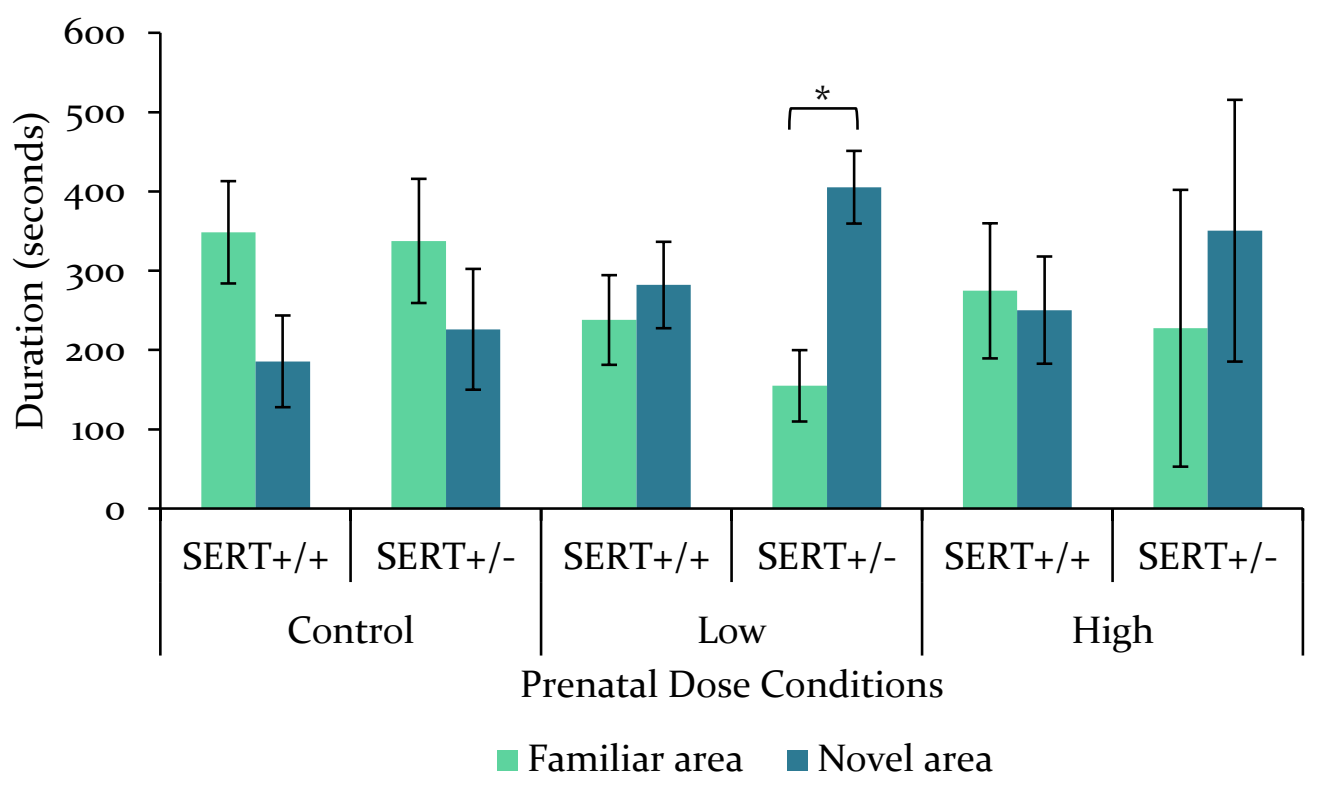

Figure 3.14. The average duration of time spent for in the familiar or novel areas for SERT ${ }^{+/+}$and $\mathrm{SERT}^{+/-}$females that were prenatally exposed to control, low, or high VPA doses. Prenatal VPA exposure disrupts the social preference for SERT ${ }^{+/}$females. ${ }^{*}$ indicates significance to $p<.05$. Error bars represent \pm SEM. 


\subsubsection{Locomotor Activity}

In addition to the SAA measures, the overall locomotor activity of the test animals was measured with the total distance travelled during the testing sessions (phase one and two).

\section{Distance travelled for males}

This analysis showed no significant effect for male SERT ${ }^{+/+}$and SERT ${ }^{+/-}$prenatally exposed to VPA, see figure 3.15. That is, the distance travelled for males did not significantly change between the two phases $\left(F(1,58)=.76, p=.386, \eta_{\mathrm{p}}^{2}=.01, n s\right)$, there was also no interaction between phase and prenatal dose $\left(F(2,58)=1.26, p=.293, \eta_{\mathrm{p}}{ }^{2}=.04, n s\right)$ or genotype $\left(F(1,58)=1.54, p=.224, \eta_{\mathrm{p}}^{2}=.05, n s\right)$. Furthermore, there were no overall effects of prenatal dose $\left(F(2,58)=.38, p=.687, \mathrm{\eta}_{\mathrm{p}}^{2}=.01, n s\right)$ or genotype $\left(F(1,58)=.32, p=.575, \mathrm{\eta}_{\mathrm{p}}{ }^{2}=.00, n s\right)$, nor was there an interaction $\left(F(2,58)=.40, p=.673, \mathrm{\eta}_{\mathrm{p}}^{2}=.01, n s\right)$.

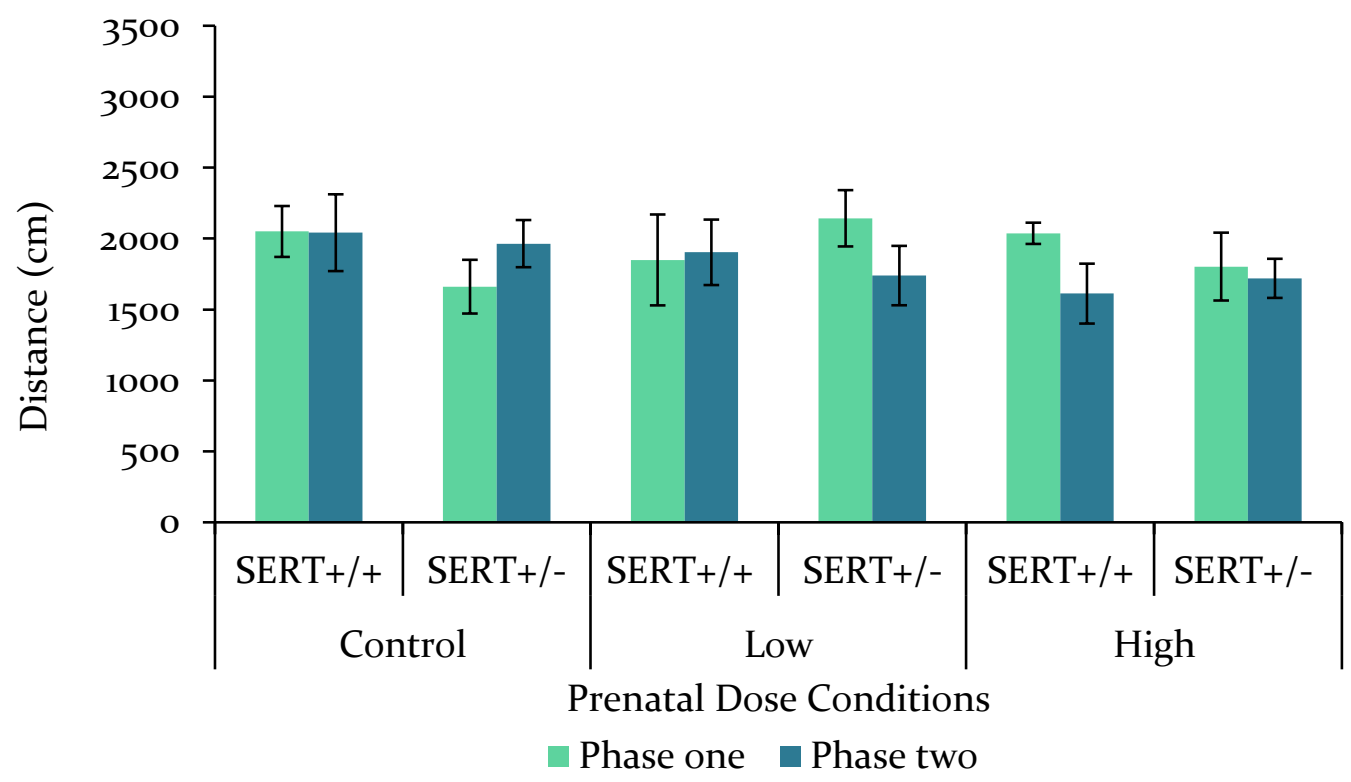

Figure 3.15. The average distance travelled of SERT ${ }^{+/+}$and SERT ${ }^{+-}$males prenatally exposed to control, low, and high doses of VPA. There were no significant differences in the locomotor activity of males between phase one and two. Error bars represent \pm SEM. 


\section{Distance travelled for females}

The distance travelled by female SERT ${ }^{+/+}$and $\mathrm{SERT}^{+/-}$was close to showing a significant effect of phase $\left(F(1,33)=3.38, p=.075, \mathrm{\eta}_{\mathrm{p}}^{2}=.09\right.$, ns, see figure 3.16). There was no interaction between phase and prenatal dose $\left(F(2,33)=.80, p=.459, \eta_{\mathrm{p}}{ }^{2}=.04, n s\right)$ or genotype $(F(1,33)=$ $\left.1.85, p=.183, \mathrm{\eta}_{\mathrm{p}}^{2}=.05, n s\right)$. Furthermore, there were no overall effects of genotype $(F(1,33)=$ $\left.2.64, p=.114, \mathrm{n}_{\mathrm{p}}{ }^{2}=.07, n s\right)$, nor was there an interaction (prenatal dose $\times$ genotype: $F(2,33)=$ $\left..37, p=.691, \eta_{\mathrm{p}}^{2}=.02, n s\right)$.

However, there was an overall effect of prenatal dose $\left(F(2,33)=4.70, p=.016, \eta_{\mathrm{p}}^{2}=.22\right)$. The post-hoc analysis showed that the females exposed to a low dose travelled a significantly greater distance than the females exposed to the control dose ( $p=.038$; see figure 3.16).

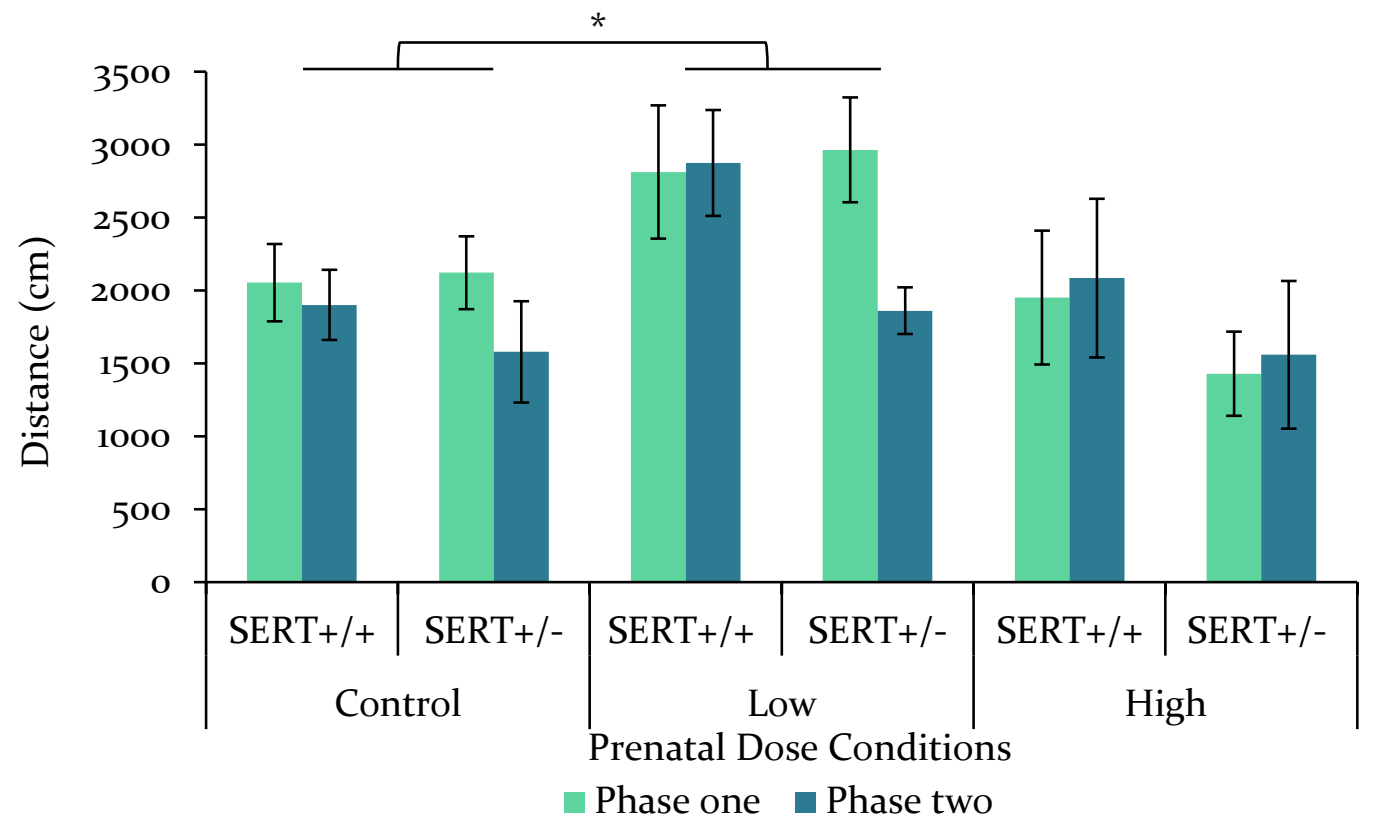

Figure 3.16. The average distance travelled of SERT ${ }^{+/+}$and SERT ${ }^{+/}$females prenatally exposed to control, low, and high doses of VPA. Displaying the increased distance of SERT ${ }^{+/-s}$ s prenatally exposed to a low dose. ${ }^{*}$ indicates significance to $p<.05$. Error bars represent \pm SEM. 


\subsubsection{SAA Results for Post-Pubescent Rats}

A total of 99 rats were tested in the SAA paradigm, the test session occurring between PND 60 and 65. Five animals were excluded from the analysis due to lack of exploration in one or both phases. Of the control dose litters, one SERT ${ }^{+/+}$male and two $\mathrm{SERT}^{+/-}$males were removed, and one $\mathrm{SERT}^{+/+}$male and one $\mathrm{SERT}^{+/-}$male exposed to a high dose, were also excluded. Table 3.5 provides the number of samples for each condition, sex, genotype.

Table 3.5. The number of test animals used in SAA PND 60-65 divided by dose, sex and genotype.

\begin{tabular}{c|c|c|c|c|}
\hline \hline \multirow{3}{*}{ Control } & & SERT $^{+/+}$ & SERT $^{+/}$ & Combined \\
\hline & Males & 8 & 10 & 18 \\
\cline { 2 - 5 } & Females & 8 & 7 & 15 \\
\cline { 2 - 5 } Low & Combined & 16 & 17 & 33 \\
\hline \multirow{3}{*}{ High } & Males & 9 & 12 & 21 \\
\cline { 2 - 5 } & Females & 6 & 11 & 17 \\
\cline { 2 - 5 } & Combined & 15 & 23 & 38 \\
\hline \hline & Males & 7 & 10 & 17 \\
\hline & Females & 5 & 4 & 26 \\
\hline
\end{tabular}




\subsubsection{Phase One Analysis for Post-Puberty}

Following the procedures used in chapter two and this study at PND 30, phase one of SAA examines the social preference of SERT ${ }^{+/}$and SERT ${ }^{+/-}$rats prenatally exposed to VPA.

\section{Male sociability score}

There was a significant effect of prenatal doses on the sociability score for males $(F(2$, $\left.50)=4.39, p=.018, \eta_{\mathrm{p}}^{2}=.15\right)$. That is, the males prenatally exposed to the high dose exhibited a sociability score that was significantly lower than the males exposed to the low dose ( $p=.011)$, but there was no difference from the control dose males, see figure 3.17. There was no main effect of genotype $\left(F(1,50)=.32, p=.577, \eta_{\mathrm{p}}{ }^{2}=.00, n s\right)$ nor interactions between prenatal doses and genotypes $\left(F(2,50)=.10, p=.909, \mathrm{\eta}_{\mathrm{p}}^{2}=.00, n s\right)$.

\section{Female sociability score}

The female SERT ${ }^{+/+}$and SERT ${ }^{+/-}$prenatally exposed to VPA showed no significant effects of prenatal dose $\left(F(2,35)=.35, p=.704, \mathrm{\eta}_{\mathrm{p}}{ }^{2}=.02, n s\right)$, genotype $\left(F(1,35)=.33, p=.569, \mathrm{\eta}_{\mathrm{p}}{ }^{2}=\right.$ $.00, n s)$, nor were there any interactions $\left(F(2,35)=1.19, p=.318, \mathrm{n}_{\mathrm{p}}^{2}=.06, n s\right.$, see figure 3.17).

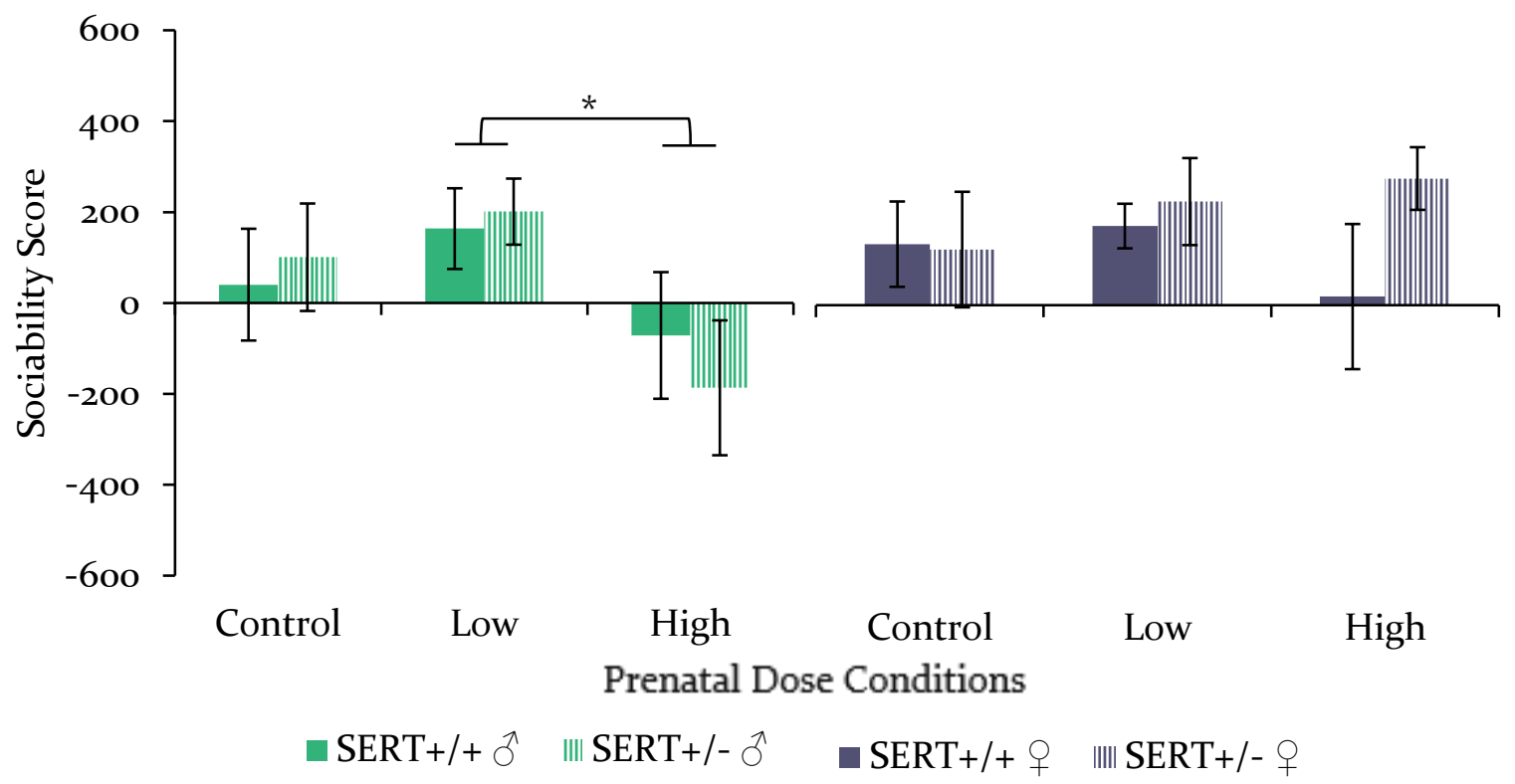

Figure 3.17. The average sociability score for males (left) and females (right) SERT ${ }^{+/+}$and $\mathrm{SERT}^{+/-}$ prenatally exposed to control, low, and high doses of VPA. Negative scores indicate interest in non-social areas. Male exposed to the high dose exhibited a significant preference for the nonsocial areas compared with the low dose males, and no effect for females. * indicates significance to $p<.05$. Error bars represent \pm SEM. 


\section{Males time spent in social and non-social areas}

The males exposure to the prenatal doses differentially affected the duration of time they spent in the social or non-social areas $\left(F(2,50)=4.39, p=.018, \mathrm{\eta}_{\mathrm{p}}^{2}=.15\right.$, see figure 3.18). However, there was no overall preference for the social or non-social areas $(F(1,50)=1.84, p=$ $\left..181, \mathrm{\eta}_{\mathrm{p}}{ }^{2}=.03, n s\right)$, nor was there a differential effect of the genotypes and areas $(F(1,50)=.32, p$ $\left.=.577, \mathrm{\eta}_{\mathrm{p}}{ }^{2}=.01, n s\right)$. There were no overall effects of prenatal dose $\left(F(2,50)=.59, p=.556, \mathrm{\eta}_{\mathrm{p}}^{2}\right.$ $=.02, n s)$, or genotype $\left(F(1,50)=.55, p=.463, \eta_{\mathrm{p}}^{2}=.01, n s\right)$, nor an three way interaction between genotype, prenatal dose and preference $\left(F(2,50)=2.01, p=.144, \eta_{\mathrm{p}}{ }^{2}=.08, n s\right)$.

To examine the prenatal dose effect on social and non-social area preference, the duration of time in either area was analysed within each prenatal dose condition. The males prenatally exposed to the low dose exhibited a significant preference for the social area compared with the non-social $(p=.001)$, no other dose conditions exhibited this preference, see figure 3.18.

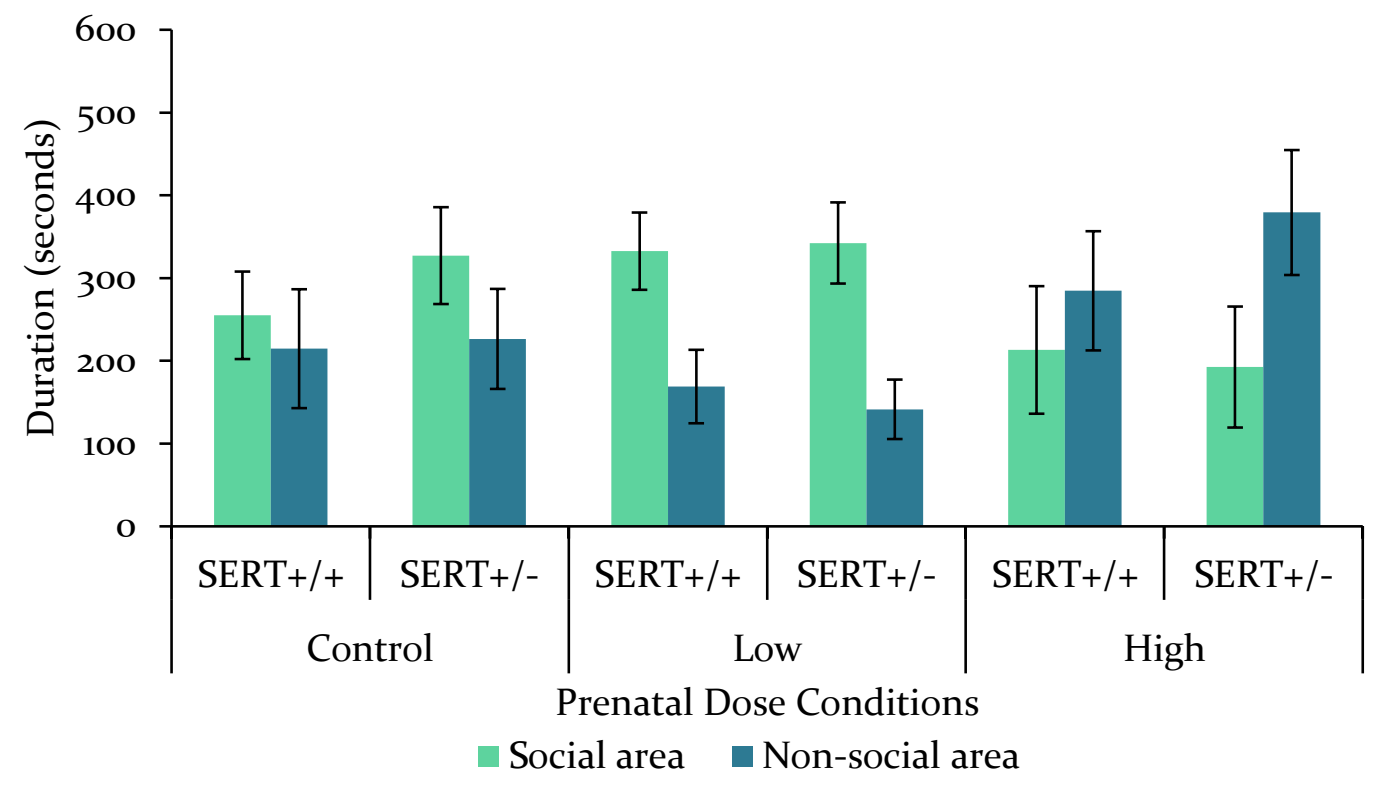

Figure 3.18. The average duration of time spent in the social or non-social areas for male SERT ${ }^{+/+}$ and SERT ${ }^{+/}$prenatally exposed to control, low, and high doses of VPA, with low dose offspring displaying increase time in the social area. Error bars represent \pm SEM. 


\section{Females time spent in social and non-social areas}

There was a significant area preference for all females, regardless of the prenatal dose or genotype $\left(F(2,35)=8.90, p=.005, \eta_{\mathrm{p}}{ }^{2}=.20\right.$, see figure 3.19). However, there was no differential effect of the prenatal dose on the preference for the social or non-social areas $(F(2,35)=.35, p$ $\left.=.704, \eta_{\mathrm{p}}{ }^{2}=.02, n s\right)$, nor was there a differential effect of the genotypes and areas $(F(1,35)=$ $\left..33, p=.569, \mathrm{\eta}_{\mathrm{p}}^{2}=.01, n s\right)$. There were no overall effects of prenatal dose $(F(2,35)=.50, p=.611$, $\left.\eta_{\mathrm{p}}{ }^{2}=.02, n s\right)$, or genotype $\left(F(1,35)=.69, p=.413, \eta_{\mathrm{p}}{ }^{2}=.02, n s\right)$, nor a three way interaction between genotype, prenatal dose and preference $\left(F(2,35)=.16, p=.852, \mathrm{\eta}_{\mathrm{p}}^{2}=.01, n s\right)$.

To further examine the overall effect of area preference, the duration spent in the social and non-social areas was analysis in each prenatal dose condition separately. The females $\mathrm{SERT}^{+/+}$and SERT ${ }^{+/}$exposed to the low dose were the only conditions to show a significant social preference ( $p=.008$, and $p=.047$, respectively). Furthermore, planned comparisons showed a significant social preference for the SERT ${ }^{+-}$exposed to the high dose $(p=.014)$, and there was a trend of social area preference for the SERT ${ }^{+/}$exposed to the control dose $(p=.09, n s)$.

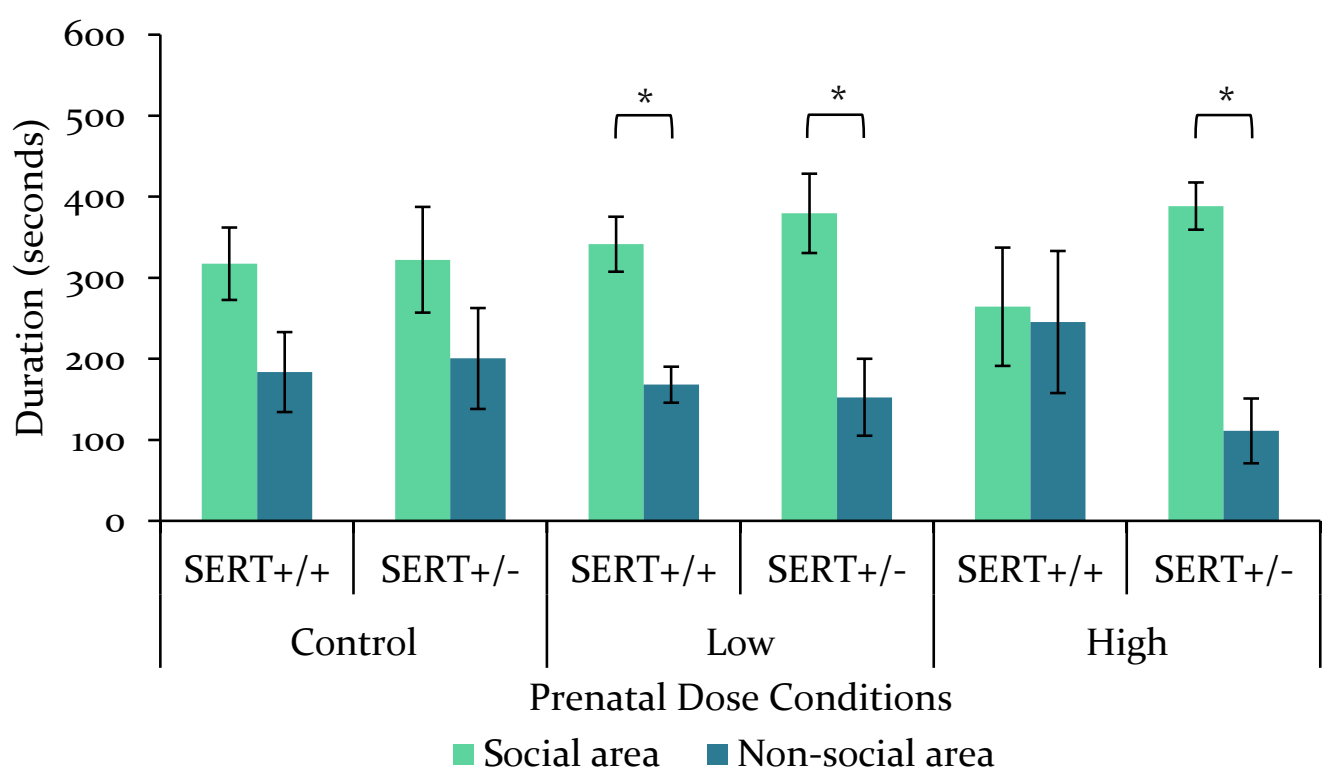

Figure 3.19. The average duration time spent in the social and non-social areas for female SERT $^{+/+}$and SERT ${ }^{+/-}$prenatally exposed to control, low, and high doses of VPA, with low dose offspring displaying increase time in the social area. * indicate significance to $p<.05$. Error bars represent \pm SEM. 


\subsubsection{Phase Two Analysis for Post-Puberty}

Phase two of SAA introduced a new social stimulus, which allowed the test animal to interact with the familiar stimulus (introduced in phase one) or the new social stimulus (referred to as the 'novel').

\section{Males sociability score}

In phase two, positive values in the sociability score reflect time spent with the novel social stimulus and negative values reflect time with the familiar.

The male SERT ${ }^{+/+}$and SERT ${ }^{+/-}$prenatally exposed to VPA showed no significant effects of prenatal dose $\left(F(2,50)=.30, p=.743, \eta_{\mathrm{p}}{ }^{2}=.01, n s\right)$, genotype $\left(F(1,50)=.79, p=.378, \eta_{\mathrm{p}}{ }^{2}=\right.$ $.02, n s)$, nor were there any interactions $\left(F(2,35)=.94, p=.398, \mathrm{n}_{\mathrm{p}}{ }^{2}=.04, n s\right.$, see figure 3.20). As figure 3.20 displays, the $\mathrm{SERT}^{+/+}$and $\mathrm{SERT}^{+/-}$offspring did not demonstrate a statistically significant preference for either social familiarity or novelty.

\section{Females sociability score}

The female SERT ${ }^{+/}$and SERT ${ }^{+/}$prenatally exposed to VPA showed no significant effects of prenatal dose $\left(F(2,35)=.44, p=.651, \eta_{\mathrm{p}}^{2}=.02, n s\right)$, genotype $\left(F(1,35)=2.43, p=.128, \eta_{\mathrm{p}}^{2}=\right.$ $.06, n s)$, nor were there any interactions $\left(F(2,35)=.04, p=.964, \mathrm{n}_{\mathrm{p}}{ }^{2}=.00, n s\right.$, see figure 3.20).

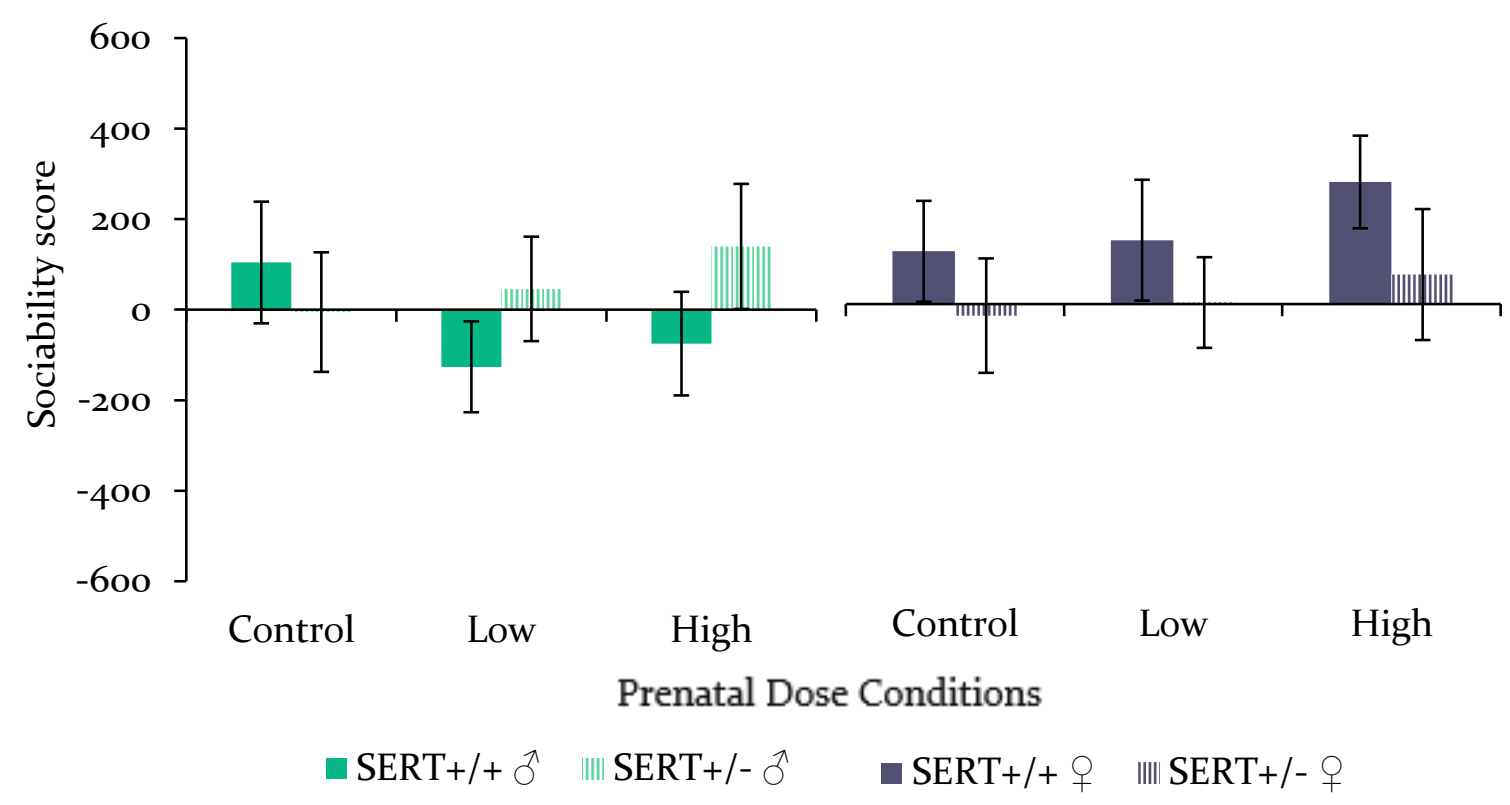

Figure 3.20. The average sociability scores for male (left) and female (right) SERT ${ }^{+/+}$and SERT ${ }^{+/-}$ prenatally exposed to VPA, displaying no significant differences between groups. Positive values correspond to time with the novel stimulus, and all rats exhibiting similar sociability regardless of prenatal treatment. Error bars represent \pm SEM. 


\section{Males time spent in the familiar and novel areas}

This analysis revealed no statistically significant interactions or main effects in the analysis of male $\mathrm{SERT}^{+/+}$and $\mathrm{SERT}^{+/-}$prenatally exposed to VPA. There was no significant preference for the either the familiar or novel stimulus (area: $F(2,50)=.07, p=.791, \eta_{\mathrm{p}}{ }^{2}=.00$, $n s$ ), nor was there an interaction between the prenatal dose and area preference (area $\times$ prenatal dose: $\left.F(2,50)=.30, p=.743, \mathrm{\eta}_{\mathrm{p}}^{2}=.01, n s\right)$. There was no effect of genotype $(F(1,50)=.79, p=$ $.378, \eta_{\mathrm{p}}{ }^{2}=.02, n s$ ), or any interaction (area $\times$ genotype: $F(2,50)=.94, p=.398, \eta_{\mathrm{p}}^{2}=.04, n s$ ). Furthermore, the prenatal dose did not have a main effect $\left(F(2,50)=1.34, p=.271, \eta_{\mathrm{p}}{ }^{2}=.05\right.$, $n s)$, but, there was a close to significant effect of genotype $\left(F(1,50)=3.63, p=.063, \eta_{\mathrm{p}}^{2}=.07\right.$, $n s)$, however, no further effects were found. The average time spent in the familiar and novel social area is represented in figure 3.21 .

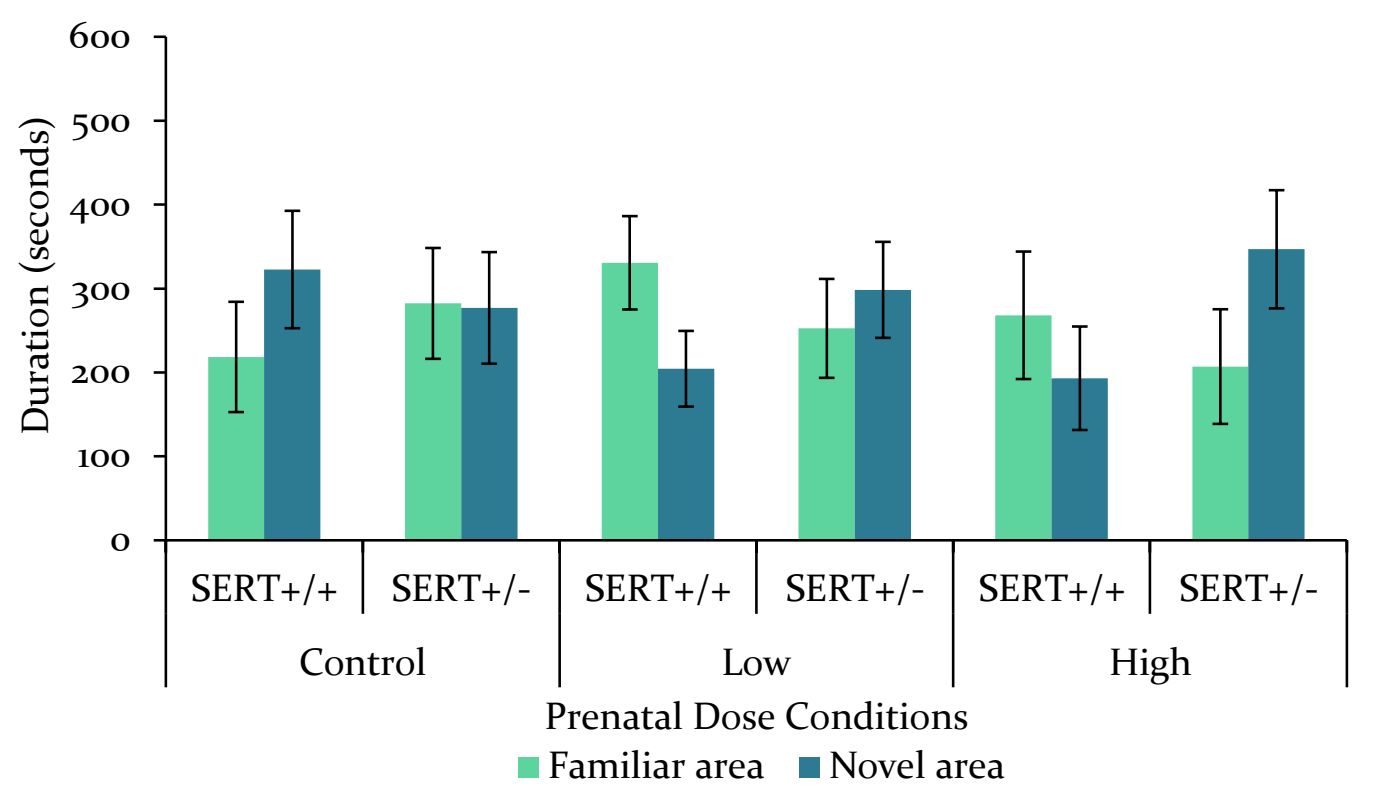

Figure 3.21. The average duration of time spent in the familiar and novel areas for male SERT ${ }^{+/+}$ and SERT ${ }^{+-}$offspring exposed to control, low, and high doses of VPA, no statistically significant results were found. Error bars represent \pm SEM. 


\section{Females time spent in the familiar and novel social area}

The female SERT ${ }^{+/}$and SERT ${ }^{+/}$prenatally exposed to VPA showed a trend towards significance for area preference (area: $F(2,35)=3.38, p=.075, \eta_{\mathrm{p}}{ }^{2}=.09, n s$, see figure 3.22). There was no interaction between the prenatal dose and area preference (area $\times$ prenatal dose: $\left.\mathrm{F}(2,35)=.44, \mathrm{p}=.651, \mathrm{\eta}_{\mathrm{p}}^{2}=.02, \mathrm{~ns}\right)$, or the genotype (area $\times$ genotype: $F(1,35)=2.43, p=.128$, $\eta_{\mathrm{p}}{ }^{2}=.07, n s$ ), or any interaction (area $\times$ prenatal dose $\times$ genotype: $F(2,35)=.04, p=.964, \eta_{\mathrm{p}}{ }^{2}=$ $.00, n s)$. Furthermore, there were no overall effects of prenatal dose (prenatal dose: $F(2,35)=$ $\left..32, p=.731, \eta_{\mathrm{p}}^{2}=.02, n s\right)$.

While the overall analyses revealed no genotype effect, a review of the time spent (represented in figure 3.22) is suggestive of a genotype effect, but it was perhaps disguised by multiple comparisons. As such, SERT ${ }^{+/+}$and SERT ${ }^{+/-}$females were examined for area preference. The SERT ${ }^{+/+}$females showed a significant area preference for the novel social stimulus $(F(1,16)$ $\left.=6.30, p=.023, \mathrm{\eta}_{\mathrm{p}}^{2}=.28\right)$, whereas, the SERT ${ }^{+-}$showed no preference $(F(1,19)=.04, p=.851$, $\left.\eta_{\mathrm{p}}^{2}=.00, n s\right)$.

Thus, the SERT genotype, particularly in combination with the prenatal exposure to VPA, disrupts the preference for familiar or novel stimuli.

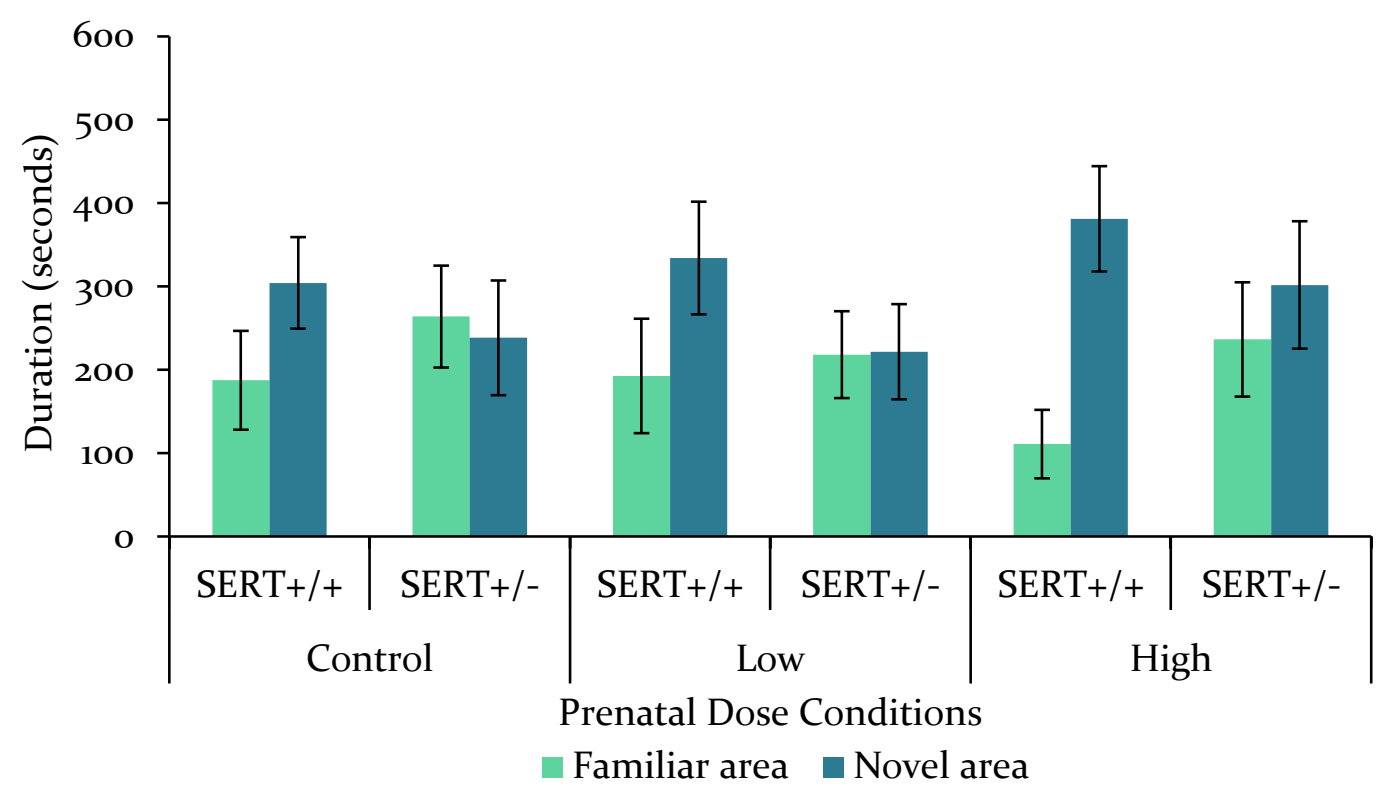

Figure 3.22. The average duration of time spent in familiar and novel areas for female SERT ${ }^{+/+}$ and SERT ${ }^{+/-}$offspring exposed to control, low, and high doses of VPA. SERT ${ }^{+/+}$females exhibited increased duration with the novel stimulus. Error bars represent \pm SEM. 


\subsubsection{Locomotor Activity}

The overall locomotor activity of the test animals was measured with the total distance travelled in phase one and two at PND 60.

\section{Distance travelled for males}

$\mathrm{SERT}^{+/+}$and SERT ${ }^{+/-}$males prenatally exposed to VPA showed no difference in locomotor activity see figure 3.23. There was no difference in distance travelled between the first and second phase $\left(F(1,50)=1.10, p=.300, \mathrm{n}_{\mathrm{p}}{ }^{2}=.02, n s\right)$. There was no interaction between prenatal dose exposure and the distance travelled $\left(F(2,50)=.05, p=.949, \eta_{\mathrm{p}}{ }^{2}=.00, n s\right)$, and nor did any of the prenatal dose conditions show an effect overall $\left(F(2,50)=.69, p=.505, \eta_{\mathrm{p}}^{2}=.03, n s\right)$. Additionally, the genotypes did not have a differential impact $\left(F(1,50)=.31, p=.581, \eta_{\mathrm{p}}{ }^{2}=.00\right.$, $n s)$, nor did it have an overall effect $\left(F(1,50)=1.64, p=.207, \mathrm{\eta}_{\mathrm{p}}^{2}=.03, n s\right)$. Furthermore, there were no interactions between the distance travelled in phase one and two, with the prenatal dose conditions and genotypes $\left(F(2,50)=.08, p=.927, \mathrm{\eta}_{\mathrm{p}}^{2}=.00, n s\right)$.

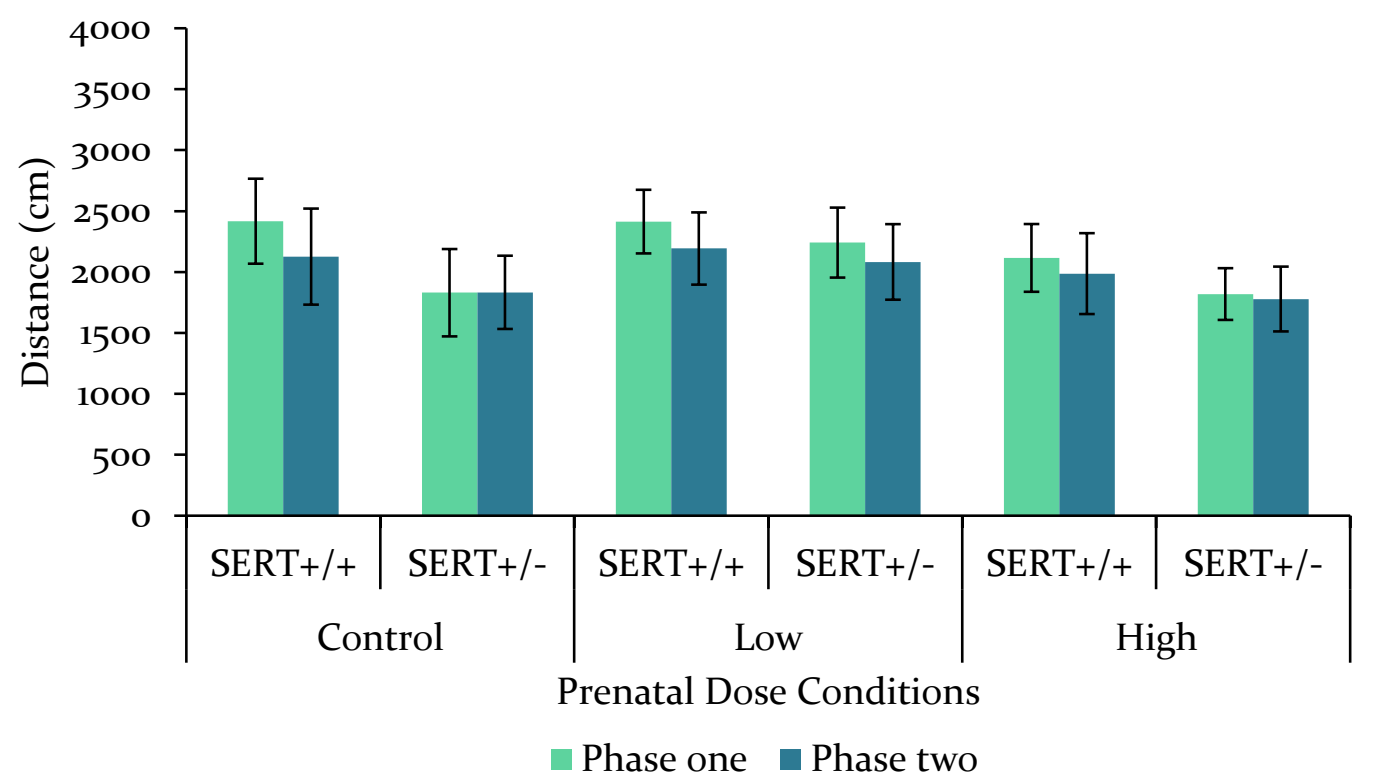

Figure 3.23. The average distance travelled in the first and second phases of SAA by male $\mathrm{SERT}^{+/+}$ and SERT ${ }^{+/-}$prenatally exposed to control, low, and high doses of VPA, showing no effects. Error bars represent \pm SEM. 


\section{Distance travelled for females}

SERT $^{+/+}$and SERT ${ }^{+/-}$females prenatally exposed to VPA showed a significant effect of phase $\left(F(1,35)=10.35, p=.003, \eta_{\mathrm{p}}^{2}=.23\right)$, see figure 3.24. This effect was due to the overall significant decrease in locomotor activity from the first phase to the second phase $(p=.003)$. To explore this effect further, the distance travelled for females was examined within each of the prenatal dose conditions. There was a significant decrease in activity for the females exposed to the control $\left(F(1,14)=4.74, p=.047, \eta_{\mathrm{p}}^{2}=.25\right)$ and low doses $\left(F(1,16)=7.26, p=.016, \mathrm{\eta}_{\mathrm{p}}^{2}=\right.$ .31) but not for the high dose females $\left(F(1,8)=1.92, p=.204, \eta_{\mathrm{p}}{ }^{2}=.19, n s\right)$.

Additionally, the genotypes did not have a differential impact $\left(F(1,50)=.31, p=.581, \mathrm{n}_{\mathrm{p}}^{2}\right.$ $=.01, n s)$, nor did it have an overall effect $\left(F(1,35)=.01, p=.913, \mathrm{\eta}_{\mathrm{p}}^{2}=.00, n s\right)$. Furthermore, there were no interactions between the distance travelled in phase one and two, with the prenatal dose conditions and genotypes $\left(F(2,35)=.22, p=.805, \mathrm{\eta}_{\mathrm{p}}{ }^{2}=.01, n s\right)$.

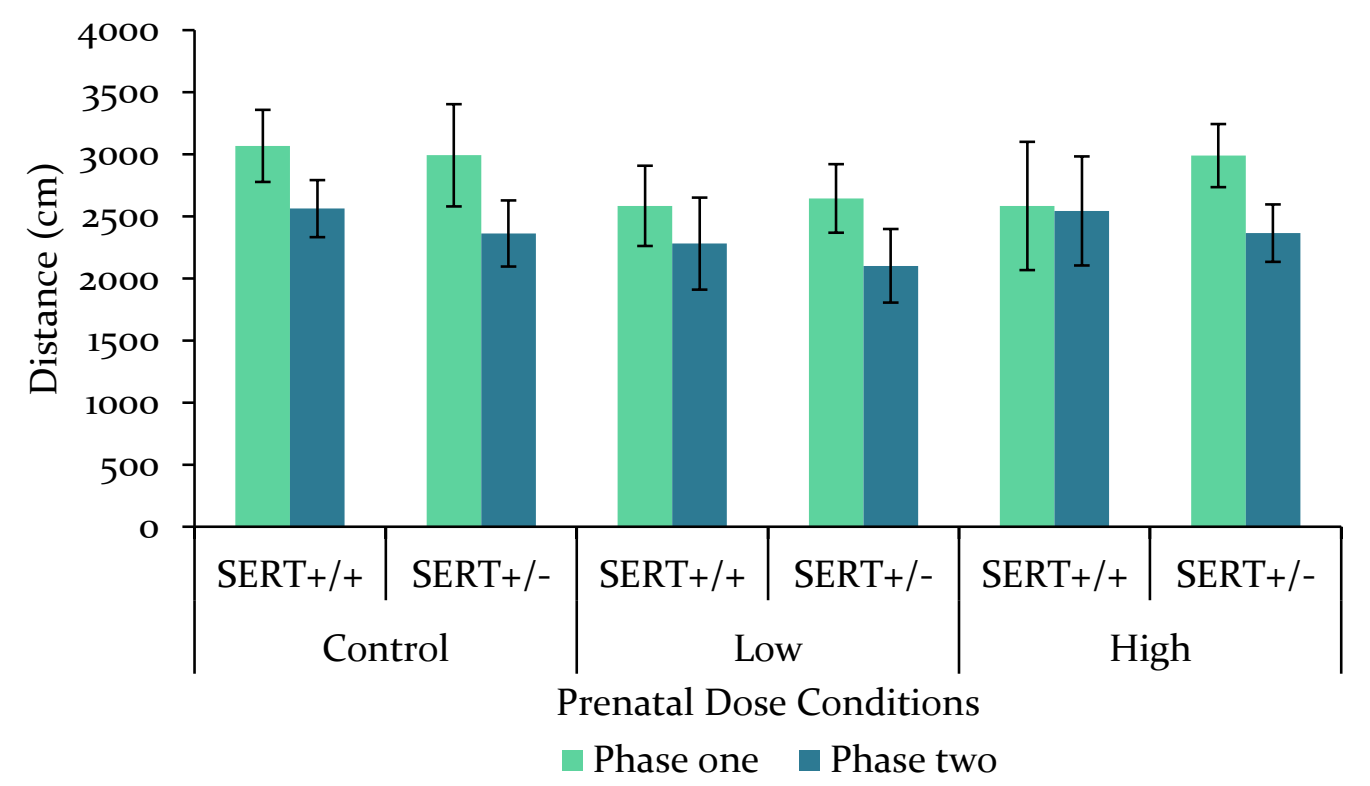

Figure 3.24. The average distance travelled in the first and second phases of SAA by SERT ${ }^{+/+}$and SERT $^{+/-}$females prenatally exposed to control, low, and high doses of VPA, showing increased activity in the first phase and the increased distance for the females. Error bars represent \pm SEM. 


\subsubsection{Pre- \& Post-Pubescent Comparison Results}

The social behaviour of SERT ${ }^{+/+}$and SERT ${ }^{+/}$that were prenatally exposed to VPA was examined pre-puberty (PND 30-35) and post-puberty (PND 60-65). A 2 (PND: 30, 60), by 3(prenatal dose: control, low, high), by 2(genotype: $\mathrm{SERT}^{+/+}, \mathrm{SERT}^{+/-}$) repeated measures ANOVA was run on the sociability score. 


\section{Males sociability score in phase one from Pre- E Post-Puberty}

The prenatal doses differentially affected the sociability score in phase one over time $\left(P N D \times\right.$ prenatal dose: $\left.F(2,44)=4.00, p=.026, \eta_{\mathrm{p}}^{2}=.15\right)$. To explore this effect, the sociability score on PND 30 and 60 were compared within each of the prenatal dose conditions. This revealed a significant decrease from PND 30 to 60 for the offspring exposed to the low dose ( $p$ $=.001)$.

There were no further statistically significant effects. There was no difference in the sociability score on between PND 30 and $60\left(F(2,44)=1.37, p=.248, \mathrm{n}_{\mathrm{p}}{ }^{2}=.03, n s\right)$, and there was no genotype effect on the sociability score between these days $\left(F(1,44)=.39, p=.537, \mathrm{n}_{\mathrm{p}}{ }^{2}=\right.$ $.01, n s)$, and there was no interaction between prenatal doses, genotypes, and the PNDs $(F(2$, 44) $\left.=1.43, p=.251, \mathrm{\eta}_{\mathrm{p}}^{2}=.06, n s\right)$.

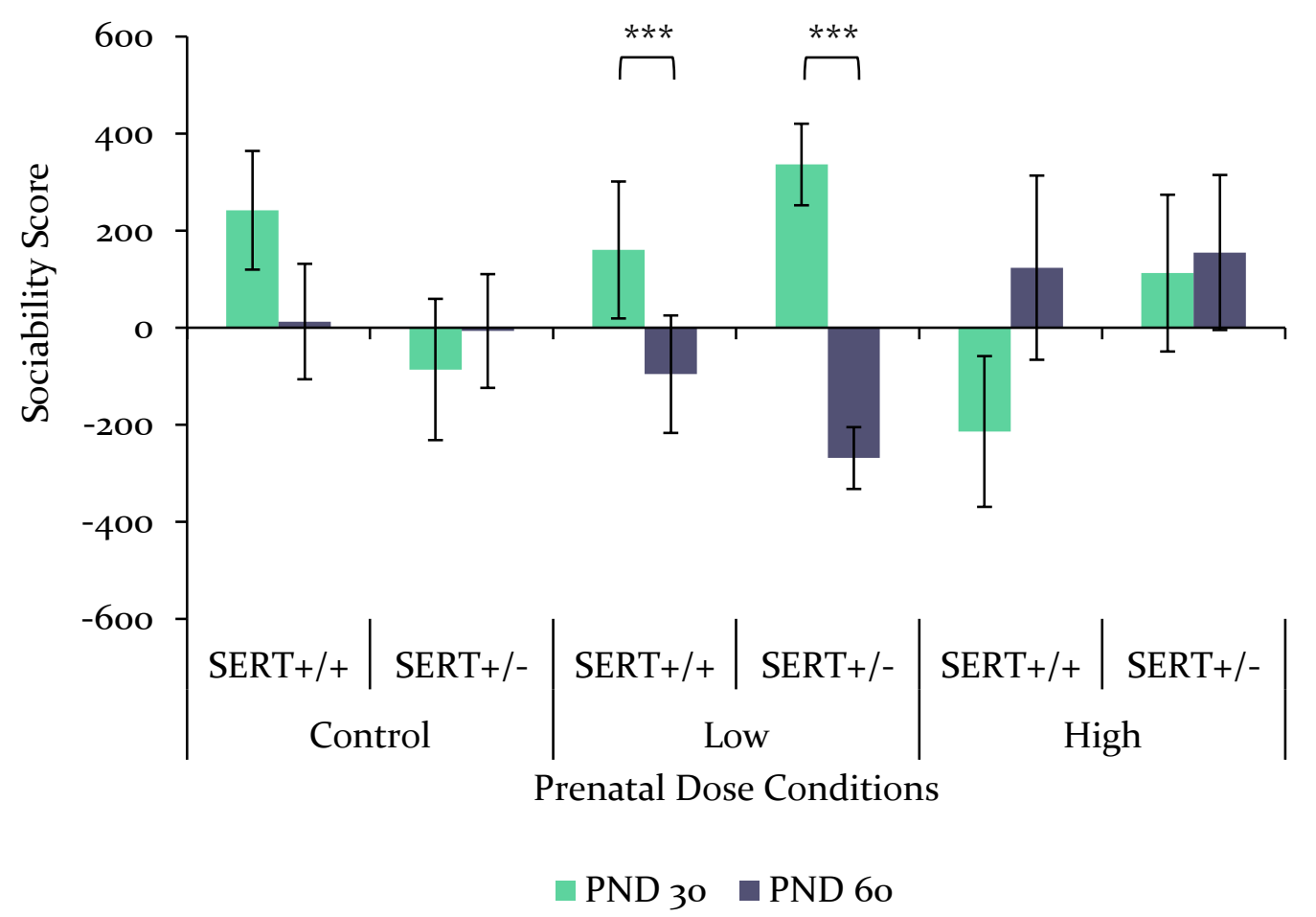

Figure 3.25. Phase one sociability scores measured on PND 30 and 60 for $\mathrm{SERT}^{+/+}$and SERT ${ }^{+/-}$ males prenatally exposed to control, low, and high doses of VPA. Significant decrease from PND 30 to 60 in sociability for low dose males (represented individually). Error bars represent \pm SEM. 


\section{Females sociability score in phase one from Pre- E Post-Puberty}

Overall, there was no interaction of prenatal dose, genotype and PND on the sociability score of females $\left(P N D \times\right.$ prenatal dose $\times$ genotype: $\left.F(2,31)=.99, p=.385, \eta_{\mathrm{p}}{ }^{2}=.06, n s\right)$. However, there was a trend for the different effect of prenatal doses on the PND (PND $\times$ prenatal dose: $\left.F(2,31)=2.52, p=.097, \eta_{\mathrm{p}}{ }^{2}=.14\right)$, and there was a close to significant effect of the prenatal dose on sociability score regardless of PND (main effect prenatal dose: $F(2,31)=3.05, p=.062, \eta_{\mathrm{p}}{ }^{2}=$ .16). The post hoc test indicated that a (non-significant) prenatal dose effect was attributed to the decreased sociability score in the high dose females compared with the low dose females ( $p$ $=.088, n s$, see figure 3.26).

The genotype of females was also trending towards a significant interaction with PND $\left(P N D \times\right.$ genotype: $\left.F(1,31)=2.30, p=.093, \mathrm{\eta}_{\mathrm{p}}^{2}=.09\right)$. This analysis confirmed that the $\mathrm{SERT}^{+/-}$ prenatally exposed to the control dose showed a significant effect of PND, where their sociability score was high on PND 30 and then substantially decreased on PND 60 ( $p=.001)$.

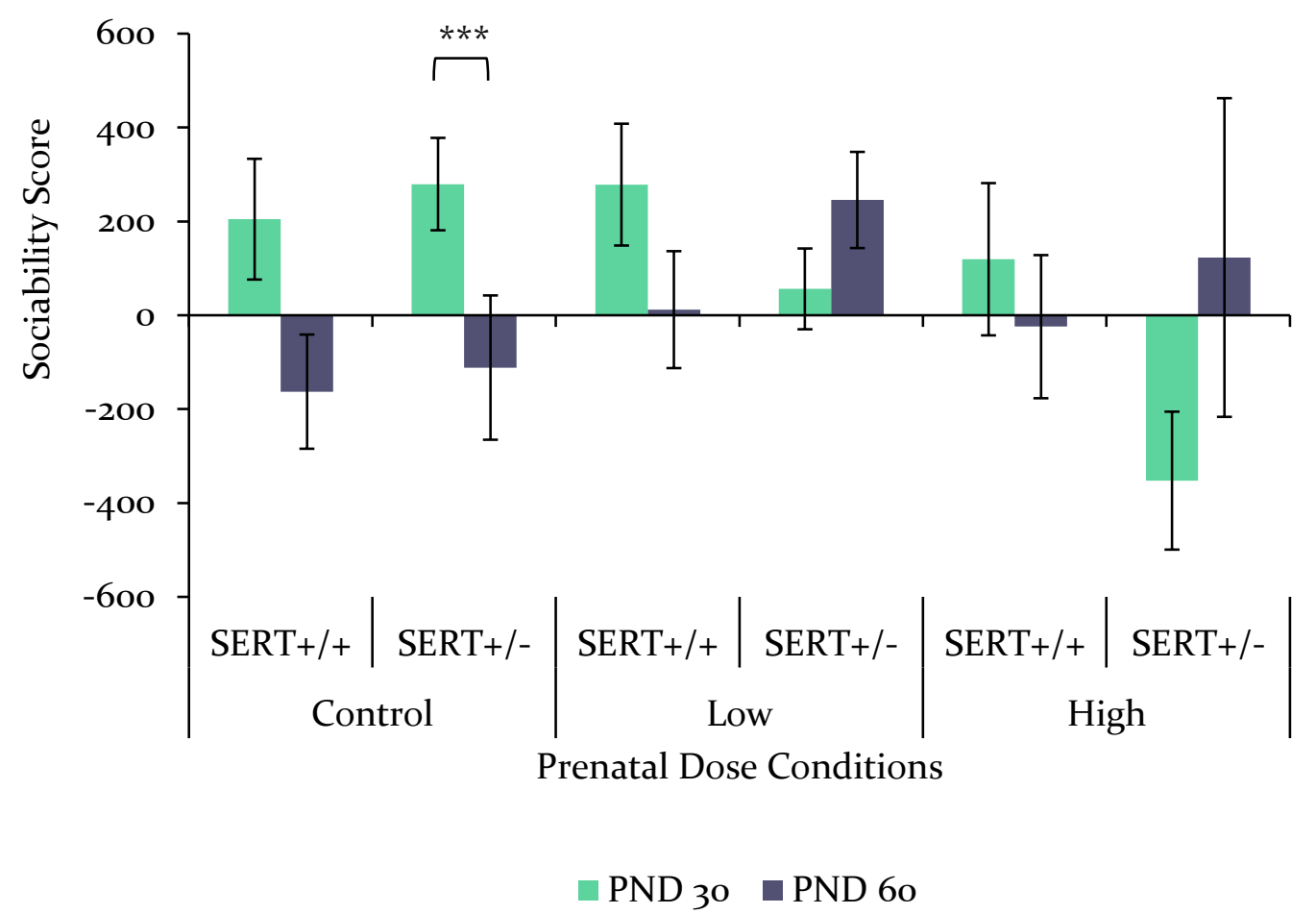

Figure 3.26. Phase one sociability scores measured on PND 30 and 60 for $\mathrm{SERT}^{+/+}$and SERT ${ }^{+/-}$ females prenatally exposed to control, low, and high doses of VPA. Decreased sociability with age, with the exception of the SERT ${ }^{+/-}$exposed to the high which exhibited an increase. ${ }^{* * *}$ indicates significance to $p<.001$. Error bars represent \pm SEM. 


\section{Males sociability score in phase two from Pre- E Post-Puberty}

The male $\mathrm{SERT}^{+/+}$and $\mathrm{SERT}^{+/-}$prenatally exposed to VPA showed no significant differences in their familiarity or novelty preference over time, see figure 3.27. There were no differences in the sociability score on between PND 30 and $60\left(F(1,44)=.71, p=.404, \eta_{\mathrm{p}}{ }^{2}=.02\right.$, $n s)$, there was no prenatal dose effect $\left(F(2,44)=2.19, p=.124, \mathrm{\eta}_{\mathrm{p}}^{2}=.09, n s\right)$, and there was no genotype effect $\left(F(1,44)=.77, p=.386, \eta_{\mathrm{p}}^{2}=.02, n s\right)$, as well as no interaction between prenatal doses, genotypes, and the PNDs $\left(F(2,44)=1.49, p=.236, \eta_{\mathrm{p}}^{2}=.06, n s\right)$. No significant main effects were found.

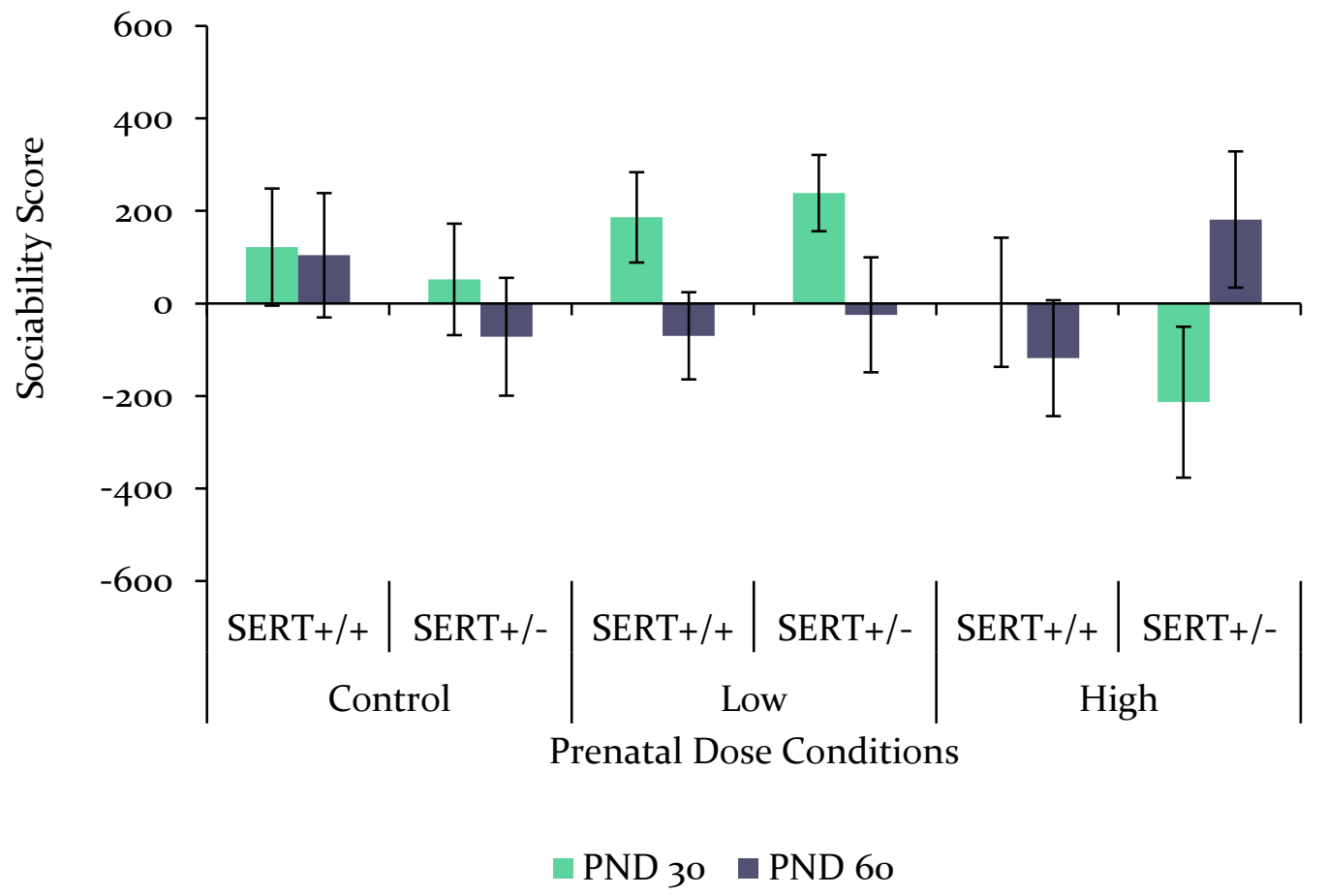

Figure 3.27. Phase two sociability scores measured on PND 30 and 60 for SERT ${ }^{+/+}$and SERT ${ }^{+/-}$ males prenatally exposed to control, low, and high doses of VPA, with no significant statistical effects. Error bars represent \pm SEM. 


\section{Females sociability score in phase two from Pre- E Post-Puberty}

The female SERT ${ }^{+/+}$and SERT ${ }^{+/-}$prenatally exposed to VPA showed no significant differences in their familiarity or novelty preference overtime, see figure 3.28. There was no difference in the sociability score on between PND 30 and $60\left(F(1,31)=.09, p=.764, \mathrm{\eta}_{\mathrm{p}}^{2}=.00\right.$, $n s)$, there was no genotype effect on the sociability score between these days $(F(1,31)=1.28, p=$ $\left..267, \mathrm{\eta}_{\mathrm{p}}{ }^{2}=.04, n s\right)$ and there was no interaction between prenatal doses, genotypes, and the PNDs $\left(F(2,31)=.47, p=.628, \eta_{\mathrm{p}}{ }^{2}=.03, n s\right)$. No significant main effects were found.

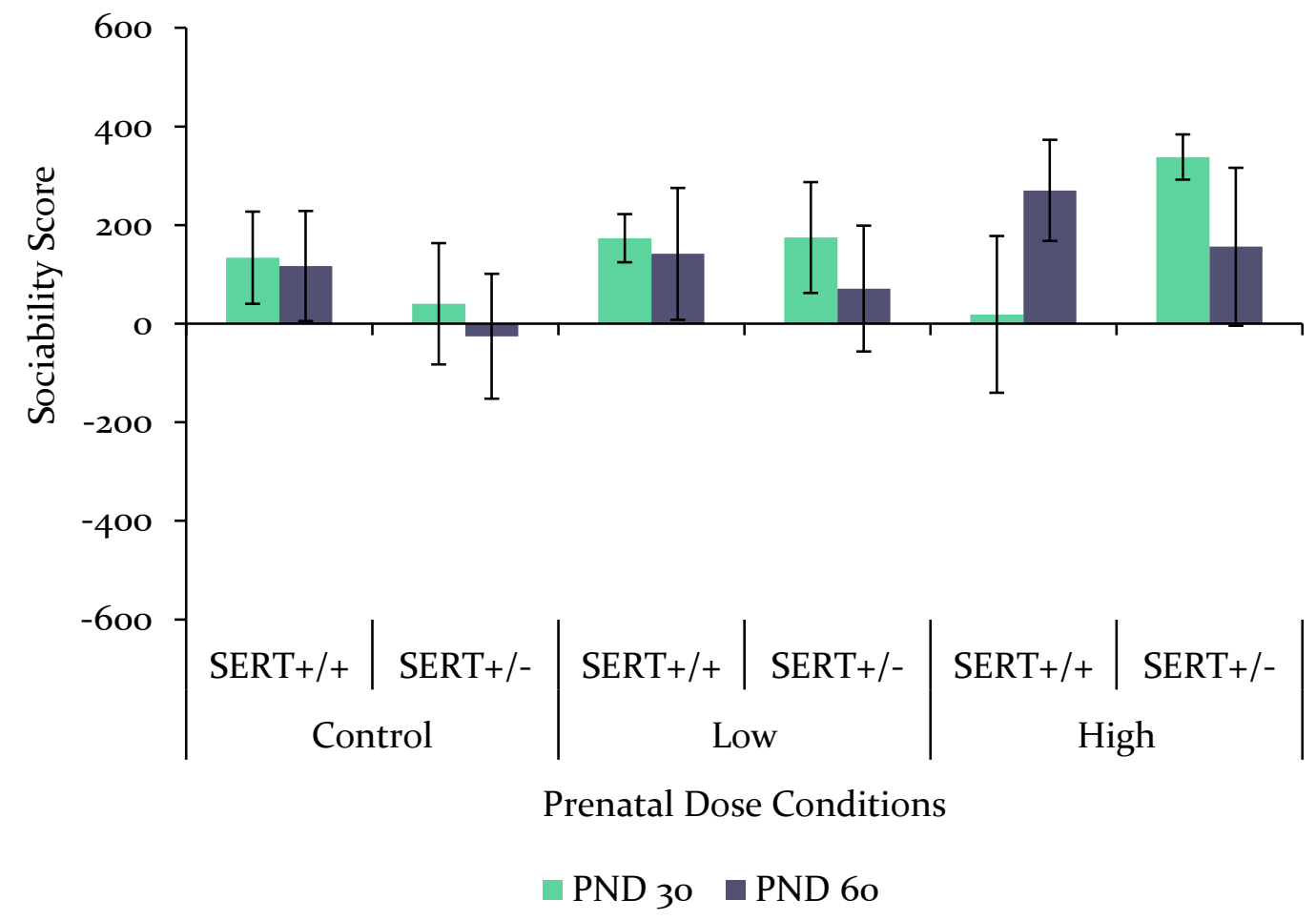

Figure 3.28. Phase two sociability scores measured on PND 30 and 60 for SERT ${ }^{+/+}$and SERT ${ }^{+/-}$ females prenatally exposed to control, low, and high doses of VPA, with no statistically significant effects. Error bars represent \pm SEM. 


\subsection{Experiment Four: Successive Alleys Test}

The introduction to this chapter (section 3.1) highlighted the co-existence of anxiety symptoms for people with ASD, and this experiment examines the influence of anxiety-like behaviours for $\mathrm{SERT}^{+/+}$and SERT ${ }^{+/-}$prenatally exposed to VPA.

Anxiety assays have revealed changes in both SERTs and animals prenatally exposed to VPA. Olivier et al. (2008) demonstrated that SERT ${ }^{-/}$consistently showed increased anxiety-like behaviours in the open field, elevated plus maze, novelty suppressed feeding, and home cage emergence assays. Animals prenatally exposed to VPA likewise exhibit anxiety-like behaviours, as demonstrated by Schneider, Turczak, and Przewłocki (2006) who found that VPA exposed offspring showed significant decreases in the entries and exploration of the open arms in the elevated plus maze. Furthermore, Ellenbroek et al. (2016) found VPA exposed offspring spent less time in the open arms of the elevated plus maze, and increased latency in the novelty suppressed feeding task, thus exhibiting increased anxiety-like behaviour.

These studies exemplify the range of behavioural assays used to measure the anxietylike behaviour of rodents. Most, if not all, of the assays, are based on the animal choosing between conflicting options of safety or exploration. The elevated plus maze, open field, and home cage emergence, all measure the time an animal spends in open, exposed areas compared with enclosed areas, as well as the entries to open or enclosed areas. These behavioural assays require the animal to make a binary choice. Where it is a choice of safety (thus exhibiting 'fearful' and 'anxious' behaviours) or exploration (thus 'explorative'). This binary choice excludes options for measuring a spectrum of anxiety-like behaviours. Ideally, anxiety-like behaviours are in a range of more or less severe. The Successive Alleys Test (SAT) attempts to overcome the binary nature of anxiety assays.

SAT is derived from the elevated plus maze with enclosed, and exposed areas and the whole apparatus is elevated off the floor. The SAT apparatus is divided into four "alleys", and each alley has progressively lower walls and narrower floors (see figure 3.30). This makes each subsequent alley more anxiogenic. The SAT was designed by Deacon (2013), who found that mice with lesions of the medial prefrontal cortex exhibited increased duration in the distant (more anxiogenic) alleys (alleys two, three, and four) compared with controls. The same mice with $\mathrm{mPFC}$ lesions showed increased duration in the open arms of the elevated plus maze compared with control. The results of both experiments indicate that mPFC lesions in mice reduce anxiety-like behaviours. SAT has been used in our laboratory, and the SERT ${ }^{-/}$animals have shown increased latencies in reaching the most anxiogenic alleys, coupled with decreased 
duration in the same alleys, compared with SERT ${ }^{+/+}$(Kidwell, 2018). Thus, $\mathrm{SERT}^{-/-}$demonstrated a more severe anxious phenotype compared with SERT ${ }^{+/+}$or SERT ${ }^{+/}$, and the SAT shows effects similar to the elevated plus maze and other anxiety tests (Olivier et al., 2008).

This experiment aims to examine the anxious phenotype of SERT ${ }^{+/}$and $\mathrm{SERT}^{+/+}$males and females prenatally exposed to VPA throughout gestation. The anxious phenotype is clearly found in the SERT ${ }^{-1-}$ rats, but it is not clear in the SERT ${ }^{+-}$rats. As such, it is uncertain if the $\mathrm{SERT}^{+/-}$in this experiment will exhibit an anxious phenotype, although it is worthy of investigation in the context of the gene $\times$ environment interaction. Thus, it is hypothesised that:

i. Male and female offspring prenatally exposed to VPA will exhibit increased anxiety-like behaviour compared with rats prenatally exposed to the control dose,

ii. Furthermore, there will be an interaction between SERT reduction and chronic exposure to VPA. Such that, SERT ${ }^{+-}$prenatally exposed to VPA will exhibit increased anxiety-like behaviours compared with SERT ${ }^{+/+}$exposed to the control dose. 


\subsubsection{SAT Methods}

\subsubsection{SAT Apparatus}

The SAT apparatus was constructed from black or grey polycarbonate panels. Alleys progressively reduce in wall height and width. The colour of each alley was progressively lighter, alley one was black, alley two was dark grey, alley three was grey, and alley four was light grey. Each alley is $45 \mathrm{~cm}$ in length, and the total length is $180 \mathrm{~cm}$. See table 3.6 and figure 3.29 for wall height and apparatus representation.

Table 3.6. Dimensions of the Successive Alleys Apparatus

\begin{tabular}{|c|c|c|c|}
\hline Alley & $\begin{array}{c}\text { Width } \\
(\mathrm{cm})\end{array}$ & $\begin{array}{c}\text { Wall height } \\
(\mathrm{cm})\end{array}$ & Hypothesised Anxiogenic Effect \\
\hline One & 9 & 29 & Low \\
\hline Two & 8 & 6 & Modest \\
\hline Three & 6.5 & 2.5 & Moderate \\
\hline Four & 3.5 & 1 & High \\
\hline
\end{tabular}
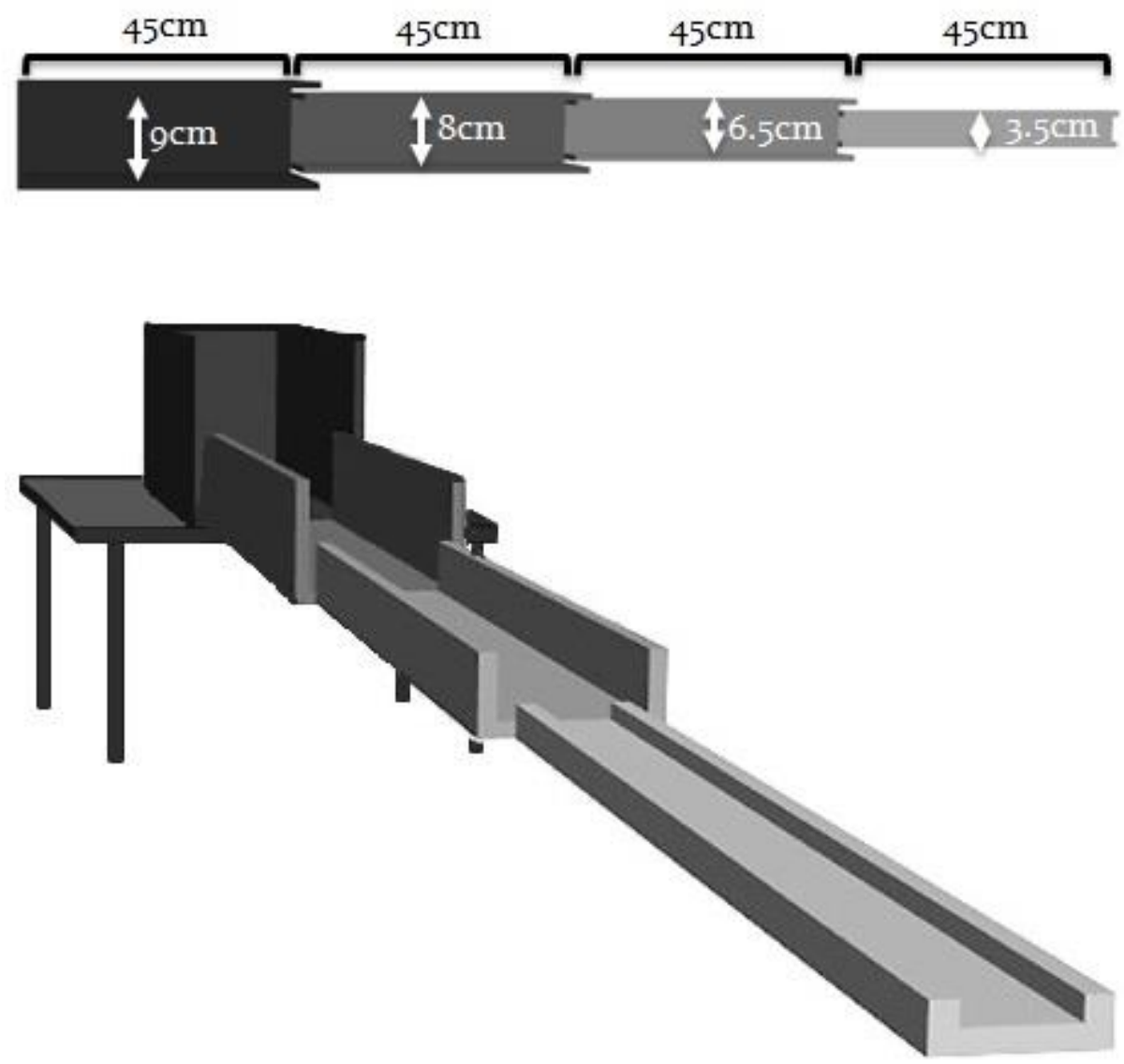

Figure 3.29. Representation and dimensions of the Successive Alleys Apparatus 


\subsubsection{SAT Procedure}

On or after PND 80, animals were selected from their home cage, transferred into a temporary cage, and transported into the experimental room. Animals were habituated to the room for 10 minutes.

The trial commenced when the rat was placed into the apparatus facing the back wall. The rat's activity was recorded with EthoVision $\left(\mathrm{XT}^{\mathrm{TM}}\right)$ for 5 minutes, while the experimenter manually coded the entries into each of the alleys with the use of EthoVision $\left(\mathrm{XT}^{\mathrm{TM}}\right)$ manual scoring function. An entry was coded when the hindlegs of the rat crossed the boundary between the two alleys. The duration in each alley and the latency to enter an alley was measured from the manually coded entries. Manually coding the rat's activity was required as EthoVision $\left(\mathrm{XT}^{\mathrm{TM}}\right)$ was unable to automatically track the rat's movement due to inference from the overhead lights in the experimental room.

At the end of the 5-minute trial, rats were weighed and returned to their home cage. The alleys were wiped down with 70\% ethanol and paper towels. The alleys were dried before the next rat was tested.

\subsubsection{SAT Data Analysis}

To analyse the effect of prenatal exposure to VPA in SERT ${ }^{+/+}$and $\mathrm{SERT}^{+/-}$, factorial ANOVAs 3 (Dose; control, low, high) by 2 (Genotype; SERT ${ }^{+/+}$, SERT ${ }^{+-}$) were run separately for male and female adults. The total duration in each alley, and the latency to reach alleys two, three, and four were the variable of interest. To further examine the duration of time in the alleys, a repeated measures 3(Dose: control, low, high), 2(Genotype: SERT ${ }^{+/+}$and SERT ${ }^{+/}$), 3(Alley: Alley two, three, four) ANOVA was used; with alley as the repeated factor.

All data were analysed for statistical significance with SPSS (version 22-25). Partial eta squared $\left(\mathrm{n}_{\mathrm{p}}^{2}\right)$ was used for the effect size. All significant analyses were followed up with Tukey HSD post hoc tests, and/or planned comparisons were used to follow up potential effect comparing specific prenatal dose or genotype conditions with others using Student's t-test. The alpha level for statistical significance was set at $p<.05$. 


\subsubsection{SAT Results}

A total of 124 rats were tested in the SAT paradigm, the test session occurred on or after PND 80. One SERT ${ }^{+/+}$male exposed to a low dose was excluded from the analysis as it did not leave alley one and did not explore the apparatus. Table 3.7 provides the number of samples for each condition, sex, genotype.

Table 3.7. The number of test animals used in SAT PND 80+ divided by dose, sex and genotype.

\begin{tabular}{c|c|c|c|c|}
\hline \hline \multirow{3}{*}{ Control } & Males & SERT $^{+/+}$ & SERT $^{+/-}$ & Combined \\
\cline { 2 - 5 } & Females & 10 & 15 & 25 \\
\cline { 2 - 5 } & Combined & 19 & 6 & 15 \\
\hline \multirow{4}{*}{ Low } & Males & 12 & 21 & 40 \\
\cline { 2 - 5 } & Females & 11 & 8 & 20 \\
\cline { 2 - 5 } & Combined & 23 & 19 & 30 \\
\hline High & Males & 12 & 10 & 22 \\
& Females & 7 & 5 & 12 \\
\cline { 2 - 5 } & Combined & 19 & 15 & 34 \\
\hline \hline
\end{tabular}




\subsubsection{Alley Entry Latencies}

Since all rats were placed in alley one, only the latency to enter the remaining three alleys is considered. Firstly, the effects of prenatal VPA exposure and genotypes on the latency to enter all alleys was examined in one analysis, and significant effects for particular alleys are assessed subsequently.

\section{Repeated measure analysis on latency to enter alley two, three, and four for males}

The repeated measures ANOVA on the latency to enter each alley showed there was a significant effect of alleys $\left(F(1.71,102.79)=67.71, p=.001, \eta_{\mathrm{P}}^{2}=.53\right.$, see figure 3.31$)$, although this is unsurprising as all rats had to transverse alley two and alley three in order to reach alley four. The post hoc analysis confirmed there was a significant delay in reaching alley four compared with the latency to enter alley two $(p<.05$, see appendix three, section B for a table of the comparison and significance values).

There were no further effects of the prenatal dose conditions (alleys $\times$ prenatal dose: $F(3.43,102.79)=.92, p=.455, \mathrm{\eta}_{\mathrm{p}}^{2}=.03, n s ;$ main effect of prenatal dose: $F(2,60)=.78, p=.462$, $\eta_{\mathrm{p}}{ }^{2}=.03, n s ;$ ), nor with genotype (alley $\times$ genotype: $F(1.71,102.79)=.64, p=.505, \eta_{\mathrm{p}}{ }^{2}=.01, n s$; main effect of genotype: $\left.F(1,60)=.36, p=.551, \eta_{\mathrm{p}}{ }^{2}=.01, n s\right)$ and no interaction between the prenatal doses and genotypes (and alley: $F(2,102.79)=1.64, p=.171, \eta_{\mathrm{p}}{ }^{2}=.05$, $n s$; or as a main effect: $\left.F(2,60)=.41, p=.665, \mathrm{n}_{\mathrm{p}}^{2}=.01, n s\right)$.

To further explore the overall effect of delayed latency to enter the alleys (found in the overall repeated measures analysis), the latency to enter each of the alleys was assessed separately.

\section{Latency to enter individual alleys for males}

Latency to enter alley two: Males prenatally exposed to VPA showed a trend towards significance for the latency to enter alley two $\left(F(2,60)=2.49, p=.091, \eta_{\mathrm{p}}^{2}=.08, n s\right)$. The post hoc analysis indicated that the non-significant prenatal dose effect was attributed to a significantly increased latency of the low dose males compared with the control dose males ( $p$ $=.040)$. Furthermore, there was no interaction between the prenatal doses and genotypes $(F(2$, $\left.60)=.82, p=.444, \mathrm{\eta}_{\mathrm{p}}^{2}=.03, n s\right)$, but there was an overall effect of genotype $(F(1,60)=4.13, p=$ $\left..047, \mathrm{\eta}_{\mathrm{p}}{ }^{2}=.06\right)$. The follow up analysis revealed that $\mathrm{SERT}^{+/-}$males were significantly slower to enter alley two compared with SERT ${ }^{+/+}$males $(p=.030$, see figure 3.30). 


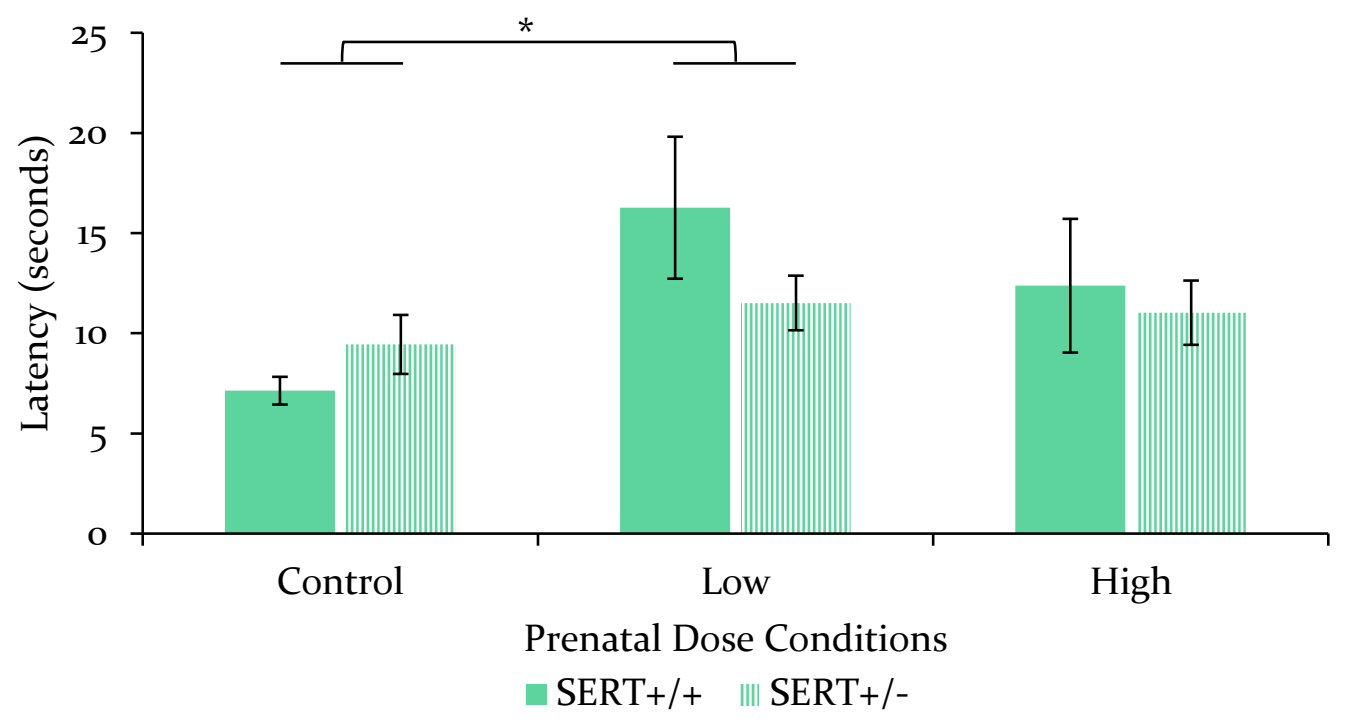

Figure 3.30. The average latency to enter alley two for $\mathrm{SERT}^{+/+}$and $\mathrm{SERT}^{+/-}$males exposed to control, low, and high VPA doses, displaying the decreased latency of the low SERT ${ }^{+/-} \mathrm{s}$. * indicates significance to $p<.05$. Error bars represent \pm SEM.

Latency to enter alley three and four: No statistically significant results were found. There were no effects of the prenatal dose conditions (alley three: $F(2,60)=.21, p=.813, \mathrm{\eta}_{\mathrm{p}}{ }^{2}=.01, n s$; alley four: $\left.F(2,60)=1.17, p=.318, \eta_{\mathrm{p}}^{2}=.04, n s\right)$, nor with genotype (alley three: $F(1,60)=.02, p$ $=.881, \mathrm{\eta}_{\mathrm{p}}^{2}=.01, n s ;$ alley four: $\left.F(1,60)=.74, p=.395, \mathrm{\eta}_{\mathrm{p}}{ }^{2}=.01, n s\right)$ and no interaction between the prenatal doses and genotypes (alley three: $F(2,60)=.27, p=.761, \eta_{\mathrm{p}}{ }^{2}=.01$, ns; alley four: $\left.F(2,60)=1.45, p=.243, \mathrm{n}_{\mathrm{p}}^{2}=.05, n s\right)$. The averages for the latency to enter alley three (green) and four (purple) is represented the repeated measures figure 3.31.

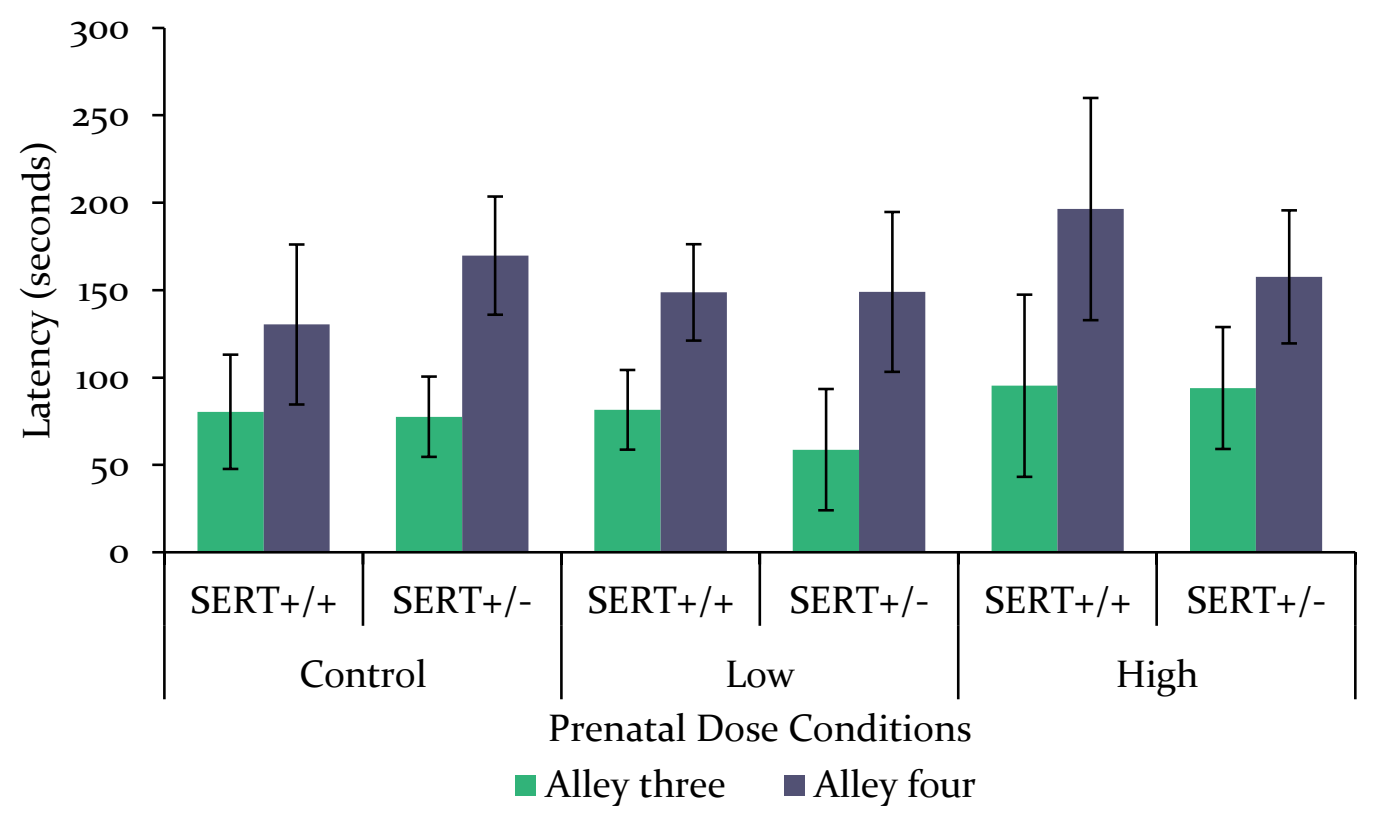

Figure 3.31. The average latency to enter alley three and four, for male SERT ${ }^{+/+}$and $\mathrm{SERT}^{+/-}$ prenatally exposed to control, low, and high VPA doses, with an overall effect of delayed latency to enter alley three and four. Error bars represent \pm SEM. 


\section{Repeated measure analysis on latency to enter alley two, three, and four for females}

There was a significant effect for the repeated measures analysis in the latency to enter all alleys for females $\left(F(1.78,90.80)=41.72, p=.001, \eta_{\mathrm{p}}{ }^{2}=.45\right.$, see figure 3.33), again unsurprising as all rats had to transverse less anxiogenic alleys in order to reach the more anxiogenic alley four. The post hoc analysis confirmed there was a significant delay in reaching alley four compared with the latency to enter alley two $(p<.05$, see appendix three, section B for a table of the comparison and significance values).

There were no further effects of the prenatal dose conditions (alleys $\times$ prenatal dose: $F(3.56,90.80)=.86, p=.481, \eta_{\mathrm{p}}^{2}=.03, n s$; main effect of prenatal dose: $F(2,51)=1.25, p=.295$, $\left.\eta_{\mathrm{p}}{ }^{2}=.05, n s ;\right)$, nor with genotype (area $\times$ genotype: $F(1.78,90.80)=.16, p=.854, \eta_{\mathrm{p}}{ }^{2}=.01, n s$; main effect of genotype: $\left.F(1,51)=.03, p=.861, \mathrm{\eta}_{\mathrm{p}}{ }^{2}=.01, n s\right)$ and no interaction between the prenatal doses and genotypes (and area: $F(3.56,90.80)=.06, p=.989, \mathrm{\eta}_{\mathrm{p}}{ }^{2}=.01$, $n s$; or as a main effect: $\left.F(2,51)=.03, p=.997, \eta_{\mathrm{p}}^{2}=.01, n s\right)$.

\section{Latency to enter alley two for females}

Females prenatally exposed to VPA showed a close to significant effect on the latency to enter alley two $\left(F(2,51)=3.01, p=.058, \eta_{\mathrm{p}}^{2}=.11, n s\right.$, see figure 3.32$)$. The post hoc analysis indicated a non-significant effect, which was attributed to increased latency in the low dose females, compared with the control dose females $(p=.074, n s)$. Furthermore, there was no overall effects of genotype $\left(F(1,51)=1.67, p=.202, \mathrm{\eta}_{\mathrm{p}}{ }^{2}=.03, n s\right)$ and no interaction between the prenatal doses and genotypes $\left(F(2,51)=.36, p=.701, \mathrm{\eta}_{\mathrm{p}}^{2}=.01, n s\right)$.

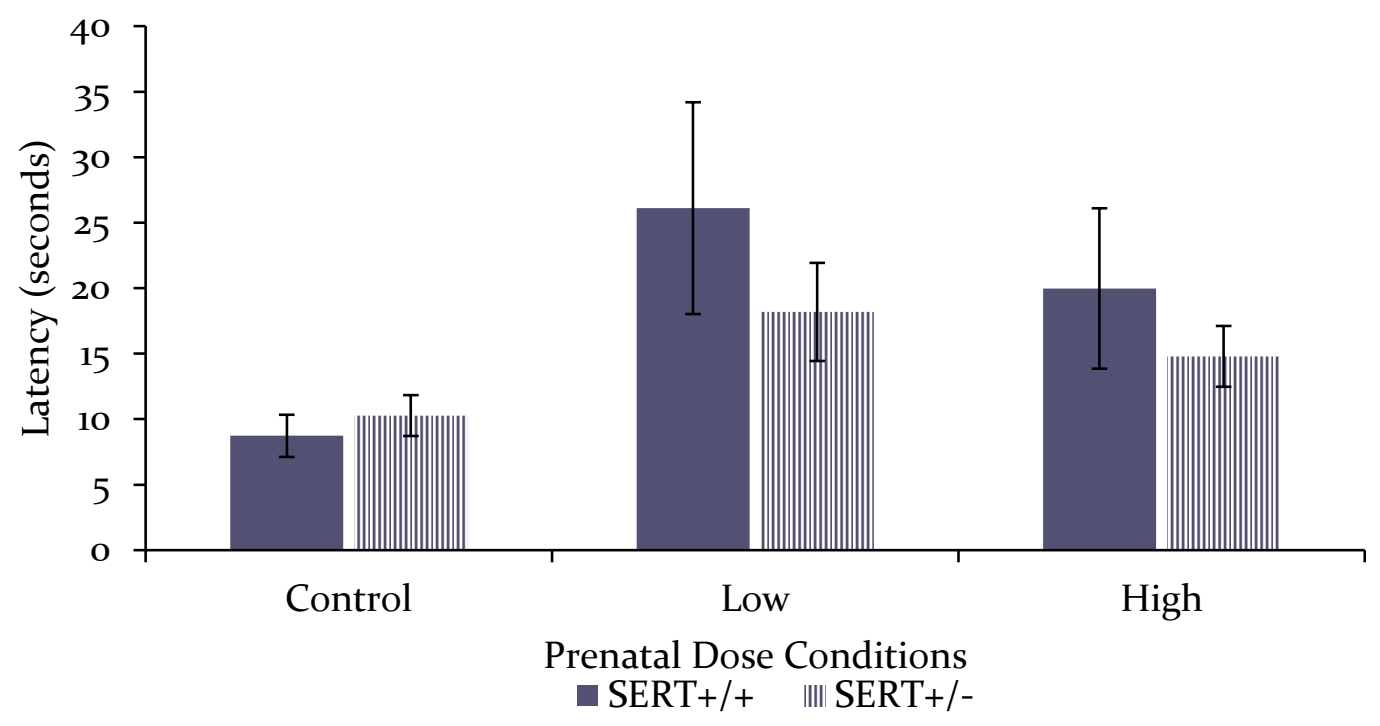

Figure 3.32. The average latency to enter alley two for SERT ${ }^{+/+}$and $\mathrm{SERT}^{+/-}$females exposed to control, low, and high doses of VPA. Displaying an increased latency for low dose females. Error bars represent \pm SEM. 
Latency to enter alley three and four: No statistically significant results were found. There were no effects of the prenatal dose conditions (alley three: $F(2,51)=.47, p=.631, \eta_{\mathrm{p}}^{2}=.02, n s$; alley four: $\left.F(2,51)=1.40, p=.256, \eta_{\mathrm{p}}^{2}=.05, n s\right)$, nor with genotype (alley three: $F(1,51)=.09, p$ $=.761, \mathrm{\eta}_{\mathrm{p}}^{2}=.01$, ns; alley four: $\left.F(1,51)=.05, p=.818, \mathrm{\eta}_{\mathrm{p}}^{2}=.01, n s\right)$ and no interaction between the prenatal doses and genotypes (alley three: $F(2,51)=.03, p=.963, \eta_{\mathrm{p}}{ }^{2}=.01$, $n$; ; alley four: $\left.F(2,51)=.02, p=.983, \eta_{\mathrm{p}}{ }^{2}=.01, n s\right)$. The averages for the latency to enter alley three (green) and four (purple) is represented in repeated measure figure 3.33 .

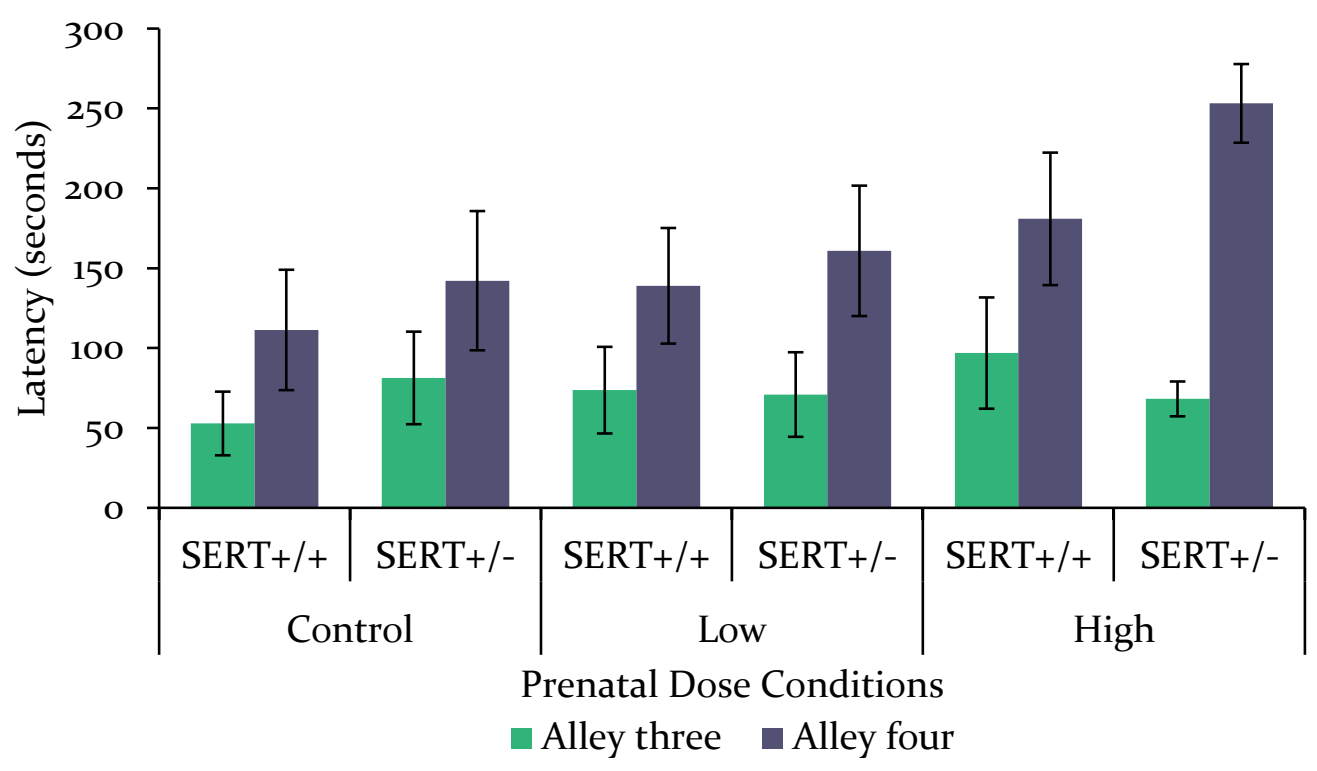

Figure 3.33. The average latency to enter alleys three and four for female SERT ${ }^{+/+}$and SERT ${ }^{+/-}$ prenatally exposed to control, low, and high doses of VPA, with an overall effect of alleys and not prenatal conditions. Error bars represent \pm SEM 


\subsubsection{Duration of Time in the Alleys}

\section{Time spent alley one, two, three, and four, for males}

An analysis of duration in all the alleys assesses the anxiety-like behaviour of the rats (decrease duration representing increase anxiety). Using a repeated measures ANOVA, with the duration in each alley as the repeated measure, there was a significant effect of alley, regardless of prenatal dose and genotypes $\left(F(1.38,83.28)=126.63, p=.001, \mathrm{\eta}_{\mathrm{p}}{ }^{2}=.68\right.$, see figure 3.34$)$. The follow up analyses confirmed that the duration spent in alley one was significantly longer than the duration in alley two $(p=.012)$, and the duration in alley two was longer than alley three ( $p$ $=.001)$, and three was increased over the duration in alley four $(p=.001)$.

For the male SERT ${ }^{+/+}$and SERT ${ }^{+/-}$prenatally exposed to VPA, there was no interaction between the prenatal dose and the duration spent in the alleys (alley $\times$ prenatal dose: $F(2.78$, $\left.83.28)=.63, p=.584, \eta_{\mathrm{p}}{ }^{2}=.02, n s\right)$, nor was there an interaction between the genotypes and duration in the alleys (alley $\times$ genotype: $\left.F(1.39,83.28)=.34, p=.634, \eta_{\mathrm{p}}{ }^{2}=.01, n s\right)$. Further, there was no interaction between the prenatal doses, genotypes and the duration in the alleys $(F(2.78$, 83.28) $\left.=1.18, p=.322, \eta_{\mathrm{p}}{ }^{2}=.04, n s\right)$. Finally, there was no overall effects of prenatal dose (prenatal dose: $\left.F(2,60)=2.01, p=.143, \mathrm{\eta}_{\mathrm{p}}{ }^{2}=.06, n s\right)$, and no overall effects of (genotype: $F(1$, $\left.60)=.08, p=.782, \eta_{\mathrm{p}}^{2}=.00, n s\right)$.

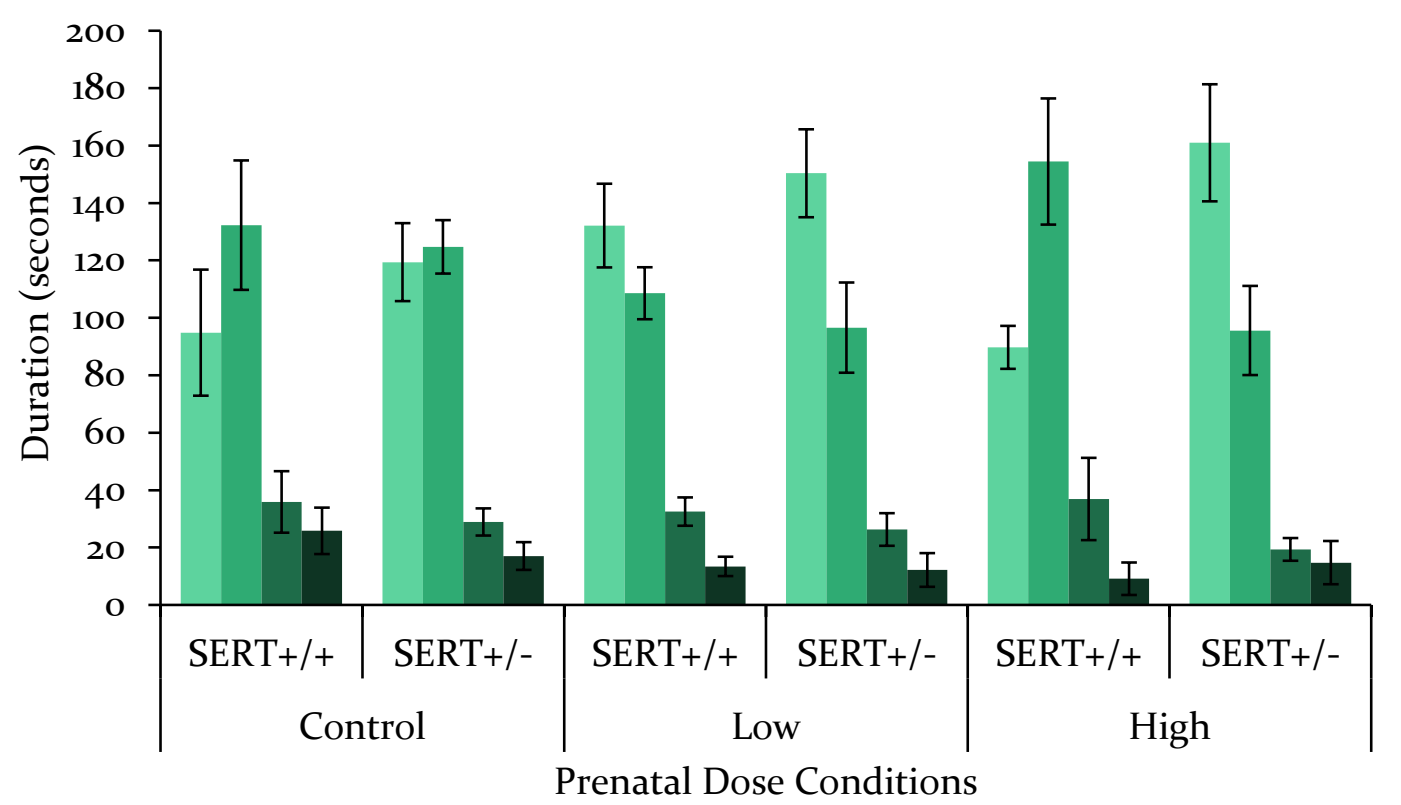

$\square$ Alley one $\quad$ Alley two $\quad$ Alley three $\quad$ Alley four

Figure 3.34. The average duration of time in all alleys of male SERT ${ }^{+/+}$and SERT ${ }^{+/-}$prenatally exposed to control, low, and high doses of VPA. All males show a significant decrease in time spent in alley 2,3 , and 4 . Error bars represent \pm SEM. 


\section{Time spent alley one, two, three, and four, for females}

Using a repeated measures ANOVA, with the duration in each alley as the repeated measure, there was a significant effect of alleys, regardless of prenatal dose and genotypes $\left(F(1.48,75.46)=80.41, p=.001, \eta_{\mathrm{p}}{ }^{2}=.61\right.$, see figure 3.35). The follow up analyses confirmed that the duration of time spent in alley one was significantly longer than the duration in alley three $(p=.001)$, and alley four $(p=.001)$. The duration spent in alley two was longer than the duration in alley three $(p=.001)$, and alley four $(p=.001)$. The duration of time in alley one and two were not significantly different, see figure 3.35 for graphical representation.

For the female $\mathrm{SERT}^{+/+}$and $\mathrm{SERT}^{+/-}$rats prenatally exposed to VPA, there was no interaction between the prenatal dose and the duration spent in the alleys (alley $\times$ prenatal dose: $\left.F(2.96,75.46)=1.46, p=.232, \eta_{\mathrm{p}}^{2}=.05, n s\right)$, nor was there an interaction between the genotypes and duration in the alleys (alley $\times$ genotype: $F(1.48,75.46)=2.29, p=.123, \eta_{\mathrm{p}}{ }^{2}=.04, n s$ ). Further, there was no interaction between the prenatal doses, genotypes and the duration in the alleys $\left(F(2.96,75.46)=1.70, p=.174, \eta_{\mathrm{p}}^{2}=.06, n s\right)$. Finally, there was no overall effects of prenatal dose (prenatal dose: $F(2,51)=1.45, p=.244, \mathrm{\eta}_{\mathrm{p}}{ }^{2}=.05, n s$ ), and no overall effects of (genotype: $\left.F(1,51)=.57, p=.455, \mathrm{\eta}_{\mathrm{p}}^{2}=.01, n s\right)$.

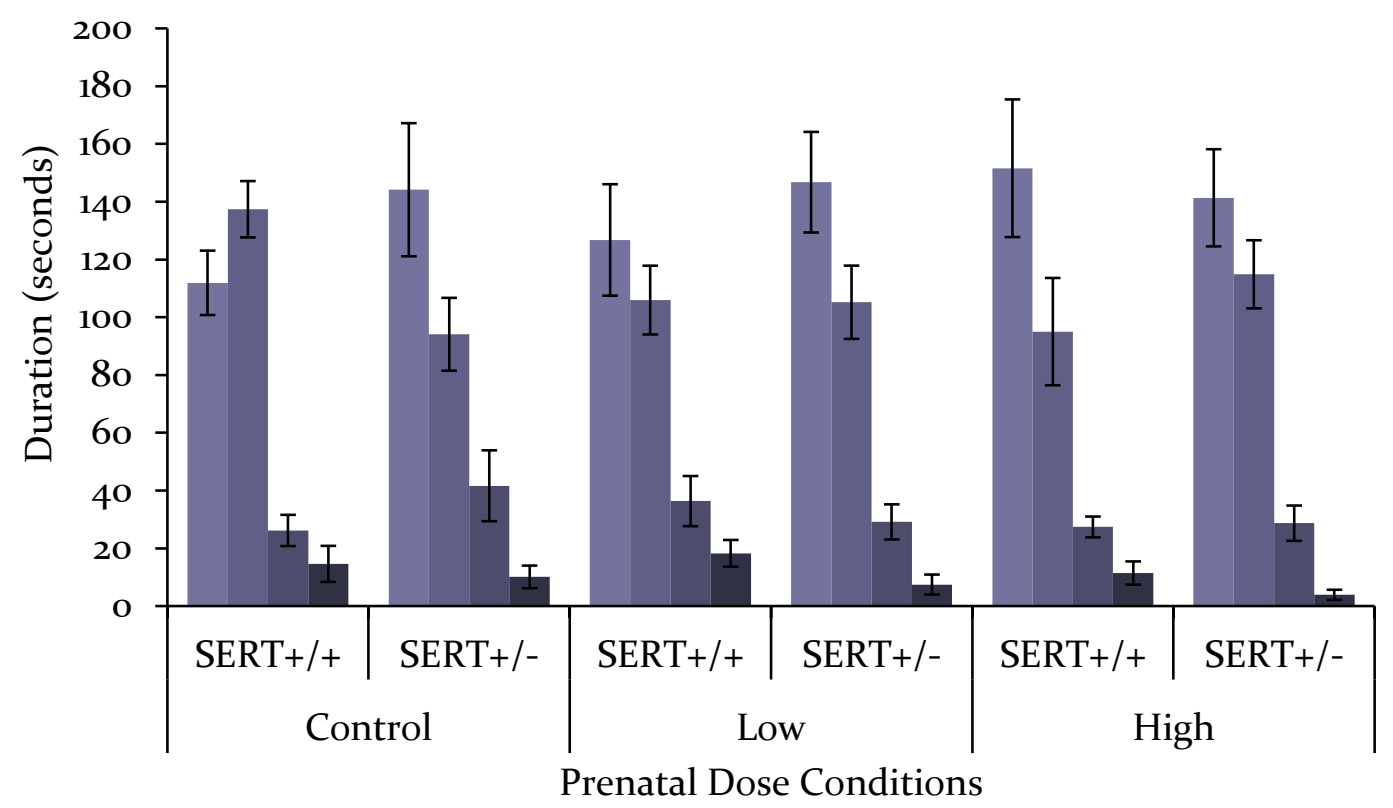

$\square$ Alley one $\quad$ Alley two $\quad$ Alley three $\quad$ Alley four

Figure 3.35. The average duration spent in all allies for SERT ${ }^{+/+}$and $\mathrm{SERT}^{+/-}$females prenatal dose conditions, displaying the significant decrease in duration in the more anxiogenic alleys. Error bars represent \pm SEM. 


\subsection{Results Summary Tables}

Table 3.8.1. This table summarises the prenatal dose and genotype differences, as well as specifying the direction of the difference observed in this chapter.

\begin{tabular}{|c|c|c|c|c|}
\hline \multirow[t]{2}{*}{ Experiment } & \multirow[t]{2}{*}{ Variable } & \multicolumn{3}{|c|}{$\begin{array}{c}\text { Significant differences in prenatal condition } \\
\text { comparisons }\end{array}$} \\
\hline & & Condition & Direction & Condition \\
\hline \multirow{2}{*}{ USVs } & Duration of USVs & Low & $<$ & High \\
\hline & Frequency Range of USVs & Low & $>$ & High \\
\hline \multirow{2}{*}{$\begin{array}{c}\text { Play } \\
\text { Behaviour } \\
\text { PND } 25\end{array}$} & $\begin{array}{c}\text { Males Chasing } \\
\text { Duration }\end{array}$ & Control & $<$ & High \\
\hline & $\begin{array}{l}\text { Males Chasing } \\
\text { Frequency }\end{array}$ & Control & $<$ & High \\
\hline $\begin{array}{c}\text { SAA } \\
\text { PND } 30 \\
\text { Phase } 1\end{array}$ & $\begin{array}{l}\text { Females } \\
\text { Sociability }\end{array}$ & Control & $>$ & High \\
\hline \multirow{2}{*}{$\begin{array}{c}\text { SAA } \\
\text { PND } 30 \\
\text { Phase } 2\end{array}$} & $\begin{array}{l}\text { Females } \\
\text { Sociability }\end{array}$ & Low & $>$ & High \\
\hline & $\begin{array}{c}\text { Females: } \\
\text { Familiar vs Novel - } \\
\text { Duration }\end{array}$ & $\mathrm{SERT}^{+/-}$ & $>$ & $\mathrm{SERT}^{+/+}$ \\
\hline $\begin{array}{c}\text { SAA } \\
\text { PND } 30 \\
\text { Locomotor }\end{array}$ & $\begin{array}{c}\text { Females: } \\
\text { Distance Travelled }\end{array}$ & Control & $<$ & Low \\
\hline $\begin{array}{c}\text { SAA } \\
\text { PND } 60 \\
\text { Phase } 1\end{array}$ & $\begin{array}{l}\text { Males: } \\
\text { Sociability Score }\end{array}$ & Low & $>$ & High \\
\hline $\begin{array}{c}\text { SAA } \\
\text { PND } 60 \\
\text { Phase } 2\end{array}$ & $\begin{array}{c}\text { Females: } \\
\text { Social vs Non-Social - } \\
\text { Duration }\end{array}$ & SERT $^{+/+}$ & $>$ & SERT $^{+/-}$ \\
\hline $\begin{array}{c}\text { SAT } \\
\text { PND 80+ }\end{array}$ & $\begin{array}{c}\text { Males: } \\
\text { Latency to Enter } \\
\text { Alley Two }\end{array}$ & $\begin{array}{l}\text { Control } \\
\text { SERT }^{+/-}\end{array}$ & $>$ & $\begin{array}{l}\text { Low } \\
\text { SERT }^{+/+}\end{array}$ \\
\hline
\end{tabular}

Note: '<' refers to less than, e.g. the control males chasing duration was less than high males. 
Table 3.8.2. This table summarises the significant time effects and specifies the direction of the difference.

\begin{tabular}{|c|c|c|c|c|}
\hline \multirow{2}{*}{ Experiment } & \multirow{2}{*}{ Variable } & \multicolumn{3}{|c|}{ Significant differences in dose comparisons } \\
\hline & & PND & Direction & PND \\
\hline \multirow{4}{*}{$\begin{array}{c}\text { USVs } \\
\text { PND } 7 \text { vs } 14\end{array}$} & Number & \multirow{4}{*}{7} & \multirow{4}{*}{$<$} & \multirow{4}{*}{14} \\
\hline & Duration of USVs & & & \\
\hline & High Frequency & & & \\
\hline & $\begin{array}{l}\text { Range of } \\
\text { Frequency }\end{array}$ & & & \\
\hline $\begin{array}{c}\text { SAA } \\
\text { PND } 30 \mathrm{v} \\
60\end{array}$ & $\begin{array}{c}\text { Males: } \\
\text { Sociability Score } \\
\text { Phase } 1\end{array}$ & 30 & $>$ & 60 \\
\hline $\begin{array}{c}\text { SAA } \\
\text { PND } 30 \mathrm{v} \\
60\end{array}$ & $\begin{array}{c}\text { Females } \\
\text { Sociability score } \\
\text { Phase } 1\end{array}$ & $\begin{array}{c}\text { Control SERT } \\
30\end{array}$ & $>$ & $\begin{array}{c}\text { Control SERT }{ }^{+/-} \\
60\end{array}$ \\
\hline
\end{tabular}

Note: '<' refers to less than; e.g. The number of USVs at PND 7 was less than the number of USVs at PND 14. 


\subsection{Discussion}

Before discussing the specific results of the experiment, it is relevant to briefly summarise the main findings in this chapter. This chapter intended to assess the social behaviours of rats with genetic deficits in the SERT following chronic prenatal exposure to VPA. It was hypothesised that an interaction would occur between the SERT reduction and the chronic VPA exposure which would result in a pronounced ASD-like phenotype in the offspring. Overall, we were unable to confirm this hypothesis, although chronic VPA administration did induce several ASD-like behavioural changes. The offspring prenatally exposed to VPA exhibited a significant increase in USV length and reductions in the frequency range, as well as increased chasing behaviour. Furthermore, prenatal exposure to VPA disrupted the social preference formation and approach to social stimuli. However, the anxiety-like behaviour of these rats was not seen.

It is also important to mention the large degree of variance seen in most experiments. Care was taken to reduce the effects of variance on the statistical tests (e.g. removing outliers, investigating transformations, combining dependent measures like the sociability score). It is likely that the variance is in part attributed to the small sample size, particularly with separation of sex, then prenatal dose and genotype. It is also possible that the variance is contributed to the amount of exposure to VPA in utero. Within a litter, the pups may have been differentially exposed to VPA concentrations, depending on their uterine position (Lipton, Robie, Ling, Weese-Mayer, \& Carvey, 1998; Semmler et al., 2017), which may have induced different behavioural effects.

Perhaps more importantly, as shown in table 3.1, while we aimed for 300 and $600 \mathrm{mg} / \mathrm{kg} /$ day, the actual amount consumed by the mother was typically less and varied quite a bit between animals. While the gelatine administration procedure is clearly superior to injections from a stress exposure point of view, it is less accurate in the dose administration. Importantly, the administration method and doses resulted in no fatalities to the dams (as identified in previous literature; Vorhees, 1987). However, there were two stillborn litters from dams that were given $600 \mathrm{mg} / \mathrm{kg} /$ day (consumed on average $355-410 \mathrm{mg} / \mathrm{kg} /$ day). Another dam that was given $600 \mathrm{mg} / \mathrm{kg} /$ day (consumed $394 \mathrm{mg} / \mathrm{kg} /$ day) birthed 15 offspring and only one survived. Thus, the high dose was clearly toxic for the offspring, a finding that aligns with previous literature (Nau et al., 1981; Vorhees, 1987). Furthermore, the stillborn litters and reduced pup survival raises concerns about the application of a dose within the toxicity range (400-800mg/kg as specified by Nau, et al., 1981). 
On the other hand, other dams (identifiers 011, 014, and 018; see table 3.1) consumed similar amounts of VPA, and their litters survived normally into adulthood. Interestingly, we did not observe any other neurodevelopmental complications often described after VPA (kinked tail etc). Thus, it seems that in these animals, the dose either led to premature death or no gross abnormalities.

In addition to the aversive impact of the chronic administration method, the gelatine pellets meant that litters and offspring could be grouped into low and high dose groups. This was based on the average amount of VPA consumed throughout gestation. In comparison with the methods used in chapter two and the use of drinking water (Frisch et al., 2009), the gelatine pellets afforded a more accurate measurement of the total intake. Unfortunately, there were only relatively small differences between the two doses, as the highest amount consumed in the low dose group was $299 \mathrm{mg} / \mathrm{kg} /$ day and the lowest amount consumed in the high dose group was $351 \mathrm{mg} / \mathrm{kg} /$ day. The narrow difference between low and high dose conditions is related to the inconsistency of dams consuming pellets containing VPA, which may have been due to the unpleasant taste of the pellets, or other factors like psychodynamic drug effects, such as sedation (as seen in dams following a 600mg/kg VPA dose; Ong et al., 1983). The narrow dose division may further explain the unclear dose-related behaviours in the phenotype of the offspring. Further investigation is needed to enhance and refine the gelatine pellet recipe and administration. In particular, future research should look to examine taste aversions in pregnant rats to determine levels of tolerance and optimal dose.

\subsubsection{USVs}

The total number of vocalisations is often used as an indicator of general communication, and prenatal exposure to VPA in rodents has consistently resulted in fewer USVs (Bronzuoli et al., 2018; Melancia et al., 2018; Servadio et al., 2018). It was hypothesised that rats prenatally exposed to VPA would exhibit this reduction, which was not supported (figure 3.2). The number of USVs emitted by pre-weaning pups exposed to VPA was not statistically different from the number of USVs emitted by pups exposed to the control dose. Interestingly, there was a significant increase in the number of USVs from PND 7 to 14 for the pups exposed to the low dose of VPA, which was not found in the control animals, nor in the high dose group (although the same tendency was found). Inspection of figure 3.2 showed that both the low and high dose rats vocalised somewhat less on PND 7 than controls (although this failed to reached significance). 
The USVs emitted during maternal isolation are 'distress calls' to elicit retrieval behaviour from the mother (Hofer, 1996; Noirot, 1972; Smotherman, Bell, Starzec, Elias, \& Zachman, 1974). The number of isolation calls peak around PND 7 and reduce to 'adult-like' calling around PND 14 (PND 12-18), as pups are less dependent on their mother for survival and temperature control (as seen in chapter two; and Branchi et al., 2001; Kromkhun et al., 2013). However, the decline in the number of USVs was not found in this chapter, as there was a significant increase of USVs on PND 14 for controls and VPA exposed pups, particularly for the pups exposed to the low dose. The sustained increased in number of USVs at PND 14 may be indicative of a delay in maturation, similar to what was discussed in the previous chapter. The increased number of USVs, is typical for an earlier developmental stage (PND 7 to 10) where rats would be more dependent on the dam and nest (Hofer, Masmela, Brunelli, \& Shair, 1998).

In addition to the common measure of the number of USVs, qualitative measures were also investigated, and the pre-weaning pups showed substantial changes in their communication. The length of USVs increased with age, and the pups prenatally exposed to the high dose showed a significant increase, although it was only in comparison to the length of USVs emitted by the low dose pups, rather than the controls (figure 3.3). Also, the high frequency and range of frequency measures showed an effect of PND. The highest pitch of vocalisations increased from PND 7 to 14, for the control and low dose pups, but not for the pitch of USVs emitted pups exposed to the high dose, which stayed relatively low. This is very similar to what was observed in chapter two. The range frequencies in the USVs showed an increase from PND 7 to 14, but the range was significantly smaller for the USVs emitted pups exposed to the high dose compared to the low dose (but not the controls). Overall, the preweaning VPA exposed offspring emitted abnormal vocalisations. However, as the current literature tends to focus only the total number of vocalisations, it is difficult to assess the extent of the abnormality. As explored in the discussion of chapter two, there are advances in software that would allow for automated classifications of USVs such as MUPET or DeepSqueak, which will hopefully lead to investigations that look beyond the total number of USVs

\subsubsection{Play Behaviour}

Juvenile play behaviour is critical for normal development (Kiser, Steemers, Branchi, \& Homberg, 2012), and alterations in such behaviour are indicative of abnormal neurodevelopment. Rats chronically exposed to VPA throughout gestation exhibited abnormal play behaviour, particularly in chasing behaviour. Chasing is a behaviour that initiates a play bout (Vanderschuren, Spruijt, Hol, Niesink, \& Van Ree, 1995), and the offspring exposed to VPA 
exhibited increases in both duration and frequency of this behaviour (figure 3.6). The prenatal dose effect on the increased chasing behaviour was clearly seen in the male offspring exposed to low and high doses of VPA, whereas the only the low dose females showed an increase in chasing. This finding is indicative of the differential effects of chronic exposure to VPA in male and female offspring.

From chasing a play bout typically moves to a pinning behaviour (Vanderschuren et al., 1995). A null effect in pinning behaviour was surprising as a decrease in pinning was expected for males and females. Particularly, as previous studies that used a single VPA exposure found decreased pinning behaviours of the exposed rats (Chomiak, Karnik, Block, \& $\mathrm{Hu}, 2010$; Schneider \& Przewlocki, 2005; Servadio et al., 2018), although some have found no change or an increase in pinning behaviours as well (Raza et al., 2015). Interestingly, the VPA exposed offspring did not show an increase in pinning behaviour, suggesting a disturbance in the normal pattern of social play behaviour. One could even suggest that the increase in chasing (initiation behaviour) is a consequence of a reduced ability to complete a normal cycle of play behaviour. From the play behaviour experiment with juveniles that were chronically exposed to VPA in utero, it is likely that VPA has affected the overall structure of play behaviour. This clearly needs further investigation with a special focus on the structure and sequence of behaviour. Further research should utilise software like Theme (patternvision.com) that measures the order and latency between behavioural events, such as the progression of pouncing to pinning and other behaviours that follow in the play fighting sequence (Casarrubea et al., 2015; Pellis \& Pellis, 2007).

The non-active social interaction behaviour (passive interaction) showed no significant effect for males and females. The passive behaviours of females were consistent across control and VPA dose conditions. However, there appeared to be an effect of VPA exposure for males. The male offspring exposed to the high dose of VPA showed more passive behaviours than males exposed to the low dose, although this was non-significant (see figure 3.8).

Similar play behaviour experiments have shown no effects in the social behaviour of juvenile rats that were exposed to a single dose of VPA (Narita et al., 2010; Schneider \& Przewlocki, 2005), but have also shown increased passive behaviours (Olde Loohuis et al., 2015). The discrepancy in findings between the play behaviour experiment in this chapter and Narita et al. (2010) and Schneider \& Przewlocki (2005) is perhaps related to the chronic prenatal exposure to VPA compared with the one-off exposure used in previous studies. Alternatively, it may be related to the variability in experimental coding. Nevertheless, it would be worthy of 
examining the play bout sequence of the VPA exposed offspring, as the passive interaction is perhaps replacing more active behaviours, as the frequency of passive interaction is nearly double the frequency of chasing and pinning.

It is worthy of mentioning, the impact of the SERT ${ }^{+/+}$and $\mathrm{SERT}^{+/-}$genotypes could not be determined in this experiment (as outlined in section 3.3 and 3.3.3, see appendix A.a), and thus the influence of the genotype differences are an unknown factor in these results. While attempts were made to create a sample size large enough to allow for comparisons, unfortunately, as pairing occurred prior to receiving the genotype information, the sample sizes were not large enough. It is possible that the prenatal exposure to VPA for SERT ${ }^{+/-}$may have lead to opposing effects in comparison to control SERT ${ }^{+/+}$(based on examination of figures Al.1, $\mathrm{Al}, 2$, and Al.3). However, it likely that any genetic or interaction effects would be secondary to findings that indicate abnormal structure and sequence of play behaviour for these animals.

\subsubsection{SAA}

SAA allows for the investigation of social behaviour of VPA exposed offspring in a context where the conspecifics cannot physically affect the offspring's behaviour. The following section reviews the findings of the pre- and post-puberty measures recorded between PND 3035, and PND 60-65.

\section{Sociability score}

The sociability score encompassed the measures of social approach (or novelty in phase two) and social avoidance (or familiarity in phase two) into a single measure and derived from (Brodkin et al., 2004). The sociability findings at PND 30-35 and PND 60-65 demonstrate a large degree of variance (figure 3.9 and 3.12), which has likely contributed to the lack of significant differences between conditions in this measure. The sociability score was useful for detecting large effects, such as the low sociability score of the SERT ${ }^{+/-}$females exposed to the high dose, compared with the SERT ${ }^{+/+}$females (figure 3.9). The area preference for social (or novel in phase two) and non-social (or familiar in phase two) stimuli was more suitable for detecting social behaviour changes for VPA exposed rats with reductions in SERT activity.

\section{Area preference}

The area preference findings were more revealing about changes in rats behaviour following VPA exposure. It was expected that $\mathrm{SERT}^{+/+}$exposed to the control dose would exhibit a preference for the social area in phase one and the novel area in phase two. The experiments 
at PND 30-35 demonstrated non-significant trends towards the expected behaviours, whereas PND 60-65 experiments demonstrated that these rats tended towards the non-social or novel areas. The opposing effects of the control dose SERT ${ }^{+/+}$rats suggest concerns with the methods for the adult rats, such as the use of the T-maze and the distance between areas or stimuli. These concepts were raised in the discussion of chapter two, but briefly, at PND 60 rats are about three times the size of PND 30 rats, and with the larger size they can remain in proximity to an area while showing interest in the opposing area. Measuring the sniffing behaviour of the test animal with the stimuli would overcome this flaw, and it is proposed as a more sensitive measure of social interest (Moy et al., 2008).

Turning to the effects of VPA on the area preference. Based on previous literature, it was expected that males and females that were prenatally exposed to VPA would spend less time with the social stimuli, thus exhibiting social aversion (Bambini-Junior et al., 2011; DufourRainfray et al., 2010; Kim et al., 2011). There was some support for this, where females spent more time in the non-social areas, exhibited social aversion (figure 3.11). Interestingly, both sexes showed a preference for the novel stimuli (figures 3.12 and 3.13) or displayed no preference (figures 3.18 and 3.19), therefore exhibiting an opposing pattern to that described in previous literature. The findings in this experiment indicate that prenatal exposure to VPA indeed disrupted social preference formation. However, not all conditions showed this disruption, such as PND 60-65 SERT $^{+/}$females exposed to the high dose of VPA preferred the social area, whereas the $\mathrm{SERT}^{+/+}$exhibited no preference (figures 3.19 and 3.22). So, while this is some indication that VPA disrupts social preference, particularly in females, the effects seem relatively moderate. As discussed above, and in chapter two, this may, at least in part, be due to lack of a clear preference in the controls.

More importantly, a gene $\times$ environment interaction was predicted. The $\mathrm{SERT}^{+/-}$rats at PND 30-35 showed no preference after a low dose and even a preference for the non-social area at a high dose of VPA (see figure 3.11 for phase one and figure 3.14 for phase two). This was the first indication of gene $\times$ environment interaction, as $\mathrm{SERT}^{+/+}$females were significantly less impacted by prenatal VPA (though the interaction just missed significance). As discussed in the introduction, there is substantial evidence that prenatal VPA impacts on the serotonergic system, particularly on its development, and therefore a gene $\times$ environment interaction is not unexpected. Nonetheless, the effect is mild, perhaps due to the lower than anticipated dose of VPA that the dams actually ingested or the fact that only SERT ${ }^{+/-}$rats were used. Future studies using higher VPA doses and perhaps including the $\mathrm{SERT}^{-/-}$rats may lead to stronger interactive effects. 
Furthermore, sociability score in phase one and two were examined pre- and postpuberty. It was predicted that sociability decreases exhibited by $\mathrm{SERT}^{+/-}$and $\mathrm{SERT}^{+/+}$prenatally exposed to VPA would be seen both pre- and post-puberty. However, the control and low dose offspring exhibited decreases in sociability with age (figure 3.25 ), and perhaps 'normal' social decrease with age (Salchner, Lubec, \& Singewald, 2004). On the other hand, the high dose females had increased sociability (figure 3.26), which is similar to overall increases in sociability as found by Melancia et al. (2018). The increased sociability with the age for females exposed to the high dose suggests a continuation of VPA effects on social behaviour for these animals.

\section{Locomotor activity}

In addition to the sociability measures, a general locomotor activity measure was taken. While males showed no differences in the general activity, the female offspring moved more in the first phase (social vs non-social areas), compared with the second phase. Furthermore, the prenatal exposure to the low dose of VPA increased overall activity, particularly for the females (see figure 3.17). Similar effects were found in Schneider et al. (2008) were females prenatally exposed to $600 \mathrm{mg} / \mathrm{kg}$ exhibited increased locomotor activity in an open field compared with control and VPA exposed males.

The increased locomotor activity is unlikely that this was in response to a novel environment, as the rats were habituated to the room and then the empty apparatus (for a total of 20 minutes) before the first testing phase. As such, the increased activity is attributed to the inclusion of the social stimulus. Locomotor activity is rarely examined within the context SAA experiment, therefore, the impact social stimulus on locomotor activity is not clear. However, there is research to suggest that female rats are more exploratory and therefore locomote more than males (Lynn \& Brown, 2009; Valle \& Gorzalka, 1980), although this finding is not consistent (Masur, Schutz, \& Boerngen, 1980; Slob, Huizer, \& Ten Bosch, 1986).

\subsubsection{SAT}

Anxiety behaviours were examined in male and female SERT ${ }^{+/+}$and $\mathrm{SERT}^{+/}$, that were chronically exposed to control, low, or high doses of VPA throughout gestation. It was hypothesised that the prenatal exposure to VPA would lead to an anxious phenotype and SERT reductions might exacerbate the severity of the behaviours. It was therefore expected that the SERT $^{+/}$exposed to the high dose of VPA would show the longest latency to reach the more anxiogenic alleys and they would spend more time in the less anxiogenic alley (alley one). 
On the whole, the offspring chronically exposed to VPA during gestation displayed an increased anxious phenotype. The low dose males and females exhibited increased delays to enter the more anxiogenic areas of the alleys, although this seemed to be restricted to alley two (see figure 3.30 and 3.32). The SERT reduction exacerbated the delay for the SERT ${ }^{+/}$females that had been exposed to low dose, which provides support for the gene $\times$ environment interaction hypothesis (figure 3.32). These findings extend the work of Ellenbroek et al. (2016). The authors found that a single exposure of VPA during pregnancy decreased the time spent in the open arms of the elevated plus maze in the offspring (therefore exhibiting an anxious phenotype), as well as increase the latency to feed in the novelty suppressed feeding paradigm. However, in contrast to the results in this chapter, they did not find a gene $\times$ environment interaction effect. This could be due to the differences in VPA administration, or to the fact that Ellenbroek et al. only investigated males.

In addition, rats exposed to a high dose displayed increased variability, particularly with the time spent in alley one and two. However, a clear behavioural pattern did not emerge, where the latency and duration were matched with the anxiogenic status of the alleys. Therefore, the pattern of anxious behaviour is not clear, and future investigations are needed to examine this phenotype in male and females rats that are prenatally exposed to VPA. One such way would be to use repeated trials in the SAT to depict the persistent behavioural patterns, in combination with other established and validated behavioural assays. 


\subsubsection{Summary}

Together these experiments have confirmed that the chronic prenatal exposure to VPA in rats with the reduction in the SERT considerably alters their communication and social behaviour, and to some extent their anxiety-like behaviour. The behavioural impacts were enhanced with the accuracy and non-invasive method of administering VPA in gelatine pellets which afforded well-defined dose groups based on the VPA consumed. From this basis, the $\mathrm{SERT}^{+/+}$and $\mathrm{SERT}^{+/-}$males and females that were exposed to VPA consistently exhibited abnormalities that align with an ASD-like phenotype, such as changes in vocalisations, play bout sequences, social avoidance.

Overall, the SERT ${ }^{+/+}$and SERT ${ }^{+/}$males and females that were exposed to VPA exhibit a phenotype that aligns with the ASD model; however, the underlying origin of these behaviours is unclear. Previous research has demonstrated neural abnormalities in ASD patients (Hashemi et al., 2016) as well as VPA-induced ASD (Gogolla et al., 2009; Zimmerman, Patel, Smith, Pasos, \& Kulesza Jr, 2018). To further explore the gene and environment interaction in light of this ASD rat model, the next study will investigate abnormalities in the adult brain of the rats described in this chapter, and the focus will be on regions associated with social behaviour (amygdala and hippocampus). 


\section{Chapter Four}

PV Neurons of SERT Deficient Rats

that were Prenatally Exposed to

VPA Throughout Gestation 


\section{Chapter Four Contents}

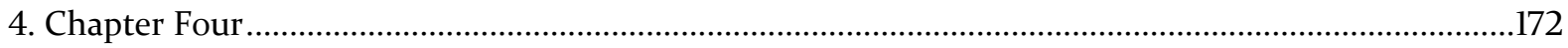

4.1.1. Neuronal changes found in ASD populations .............................................172

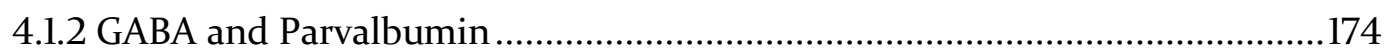

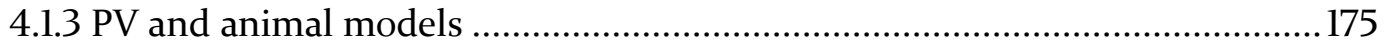

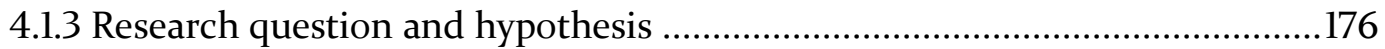

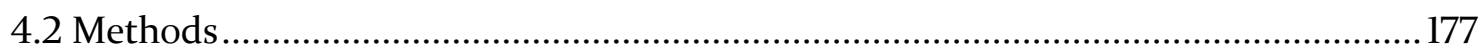

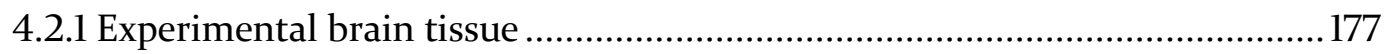

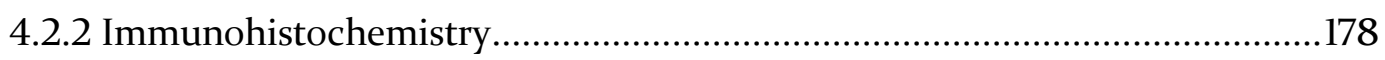

4.2.3 Cell Counts and Analysis of Immunohistochemical Data .............................179

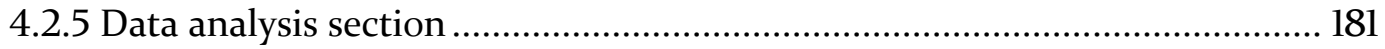

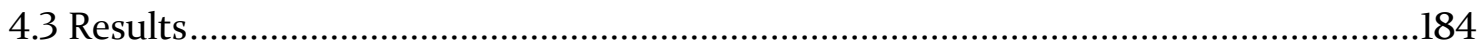

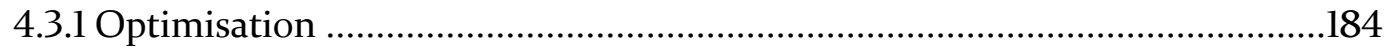

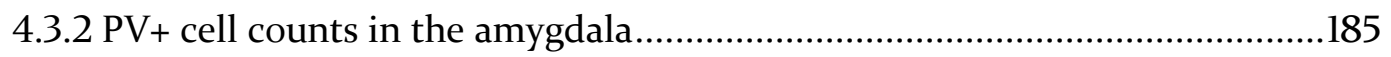

4.3.4 PV+ cell counts in the ventral hippocampus ............................................192

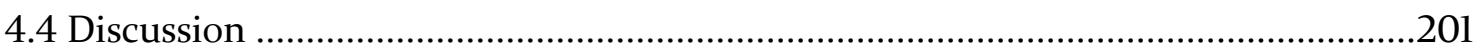

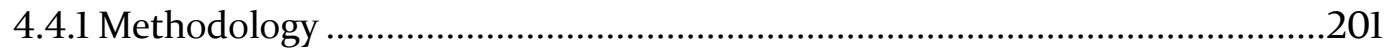

4.4.2 Amygdala and ventral hippocampus findings ...........................................202

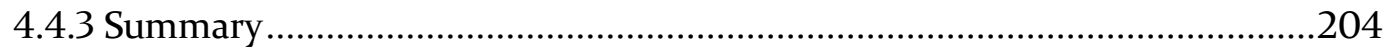




\section{Chapter Four}

As discussed in chapter one and the beginning of chapter three, interactions between genetic and environmental factors (such as prenatal challenges) have a role in initiating neuronal changes found in ASD populations. To explore the validity of chronic prenatal administration of VPA in rats with SERT deficits, it is worthy to further investigate the neuronal changes in these animals. Chapter three examined the social behaviour of male and female $\mathrm{SERT}^{+/+}$and SERT ${ }^{+/-}$rats that were prenatally exposed to VPA, and the findings were indicative of ASD-like behaviours (altered communication, aberrant play behaviour, and social avoidance). To complement chapter three, this chapter will examine the neuronal changes of the rats that were bred and tested in chapter three. Chapter four is focused on alterations in the gamma-aminobutyric acid (GABA) interneurons as a method for examining the impact of gene and environment interactions on the development of the central nervous system. The following introduction highlights research relevant to the regions of interest and GABA interneurons findings in ASD populations and animal models.

\subsubsection{Neuronal Changes Found in ASD Populations}

There are multiple neuronal changes found in ASD populations, indicating that the symptoms experienced by ASD individuals are the result of neuronal changes. A subset of individuals with ASD have an increased head size (Aylward et al., 2002). There is evidence suggesting that this increased size is the result of changes to the CNS, such as increased myelination or decreased synaptic pruning (Courchesne et al., 2004; Herbert, 2005). Additionally, some ASD individuals show structural changes in specific brain regions. Schumann et al. (2004) found that ASD individuals showed an enlarged hippocampus and amygdala. These findings are indicative of underlying structural and neuronal differences in ASD populations.

Further support for neuronal abnormalities underlying ASD symptoms is drawn from co-occurring disorders, such as intellectual disability and epilepsy. There is approximately $70 \%$ of ASD individuals experiencing intellectual disability (Fombonne, 1999; Kelleher \& Bear, 2008). Also, there is a high co-occurrence of ASD with epileptic syndromes (Amiet et al., 2008; Danielsson, Gillberg, Billstedt, Gillberg, \& Olsson, 2005; Jeste \& Tuchman, 2015). The cooccurrence of intellectual disability and epilepsy suggests there are significant changes that affect normal functioning. Furthermore, the co-occurrence with epilepsy is strongly indicative of excitation and inhibition imbalances for ASD individuals. 
The amygdala and the hippocampus are brain regions associated with emotional and social processes, and deficits in social processing is a core symptom of ASD. Therefore, these regions are of particular interest in this thesis and chapter. Studies which have examined ASD populations have reported an increased volume of the hippocampus (Bauman \& Kemper, 1985; Saitoh, Karns, \& Courchesne, 2001; Sparks et al., 2002), and reduced complexity and dendritic arborizations in specific regions (CAl and CA4) of the hippocampus (Raymond et al., 1995). However, other studies have found no differences in the hippocampus of ASD individuals compared to neurotypicals (Piven, Bailey, Ranson, \& Arndt, 1998; Saitoh, Courchesne, Egaas, Lincoln, \& Schreibman, 1995). As Saitoh et al. (2001) mentioned, the variance in findings regarding brain regions can be attributed to individual variability, and uncertain diagnosis, particularly when the evidence is based on post-mortem data.

Additionally, studies have also reported overgrowth in the amygdala of ASD individuals (Aylward et al., 1999; Schumann et al., 2004; Won, Mah, \& Kim, 2013). This overgrowth appears to be associated with an abnormal developmental pattern, as children with ASD display increased amygdala volumes (Schumann, Barnes, Lord, \& Courchesne, 2009), and ASD children followed up after a year, exhibit greater amygdala growth than neurotypicals (Nordahl et al., 2012). However, adult brains do not show the same overgrowth. Schumann and Amaral (2006) examined the amygdala in the post-mortem adult brain tissue and found no difference in volume or cell size, but there were fewer neurons in the amygdala, specifically in the lateral nucleus. Currently, it is unclear what neuroanatomical changes are specific to ASD symptomology, but the neuronal changes in the amygdala and hippocampus provide neuronal correlates for aberrant social behaviour.

Cellular changes are also found in ASD brains. The abnormal neural activity in ASD brains may be attributed to (at least in part) abnormalities at a cellular level. Post-mortem ASD brain tissue is found to have low numbers of Purkinje (GABAergic neurons involved in inhibitory processes) and granule cells (small neurons involved in excitation) in the cerebellum (Bailey et al., 1998; Casanova, Buxhoeveden, Switala, \& Roy, 2002; Casanova et al., 2002; Ritvo et al., 1986). Other cellular abnormalities are found in the cortical minicolumns, which are vertical layers of neurons (Buxhoeveden et al., 2006; Casanova et al., 2002). The number and size of columns are decreased in ASD brains (Casanova et al., 2002), although the reduction may be region-specific. Buxhoeveden et al. (2006) found smaller columns in the frontal cortex but not in the primary visual cortex. Casanova et al. (2002) showed cell dispersion within the columns, and Bailey et al. (1998) confirmed the cell dispersion and showed abnormal neuronal 
migration. These findings indicate that the neurons are not in their expected locations, and this impacts neuronal connectivity.

\subsubsection{GABA and Parvalbumin}

GABA is the primary inhibitory neurotransmitter in adult brains. GABA is synthesised and released from inhibitory projection and interneurons (Coghlan et al., 2012; Lübkemann et al., 2015). Inhibitory interneurons are functionally and anatomically diverse (García, Karayannis, \& Fishell, 2011), and can be classified by cytoarchitecture properties (basket, chandelier, stellate), or molecular properties (parvalbumin-, cholecystokinin-, calbindin-, calretinin-, vasoactive intestinal polypeptide-, somatostatin- expressing). Further classifications can be made based on the electrophysiological or functional state of the cells (Coghlan et al., 2012; Dehorter, Marichal, Marín, \& Berninger, 2017). There is emerging evidence suggesting that Parvalbumin-expressing $(\mathrm{PV}+)$ interneurons may be critically related to the dysfunctional GABAergic systems implicated in the aetiology of ASD (Coghlan et al., 2012; Hashemi et al., 2016).

PV+ cells are fast-spiking basket cells that form synapses with the soma (cell body) of excitatory pyramidal cells (Dehorter et al., 2017). The synapse with pyramidal cells allows the $\mathrm{PV}+$ cells to regulate the duration and speed of excitatory spikes in the connected cell (Taniguchi, 2014). PV+ cells also form synapses with other interneurons, which contributes to neuronal synchronisation within the region (Tamás, Lörincz, Simon, \& Szabadics, 2003; Taniguchi, 2014). PV+ neurons are mainly found in the hippocampus, thalamus, cortex, and cerebellum (Celio, 1990; Vreugdenhil, Jefferys, Celio, \& Schwaller, 2003). PV+ neurons are also found in the amygdala, where it has a role in regulating the excitatory action of the $5-\mathrm{HT}_{2 \mathrm{~A}}$ receptor (McDonald, 1982; McDonald \& Mascagni, 2007), and as established earlier, the amygdala has a critical role in regulating social and emotional behaviour (Hale et al., 2010). To investigate the link between ASD and PV, Hashemi et al. (2016) examined the interneuron populations in the prefrontal cortex (PFC) of post-mortem tissue from ASD and age-matched controls. They showed that the PV+ interneurons were significantly reduced in the PFC, but other (calbindin-and calretinin-expressing) interneurons did not show the same reductions in the same regions. This selective decrease in PV+ cells and signalling could lead to changes in the GABAergic inhibition and therefore shifts in the excitation : inhibition (E:I) balance in the neuronal network (Hashemi et al., 2016; Inui, Kumagaya, \& Myowa-Yamakoshi, 2017; Selten et al., 2018). 


\subsubsection{PV \& Animal Models of ASD}

There is emerging evidence to suggest that similar reductions of PV occur in the brains of rodents prenatally exposed to VPA. Gogolla et al. (2009) showed that mice prenatally exposed to VPA had significantly reduced PV+ cells in the neocortex, but not in the hippocampus. PV was decreased in the striatum, but not in the medial PFC and somatosensory cortex of mice prenatally exposed to VPA (Lauber, Filice, \& Schwaller, 2016). Dendrinos et al. (2011) examined the superior colliculus (a brainstem region involved in visual, auditory and somatosensory integrations and processing) and found a 30\% decrease in PV+ cells in the superficial layer of the superior colliculus of rats prenatally exposed to VPA. Additionally, Ingram, Peckham, Tisdale, and Rodier (2000) found that VPA exposed rats had reductions in Purkinje cells of the cerebellum. A subpopulation of Purkinje cells express PV, making these cells an indirect measure of PV expression (Fortin, Marchand, \& Parent, 1998; Soghomonian, Zhang, Reprakash, \& Blatt, 2017).

The findings from prenatally exposed VPA rodents, in combination with the above mentioned alterations in the GABA/PV signalling in ASD brains, shows that prenatal exposure to VPA results in similar molecular changes. The following experiment examines PV+ cells in the amygdala and hippocampus of SERT ${ }^{+/+}$and SERT ${ }^{+/-}$adult rats that were prenatally exposed VPA. While there is evidence to indicate that VPA exposure has an impact on PV+ cells of rodents, there is a lack of evidence relating to the SERT genotype and PV+ expression. To our knowledge, there are no published studies on PV levels in the SERT KO rats. As such, this study will examine PV in animals prenatally exposed to VPA with SERT reduction. This study intends to validate the behavioural findings of chapter three and begin to elucidate the molecular mechanisms of ASD-like deficits in this ASD animal model. 


\subsubsection{Overall Aims of Chapter Four}

The experiment in this chapter examines the neuronal deficits of rats with a genetic reduction in the SERT that were chronically exposed to VPA throughout gestation. This chapter investigates the neuronal effects that may underlie the behavioural aberrations seen in chapter three, with a focus on the brain regions that are associated with social behaviour (the amygdala and the hippocampus). It is hypothesised that rats with a genetic reduction in SERT that were prenatally exposed to a high dose would show reductions in PV+ cells. Thus, it is predicted that:

i. Rats prenatally exposed to VPA will have decreased PV+ GABAergic interneurons in the amygdala and hippocampus when compared with rats that were prenatally exposed to the control dose,

ii. The effects will differ as a function of prenatal dose, such that the rats prenatally exposed to the high dose of VPA will show a reduction in cells beyond what is seen in the rats exposed to the low dose,

iii. There will be an interaction between SERT reduction and chronic exposure to VPA. As such, SERT ${ }^{+/-}$prenatally exposed to VPA will exhibit a larger decrease in $\mathrm{PV}+$ expressing cells in the amygdala and hippocampus, compared with SERT ${ }^{+/+}$ exposed to VPA. 


\subsection{Parvalbumin Staining Methods}

\subsubsection{Experimental brain tissue}

\section{Experimental animals}

Fifty-nine offspring were selected based on their completion of the behavioural tasks in chapter three (see section 3.1.3 for full details on breeding and VPA administration). The tissue from three brains was excluded from analysis due to significant tissue damage, thus resulting in brain tissue from 56 offspring. Table 4.1 shows the number of brains per condition used in the data analysis.

Table 4.1. The number of brain samples from experimental animals, divided by prenatal dose, sex and genotype. The grey cells refer to the combined (either of sex or genotype) totals.

\begin{tabular}{c|c|c|c|c|}
\hline \hline \multirow{4}{*}{ Control } & & SERT $^{+/+}$ & SERT $^{+/-}$ & Combined \\
\hline & Males & 6 & 5 & 11 \\
\cline { 2 - 5 } & Females & 5 & 3 & 8 \\
\cline { 2 - 5 } & Combined & 11 & 8 & 19 \\
\hline \multirow{4}{*}{ Low } & Males & 5 & 5 & 10 \\
& Females & 4 & 7 & 11 \\
\cline { 2 - 5 } & Combined & 9 & 12 & 21 \\
\hline \hline & Males & 5 & 4 & 9 \\
& Females & 5 & 2 & 7 \\
\hline \hline
\end{tabular}

\section{Brain extraction}

Rats were deeply anaesthetised with an intraperitoneal injection (IP) of sodium pentobarbital $(50 \mathrm{mg} / \mathrm{kg})$. The rats were trans-cardially perfused with $200 \mathrm{ml}$ of $0.9 \%$ saline solution with $0.1 \%$ heparin, followed by $250 \mathrm{ml}$ of $4 \%$ paraformaldehyde solution (PFA) in $0.1 \mathrm{M}$ phosphate buffer (PB, pH 7.4). All perfusions were completed with a perfusion pump (EYLA microtube pump MP-3, Tokyo Rikakikai Co., LTD Tokyo, Japan) at a speed of $24 \mathrm{~mL} / \mathrm{min}$. Brains were extracted and immersed in $4 \%$ PFA overnight at $4{ }^{\circ} \mathrm{C}$. The following day the brains were placed in a solution of $30 \%$ sucrose in $0.1 \mathrm{M} \mathrm{PB}$ ( $\mathrm{pH} 7.4$ ) for $4-6$ days at $4^{\circ} \mathrm{C}$ until they sunk. The brains were then snap frozen with isopentane and dry ice and stored at $-80^{\circ} \mathrm{C}$ until slicing.

\section{Brain tissue slicing}

Prior to slicing, the cerebellum was sliced coronally to provide a flat surface, and the tissue was mounted on a cryostat stand with embedding medium (Tissue-Tek ${ }^{\circledR}$ O.C.T. Compound, Sakura Finetek Japan, Tokyo, Japan). Coronal sections were cut at 30 $\mu \mathrm{m}$ using a Leica CM 3050 S Research Cryostat (Leica Biosystems, Wetzlar, Germany). The slices were 
collected in $10 \mathrm{mM}$ phosphate-buffered saline (PBS) with $0.1 \%$ sodium azide in a series of six slices and stored at $4^{\circ} \mathrm{C}$ until use.

\subsubsection{Immunohistochemistry for Parvalbumin Staining}

The tissue used in the following protocol was one of the six-series of free-floating sections that was sliced from each brain. The following protocol was carried out on brain tissue in a pseudo-random order to ensure consistent staining across all treatment conditions. Additionally, there was randomly selected tissue from non-experimental brains, which was used as the negative control tissue, and the negative controls were used for each staining session.

All washes and incubations were performed in 6-well plates with mesh well inserts, and at room temperature $\left(20-22^{\circ} \mathrm{C}\right)$ on a gently rocking platform to produce mild agitation.

The tissue was washed three times for five minutes each in PBS with Triton X-100 (PBST). The tissue was incubated in 3\% hydrogen peroxide $\left(\mathrm{H}_{2} \mathrm{O}_{2}\right)$ for 10 minutes to prevent endogenous peroxidase activity, then the tissue was washed three times for five minutes in PBST. The tissue was then blocked with 1\% bovine serum albumin (BSA) in PBST for 60 minutes to reduce non-specific background staining and then washed three times for five minutes in PBST. Next, the tissue was incubated with the mouse monoclonal anti-parvalbumin antibody (Sigma-Aldrich, Auckland, New Zealand; \#P3088) antibody (1: 80,000) in 1\% BSA in PBST for 90 minutes, and the tissue was washed three times for five minutes in PBST. Then, the tissue was incubated for 90 minutes with biotinylated goat anti-mouse (Vector laboratories, Inc., Burlingame, CA, USA; \#BA-9200) antibody (1: 5000) in PBST and washed three times for five minutes in PBST. Following the secondary antibody, the tissue was incubated for 60 minutes with avidin-biotin-peroxidase complex (ABC; Vector Laboratories, Inc., Burlingame, CA, USA; 1: 1000) in PBST to increase the signal. The tissue was washed three times for five minutes in PBST. The final step was to incubate the tissue in 3,3'-diaminobenzidine (DAB; $4 \mathrm{mg} / \mathrm{mL}$ ) with nickel(II) chloride $\left(\mathrm{NiCl}_{2} ; 8 \mathrm{mg} / \mathrm{mL}\right)$ and $0.3 \% \mathrm{H}_{2} \mathrm{O}_{2}$ in $50 \mathrm{mM}$ Tris $\mathrm{HCl} \mathrm{pH} 7.4$, for 10 minutes. The resulting reaction allowed visualisation of PV+ cells. Finally, the tissue was washed three times for five minutes in PBST.

The slices were stored for no more than three days in PBST at $4^{\circ} \mathrm{C}$ until the slices were mounted on gelatinised slides. After allowing the mounted slices to dry for 6 to 12 hours, the tissue was dehydrated in successive baths of Ethanol (70\%, 95\% x 2, 100\% x 2), and then cleared in Neo-clear ${ }^{\circledast}$ (15 minutes x 2; Sigma-Aldrich, Auckland, New Zealand; \#109843), before being mounted in Neo-mount ${ }^{\circledR}$ (Sigma-Aldrich, Auckland, New Zealand; \#109016) and allowed to dry. 
Every batch included tissue that was nominated as the negative control. The negative control tissue was subjected to every treatment except the primary antibody, to ensure the visible staining was the result of the primary antibody. Furthermore, representative images of negative control tissue, in comparison to positively stained tissue are displayed in figure 4.1. The negative control tissue was checked at the end of each batch treatment to ensure there were no false-positive results.

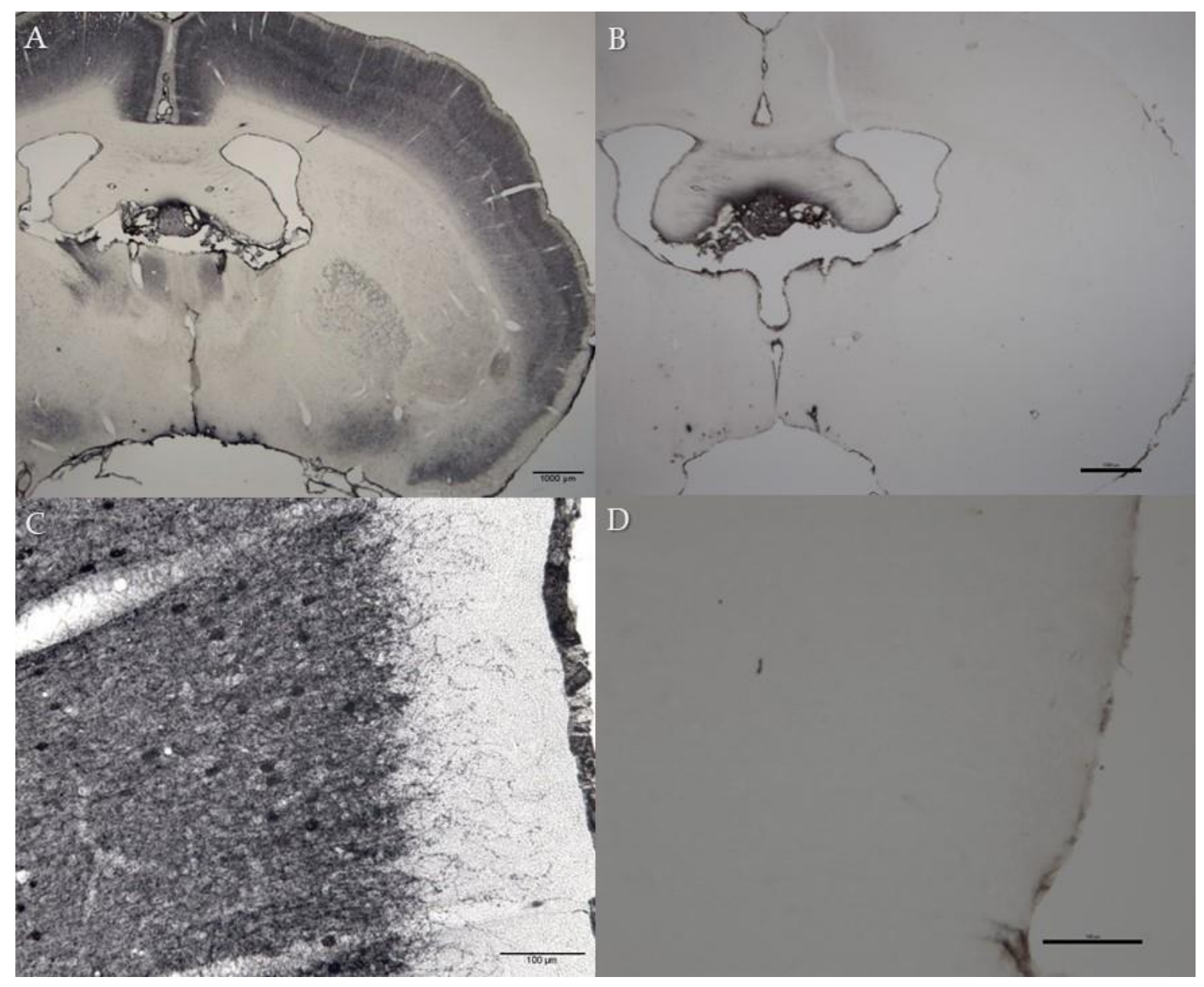

Figure 4.1. Representative light microscopy images of PV+ stain and negative control tissue at bregma -1.08, no regions of interest at this location. A. Extensive PV+ staining in the cerebral cortex and minimal staining in the anterior thalamus. B. A complete absence of staining. A-B. $1.25 \mathrm{X}$ objective with scale bar $1000 \mu \mathrm{m}$. C. Dense PV+ staining in the cerebral cortex, with visible cell bodies. D. A complete absence of staining. C-D. 20X objective with $100 \mu \mathrm{m}$ scale.

\subsubsection{Cell Counts and Analysis of Immunohistochemical Data}

The DAB-Nickel reaction resulted in a black-purple colouration of the PV+ cells, and immunostaining was observed in the neuronal fibres and cell bodies. PV-immunoreactive cells 
were manually counted using an Olympus BX51 microscope equipped with a Lumenera camera (Lt665R) connected to the image analysis software Neurolucida 2017 (MBF Bioscience, Williston, Vermont, USA).

The cell densities for each region were calculated as $\mathrm{PV}+$ cells $/ \mathrm{mm}^{2}$, and the averages for each region of interest (ROI) were then calculated for each brain. See figures 4.2 and 4.3 for a representation of ROIs.

\section{Amygdala}

The cell counting method for the amygdala was derived from Hale et al. (2010). To capture representative PV+ cell counts throughout the amygdala, three anteroposterior slices (Bregma; B -2.04 mm; -3.12 mm, -3.72 mm) were selected. The PV+ neurons were counted in both hemispheres on one slice of tissue per anteroposterior slice.

Further to this, the cell counts were separated by the ROIs, to capture any changes within the anteroposterior range as a result of prenatal conditions. On the anterior slice (B $2.04 \mathrm{~mm}$ ) two separate ROIs, the dorsolateral part of the lateral amygdala (LaDL) and the anterior basolateral amygdala (BLA), were identified (see left panel figure 4.1). The midanteroposterior slice (B -3.12 mm) had five ROIs: the LaDL and the BLA continued, and the ventromedial $(\mathrm{LaVM})$ and ventrolateral $(\mathrm{LaVL})$ parts of the lateral amygdala and posterior basolateral amygdala (BLP; see middle panel) were identified (see figure 4.1). Finally, the posterior slice (B -3.72 mm) has four ROIs which includes the LaDL, LaVM, BLP, and posterior part of the basomedial amygdala (BMP; see right panel figure 4.2).

\section{Ventral hippocampus}

The cell counting method for the ventral hippocampus was based on from Honeycutt, Keary, Kania, and Chrobak (2016) and Gogolla et al. (2009). To capture representative PV+ cell counts throughout the ventral hippocampus, three anteroposterior slices were selected (B 5.0mm, $-5.3 \mathrm{~mm}$, and $-5.8 \mathrm{~mm}$ ). The PV+ neurons were counted on both hemispheres on one slice of tissue per anteroposterior slice, in six ROIs. See figure 4.3 for a full representation of the ventral hippocampus ROIs.

The ROIs for the ventral hippocampus were consistent across all slices. Five regions focused on the hippocampus (dorsal Dentate Gyrus (DG), dorsal CAl, dorsal CA3, as well as ventral CAl and ventral CA3). 


\subsubsection{Parvalbumin Staining Data Analysis}

Data were analysed using SPSS (version 25) and using 3(Prenatal Dose: Control, Low, High) by 2(Genotype: SERT ${ }^{+/}$, SERT ${ }^{+/}$) factorial ANOVAs with Tukeys post hoc test to follow up significant main effects. Partial eta squared $\left(\eta_{\mathrm{p}}^{2}\right)$ was used for the effect size. The alpha level for statistical significance was set at $p<.05$. PV+ cell counts from each hemisphere were averaged to provide a single $\mathrm{PV}+$ cell count $\left(\right.$ in $\mathrm{mm}^{2}$ ) for each ROI.

Firstly, to identify changes in PV+ cells as a result of prenatal conditions, the cell counts from the entire amygdala or ventral hippocampus were combined. That is, the cell counts from the three anteroposterior slices and all ROIs within the slices were combined to provide a total PV+ cell count. This was analysed with a 3(Prenatal Dose: Control, Low, High) by 2(Genotype: $\mathrm{SERT}^{+/+}, \mathrm{SERT}^{+/-}$) factorial ANOVA.

Then, to investigate ROI specific changes as a result of prenatal conditions, the cell counts of each ROI in the amygdala or ventral hippocampus were combined over different the anteroposterior slices, to provide a total count within each ROI. This was analysed with a 3(Control, Low, and High) and genotype 2(SERT ${ }^{+/+}$, and SERT $\left.{ }^{+/}\right)$factorial ANOVA. 


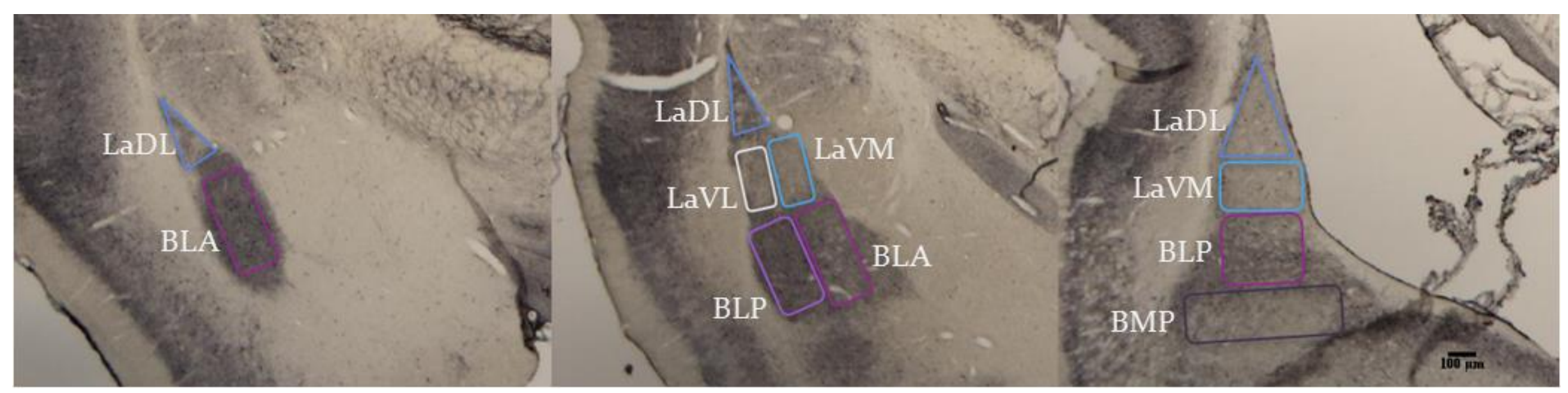

Figure 4.2. Amygdala regions of interest overlayed onto images selected at $2 \mathrm{x}$ objective from a SERT ${ }^{+/+}$control brain. The left panel is B -2.04mm with lateral amygdala (LaDL, blue) and the anterior basolateral amygdala (BLA, purple). The middle panel is B $-3.12 \mathrm{~mm}$ with contained the LaDL (blue), the ventromedial (LaVM, light blue) and ventrolateral (LaVL, white), BLA (purple) and posterior basolateral amygdala (BLP, pink). The right panel is B -3.72mm LaDL (blue), LaVM (light blue), BLP (purple) and posterior part of the basomedial amygdala (BMP, dark purple). 


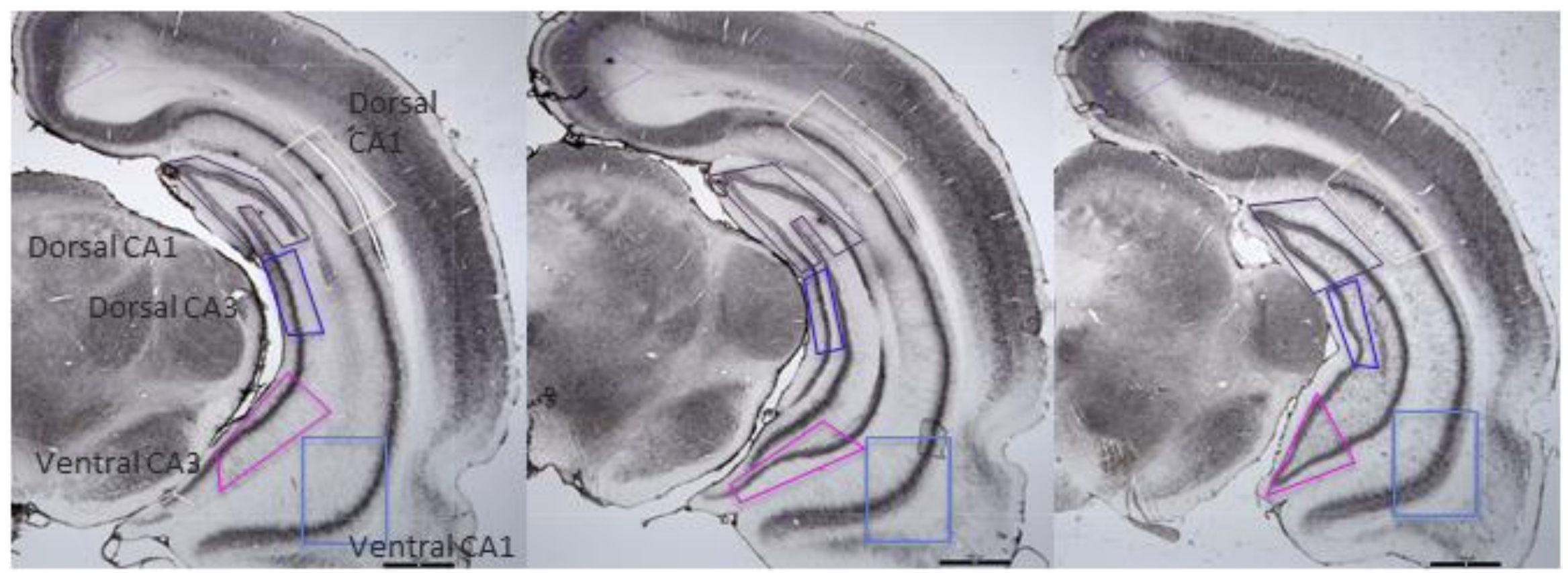

Figure 4.3. Hippocampus regions of interest overlayed onto images selected at $1.25 \mathrm{x}$ objective from a SERT ${ }^{+/+}$control brain. The left panel is $-5.00 \mathrm{~mm}$ from bregma, the central panel is $-5.30 \mathrm{~mm}$ and the right panel is $-5.80 \mathrm{~mm}$ from bregma. All three panels show dorsal dentate gyrus (dark purple), dorsal CAl (white), dorsal CA3 (blue), as well as ventral CAl (light blue) and ventral CA3 (pink). 


\subsection{Parvalbumin Staining Results}

\subsubsection{Parvalbumin Staining Optimisation}

The immunohistochemistry protocol detailed in section 4.2.2 was developed after a series of optimisation tests. The final protocol resulted in successful full cell body staining, which extended to axon branches which were aligned with the originating cell body, and minimal background staining, see figures 4.9 and 4.10 for illustrative images of amygdala staining and figures 4.16 and 4.17 for hippocampus staining. Additionally, Appendix B details the full optimisation process and the original protocol.

The original protocol which was developed in our laboratory called for overnight incubation in the primary antibody (I-Ab) at a high concentration (1: 5000). This step resulted in dark staining, increased background noise, low visualisation of the PV+ cells, and staining was visible in the negative control tissue. The I-Ab was tested at 1: 3500, 1: 10,000, 1: 20,000, 1: 80,000, and 1: 160,000. A concentration of 1: 80,000 produced the best results. Furthermore, the overnight incubation was reduced to 90 minutes, following testing at 120 and 60 minute incubation times.

While optimising the I-Ab concentration, the secondary antibody (II-Ab) was also tested, to ensure the compatibility between the I-Ab and II-Ab. The original protocol required the II-Ab concentration to be $1: 1000$, and the concentration was tested at 1: 1000, 1:2000, and 1: 5000 in combination with the I-Ab. The lowest concentration of II-Ab (1: 5000) was optimal for the I-Ab, which was a 1: 80,000 concentration. The final target to reduce staining was the $\mathrm{DAB}$ reaction, which enables the visualisation of PV+ cells. The original protocol specified a 30 minute incubation with DAB-Nickle, and following multiple tests this was reduced to 10 minutes.

Additionally, the original protocol included a secondary stain of Neutral Red to aid visualisation and region localisation. The initial tests indicated that counterstains of Neutral Red and Haematoxylin (at varying ranges of exposure time five seconds to two minutes) obscured the visualisation of PV+ cells. Thus no counterstain was used for the final protocol. 


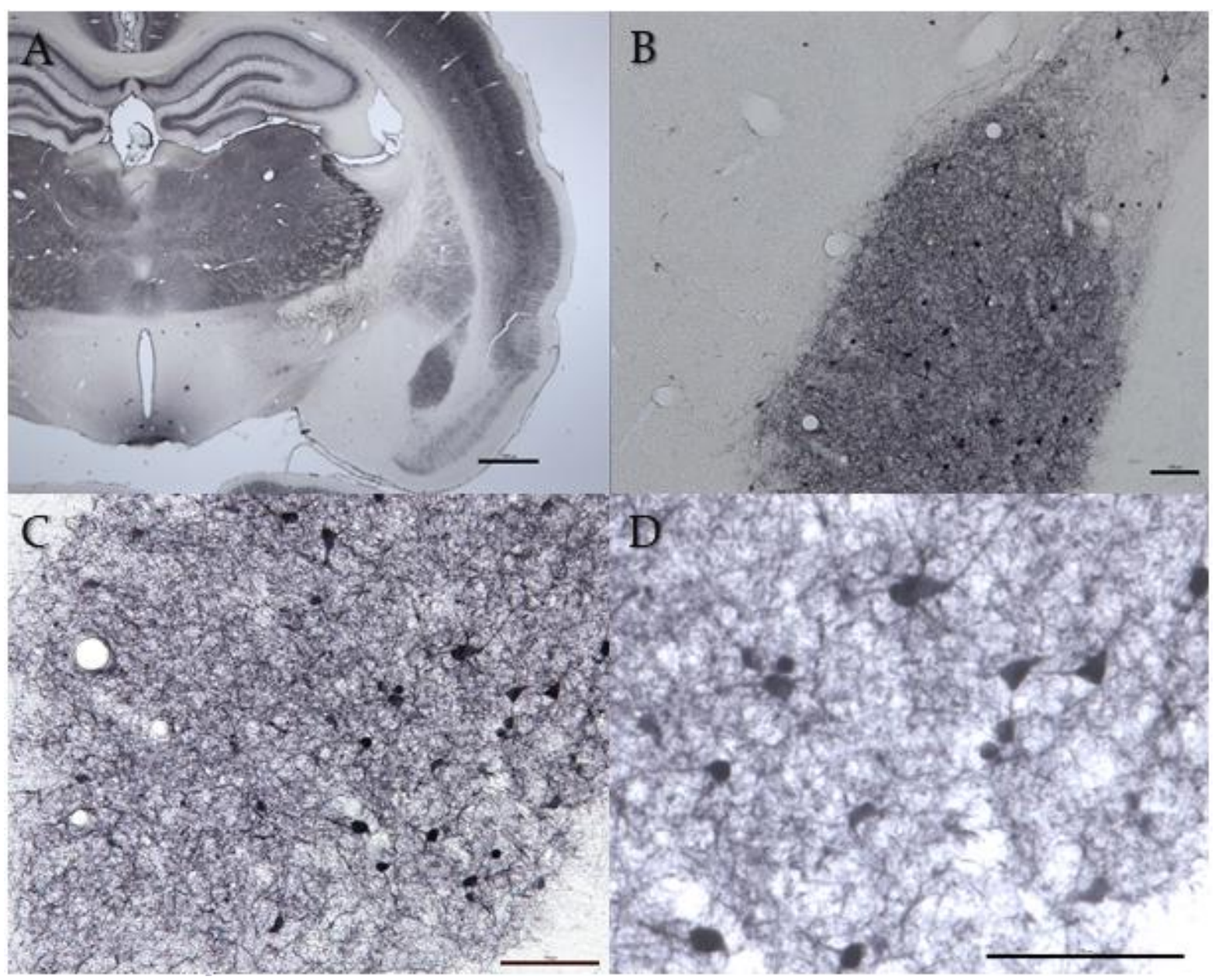

Figure 4.9. Representative light microscopy images of PV+ cells in the amygdala from a $\mathrm{SERT}^{+/+}$ male prenatally exposed to the control dose, at bregma -2.04. A. Extensive PV+ staining in the amygdala, hippocampus, thalamus, and cerebral cortex, $1.25 \mathrm{X}$ objective, with scale bar $1000 \mu \mathrm{m}$. B. Dense PV+ staining in the basolateral amygdala (BLA), compared with the lateral dorsal amygdala. No PV+ staining in the central amygdala located medial to the BLA, 10X objective with $100 \mu \mathrm{m}$ scale bar. C. Dense PV+ staining in the BLA, with clearly defined cell bodies, 10X objective with $100 \mu \mathrm{m}$ scale bar. D. PV+ cell staining with connected dendritic branches, $40 \mathrm{X}$ objective with $100 \mu \mathrm{m}$ scale bar. 


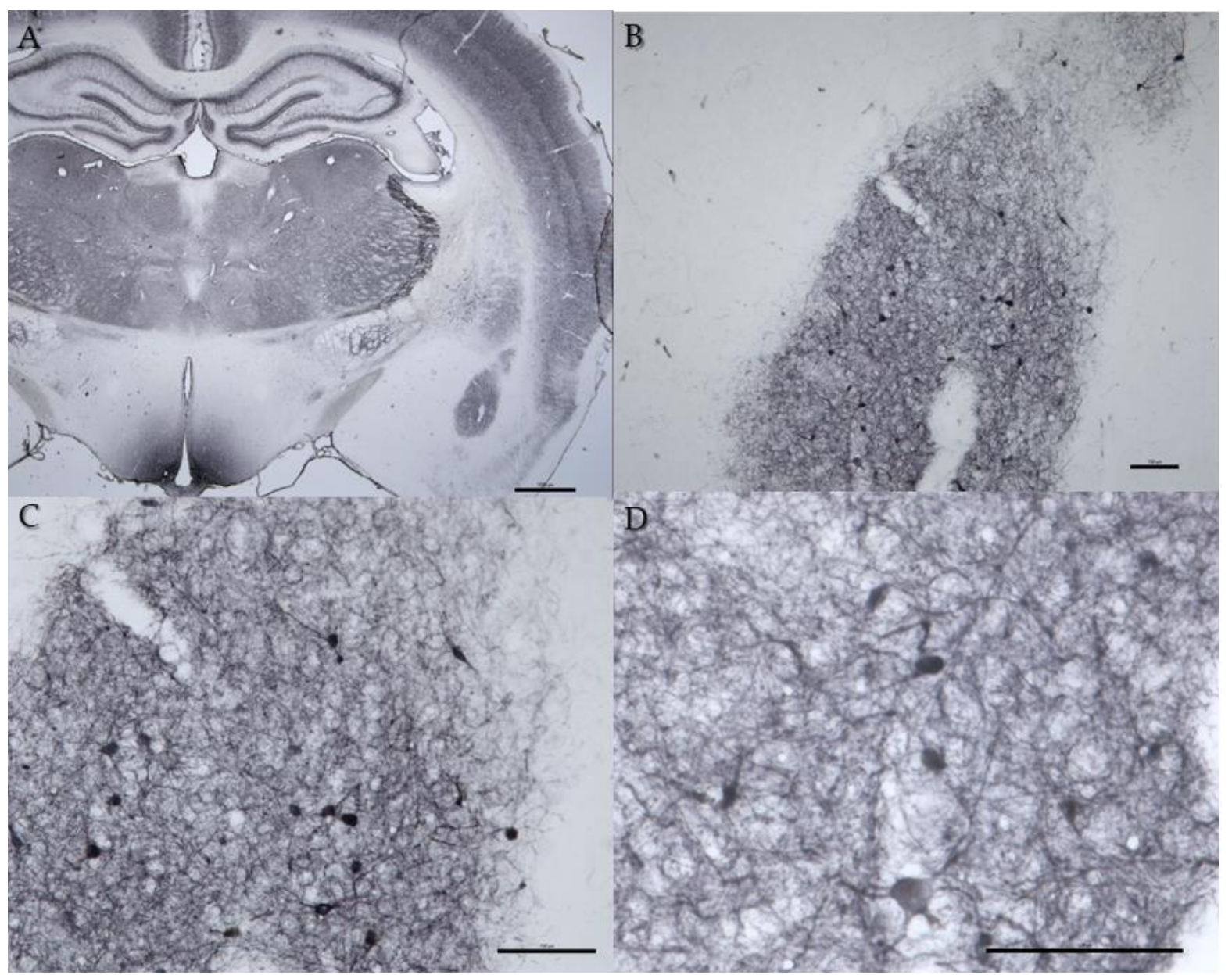

Figure 4.10. Representative light microscopy images of PV+ cells in the amygdala from a SERT ${ }^{+/-}$ male prenatally exposed to the high dose, at bregma -2.04. A. Extensive PV+ staining in the amygdala, hippocampus, thalamus, and cerebral cortex, $1.25 \mathrm{X}$ objective, with scale bar $1000 \mu \mathrm{m}$. B. Dense PV+ staining in the basolateral amygdala (BLA), compared with the lateral dorsal amygdala. No PV+ staining in the central amygdala located medial to the BLA, 10X objective with $100 \mu \mathrm{m}$ scale bar. C. Dense PV+ staining in the BLA, with clearly defined cell bodies, 10X objective with $100 \mu \mathrm{m}$ scale bar. D. Well defined PV+ cell bodies with connected dendritic branches, $40 \mathrm{X}$ objective with $100 \mu \mathrm{m}$ scale bar. 


\section{$P V+$ cell counts in the amygdala}

There was no effect of prenatal dose or genotype on PV+ cell counts in the amygdala, see figure 4.4. That is, the prenatal dose had no effect $\left(F(2,50)=1.56, p=.221, \eta_{\mathrm{p}}^{2}=.06, n s\right)$, nor did the genotype $\left(F(1,50)=.06, p=.807, \mathrm{\eta}_{\mathrm{p}}{ }^{2}=.01, n s\right)$, and there was no interaction between prenatal dose and genotype $\left(F(2,50)=1.18, p=.315, \mathrm{\eta}_{\mathrm{p}}^{2}=.05, n s\right)$.

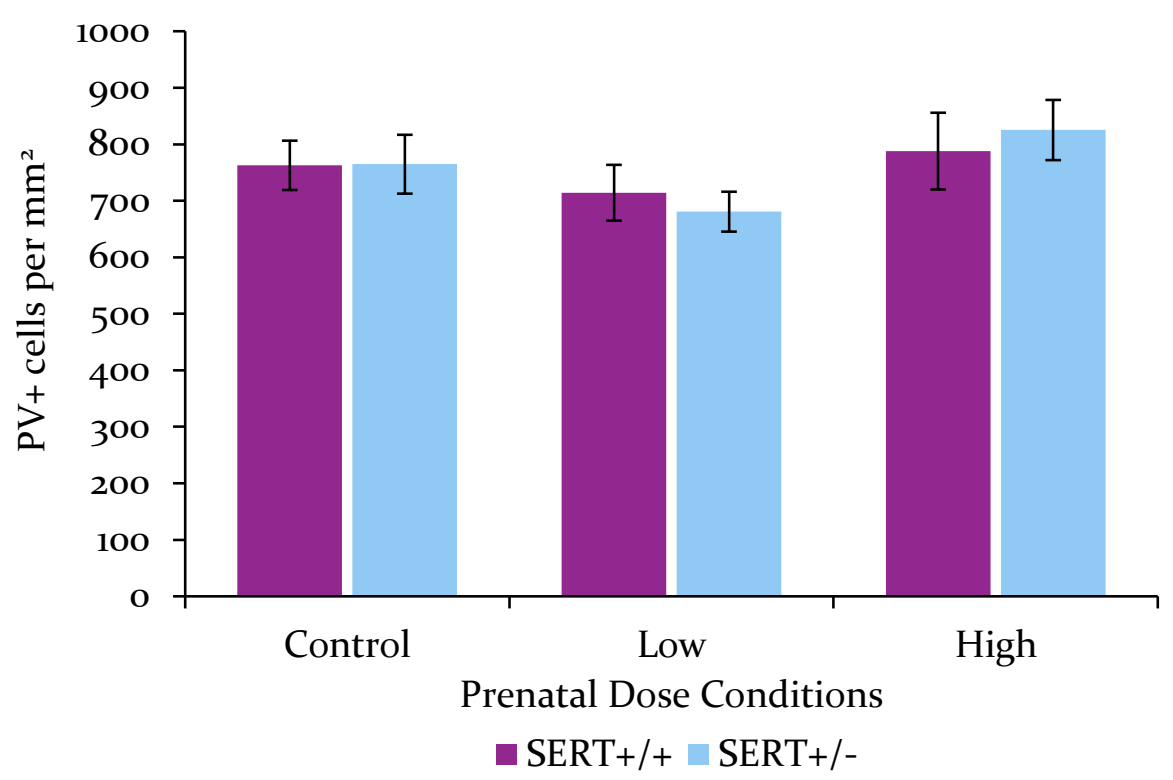

Figure 4.4. The average PV+ cell counts in the amygdala for adult $\mathrm{SERT}^{+/+}$and $\mathrm{SERT}^{+/-}$rats prenatally exposed to control, low, and high doses of VPA. No detectable differences between the conditions. Error bars represent \pm SEM. 


\section{$P V+$ cell counts in the dorsolateral amygdala}

There was a near to significant effect of prenatal dose on the PV+ cell counts in dorsolateral amygdala $\left(F(2,50)=2.61, p=.084, \mathrm{n}_{\mathrm{p}}^{2}=.09, n s\right.$, see figure 4.5). This effect was due to the reduced PV+ cell count in the dorsolateral amygdala of rats that were prenatally exposed to the low dose of VPA, compared with the control dose rats $(p=.075, n s)$. However, there was no effect of the genotype $\left(F(1,50)=.16, p=.688, \mathrm{n}_{\mathrm{p}}^{2}=.01, n s\right)$, and there was no interaction between prenatal dose and genotype $\left(F(2,50)=.28, p=.761, \eta_{\mathrm{p}}^{2}=.01, n s\right)$.

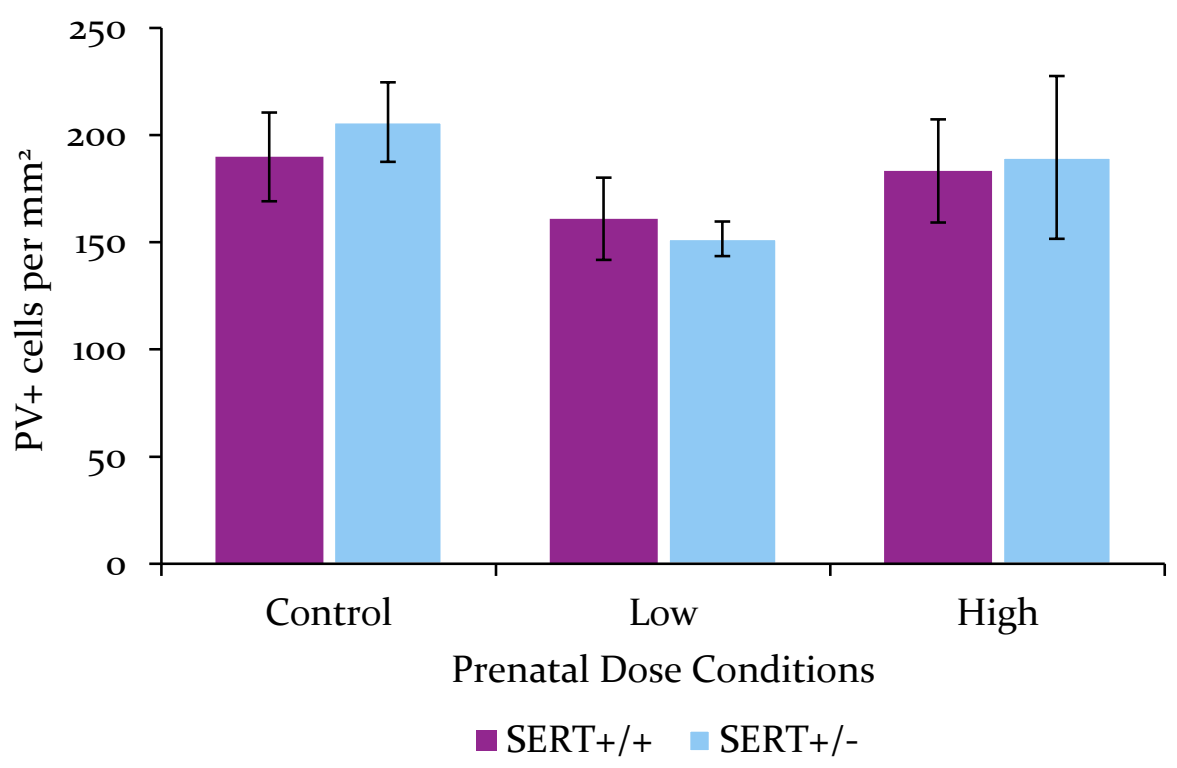

Figure 4.5. The average PV+ cell counts in the dorsolateral amygdala for adult SERT ${ }^{+/+}$and $\mathrm{SERT}^{+/-}$rats prenatally exposed to control, low, and high doses of VPA. There was a trend toward a significant decrease in PV+ cells for low dose rats. Error bars represent \pm SEM. 


\section{$P V+$ cell counts in the ventromedial amygdala}

There was no effect of prenatal dose or genotype on PV+ cell counts in ventromedial amygdala, see figure 4.6. That is, the prenatal dose had no effect $\left(F(2,50)=.14, p=.866, \eta_{\mathrm{p}}^{2}=\right.$ $.01, n s)$, nor did the genotype $\left(F(1,50)=.74, p=.395, \mathrm{\eta}_{\mathrm{p}}^{2}=.02, n s\right)$, and there was no interaction between prenatal dose and genotype $\left(F(2,50)=.72, p=.493, \mathrm{n}_{\mathrm{p}}{ }^{2}=.03, n s\right)$.

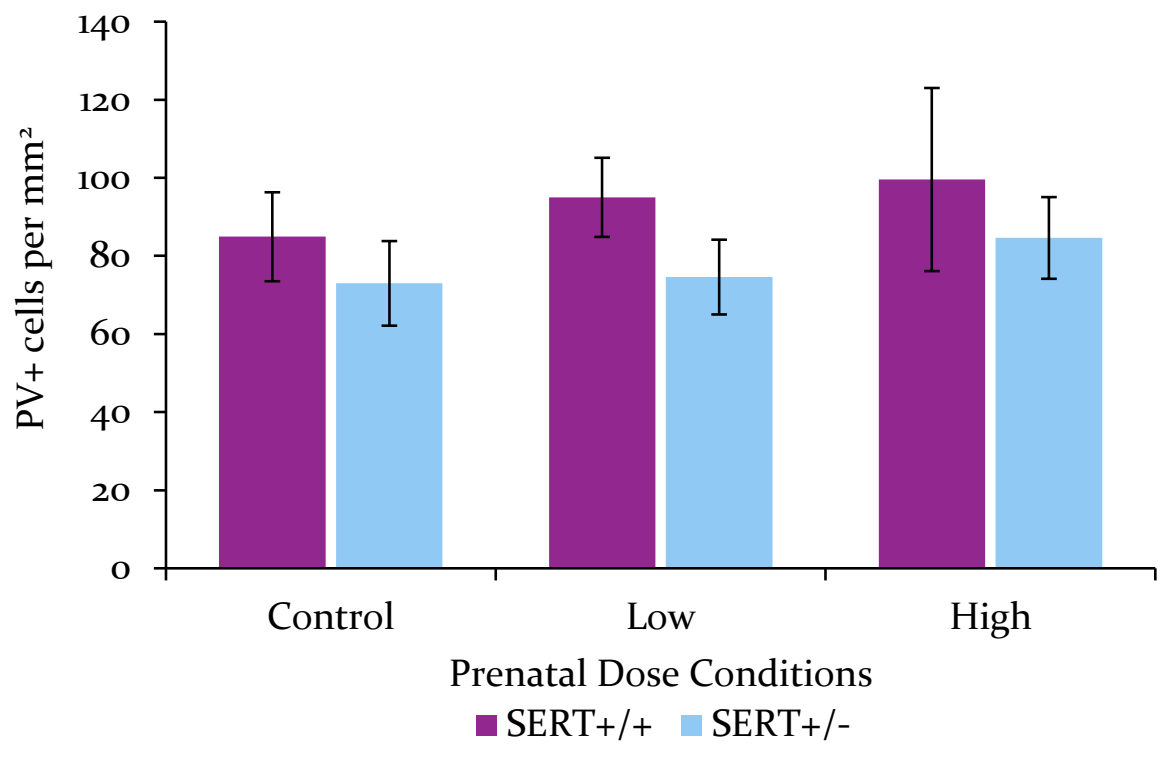

Figure 4.6. The average PV+ cell counts in the ventromedial amygdala for adult SERT ${ }^{+/+}$and $\mathrm{SERT}^{+/-}$rats prenatally exposed to control, low, and high doses of VPA. No statistical differences detected. Error bars represent \pm SEM. 


\section{$P V+$ cell counts in the ventrolateral amygdala}

There was no effect of prenatal dose or genotype on PV+ cell counts in ventrolateral amygdala, see figure 4.7. That is, the prenatal dose had no effect $\left(F(2,50)=2.04, p=.141, \mathrm{\eta}_{\mathrm{p}}{ }^{2}=\right.$ $.08, n s)$, nor did the genotype $\left(F(1,50)=.96, p=.331, \mathrm{\eta}_{\mathrm{p}}^{2}=.02, n s\right)$, and there was no interaction between prenatal dose and genotype $\left(F(2,50)=2.47, p=.095, \mathrm{\eta}_{\mathrm{p}}^{2}=.09, n s\right)$.

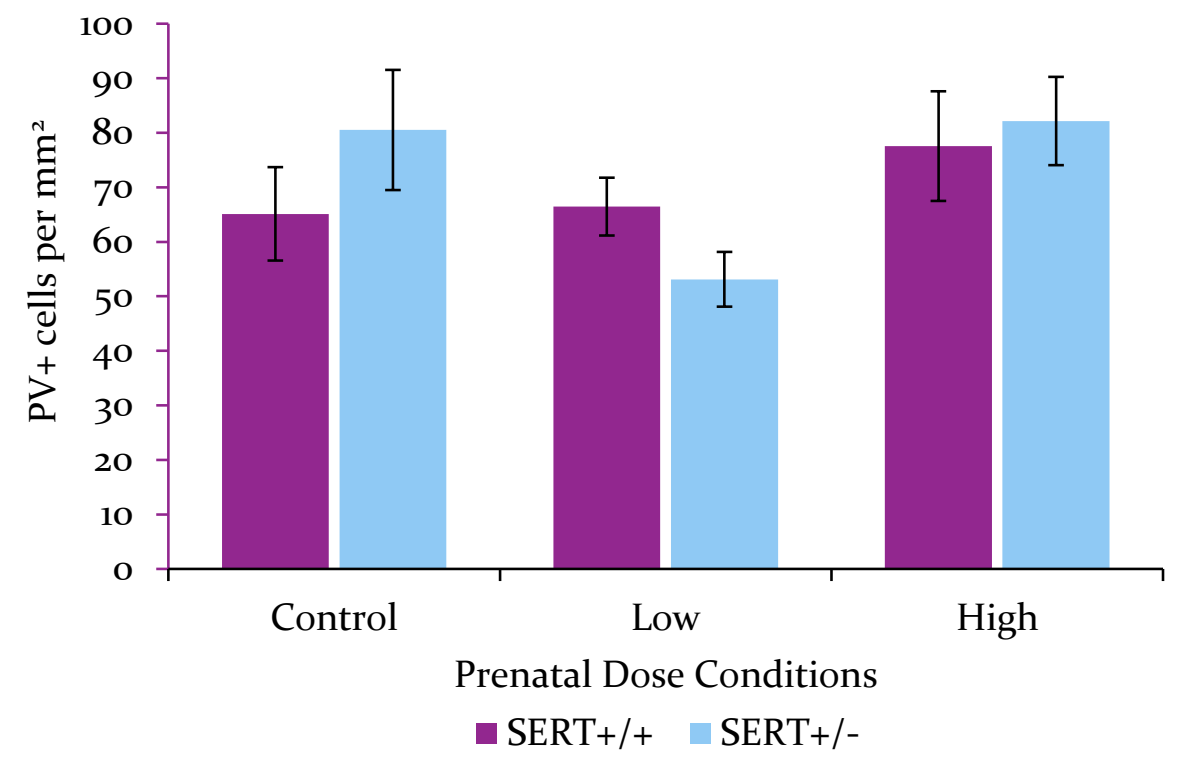

Figure 4.7. The average PV+ cell counts in the ventrolateral amygdala for adult SERT ${ }^{+/+}$and $\mathrm{SERT}^{+/-}$rats prenatally exposed to control, low, and high doses of VPA. No statistical differences detected. Error bars represent \pm SEM. 


\section{$P V+$ cell counts in the basolateral/basomedial amygdala}

There was no effect of prenatal dose or genotype on PV+ cell counts in basolateral/basomedial amygdala, see figure 4.8 . The prenatal dose had no effect $(F(2,50)=$ $\left.1.42, p=.251, \mathrm{n}_{\mathrm{p}}^{2}=.05, n s\right)$, nor did the genotype $\left(F(1,50)=.05, p=.820, \mathrm{\eta}_{\mathrm{p}}^{2}=.01, n s\right)$, and there was no interaction between prenatal dose and genotype $\left(F(2,50)=1.02, p=.368, \mathrm{n}_{\mathrm{p}}{ }^{2}=.04, n s\right)$.

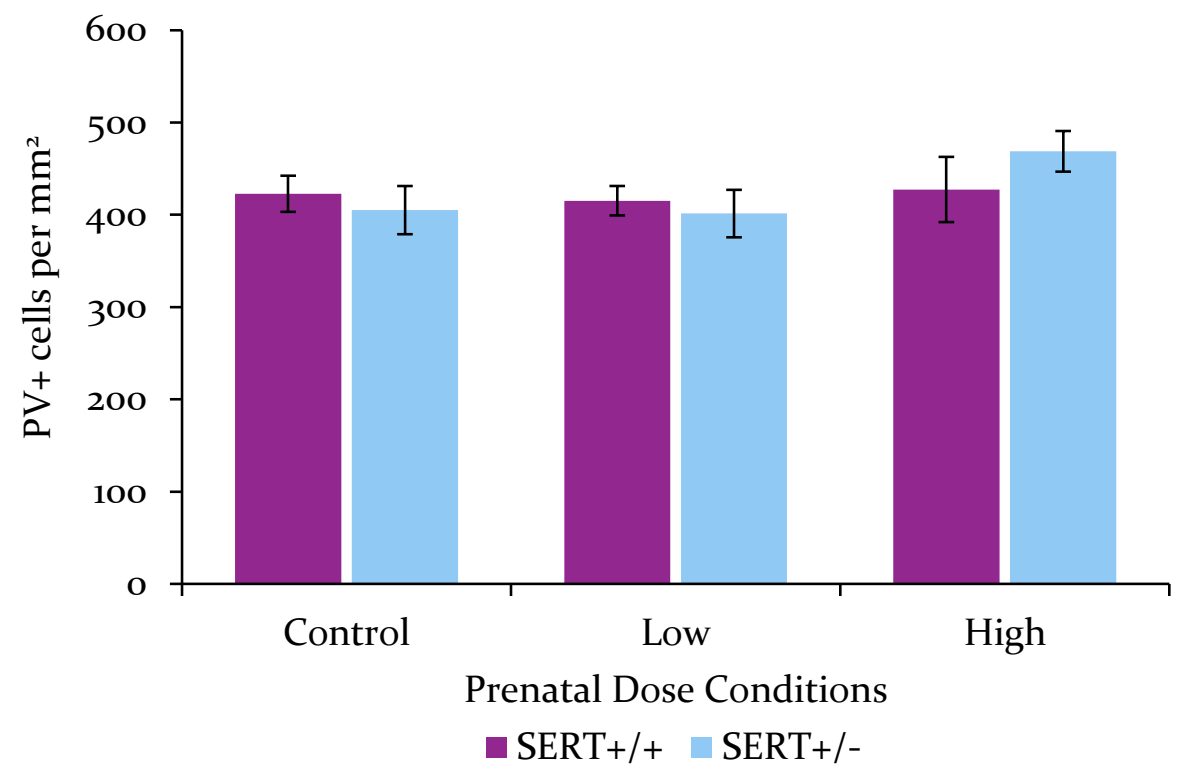

Figure 4.8. The average PV+ cell counts in the basolateral/basomedial amygdala for adult $\mathrm{SERT}^{+/+}$and SERT ${ }^{+/-}$rats prenatally exposed to control, low, and high doses of VPA. No statistical differences detected. Error bars represent \pm SEM. 


\section{Sample elimination and sample size}

From the original 56 brains that were sliced and stained for PV+ cell, 50 brains were used in the analysis of the hippocampus. Six brains were excluded from the analysis as the tissue was damaged, leading to unreliable cell counts. As such, the sample sizes for the hippocampus analysis are drawn from the grey cells in table 4.2.

Table 4.2. The number of brain samples from experimental animals used for the analysis of PV+ cells in the hippocampus, divided by prenatal dose, sex and genotype. The grey cells refer to the combined (either of sex or genotype) totals.

\begin{tabular}{c|c|c|c|c|}
\hline \hline \multirow{4}{*}{ Control } & & SERT $^{+/+}$ & SERT $^{+/-}$ & Combined \\
\hline \multirow{5}{*}{ Low } & Males & 5 & 5 & 10 \\
\cline { 2 - 5 } & Females & 4 & 3 & 7 \\
\cline { 2 - 5 } & Combined & 9 & 8 & 17 \\
\hline \multirow{3}{*}{ High } & Males & 4 & 5 & 9 \\
\cline { 2 - 5 } & Females & 3 & 7 & 10 \\
\cline { 2 - 5 } & Combined & 7 & 12 & 19 \\
\hline \hline & Males & 5 & 3 & 6 \\
\hline & Females & 4 & 2 & 14 \\
\hline
\end{tabular}




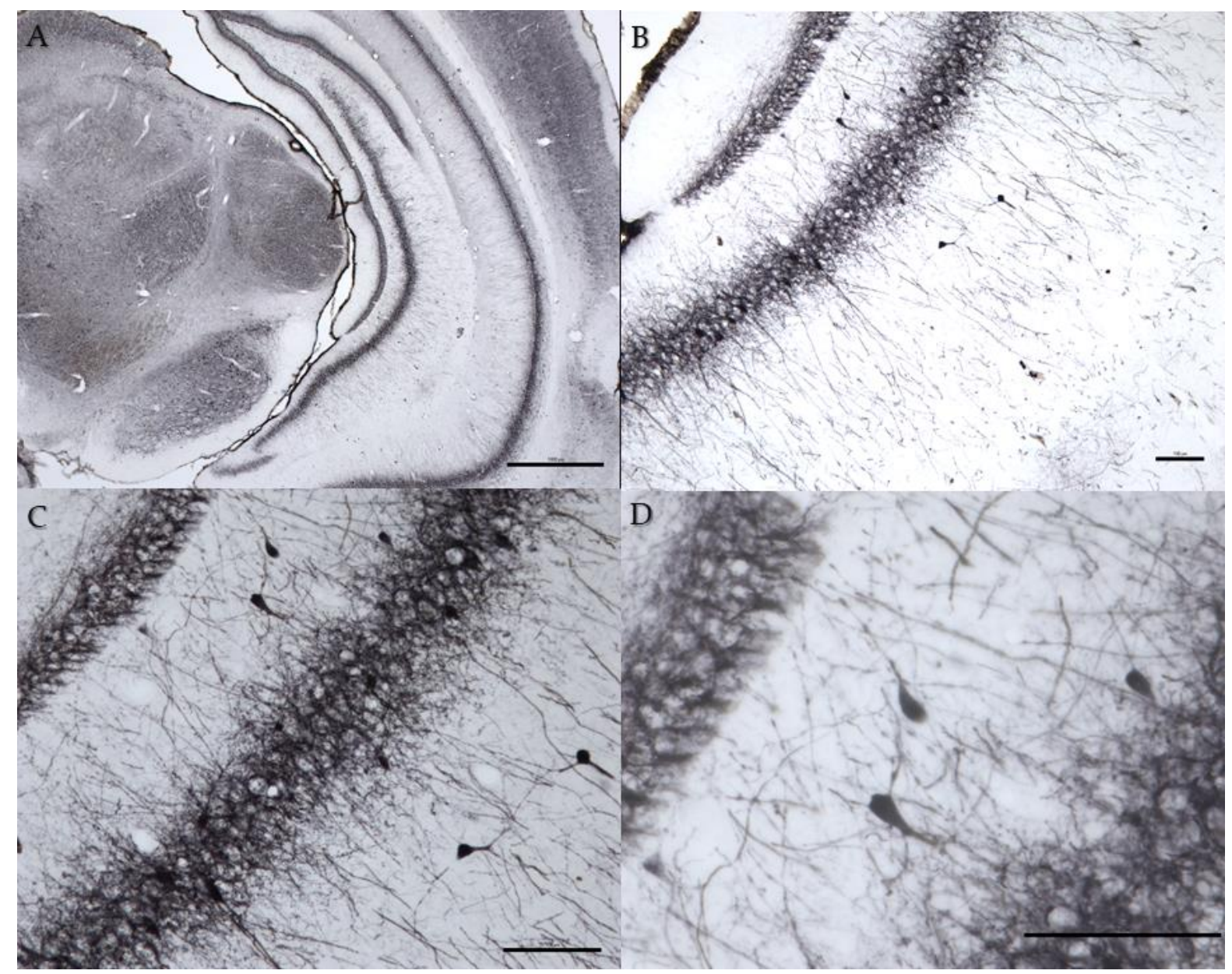

Figure 4.16. Representative light microscopy images of PV+ cells in the ventral hippocampus from a SERT ${ }^{+/+}$male prenatally exposed to the control dose, at bregma -5.30. A. Extensive PV+ staining in the hippocampus, thalamus, and cerebral cortex, $1.25 \mathrm{X}$ objective, with scale bar $1000 \mu \mathrm{m}$. B. Dense PV+ staining in the CA3 with dendritic branches projecting laterally, 10X objective with $100 \mu \mathrm{m}$ scale bar. C. Dense PV+ staining in the CA3, with clearly defined cell bodies, 10X objective with $100 \mu \mathrm{m}$ scale bar. D. PV+ cell staining with connected dendritic branches, $40 \mathrm{X}$ objective with $100 \mu \mathrm{m}$ scale bar. 


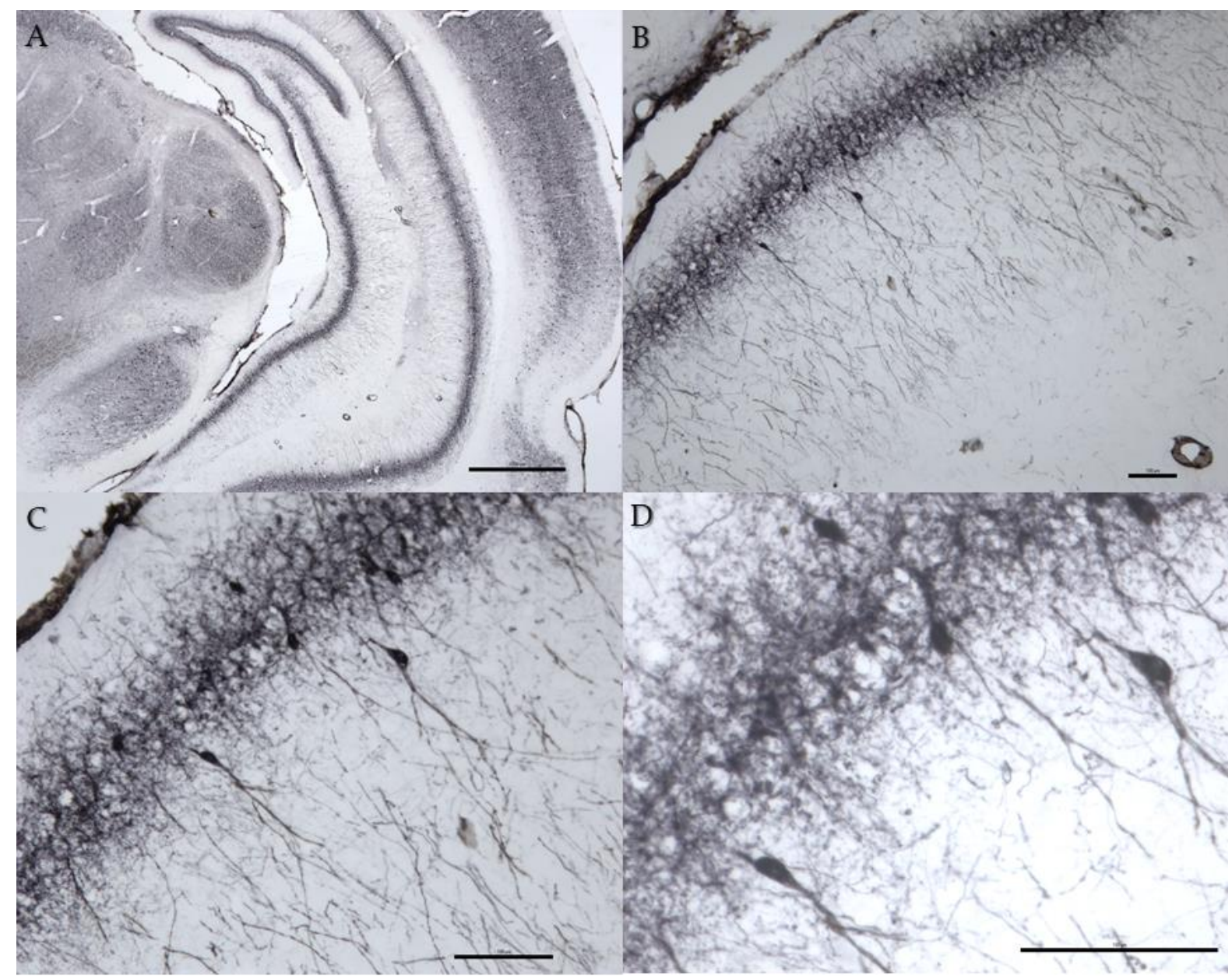

Figure 4.17. Representative light microscopy images of PV+ cells in the ventral hippocampus from a SERT ${ }^{+-}$male prenatally exposed to the high dose, at bregma -5.30 . A. Extensive PV+ staining in the hippocampus, thalamus, and cerebral cortex, $1.25 \mathrm{X}$ objective, with scale bar $1000 \mu \mathrm{m}$. B. Dense PV+ staining in the CA3 with dendritic branches projecting laterally, 10X objective with $100 \mu \mathrm{m}$ scale bar. C. Dense PV+ staining in the CA3, with clearly defined cell bodies, 10X objective with $100 \mu \mathrm{m}$ scale bar. D. PV+ cell staining with connected dendritic branches, $40 \mathrm{X}$ objective with $100 \mu \mathrm{m}$ scale bar. 


\section{$P V+$ cell counts in the ventral hippocampus}

There was no effect of prenatal dose or genotype on PV+ cell counts in ventral hippocampus when all cell counts are considered together, see figure 4.11. That is, the prenatal dose had no effect $\left(F(2,44)=1.29, p=.285, \eta_{\mathrm{p}}^{2}=.06, n s\right)$, nor did the genotype $(F(1,44)=.02$, $\left.p=.904, \eta_{\mathrm{p}}{ }^{2}=.01, n s\right)$, and there was no interaction between prenatal dose and genotype $(F(2$, $\left.44)=.12, p=.886, \mathrm{\eta}_{\mathrm{p}}^{2}=.01, n s\right)$.

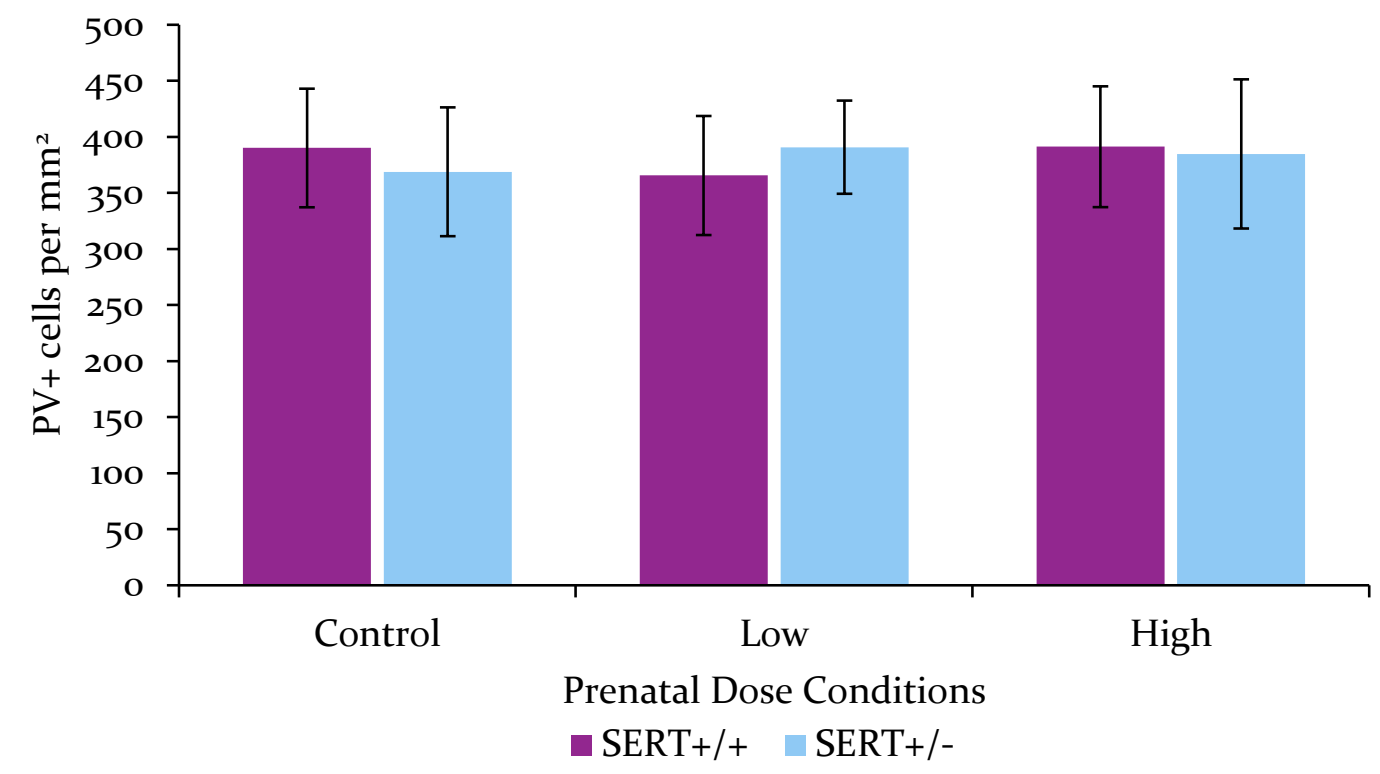

Figure 4.11. The average PV+ cells in the ventral hippocampus for adult SERT ${ }^{+/+}$and SERT ${ }^{+/-}$ prenatally exposed to control, low, and high doses of VPA. No detectable differences were found. Error bars represent \pm SEM. 
There was no effect of prenatal dose or genotype on PV+ cell counts in the dentate gyrus, see figure 4.12. That is, the prenatal dose had no effect $\left(F(2,44)=.45, p=.640, \mathrm{\eta}_{\mathrm{p}}{ }^{2}=.02, n s\right)$, nor did the genotype $\left(F(1,44)=.07, p=.799, \mathrm{\eta}_{\mathrm{p}}^{2}=.01, n s\right)$, and there was no interaction between prenatal dose and genotype $\left(F(2,44)=.67, p=.517, \mathrm{\eta}_{\mathrm{p}}{ }^{2}=.03, n s\right)$.

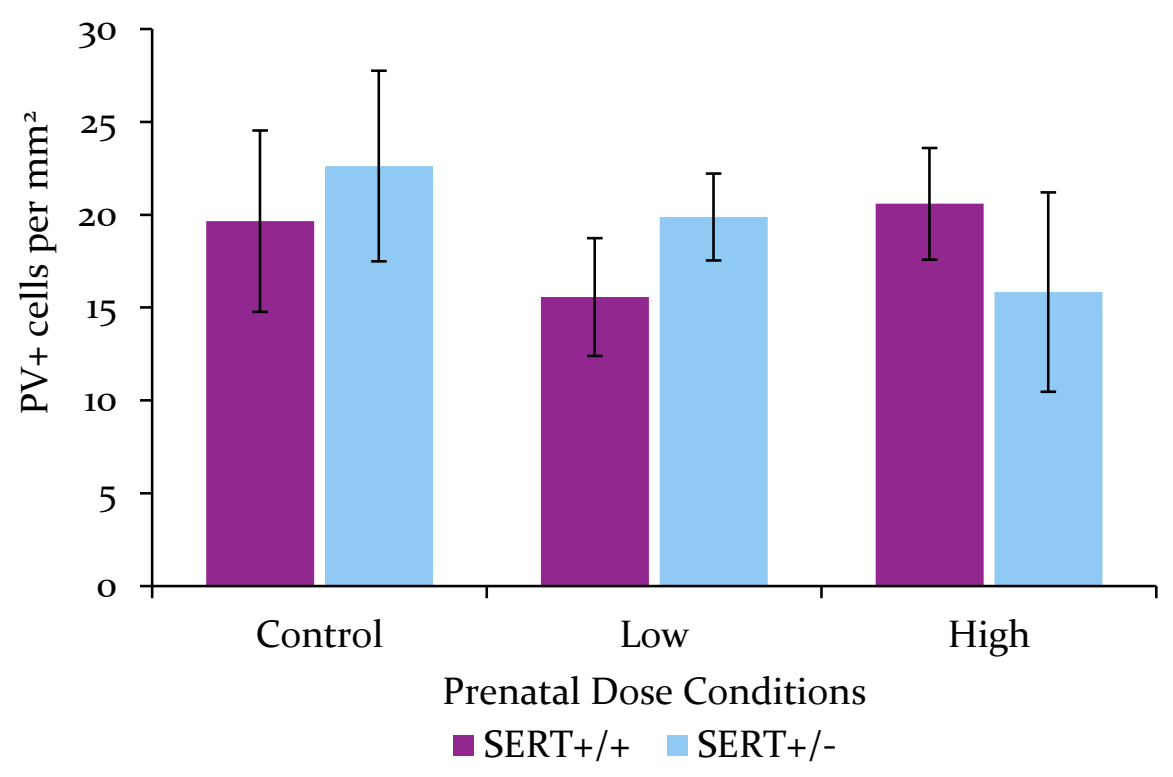

Figure 4.12. The average PV+ cells in the dentate gyrus hippocampus for adult $\mathrm{SERT}^{+/+}$and $\mathrm{SERT}^{+/-}$prenatally exposed to control, low, and high doses of VPA. No detectable differences were found. Error bars represent \pm SEM. 


\section{$P V+$ cell counts in the Dorsal CAl}

There was no effect of prenatal dose or genotype on PV+ cell counts in the dorsal CAl, see figure 4.13. That is, the prenatal dose had no effect $\left(F(2,44)=.02, p=.978, \mathrm{\eta}_{\mathrm{p}}{ }^{2}=.01, n s\right)$, nor did the genotype $\left(F(1,44)=.02, p=.889, \mathrm{\eta}_{\mathrm{p}}^{2}=.01, n s\right)$, and there was no interaction between prenatal dose and genotype $\left(F(2,44)=.58, p=.563, \mathrm{\eta}_{\mathrm{p}}^{2}=.03, n s\right)$.

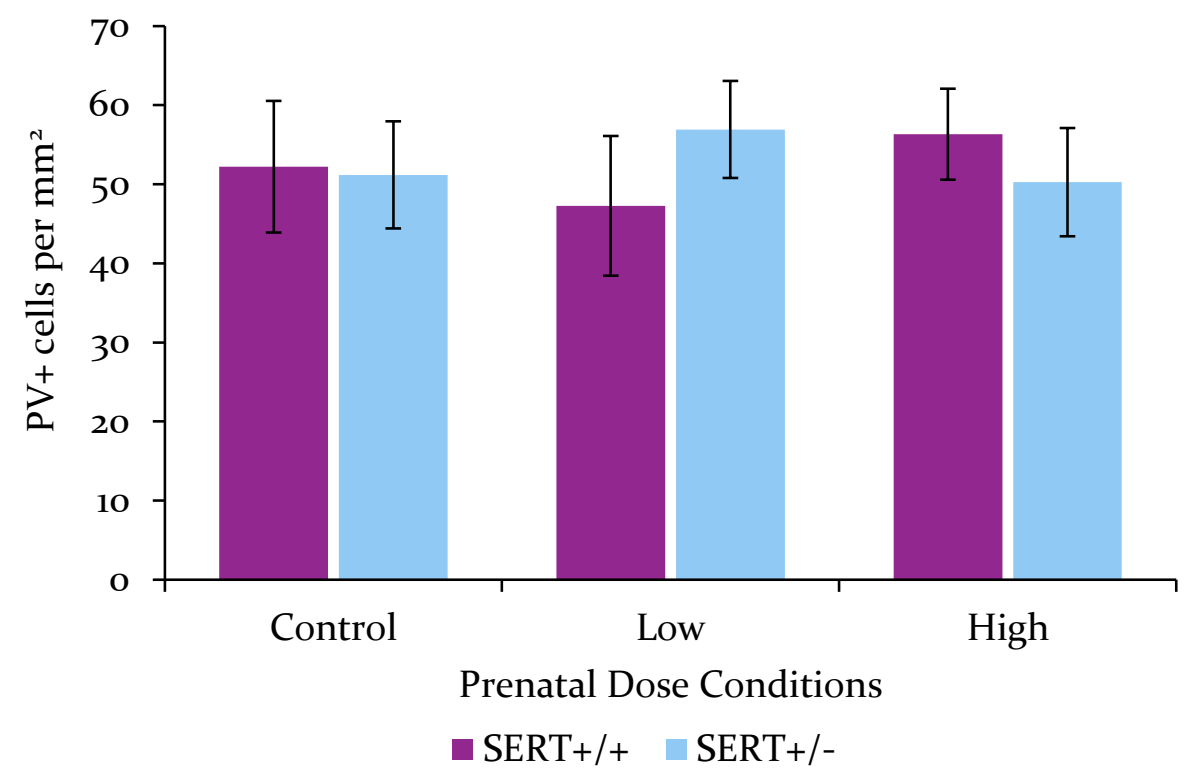

Figure 4.13. The average PV+ cells in the dorsal CAl hippocampus for adult SERT ${ }^{+/+}$and $\mathrm{SERT}^{+/-}$ prenatally exposed to control, low, and high doses of VPA. No detectable differences were found. Error bars represent \pm SEM. 
There was no effect of prenatal dose or genotype on PV+ cell counts in the dorsal CA3, see figure 4.14. That is, the prenatal dose had no effect $\left(F(2,44)=.32, p=.725, \mathrm{\eta}_{\mathrm{p}}^{2}=.02, n s\right)$, nor did the genotypes $\left(F(1,44)=1.05, p=.311, \mathrm{\eta}_{\mathrm{p}}{ }^{2}=.02, n s\right)$, and there was no interaction between prenatal dose and genotype $\left(F(2,44)=.79, p=.459, \eta_{\mathrm{p}}{ }^{2}=.04, n s\right)$.

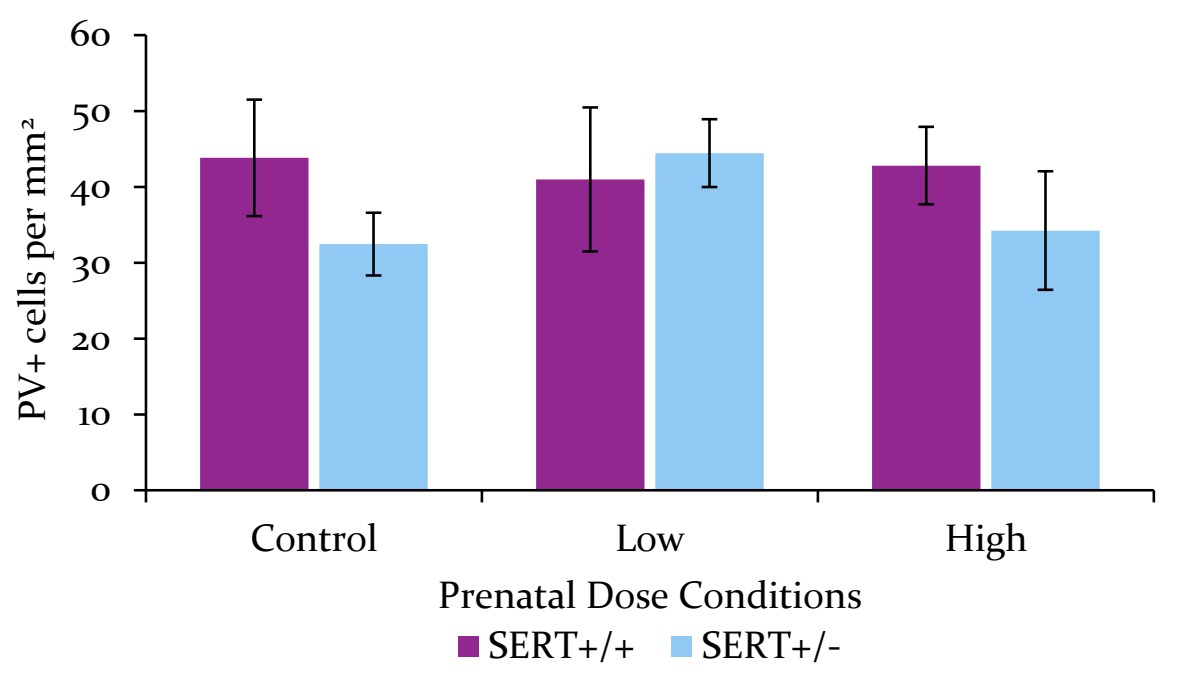

Figure 4.14. The average PV+ cells in the dorsal CA3 hippocampus for adult $\mathrm{SERT}^{+/+}$and $\mathrm{SERT}^{+/-}$ prenatally exposed to control, low, and high doses of VPA. No detectable differences were found. Error bars represent \pm SEM. 
There was no effect of prenatal dose or genotype on PV+ cell counts in the ventral CAl, see figure 4.15. That is, the prenatal dose had no effect $\left(F(2,44)=1.08, p=.347, \mathrm{\eta}_{\mathrm{p}}{ }^{2}=.05, n s\right)$, nor did the genotypes $\left(F(1,44)=.86, p=.358, \eta_{\mathrm{p}}^{2}=.02, n s\right)$, and there was no interaction between prenatal dose and genotype $\left(F(2,44)=1.28, p=.287, \mathrm{\eta}_{\mathrm{p}}^{2}=.06, n s\right)$.

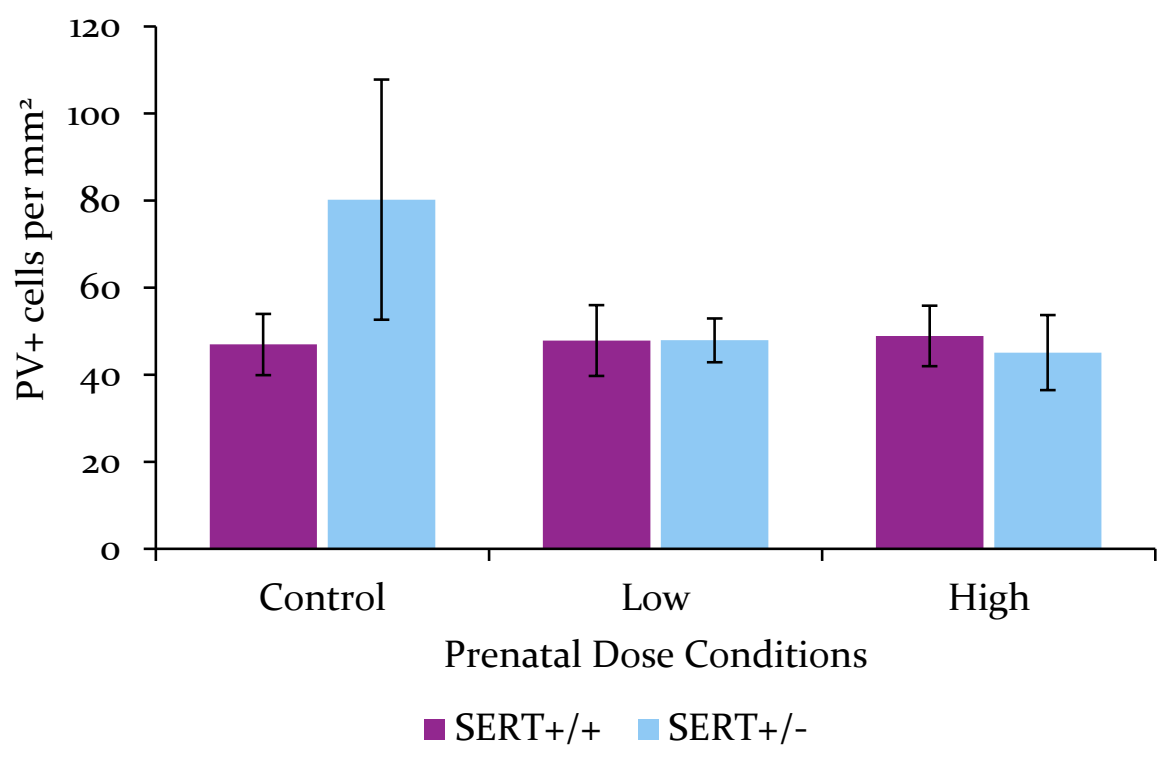

Figure 4.15. The average PV+ cells in the ventral CAl hippocampus for adult SERT ${ }^{+/+}$and SERT ${ }^{+/-}$ prenatally exposed to control, low, and high doses of VPA. No detectable differences were found. Error bars represent \pm SEM. 
There was no effect of prenatal dose or genotype on PV+ cell counts in the ventral CA3, see figure 4.16. That is, the prenatal dose had no effect $\left(F(2,44)=1.92, p=.158, \mathrm{\eta}_{\mathrm{p}}^{2}=.08, n s\right)$, nor did the genotypes $\left(F(1,44)=.09, p=.765, \eta_{\mathrm{p}}^{2}=.01, n s\right)$, and there was no interaction between prenatal dose and genotype $\left(F(2,44)=.18, p=.838, \mathrm{\eta}_{\mathrm{p}}{ }^{2}=.01, n s\right)$.

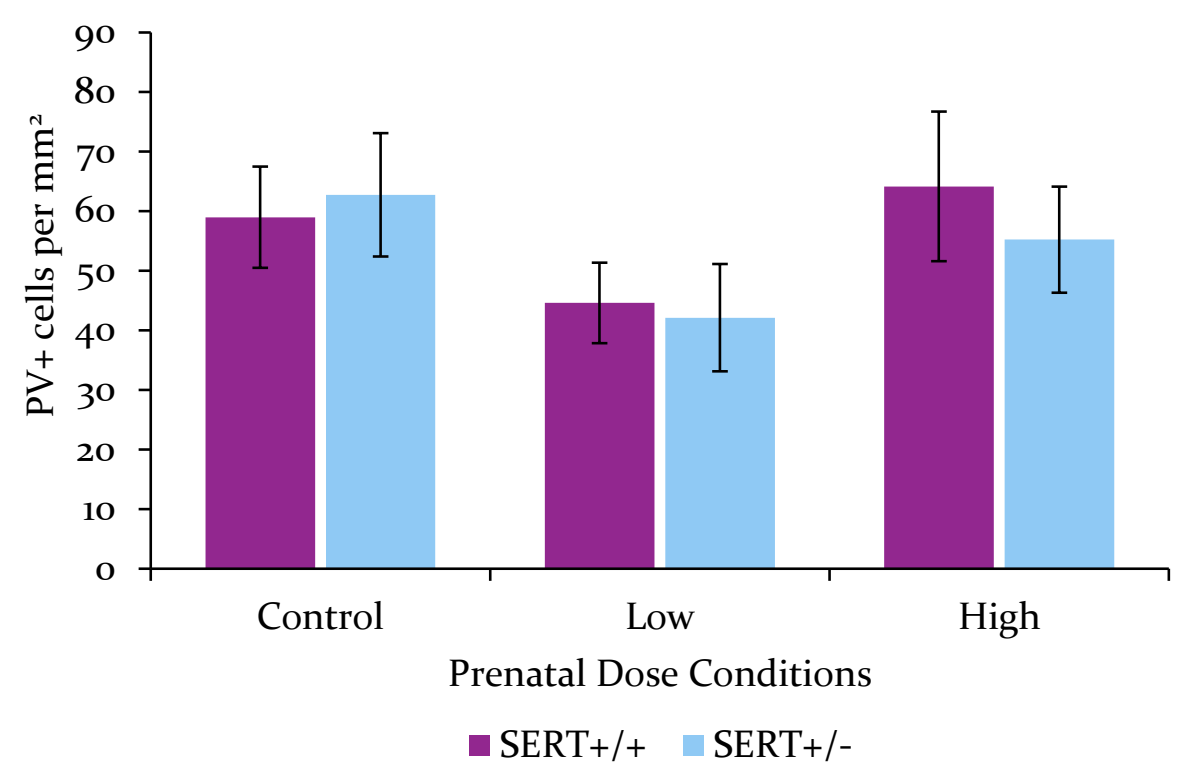

Figure 4.16. The average PV+ cells in the ventral CA3 hippocampus for adult $\mathrm{SERT}^{+/+}$and SERT ${ }^{+/-}$ prenatally exposed to control, low, and high doses of VPA. No detectable differences were found. Error bars represent \pm SEM. 


\subsection{Discussion}

This chapter examined PV neurons in the amygdala and ventral hippocampus of SERT ${ }^{+/+}$ and $\mathrm{SERT}^{+/-}$rats that were prenatally exposed to VPA throughout gestation. PV+ cells were examined as a measure of GABA interneuron expression and neuronal changes in the animal model of ASD, in regions that are vital for social functioning. It was hypothesised that the SERT deficiency and prenatal exposure to VPA would lead to a decrease in PV+ expression. The overall findings demonstrated that PV+ cells in the amygdala and the ventral hippocampus were not affected by either the SERT genotype nor the prenatal exposure to VPA. The following discussion will explore the methodology and contributing factors for the lack of findings.

\subsubsection{Methodology}

As highlighted in the optimisation section of the results (section 4.3.1), the experiment outlined in this chapter was preceded by extensive optimisation (as detailed in appendix B). The protocol for $\mathrm{PV}+$ staining had in part been developed in our laboratory but was yet to be optimised for experiments. The initial tests of the protocol revealed pale and incomplete staining, and with changes to the primary and secondary antibody, the staining became dense, and cell bodies were visible. With further tests on the primary antibody concentrations and the DAB revelation time, optimal and replicable staining was achieved.

The PV+ staining was visible in expected regions, such as the lateral and ventral amygdala, but was absent from the central amygdala nucleus (as displayed in figures 4.9 and 4.10; also established in Kemppainen \& Pitkänen, 2000). While it was not examined in this experiment, $\mathrm{PV}+$ staining was visible in the striatum (figures 4.9 and 4.10 , image A), and the cortex (figure 4.9 and 4.10, image A). Furthermore, the PV+ staining resulted in successful full cell body staining, which extended to the dendritic branches and these were aligned with the originating cell body, and minimal background staining (see figures 4.9 and 4.10 , as well as 4.16 and 4.17). Finally, there was no visible staining in the negative control tissue, and this was confirmed for each repetition of the protocol when processing the experimental tissue (see figure 4.1). As a result of the described methodology, the procedure used in this experiment led to successful staining of the PV+ cells in the amygdala and ventral hippocampus. Moreover, as indicated by the graphs, in most cases, that variability between the biological samples was small, thus adding to the confidence in the overall results. 


\subsubsection{Amygdala and Ventral Hippocampus Findings}

As mentioned before, there were no significant differences relating to the genotypes $\left(\mathrm{SERT}^{+/+}\right.$and SERT $\left.{ }^{+/}\right)$nor the prenatal exposure to VPA, in the amygdala and ventral hippocampus. Additionally, there was no difference between males and females. Although, the lack of sex differences, may be in part due to the relatively small size, especially in the number of female $\mathrm{SERT}^{+/-}$rats was relatively low (see table 4.1).

The overall the lack of impact by the SERT genotype and prenatal exposure to VPA, may also be attributed to the age of rats at the time of brain extraction. Studies that have examined older rats that were prenatally exposed to VPA and have found normalisation of synaptic physiology (Martin \& Manzoni, 2014; Walcott, Higgins, \& Desai, 2011), although these studies had not examined PV+ expression. Previous PV+ expression experiments in the VPA-induced ASD animal model have looked at the brains of preweaning mice (Lauber et al., 2016) and young adults rats (Dendrinos et al., 2011) and young adult mice (Gogolla et al., 2009), rather than the much older 4-8-month-old rats that were used in this study. Older rats in this study were used as the rats were required for behavioural testing between PND 7 and PND 80+, thus earlier extraction of brains was not possible without significantly increasing the litters and animals used in this research. Furthermore, there are indications of developmental neuronal changes in ASD populations (as discussed in relation to the amygdala in section 4.1.2; Courchesne, Campbell, \& Solso, 2011; Nomi \& Uddin, 2015). Therefore, examining adulthood/late adulthood is perhaps missing the window to see the changes that originate from the interaction of prenatal exposure to VPA and the SERT reduction, as such research following this thesis, should examine the neuronal changes of younger animals.

The results of this experiment are surprising as decreases in PV+ expression were expected. Previous studies demonstrated that single prenatal exposure to VPA reduces the PV+ expression (Gogolla et al., 2009; Lauber et al., 2016; Wöhr et al., 2015). However, it is important to keep in mind that this has typically been observed in other brain regions, such as the cortex (Gogolla et al., 2009; Lauber et al., 2016), the striatum (Lauber et al., 2016) and the superior colliculus (Dendrinos et al., 2011). Only one study, to the best of our knowledge, has investigated the hippocampus. In this study, Gogolla and colleagues exposed mice to $500 \mathrm{mg} / \mathrm{kg}$ of VPA at GD 10, and this led to significantly reduced PV+ cells in the neocortex, but not in the hippocampus. It is currently unclear why we did not find any changes in the hippocampus. As discussed before, the differences in VPA administration (IP or SC injection, acute or chronic, and the level of VPA dose) may all have contributed to these discrepancies. So far, no study has 
focused on the role of the amygdala, in spite of its prominent role in social and emotional behaviour, as well as in ASD.

The results of this experiment show that neither the SERT genotype nor the prenatal exposure significantly impacted the number of PV+ cells in the amygdala and ventral hippocampus. The lack of effect is perhaps due to the 'dose' effects, of both gene and prenatal VPA exposure. In the case of the genotype, the SERT ${ }^{+-}$genotype is deemed as a mild phenotype, suggesting that the one functional allele may be substantially compensating for the lack of SERT activity of the inactive allele (Homberg et al., 2007). In this respect, there is indeed some evidence pointing to disruptions of the GABAergic system in homozygous SERT ${ }^{-/}$rats. Calabrese et al. (2013) showed that SERT ${ }^{-/-}$rats (compared to SERT ${ }^{+/+}$) exhibited significant decreases in the mRNA for $\mathrm{GABA}_{\mathrm{A}}$ receptor and glutamic acid decarboxylase 67 (GAD 6 ; which is required for the synthesis of GABA). The mRNA of $G_{A B A}$ receptor was significantly decreased in the ventral hippocampus and the prefrontal cortex, whereas $\mathrm{GAD}_{67}$ was only decreased in the ventral hippocampus. These findings suggest that SERT ${ }^{-/-}$rats have decreased GABA function in the ventral hippocampus, and further research is needed to understand the GABA function in the prefrontal cortex. The work of Calabrese and colleagues did not include the SERT ${ }^{+/-}$genotype. Recently, our laboratory undertook an RNA sequencing study of the frontal cortex, data of which have not been published yet. This study also provided some indication of reduced GABAergic functioning. Thus, compared to SERT ${ }^{+/+}$rats, $\mathrm{SERT}^{-/-}$rats had lower expression of the Pvalb gene, which codes for parvalbumin. In line with the data in this chapter, the SERT ${ }^{+/}$did not differ from SERT ${ }^{+/}$. It would, therefore, be relevant to investigate if the full genetic deletion of SERT alters the expression of the PV+ in rats. Such research would further our knowledge of genetic SERT deletion and GABA function in the brain.

The results of this experiment have established that the amygdala and the hippocampus may not have been the ideal for detecting deficits in PV+ expression in the ASD animal model. For this experiment, it was pertinent to focus on the amygdala and ventral hippocampus to investigate neuronal changes underlying social functioning. However, there is evidence to suggest that the amygdala and the ventral hippocampus are tightly connected (Lübkemann et al., 2015), and vital for social processes, as such there may be ceiling effects involved. Therefore, research following this thesis should look to examine brains that express PV, such as thalamus, cortex, and cerebellum (Celio, 1990). Future investigations into the PV expression these regions could provide a better estimate of the PV/GABA deficits in the animal model of ASD. 


\subsubsection{Summary}

Overall, the PV+ expression in the amygdala and ventral hippocampus was not affected by the SERT genotype, nor the prenatal exposure to VPA throughout gestation. These results were surprising given the indications that, at least, the prenatal VPA exposure would reduce the $\mathrm{PV}+$ expression. However, this experiment was limited by the advanced age of the rats at the time of brain extraction, when compared to the previous studies. The findings were also limited by the reduced prenatal VPA doses and the examined brain regions. Despite the limitations of the experiment, the methodology was thorough, and the PV+ staining was identified in the expected brain regions, and there was no identifiable false-positive staining. 


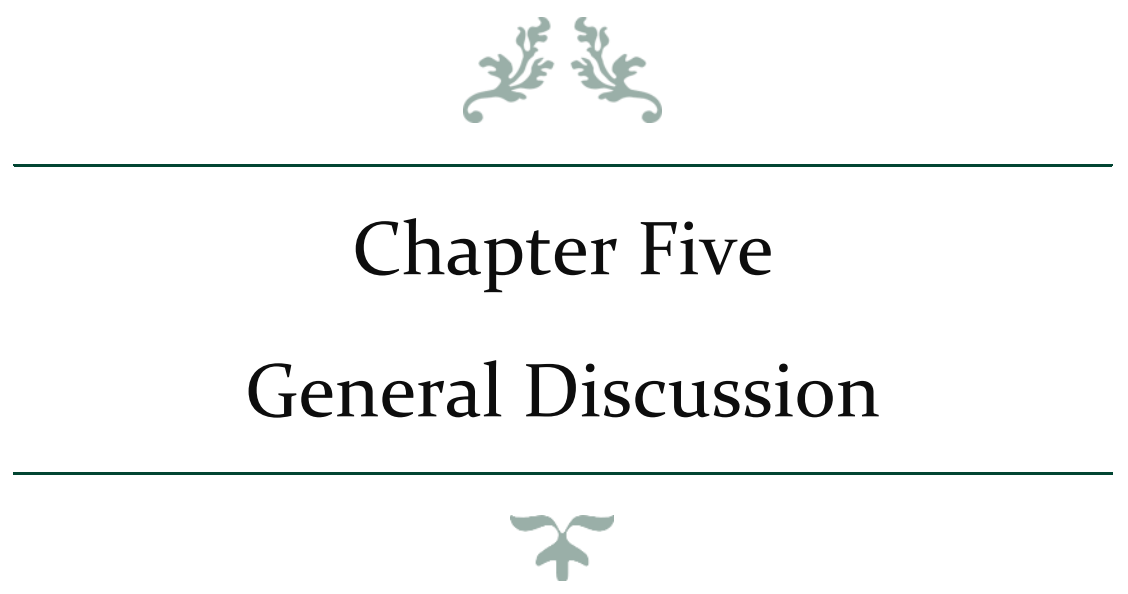




\section{Chapter Five Contents}

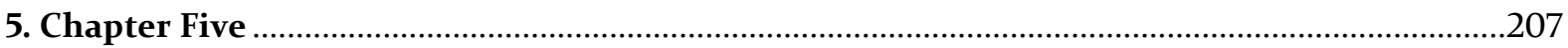

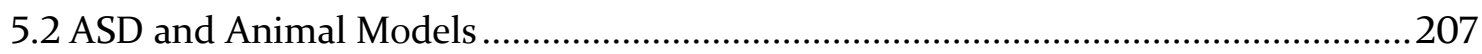

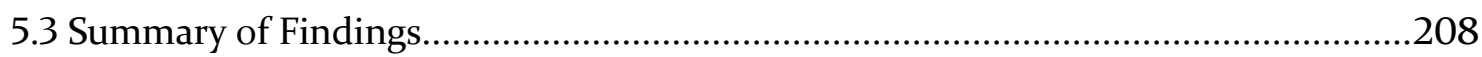

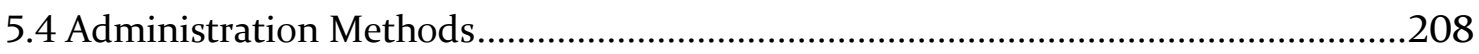

5.5 Gene-Environment Interaction Approach to the ASD animal model........................210

5.5.2 Contribution of Reduced SERT Function to the ASD Animal Model ............211

5.5.3 Contribution of the Prenatal VPA Exposure to the ASD Animal Model.......214

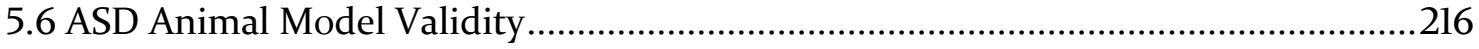

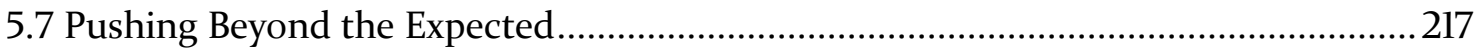

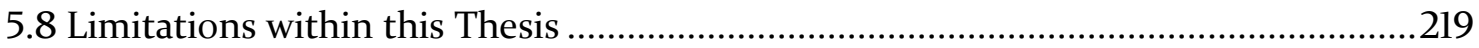

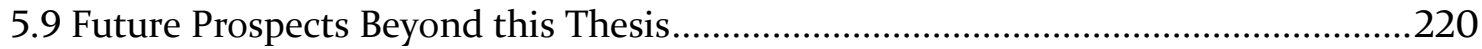

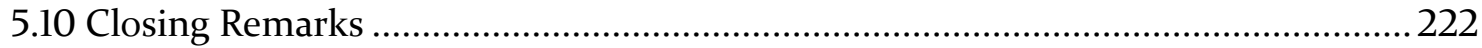




\section{Chapter Five}

\subsection{ASD and Animal Models}

ASD is a complex heterogeneous disorder with multiple behavioural and cognitive abnormalities, which are clinically present from childhood. It has been more than 75 years since ASD was first described, and our understanding of the symptoms and neurological underpinning is still evolving. Over time ASD has been consistently characterised by the deficits in communication, social interaction, and repetitive behaviours. The prevalence of ASD is increasing, with current estimates of one in 68 children diagnosed (Baio et al., 2018). With these increasing number of patients comes the substantial lifelong impact on the individual, their family, and the wider community. In addition, the aetiology is mostly unknown, with continued research identifying a multitude of genetic and non-genetic factors, including maternal infection and teratogen exposure. However, it is increasingly becoming clear that these factors alone are not the direct cause of ASD. Instead, it is more likely that the interaction between environmental factors and the genetic background of an individual ultimately leads to ASD (or psychiatric disorders in general).

There is an urgent need for improved animal models of ASD to further our understanding of aetiology and particularly its pathophysiology (South, Rodgers, \& Van Hecke, 2017), which would aid in the identification of new therapeutic targets (Caraci, Leggio, Salomone, \& Drago, 2017; Kaiser \& Feng, 2015). However, the animal models of ASD are in their infancy, as most models examine only the genetic (Ellegood et al., 2015; McFarlane et al., 2008) or the environmental (Malkova et al., 2012; Shi et al., 2009) contributions, and very rarely a combination of the two. The consistent lack of gene $x$ environment interaction research in the animal models of ASD leads to reduced construct validity, particularly, in regard to the aetiological process involved with the development of the ASD-like phenotype. Given the background, this thesis aimed to improve the VPA-induced ASD animal model with a geneenvironment interaction approach, as well as optimising the chronic administration method to enhance its construct validity. 


\subsection{Summary of Findings}

The chronic administration of VPA throughout gestation was investigated using genetically normal (wildtype) rats (chapter two) as well as rats with genetically compromised SERT (chapter three and four). In chapter two, daily doses of $0,100,200,300 \mathrm{mg} / \mathrm{kg} /$ day were administered in sweetened condensed milk to pregnant Sprague-Dawley rats, to examine the efficacy of administering chronic doses of VPA as well as the impact of these doses on the communication and social behaviour of the offspring. The findings of chapter two confirmed that the 100,200 , and $300 \mathrm{mg} / \mathrm{kg} /$ day VPA doses were below the toxic levels for the dam, and the offspring exhibited a mild phenotype (such as increased social behaviour in play behaviour paradigm, and social aversion in the SAA paradigm). The results for chapter two confirm that the chronic voluntary ingestion of VPA was a viable administration method, although further optimisation was required. Thus, the administration method was optimised for chapter three using gelatine pellets, along with increased doses of 300 and $600 \mathrm{mg} / \mathrm{kg} / \mathrm{day}$ which were ingested by SERT ${ }^{+/+}$dams that had mated with SERT ${ }^{+/-}$males. The experiments in chapter three demonstrate the behavioural impact of control, low, and high doses of VPA on SERT ${ }^{+/+}$and $\mathrm{SERT}^{+/}$offspring. The findings of chapter three confirmed that the chronic prenatal exposure to low and high doses of VPA in rats with the reduction in the SERT alters their communication maturation, leads to an increase in active behaviours, disrupts social preference formation, and increases their anxiety-like behaviour. To further explore the underlying origins of the altered behaviours of the offspring, the GABA interneurons were examined in the amygdala and ventral hippocampus, as seen in chapter four. The lack of significant effects in chapter four is perhaps indicative of low prenatal VPA doses. That is, the VPA exposure during gestation did not lead to neurobiological alterations in the amygdala and ventral hippocampus of adult rats.

Overall, the SERT ${ }^{+/+}$and SERT ${ }^{+/}$rats prenatally exposed to VPA, exhibit a mild ASD phenotype, but one that shows deficits in the core symptoms of ASD, particularly communication and social interaction.

\subsection{Administration Methods}

This thesis aimed to strengthen the construct validity of the VPA-induced animal model of ASD, particularly in regard to the chronic administration of VPA in a non-stressful manner for pregnant female rodents. The VPA-induced animal model was derived from the observation that when pregnant women were medicated with VPA, their children were more likely to develop ASD (Christensen et al., 2013; Rasalam et al., 2005). Previous studies have typically 
used a single exposure of VPA to induce ASD-like behaviours in rodents (Rodier et al., 1997; Schneider \& Przewlocki, 2005). The single exposure to VPA is, of course, at odds with the clinical situation, as pregnant women ingest VPA daily throughout pregnancy. As a result, while the face validity of the single administration of VPA is well-established, the construct validity is substantially less. Studies that have attempted to increase the validity and by repeatedly exposing pregnant rodents to VPA have been few, and both the details of the administration and the results have been very varied (see literature summary in table 2.1).

The administration methods in this thesis were aimed to increase the construct validity of this model by using daily oral administration. Chapter two implemented the use of sweetened condensed milk as the vehicle for VPA. The findings of chapter two confirmed that dams consistently and voluntarily ingested 100,200 , and $300 \mathrm{mg} / \mathrm{kg} /$ day of VPA. These doses were below the level of toxicity for the dams and the offspring and led to observable behavioural changes in the offspring. However, the administration method used in chapter two was limited, as there was no quantifiable method for confirming the consumed dose, at times, different amounts of milk remained in the bowls. Therefore, the administration method was optimised and implemented in chapter three, with the use of gelatine pellets.

Gelatine pellets containing VPA was a superior option compared to syringe feeding (Atcha et al., 2010), peanut butter (Main \& Kulesza, 2017), and SCM methods (chapter two). The gelatine pellet administration method for VPA delivery induced ASD-like behavioural changes in the offspring, without causing dam fatalities. The pellets provided a more precise and less invasive method for administering VPA doses to rodents (Zhang, 2011). Furthermore, the given and consumed doses were quantifiable and allowed for dose-related groups of low and high doses (see consumption summary in table 3.1). However, there were inconsistencies in the consumption of VPA pellets, which was likely due to the unpleasant taste of the VPA. Nonetheless, the gelatine pellets of VPA provided a non-stressful method for administering VPA to pregnant dams for them to consume voluntarily in a short timeframe. Thus, the gelatine pellets were comparable to the ingestion of VPA as medication for pregnant women.

With all of this said, the administration methods for chronic VPA exposure during gestation requires further development. As shown in the consumption summary information (table 3.1), the consumed doses of VPA are not equivalent to the administered doses; thus, the palatability of the doses needs to be increased. Attempts were made in this thesis to increase this by adding different artificial flavours and subdividing the dose into two administrations daily. However, these methods would be enhanced with an investigation into taste aversion and 
palatability of doses. Furthermore, oral consumption does not achieve blood plasma levels that are comparable to IP or SC injection administration levels (Klotz \& Antonin, 1977; Löscher, 2007). As such, the chronic administration methods to achieve dam plasma levels high enough to cause substantial neurodevelopment effects must be further developed. Preliminary data from our animals indeed seem to suggest that the doses used in this study, while clearly leading to substantial amounts of VPA in the blood of the mother, might have fallen short of the doses typically obtained with a single IP injection. Thus, further pharmacokinetic investigation in a larger group of animals is warranted.

Prior to the selection of the gelatine administration technique, the use of osmotic pumps was investigated. These devices have a semipermeable membrane which allows the desired compound (like VPA) to continuously diffuse into the circulation to maintain steady plasma concentrations when they are subcutaneously implanted (Herrlich, Spieth, Messner, \& Zengerle, 2012). However, none of the osmotic pumps currently available would be large enough to contain the required dose of VPA needed throughout pregnancy. Hence, they would require replacement (involving anaesthesia and surgery) halfway through gestation which was not considered a viable option for this study, as it would induce considerable stress to the dams. The osmotic pumps could be a viable option in the future when the reservoirs are large enough to carry high quantities of VPA (the estimated maximum volume was two grams of VPA (not including vehicle) for 21 days, depending on dam weight). Alternatively, the gelatine pellet administration would benefit from pharmaceutical compounding development, mainly to increase the palatability. Ultimately, it is clear that the administration methods for chronic doses of VPA need to be further optimised, however, this thesis has clearly made a step in that direction.

\subsection{Gene $\times$ Environment Interaction Approach to the ASD animal model}

Overall, the administration methods and experiments in this thesis have advanced the VPA induced animal model for ASD, with a gene $x$ environment approach. The gene $x$ environment approach to inducing an ASD phenotype has demonstrated that SERT ${ }^{+/+}$and $\mathrm{SERT}^{+/-}$males and females that were continuously exposed to VPA throughout gestation, exhibited abnormalities that align with an ASD-like phenotype. This supports previous work undertaken in our lab, whereby Ranger (2016) found interactions between the SERT ${ }^{+/}$genotype and the single prenatal exposure to VPA, particularly in social and repetitive behaviours of adult offspring. 
Surprisingly, there was not a consistent genotype and dose interaction, in chapter three and four. An interaction had been expected as the single VPA prenatal exposure had resulted in communication and social deficits (Olde Loohuis et al., 2015; Schneider \& Przewlocki, 2005). Similarly, reductions in social behaviour were also found in the $\mathrm{SERT}^{-/-}$genotype (Homberg et al., 2007). Therefore, it was expected that reduced function of the SERT in the SERT ${ }^{+/}$would potentiate the susceptibility to the teratogenic effects of prenatal VPA exposure, which had been speculated by Ellenbroek et al. (2016). As such, the conservative approach to avoid potential ceiling effects (due to the SERT ${ }^{-/}$) may have prevented the demonstration of a clear gene and environment interaction between SERT function and chronic prenatal exposure to a high dose of VPA. Although, chapter three demonstrated deficits in communication, social, and anxiety behaviours, the inclusion of the SERT ${ }^{-/-}$genotype would likely confirm this interaction.

The phenotype exhibited by the offspring in this thesis differs from previous research, notably where the single prenatal VPA administration led to offspring that have considerable reductions in communication and social behaviour (Dufour-Rainfray et al., 2010; Kim et al., 2011; Schneider \& Przewlocki, 2005). As such, the phenotype in this thesis may be a result of low VPA doses combined with an administration method that limited VPA blood levels rather than an abrupt onset achieved with an injection or oral gavage. As discussed above, preliminary data has examined this premise further (not included in the thesis). The pilot examined VPA in the serum of the dam that ingested VPA gelatine pellets $(600 \mathrm{mg} / \mathrm{kg} /$ day $)$ or received a single SC injection of $400 \mathrm{mg} / \mathrm{kg}$ VPA. The preliminary results show that gelatine pellets result in a dose that is one-seventh of an SC injection. As such, the mild phenotype identified in this thesis is aligned with previous research (Chapman \& Cutler, 1984; Vorhees, 1987; Wellmann et al., 2014) but is limited by the low VPA doses.

\subsubsection{Contribution of Reduced SERT Function to the ASD Animal Model}

The SERT $^{+-}$genotype was used to provide genetic vulnerability in the ASD phenotype, rather than providing a complete ASD phenotype. Animals with reduced SERT function were selected to further the construct validity of the VPA-induced ASD animal model, as the developments in the animal models of ASD too often examine the genetic or environmental contributions to ASD in isolation. Furthermore, the reduced SERT function of SERT ${ }^{+-}$rats is more similar to the 5-HTTLPR which has been proposed as a contributing factor in the aetiology of ASD (Cook et al., 1997; Devlin et al., 2005).

The findings of this thesis show that reduced SERT function does not substantially impact communication and social behaviours. In particular, the social behaviours exhibited in 
SAA show very few differences between $\mathrm{SERT}^{+/+}$and SERT ${ }^{+/}$, and when they do occur, it is only the female SERT ${ }^{+/}$and SERT ${ }^{+/-}$that exhibit significant effects (see results summary for chapter three, table 3.8.1). Similarly, Ellenbroek et al. (2016) found no evidence of a genotype difference between (males) $\mathrm{SERT}^{+/+}$and SERT ${ }^{+/-}$rats in relation to cognitive and anxiety-like behaviours. So, while the $\mathrm{SERT}^{+/}$genotype seemed a sensible choice to investigate gene $\times$environment interactions (since reduced SERT activity has repeatedly been associated with increased vulnerability for ASD in clinical studies), it was disappointing to find very few gene-related deficits.

However, it should be acknowledged that, on the whole, the exact contribution of reduced SERT function to the ASD phenotype is still not fully understood. Previous literature has indicated that the SERT ${ }^{+/-}$genotype does not have an overt phenotype, as the $\mathrm{SERT}^{+/-}$ behaviours are much closer to SERT ${ }^{+/+}$animals (Homberg et al., 2007; Kalueff et al., 2007). Previous studies have used the SERT ${ }^{-/}$genotype to demonstrate the ASD-like deficits of these animals with studies showing significant reductions in play behaviour, and abnormal play behaviour patterns (Homberg et al., 2007; Muller et al., 2010). However, the full genetic deletion of the SERT is not a direct model of the genetic variants found in ASD populations (refer to chapter one section 1.6). As such, the SERT ${ }^{+/}$genotype was used to more closely align with reduced SERT function found in a subset of the ASD population, in particular, those with the s-allele of the 5-HTTLPR.

A possible explanation for the minimal genotype findings and possibly the lack of gene $x$ environment interactions may lie in the complex development of the 5-HTergic system. During the initial prenatal development, 5-HT is solely determined and regulated by the maternal environment until endogenous $5-\mathrm{HT}$ is formed in the foetus, roughly from GD 9 onwards (Bonnin \& Levitt, 2011). Interestingly, SERT develops before the foetus can produce 5HT (Narboux-Nême, Pavone, Avallone, Zhuang, \& Gaspar, 2008). Moreover, during neurogenesis, the SERT protein is spread throughout the brain, compared with later stages of development where is it restricted to 5-HTergic fibres, where 5-HT is primarily a neurotransmitter (Buznikov, Lambert, \& Lauder, 2001; Narboux-Nême et al., 2008). Thus, during the early stages of development (i.e. when 5-HT plays a dominant role in neurodevelopment), the SERT appears to be primarily stimulated by maternal 5-HT levels. In order to circumvent the potential influence of altered maternal behaviour on subsequent behaviour in the offspring, we opted, in our study to mate SERT ${ }^{+-}$males with normal SERT $\mathrm{SE}^{+/+}$ females. These mothers had normal levels of 5-HT, but the 5-HT influence on the pups differed, as $\mathrm{SERT}^{+/-}$pups would have been less affected. Therefore, research that follows this thesis should 
aim to include SERT ${ }^{+/-}$mothers, and perhaps include cross fostering after birth, as this would control for potential changes in maternal behaviour and the subsequent influences on the pups.

It is also important to remember that prenatal exposure to VPA not only increases 5-HT in the dam (Löscher, 1999) but can also cross the blood placental barrier (Narita et al., 2002; Nau et al., 1984), where it likely increases 5-HT levels in the pups (possibly in a SERT genotypedependent manner). Taken all together, a highly complex picture emerges comprising of different SERT genotypes of mothers and pups (leading to different levels of 5-HT) combined with the effects of VPA which are likely dependent on both the maternal and foetal genotype as well. Moreover, specifically related to the continuous treatment of VPA used in the present thesis, the effects are likely to differ between the early (before the foetuses produce their own 5-HT) and the later stages of development (when SERT is restricted to 5-HTergic fibres). It is therefore perhaps not surprising that the exact interaction between the SERT and VPA could not be resolved in the present thesis.

Intriguingly, some research has suggested that a genetic increase in SERT activity may also lead to ASD-like changes. Veenstra-VanderWeele et al. (2012), for instance, used the SERT knock-in mice, where a single amino acid Gly (at position 56) was converted to Ala, that led to enhanced SERT activity and increased 5-HT clearance in the synapse. Like the s-allele of the SERT, this genetic variation has also been shown to be linked to ASD (Glatt et al., 2001; Sutcliffe et al., 2005). Interestingly, the homozygous SERT ${ }^{\text {Gly56Ala }}$ knock-in mice exhibited no preference for social or non-social stimuli in an SAA paradigm as well as decreased pre-weaning USVs, thus exhibiting an ASD-like phenotype. Together, this suggests that there may be an optimal level of 5-HT for typical development, and both too little or too much would lead to ASD-like behavioural changes. As such, the SERT ${ }^{+-}$genotype may have maintained 5-HT levels within the optimal levels, thus not providing enough of tipping point for the ASD animal model.

While, as mentioned above, VPA affects 5-HT levels, the interaction is complex, and it might even be the case that VPA actually induced effects on 5-HT that are opposite of those induced by the SERT genotype. While in the present thesis, we did not find many indications for that, Peter Ranger, in his thesis did find a few examples where the $\mathrm{SERT}^{+/}$genotype seemed to protect against the effects of a single VPA injection (e.g. SERT+/- rats prenatally exposed to VPA showed increase in social behaviours compared with SERT deficit or VPA exposure alone; Ranger, 2016). Future research should look therefore look at different genetic models that impact 5-HT availability (including the SERT ${ }^{\text {Gly56Ala }}$ knock-in model) in combination with the 
prenatal VPA exposure. This would allow us to answer the question of whether there is an interaction between genetically altered 5-HT and prenatal exposure to VPA.

\subsubsection{Contribution of the Prenatal VPA Exposure to the ASD Animal Model}

The chronic prenatal exposure to VPA led to a mild ASD-like phenotype. Chapter two findings showed that prenatal exposure to VPA at 200 and $300 \mathrm{mg} / \mathrm{kg} /$ day disrupted communication maturation, increased active behaviours, and social novelty aversion. Chapter three demonstrated that increased doses led to more consistent alterations in behaviour. The offspring prenatally exposed to VPA in chapter three exhibited disruptions in the communication, and increased chasing behaviour. Importantly, chapter three showed that prenatal exposure to VPA clearly disrupts the social preference formation and approach to social stimuli. Interestingly, the prenatal exposure to VPA did not have consistent impacts on the PV+ interneurons in the amygdala and hippocampus, as seen in chapter four.

As previously established, a single prenatal exposure to VPA leads to ASD-like deficits; therefore, it was expected that chronic exposure and increased doses would have a greater impact on communication and social behaviour deficits. While ASD-like deficits were identified, it appears that chronic VPA exposure leads to different (and even opposite) effects compared to a single exposure. Previous studies that used a single prenatal exposure found that the offspring exhibited decreased play behaviour (Olde Loohuis et al., 2015; Schneider \& Przewlocki, 2005) and reduced social stimulus visits (Dufour-Rainfray et al., 2010). On the other hand, experiments in this thesis showed that the prenatal exposure to chronic doses leads to increased play behaviour paradigm (figures 2.7 and 3.6), as well as an overall preference for the social stimulus (figures 3.10 and 3.11). As such, the chronic administration VPA during gestation differentially impacts behaviour, compared with acute prenatal exposure.

It is unclear what the exact cause is of the differential effects. One consideration is VPA's effects on GABA in the prenatal brain. In particular, VPA crosses the placental barrier leading to direct VPA exposure in the offspring (Nau et al., 1984), leading to increases in GABA in the foetus and (Löscher, 1982). It is possible that chronic exposure to VPA continuously increases GABA in the foetus, which may lead to habituation. Acute exposure to VPA, on the other hand, is unlikely to cause such effects as the single administration leads to a spike in GABA that is normalised over time (Nau \& Loscher, 1984; Nau et al., 1984), which poses a significant challenge for the foetus but is unlikely to cause cellular regulation changes. If chronic VPA exposure indeed led to habituation of its effect on the GABAergic system, this may also explain why this study failed to see a change in GABAergic interneurons. 
Another consideration related to the chronic prenatal exposure to VPA is the epigenetic changes of VPA. The teratogenic mechanism of VPA is attributed to its ability to inhibit histone deacetylase (HDAC), typically leading to increased gene expression in the developing foetus (Gurvich et al., 2005; Moldrich et al., 2013; Ornoy, 2009). This mechanism has been suggested to underlie the congenital malformations (Giavini \& Menegola, 2014) and spontaneous abortions typically associated with high levels of prenatal VPA exposure (Lloyd, 2013). Therefore, this HDAC inhibition may be a contributing factor to the loss of litters following exposure to $600 \mathrm{mg} / \mathrm{kg} /$ day (see table 3.1 ).

Interestingly, other malformations (tail kinks or fused digits) often associated with prenatal VPA exposure (Edalatmanesh et al., 2013; Favre et al., 2013; Foley et al., 2012; Nau et al., 1981; Vorhees, 1987) were never found in the experiments detailed in this thesis. The lack of characteristic malformation is perhaps related to the lower VPA doses, such that the doses were below the toxic levels to induce significant neurodevelopmental changes. However, the high dose $(350-410 \mathrm{mg} / \mathrm{kg} /$ day) in chapter three demonstrated that there were toxic effects for the embryos, with a 50\% loss of litters (table 3.1). An alternative explanation may be related to the bioavailability and the slower onset of the VPA plasma levels with ingestion of the gelatine pellets. Löscher (1999) identified the bioavailability for rodents to be $34-47 \%$, compared with 70-100\% in humans. Thus, rodents require a much higher dose to achieve comparable 'active' levels of VPA. Furthermore, oral gavage and IP/SC injection methods lead to much faster increases in plasma levels. It is possible that this more rapid increase in VPA in the offspring may underlie these obvious malformations. Reasoning along the same lines, the much slower (but stable) increase in VPA levels in the mother and pups may have allowed the brain of the offspring to adapt, which could be an explanation of the much more subtle behavioural changes observed in this thesis.

Additionally, the chronic administration may have initiated regulation effects (up- or down-regulation) which mitigate the teratogenic impact of HDAC inhibition. The continuous exposure to VPA during development may have up-regulated other HDACs, as VPA only inhibits class I HDACs, whereas there are four classes and nine other HDAC types (Krämer et al., 2003). The continuous administration during development may have up-regulated other mechanisms that regulate HDAC activity (such as protein-protein interactions, or posttranslational modifications; Hassig et al., 1998) which may have contributed to the mild presence of a milder phenotype. 


\subsection{ASD Animal Model Validity}

This thesis and all included experiments were aimed at advancing animal modelling of ASD. Different models were explored in chapter one (section 1.7), all leading to a specific focus on the VPA-induced animal model. The following section will explore the validity of the VPAinduced ASD animal model with the gene $\times$ environment approach demonstrated in this thesis.

Conventionally, the validity of animal models of psychiatric disorders has been assessed on three criteria. First, construct validity refers to how the disorder and its aetiology is manifested in the animal model (Hulbert \& Jiang, 2016; Willner, 1984). Second, face validity refers to the animal model's representation of the anatomical, behavioural, and biochemical characteristics of the disorder (Nestler \& Hyman, 2010). Third, predictive validity requires the model to respond to pharmacological treatments as a human would respond (Nestler \& Hyman, 2010). However, the third criterion is exceptionally difficult to assess at this time as there are no effective pharmacological treatments for ASD patients.

Overall, the VPA-induced ASD animal models that use an acute or single prenatal exposure to VPA to induced ASD-like behaviours exhibit weak construct validity, as the acute exposure is dissimilar to pregnant women ingesting VPA every day of gestation. The dissimilarities between acute exposure and the daily ingestion of medication dissociate the causal factors of the disorder, as a one-off challenge is distinct from continual exposure to a teratogen. The use of gelatine pellets has increased the construct validity of the VPA-induced ASD animal model. The gelatine pellets allow the dams to consume VPA voluntarily in a short timeframe, which can be considered comparable to the daily medication that women take while pregnant.

The construct validity of the VPA-induced ASD animal model has been furthered strengthened by the inclusion of the SERT genotype. As explored in chapter one, the genetic makeup of an individual with ASD likely has a critical role in the development and severity of their disorder. Even more so, are the alterations in the 5-HTergic system and the role it has in ASD. The gene $x$ environment interactions have been previously proposed in the VPA-induced animal model (Mabunga, Gonzales, Kim, Kim, \& Shin, 2015; Roullet et al., 2013), and very rarely the prenatal exposure to VPA is combined with a genetic vulnerability (Kim et al., 2019). To our knowledge, two studies have examined the behaviours of SERT animals prenatally exposed to VPA (Ellenbroek et al., 2016; Ranger, 2016). As such, genetic and environmental contribution to the VPA-induced ASD animal model has enhanced the construct validity of this model. 
The VPA-induced ASD animal model, with acute prenatal exposure to VPA, has strong face validity (Mabunga et al., 2015). The face validity is a criterion that assesses anatomical, behavioural, and biochemical characteristics of the disorder. The VPA-induced ASD animal model with the gene $\times$ environment approach in this thesis demonstrated some alterations in communication and social interaction. However, it was not possible to confirm anatomical underpinnings of the behavioural changes. Therefore, while the face validity of this model, at this point in time, might be somewhat limited, it makes an important contribution to the advancement for the VPA-induced ASD animal model with the gene $\times$ environment approach.

\subsection{Pushing Beyond the Expected}

This thesis has highlighted the need to go beyond the simple quantitative measures when examining the behavioural phenotypes in animal models of psychiatric disorders, particularly considering the heterogeneous nature of ASD. Published literature typically present relatively simple quantitative results. For instance in USV studies, most papers only report a total number of vocalisations (Bronzuoli et al., 2018; Melancia et al., 2018; Raza et al., 2015), rather than duration, or frequency measures (as displayed in section two of chapter two and chapter three; and see in Felix-Ortiz \& Febo, 2012). The results of this thesis suggest that much more information can be obtained by a more detailed characterisation of the calls. Wright et al. (2010) studied predominantly adult communication and classified USVs into at least 14 different types of call categories. Moreover, they showed that depending on the circumstances, only some of these calls may be affected. Using recently developed software, it is now possible to examine the sonographic features of these calls (Coffey et al., 2019). Preliminary studies indicate that prenatal VPA may, in fact, alter specific calls; thus, further research should be undertaken to identify the extent and impacted of altered USVs.

The play behaviour experiments in chapter two and three, further highlight the need to examine more than a single measure of behaviour. Studies have often reported only pinning behaviour (Olde Loohuis et al., 2015; Wang et al., 2013), rather than additional measures (as seen in section three in chapter two and chapter three; as well as Markram et al., 2008; Sandhya et al., 2012). Rodent play behaviour is another area where software developments are allowing for a more detailed analysis. Play behaviour is very ritualistic with chasing typically followed by wrestling followed by pinning followed by non-social behaviour. The findings of chapter three are suggestive of disrupted play behaviour sequences whereby the offspring exposed to VPA exhibited an increased chasing behaviour, yet there were no changes in pinning behaviours, which should have followed a chasing behaviour, and thus indicative of aberrant play behaviour 
sequences. Future research into chronic prenatal exposure to VPA should utilise software like Theme to examine the progression of behaviours (Casarrubea et al., 2015). Theme and other software like it examine the sequences or pattern of behaviour, thus confirming that chasing behaviour leads to pinning behaviour, and allows for analysis of other behavioural patterns. The examination of behavioural sequences is going beyond the quantification of pinning, pouncing, wrestling, and passive behaviour, and investigation the holistic behaviours.

Furthermore, chapters two and three demonstrated that the combined measure of sociability (derived from Brodkin et al., 2004) was more accurate as a stand-alone measure of the behaviour of the offspring, particularly in regards to SAA in chapter three (section 3.4.2, figures 3.9 and 3.12). It was the additional measures of duration with social stimuli that highlighted a more detailed pattern of disrupted social preference in the offspring that been exposed to VPA (figures 3.11 and 3.14). The social behaviours demonstrated in SAA could have been extended by examining the sniffing behaviour of the test animal. Sniffing behaviour has been deemed to be a more sensitive measure of sociability (Moy et al., 2008), and future studies should examine this behaviour as it provides a more direct measure of social interest and investigation.

Finally, the experiments in this thesis have examined sex differences, where possible. This thesis has demonstrated a different phenotype for males and females that were SERT ${ }^{+/+}$ and SERT ${ }^{+-}$and prenatally exposed to VPA. The findings in the play behaviour and SAA experiments demonstrate that males exposed to VPA show increased active play behaviours but tend to be ambivalent between social and non-social stimuli, or familiar and novel stimuli. Females, on the other hand, while showing similar active play behaviours, exhibited social aversion and a preference for social familiarity. These sex differences in social behaviours are reminiscent of findings in ASD populations, however, males and females exhibit different ASD profiles. Hull, Mandy, and Petrides (2017) identified that there are phenotype differences for male and female individuals that were diagnosed with ASD, with males exhibiting more physical behaviours (repetitive behaviours), whereas females exhibit more cognitive deficits. As such, future research into the VPA-induced ASD model, should examine males and females as the outcomes of the prenatal exposure to VPA leads to different behavioural outcomes.

Too often, animal models of psychiatric disorders only examine males, with the reason that the estrous cycle makes females too variable (despite evidence that indicates otherwise; Prendergast, Onishi, \& Zucker, 2014). Others have cited that the estrous cycle leads females to react differently to pharmacological interventions (Hughes, 2007). In 2014, the National 
Institute of Health (NIH) in the United States set forth a directive that grant applications in the pre-clinical field must include both sexes (Clayton \& Collins, 2014). The NIH policy change encourages a shift in convention and to ensure the pre-clinical research meets the needs of the population it aims to support. This thesis has examined beyond the conventional the male-only view and shown differential effects in the males and females.

\subsection{Limitations within this Thesis}

This thesis has focused on chronic exposure to VPA throughout gestation, as a method for inducing ASD-like behaviours in rats. The prenatal exposure to VPA provides the environmental factor and is derived from unfortunate events in the 1980s when women were taking this medication while pregnant. As described in chapter one, when women ingest this medication while pregnant, their children are at a higher risk of developing ASD (Christensen et al., 2013). As such, the environmental situation modelled in this thesis is relevant for a subset of individuals that were exposed to VPA (or tetragons) and have alterations in the 5-HTTLPR gene. That is, the prenatal VPA exposure, this is not a causal link, and the same is likely true for rodents that exposed to VPA throughout gestation. That is, the prenatal exposure to a pregnant rat, does not cause ASD in the offspring but rather increases the risk. Some of the offspring may exhibit more ASD like behaviours than their siblings, and it may also be dose or exposure dependent (as explored in the discussion of chapter three, section 3.7). I suggest this is an underlying cause of the high variability seen the behavioural assays in this thesis.

Furthermore, the animal model described in this thesis has been limited by the amount of VPA the dams would voluntarily and reliably consume. As described earlier in this chapter, all attempts were undertaken to increase the doses while maintaining the health of the dams to get the offspring to full gestation. Without further compounding techniques, it is unlikely that chronic oral doses could be increased to equivalent levels found with injection methods. It may be possible to suspend VPA in a compound to prevent the dam's experience of the unpleasant taste, and pharmaceutical compounding would be a worthy avenue to investigate for research following this thesis.

While this thesis and experiments have sought to advance the animal model of ASD, some restrictions prevent the progress of this model. ASD is a highly heterogeneous and complex disorder, as such, it is difficult to encapsulate the vast range of ASD in an animal, or to model the full spectrum of disorders within a single gene $\times$ environment approach. As established in chapter one, the manifestation of ASD is more likely due to complex interactions of multiple genes and environmental influences, both prenatal and postnatal. The complexity 
of ASD is an inherent limitation for ASD animal models, however, it is important and relevant to continue to explore the contributing factors to ASD, and the interactions that underlie the complexity. The continued expansion of knowledge allows for exploration of new treatment and intervention options at the pre-clinical and clinical level, for a growing population that is so severely impacted by a complex disorder.

\subsection{Future Prospects Beyond this Thesis}

It has been more than 75 years since ASD was first described, and while our understating of the symptoms and neurological underpinnings has evolved, we are still unravelling the complexities of this disorder. It is clear that ASD is the result of complex genetic and environmental interactions. The complex interactions need to inform the animal models of ASD, not only to further our understanding of aetiology (South et al., 2017) but to aid in the pre-clinical trials for new pharmaceutical treatments (Caraci et al., 2017). As we move forward, it is important and relevant to consider the complex interactions that lead to ASD, rather than the focus on isolated contributing factors.

Moreover, we need to examine the underlying assumptions we make as pre-clinical researchers and the approach we take to developing animal models. In the case of ASD, the assumption is that the animal model will exhibit deficits in communication and social behaviour with repetitive behaviours and interests, which are the core symptoms of ASD. This assumption focuses on the requirement that all core symptoms must occur together, at the exclusion of cooccurring disorders like anxiety or epilepsy. In complying with this assumption, research overlooks findings on the individual symptoms, for example, social deficits. A comprehensive analysis of social deficits could inform interventions that apply to ASD and associated disorders. A symptom-based approach would have implications for multiple disorders, rather than findings that are deeply rooted in a single disorder.

In 2010, the National Institute of Mental Health (NIHM) in the United States, launched the Research Domain Criteria ( $\mathrm{RDoC}$ ) project (Insel et al., 2010). The RDoC project developed a research framework for examining disorders like ASD, in a way that integrates multi-level information (genetics and behaviour, to self-reported data from individuals with ASD). While the $\mathrm{RDoC}$ has its own assumptions, it has moved away from the restrictive nature of disorderbased approaches, and more into the symptom-based approach. As the RDoC has helped to conceptualise disorders in a dimensional manner, which has allowed re-grouping of symptoms that had not previously been considered, such as the commonality of working memory deficits in schizophrenia and bipolar (Schwarz, Tost, \& Meyer-Lindenberg, 2016). However, RDoC has 
also been met with criticism and disagreement (Weinberger, Glick, \& Klein, 2015). In particular, Ross and Margolis (2019) disagree with RDoC's assumption that disorders (like ASD) are a deviation from neurotypical, and perhaps with correct interventions, the individual would return to neurotypical functioning. Ross and Margolis (2019) suggest that disorders are the result of complex interactions that fundamentally change neuronal function, and thereby altering cognition, emotion, and behaviour, which may never return to neurotypical function. Despite, the criticism of RDoC, it provides another lens through which pre-clinical researchers can assess and understand findings from animal research. 


\subsection{Closing Remarks}

This thesis sought to advance the animal model of ASD and further the construct validity of the model. To this aim, genetically normal rats were exposed to VPA throughout gestation, then their communication and social behaviour were examined. The data showed that the administration method had some effects, with animals showing communication maturation delays, increased active behaviours, and social novelty aversion. This was expanded upon, with

the use of genetically compromised SERT animals. SERT ${ }^{+/+}$females were exposed to VPA throughout gestation, with a more controlled method of administration. Their SERT ${ }^{+/+}$and SERT $^{+/-}$offspring exhibited a mild ASD-like phenotype, particularly with the alterations in communication and the social aversion. The social processing regions of the offspring brains were also examined, and the findings do not show consistent changes in the PV+ interneuron levels in the amygdala and hippocampus (in late adulthood); thus, alterations in the PV+ or GABAergic system is unlikely to be the underlying cause of the behavioural changes demonstrated in chapter three. Ultimately, this thesis has furthered the VPA-induced ASD animal model with a gene $\times$ environment interaction approach, as well as optimising the administration method to align with the situation where women ingest VPA throughout pregnancy. Research following this thesis should attempt higher VPA doses with the gelatine pellets and continue to develop the gene $\times$ environment interaction approach to delineate the exhibited phenotype, with a focus on a symptom-based lens for assessing and reviewing the findings. 


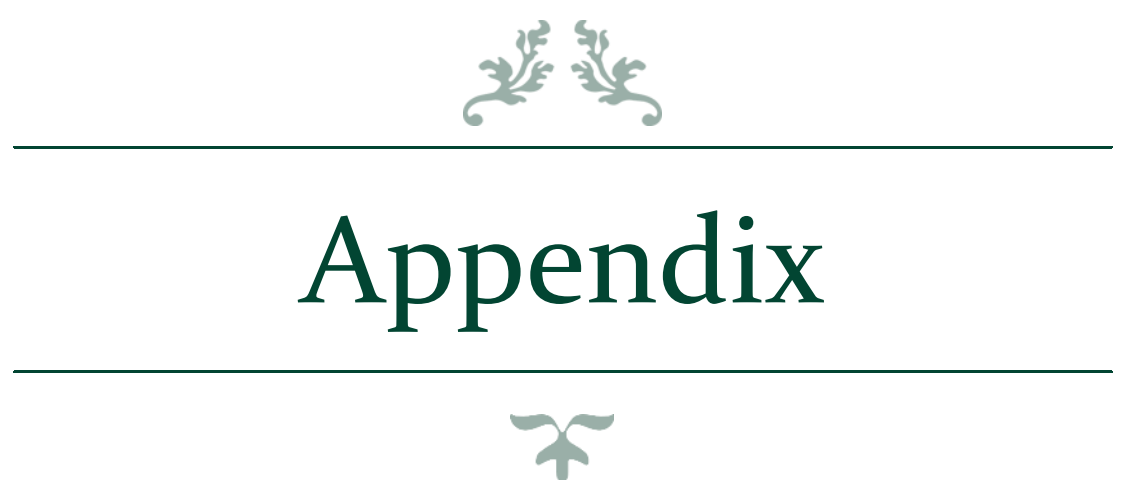




\section{Appendix A - Information Relating to Chapter Three}

\section{Al. Play Behaviour with Genotypes included}

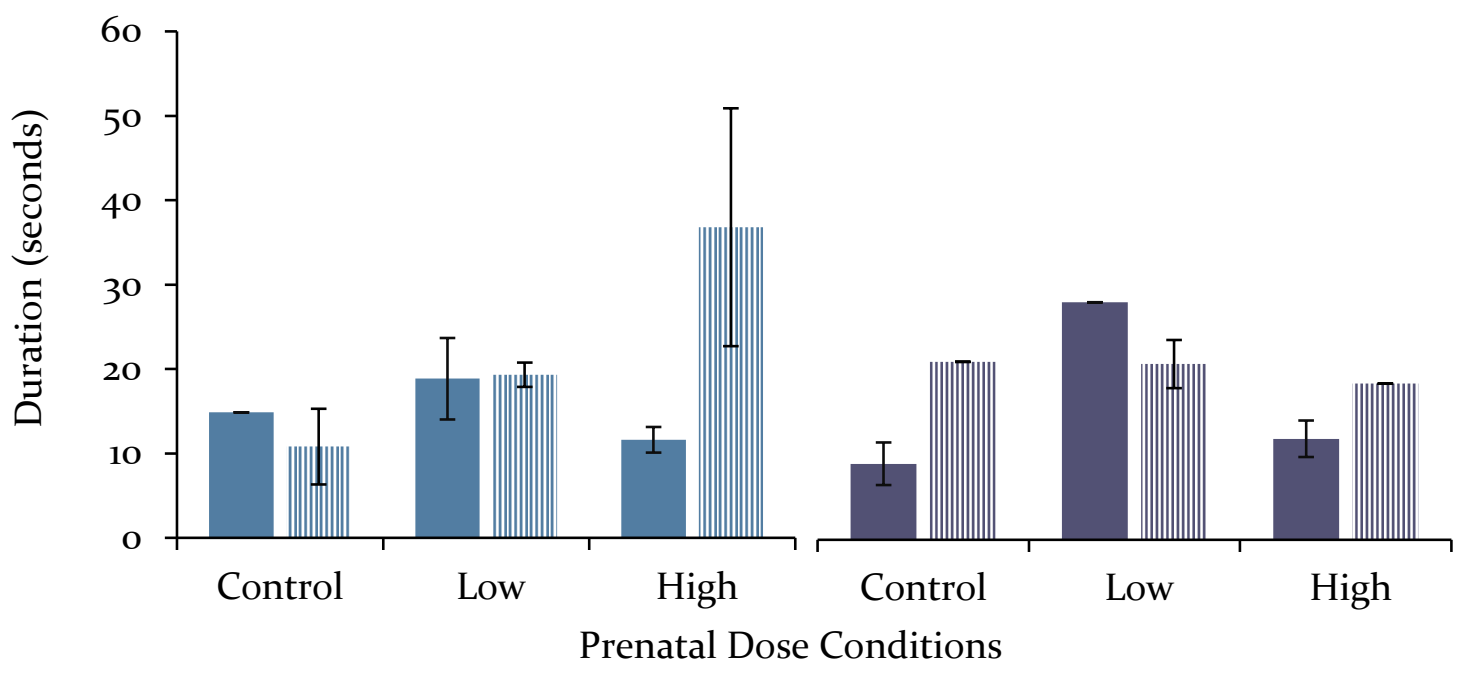

nSERT+/+ pairs IIIISERT+/- pairs —SERT+/+ pairs IIIISERT+/- pairs

Figure A1.1. The average chasing duration of males (blue) and females (purple) separated by prenatal dose conditions and genotype pairs. Error bars represent \pm SEM, and conditions without error bars indicate a single pair.

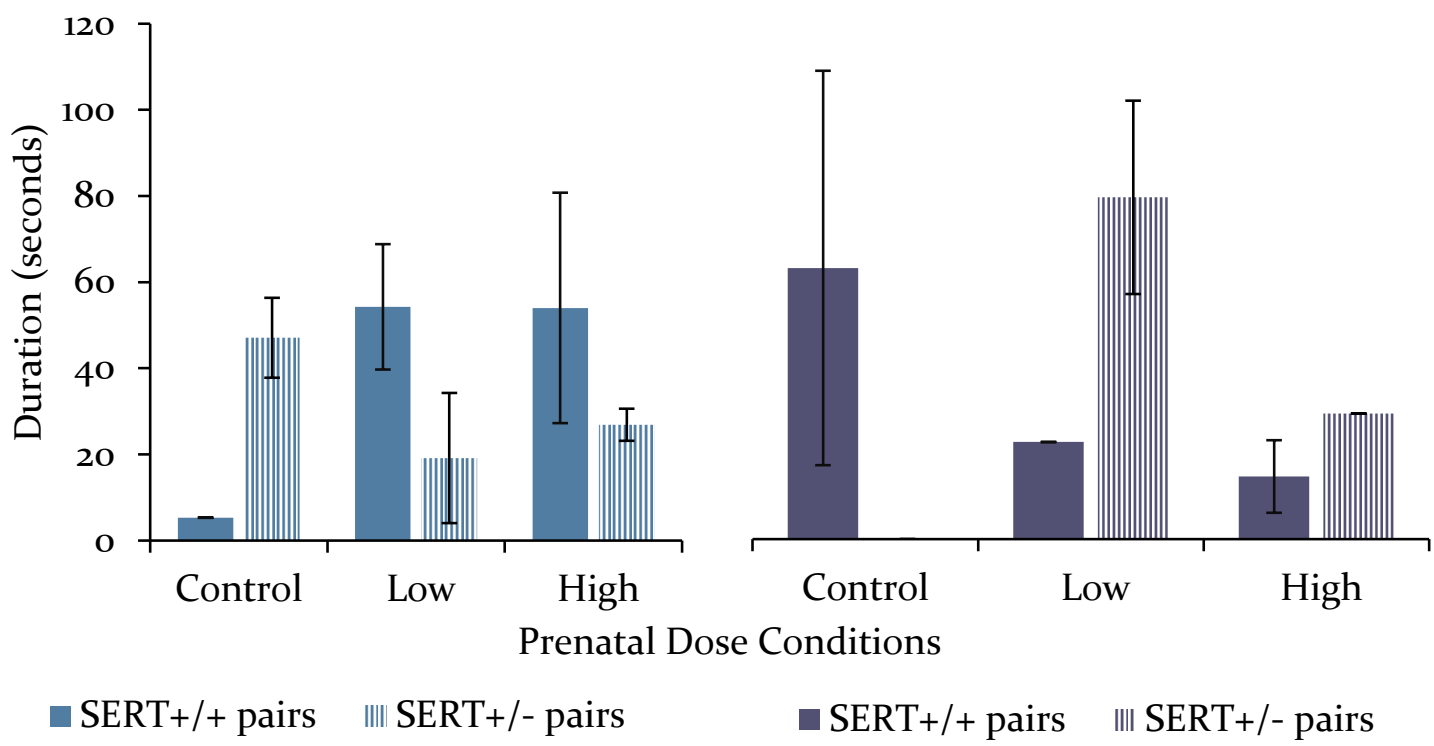

Figure A1.2. The average pinning duration of males (blue) and females (purple) separated by prenatal dose conditions and genotype pairs. Note, there was no recorded pinning behaviour for the female SERT ${ }^{+/} \mathrm{SERT}^{+/-}$control dose pairs. Error bars represent \pm SEM, and conditions without error bars indicate a single pair. 


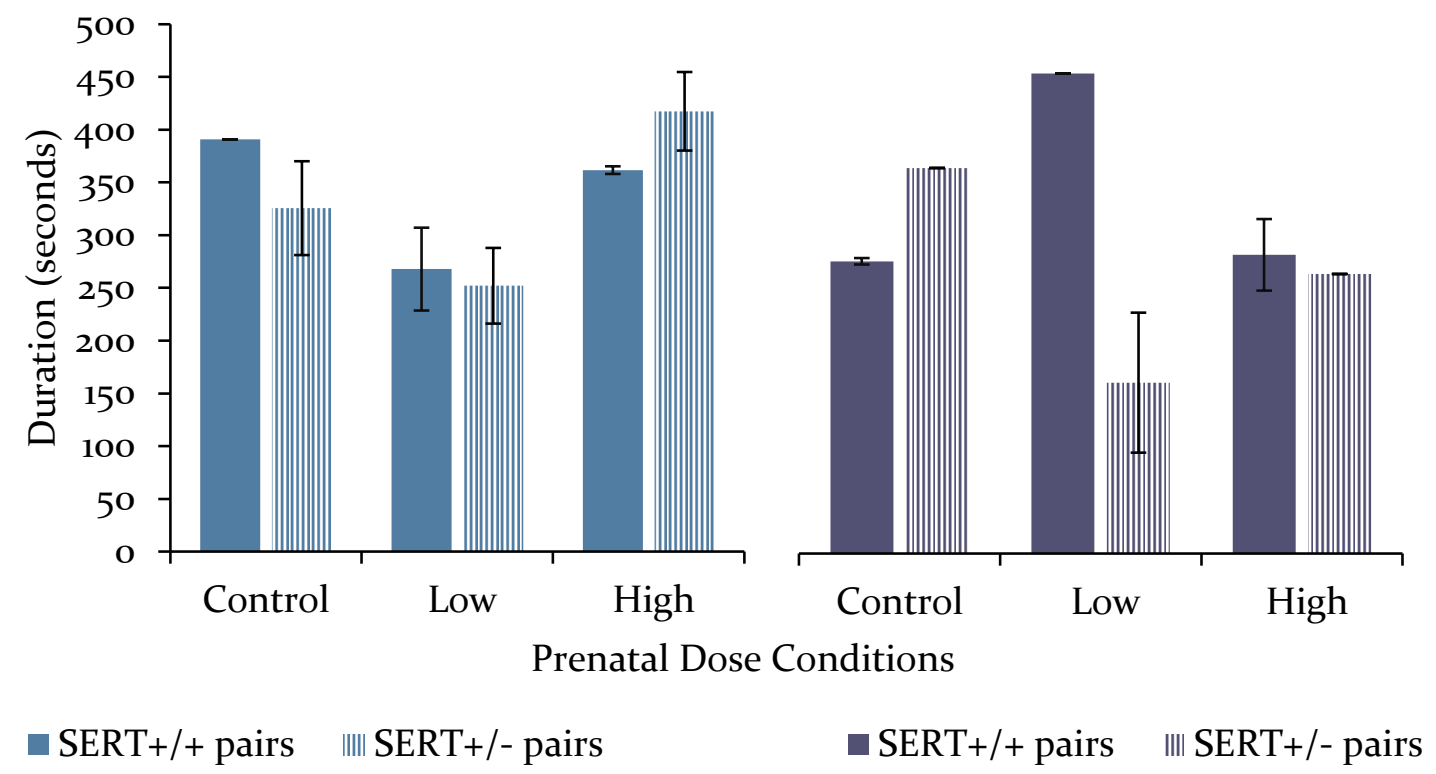

Figure A1.3. The average passive duration of males (blue) and females (purple) separated by prenatal dose conditions and genotype pairs. Error bars represent \pm SEM, and conditions without error bars indicate a single pair. 


\section{A2. SAT Results}

Relating to section 3.5.5.1 - SAT males latency to entry alleys.

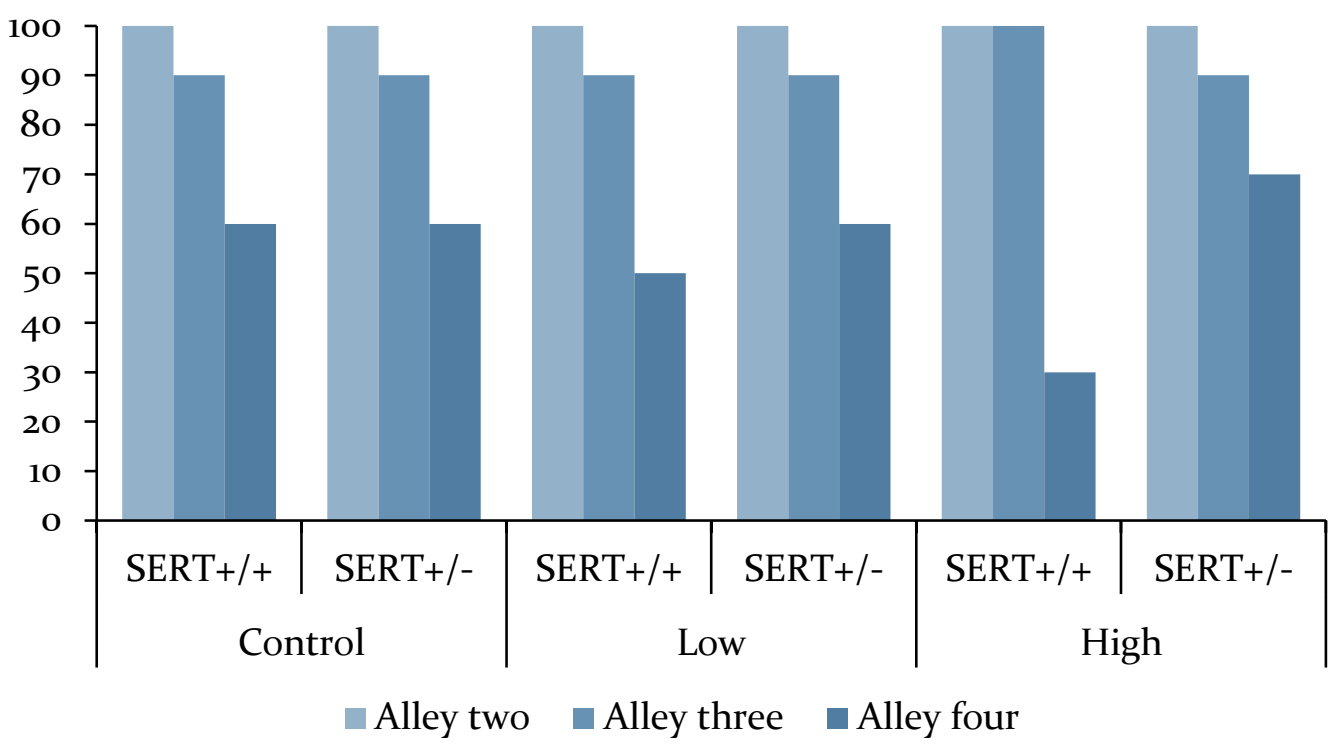

Figure A2.1. The percentage of male offspring in alleys 2, 3, \& 4 separated by prenatal doses and genotypes. All rats are placed in alley one (therefore 100\%) and all rats move from alley one into two, but fewer rats enter alley three and even less enter for alley 4 . Each condition has significantly fewer animals in alley four compared with alley 1 . (no error bars due to a comparison of total percentage in each alley).

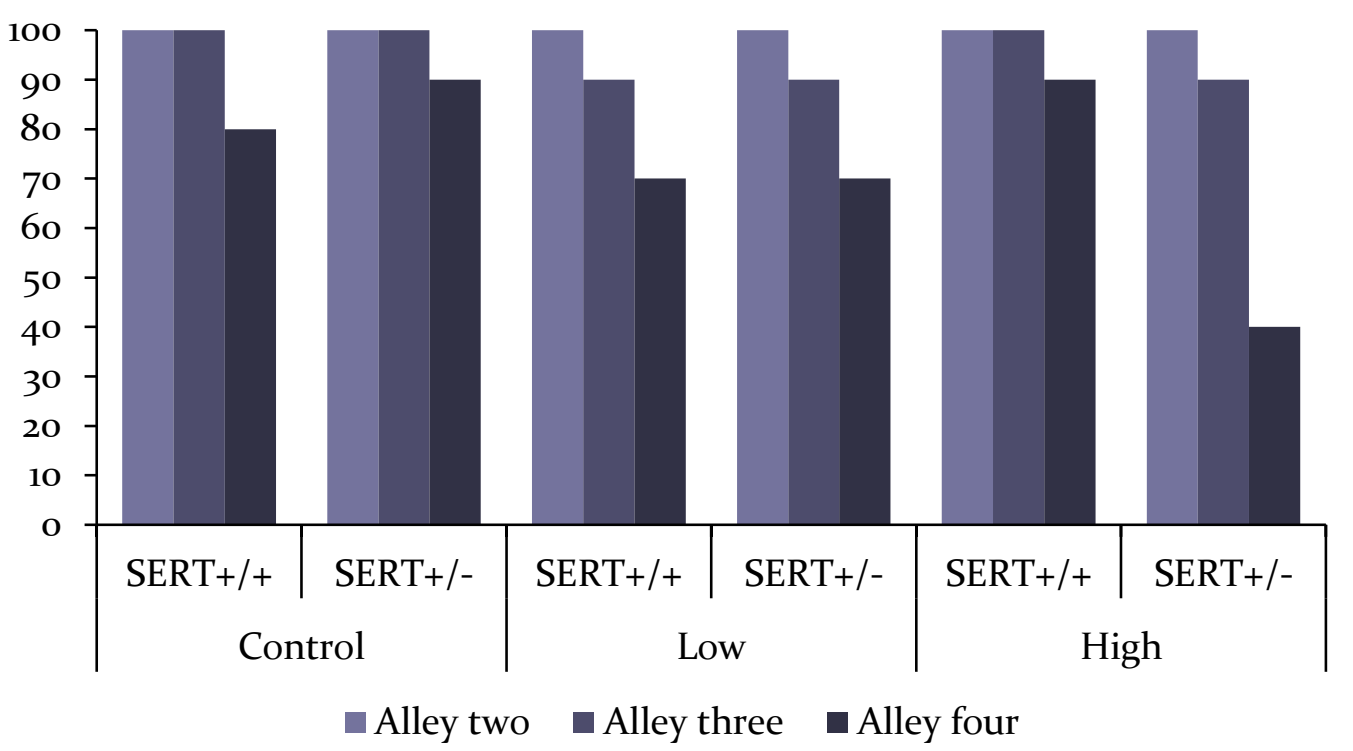

Figure A2.2. The percentage of female offspring in alleys 2,3 , \& 4 separated by prenatal doses and genotypes. All rats are placed in alley one (therefore 100\%) and all rats move from alley one into two, but fewer rats enter alley three and even less enter for alley 4 . Only low and high dose conditions had significantly fewer animals in alley four compared with alley $1(p<.05)$. (no error bars due to a cumulative totals). 
Table A1.1. Male SERT ${ }^{+/+}$and SERT ${ }^{+/-}$follow up comparisons of delayed entry into alleys 2, 3, and 4. Confirming the overall increase in latency. ${ }^{*}$ refers to $p<.05$ and ${ }^{* * *}$ is $p=.001$.

\begin{tabular}{c|c|c|c|c|}
\hline \multirow{2}{*}{ Control } & SERT+/+ & Alley 2 v & Alley 3v 4 & Alley 2 v 4 \\
& SERT+/- & - & - & $*$ \\
\hline \multirow{2}{*}{ Low } & SERT+/+ & - & $*$ & $* * *$ \\
& SERT+/- & - & - & $*$ \\
\cline { 2 - 5 } High & SERT+/+ & $* * *$ & - & - \\
\cline { 2 - 5 } & SERT+/- & - & $* * *$ & $* * *$ \\
\cline { 2 - 5 } & & & - & $*$ \\
\hline
\end{tabular}

Table A1.2. Female SERT ${ }^{+/+}$and SERT ${ }^{+/}$follow up comparisons of delayed entry into alleys 2,3 , and 4. Confirming the overall increase in latency. ${ }^{*}$ refers to $p<.05$ and ${ }^{* * *}$ is $p=.001$.

\begin{tabular}{|c|c|c|c|c|}
\hline & & Alley 2 v 3 & Alley 3 v 4 & Alley 2 v 4 \\
\hline \multirow[t]{2}{*}{ Control } & SERT+/+ & - & - & - \\
\hline & SERT+/- & - & - & - \\
\hline \multirow[t]{2}{*}{ Low } & SERT+/+ & - & - & * \\
\hline & SERT+/- & * & * & $* * *$ \\
\hline \multirow[t]{2}{*}{ High } & SERT+/+ & - & - & $* * *$ \\
\hline & SERT+/- & - & - & - \\
\hline
\end{tabular}




\section{Appendix B - Information Relating to Chapter Four}

\section{A4.1 Original Protocol for Parvalbumin Staining}

All washes and incubations were performed in 6-well plates with mesh well inserts, and at room temperature $\left(20-22^{\circ} \mathrm{C}\right)$ on a gently rocking platform to produce mild agitation.

The tissue was washed three times for five minutes each in $10 \mathrm{mM}$ phosphate buffered saline Triton X-100 (PBST). The tissue was bathed in 3\% hydrogen peroxide $\left(\mathrm{H}_{2} \mathrm{O}_{2}\right)$ for 10 minutes to prevent endogenous peroxidase activity, then the tissue was washed three times for five minutes in PBST. The tissue was then blocked with $1 \%$ bovine serum albumin (BSA) in PBST for 60 minutes to reduce non-specific background staining and then washed three times for five minutes in PBST. Next, the tissue was incubated in the primary anti-parvalbumin mouse (Sigma-Aldrich, Auckland, New Zealand; \#P3088) antibody diluted 1:5000 in 1\% BSA in 1 x PBST overnight, and the next morning the tissue was washed three times for five minutes in PBST. Then, the tissue was incubated for 90 minutes with biotinylated goat anti-mouse antibody (Vector laboratories, Inc., Burlingame, CA, USA; \#BA-9200) diluted 1:1000 in PBST and washed three times for five minutes in PBST. Following the secondary antibody, the tissue was incubated for 60 minutes with avidin-biotin-peroxidase complex (ABC; Vector Laboratories, Inc., Burlingame, CA, USA) diluted 1:1000 in PBST to increase the signal. The tissue was washed three times for five minutes in PBST. The final step was to incubate the tissue in 3,3'diaminobenzidine (DAB), nickel(II) chloride $\left(\mathrm{NiCl}_{2}\right)$ and, $0.3 \% \mathrm{H}_{2} \mathrm{O}_{2}$ in Tris $\mathrm{HCl} 50 \mathrm{mM}$ buffer $\mathrm{pH}$ 7.4, for 30 minutes. The resulting reaction allowed visualisation of PV+ cells. The tissue was washed three times for five minutes in PBST.

Every batch included a tissue nominated as the negative control, and the tissue was subjected to every treatment except the primary antibody at this step to ensure the visible staining result is a result of the primary antibody. 


\section{A4.1 Baseline \& Initial Testing}

Baseline testing showed that the staining was exceptionally pale in comparison to previous work in our laboratory and the literature. It was difficult to detect PV+ cells. Neutral red (figure A4.1 C and D) and haematoxylin (figure A4.1 E and F) were tested, and the tissue was stained for 30 or 60 seconds (rather than the original protocol which called for two minutes).

$\mathrm{PV}+$ cells were more clearly identified in the absence of the counterstain (figure A4.1, AB). Neutral red and haematoxylin diminished the clarity of the PV staining, which reduced the visibility of the cell bodies and axons. Additionally, positive staining was detected in the negative control tissue, which was attributed to omission of the pre-quenching step (not shown). Following protocol would ensure the use of pre-quenching with $\mathrm{H}_{2} \mathrm{O}_{2}$ is required to remove the endogenous peroxidases.
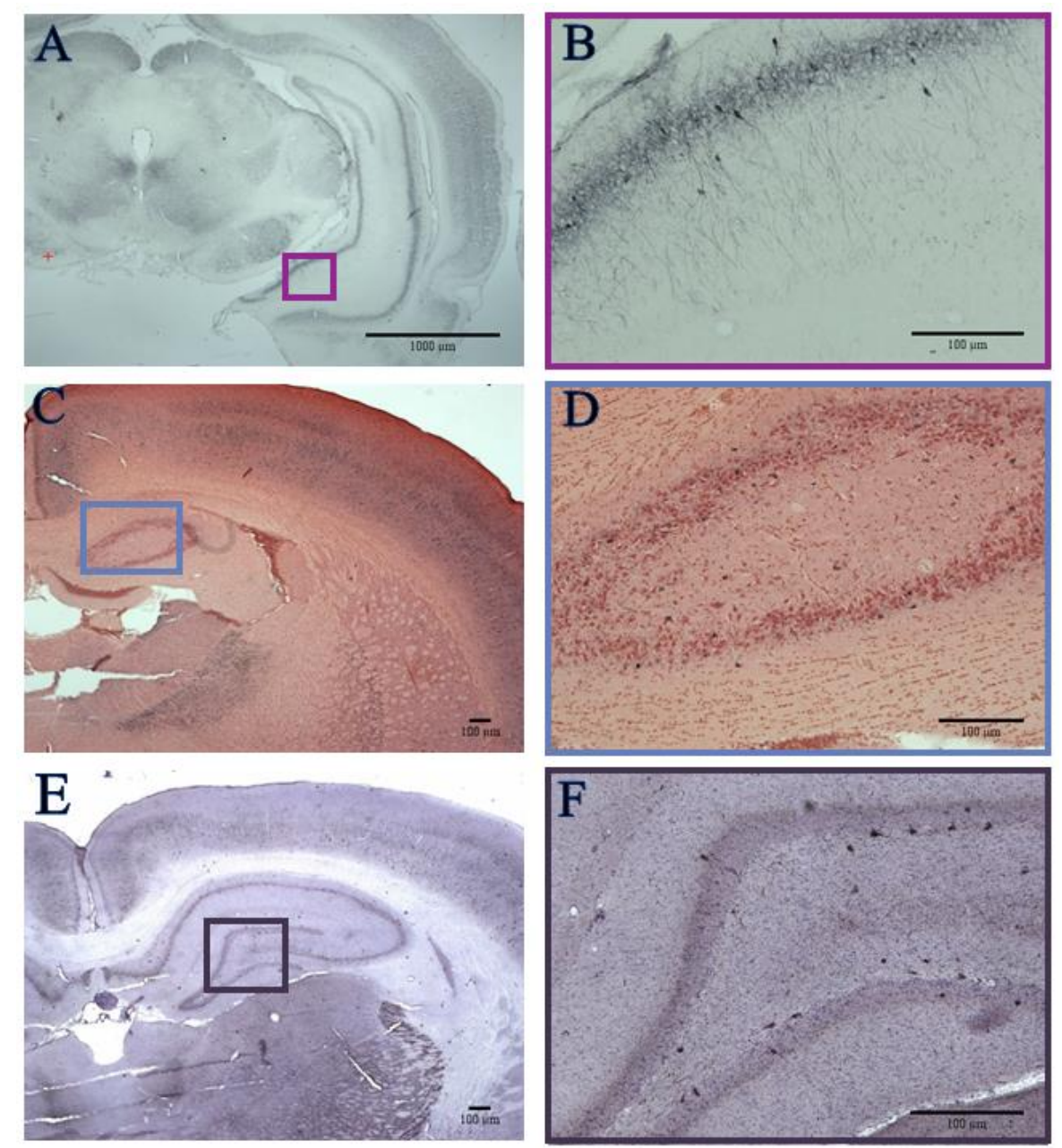

Figure A4.1. Representative image of the counterstain test. Images A (1.25x objective with scale bar $1000 \mu \mathrm{m})$ and B (10x with $100 \mu \mathrm{m}$ scale bar) are without counterstaining and showing clear cell bodies in the lateral posterior CA3. Image C (2x) and D (10x) display PV+ staining with neutral red counterstain for 30 seconds. Image $\mathrm{E}(2 \mathrm{x})$ and $\mathrm{F}(10 \mathrm{x})$ display PV+ cell staining with haematoxylin as the counterstain. 


\section{A4.2 Pursuit of Darker Staining}

The original protocol stipulated that the primary antibody (I-ab) concentration should be 1: 5000 . To ensure this was the optimal concentration, the I-ab was tested at increased (1: 3500 ) and decreased concentrations (1:10,000, and 1:20,000). Additionally, the DAB revelation time was originally 30 minutes, and this was reduced to 10 minutes.

Higher I-ab concentrations led to dark and dense staining (figure 4.2 A-D). The lower primary antibody concentration appeared to be slightly better (Figure $4.3 \mathrm{C}$-D). The 30 minute $\mathrm{DAB}$ revelation was too long (figure A4.3 A-D), and it was possible that 10 minutes was too long as well (figure A4.3 A-H).
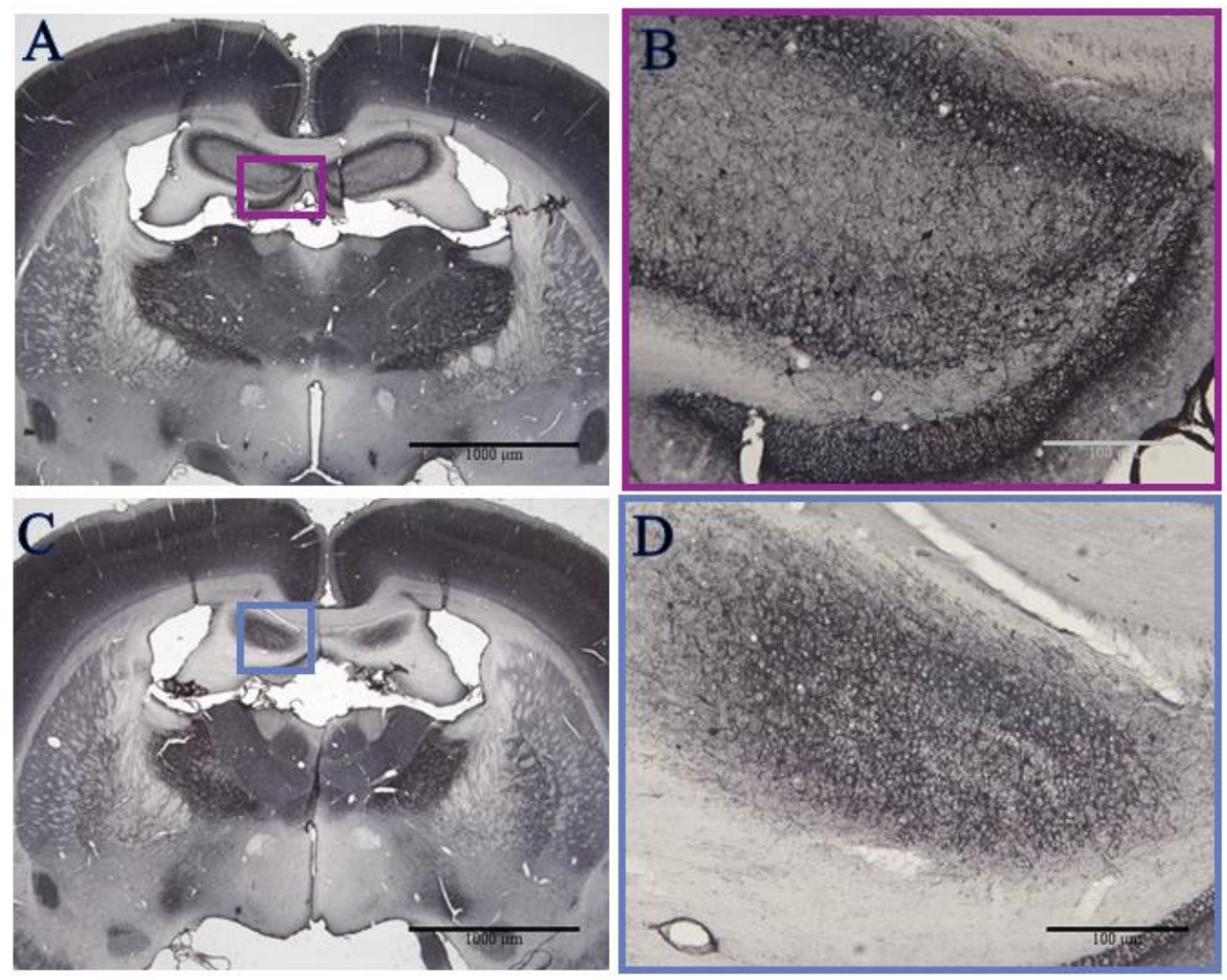

Figure A4.2. Images from tissue with primary antibody concentration testing. Tissue was exposed to DAB for 30 minutes. Primary antibody concentration of 1:3500, (A; 1.25x objective with $100 \mu \mathrm{m}$ scale bar and $\mathrm{B}$; hippocampus $10 \mathrm{x}$ with scale bar $1000 \mu \mathrm{m})$. A lower concentration of 1:10,000 show little change in density (C and D). 

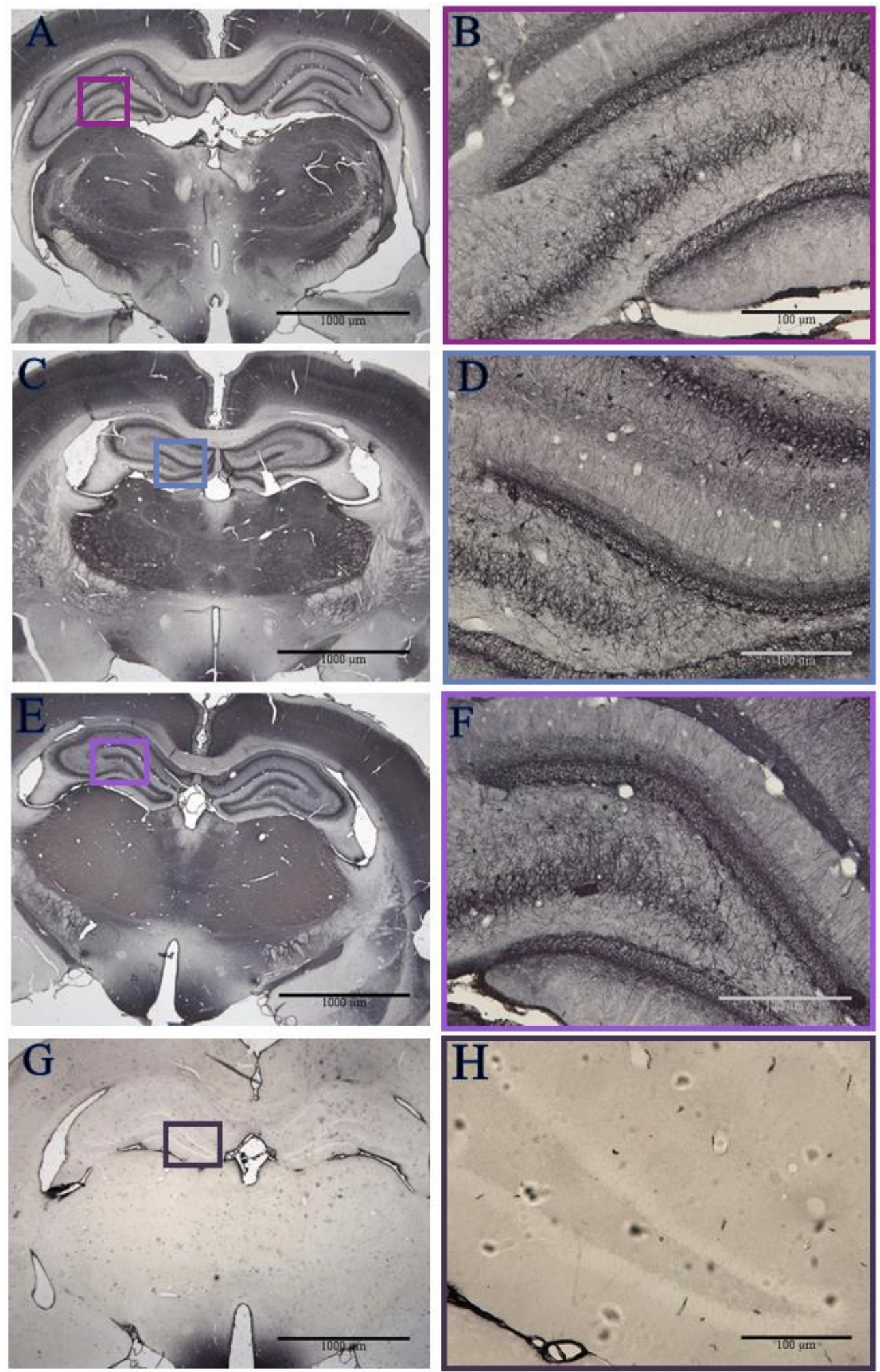

Figure A4.3. Representative images for primary antibody concentration testing. All tissue was exposed to DAB for 10 minutes. Primary antibody concentration of 1:3500, (A; 1.25x objective and $\mathrm{B}$; hippocampus 10x). A lower concentration of 1:10,000 show little change in density (C and $\mathrm{D}$ ). Also 1:20,000 shows clear cell and dendrite staining ( $\mathrm{E}$ and $\mathrm{F}$ ). The negative controls are revealed some endogenous reactivity $(G$ and $H)$. A, C, E, G were captured at $1.25 \mathrm{X}$ magnification with scale bar $1000 \mu \mathrm{m}$, and B, D, F, H were captured at 10X objective with $100 \mu \mathrm{m}$ scale bar. 


\section{A4.3 Further Pursuit of Darker Staining}

The concentration of the secondary antibody (II-ab) was verified with a comparison of 1: 1000 vs $1: 2000$ while maintaining the primary antibody concentration of $1: 10,000$ and DAB for 10 minutes. The II-ab concentration of 1:2000 resulted in dense staining of the neuronal branches and background signal (figure A4.4 A-D).
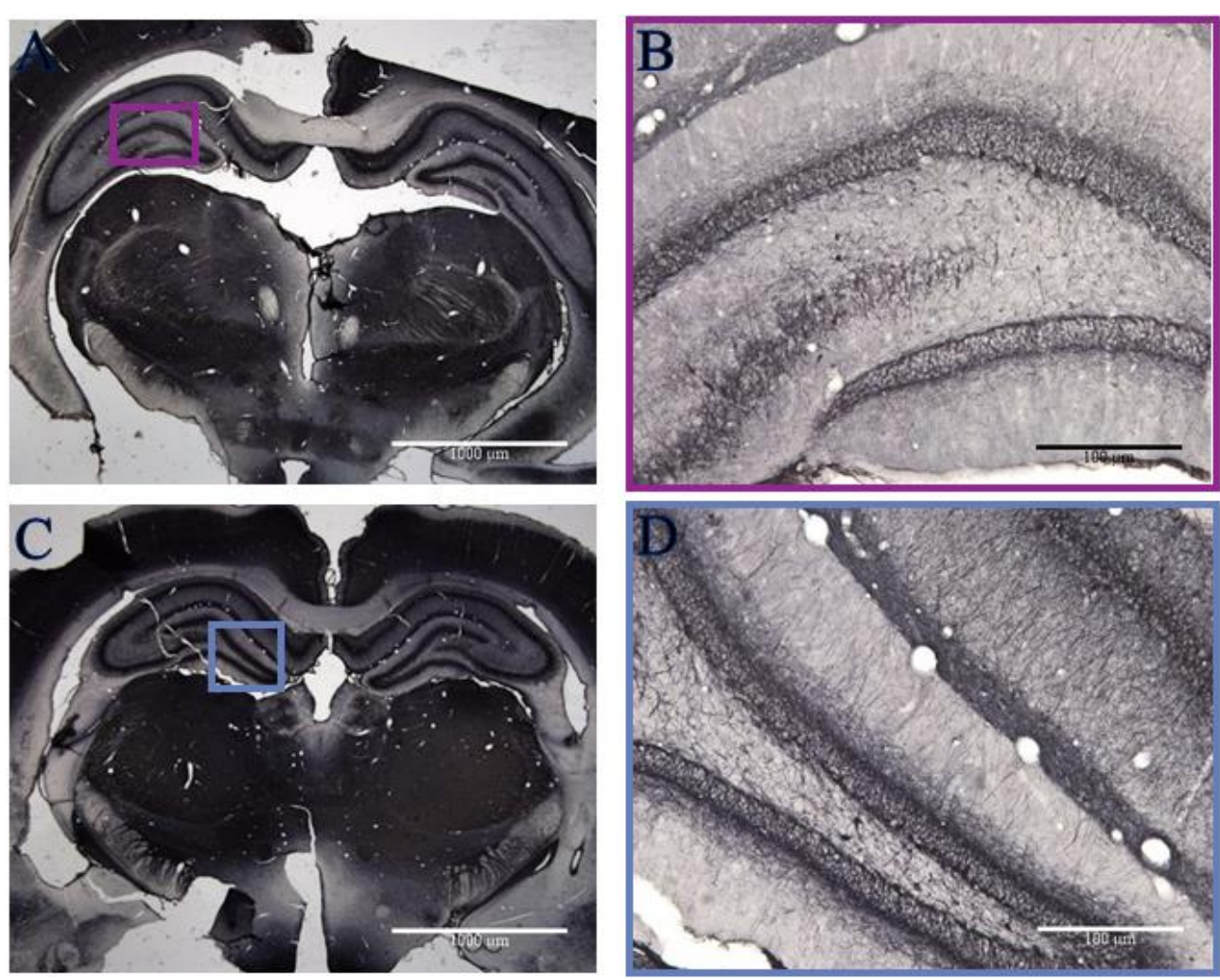

Figure A4.4. Images from tissue with secondary antibody concentration testing. All tissue in this figure was exposed to the primary antibody concentration of 1: 10,000 and DAB for 10 minutes. The original concentration of secondary antibody concentration of 1:1000 (A; 1.25x objective with $1000 \mu \mathrm{m}$ scale bar and B; hippocampus $10 \mathrm{x}$ objective with $100 \mu \mathrm{m}$ scale bar) is compared with a lower concentration of 1:2000 show no differences in cell staining or density (C and D). 


\section{A4.1.8 Primary Antibody Concentration Test \& DAB Strength}

So far, the optimisation testing had led us to confirm that tissue will be pre-quenched with $\mathrm{H}_{2} \mathrm{O}_{2}$, with I-ab concentration of 1:10,000, the II-ab concentration of 1:1000 and tissue would be incubated in DAB for 10 minutes, along with no counterstaining. These steps resulted in staining that was still dark and dense, therefore difficult to easily identify the cell bodies in the cortex and denser regions of the hippocampus.

Therefore, we tested I-ab concentrations of 1: 10,000 (to serve as the baseline), 1: 80,000 and 1: 160,000. At each of these concentrations, we also tested the DAB dilution of 50 or $100 \%$. Overall the results showed clear consistent dark staining. Lowering the concentration of the PV antibody did not reduce the strength of the stain (figure A4.5 E-H and figure A4.6 A-D). A half dilution of $\mathrm{DAB}$ allows for better visualisation of the cells in the denser regions of the hippocampus (figure A4.5 C-D, G-H and figure A4.6 C-D). 

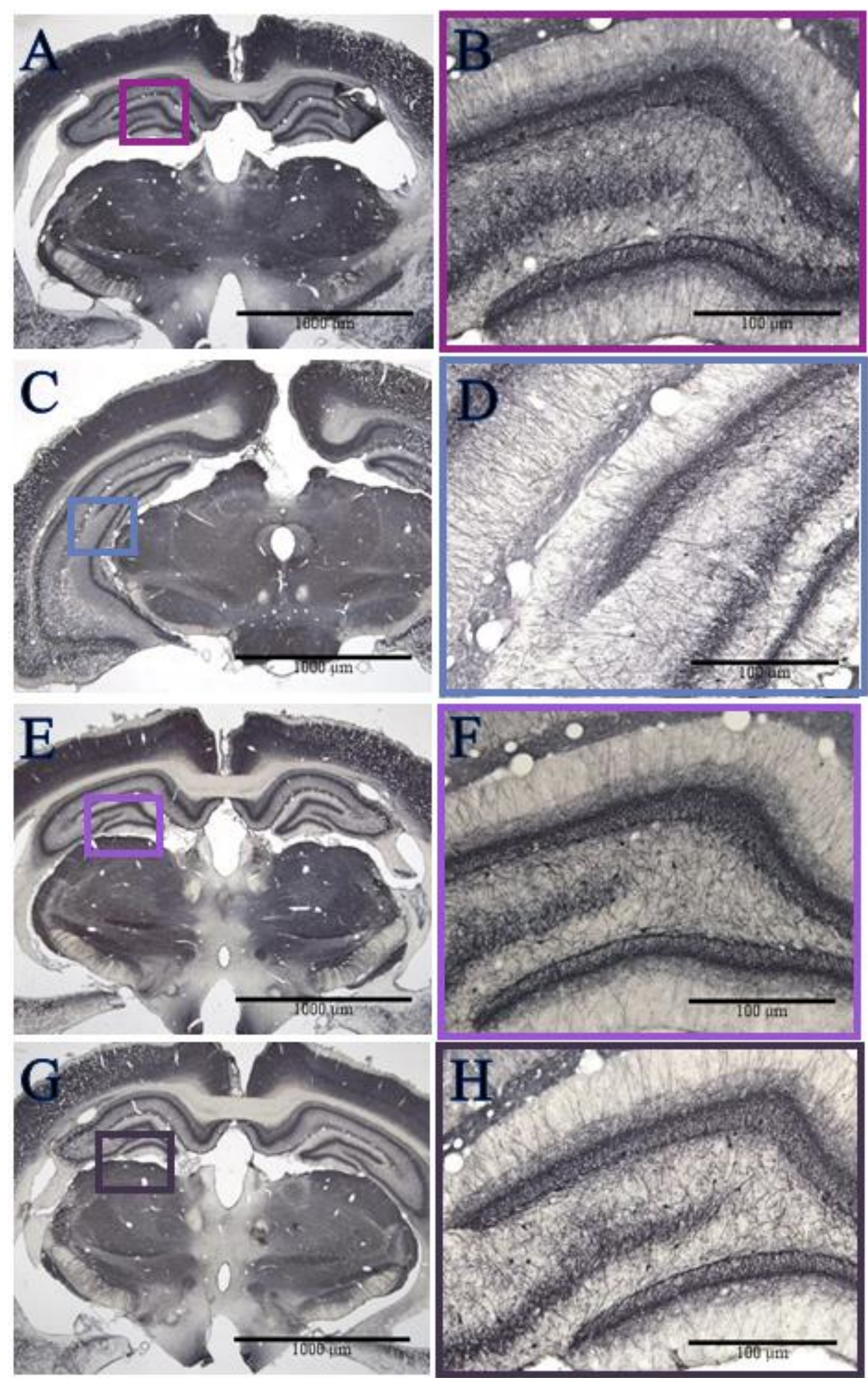

Figure A4.5. Primary antibody and DAB concentration comparison images. A 1: 10,000 primary antibody was used as the baseline (A; 1.25x objective, B; hippocampus 10x) with the full concentration of DAB which is compared with 1: 10,000 and a half dilution of DAB (C D). This is also matched with the primary antibody concentration of 1: 80,000 with full DAB (E F) matched with 1: 80,000 with half dilutions of DAB $(\mathrm{G} \mathrm{H})$. A, C, E, G were captured at 1.25X objective with scale bar $1000 \mu \mathrm{m}$, and B, D, F, H were captured at $10 \mathrm{X}$ objective with $100 \mu \mathrm{m}$ scale bar. 

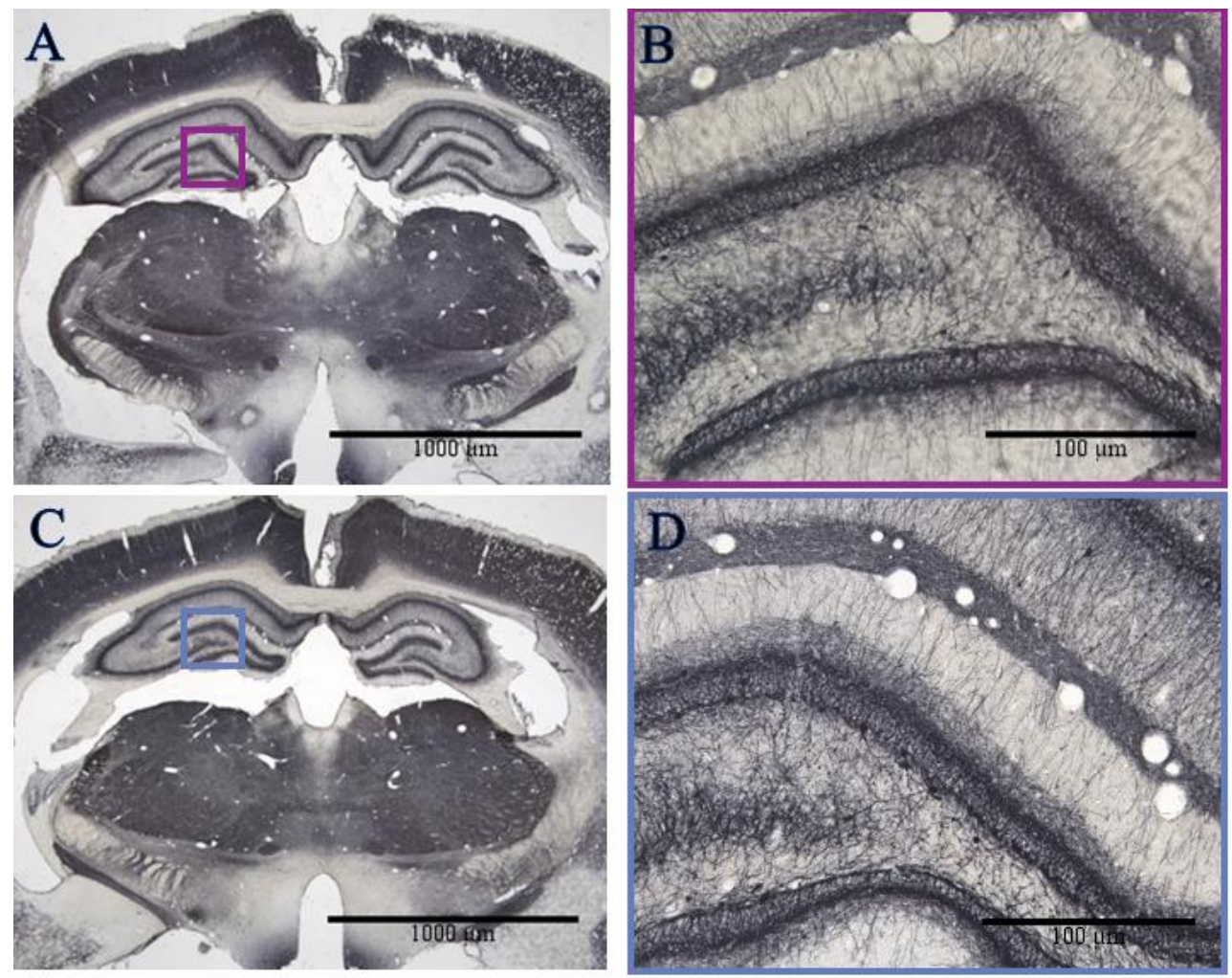

Figure A4.6. Primary antibody and DAB concentration comparison images. A 1:160,000 primary antibody was used $(\mathrm{A} ; 1.25 \mathrm{x}$ objective, $\mathrm{B}$; hippocampus $10 \mathrm{x}$ ) with the full concentration of DAB which is compared with 1: 160,000 and a half dilution of DAB (D-F). ). A, C were captured at $1.25 \mathrm{X}$ objectivewith scale bar $1000 \mu \mathrm{m}$, and $\mathrm{B}, \mathrm{D}$ at $10 \mathrm{X}$ objective with $100 \mu \mathrm{m}$ scale bar. 


\section{A4.1.10 Shorter Primary Antibody Incubation}

Prior to this point, all I-ab incubations had been for $8+$ hours or overnight incubation. In order to identify if a shorter incubation time was suitable and replicable, the I-ab incubation times were tested at 120,90, and 60 minutes. Given that the concentration of the II-ab also impacts the non-specific staining and the density of the stain, it was apparent that we needed to test different concentrations of the II-ab.

As such, we used the optimised steps so far $\left(\mathrm{H}_{2} \mathrm{O}_{2} 10\right.$ minutes, BSA $1 \% 60$ minutes, I-ab 1: 80,000 for 120 minutes or 90 minutes or 60 minutes, II-Ab 1: 1000, or 1:5000, or 1: 10,000 for 90 minutes, $\mathrm{ABC} 60$ minutes and $\mathrm{DAB} 10$ minutes - full or half concentration).

The II-ab concentration of 1:10,000 (figure A4.9) led to the poorest results, with cell bodies pale in comparison to other secondary concentrations and the dendrites were not aligned with cell bodies.

The II-ab concentrations of 1:1000 (figure A4.7) or 1:5000 (figure A4.8) gave similar results but at different I-ab incubations. That is 120 minutes of I-ab incubation, with secondary at 1:5000 with the full DAB may have provided the best overall results (figure A4.8 A-B). However, this is highly comparable with 90 minutes primary incubation with secondary at 1:1000 and a half dilution of DAB (figure A4.7 G-H).

Ultimately, we settled on I-ab incubation of 90 minutes with 1:5000 II-ab concentration with a full dilution of DAB. This appears to be a suitable compromise for visualisation in the and the amygdala and hippocampus. The full and final description of the protocol is detailed in section 4.2.2 of chapter four. 


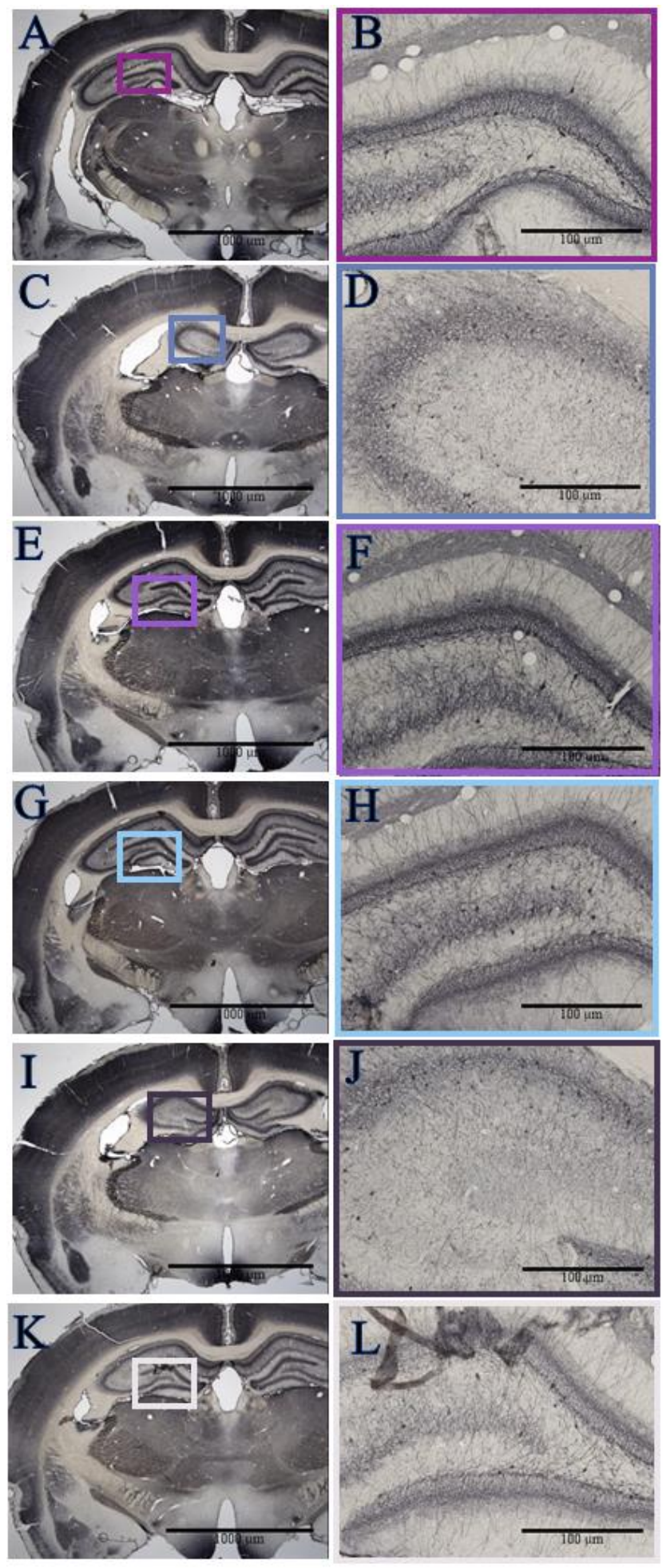

Figure A4.7. Secondary antibody at 1:1000. Primary antibody for 120 minutes with full DAB concentration (A; 1.25x objective, B; hippocampus 10x). Primary antibody for 120 minutes, with half DAB (C-D). Primary antibody for 90 minutes with full DAB concentration (E-F), primary antibody for 90 minutes with half DAB $(\mathrm{G}-\mathrm{H})$. Primary antibody for 60 minutes with full DAB (I-J) and primary antibody for 60 minutes and half DAB (K-L). A, C, E, G, I, K were captured at $1.25 \mathrm{X}$ objective with scale bar $1000 \mu \mathrm{m}$, and B, D, F, H, J, L were captured at 10X objective with $100 \mu \mathrm{m}$ scale bar. 

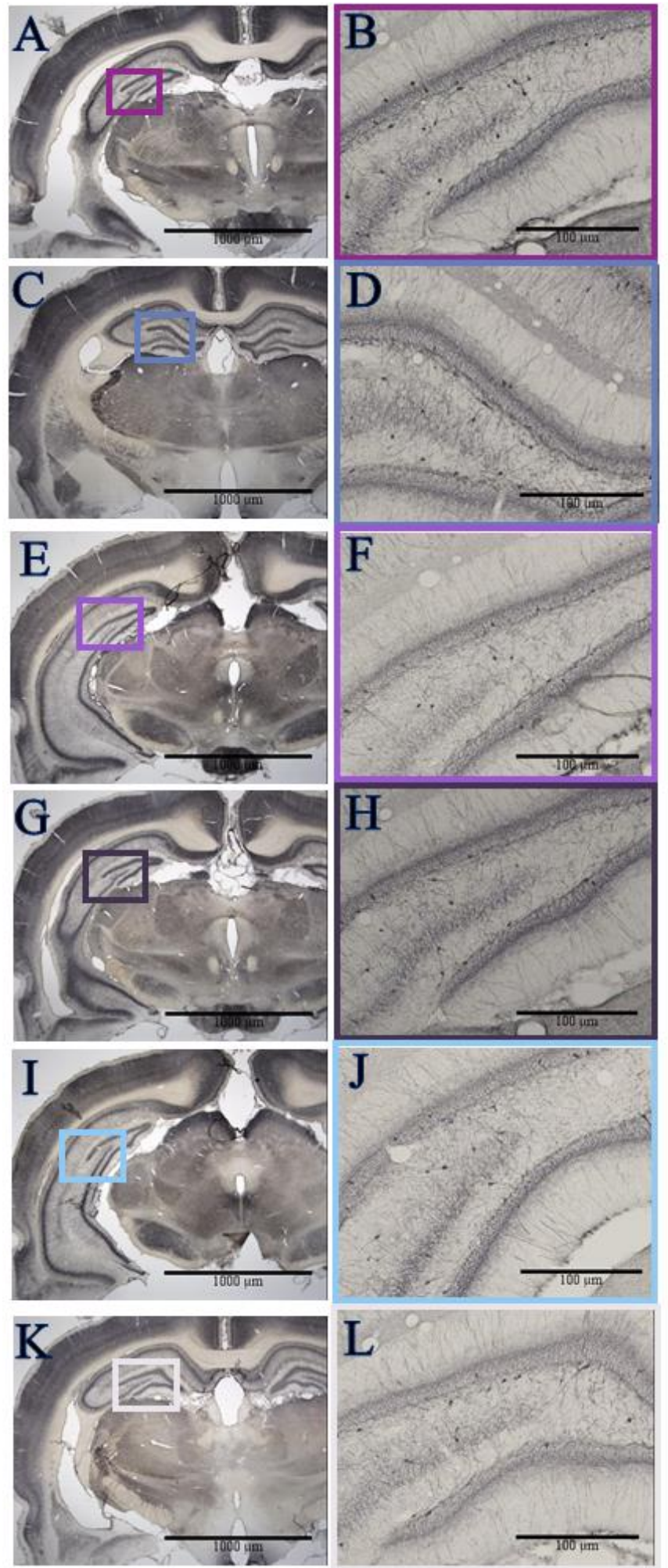

Figure A4.8. Secondary antibody at 1:5000. Primary antibody for 120 minutes with full concentration of $\mathrm{DAB}(\mathrm{A} ; 1.25 \mathrm{x}$ objective, B; hippocampus $10 \mathrm{x})$. Primary antibody for 120 minutes, with half DAB (C-D). Primary antibody for 90 minutes with full DAB (E-F), primary antibody for 90 minutes, with half DAB $(\mathrm{G}-\mathrm{H})$. Primary antibody for 60 minutes with full concentration of DAB (I-J), and primary antibody for 60 minutes with half DAB (K-L). A, C, E, G, I, K were captured at $1.25 \mathrm{X}$ objective with scale bar $1000 \mu \mathrm{m}$, and B, D, F, H, J, L were captured at $10 \mathrm{X}$ objective with $100 \mu \mathrm{m}$ scale bar. 

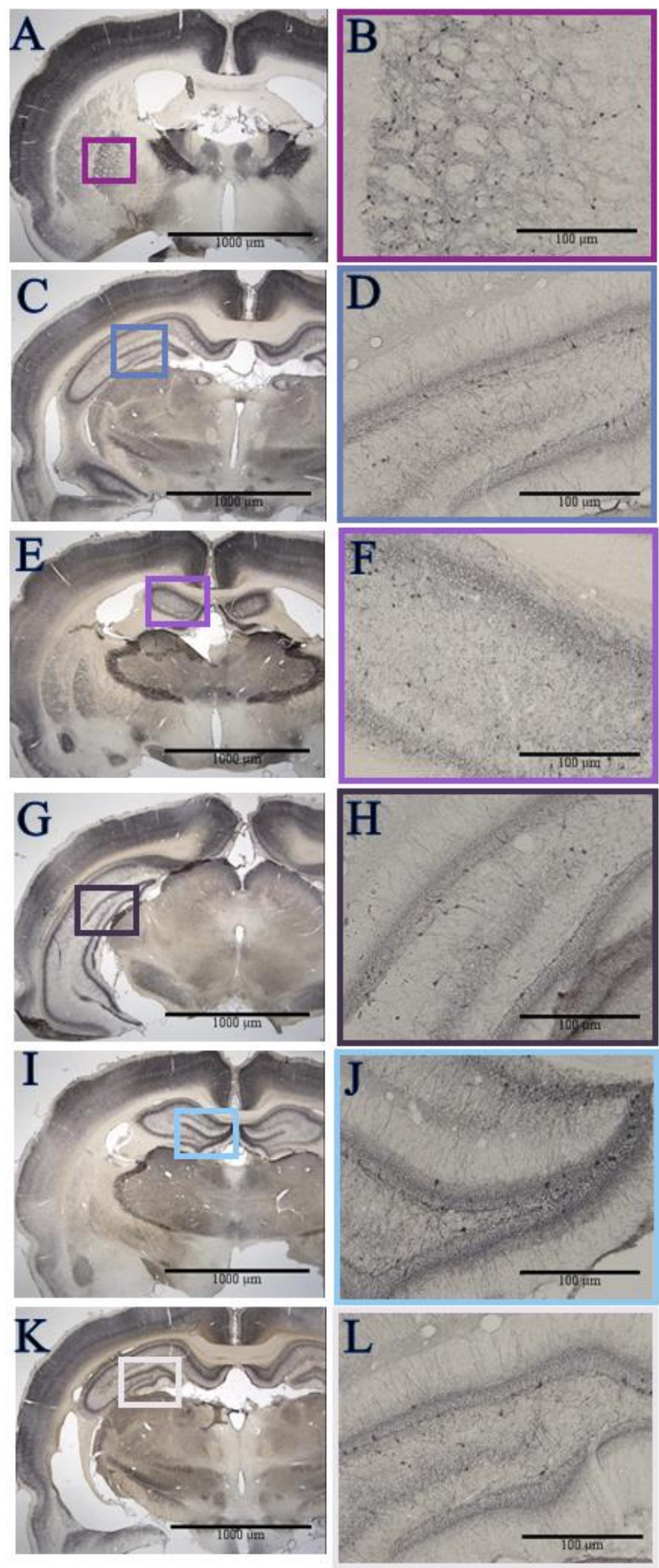

Figure A4.9. Secondary antibody at 1:10,000. Primary antibody for 120 minutes, with full concentration of DAB (A; 1.25x objective, B; striatum, 10x, C; cortex 10x). Primary antibody for 120 minutes, half concentration of DAB (D-F). Primary antibody for 90 minutes, with full concentration of DAB (G-I), Primary antibody for 90 minutes, with half concentration of DAB (J-L). Primary antibody for 60 minutes, full concentration of DAB (M-O), and primary antibody for 60 minutes, with half DAB (P-R). A, C, E, G, I, K were captured at 1.25X objective with scale bar $1000 \mu \mathrm{m}$, and B, D, F, H, J, L were captured at $10 \mathrm{X}$ objective with $100 \mu \mathrm{m}$ scale bar. 


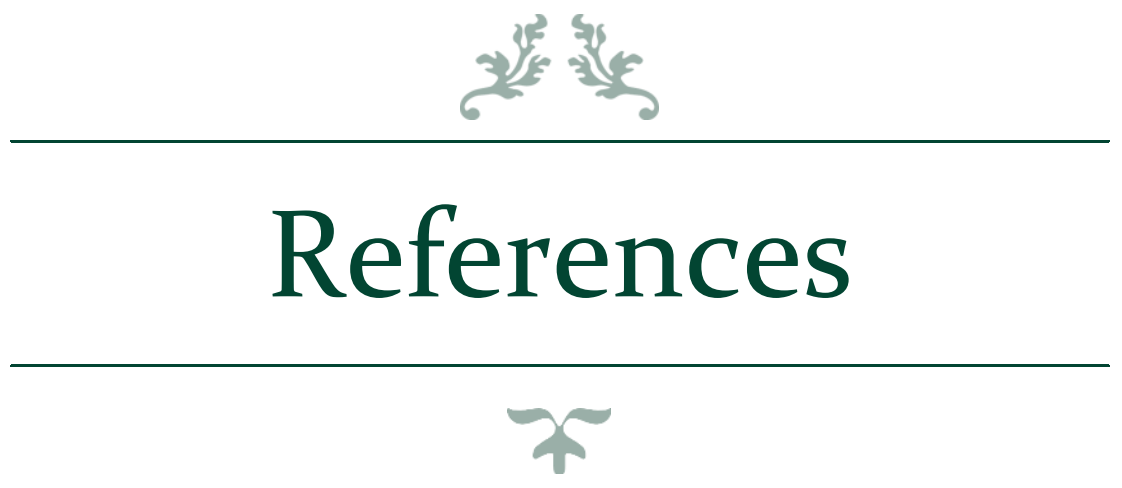




\section{References}

Adab, N., Kini, U., Vinten, J., Ayres, J., Baker, G., Clayton-Smith, J., . . Gregg, J. (2004). The longer term outcome of children born to mothers with epilepsy. Journal of Neurology, Neurosurgery \& Psychiatry, 75(11), 1575-1583.

Adamec, R., Burton, P., Blundell, J., Murphy, D. L., \& Holmes, A. (2006). Vulnerability to mild predator stress in serotonin transporter knockout mice. Behavioural brain research, 170(1), 126-140.

Adams, J. B., Audhya, T., McDonough-Means, S., Rubin, R. A., Quig, D., Geis, E., . . Atwood, S. (2011). Nutritional and metabolic status of children with autism vs. neurotypical children, and the association with autism severity. Nutrition $\mathcal{E}$ metabolism, 8(1), 34.

Alenina, N., \& Klempin, F. (2015). The role of serotonin in adult hippocampal neurogenesis. Behavioural brain research, 277, 49-57.

Amaral, D. G., Schumann, C. M., \& Nordahl, C. W. (2008). Neuroanatomy of autism. Trends in neurosciences, 31(3), 137-145.

Amiet, C., Gourfinkel-An, I., Bouzamondo, A., Tordjman, S., Baulac, M., Lechat, P., . . Cohen, D. (2008). Epilepsy in autism is associated with intellectual disability and gender: evidence from a meta-analysis. Biological psychiatry, 64(7), 577-582.

Anderson, G. M. (2004). Peripheral and central neurochemical effects of the selective serotonin reuptake inhibitors (SSRIs) in humans and nonhuman primates: assessing bioeffect and mechanisms of action. International Journal of Developmental Neuroscience, 22(5-6), 397-404.

Anney, R. J. L. (2013). Common Genetic Variants in Autism Spectrum Disorders (J. D. Buxbaum \& P. R. Hof Eds.): Academic Press.

APA. (2013). Diagnostic and Statistical Manual of mental disorders. Fifth Edition. Arlington VA: American Psychiatric Publishing.

Ashdown, H., Dumont, Y., Ng, M., Poole, S., Boksa, P., \& Luheshi, G. (2006). The role of cytokines in mediating effects of prenatal infection on the fetus: implications for schizophrenia. Molecular Psychiatry, 11(1), 47.

Asperger, H. (1944). Problem with infantile autism. Medical faculty of the Viennese University.

Atcha, Z., Rourke, C., Neo, A. H., Goh, C. W., Lim, J. S., Aw, C.-C., . . Pemberton, D. J. (2010). Alternative method of oral dosing for rats. Journal of the American Association for Laboratory Animal Science, 49(3), 335-343.

Atladottir, H. O., Thorsen, P., Ostergaard, L., Schendel, D. E., Lemcke, S., Abdallah, M., \& Parner, E. T. (2010). Maternal Infection Requiring Hospitalization During Pregnancy and Autism Spectrum Disorders. Journal Of Autism And Developmental Disorders, 40(12), 1423-1430. Retrieved from <Go to ISI>://000284158200001. doi:DOI 10.1007/s10803-010-1006-y

Aylward, E., Minshew, N., Goldstein, G., Honeycutt, N., Augustine, A., Yates, K., . . Pearlson, G. (1999). MRI volumes of amygdala and hippocampus in non-mentally retarded autistic adolescents and adults. Neurology, 53(9), 2145-2145.

Aylward, E. H., Minshew, N. J., Field, K., Sparks, B., \& Singh, N. (2002). Effects of age on brain volume and head circumference in autism. Neurology, 59(2), 175-183.

Bachevalier, J. (1996). Brief report: medial temporal lobe and autism: a putative animal model in primates. Journal Of Autism And Developmental Disorders, 26(2), 217-220.

Bailey, A., Le Couteur, A., Gottesman, I., Bolton, P., Simonoff, E., Yuzda, E., \& Rutter, M. (1995). Autism as a strongly genetic disorder: evidence from a British twin study. Psychological medicine, 25(1), 63-77.

Bailey, A., Luthert, P., Dean, A., Harding, B., Janota, I., Montgomery, M., . . Lantos, P. (1998). A clinicopathological study of autism. Brain: a journal of neurology, 121(5), 889-905. 
Baio, J., Wiggins, L., Christensen, D. L., Maenner, M. J., Daniels, J., Warren, Z., . . White, T. (2018). Prevalence of autism spectrum disorder among children aged 8 years-Autism and Developmental Disabilities Monitoring Network, 11 Sites, United States, 2014. MMWR Surveillance Summaries, 67(6), 1.

Baird, G., Douglas, H. R., \& Murphy, M. S. (2011). Recognising and diagnosing autism in children and young people: summary of NICE guidance. BMJ, 343(d6360), 10.1136.

Baird, G., Simonoff, E., Pickles, A., Chandler, S., Loucas, T., MEldrum, D., \& Charman, T. (2006). Prevalence of disorders of the autism spectrum in a population cohort of children in South Thames: the Special Needs and Autism Project (SNAP). The Lancet, $368,210-215$.

Bambini-Junior, V., Rodrigues, L., Behr, G. A., Moreira, J. C., Riesgo, R., \& Gottfried, C. (2011). Animal model of autism induced by prenatal exposure to valproate: behavioral changes and liver parameters. Brain Res, 1408, 8-16. Retrieved from http://www.ncbi.nlm.nih.gov/pubmed/21767826. doi:10.1016/j.brainres.2011.06.015

Banasr, M., Hery, M., Printemps, R., \& Daszuta, A. (2004). Serotonin-induced increases in adult cell proliferation and neurogenesis are mediated through different and common 5 -HT receptor subtypes in the dentate gyrus and the subventricular zone. Neuropsychopharmacology, 29(3), 450.

Banerjee-Basu, S., \& Packer, A. (2010). SFARI Gene: an evolving database for the autism research community. In: The Company of Biologists Ltd.

Barnea-Goraly, N., Frazier, T. W., Piacenza, L., Minshew, N. J., Keshavan, M. S., Reiss, A. L., \& Hardan, A. Y. (2014). A preliminary longitudinal volumetric MRI study of amygdala and hippocampal volumes in autism. Progress in Neuro-Psychopharmacology and Biological Psychiatry, 48, 124-128.

Baron-Cohen, S. (2000). Theory of mind and autism: A fifteen year review. Understanding other minds: Perspectives from developmental cognitive neuroscience, 2, 3-20.

Baron-Cohen, S. (2016). Autism and the Empathizing-Systemizing (ES) theory. In Developmental social cognitive neuroscience (pp. 139-152): Psychology Press.

Baron-Cohen, S., Leslie, A. M., \& Frith, U. (1985). Does the autistic child have a "theory of mind"? Cognition, 21(1), 37-46.

Bauman, M., \& Kemper, T. L. (1985). Histoanatomic observations of the brain in early infantile autism. Neurology, 35(6), 866-866.

Bauman, M. D., Iosif, A.-M., Smith, S. E., Bregere, C., Amaral, D. G., \& Patterson, P. H. (2014). Activation of the maternal immune system during pregnancy alters behavioral development of rhesus monkey offspring. Biological psychiatry, 75(4), 332-341.

Bauman, M. D., Iosif, A., Ashwood, P., Braunschweig, D., Lee, A., Schumann, C., . . Amaral, D. (2013). Maternal antibodies from mothers of children with autism alter brain growth and social behavior development in the rhesus monkey. Translational psychiatry, 3(7), e278.

Bauman, M. L., \& Kemper, T. L. (2005). Structural brain anatomy in autism: what is the evidence. The neurobiology of autism, 2nd edn. Johns Hopkins University Press, Baltimore, 121-135.

Baxter, A. J., Brugha, T., Erskine, H., Scheurer, R., Vos, T., \& Scott, J. (2015). The epidemiology and global burden of autism spectrum disorders. Psychological medicine, 45(3), 601613.

Belmonte, M. K., Cook Jr, E. H., Anderson, G. M., Rubenstein, J. L., Greenough, W. T., BeckelMitchener, A., . . . Levitt, P. R. (2004). Autism as a disorder of neural information processing: directions for research and targets for therapy. Molecular Psychiatry, 9(7), 646.

Belzung, C., Leman, S., Vourc'h, P., \& Andres, C. (2005). Rodent models for autism: a critical review. Drug discovery today: Disease models, 2(2), 93-101. 
Belzung, C., \& Lemoine, M. (2011). Criteria of validity for animal models of psychiatric disorders: focus on anxiety disorders and depression. Biology of mood $\mathcal{E}$ anxiety disorders, 1(1), 9.

Bernardet, M., \& Crusio, W. E. (2006). Fmrl KO mice as a possible model of autistic features. The Scientific World Journal, 6, 1164-1176.

Bertelsen, F., Folloni, D., Møller, A., Landau, A. M., Scheel-Krüger, J., \& Winterdahl, M. (2017). Suppressed play behaviour and decreased oxytocin receptor binding in the amygdala after prenatal exposure to low-dose valproic acid. Behavioural pharmacology, 28(6), 450-457.

Betancur, C., Corbex, M., Spielewoy, C., Philippe, A., Laplanche, J.-L., Launay, J.-M., . . G Giros, B. (2002). Serotonin transporter gene polymorphisms and hyperserotonemia in autistic disorder. Molecular Psychiatry, 7(1), 67.

Blakely, R. D., \& Veenstra-VanderWeele, J. (2011). Genetic indeterminism, the 5-HTTLPR, and the paths forward in neuropsychiatric genetics. Archives of General Psychiatry, 68(5), 457-458.

Blatt, G. J., \& Fatemi, S. H. (2011). Alterations in GABAergic biomarkers in the autism brain: research findings and clinical implications. The Anatomical Record, 294(10), 1646-1652.

Blatt, G. J., Fitzgerald, C. M., Guptill, J. T., Booker, A. B., Kemper, T. L., \& Bauman, M. L. (2001). Density and distribution of hippocampal neurotransmitter receptors in autism: an autoradiographic study. Journal Of Autism And Developmental Disorders, 31(6), 537543.

Blundell, J., Blaiss, C. A., Etherton, M. R., Espinosa, F., Tabuchi, K., Walz, C., . . Powell, C. M. (2010). Neuroligin-1 deletion results in impaired spatial memory and increased repetitive behavior. Journal of Neuroscience, 30(6), 2115-2129.

Boccuto, L., Chen, C.-F., Pittman, A. R., Skinner, C. D., McCartney, H. J., Jones, K., . . . Schwartz, C. E. (2013). Decreased tryptophan metabolism in patients with autism spectrum disorders. Molecular autism, 4(1), 16.

Bolles, R. C., \& Woods, P. J. (1964). The onotogeny of behavior in the albino rat. Animal behaviour.

Bollino, D., Balan, I., \& Aurelian, L. (2015). Valproic acid induces neuronal cell death through a novel calpain-dependent necroptosis pathway. Journal of neurochemistry, 133(2), 174186.

Bolton, P., Macdonald, H., Pickles, A., Rios, P. a., Goode, S., Crowson, M., . . Rutter, M. (1994). A case-control family history study of autism. Journal of Child Psychology and Psychiatry, 35(5), 877-900.

Bonnin, A., \& Levitt, P. (2011). Fetal, maternal, and placental sources of serotonin and new implications for developmental programming of the brain. Neuroscience, 197, 1-7.

Border, R., Johnson, E. C., Evans, L. M., Smolen, A., Berley, N., Sullivan, P. F., \& Keller, M. C. (2019). No support for historical candidate gene or candidate gene-by-interaction hypotheses for major depression across multiple large samples. American Journal of Psychiatry, 176(5), 376-387.

Branchi, I., Santucci, D., \& Alleva, E. (2001). Ultrasonic vocalisation emitted by infant rodents: a tool for assessment of neurobehavioural development. Behavioural brain research, 125(1-2), 49-56.

Bregman, T., Nona, C., Volle, J., Diwan, M., Raymond, R., Fletcher, P. J., . . Hamani, C. (2018). Deep brain stimulation induces antidepressant-like effects in serotonin transporter knockout mice. Brain stimulation, 11(2), 423-425.

Bringas, M., Carvajal-Flores, F., López-Ramírez, T., Atzori, M., \& Flores, G. (2013). Rearrangement of the dendritic morphology in limbic regions and altered exploratory behavior in a rat model of autism spectrum disorder. Neuroscience, 241, 170-187. 
Brodkin, E. S., Hagemann, A., Nemetski, S. M., \& Silver, L. M. (2004). Social approachavoidance behavior of inbred mouse strains towards DBA/2 mice. Brain research, 1002(1-2), 151-157.

Bromley, R., Mawer, G., Clayton-Smith, J., Baker, G., Liverpool, \& Group, M. N. (2008). Autism spectrum disorders following in utero exposure to antiepileptic drugs. Neurology, 71(23), 1923-1924.

Bronzuoli, M. R., Facchinetti, R., Ingrassia, D., Sarvadio, M., Schiavi, S., Steardo, L., . . . Scuderi, C. (2018). Neuroglia in the autistic brain: evidence from a preclinical model. Molecular autism, 9(1), 66.

Brudzynski, S. M. (2005). Principles of rat communication: quantitative parameters of ultrasonic calls in rats. Behavior genetics, 35(1), 85-92.

Brudzynski, S. M. (2015). Pharmacology of ultrasonic vocalizations in adult rats: significance, call classification and neural substrate. Current neuropharmacology, 13(2), 180-192.

Brudzynski, S. M., Kehoe, P., \& Callahan, M. (1999). Sonographic structure of isolationinduced ultrasonic calls of rat pups. Developmental Psychobiology: The Journal of the International Society for Developmental Psychobiology, 34(3), 195-204.

Brune, C. W., Kim, S.-J., Salt D Clin Psy, J., Leventhal, B. L., Lord, C., \& Cook Jr, M., Edwin H. (2006). 5-HTTLPR genotype-specific phenotype in children and adolescents with autism. American Journal of Psychiatry, 163(12), 2148-2156.

Buescher, A. V., Cidav, Z., Knapp, M., \& Mandell, D. S. (2014). Costs of autism spectrum disorders in the United Kingdom and the United States. JAMA pediatrics, 168(8), 721728.

Buxbaum, J. D., Daly, M. J., Devlin, B., Lehner, T., Roeder, K., \& Consortium, A. S. (2012). The autism sequencing consortium: large-scale, high-throughput sequencing in autism spectrum disorders. Neuron, 76(6), 1052-1056.

Buxhoeveden, D., Semendeferi, K., Buckwalter, J., Schenker, N., Switzer, R., \& Courchesne, E. (2006). Reduced minicolumns in the frontal cortex of patients with autism. Neuropathology and applied neurobiology, 32(5), 483-491.

Buznikov, G. A., Lambert, W. H., \& Lauder, J. M. (2001). Serotonin and serotonin-like substances as regulators of early embryogenesis and morphogenesis. Cell and tissue research, 305(2), 177-186.

Calabrese, F., Guidotti, G., Middelman, A., Racagni, G., Homberg, J., \& Riva, M. A. (2013). Lack of serotonin transporter alters BDNF expression in the rat brain during early postnatal development. Molecular neurobiology, 48(1), 244-256.

Caraci, F., Leggio, G. M., Salomone, S., \& Drago, F. (2017). New drugs in psychiatry: focus on new pharmacological targets. F1000Research, 6 .

Carew, J. S., Giles, F. J., \& Nawrocki, S. T. (2008). Histone deacetylase inhibitors: mechanisms of cell death and promise in combination cancer therapy. Cancer letters, 269(1), 7-17.

Carper, R. A., \& Courchesne, E. (2005). Localized enlargement of the frontal cortex in early autism. Biological psychiatry, 57(2), 126-133.

Carroll, J. C., Boyce-Rustay, J. M., Millstein, R., Yang, R., Wiedholz, L. M., Murphy, D. L., \& Holmes, A. (2007). Effects of mild early life stress on abnormal emotion-related behaviors in 5-HTT knockout mice. Behavior genetics, 37(1), 214-222.

Casanova, M. F., Buxhoeveden, D. P., Switala, A. E., \& Roy, E. (2002). Asperger's syndrome and cortical neuropathology. Journal of child neurology, 17(2), 142-145.

Casanova, M. F., Buxhoeveden, D. P., Switala, A. E., \& Roy, E. (2002). Minicolumnar pathology in autism. Neurology, 58(3), 428-432.

Casarrubea, M., Jonsson, G., Faulisi, F., Sorbera, F., Di Giovanni, G., Benigno, A., . . . Magnusson, M. (2015). T-pattern analysis for the study of temporal structure of animal and human behavior: a comprehensive review. Journal of neuroscience methods, 239, 34-46. 
Celio, M. (1990). Calbindin D-28k and parvalbumin in the rat nervous system. Neuroscience, 35(2), 375-475.

Chadman, K., \& Guariglia, S. (2012). The BTBR T+ tf/J (BTBR) mouse model of autism. Autism Sl, 9, 2.

Chadman, K. K. (2011). Fluoxetine but not risperidone increases sociability in the BTBR mouse model of autism. Pharmacology Biochemistry and Behavior, 97(3), 586-594.

Chapman, J. B., \& Cutler, M. G. (1984). Sodium valproate: Effects on social behaviour and physical development in the mouse. Psychopharmacology, 83, 390-396.

Cheng, D., Spiro, A. S., Jenner, A. M., Garner, B., \& Karl, T. (2014). Long-term cannabidiol treatment prevents the development of social recognition memory deficits in Alzheimer's disease transgenic mice. Journal of Alzheimer's Disease, 42(4), 1383-1396.

Chess, S. (1971). Autism in children with congenital rubella. Journal of autism and childhood schizophrenia, 1(1), 33-47.

Chevallier, C., Kohls, G., Troiani, V., Brodkin, E. S., \& Schultz, R. T. (2012). The social motivation theory of autism. Trends in cognitive sciences, 16(4), 231-239.

Chomiak, T., Karnik, V., Block, E., \& Hu, B. (2010). Altering the trajectory of early postnatal cortical development can lead to structural and behavioural features of autism. BMC neuroscience, $11(1), 102$.

Christensen, D. L., Bilder, D. A., Zahorodny, W., Pettygrove, S., Durkin, M. S., Fitzgerald, R. T., ... Yeargin-Allsopp, M. (2016). Prevalence and characteristics of autism spectrum disorder among 4-year-old children in the autism and developmental disabilities monitoring network. Journal of Developmental $\mathcal{E}$ Behavioral Pediatrics, 37(1), 1-8.

Christensen, J., Gronborg, T. K., Sorensen, M. J., Schendel, D., Parner, E. T., Pedersen, L. H., \& Vestergaard, M. (2013). Prenatal valproate exposure and risk of autism spectrum disorders and childhood autism. JAMA, 309(16), 1696-1703. Retrieved from http://www.ncbi.nlm.nih.gov/pubmed/23613074. doi:10.1001/jama.2013.2270

Christianson, A. L., Chester, N., \& Kromberg, J. G. (1994). Fetal valproate syndrome: clinical and neuro-developmental features in two sibling pairs. Developmental Medicine $\mathcal{E}$ Child Neurology, 36(4), 361-369.

Cidav, Z., Marcus, S. C., \& Mandell, D. S. (2012). Implications of childhood autism for parental employment and earnings. Pediatrics, peds. 2011-2700.

Clayton, J. A., \& Collins, F. S. (2014). Policy: NIH to balance sex in cell and animal studies. Nature News, 509(7500), 282.

Cloyd, J. C., Kriel, R. L., Jones-Saete, C. M., Ong, B. Y., Jancik, J. T., \& Remmel, R. P. (1992). Comparison of sprinkle versus syrup formulations of valproate for bioavailability, tolerance, and preference. The Journal of pediatrics, 120(4), 634-638.

Coffey, K. R., Marx, R. G., \& Neumaier, J. F. (2019). DeepSqueak: a deep learning-based system for detection and analysis of ultrasonic vocalizations. Neuropsychopharmacology, $44(5), 859$.

Coghlan, S., Horder, J., Inkster, B., Mendez, M. A., Murphy, D. G., \& Nutt, D. J. (2012). GABA system dysfunction in autism and related disorders: from synapse to symptoms. Neuroscience E Biobehavioral Reviews, 36(9), 2044-2055.

Cohen, O. S., Varlinskaya, E. I., Wilson, C. A., Glatt, S. J., \& Mooney, S. M. (2013). Acute prenatal exposure to a moderate dose of valproic acid increases social behavior and alters gene expression in rats. International Journal of Developmental Neuroscience, 31(8), 740-750. Retrieved from <Go to ISI >://WOS:000329270200005.

Conroy, J., Meally, E., Kearney, G., Fitzgerald, M., Gill, M., \& Gallagher, L. (2004). Serotonin transporter gene and autism: a haplotype analysis in an Irish autistic population. Molecular Psychiatry, 9(6), 587. 
Cook, E. H., Courchesne, R., Lord, C., Cox, N. J., Yan, S., Lincoln, A., . . Leventhal, B. L. (1997). Evidence of linkage between the serotonin transporter and autistic disorder. Molecular Psychiatry, 2(3), 247.

Cook, E. H., \& Leventhal, B. L. (1996). The serotonin system in autism. Current opinion in pediatrics, 8(4), 348-354.

Cooper, S. J. (1992). Schizophrenia after prenatal exposure to 1957 A2 influenza epidemic. The British Journal of Psychiatry, 161(3), 394-396.

Courchesne, E., Campbell, K., \& Solso, S. (2011). Brain growth across the life span in autism: age-specific changes in anatomical pathology. Brain research, 1380, 138-145.

Courchesne, E., Karns, C., Davis, H., Ziccardi, R., Carper, R., Tigue, Z., . . Lord, C. (2001). Unusual brain growth patterns in early life in patients with autistic disorder: an MRI study. Neurology, 57(2), 245-254.

Courchesne, E., Redcay, E., \& Kennedy, D. P. (2004). The autistic brain: birth through adulthood. Current opinion in neurology, 17(4), 489-496.

Coutinho, A., Oliveira, G., Morgadinho, T., Fesel, C., Macedo, T., Bento, C., . . Borges, L. (2004). Variants of the serotonin transporter gene (SLC6A4) significantly contribute to hyperserotonemia in autism. Molecular Psychiatry, 9(3), 264.

Coutinho, A. M., Sousa, I., Martins, M., Correia, C., Morgadinho, T., Bento, C., . . Moore, J. H. (2007). Evidence for epistasis between SLC6A4 and ITGB3 in autism etiology and in the determination of platelet serotonin levels. Human Genetics, 121(2), 243-256.

Crawley, J. N. (2004). Designing mouse behavioral tasks relevant to autistic-like behaviors. Mental retardation and developmental disabilities research reviews, 10(4), 248-258.

Cridland, E. K., Jones, S. C., Caputi, P., \& Magee, C. A. (2014). Being a girl in a boys' world: Investigating the experiences of girls with autism spectrum disorders during adolescence. Journal Of Autism And Developmental Disorders, 44(6), 1261-1274.

Croonenberghs, J., Verkerk, R., Scharpe, S., Deboutte, D., \& Maes, M. (2005). Serotonergic disturbances in autistic disorder: L-5-hydroxytryptophan administration to autistic youngsters increases the blood concentrations of serotonin in patients but not in controls. Life sciences, 76(19), 2171-2183.

Daenen, E. W., Wolterink, G., Gerrits, M. A., \& Van Ree, J. M. (2002). The effects of neonatal lesions in the amygdala or ventral hippocampus on social behaviour later in life. Behavioural brain research, 136(2), 571-582.

Danielsson, S., Gillberg, I. C., Billstedt, E., Gillberg, C., \& Olsson, I. (2005). Epilepsy in young adults with autism: a prospective population-based follow-up study of 120 individuals diagnosed in childhood. Epilepsia, 46(6), 918-923.

De Rubeis, S., He, X., Goldberg, A. P., Poultney, C. S., Samocha, K., Cicek, A. E., . . W Walker, S. (2014). Synaptic, transcriptional and chromatin genes disrupted in autism. Nature, 515(7526), 209.

Deacon, R. M. (2013). The successive alleys test of anxiety in mice and rats. Journal of visualized experiments: JoVE(76).

Dean, J., Hailey, H., Moore, S., Lloyd, D. J., Turnpenny, P., \& Little, J. (2002). Long term health and neurodevelopment in children exposed to antiepileptic drugs before birth. Journal of Medical Genetics, 39(4), 251-259.

Defensor, E. B., Pearson, B. L., Pobbe, R. L., Bolivar, V. J., Blanchard, D. C., \& Blanchard, R. J. (2011). A novel social proximity test suggests patterns of social avoidance and gaze aversion-like behavior in BTBR T+ tf/J mice. Behavioural brain research, 217(2), 302308.

Defoort, E. N., Kim, P. M., \& Winn, L. M. (2006). Valproic acid increases conservative homologous recombination frequency and reactive oxygen species formation: a potential mechanism for valproic acid-induced neural tube defects. Molecular pharmacology, 69(4), 1304-1310. 
Dehorter, N., Marichal, N., Marín, O., \& Berninger, B. (2017). Tuning neural circuits by turning the interneuron knob. Current opinion in neurobiology, 42, 144-151.

Dendrinos, G., Hemelt, M., \& Keller, A. (2011). Prenatal VPA exposure and changes in sensory processing by the superior colliculus. Frontiers in integrative neuroscience, 5, 68.

Deoni, S. C., Zinkstok, J., Daly, E., Ecker, C., Williams, S. C., Murphy, D. G., \& Consortium, M. A. (2015). White-matter relaxation time and myelin water fraction differences in young adults with autism. Psychological medicine, 45(4), 795-805.

Detrait, E. R., George, T. M., Etchevers, H. C., Gilbert, J. R., Vekemans, M., \& Speer, M. C. (2005). Human neural tube defects: developmental biology, epidemiology, and genetics. Neurotoxicology and teratology, 27(3), 515-524.

Devlin, B., Cook Jr, E., Coon, H., Dawson, G., Grigorenko, E., McMahon, W., . . Spence, M. (2005). Autism and the serotonin transporter: the long and short of it. Molecular Psychiatry, 10(12), 1110.

Dhossche, D., Applegate, H., Abraham, A., Maertens, P., Bland, L., Bencsath, A., \& Martinez, J. (2002). Elevated plasma gamma-aminobutyric acid (GABA) levels in autistic youngsters: stimulus for a GABA hypothesis of autism. Medical Science Monitor, 8(8), PR1-PR6.

Di Martino, A., \& Castellanos, F. X. (2003). Functional neuroimaging of social cognition in pervasive developmental disorders: a brief review. Annals of the New York Academy of Sciences, 1008(1), 256-260.

Di Martino, A., Yan, C.-G., Li, Q., Denio, E., Castellanos, F. X., Alaerts, K., . . Dapretto, M. (2014). The autism brain imaging data exchange: towards a large-scale evaluation of the intrinsic brain architecture in autism. Molecular Psychiatry, 19(6), 659.

Dickinson, R. G., Harland, R. C., Lynn, R. K., Smith, W. B., \& Gerber, N. (1979). Transmission of valproic acid (Depakene) across theplacenta: Half-life of the drug in mother and baby. The Journal of pediatrics, 94(5), 832-835.

Donaldson, Z. R., \& Young, L. J. (2008). Oxytocin, vasopressin, and the neurogenetics of sociality. Science, 322(5903), 900-904.

Dong, S., Walker, M. F., Carriero, N. J., DiCola, M., Willsey, A. J., Adam, Y. Y., . . Frahm, S. (2014). De novo insertions and deletions of predominantly paternal origin are associated with autism spectrum disorder. Cell reports, 9(1), 16-23.

Dufour-Rainfray, D., Vourc'h, P., Le Guisquet, A.-M., Garreau, L., Ternant, D., Bodard, S., ... Andres, C. R. (2010). Behavior and serotonergic disorders in rats exposed prenatally to valproate: a model for autism. Neuroscience Letters, 470(1), 55-59.

Ecker, C., Shahidiani, A., Feng, Y., Daly, E., Murphy, C., D’Almeida, V., . . G Gudbrandsen, M. (2014). The effect of age, diagnosis, and their interaction on vertex-based measures of cortical thickness and surface area in autism spectrum disorder. Journal of Neural Transmission, 121(9), 1157-1170.

Edalatmanesh, M. A., Nikfarjam, H., Vafaee, F., \& Moghadas, M. (2013). Increased hippocampal cell density and enhanced spatial memory in the valproic acid rat model of autism. Brain research, 1526, 15-25.

Ellegood, J., Anagnostou, E., Babineau, B., Crawley, J., Lin, L., Genestine, M., . . Penagarikano, O. (2015). Clustering autism: using neuroanatomical differences in 26 mouse models to gain insight into the heterogeneity. Molecular Psychiatry, 20(1), 118.

Ellenbroek, B., August, C., \& Youn, J. (2016). Does prenatal valproate interact with a genetic reduction in the serotonin transporter? A rat study on anxiety and cognition. Frontiers in neuroscience, 10, 424.

Elsner, J., Suter, D., \& Alder, S. (1990). Microanalysis of ultrasound vocalizations of young rats: assessment of the behavioral teratogenicity of methylmercury. Neurotoxicology and teratology, 12(1), 7-14. 
Ergaz, Z., Weinstein-Fudim, L., \& Ornoy, A. (2016). Genetic and non-genetic animal models for autism spectrum disorders (ASD). Reproductive Toxicology, 64, 116-140.

Fairbanks, L. A., Melega, W. P., Jorgensen, M. J., Kaplan, J. R., \& McGuire, M. T. (2001). Social impulsivity inversely associated with CSF 5-HIAA and fluoxetine exposure in vervet monkeys. Neuropsychopharmacology, 24(4), 370.

Farley, M., Cottle, K. J., Bilder, D., Viskochil, J., Coon, H., \& McMahon, W. (2018). Mid-life social outcomes for a population-based sample of adults with ASD. Autism Research, $11(1), 142-152$.

Farrant, K., \& Uddin, L. Q. (2016). Atypical developmental of dorsal and ventral attention networks in autism. Developmental science, 19(4), 550-563.

Fatemi, S. H., Halt, A. R., Realmuto, G., Earle, J., Kist, D. A., Thuras, P., \& Merz, A. (2002). Purkinje cell size is reduced in cerebellum of patients with autism. Cellular and molecular neurobiology, 22(2), 171-175.

Fatemi, S. H., Reutiman, T. J., Folsom, T. D., Huang, H., Oishi, K., Mori, S., . . Sohr, R. (2008). Maternal infection leads to abnormal gene regulation and brain atrophy in mouse offspring: implications for genesis of neurodevelopmental disorders. Schizophrenia research, 99(1-3), 56-70.

Fatemi, S. H., Reutiman, T. J., Folsom, T. D., \& Thuras, P. D. (2009). GABA A receptor downregulation in brains of subjects with autism. Journal Of Autism And Developmental Disorders, 39(2), 223.

Favre, M. R., Barkat, T. R., Mendola, D. L., Khazen, G., Markram, H., \& Markram, K. (2013). General developmental health in the VPA-rat model of autism. Frontiers in behavioral neuroscience, 7, 88.

Favre, M. R., La Mendola, D., Meystre, J., Christodoulou, D., Cochrane, M. J., Markram, H., \& Markram, K. (2015). Predictable enriched environment prevents development of hyper-emotionality in the VPA rat model of autism. Frontiers in neuroscience, 9, 127.

Feinberg, J. I., Bakulski, K. M., Jaffe, A. E., Tryggvadottir, R., Brown, S. C., Goldman, L. R., .. . Daniele Fallin, M. (2015). Paternal sperm DNA methylation associated with early signs of autism risk in an autism-enriched cohort. International journal of epidemiology, 44(4), 1199-1210.

Felix-Ortiz, A. C., \& Febo, M. (2012). Gestational valproate alters BOLD activation in response to complex social and primary sensory stimuli. PLoS One, 7(5), e37313.

File, S. E., \& Hyde, J. (1978). Can social interaction be used to measure anxiety? British journal of pharmacology, 62(1), 19-24.

Foley, A. G., Gannon, S., Rombach-Mullan, N., Prendergast, A., Barry, C., Cassidy, A. W., \& Regan, C. M. (2012). Class I histone deacetylase inhibition ameliorates social cognition and cell adhesion molecule plasticity deficits in a rodent model of autism spectrum disorder. Neuropharmacology, 63(4), 750-760.

Folstein, S., \& Rutter, M. (1977). Infantile autism: a genetic study of 21 twin pairs. Journal of Child Psychology and Psychiatry, 18(4), 297-321.

Fombonne, E. (1999). The epidemiology of autism: a review. Psychological medicine, 29(4), 769-786.

Fortin, M., Marchand, R., \& Parent, A. (1998). Calcium-binding proteins in primate cerebellum. Neuroscience research, 30(2), 155-168.

Frisch, C., Hüsch, K., Angenstein, F., Kudin, A., Kunz, W., Elger, C. E., \& Helmstaedter, C. (2009). Dose-dependent memory effects and cerebral volume changes after in utero exposure to valproate in the rat. Epilepsia, 50(6), 1432-1441.

Frith, U. (2003). Autism: Explaining the enigma: Blackwell Publishing.

Frith, U., \& Happé, F. (1994). Autism: Beyond "theory of mind". Cognition, 50(1-3), 115-132.

Fujimura, K., Mitsuhashi, T., Shibata, S., Shimozato, S., \& Takahashi, T. (2016). In utero exposure to valproic acid induces neocortical dysgenesis via dysregulation of neural 
progenitor cell proliferation/differentiation. Journal of Neuroscience, 36(42), 1090810919.

Furman, D. J., Chen, M. C., \& Gotlib, I. H. (2011). Variant in oxytocin receptor gene is associated with amygdala volume. Psychoneuroendocrinology, 36(6), 891-897.

Gabriele, S., Sacco, R., \& Persico, A. M. (2014). Blood serotonin levels in autism spectrum disorder: a systematic review and meta-analysis. European Neuropsychopharmacology, 24(6), 919-929.

Gabrielsen, T. P., Farley, M., Speer, L., Villalobos, M., Baker, C. N., \& Miller, J. (2015). Identifying autism in a brief observation. Pediatrics, peds. 2014-1428.

Gandal, M. J., Edgar, J. C., Ehrlichman, R. S., Mehta, M., Roberts, T. P., \& Siegel, S. J. (2010). Validating $\gamma$ oscillations and delayed auditory responses as translational biomarkers of autism. Biological psychiatry, 68(12), 1100-1106.

Gao, R., \& Penzes, P. (2015). Common mechanisms of excitatory and inhibitory imbalance in schizophrenia and autism spectrum disorders. Current molecular medicine, 15(2), 146167.

García, N. V. D. M., Karayannis, T., \& Fishell, G. (2011). Neuronal activity is required for the development of specific cortical interneuron subtypes. Nature, 472(7343), 351.

Gauthier, J., Spiegelman, D., Piton, A., Lafreniere, R. G., Laurent, S., St-Onge, J., . . Mottron, L. (2009). Novel de novo SHANK3 mutation in autistic patients. American Journal of Medical Genetics Part B: Neuropsychiatric Genetics, 150(3), 421-424.

Gelernter, J., Pakstis, A., \& Kidd, K. (1995). Linkage mapping of serotonin transporter protein gene SLC6A4 on chromosome 17. Human Genetics, 95(6), 677-680.

Gemelli, T., Berton, O., Nelson, E. D., Perrotti, L. I., Jaenisch, R., \& Monteggia, L. M. (2006). Postnatal loss of methyl-CpG binding protein 2 in the forebrain is sufficient to mediate behavioral aspects of Rett syndrome in mice. Biological psychiatry, 59(5), 468-476.

Giavini, E., \& Menegola, E. (2014). Teratogenic activity of HDAC inhibitors. Current pharmaceutical design, 20(34), 5438-5442.

Gillberg, C., Cederlund, M., Lamberg, K., \& Zeijlon, L. (2006). Brief report:"the autism epidemic". The registered prevalence of autism in a Swedish urban area. Journal of Autism And Developmental Disorders, 36(3), 429.

Glatt, C. E., DeYoung, J. A., Delgado, S., Service, S. K., Giacomini, K. M., Edwards, R. H., . . . Freimer, N. B. (2001). Screening a large reference sample to identify very low frequency sequence variants: comparisons between two genes. Nature genetics, 27(4), 435.

Glessner, J. T., Wang, K., Cai, G., Korvatska, O., Kim, C. E., Wood, S., . . Bradfield, J. P. (2009). Autism genome-wide copy number variation reveals ubiquitin and neuronal genes. Nature, 459(7246), 569.

Gogolla, N., LeBlanc, J. J., Quast, K. B., Südhof, T. C., Fagiolini, M., \& Hensch, T. K. (2009). Common circuit defect of excitatory-inhibitory balance in mouse models of autism. Journal of neurodevelopmental disorders, 1(2), 172.

Gonchar, Y., Wang, Q., \& Burkhalter, A. H. (2008). Multiple distinct subtypes of GABAergic neurons in mouse visual cortex identified by triple immunostaining. Frontiers in neuroanatomy, 2, 3.

Gotham, K., Marvin, A. R., Taylor, J. L., Warren, Z., Anderson, C. M., Law, P. A., . . Lipkin, P. H. (2015). Characterizing the daily life, needs, and priorities of adults with autism spectrum disorder from Interactive Autism Network data. Autism, 19(7), 794-804.

Gould, G. G., Hensler, J. G., Burke, T. F., Benno, R. H., Onaivi, E. S., \& Daws, L. C. (2011). Density and function of central serotonin (5-HT) transporters, 5-HTlA and 5-HT2A receptors, and effects of their targeting on BTBR $\mathrm{T}+\mathrm{tf} / \mathrm{J}$ mouse social behavior. Journal of neurochemistry, 116(2), 291-303. 
Grant, E. (1963). An analysis of the social behaviour of the male laboratory rat. Behaviour, 21(34), 260-281.

Green, L., Fein, D., Modahl, C., Feinstein, C., Waterhouse, L., \& Morris, M. (2001). Oxytocin and autistic disorder: alterations in peptide forms. Biological psychiatry, 50(8), 609613.

Gurvich, N., Berman, M. G., Wittner, B. S., Gentleman, R. C., Klein, P. S., \& Green, J. B. (2005). Association of valproate-induced teratogenesis with histone deacetylase inhibition in vivo. The FASEB journal, 19(9), 1166-1168.

Ha, S., Sohn, I.-J., Kim, N., Sim, H. J., \& Cheon, K.-A. (2015). Characteristics of brains in autism spectrum disorder: structure, function and connectivity across the lifespan. Experimental neurobiology, 24(4), 273-284.

Hahn, M. E., \& Lavooy, M. J. (2005). A review of the methods of studies on infant ultrasound production and maternal retrieval in small rodents. Behavior genetics, 35(1), 31-52.

Hale, M. W., Johnson, P. L., Westerman, A. M., Abrams, J. K., Shekhar, A., \& Lowry, C. A. (2010). Multiple anxiogenic drugs recruit a parvalbumin-containing subpopulation of GABAergic interneurons in the basolateral amygdala. Progress in NeuroPsychopharmacology and Biological Psychiatry, 34(7), 1285-1293.

Hali, S., Kim, J., Kwak, T. H., Lee, H., Shin, C. Y., \& Han, D. W. (2020). Modelling monogenic autism spectrum disorder using mouse cortical organoids. Biochemical and Biophysical Research Communications, 521(1), 164-171.

Hallmayer, J., Cleveland, S., Torres, A., Phillips, J., Cohen, B., Torigoe, T., . . Smith, K. (2011). Genetic heritability and shared environmental factors among twin pairs with autism. Archives of General Psychiatry, 68(11), 1095-1102.

Hanley, H. G., Stahl, S. M., \& Freedman, D. X. (1977). Hyperserotonemia and amine metabolites in autistic and retarded children. Archives of General Psychiatry, 34(5), 521-531.

Harrington, R. A., Lee, L. C., Crum, R. M., Zimmerman, A. W., \& Hertz-Picciotto, I. (2013). Serotonin hypothesis of autism: implications for selective serotonin reuptake inhibitor use during pregnancy. Autism Research, 6(3), 149-168.

Hashemi, E., Ariza, J., Rogers, H., Noctor, S. C., \& Martínez-Cerdeño, V. (2016). The number of parvalbumin-expressing interneurons is decreased in the prefrontal cortex in autism. Cerebral cortex, 27(3), 1931-1943.

Hassan, T. H., Abdelrahman, H. M., Fattah, N. R. A., El-Masry, N. M., Hashim, H. M., ElGerby, K. M., \& Fattah, N. R. A. (2013). Blood and brain glutamate levels in children with autistic disorder. Research in Autism Spectrum Disorders, 7(4), 541-548.

Hassig, C. A., Tong, J. K., Fleischer, T. C., Owa, T., Grable, P. G., Ayer, D. E., \& Schreiber, S. L. (1998). A role for histone deacetylase activity in HDACl-mediated transcriptional repression. Proceedings of the National Academy of Sciences, 95(7), 3519-3524.

Hasson, U., Avidan, G., Gelbard, H., Vallines, I., Harel, M., Minshew, N., \& Behrmann, M. (2009). Shared and idiosyncratic cortical activation patterns in autism revealed under continuous real-life viewing conditions. Autism Research, 2(4), 220-231.

Hazlett, H. C., Poe, M., Gerig, G., Smith, R. G., Provenzale, J., Ross, A., . . Piven, J. (2005). Magnetic resonance imaging and head circumference study of brain size in autism: birth through age 2 years. Archives of General Psychiatry, 62(12), 1366-1376.

Hazlett, H. C., Poe, M. D., Gerig, G., Styner, M., Chappell, C., Smith, R. G., . . Piven, J. (2011). Early brain overgrowth in autism associated with an increase in cortical surface area before age 2 years. Archives of General Psychiatry, 68(5), 467-476.

Health, M. o. (2011). New Zealand Autism Spectrum Disorder Guideline: Supplementary paper on three pharmacological interventions. New Zealand Guidelines Group. 
Hecht, P. M., Hudson, M., Connors, S. L., Tilley, M. R., Liu, X., \& Beversdorf, D. Q. (2016). Maternal serotonin transporter genotype affects risk for ASD with exposure to prenatal stress. Autism Research, 9(11), 1151-1160.

Heils, A., Teufel, A., Petri, S., Stöber, G., Riederer, P., Bengel, D., \& Lesch, K. P. (1996). Allelic variation of human serotonin transporter gene expression. Journal of neurochemistry, 66(6), 2621-2624.

Heinrichs, M., \& Domes, G. (2008). Neuropeptides and social behaviour: effects of oxytocin and vasopressin in humans. Progress in brain research, 170, 337-350.

Herbert, M. R. (2005). Large brains in autism: the challenge of pervasive abnormality. The Neuroscientist, 11(5), 417-440.

Herrlich, S., Spieth, S., Messner, S., \& Zengerle, R. (2012). Osmotic micropumps for drug delivery. Advanced drug delivery reviews, 64(14), 1617-1627.

Higley, J., \& Linnoila, M. (1997). Low central nervous system serotonergic activity is traitlike and correlates with impulsive behavior. Annals of the New York Academy of Sciences, 836(1), 39-56.

Hofer, M., Masmela, J., Brunelli, S., \& Shair, H. (1998). The ontogeny of maternal potentiation of the infant rats' isolation call. Developmental Psychobiology: The Journal of the International Society for Developmental Psychobiology, 33(3), 189-201.

Hofer, M. A. (1996). Multiple regulators of ultrasonic vocalization in the infant rat. Psychoneuroendocrinology, 21(2), 203-217.

Hollander, E., Soorya, L., Chaplin, W., Anagnostou, E., Taylor, B. P., Ferretti, C. J., . . Settipani, C. (2012). A double-blind placebo-controlled trial of fluoxetine for repetitive behaviors and global severity in adult autism spectrum disorders. American Journal of Psychiatry, 169(3), 292-299.

Holmes, A., Li, Q., Murphy, D., Gold, E., \& Crawley, J. (2003). Abnormal anxiety-related behavior in serotonin transporter null mutant mice: the influence of genetic background. Genes, Brain and Behavior, 2(6), 365-380.

Holmes, A., Murphy, D. L., \& Crawley, J. N. (2003). Abnormal behavioral phenotypes of serotonin transporter knockout mice: parallels with human anxiety and depression. Biological psychiatry, 54(10), 953-959.

Homberg, J., Olivier, J., Smits, B., Mul, J., Mudde, J., Verheul, M., . . Cremers, T. (2007). Characterization of the serotonin transporter knockout rat: a selective change in the functioning of the serotonergic system. Neuroscience, 146(4), 1662-1676.

Homberg, J. R., Schiepers, O. J., Schoffelmeer, A. N., Cuppen, E., \& Vanderschuren, L. J. (2007). Acute and constitutive increases in central serotonin levels reduce social play behaviour in peri-adolescent rats. Psychopharmacology, 195(2), 175-182.

Honeycutt, J. A., Keary, K. M., Kania, V. M., \& Chrobak, J. J. (2016). Developmental age differentially mediates the calcium-binding protein parvalbumin in the rat: Evidence for a selective decrease in hippocampal parvalbumin cell counts. Developmental neuroscience, 38(2), 105-114.

Horlin, C., Falkmer, M., Parsons, R., Albrecht, M. A., \& Falkmer, T. (2014). The cost of autism spectrum disorders. PLoS One, 9(9), el06552.

Horwitz, B., Rumsey, J. M., Grady, C. L., \& Rapoport, S. I. (1988). The cerebral metabolic landscape in autism: intercorrelations of regional glucose utilization. Archives of neurology, 45(7), 749-755.

Huang, C. H., \& Santangelo, S. L. (2008). Autism and serotonin transporter gene polymorphisms: A systematic review and meta-analysis. American Journal of Medical Genetics Part B: Neuropsychiatric Genetics, 147(6), 903-913.

Hughes, R. N. (2007). Sex does matter: comments on the prevalence of male-only investigations of drug effects on rodent behaviour. Behavioural pharmacology, 18(7), 583-589. 
Hulbert, S. W., \& Jiang, Y.-H. (2016). Monogenic mouse models of autism spectrum disorders: common mechanisms and missing links. Neuroscience, 321, 3-23.

Hull, L., Mandy, W., \& Petrides, K. (2017). Behavioural and cognitive sex/gender differences in autism spectrum condition and typically developing males and females. Autism, 21(6), 706-727.

Humphrey, N., \& Symes, W. (2010). Perceptions of social support and experience of bullying among pupils with autistic spectrum disorders in mainstream secondary schools. European Journal of Special Needs Education, 25(1), 77-91.

Hussman, J. P. (2001). Letters to the editor: suppressed GABAergic inhibition as a common factor in suspected etiologies of autism. Journal Of Autism And Developmental Disorders, 31(2), 247-248.

Ingram, J. L., Peckham, S. M., Tisdale, B., \& Rodier, P. M. (2000). Prenatal exposure of rats to valproic acid reproduces the cerebellar anomalies associated with autism. Neurotoxicology and teratology, 22(3), 319-324.

Inoue, H., Yamasue, H., Tochigi, M., Abe, O., Liu, X., Kawamura, Y., . . Rogers, M. A. (2010). Association between the oxytocin receptor gene and amygdalar volume in healthy adults. Biological psychiatry, 68(11), 1066-1072.

Insel, T., Cuthbert, B., Garvey, M., Heinssen, R., Pine, D. S., Quinn, K., . . Wang, P. (2010). Research domain criteria (RDoC): toward a new classification framework for research on mental disorders. In: Am Psychiatric Assoc.

Inui, T., Kumagaya, S., \& Myowa-Yamakoshi, M. (2017). Neurodevelopmental hypothesis about the etiology of autism spectrum disorders. Frontiers in human neuroscience, 11 , 354.

Janušonis, S. (2008). Origin of the blood hyperserotonemia of autism. Theoretical Biology and Medical Modelling, 5(1), 10.

Järbrink, K. (2007). The economic consequences of autistic spectrum disorder among children in a Swedish municipality. Autism, 11(5), 453-463.

Jeste, S. S., \& Tuchman, R. (2015). Autism spectrum disorder and epilepsy: two sides of the same coin? Journal of child neurology, 30(14), 1963-1971.

Jones, K. L., Smith, R. M., Edwards, K. S., Givens, B., Tilley, M. R., \& Beversdorf, D. Q. (2010). Combined effect of maternal serotonin transporter genotype and prenatal stress in modulating offspring social interaction in mice. International Journal of Developmental Neuroscience, 28(6), 529-536.

Juliandi, B., Tanemura, K., Igarashi, K., Tominaga, T., Furukawa, Y., Otsuka, M., . . Sanosaka, T. (2015). Reduced adult hippocampal neurogenesis and cognitive impairments following prenatal treatment of the antiepileptic drug valproic acid. Stem cell reports, 5(6), 996-1009.

Juranek, J., Filipek, P. A., Berenji, G. R., Modahl, C., Osann, K., \& Spence, M. A. (2006). Association between amygdala volume and anxiety level: magnetic resonance imaging (MRI) study in autistic children. Journal of child neurology, 21(12), 1051-1058.

Just, M. A., Cherkassky, V. L., Keller, T. A., Kana, R. K., \& Minshew, N. J. (2006). Functional and anatomical cortical underconnectivity in autism: evidence from an FMRI study of an executive function task and corpus callosum morphometry. Cerebral cortex, 17(4), 951-961.

Kaiser, T., \& Feng, G. (2015). Modeling psychiatric disorders for developing effective treatments. Nature medicine, 21(9), 979.

Kalueff, A., Fox, M., Gallagher, P., \& Murphy, D. (2007). Hypolocomotion, anxiety and serotonin syndrome-like behavior contribute to the complex phenotype of serotonin transporter knockout mice. Genes, Brain and Behavior, 6(4), 389-400. 
Kana, R. K., Keller, T. A., Cherkassky, V. L., Minshew, N. J., \& Just, M. A. (2006). Sentence comprehension in autism: thinking in pictures with decreased functional connectivity. Brain, 129(9), 2484-2493.

Kana, R. K., Keller, T. A., Cherkassky, V. L., Minshew, N. J., \& Just, M. A. (2009). Atypical frontal-posterior synchronization of Theory of Mind regions in autism during mental state attribution. Social neuroscience, 4(2), 135-152.

Kana, R. K., Uddin, L. Q., Kenet, T., Chugani, D., \& Müller, R.-A. (2014). Brain connectivity in autism. Frontiers in human neuroscience, 8, 349.

Kanner, L. (1943). Autistic disturbances of affective contact. Nervous child, 2(3), 217-250.

Karlsson, H., \& Dalman, C. (2019). Epidemiological Studies of Prenatal and Childhood Infection and Schizophrenia.

Kataoka, S., Takuma, K., Hara, Y., Maeda, Y., Ago, Y., \& Matsuda, T. (2013). Autism-like behaviours with transient histone hyperacetylation in mice treated prenatally with valproic acid. International Journal of Neuropsychopharmacology, 16(1), 91-103.

Kelleher, R. J., \& Bear, M. F. (2008). The autistic neuron: troubled translation? Cell, 135(3), 401-406.

Kemppainen, S., \& Pitkänen, A. (2000). Distribution of parvalbumin, calretinin, and calbindin-D28k immunoreactivity in the rat amygdaloid complex and colocalization with $\gamma$-aminobutyric acid. Journal of Comparative Neurology, 426(3), 441-467.

Kern, J. K. (2003). Purkinje cell vulnerability and autism: a possible etiological connection. Brain and Development, 25(6), 377-382.

Kerr, D., Downey, L., Conboy, M., Finn, D., \& Roche, M. (2013). Alterations in the endocannabinoid system in the rat valproic acid model of autism. Behavioural brain research, 249, 124-132.

Kidwell, M. (2018). Establishment of an Animal Model of Depression: The Serotonin Transporter Knockout Rat. (Master's ), Victoria University of Wellington, Wellington.

Kim, J.-W., Park, K., Kang, R. J., Gonzales, E. L., Oh, H. A., Seung, H., . . Shin, C. Y. (2019). Gene-environment interaction counterbalances social impairment in mouse models of autism. Scientific reports, 9(1), 1-10.

Kim, J. W., Seung, H., Kwon, K. J., Ko, M. J., Lee, E. J., Oh, H. A., . . You, J. S. (2014). Subchronic treatment of donepezil rescues impaired social, hyperactive, and stereotypic behavior in valproic acid-induced animal model of autism. PLoS One, 9(8), el04927.

Kim, K. C., Kim, P., Go, H. S., Choi, C. S., Yang, S.-I., Cheong, J. H., . . Ko, K. H. (2011). The critical period of valproate exposure to induce autistic symptoms in Sprague-Dawley rats. Toxicology letters, 201(2), 137-142.

Kim, S.-Y., Choi, U.-S., Park, S.-Y., Oh, S.-H., Yoon, H.-W., Koh, Y.-J., . . Cheon, K.-A. (2015). Abnormal activation of the social brain network in children with autism spectrum disorder: an FMRI study. Psychiatry investigation, 12(1), 37.

Kim, Y. S., \& Leventhal, B. L. (2015). Genetic epidemiology and insights into interactive genetic and environmental effects in autism spectrum disorders. Biological psychiatry, 77(1), 66-74.

Kim, Y. S., Leventhal, B. L., Koh, Y.-J., Fombonne, E., Laska, E., Lim, E.-C., . . Lee, H. (2011). Prevalence of autism spectrum disorders in a total population sample. American Journal of Psychiatry, 168(9), 904-912.

Kinney, D. K., Miller, A. M., Crowley, D. J., Huang, E., \& Gerber, E. (2008). Autism prevalence following prenatal exposure to hurricanes and tropical storms in Louisiana.J Autism Dev Disord, 38(3), 481-488. Retrieved from http://www.ncbi.nlm.nih.gov/pubmed/17619130. doi:10.1007/s10803-007-0414-0 
Kinney, D. K., Munir, K. M., Crowley, D. J., \& Miller, A. M. (2008). Prenatal stress and risk for autism. Neurosci Biobehav Rev, 32(8), 1519-1532. Retrieved from http://www.ncbi.nlm.nih.gov/pubmed/18598714. doi:10.1016/j.neubiorev.2008.06.004

Kirsten, T. B., Queiroz-Hazarbassanov, N., Bernardi, M. M., \& Felicio, L. F. (2015). Prenatal zinc prevents communication impairments and BDNF disturbance in a rat model of autism induced by prenatal lipopolysaccharide exposure. Life sciences, 130, 12-17.

Kirsten, T. B., Taricano, M., Maiorka, P. C., Palermo-Neto, J., \& Bernardi, M. M. (2010). Prenatal lipopolysaccharide reduces social behavior in male offspring. Neuroimmunomodulation, 17(4), 240-251.

Kiser, D., Steemers, B., Branchi, I., \& Homberg, J. R. (2012). The reciprocal interaction between serotonin and social behaviour. Neuroscience E Biobehavioral Reviews, 36(2), 786-798.

Klotz, U., \& Antonin, K. (1977). Pharmacokinetics and bioavailability of sodium valproate. Clinical Pharmacology \& Therapeutics, 21(6), 736-743.

Kluck, S. M., Poustka, F., Benner, A., Lesch, K.-P., \& Poustka, A. (1997). Serotonin transporter (5-HTT) gene variants associated with autism? Human molecular genetics, 6(13), 22332238.

Knutson, B., Burgdorf, J., \& Panksepp, J. (2002). Ultrasonic vocalizations as indices of affective states in rats. Psychological bulletin, 128(6), 961.

Koegel, L. K., Park, M. N., \& Koegel, R. L. (2014). Using self-management to improve the reciprocal social conversation of children with autism spectrum disorder. Journal of Autism And Developmental Disorders, 44(5), 1055-1063.

Kogan, J. H., Frankland, P. W., \& Silva, A. J. (2000). Long-term memory underlying hippocampus-dependent social recognition in mice. Hippocampus, 10(1), 47-56.

Kosaka, H., Okamoto, Y., Munesue, T., Yamasue, H., Inohara, K., Fujioka, T., . . Jung, M. (2016). Oxytocin efficacy is modulated by dosage and oxytocin receptor genotype in young adults with high-functioning autism: a 24-week randomized clinical trial. Translational psychiatry, 6(8), e872.

Koul, O. (2005). Myelin and autism. The neurobiology of autism, 150-163.

Krämer, O. H., Zhu, P., Ostendorff, H. P., Golebiewski, M., Tiefenbach, J., Peters, M. A., . . . Heinzel, T. (2003). The histone deacetylase inhibitor valproic acid selectively induces proteasomal degradation of HDAC2. The EMBO journal, 22(13), 3411-3420.

Kromkhun, P., Katou, M., Hashimoto, H., Terada, M., Moon, C., \& Saito, T. R. (2013).

Quantitative and qualitative analysis of rat pup ultrasonic vocalization sounds induced by a hypothermic stimulus. Laboratory animal research, 29(2), 77-83.

Kuwagata, M., Ogawa, T., Shioda, S., \& Nagata, T. (2009). Observation of fetal brain in a rat valproate-induced autism model: a developmental neurotoxicity study. International Journal of Developmental Neuroscience, 27(4), 399-405.

La Malfa, G., Lassi, S., Bertelli, M., Salvini, R., \& Placidi, G. (2004). Autism and intellectual disability: a study of prevalence on a sample of the Italian population. Journal of intellectual disability research, 48(3), 262-267.

Laplante, D. P., Brunet, A., Schmitz, N., Ciampi, A., \& King, S. (2008). Project Ice Storm: prenatal maternal stress affects cognitive and linguistic functioning in 51 /2-year-old children. J Am Acad Child Adolesc Psychiatry, 47(9), 1063-1072. Retrieved from http://www.ncbi.nlm.nih.gov/pubmed/18665002. doi:10.1097/CHI.0b013e31817eec80

Lauber, E., Filice, F., \& Schwaller, B. (2016). Prenatal valproate exposure differentially affects parvalbumin-expressing neurons and related circuits in the cortex and striatum of mice. Frontiers in molecular neuroscience, 9, 150.

LeClerc, S., \& Easley, D. (2015). Pharmacological therapies for autism spectrum disorder: a review. Pharmacy and Therapeutics, 40(6), 389. 
Leigh, J. P., \& Du, J. (2015). Brief report: Forecasting the economic burden of autism in 2015 and 2025 in the United States. Journal Of Autism And Developmental Disorders, 45(12), 4135-4139.

Lemaire, V., Koehl, M., Le Moal, M., \& Abrous, D. N. (2000). Prenatal stress produces learning deficits associated with an inhibition of neurogenesis in the hippocampus. Proceedings of the National Academy of Sciences, 97(20), 11032-11037.

Lesch, K.-P., Balling, U., Gross, J., Strauss, K., Wolozin, B., Murphy, D., \& Riederer, P. (1994). Organization of the human serotonin transporter gene. Journal of Neural Transmission/General Section JNT, 95(2), 157-162.

Lesch, K.-P., Bengel, D., Heils, A., Sabol, S. Z., Greenberg, B. D., Petri, S., . . Murphy, D. L. (1996). Association of anxiety-related traits with a polymorphism in the serotonin transporter gene regulatory region. Science, 274(5292), 1527-1531.

Lesch, K.-P., \& Gutknecht, L. (2005). Pharmacogenetics of the serotonin transporter. Progress in Neuro-Psychopharmacology and Biological Psychiatry, 29(6), 1062-1073.

Lester, B. M. (1987). Developmental outcome prediction from acoustic cry analysis in term and preterm infants. Pediatrics, 80(4), 529-534.

Li, Y., Missig, G., Finger, B. C., Landino, S. M., Alexander, A. J., Mokler, E. L., . . Kim, K.-S. (2018). Maternal and early postnatal immune activation produce dissociable effects on neurotransmission in mPFC-amygdala circuits. Journal of Neuroscience, 38(13), 33583372.

Libbey, J. E., Sweeten, T. L., McMahon, W. M., \& Fujinami, R. S. (2005). Autistic disorder and viral infections. Journal of neurovirology, 11(1), 1-10.

Lipton, J. W., Robie, H. C., Ling, Z., Weese-Mayer, D. E., \& Carvey, P. M. (1998). The magnitude of brain dopamine depletion from prenatal cocaine exposure is a function of uterine position. Neurotoxicology and teratology, 20(4), 373-382.

Lira, A., Zhou, M., Castanon, N., Ansorge, M. S., Gordon, J. A., Francis, J. H., . . Arango, V. (2003). Altered depression-related behaviors and functional changes in the dorsal raphe nucleus of serotonin transporter-deficient mice. Biological psychiatry, 54(10), 960-971.

Lloyd, K. A. (2013). A scientific review: mechanisms of valproate-mediated teratogenesis. Bioscience Horizons, 6, hzt003.

Lloyd, K. A. (2013). A scientific review: mechanisms of valproate-mediated teratogenesis. Bioscience Horizons: The International Journal of Student Research, 6.

Loohuis, N. O., Martens, G. J., van Bokhoven, H., Kaplan, B. B., Homberg, J. R., \& Aschrafi, A. (2017). Altered expression of circadian rhythm and extracellular matrix genes in the medial prefrontal cortex of a valproic acid rat model of autism. Progress in NeuroPsychopharmacology and Biological Psychiatry, 77, 128-132.

LoParo, D., \& Waldman, I. (2015). The oxytocin receptor gene (OXTR) is associated with autism spectrum disorder: a meta-analysis. Molecular Psychiatry, 20(5), 640.

Löscher, W. (1982). Anticonvulsant and biochemical effects of inhibitors of GABA aminotransferase and valproic acid during subchronic treatment in mice. Biochemical pharmacology, 31(5), 837-842.

Löscher, W. (1999). Valproate: a reappraisal of its pharmacodynamic properties and mechanisms of action. Progress in neurobiology, 58(1), 31-59.

Löscher, W. (2002). Basic pharmacology of valproate. CNS drugs, 16(10), 669-694.

Löscher, W. (2007). The pharmacokinetics of antiepileptic drugs in rats: consequences for maintaining effective drug levels during prolonged drug administration in rat models of epilepsy. Epilepsia, 48(7), 1245-1258.

Lübkemann, R., Eberhardt, J., Röhl, F.-W., Janitzky, K., Nullmeier, S., Stork, O., . . Linke, R. (2015). Identification and characterization of GABAergic projection neurons from ventral hippocampus to amygdala. Brain sciences, 5(3), 299-317. 
Lynn, D. A., \& Brown, G. R. (2009). The ontogeny of exploratory behavior in male and female adolescent rats (Rattus norvegicus). Developmental psychobiology, 51(6), 513-520.

Mabunga, D. F. N., Gonzales, E. L. T., Kim, J.-w., Kim, K. C., \& Shin, C. Y. (2015). Exploring the validity of valproic acid animal model of autism. Experimental neurobiology, 24(4), 285-300.

Machado, C. J., Whitaker, A. M., Smith, S. E., Patterson, P. H., \& Bauman, M. D. (2015). Maternal immune activation in nonhuman primates alters social attention in juvenile offspring. Biological psychiatry, 77(9), 823-832.

Macintosh, K. E., \& Dissanayake, C. (2004). Annotation: the similarities and differences between autistic disorder and Asperger's disorder: a review of the empirical evidence. Journal of Child Psychology and Psychiatry, 45(3), 421-434.

Maestrini, E., Lai, C., Marlow, A., Matthews, N., Wallace, S., Bailey, A., . . Monaco, A. P. (1999). Serotonin transporter (5-HTT) and $\gamma$-aminobutyric acid receptor subunit $\beta 3$ (GABRB3) gene polymorphisms are not associated with autism in the IMGSA families. American Journal of Medical Genetics, 88(5), 492-496.

Maestripieri, D., McCormack, K., Lindell, S. G., Higley, J. D., \& Sanchez, M. M. (2006). Influence of parenting style on the offspring's behaviour and CSF monoamine metabolite levels in crossfostered and noncrossfostered female rhesus macaques. Behavioural brain research, 175(1), 90-95.

Main, S. L., \& Kulesza, R. J. (2017). Repeated prenatal exposure to valproic acid results in cerebellar hypoplasia and ataxia. Neuroscience, 340, 34-47.

Malkova, N. V., Collin, Z. Y., Hsiao, E. Y., Moore, M. J., \& Patterson, P. H. (2012). Maternal immune activation yields offspring displaying mouse versions of the three core symptoms of autism. Brain, behavior, and immunity, 26(4), 607-616.

Mandy, W., Chilvers, R., Chowdhury, U., Salter, G., Seigal, A., \& Skuse, D. (2012). Sex differences in autism spectrum disorder: evidence from a large sample of children and adolescents. Journal Of Autism And Developmental Disorders, 42(7), 1304-1313.

Markram, H., Rinaldi, T., \& Markram, K. (2007). The intense world syndrome-an alternative hypothesis for autism. Frontiers in neuroscience, 1, 6 .

Markram, K., Rinaldi, T., La Mendola, D., Sandi, C., \& Markram, H. (2008). Abnormal fear conditioning and amygdala processing in an animal model of autism. Neuropsychopharmacology, 33(4), 901.

Martin, H. G. S., \& Manzoni, O. (2014). Late onset deficits in synaptic plasticity in the valproic acid rat model of autism. Frontiers in cellular neuroscience, 8, 23.

Masur, J., Schutz, M. T., \& Boerngen, R. (1980). Gender differences in open-field behavior as a function of age. Developmental Psychobiology: The Journal of the International Society for Developmental Psychobiology, 13(2), 107-110.

Matson, J. L., \& Shoemaker, M. (2009). Intellectual disability and its relationship to autism spectrum disorders. Research in developmental disabilities, 30(6), 1107-1114.

Matsuo, M., Maeda, T., Sasaki, K., Ishii, K., \& Hamasaki, Y. (2010). Frequent association of autism spectrum disorder in patients with childhood onset epilepsy. Brain and Development, 32(9), 759-763.

McAlonan, G. M., Li, Q., \& Cheung, C. (2010). The timing and specificity of prenatal immune risk factors for autism modeled in the mouse and relevance to schizophrenia. Neurosignals, 18(2), 129-139.

McBride, P. A., Anderson, G. M., Hertzig, M. E., Snow, M. E., Thompson, S. M., Khait, V. D., . . . Cohen, D. J. (1998). Effects of diagnosis, race, and puberty on platelet serotonin levels in autism and mental retardation. Journal of the American Academy of Child $\mathcal{E}$ Adolescent Psychiatry, 37(7), 767-776.

McDonald, A. J. (1982). Neurons of the lateral and basolateral amygdaloid nuclei: a Golgi study in the rat. Journal of Comparative Neurology, 212(3), 293-312. 
McDonald, A. J., \& Mascagni, F. (2007). Neuronal localization of 5-HT type 2A receptor immunoreactivity in the rat basolateral amygdala. Neuroscience, 146(1), 306-320.

McDougle, C., Naylor, S. T., Cohen, D. J., Aghajanian, G. K., Heninger, G. R., \& Price, L. H. (1996). Effects of tryptophan depletion in drug-free adults with autistic disorder. Archives of General Psychiatry, 53(11), 993-1000.

McDougle, C. J., Naylor, S. T., Cohen, D. J., Volkmar, F. R., Heninger, G. R., \& Price, L. H. (1996). A double-blind, placebo-controlled study of fluvoxamine in adults with autistic disorder. Archives of General Psychiatry, 53(11), 1001-1008.

McFarlane, H. G., Kusek, G., Yang, M., Phoenix, J., Bolivar, V., \& Crawley, J. (2008). Autismlike behavioral phenotypes in BTBR T+ tf/J mice. Genes, Brain and Behavior, 7(2), 152163.

McGraw, L. A., \& Young, L. J. (2010). The prairie vole: an emerging model organism for understanding the social brain. Trends in neurosciences, 33(2), 103-109.

Meaney, M. J., \& Stewart, J. (1979). Environmental factors influencing the affiliative behavior of male and female rats (Rattus norvegicus). Animal Learning $\mathcal{E}$ Behavior, 7(3), 397405.

Mednick, S., Huttunen, M. O., \& Machón, R. A. (1994). Prenatal influenza infections and adult schizophrenia. Schizophrenia bulletin, 20(2), 263-267.

Melancia, F., Schiavi, S., Servadio, M., Cartocci, V., Campolongo, P., Palmery, M., . . Trezza, V. (2018). Sex-specific autistic endophenotypes induced by prenatal exposure to valproic acid involve anandamide signalling. British journal of pharmacology, 175(18), 3699-3712.

Meng, Q., Li, N., Han, X., Shao, F., \& Wang, W. (2010). Peri-adolescence isolation rearing alters social behavior and nociception in rats. Neuroscience Letters, 480(1), 25-29.

Meyza, K. Z., Defensor, E. B., Jensen, A. L., Corley, M. J., Pearson, B. L., Pobbe, R. L., . . . Blanchard, R. J. (2013). The BTBR T+tf/J mouse model for autism spectrum disordersin search of biomarkers. Behavioural brain research, 251, 25-34.

Miller, M. T., Strömland, K., Gillberg, C., Johansson, M., \& Nilsson, E. W. (1998). The puzzle of autism: an ophthalmologic contribution. Transactions of the American Ophthalmological Society, 96, 369.

Mineur, Y. S., Huynh, L. X., \& Crusio, W. E. (2006). Social behavior deficits in the Fmrl mutant mouse. Behavioural brain research, 168(1), 172-175.

Miyazaki, K., Narita, N., \& Narita, M. (2005). Maternal administration of thalidomide or valproic acid causes abnormal serotonergic neurons in the offspring: implication for pathogenesis of autism. International Journal of Developmental Neuroscience, 23(2-3), 287-297.

Mizuno, A., Villalobos, M. E., Davies, M. M., Dahl, B. C., \& Müller, R.-A. (2006). Partially enhanced thalamocortical functional connectivity in autism. Brain research, 1104(1), $160-174$.

Modahl, C., Green, L. A., Fein, D., Morris, M., Waterhouse, L., Feinstein, C., \& Levin, H. (1998). Plasma oxytocin levels in autistic children. Biological psychiatry, 43(4), 270-277.

Moldrich, R. X., Leanage, G., She, D., Dolan-Evans, E., Nelson, M., Reza, N., \& Reutens, D. C. (2013). Inhibition of histone deacetylase in utero causes sociability deficits in postnatal mice. Behavioural brain research, 257, 253-264.

Moore, M., Eichner, S. F., \& Jones, J. R. (2004). Treating functional impairment of autism with selective serotonin-reuptake inhibitors. Annals of Pharmacotherapy, 38(9), 1515-1519.

Moore, S., Turnpenny, P., Quinn, A., Glover, S., Lloyd, D. J., Montgomery, T., \& Dean, J. C. S. (2000). A clinical study of 57 children with fetal anticonvulsant syndromes. Journal of Medical Genetics, 37(7), 489-497. Retrieved from <Go to ISI>://000088157200002. doi:Doi 10.1136/Jmg.37.7.489 
Moretti, P., Bouwknecht, J. A., Teague, R., Paylor, R., \& Zoghbi, H. Y. (2004). Abnormalities of social interactions and home-cage behavior in a mouse model of Rett syndrome. Human molecular genetics, 14(2), 205-220.

Moy, S. S., Nadler, J. J., Young, N. B., Nonneman, R. J., Grossman, A. W., Murphy, D. L., . . Lauder, J. M. (2009). Social approach in genetically engineered mouse lines relevant to autism. Genes, Brain and Behavior, 8(2), 129-142.

Moy, S. S., Nadler, J. J., Young, N. B., Nonneman, R. J., Segall, S. K., Andrade, G. M., . . Magnuson, T. R. (2008). Social approach and repetitive behavior in eleven inbred mouse strains. Behavioural brain research, 191(1), 118-129.

Moy, S. S., Nadler, J. J., Young, N. B., Perez, A., Holloway, L. P., Barbaro, R. P., . . Lauder, J. M. (2007). Mouse behavioral tasks relevant to autism: phenotypes of 10 inbred strains. Behavioural brain research, 176(1), 4-20.

Muller, M., Olivier, J., \& Homberg, J. (2010). Knockout and mutant rats. In Neuromethods (Vol. 44, pp. 13-31).

Munson, J., Dawson, G., Abbott, R., Faja, S., Webb, S. J., Friedman, S. D., . . Dager, S. R. (2006). Amygdalar volume and behavioral development in autism. Archives of General Psychiatry, 63(6), 686-693.

Na, L., Wartenberg, M., Nau, H., Hescheler, J., \& Sauer, H. (2003). Anticonvulsant valproic acid inhibits cardiomyocyte differentiation of embryonic stem cells by increasing intracellular levels of reactive oxygen species. Birth Defects Research Part A: Clinical and Molecular Teratology, 67(3), 174-180.

Nadler, J., Moy, S., Dold, G., Trang, D., Simmons, N., Perez, A., . . Magnuson, T. (2004). Automated apparatus for quantitation of social approach behaviors in mice. Genes, Brain and Behavior, 3(5), 303-314.

Nadler, J. J., Zou, F., Huang, H., Moy, S. S., Lauder, J., Crawley, J. N., . . Magnuson, T. R. (2006). Large-scale gene expression differences across brain regions and inbred strains correlate with a behavioral phenotype. Genetics, 174(3), 1229-1236.

Nakamura, K., Sekine, Y., Ouchi, Y., Tsujii, M., Yoshikawa, E., Futatsubashi, M., . . Suzuki, K. (2010). Brain serotonin and dopamine transporter bindings in adults with highfunctioning autism. Archives of General Psychiatry, 67(1), 59-68.

Narboux-Nême, N., Pavone, L. M., Avallone, L., Zhuang, X., \& Gaspar, P. (2008). Serotonin transporter transgenic (SERTcre) mouse line reveals developmental targets of serotonin specific reuptake inhibitors (SSRIs). Neuropharmacology, 55(6), 994-1005.

Narita, M., Oyabu, A., Imura, Y., Kamada, N., Yokoyama, T., Tano, K., . . Narita, N. (2010). Nonexploratory movement and behavioral alterations in a thalidomide or valproic acid-induced autism model rat. Neuroscience research, 66(1), 2-6.

Narita, N., Kato, M., Tazoe, M., Miyazaki, K., Narita, M., \& Okado, N. (2002). Increased monoamine concentration in the brain and blood of fetal thalidomide- and valproic acid-exposed rat: putative animal models for autism. Pediatr Res, 52(4), 576-579. Retrieved from http://www.ncbi.nlm.nih.gov/pubmed/12357053. doi:10.1203/00006450-200210000-00018

Nau, H., Koch, S., Häuser, I., \& Helge, H. (1981). Valproic acid and its metabolites: placental transfer, neonatal pharmacokinetics, transfer via mother's milk and clinical status in neonates of epileptic mothers. Journal of Pharmacology and Experimental Therapeutics, 219(3), 768-777.

Nau, H., \& Loscher, W. (1984). Valproic acid and metabolites: pharmacological and toxicological studies. Epilepsia, 25, S14-S22.

Nau, H., Merker, H., Brendel, K., Gansau, C., Hauser, I., \& Wittfoht, W. (1984). Disposition, embryotoxicity and teratogenicity of valproic acid in the mouse as related to man. Metabolism of Antiepileptic Drugs, Raven Press, New York, 85-93. 
Nau, H., Zierer, R., Spielmann, H., Neubert, D., \& Gansau, C. (1981). A new model for embryotoxicity testing: teratogenicity and pharmacokinetics of valproic acid following constant-rate administration in the mouse using human therapeutic drug and metabolite concentrations. Life sciences, 29(26), 2803-2813.

Naushad, S. M., Jain, J. M. N., Prasad, C. K., Naik, U., \& Akella, R. R. D. (2013). Autistic children exhibit distinct plasma amino acid profile.

Neale, B. M., Kou, Y., Liu, L., Ma’Ayan, A., Samocha, K. E., Sabo, A., . . Makarov, V. (2012). Patterns and rates of exonic de novo mutations in autism spectrum disorders. Nature, 485(7397), 242.

Nestler, E. J., \& Hyman, S. E. (2010). Animal models of neuropsychiatric disorders. Nat Neurosci, 13(10), 1161-1169. Retrieved from http://www.ncbi.nlm.nih.gov/pubmed/20877280. doi:10.1038/nn.2647

Nguyen, M., Roth, A., Kyzar, E. J., Poudel, M. K., Wong, K., Stewart, A. M., \& Kalueff, A. V. (2014). Decoding the contribution of dopaminergic genes and pathways to autism spectrum disorder (ASD). Neurochemistry international, 66, 15-26.

Niesink, R. J., \& Van Ree, J. (1989). Involvement of opioid and dopaminergic systems in isolation-induced pinning and social grooming of young rats. Neuropharmacology, 28(4), 411-418.

Nijmeijer, J. S., Hartman, C. A., Rommelse, N. N., Altink, M. E., Buschgens, C. J., Fliers, E. A., . . . Sergeant, J. A. (2010). Perinatal risk factors interacting with catechol Omethyltransferase and the serotonin transporter gene predict ASD symptoms in children with ADHD. Journal of Child Psychology and Psychiatry, 51(11), 1242-1250.

Noirot, E. (1972). Ultrasounds and maternal behavior in small rodents. Developmental Psychobiology: The Journal of the International Society for Developmental Psychobiology, 5(4), 371-387.

Nomi, J. S., \& Uddin, L. Q. (2015). Developmental changes in large-scale network connectivity in autism. NeuroImage: Clinical, 7, 732-741.

Nordahl, C. W., Scholz, R., Yang, X., Buonocore, M. H., Simon, T., Rogers, S., \& Amaral, D. G. (2012). Increased rate of amygdala growth in children aged 2 to 4 years with autism spectrum disorders: a longitudinal study. Archives of General Psychiatry, 69(1), 53-61.

O'Shea, M., McGregor, I. S., \& Mallet, P. E. (2006). Repeated cannabinoid exposure during perinatal, adolescent or early adult ages produces similar longlasting deficits in object recognition and reduced social interaction in rats. Journal of psychopharmacology, 20(5), 611-621.

Oblak, A. L., Gibbs, T. T., \& Blatt, G. J. (2010). Decreased GABAB receptors in the cingulate cortex and fusiform gyrus in autism. Journal of neurochemistry, 114(5), 1414-1423.

Ohkawara, T., Katsuyama, T., Ida-Eto, M., Narita, N., \& Narita, M. (2015). Maternal viral infection during pregnancy impairs development of fetal serotonergic neurons. Brain and Development, 37(1), 88-93.

Olde Loohuis, N., Kole, K., Glennon, J., Karel, P., Van der Borg, G., Van Gemert, Y., .. . Shahabipour, F. (2015). Elevated microRNA-181c and microRNA-30d levels in the enlarged amygdala of the valproic acid rat model of autism. Neurobiology of disease, 80, 42-53.

Olivier, J., Van Der Hart, M., Van Swelm, R., Dederen, P., Homberg, J., Cremers, T., . . . Ellenbroek, B. (2008). A study in male and female 5-HT transporter knockout rats: an animal model for anxiety and depression disorders. Neuroscience, 152(3), 573-584.

Ong, L. L., Schardein, J. L., Petrere, J. A., Sakowski, R., Jordan, H., Humphrey, R. R., . . de la IGLESIA, F. A. (1983). Teratogenesis of calcium valproate in rats. Toxicological Sciences, 3(2), 121-126.

Ornoy, A. (2009). Valproic acid in pregnancy: how much are we endangering the embryo and fetus? Reproductive Toxicology, 28(1), 1-10. 
Ozonoff, S., Pennington, B. F., \& Rogers, S. J. (1991). Executive function deficits in highfunctioning autistic individuals: relationship to theory of mind. Journal of Child Psychology and Psychiatry, 32(7), 1081-1105.

Ozonoff, S., Young, G. S., Carter, A., Messinger, D., Yirmiya, N., Zwaigenbaum, L., . . Dobkins, K. (2011). Recurrence risk for autism spectrum disorders: a Baby Siblings Research Consortium study. Pediatrics, peds. 2010-2825.

Panksepp, J., \& Beatty, W. W. (1980). Social deprivation and play in rats. Behavioral and neural biology, 30(2), 197-206.

Parker, K. J., Oztan, O., Libove, R. A., Sumiyoshi, R. D., Jackson, L. P., Karhson, D. S., . . . Phillips, J. M. (2017). Intranasal oxytocin treatment for social deficits and biomarkers of response in children with autism. Proceedings of the National Academy of Sciences, $114(30), 8119-8124$.

Patterson, P. H. (2011). Maternal infection and immune involvement in autism. Trends in molecular medicine, 17(7), 389-394.

Patterson, P. H. (2011). Modeling autistic features in animals. Pediatr Res, 69(5), 34R-40R.

Peça, J., Feliciano, C., Ting, J. T., Wang, W., Wells, M. F., Venkatraman, T. N., . . Feng, G. (2011). Shank3 mutant mice display autistic-like behaviours and striatal dysfunction. Nature, 472(7344), 437.

Pellis, S. M., \& Pasztor, T. J. (1999). The developmental onset of a rudimentary form of play fighting in C57 mice. Developmental Psychobiology: The Journal of the International Society for Developmental Psychobiology, 34(3), 175-182.

Pellis, S. M., \& Pellis, V. C. (2007). Rough-and-tumble play and the development of the social brain. Current directions in psychological science, 16(2), 95-98.

Penn, H. E. (2006). Neurobiological correlates of autism: a review of recent research. Child Neuropsychology, 12(1), 57-79.

Persico, A. M., Militerni, R., Bravaccio, C., Schneider, C., Amed, R., Conciatori, M., .. . Keller, F. (2000). Lack of association between serotonin transporter gene promoter variants and autistic disorder in two ethnically distinct samples. American Journal of Medical Genetics, 96(1), 123-127.

Philip, R. C., Dauvermann, M. R., Whalley, H. C., Baynham, K., Lawrie, S. M., \& Stanfield, A. C. (2012). A systematic review and meta-analysis of the fMRI investigation of autism spectrum disorders. Neuroscience \& Biobehavioral Reviews, 36(2), 901-942.

Pickles, A., Starr, E., Kazak, S., Bolton, P., Papanikolaou, K., Bailey, A., . . Rutter, M. (2000). Variable expression of the autism broader phenotype: findings from extended pedigrees. The Journal of Child Psychology and Psychiatry and Allied Disciplines, 41(4), 491-502.

Pietropaolo, S., Guilleminot, A., Martin, B., D'Amato, F. R., \& Crusio, W. E. (2011). Geneticbackground modulation of core and variable autistic-like symptoms in Fmrl knock-out mice. PLoS One, 6(2), el7073.

Pinto, D., Delaby, E., Merico, D., Barbosa, M., Merikangas, A., Klei, L., . . Wang, Z. (2014). Convergence of genes and cellular pathways dysregulated in autism spectrum disorders. The American Journal of Human Genetics, 94(5), 677-694.

Piven, J., Bailey, J., Ranson, B. J., \& Arndt, S. (1998). No difference in hippocampus volume detected on magnetic resonance imaging in autistic individuals. Journal Of Autism And Developmental Disorders, 28(2), 105-110.

Piven, J., Palmer, P., Jacobi, D., Childress, D., \& Arndt, S. (1997). Broader autism phenotype: evidence from a family history study of multiple-incidence autism families. American Journal of Psychiatry, 154(2), 185-190.

Polleux, F., \& Lauder, J. M. (2004). Toward a developmental neurobiology of autism. Mental retardation and developmental disabilities research reviews, 10(4), 303-317. 
Poole, T. B., \& Fish, J. (1975). An investigation of playful behaviour in Rattus norvegicus and Mus musculus (Mammalia). Journal of Zoology, 175(1), 61-71.

Portfors, C. V. (2007). Types and functions of ultrasonic vocalizations in laboratory rats and mice. Journal of the American Association for Laboratory Animal Science, 46(1), 28-34.

Powell, E. M., Campbell, D. B., Stanwood, G. D., Davis, C., Noebels, J. L., \& Levitt, P. (2003). Genetic disruption of cortical interneuron development causes region-and GABA cell type-specific deficits, epilepsy, and behavioral dysfunction. Journal of Neuroscience, 23(2), 622-631.

Prendergast, B. J., Onishi, K. G., \& Zucker, I. (2014). Female mice liberated for inclusion in neuroscience and biomedical research. Neuroscience \& Biobehavioral Reviews, 40, 1-5.

Purcell, A., Jeon, O., Zimmerman, A., Blue, M., \& Pevsner, J. (2001). Postmortem brain abnormalities of the glutamate neurotransmitter system in autism. Neurology, 57(9), 1618-1628.

Radyushkin, K., Hammerschmidt, K., Boretius, S., Varoqueaux, F., El-Kordi, A., Ronnenberg, A., ... Brose, N. (2009). Neuroligin-3-deficient mice: model of a monogenic heritable form of autism with an olfactory deficit. Genes, Brain and Behavior, 8(4), 416-425.

Ramoz, N., Reichert, J. G., Corwin, T. E., Smith, C. J., Silverman, J. M., Hollander, E., \& Buxbaum, J. D. (2006). Lack of evidence for association of the serotonin transporter gene SLC6A4 with autism. Biological psychiatry, 60(2), 186-191.

Ranger, P. (2016). The development of animal models for autism: A gene-environment approach.

Ranger, P., \& Ellenbroek, B. A. (2015). Perinatal influences of valproate on brain and behaviour: an animal model for autism. In Neurotoxin Modeling of Brain DisordersLife-long Outcomes in Behavioral Teratology (pp. 363-386): Springer.

Rasalam, A. D., Hailey, H., Williams, J. H., Moore, S. J., Turnpenny, P. D., Lloyd, D. J., \& Dean, J. C. (2005). Characteristics of fetal anticonvulsant syndrome associated autistic disorder. Dev Med Child Neurol, 47(8), 551-555. Retrieved from http://www.ncbi.nlm.nih.gov/pubmed/16108456.

Raymond, G. V., Bauman, M. L., \& Kemper, T. L. (1995). Hippocampus in autism: a Golgi analysis. Acta neuropathologica, 91(1), 117-119.

Raza, S., Himmler, B. T., Himmler, S. M., Harker, A., Kolb, B., Pellis, S. M., \& Gibb, R. (2015). Effects of prenatal exposure to valproic acid on the development of juvenile-typical social play in rats. Behavioural pharmacology, 26(8-9), 707-719.

Risch, N., Herrell, R., Lehner, T., Liang, K.-y., \& Merikangas, K. R. (2009). Interaction between the serotonin transporter gene (5-HTTLPR), stressful life events, and risk of depression. Paper presented at the JAMA.

Ritvo, E. R., Freeman, B., Scheibel, A. B., Duong, T., Robinson, H., Guthrie, D., \& Ritvo, A. (1986). Lower Purkinje cell counts in the cerebella of four autistic subjects: Initial findings of the UCLA-NSAC research report. The American journal of psychiatry.

Ritvo, E. R., Jorde, L. B., Mason-Brothers, A., Freeman, B., \& Pingree, C. (1989). The UCLAUniversity of Utah epidemiological survey of autism: Recurrence risk estimates and genetic counseling. The American journal of psychiatry, 146(8), 1032.

Rodier, P. M., Ingram, J. L., Tisdale, B., \& Croog, V. J. (1997). Linking etiologies in humans and animal models: studies of autism. Reproductive Toxicology, 11(2), 417-422.

Rodier, P. M., Ingram, J. L., Tisdale, B., Nelson, S., \& Romano, J. (1996). Embryological origin for autism: developmental anomalies of the cranial nerve motor nuclei. Journal of Comparative Neurology, 370(2), 247-261.

Rosenberg, R. E., Law, J. K., Yenokyan, G., McGready, J., Kaufmann, W. E., \& Law, P. A. (2009). Characteristics and concordance of autism spectrum disorders among 277 twin pairs. Archives of pediatrics $\mathcal{E}$ adolescent medicine, 163(10), 907-914. 
Ross, C. A., \& Margolis, R. L. (2019). Research Domain Criteria: Strengths, Weaknesses, and Potential Alternatives for Future Psychiatric Research. Molecular Neuropsychiatry, 1-18.

Roullet, F. I., Lai, J. K., \& Foster, J. A. (2013). In utero exposure to valproic acid and autism--a current review of clinical and animal studies. Neurotoxicol Teratol, 36, 47-56. Retrieved from http://www.ncbi.nlm.nih.gov/pubmed/23395807. doi:10.1016/j.ntt.2013.01.004

Rubenstein, J., \& Merzenich, M. M. (2003). Model of autism: increased ratio of excitation/inhibition in key neural systems. Genes, Brain and Behavior, 2(5), 255-267.

Rumsey, J. M., Duara, R., Grady, C., Rapoport, J. L., Margolin, R. A., Rapoport, S. I., \& Cutler, N. R. (1985). Brain metabolism in autism: Resting cerebral glucose utilization rates as measured with positron emission tomography. Archives of General Psychiatry, 42(5), 448-455.

Rynkiewicz, A., \& Łucka, I. (2015). Autism spectrum disorder (ASD) in girls. Co-occurring psychopathology. Sex differences in clinical manifestation. Psychiatr. Pol, 31.

Sabers, A., Bertelsen, F. C. B., Scheel-Kruger, J., Nyengaard, J. R., \& Moller, A. (2014). Longterm valproic acid exposure increases the number of neocortical neurons in the developing rat brain. A possible new animal model of autism. Neuroscience Letters, 580, 12-16. Retrieved from <Go to ISI >://WOS:000342479000003

\section{http://ac.els-cdn.com/S030439401400617X/1-s2.0-S030439401400617X-} main.pdf? tid=f31471lc-6074-1le4-b63d00000aab0f01\&acdnat=1414701794 274479e39f9al16dlb36bel806557c5f.

Saito, Y., Suga, M., Tochigi, M., Abe, O., Yahata, N., Kawakubo, Y., . . Kasai, K. (2013). Neural correlate of autistic-like traits and a common allele in the oxytocin receptor gene. Social cognitive and affective neuroscience, 9(10), 1443-1450.

Saitoh, O., Courchesne, E., Egaas, B., Lincoln, A., \& Schreibman, L. (1995). Cross-sectional area of the posterior hippocampus in autistic patients with cerebellar and corpus callosum abnormalities. Neurology, 45(2), 317-324.

Saitoh, O., Karns, C. M., \& Courchesne, E. (2001). Development of the hippocampal formation from 2 to 42 years: MRI evidence of smaller area dentata in autism. Brain, 124(7), 13171324.

Salchner, P., Lubec, G., \& Singewald, N. (2004). Decreased social interaction in aged rats may not reflect changes in anxiety-related behaviour. Behavioural brain research, 151(1-2), 18.

Sams-Dodd, F. (1995). Distinct effects of d-amphetamine and phencyclidine on the social behaviour of rats. Behavioural pharmacology.

Sandhya, T., Sowjanya, J., \& Veeresh, B. (2012). Bacopa monniera (L.) Wettst ameliorates behavioral alterations and oxidative markers in sodium valproate induced autism in rats. Neurochemical research, 37(5), 1121-1131.

Schain, R. J., \& Freedman, D. X. (1961). Studies on 5-hydroxyindole metabolism in autistic and other mentally retarded children. The Journal of pediatrics, 58(3), 315-320.

Schipper, P., Nonkes, L. J., Karel, P., Kiliaan, A. J., \& Homberg, J. R. (2011). Serotonin transporter genotype $\mathrm{x}$ construction stress interaction in rats. Behavioural brain research, 223(1), 169-175.

Schmeisser, M. J., Ey, E., Wegener, S., Bockmann, J., Stempel, A. V., Kuebler, A., . . Spilker, C. (2012). Autistic-like behaviours and hyperactivity in mice lacking ProSAPl/Shank2. Nature, 486(7402), 256.

Schneider, M., \& Koch, M. (2005). Deficient social and play behavior in juvenile and adult rats after neonatal cortical lesion: effects of chronic pubertal cannabinoid treatment. Neuropsychopharmacology, 30(5), 944.

Schneider, T., \& Przewlocki, R. (2005). Behavioral alterations in rats prenatally exposed to valproic acid: Animal model of autism. Neuropsychopharmacology, 30(1), 80-89. Retrieved from ISI:000225819700010. 
Schneider, T., Roman, A., Basta-Kaim, A., Kubera, M., Budziszewska, B., Schneider, K., \& Przewłocki, R. (2008). Gender-specific behavioral and immunological alterations in an animal model of autism induced by prenatal exposure to valproic acid.

Psychoneuroendocrinology, 33(6), 728-740.

Schneider, T., Turczak, J., \& Przewłocki, R. J. N. (2006). Environmental enrichment reverses behavioral alterations in rats prenatally exposed to valproic acid: issues for a therapeutic approach in autism. 31(1), 36.

Schumann, C. M., \& Amaral, D. G. (2006). Stereological analysis of amygdala neuron number in autism. Journal of Neuroscience, 26(29), 7674-7679.

Schumann, C. M., Barnes, C. C., Lord, C., \& Courchesne, E. (2009). Amygdala enlargement in toddlers with autism related to severity of social and communication impairments. Biological psychiatry, 66(10), 942-949.

Schumann, C. M., Hamstra, J., Goodlin-Jones, B. L., Lotspeich, L. J., Kwon, H., Buonocore, M. H., . . Amaral, D. G. (2004). The amygdala is enlarged in children but not adolescents with autism; the hippocampus is enlarged at all ages. Journal of Neuroscience, 24(28), 6392-6401.

Schwartzer, J., Careaga, M., Onore, C., Rushakoff, J., Berman, R. F., \& Ashwood, P. (2013). Maternal immune activation and strain specific interactions in the development of autism-like behaviors in mice. Translational psychiatry, 3(3), e240.

Schwarz, E., Tost, H., \& Meyer-Lindenberg, A. (2016). Working memory genetics in schizophrenia and related disorders: an RDoC perspective. American Journal of Medical Genetics Part B: Neuropsychiatric Genetics, 171(1), 121-131.

Sebat, J., Lakshmi, B., Malhotra, D., Troge, J., Lese-Martin, C., Walsh, T., . . . Wigler, M. (2007). Strong Association of De Novo Copy Number Mutations With Autism. Science, 316, 445-449. doi:10.1126/science.1138659

Selten, M., van Bokhoven, H., \& Kasri, N. N. (2018). Inhibitory control of the excitatory/inhibitory balance in psychiatric disorders. Fl000Research, 7.

Semerdjian-Rouquier, L., Bossi, L., \& Scatton, B. (1981). Determination of 5hydroxytryptophan, serotonin and 5-hydroxyindoleacetic acid in rat and human brain and biological fluids by reversed-phase high-performance liquid chromatography with electrochemical detection. Journal of Chromatography A, 218, 663-670.

Semmler, A., Frisch, C., Bleul, C., Smith, D., Bigler, L., Prost, J.-C., . . Linnebank, M. (2017). Intrauterine valproate exposure is associated with alterations in hippocampal cell numbers and folate metabolism in a rat model of valproate teratogenicity. Seizure, 46, 7-12.

Servadio, M., Manduca, A., Melancia, F., Leboffe, L., Schiavi, S., Campolongo, P., ... Trezza, V. (2018). Impaired repair of DNA damage is associated with autistic-like traits in rats prenatally exposed to valproic acid. European Neuropsychopharmacology, 28(1), 85-96.

Servadio, M., Vanderschuren, L. J., \& Trezza, V. (2015). Modeling autism-relevant behavioral phenotypes in rats and mice: Do 'autistic'rodents exist? Behavioural pharmacology, 26(6), 522-540.

Sharma, S., Woolfson, L. M., \& Hunter, S. C. (2012). Confusion and inconsistency in diagnosis of Asperger syndrome: a review of studies from 1981 to 2010. Autism, 16(5), 465-486.

Shi, L., Fatemi, S. H., Sidwell, R. W., \& Patterson, P. H. (2003). Maternal influenza infection causes marked behavioral and pharmacological changes in the offspring. Journal of Neuroscience, 23(1), 297-302.

Shi, L., Smith, S. E., Malkova, N., Tse, D., Su, Y., \& Patterson, P. H. (2009). Activation of the maternal immune system alters cerebellar development in the offspring. Brain, behavior, and immunity, 23(1), 116-123. 
Shih, P., Shen, M., Öttl, B., Keehn, B., Gaffrey, M. S., \& Müller, R.-A. (2010). Atypical network connectivity for imitation in autism spectrum disorder. Neuropsychologia, 48(10), 2931-2939.

Shinohe, A., Hashimoto, K., Nakamura, K., Tsujii, M., Iwata, Y., Tsuchiya, K. J., . . Sugihara, G.-i. (2006). Increased serum levels of glutamate in adult patients with autism. Progress in Neuro-Psychopharmacology and Biological Psychiatry, 30(8), 1472-1477.

Shumyatsky, G. P., Tsvetkov, E., Malleret, G., Vronskaya, S., Hatton, M., Hampton, L., . . Bolshakov, V. Y. (2002). Identification of a signaling network in lateral nucleus of amygdala important for inhibiting memory specifically related to learned fear. Cell, $111(6)$, 905-918.

Sigman, M., Mundy, P., Sherman, T., \& Ungerer, J. (1986). Social interactions of autistic, mentally retarded and normal children and their caregivers. Journal of Child Psychology and Psychiatry, 27(5), 647-656.

Silverman, J. L., Turner, S. M., Barkan, C. L., Tolu, S. S., Saxena, R., Hung, A. Y., . . Crawley, J. N. (2011). Sociability and motor functions in Shankl mutant mice. Brain research, 1380, 120-137.

Simonoff, E., Pickles, A., Charman, T., Chandler, S., Loucas, T., \& Baird, G. (2008). Psychiatric disorders in children with autism spectrum disorders: prevalence, comorbidity, and associated factors in a population-derived sample. Journal of the American Academy of Child E Adolescent Psychiatry, 47(8), 921-929.

Slob, A. K., Huizer, T., \& Ten Bosch, J. V. D. W. (1986). Ontogeny of sex differences in openfield ambulation in the rat. Physiology E behavior, 37(2), 313-315.

Smith, S. E., Li, J., Garbett, K., Mirnics, K., \& Patterson, P. H. (2007). Maternal immune activation alters fetal brain development through interleukin-6. Journal of Neuroscience, 27(40), 10695-10702.

Smith, V., \& Brown, N. (2014). Prenatal valproate exposure and risk of autism spectrum disorders and childhood autism. Archives of disease in childhood. Education and practice edition, 99(5), 198.

Smits, B. M. G., Mudde, J. B., van de Belt, J., Verheul, M., Olivier, J., Homberg, J., . . Cuppen, E. (2006). Generation of gene knockouts and mutant models in the laboratory rat by ENU-driven target selected mutagenesis. Pharmacogenetics and Genomics, 16(3), 159169.

Smotherman, W. P., Bell, R. W., Starzec, J., Elias, J., \& Zachman, T. A. (1974). Maternal responses to infant vocalizations and olfactory cues in rats and mice. Behavioral biology, 12(1), 55-66.

Soghomonian, J. J., Zhang, K., Reprakash, S., \& Blatt, G. J. (2017). Decreased parvalbumin mRNA levels in cerebellar Purkinje cells in autism. Autism Research, 10(11), 1787-1796.

Son, G. H., Geum, D., Chung, S., Kim, E. J., Jo, J.-H., Kim, C.-M., . . Kim, H. T. (2006). Maternal stress produces learning deficits associated with impairment of NMDA receptor-mediated synaptic plasticity. Journal of Neuroscience, 26(12), 3309-3318.

South, M., Rodgers, J., \& Van Hecke, A. (2017). Anxiety and ASD: current progress and ongoing challenges. In: Springer.

Sparks, B., Friedman, S., Shaw, D., Aylward, E., Echelard, D., Artru, A., . . Dawson, G. (2002). Brain structural abnormalities in young children with autism spectrum disorder. Neurology, 59(2), 184-192.

Sparks, B., Friedman, S., Shaw, D., Aylward, E. H., Echelard, D., Artru, A., . . . Dawson, G. (2002). Brain structural abnormalities in young children with autism spectrum disorder. Neurology, 59(2), 184-192.

Staal, W. G. (2015). Autism, DRD3 and repetitive and stereotyped behavior, an overview of the current knowledge. European Neuropsychopharmacology, 25(9), 1421-1426. 
Stanton, M. E., Peloso, E., Brown, K. L., \& Rodier, P. (2007). Discrimination learning and reversal of the conditioned eyeblink reflex in a rodent model of autism. Behavioural brain research, 176(1), 133-140.

Steele, P. M., \& Mauk, M. D. (1999). Inhibitory control of LTP and LTD: stability of synapse strength. Journal of neurophysiology, 81(4), 1559-1566.

Stefanik, P., Olexova, L., \& Krskova, L. (2015). Increased sociability and gene expression of oxytocin and its receptor in the brains of rats affected prenatally by valproic acid. Pharmacology Biochemistry and Behavior, 131, 42-50.

Steffenburg, S., Gillberg, C., Hellgren, L., Andersson, L., Gillberg, I. C., Jakobsson, G., \& Bohman, M. (1989). A twin study of autism in Denmark, Finland, Iceland, Norway and Sweden. Journal of Child Psychology and Psychiatry, 30(3), 405-416.

Stepanova, E., Dowling, S., Phelps, M., \& Findling, R. L. (2017). Pharmacotherapy of emotional and behavioral symptoms associated with autism spectrum disorder in children and adolescents. Dialogues in clinical neuroscience, 19(4), 395.

Stromland, K., Nordin, V., Miller, M., Akerstorm, B., \& Gillberg, C. (1994). Autism in Thalidomide Embryopthay: A population study. Developmental Medicine and Child Neurology, 36, 351-356.

Sutcliffe, J. S., Delahanty, R. J., Prasad, H. C., McCauley, J. L., Han, Q., Jiang, L., . . B Blakely, R. D. (2005). Allelic heterogeneity at the serotonin transporter locus (SLC6A4) confers susceptibility to autism and rigid-compulsive behaviors. The American Journal of Human Genetics, 77(2), 265-279.

Szatmari, P., Liu, X. Q., Goldberg, J., Zwaigenbaum, L., Paterson, A. D., Woodbury-Smith, M., . .. Thompson, A. (2012). Sex differences in repetitive stereotyped behaviors in autism: implications for genetic liability. American Journal of Medical Genetics Part B: Neuropsychiatric Genetics, 159(1), 5-12.

Tabuchi, K., Blundell, J., Etherton, M. R., Hammer, R. E., Liu, X., Powell, C. M., \& Südhof, T. C. (2007). A neuroligin-3 mutation implicated in autism increases inhibitory synaptic transmission in mice. Science, 318(5847), 71-76.

Takahashi, L. K., Haglin, C., \& Kalin, N. H. (1992). Prenatal stress potentiates stress-induced behavior and reduces the propensity to play in juvenile rats. Physiology E behavior, 51(2), 319-323.

Tamás, G., Lőrincz, A., Simon, A., \& Szabadics, J. (2003). Identified sources and targets of slow inhibition in the neocortex. Science, 299(5614), 1902-1905.

Taniai, H., Nishiyama, T., Miyachi, T., Imaeda, M., \& Sumi, S. (2008). Genetic influences on the broad spectrum of autism: Study of proband-ascertained twins. American Journal of Medical Genetics Part B: Neuropsychiatric Genetics, 147(6), 844-849.

Taniguchi, H. (2014). Genetic dissection of GABAergic neural circuits in mouse neocortex. Frontiers in cellular neuroscience, $8,8$.

Taylor, S. E., Way, B. M., Welch, W. T., Hilmert, C. J., Lehman, B. J., \& Eisenberger, N. I. (2006). Early family environment, current adversity, the serotonin transporter promoter polymorphism, and depressive symptomatology. Biological psychiatry, 60(7), 671-676.

Thor, D. H., \& Holloway, W. R. (1984). Developmental analyses of social play behavior in juvenile rats. Bulletin of the Psychonomic Society, 22(6), 587-590.

Tõnissaar, M., Philips, M.-A., Eller, M., \& Harro, J. (2004). Sociability trait and serotonin metabolism in the rat social interaction test. Neuroscience Letters, 367(3), 309-312.

Tordjman, S., Gutknecht, L., Carlier, M., Spitz, E., Antoine, C., Slama, F., . . Roubertoux, P. (2001). Role of the serotonin transporter gene in the behavioral expression of autism. Molecular Psychiatry, 6(4), 434. 
Trezza, V., Baarendse, P. J., \& Vanderschuren, L. J. (2010). The pleasures of play: pharmacological insights into social reward mechanisms. Trends in pharmacological sciences, 31(10), 463-469.

Tsujino, N., Nakatani, Y., Seki, Y., Nakasato, A., Nakamura, M., Sugawara, M., \& Arita, H. (2007). Abnormality of circadian rhythm accompanied by an increase in frontal cortex serotonin in animal model of autism. Neuroscience research, 57(2), 289-295.

Tung, E. W., \& Winn, L. (2011). Valproic acid increases formation of reactive oxygen species and induces apoptosis in postimplantation embryos: A role for oxidative stress in valproic acid-induced neural tube defects. Molecular pharmacology, mol. 111.072314.

Valle, F. P., \& Gorzalka, B. (1980). Open-field sex differences prior to puberty in rats. Bulletin of the Psychonomic Society, 16(6), 429-431.

Van den Berg, C. L., Hol, T., Van Ree, J. M., Spruijt, B. M., Everts, H., \& Koolhaas, J. M. (1999). Play is indispensable for an adequate development of coping with social challenges in the rat. Developmental Psychobiology: The Journal of the International Society for Developmental Psychobiology, 34(2), 129-138.

Van der Doelen, R., Deschamps, W., D'annibale, C., Peeters, D., Wevers, R., Zelena, D., .. Kozicz, T. (2014). Early life adversity and serotonin transporter gene variation interact at the level of the adrenal gland to affect the adult hypothalamo-pituitary-adrenal axis. Translational psychiatry, 4(7), e409.

van der Staay, F. J. (2006). Animal models of behavioral dysfunctions: basic concepts and classifications, and an evaluation strategy. Brain research reviews, 52(1), 131-159.

van der Staay, F. J., Arndt, S. S., \& Nordquist, R. E. (2009). Evaluation of animal models of neurobehavioral disorders. Behavioral and Brain Functions, 5(1), 11.

Van Hasselt, F., Tieskens, J., Trezza, V., Krugers, H., Vanderschuren, L., \& Joëls, M. (2012). Within-litter variation in maternal care received by individual pups correlates with adolescent social play behavior in male rats. Physiology \& behavior, 106(5), 701-706.

van Heijst, B. F., \& Geurts, H. M. (2015). Quality of life in autism across the lifespan: A metaanalysis. Autism, 19(2), 158-167.

Van Segbroeck, M., Knoll, A. T., Levitt, P., \& Narayanan, S. (2017). MUPET-mouse ultrasonic profile extraction: a signal processing tool for rapid and unsupervised analysis of ultrasonic vocalizations. Neuron, 94(3), 465-485. e465.

Vanderschuren, L. J., Niesink, R. J., \& Van Pee, J. M. (1997). The neurobiology of social play behavior in rats. Neuroscience $\mathcal{E}$ Biobehavioral Reviews, 21(3), 309-326.

Vanderschuren, L. J., Spruijt, B. M., Hol, T., Niesink, R. J., \& Van Ree, J. M. (1995). Sequential analysis of social play behavior in juvenile rats: effects of morphine. Behavioural brain research, 72(1-2), 89-95.

Vanderschuren, L. J., Trezza, V., Griffioen-Roose, S., Schiepers, O. J., Van Leeuwen, N., De Vries, T. J., \& Schoffelmeer, A. N. (2008). Methylphenidate disrupts social play behavior in adolescent rats. Neuropsychopharmacology, 33(12), 2946.

Vasa, R. A., Mostofsky, S. H., \& Ewen, J. B. (2016). The disrupted connectivity hypothesis of autism spectrum disorders: time for the next phase in research. Biological Psychiatry: Cognitive Neuroscience and Neuroimaging, 1(3), 245-252.

Vasilopoulou, E., \& Nisbet, J. (2016). The quality of life of parents of children with autism spectrum disorder: A systematic review. Research in Autism Spectrum Disorders, 23, 36-49.

Veenstra-VanderWeele, J., Muller, C. L., Iwamoto, H., Sauer, J. E., Owens, W. A., Shah, C. R., . . . Thompson, B. J. (2012). Autism gene variant causes hyperserotonemia, serotonin receptor hypersensitivity, social impairment and repetitive behavior. Proceedings of the National Academy of Sciences, 109(14), 5469-5474.

Viinikainen, K., Eriksson, K., Mönkkönen, A., Äikiä, M., Nieminen, P., Heinonen, S., \& Kälviäinen, R. (2006). The effects of valproate exposure in utero on behavior and the 
need for educational support in school-aged children. Epilepsy E Behavior, 9(4), 636640.

Volkmar, F. R., \& Reichow, B. (2013). Autism in DSM-5: progress and challenges. Molecular autism, 4(1), 13.

Vorhees, C. (1987). Behavioral teratogenicity of valproic acid: selective effects on behavior after prenatal exposure to rats. Psychopharmacology (Berl), 92(2), 173-179. Retrieved from http://www.ncbi.nlm.nih.gov/pubmed/3110838.

Vorhees, C. (1987). Teratogenicity and developmental toxicity of valproic acid in rats. Teratology, 35(2), 195-202. Retrieved from http://www.ncbi.nlm.nih.gov/pubmed/3110992. doi:10.1002/tera.1420350205

Vreugdenhil, M., Jefferys, J. G., Celio, M. R., \& Schwaller, B. (2003). Parvalbumin-deficiency facilitates repetitive IPSCs and gamma oscillations in the hippocampus. Journal of neurophysiology, 89(3), 1414-1422.

Wagner, G. C., Reuhl, K. R., Cheh, M., McRae, P., \& Halladay, A. K. (2006). A new neurobehavioral model of autism in mice: pre-and postnatal exposure to sodium valproate. Journal Of Autism And Developmental Disorders, 36(6), 779-793.

Wahlsten, D., Metten, P., \& Crabbe, J. C. (2003). Survey of 21 inbred mouse strains in two laboratories reveals that BTBR $\mathrm{T} / \mathrm{tff} / \mathrm{tf}$ has severely reduced hippocampal commissure and absent corpus callosum. Brain research, 971(1), 47-54.

Walcott, E. C., Higgins, E. A., \& Desai, N. S. (2011). Synaptic and intrinsic balancing during postnatal development in rat pups exposed to valproic acid in utero. Journal of Neuroscience, 31(37), 13097-13109.

Wang, A. T., Lee, S. S., Sigman, M., \& Dapretto, M. (2006). Neural basis of irony comprehension in children with autism: the role of prosody and context. Brain, 129(4), 932-943.

Wang, C.-C., Lin, H.-C., Chan, Y.-H., Gean, P.-W., Yang, Y. K., \& Chen, P. S. (2013). 5-HT1Areceptor agonist modified amygdala activity and amygdala-associated social behavior in a valproate-induced rat autism model. International Journal of Neuropsychopharmacology, 16(9), 2027-2039.

Wang, X., McCoy, P. A., Rodriguiz, R. M., Pan, Y., Je, H. S., Roberts, A. C., . . Bousquet-Moore, D. (2011). Synaptic dysfunction and abnormal behaviors in mice lacking major isoforms of Shank3. Human molecular genetics, 20(15), 3093-3108.

Watanabe, T., Kuroda, M., Kuwabara, H., Aoki, Y., Iwashiro, N., Tatsunobu, N., . . Kunimatsu, A. (2015). Clinical and neural effects of six-week administration of oxytocin on core symptoms of autism. Brain, 138(11), 3400-3412.

Watson, K. K., Ghodasra, J. H., \& Platt, M. L. (2009). Serotonin transporter genotype modulates social reward and punishment in rhesus macaques. PLoS One, 4(1), e4156.

Watt, N., Wetherby, A. M., Barber, A., \& Morgan, L. (2008). Repetitive and stereotyped behaviors in children with autism spectrum disorders in the second year of life. Journal Of Autism And Developmental Disorders, 38(8), 1518-1533.

Way, B. M., \& Taylor, S. E. (2010). The serotonin transporter promoter polymorphism is associated with cortisol response to psychosocial stress. Biological psychiatry, 67(5), 487-492.

Weinberger, D. R., Glick, I. D., \& Klein, D. F. (2015). Whither Research Domain Criteria (RDoC)?: The good, the bad, and the ugly. JAMA psychiatry, 72(12), 1161-1162.

Wellmann, K. A., Varlinskaya, E. I., \& Mooney, S. M. (2014). D-Cycloserine ameliorates social alterations that result from prenatal exposure to valproic acid. Brain research bulletin, 108, 1-9.

Whitaker-Azmitia, P. M. (2001). Serotonin and brain development: role in human developmental diseases. Brain research bulletin, 56(5), 479-485. 
Whiteford, H. A., Degenhardt, L., Rehm, J., Baxter, A. J., Ferrari, A. J., Erskine, H. E., .. Johns, N. (2013). Global burden of disease attributable to mental and substance use disorders: findings from the Global Burden of Disease Study 2010. The Lancet, 382(9904), 15751586.

Williams, P. G., \& Hersh, J. H. (1997). A male with fetal valproate syndrome and autism. Developmental Medicine \& Child Neurology, 39(9), 632-634.

Willner, P. (1984). The validity of animal models of depression. Psychopharmacology, 83(1), 116.

Wing, L. (1981). Asperger's syndrome: a clinical account. Psychological medicine, 11(1), 115-129. Wingate, M., Kirby, R. S., Pettygrove, S., Cunniff, C., Schulz, E., Ghosh, T., . . Autism Dev Disabilities, M. (2014). Prevalence of Autism Spectrum Disorder Among Children Aged 8 Years - Autism and Developmental Disabilities Monitoring Network, 11 Sites, United States, 2010. MMWR Surveillance Summaries, 63(2). Retrieved from <Go to ISI>://WOS:000334352600001.

Winslow, J. T., \& Insel, T. R. (2002). The social deficits of the oxytocin knockout mouse. Neuropeptides, 36(2-3), 221-229.

Wöhr, M., Orduz, D., Gregory, P., Moreno, H., Khan, U., Vörckel, K., . . Schiffmann, S. N. (2015). Lack of parvalbumin in mice leads to behavioral deficits relevant to all human autism core symptoms and related neural morphofunctional abnormalities. Translational psychiatry, 5(3), e525.

Wöhr, M., Roullet, F. I., Hung, A. Y., Sheng, M., \& Crawley, J. N. (2011). Communication impairments in mice lacking Shankl: reduced levels of ultrasonic vocalizations and scent marking behavior. PLoS One, 6(6), e20631.

Wöhr, M., \& Scattoni, M. L. (2013). Behavioural methods used in rodent models of autism spectrum disorders: current standards and new developments. Behavioural brain research, 251, 5-17.

Wöhr, M., \& Schwarting, R. K. (2008). Maternal care, isolation-induced infant ultrasonic calling, and their relations to adult anxiety-related behavior in the rat. Behavioral Neuroscience, 122(2), 310.

Wolterink, G., Daenen, L. E., Dubbeldam, S., Gerrits, M. A., van Rijn, R., Kruse, C. G., . . Van Ree, J. M. (2001). Early amygdala damage in the rat as a model for neurodevelopmental psychopathological disorders. European Neuropsychopharmacology, 11(1), 51-59.

Won, H., Lee, H.-R., Gee, H. Y., Mah, W., Kim, J.-I., Lee, J., . . Cho, Y. S. (2012). Autistic-like social behaviour in Shank2-mutant mice improved by restoring NMDA receptor function. Nature, 486(7402), 261.

Won, H., Mah, W., \& Kim, E. (2013). Autism spectrum disorder causes, mechanisms, and treatments: focus on neuronal synapses. Frontiers in molecular neuroscience, 6, 19.

Wright, J. M., Gourdon, J. C., \& Clarke, P. B. (2010). Identification of multiple call categories within the rich repertoire of adult rat $50-\mathrm{kHz}$ ultrasonic vocalizations: effects of amphetamine and social context. Psychopharmacology, 211(1), 1-13.

Yamasue, H. (2013). Function and structure in social brain regions can link oxytocin-receptor genes with autistic social behavior. Brain and Development, 35(2), 111-118.

Yamasue, H. (2015). Using endophenotypes to examine molecules related to candidate genes as novel therapeutics: The "endophenotype-associated surrogate endpoint (EASE)" concept. Neuroscience research, 99, 1-7.

Yirmiya, N., Pilowsky, T., Nemanov, L., Arbelle, S., Feinsilver, T., Fried, I., \& Ebstein, R. P. (2001). Evidence for an association with the serotonin transporter promoter region polymorphism and autism. American Journal of Medical Genetics, 105(4), 381-386.

Yoo, J., Bakes, J., Bradley, C., Collingridge, G. L., \& Kaang, B.-K. (2014). Shank mutant mice as an animal model of autism. Philosophical Transactions of the Royal Society of London B: Biological Sciences, 369(1633), 20130143. 
Young, L., Pitkow, L., \& Ferguson, J. (2002). Neuropeptides and social behavior: animal models relevant to autism. Molecular Psychiatry, 7(S2), S38.

Young, R. L., \& Rodi, M. L. (2014). Redefining autism spectrum disorder using DSM-5: The implications of the proposed DSM-5 criteria for autism spectrum disorders. Journal of Autism And Developmental Disorders, 44(4), 758-765.

Yuen, R. K., Merico, D., Cao, H., Pellecchia, G., Alipanahi, B., Thiruvahindrapuram, B., . . . Zhang, T. (2016). Genome-wide characteristics of de novo mutations in autism. NPJ genomic medicine, $1,16027$.

Zaqout, S., \& Kaindl, A. M. (2016). Golgi-cox staining step by step. Frontiers in neuroanatomy, $10,38$.

Zhang, L. (2011). Voluntary oral administration of drugs in mice. Protocol Exchange, 10.

Zhang, L., Lee, N., Nguyen, A., Enriquez, R., Riepler, S., Stehrer, B., . . Baldock, P. (2010). Additive actions of the cannabinoid and neuropeptide $Y$ systems on adiposity and lipid oxidation. Diabetes, Obesity and Metabolism, 12(7), 591-603.

Zhou, J., \& Iacovitti, L. (2000). Mechanisms governing the differentiation of a serotonergic phenotype in culture. Brain research, 877(1), 37-46.

Zielinski, B. A., Prigge, M. B., Nielsen, J. A., Froehlich, A. L., Abildskov, T. J., Anderson, J. S., . . . Lange, N. (2014). Longitudinal changes in cortical thickness in autism and typical development. Brain, 137(6), 1799-1812.

Zikopoulos, B., \& Barbas, H. (2013). Altered neural connectivity in excitatory and inhibitory cortical circuits in autism. Frontiers in human neuroscience, 7, 609.

Zimmerman, R., Patel, R., Smith, A., Pasos, J., \& Kulesza Jr, R. J. (2018). Repeated Prenatal Exposure to Valproic Acid Results in Auditory Brainstem Hypoplasia and Reduced Calcium Binding Protein Immunolabeling. Neuroscience, 377, 53-68. 